\title{
ENTWICKLUNG UND EVALUATION VON KONZEPTEN UND FORMATEN ZUM WISSENSTRANSFER VON DER FORSCHUNG IN DIE SCHULE UND ÖFFENTLICHKEIT - AM BEISPIEL DES SFB 803 -
}

\author{
Dissertation \\ zur Erlangung des mathematisch-naturwissenschaftlichen Doktorgrades \\ „Doctor rerum naturalium“ \\ der Georg-August-Universität Göttingen \\ im Promotionsprogramm Chemie \\ der Georg-August University School of Science (GAUSS)
}

vorgelegt von

ELENA VON HOFF

aus Goslar

Göttingen, 2020 
Die vorliegende Arbeit wurde im Zeitraum von Januar 2016 bis Oktober 2020 in der Abteilung für Fachdidaktik Chemie in der Arbeitsgruppe von Herrn PROF. DR. THOMAS WAITZ am Institut für Anorganische Chemie der Georg-August-Universität Göttingen angefertigt.

\section{Betreuungsausschuss}

1. Betreuer:

PROF. DR. THOMAS WAITZ

Abteilung für Fachdidaktik Chemie

Institut für Anorganische Chemie

Georg-August-Universität Göttingen

2. Betreuer: $\quad$ PROF. Dr. Claudia SteInem

Institut für Organische und Biomolekulare Chemie

Georg-August-Universität Göttingen

\section{Mitglieder der Prüfungskommission}

Referent

Prof. Dr. ThOMAs WAITZ, Abteilung für Fachdidaktik Chemie, Institut für Anorganische Chemie, Georg-August-Universität Göttingen

Korreferentin PROF. DR. Claudia STEINEM, Institut für Organische und Biomolekulare Chemie, Georg-August-Universität Göttingen

\section{Weitere Mitglieder der Prüfungskommission}

PRof. Dr. Eva-MARIA NeHER, XLAB Göttinger Experimentallabor für junge Leute, Georg-August-Universität Göttingen

APL. Prof. Dr. SuSAnNe SchneIder, Didaktik der Physik, Fakultät für Physik, Georg-August-Universität Göttingen

PROF. DR. KERSTIN STRECKER, Didaktik der Informatik, Institut für Informatik, Georg-August-Universität Göttingen

Apl. Prof. Dr. Thomas Zeuch, Institut für Physikalische Chemie, Georg-August-Universität Göttingen

Tag der mündlichen Prüfung: 12.10.2020 


\section{DANKSAGUNG}

Naturwissenschaftliche Öffentlichkeitsarbeit ist besonders fruchtbar, wenn viele Akteure an einem Strang ziehen, um die eigene Leidenschaft für die Naturwissenschaften auch in der Gesellschaft zu entfachen. Entsprechend haben zahlreiche Menschen zum Gelingen dieser Arbeit beigetragen.

Zunächst möchte ich mich bei Prof. Dr. ThOMAs WAITZ für die Möglichkeit zur Promotion und das entgegengebrachte Vertrauen bedanken. Die von konstruktiven Gesprächen geprägten Jahre in seiner Arbeitsgruppe sowie zahlreiche Möglichkeiten zur wissenschaftlichen Erprobung und Entfaltung haben in besonderem Maße zu meiner wissenschaftlichen und persönlichen Entwicklung beigetragen.

Zudem danke ich Prof. Dr. Claudia STEINEM für die Bereitschaft, diese Arbeit als Zweitgutachterin zu betreuen, sowie für die Gelegenheit, die Forschung des SONDERFORSCHUNGSBEREICHS 803 in verschiedene Projekte der Öffentlichkeitsarbeit einzubetten. An dieser Stelle sei auch den Mitgliedern des SFB gedankt, die mir stets behilflich waren und bei denen ich mich jederzeit willkommen fühlte.

Mein besonderer Dank gilt DR. INGO MEY, der sowohl für Forschung als auch für wissenschaftliche Öffentlichkeitsarbeit brennt und diese Begeisterung auch in mir geweckt hat. Seine Tür stand mir jederzeit für fachliche Fragen sowie wertvolle Ratschläge und Ideen zur Ausarbeitung und Umsetzung der verschiedenen Projekte offen und auch sonst hatte er immer ein offenes Ohr für mich.

Bedanken möchte ich mich auch bei Florian, Franzi, Konny, Marc, Marie-Lena, Marvin, Silan und Süra, sowie Add, Conni, Dennis, HilKo, Janina, Johanna, KaI, LaURA, Malte, Nele, PhilipP, SinA, SEBASTIAN, STEFFI und TIMm für jeglichen fachlichen Austausch und Input, vor allem aber für die gemeinsamen Jahre im Büro, der Mensa oder auf Tagungen im In- und Ausland, die ich schon jetzt sehr vermisse.

DR. TABEA OSWALD und KRISTINA KRAMER danke ich für die Unterstützung bei der Präparation und Untersuchung von Zellkulturen und Vesikelfusionen.

Außerdem danke ich Benjamin BÜHRING, DirK TIMmer und AXEl CARL für die gute Zusammenarbeit auf Bildungsmessen und anderen Veranstaltungen. Bedanken möchte ich mich zudem bei GIANNI für die gemeinsamen Mittags- und Kaffeepausen sowie bei ADDI, BASTI, FloRian, Hilko, Karo, LAURA, MARC und NiKOlAS, ohne die die Betreuung der Projekte nur halb so viel Spaß gemacht hätte.

Ein großes Dankeschön auch an DR. JULIA BAIER für das ausgiebige Korrekturlesen trotz eigenen Prüfungsstresses.

JULIA JOHN-SCHEDER danke ich für eine Freundschaft, die aus gemeinsamen Erfahrungen erwachsen ist, tausende Kilometer Entfernung überstanden hat und uns schließlich in dieselbe Stadt führte.

$\mathrm{Zu}$ guter Letzt möchte ich mich bei meiner Familie bedanken. UWE und ANDREA, BETTINA und INGO, JAN, SUSANNE und meine Großeltern ERICH und GETRUD haben mich über die Jahre hinweg, während des Studiums, der Promotion oder im Ausland, immer unterstützt, mich aufgemuntert, motiviert und mir die Möglichkeit gegeben, meine Potentiale zu entfalten. Dafür danke ich euch von ganzem Herzen.

Christoph, du bist mit mir durch alle Höhen und Tiefen dieser Arbeit gegangen und hast mir jederzeit Ruhe und Kraft gespendet. Danke für deine Unterstützung und dein Verständnis, vor allem aber einfach nur dafür, dass du da bist. 


\section{ZUSAMMENFASSUNG}

In der naturwissenschaftlichen Forschung nimmt die Einbindung der Gesellschaft im Rahmen von Projekten wissenschaftlicher Öffentlichkeitsarbeit einen immer größeren Stellenwert ein. Während zahlreiche Angebote existieren, die insbesondere der allgemeinen Interessenförderung bezüglich der Naturwissenschaften dienen, widmen sich bisher nur wenige Projekte der öffentlichkeitswirksamen Aufarbeitung aktueller Forschungsinhalte. Ausgehend von der Grundlagenforschung des Sonderforschungsbereichs 803 zu Biomembranen, wurden in dieser Arbeit verschiedene Projekte entwickelt und evaluiert.

Dazu wurden Aktivitäten wissenschaftlicher Öffentlichkeitsarbeit zunächst hinsichtlich der intendierten Lernprozesse systematisiert, bevor die Forschung des Sonderforschungsbereichs anhand sechs identifizierter Themenfelder didaktisch und experimentell erschlossen wurde. Die Ergebnisse flossen im Anschluss in vier Projekte zur Öffentlichkeitsarbeit ein, die Unterrichtseinheiten, ein Schülerlabor, Adaptierte Primärliteratur sowie Konzepte für Bildungsmessen umfassen. Diese wurden im Anschluss bezüglich Ihrer Eignung, aktuelle Forschung der Öffentlichkeit und insbesondere der Zielgruppe der Schülerinnen und Schüler zu präsentieren, evaluiert.

Obwohl das Thema der Biomembranen zwar zahlreiche Anknüpfungspunkte zu den Kerncurricula der Fächer Biologie und Chemie aufweist, geht die in der Forschung untersuchte Interaktion einzelner Lipide und Proteine auf molekularer Ebene weit über die modellartige Betrachtung des Aufbaus und der Funktion von Membranen in der Schule hinaus. Im Rahmen der Weiterentwicklung der einzelnen Projekte rückten deshalb konkrete Forschungsinhalte vermehrt in den Hintergrund. Stattdessen wurde der Fokus innerhalb der Projekte stärker auf die Darstellung wissenschaftlicher Arbeitsprozesse der Membranforschung gelenkt. Mithilfe verständlicher Beispiele sollten so Forschungsprozesse aufgezeigt werden, um u. a. stereotype Vorstellungen bezüglich des Berufs und der Person der Wissenschaftlerin zu revidieren. 


\begin{abstract}
In scientific research, involvement of society within the context of science outreach projects gains increasing importance. While numerous offers which serve to promote general interest in the natural sciences exist, so far only few projects have been devoted to publicly presenting current research content. Based on the basic research of the Collaborative Research Center 803 on biomembranes, various projects have been developed and evaluated within this work.

To this end, science outreach activities were initially systemized in terms of their intended learning processes before the research of the Collaborative Research Center was didactically and experimentally adapted in accordance to six previously identified topics. Subsequently, the results were implemented in four science outreach projects comprising teaching units, a student laboratory, adapted primary literature, as well as concepts for science fairs. The projects were later evaluated regarding their suitability for presenting current research to the public and in particular to students as the main target group.

While the topic of biomembranes shows several links to the biology and chemistry core curricula, the interaction of individual lipids and proteins investigated in research goes far beyond the model-based consideration of the structure and function of membranes in school. During the continued development of the outreach projects, specific research content increasingly receded into the background. Instead, the focus within the projects was directed more toward the presentation of scientific work processes in membrane research. Thus, comprehensible examples were used illustrating typical research processes in order to, among others, revise stereotypical concepts regarding the profession and personality of scientists.
\end{abstract}




\section{Inhaltsverzeichnis}

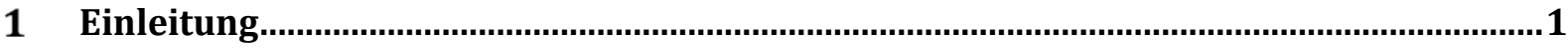

2 Zielsetzung

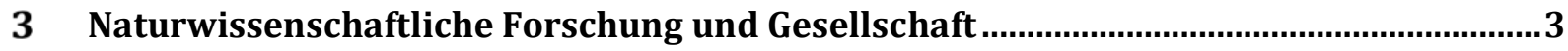

3.1 Naturwissenschaftliches Interesse in Gesellschaft und Schule ...................................5

3.1.1 Naturwissenschaftliches Interesse der Gesellschaft............................................................. 5

3.1.2 Naturwissenschaftliches Interesse von Schülerinnen und Schülern.................................... 7

3.1.3 Naturwissenschaftliches Interesse im internationalen Vergleich ........................................ 9

3.2 Konzepte zur Steigerung des naturwissenschaftlichen Interesses in der Schule. 11

3.2.1 Relevanz aktueller Forschung im naturwissenschaftlichen Unterricht. 13

3.3 Konzepte zur Steigerung des naturwissenschaftlichen Interesses in der

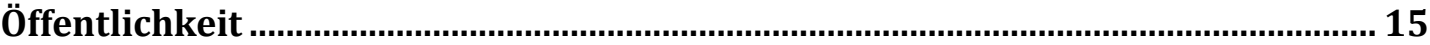

3.3.1 Naturwissenschaftliche Öffentlichkeitsarbeit - Science Outreach.................................... 16

3.3.2 Science Outreach-Akteure.............................................................................................................. 27

3.3.3 Evaluierung von Science Outreach-Aktivitäten........................................................................ 27

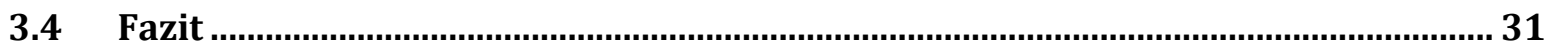

4 Sonderforschungsbereich 803

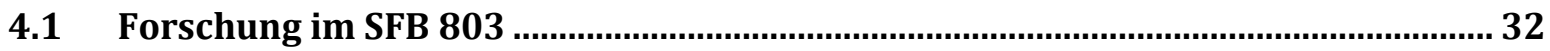

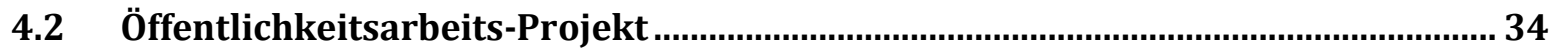

5 Didaktische Erschließung der Forschung des SFB 803

5.1 Themenfeld 1 - Struktur-Eigenschaftsbeziehungen amphiphiler Moleküle ......... 38

5.2 Themenfeld 2 - Selbstorganisation amphiphiler Moleküle .................................... 47

5.3 Themenfeld 3 - Membranen und ihre Bestandteile............................................... 58

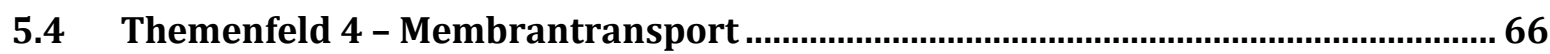

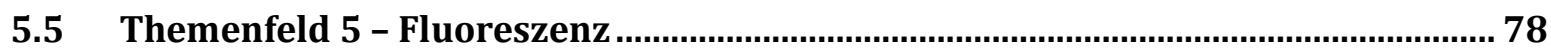

5.6 Themenfeld 6 - Untersuchungsmethoden in der Membranforschung................... 95

6 Entwicklung und Evaluation verschiedener Science Outreach-Formate......................110

6.1 Formal Intentional - Unterrichtseinheiten …..................................................111

6.1.1 Ursprüngliche Unterrichtseinheit „Vom Tensid zur Biomembran“ ................................113

6.1.2 Erweiterter Unterrichtsmaterialienpool „Vom Tensid zur Biomembran“..................117

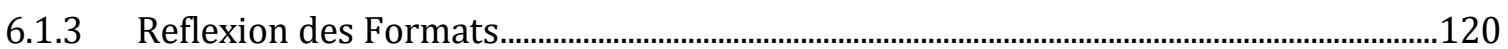

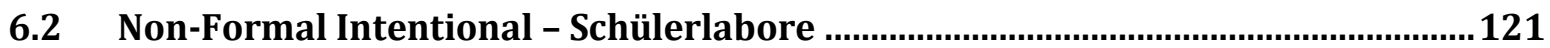

6.2.1 Schülerlabor „Gifte für die Schönheit“ .................................................................................124

6.2.2 Reflexion des Formats............................................................................................................126

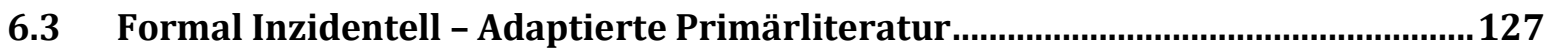

6.3.1 Adaptierte Primärliteratur „Spannungsabhängige Konformationsänderungen des Ionenkanals VDAC-1: Ein Vergleich zwischen Simulation und Experiment“. 
6.3.2 Adaptierte Primärliteratur „Synthese von Glycosphingolipiden mit fluoreszenzmarkierten Fettsäuren für die quantiative Untersuchung der Anbindung von Shiga-Toxin an Lipidmembranen “.....................................................................................140

6.3.3 Reflexion des Formats....................................................................................................145

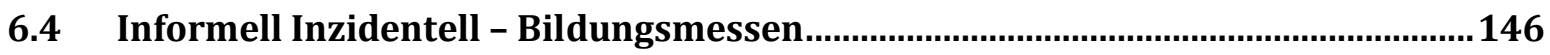

6.4.1 Das Messekonzept des SFB 803 ......................................................................................148

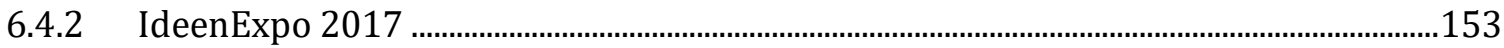

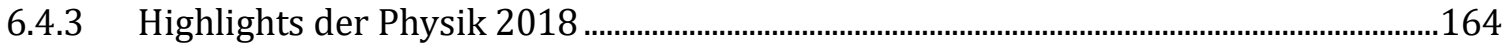

6.4.4 Reflexion des Formats.....................................................................................................172

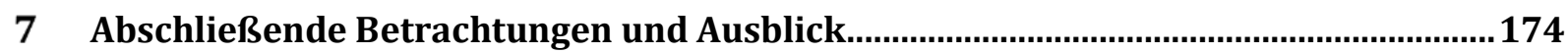

8 Literaturverzeichnis ....................................................... Fehler! Textmarke nicht definiert.

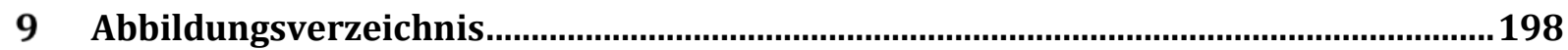

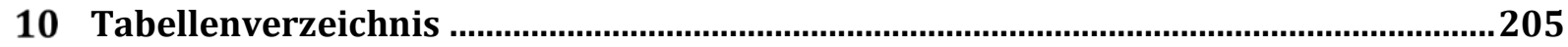




\section{Einleitung}

In einer sich kontinuierlich weiterentwickelnden, technologiegetriebenen und industrialisierten Welt, in der der wissenschaftliche und technische Fortschritt immer schneller voranschreitet, ist es umso wichtiger - und gleichzeitig umso schwieriger - die Gesellschaft mit einem angemessenen naturwissenschaftlichen Allgemeinwissen auszustatten. Insbesondere zahlreiche politische Entscheidungen, wie aktuell bspw. Fragen bezüglich der Eindämmung des Klimawandels sowie der COVID-19 Pandemie, stützen sich jedoch auf wissenschaftliche Informationen.

Aufgrund des stetigen Zuwachses an neuen Erkenntnissen gehen viele Inhalte politischer Entscheidungen schnell über die im naturwissenschaftlichen Unterricht vermittelten thematischen Inhalte hinaus. Entsprechend abhängig ist die Gesellschaft von Informationen, die sie aus Fernsehen, Zeitungen und vermehrt auch dem Internet bezieht, und umso wichtiger ist es, diese Informationen kritisch zu hinterfragen. Der Grundstein für das hierfür benötigte naturwissenschaftliche Allgemeinwissen wird in der Schule gelegt, jedoch sinkt das Interesse an den MINT-Fächern mit zunehmendem Alter und Klassenstufe [1].

Für den naturwissenschaftlich interessierten Laien ist es insgesamt jedoch schwierig, sich über Ergebnisse aktueller Forschung zu informieren. Bereits 2018 existierten über 40.000 medizinisch-naturwissenschaftliche Fachjournale, die einem Peer-Review-Prozess unterliegen. Innerhalb dieser Zeitschriften werden jährlich über drei Millionen Artikel publiziert - mit einer Wachstumsrate von vier Prozent jährlich [2]. Obwohl wissenschaftliche Artikel dabei neuerdings vermehrt auch in Open Access Journalen publiziert werden [3], sind insbesondere Journale mit hohem Impact Factor, wie u. a. NATURE oder SCIENCE, nur kostenpflichtig einsehbar. Die Artikel wenden sich zudem zumeist an spezielle Wissenschaftsgemeinden, so dass ein tiefergreifendes Verständnis des jeweiligen Themengebiets nötig ist, um deren Inhalte zu verstehen.

Alternativen bieten journalistische Artikel, die wissenschaftliche Inhalte präsentieren. Jedoch lassen sich in populärwissenschaftlicher Literatur vornehmlich Erkenntnisse zu Themen angewandter Forschung finden, u.a. aus dem medizinischen Bereich, die ein breites Publikum ansprechen sollen [4]. Obwohl Innovationen der angewandten Forschung oftmals auf Erkenntnissen aus der Grundlagenforschung basieren, nehmen hingegen gerade Inhalte aus dieser im Wissenschaftsjournalismus nur einen geringen Stellenwert ein ${ }^{[5]}$.

Um es jedoch der Gesellschaft zu ermöglichen politische Entscheidungen kritisch abzuwägen, wird u. a von Seiten der Politik vermehrt gefordert, auch über die Schule hinaus das Interesse von Schülerinnen und Schülern (SuS) sowie der Allgemeinheit an den Naturwissenschaften zu fördern und sie für eine Auseinandersetzung mit wissenschaftlichen Themen zu motivieren [6]. Dazu müssen aktuelle Forschungsergebnisse auf eine Art und Weise offengelegt werden, die für Laien sowohl zugänglich als auch verständlich ist.

Hier bieten sich Konzepte zur Öffentlichkeitsarbeit (Science Outreach, SO) an, die, je nach Ausgestaltung und Art, verschiedene Zielgruppen ansprechen können. Diesbezüglich existieren bereits zahlreiche Konzepte zur Förderung des Interesses an naturwissenschaftlich Themen, jedoch beziehen sich diese nur selten auf aktuelle Erkenntnisse aus der Forschung. 


\section{Zielsetzung}

Am Beispiel der Grundlagenforschung zu Biomembranen des Sonderforschungsbereichs 803 (SFB 803) der Georg-August-Universität Göttingen werden in dieser Arbeit vier Projekte eines forschungsbezogenen SO vorgestellt und Evaluationsergebnisse bezüglich der Implementierung der verschiedenen Projekte diskutiert.

Dazu wird zunächst das Verhältnis zwischen Forschung und Gesellschaft beleuchtet und insbesondere das Interesse von SuS an den Naturwissenschaften untersucht (Kapitel 3). Anschließend werden existierende Konzepte in Schule und Öffentlichkeit zur Steigerung des Interesses an den Naturwissenschaften dargelegt. Um ein Ableiten möglicher SO-Projekte im Rahmen der Forschung des SFB 803 zu ermöglichen, wird zudem eine Systematisierung entsprechender Konzepte hinsichtlich des Kontextes, in dem das Lernen stattfindet, vorgenommen.

An eine kurze Vorstellung des SFB 803 (Kapitel 4) schließt sich im Hauptteil der Arbeit zunächst die didaktische Erschließung dessen Forschung an Biomembranen an (Kapitel 5). Dazu werden, ausgehend von curricularen Inhalten der Fächer Chemie und Biologie, in sechs identifizierten Themenfeldern verschiedene Experimente vorgestellt, anhand derer sowohl grundlegende Konzepte des Aufbaus und der Funktion von Biomembranen erarbeitet werden können als auch Einblicke in die Forschung des SFB möglich sind. In Kapitel 6 werden schließlich vier SO-Konzepte entwickelt und evaluiert, die sich sowohl an SuS als auch an die interessierte Allgemeinheit wenden und innerhalb derer zahlreiche der Experimente verankert sind.

Die didaktische Erschließung der Forschung sowie die vorgestellten SO-Projekte werden abschließend nochmals reflektiert und eine mögliche Weiterentwicklung der Konzepte sowie eine Übertragung auf andere Öffentlichkeitsarbeitsprojekte diskutiert. 


\section{Naturwissenschaftliche Forschung und Gesellschaft}

Der Dialog zwischen Forschung und Gesellschaft gewinnt national wie international kontinuierlich an Bedeutung. Betrachtet man die Hightech-Strategie für Deutschland, die seit 2006 zu Beginn jeder Legislaturperiode von der deutschen Bundesregierung veröffentlicht und in der jeweils die strategische Ausrichtung der deutschen Forschungspolitik definiert wird, kann dieser Bedeutungszuwachs beobachtet werden. Während die Gesellschaft in den Papieren von 2006 und $2007^{1}$ noch keine Rolle spielt, lassen sich 2010 und 2014 jeweils zwei kurze Abschnitte zum „Dialog über Innovationen“ bzw. zur „Transparenz und Partizipation" finden [7-10]. In der aktuellen Ausgabe von September 2018 wird sogar in einem eigenständigen Kapitel auf fünf Seiten diskutiert, welche Maßnahmen getroffen werden müssen, um eine „Beteiligung der Gesellschaft“ zu ermöglichen und zu fördern [11].

Dieser Zuwachs ist auf internationaler Ebene unter anderem auch im Forschungsrahmenprogramm (FP) der EUROPÄISCHEN KOMMISSION zu beobachten. So wechselte die Überschrift bezüglich der Gesellschaft von Science and Society (FP 6), über Science in Society (FP 7) zu Science with and for Society (FP 8, Horizon 2020) [6,12,13]. Dabei orientiert sich die Europäische Kommission insbesondere seit Horizon 2020 am Konzept der Responsible Research and Innovation (RRI), dass sie wie folgt beschreibt:

„Responsible Research and Innovation means that societal actors work together during the whole research and innovation process in order to better align both the process and its outcomes, with the values, needs and expectations of European society. RRI is an ambitious challenge for the creation of a research and innovation policy driven by the needs of society and engaging all societal actors via inclusive participatory approaches. "[14]

Das Konzept der RRI beinhaltet die sechs Dimensionen (1) public engagement, (2) gender equality, (3) science education, (4) open access, (5) ethics und (5) governance. Neben der Teilhabe der Bürger an wissenschaftlichen Prozessen, spielen also die Förderung junger und vor allem weiblicher Talente, formale und informelle Bildungsangebote, ein erleichterter Zugang zu Forschungsergebnissen sowie die Integration gesellschaftlicher Interessen und Werte in die Forschung eine verstärkte Rolle [14,15].

Wissenschaftspolitisch setzt man also national wie international vermehrt auf eine informierte, in die Forschung involvierte Gesellschaft, mit dem Ziel innovative Lösungen für gesellschaftliche Herausforderungen gemeinsam zu erarbeiten [15]. Mit einer zunehmenden Forderung nach gesellschaftlicher Einbindung stellt sich automatisch die Frage, inwiefern diese in der Realität bereits erfolgt, bzw. ob auch die Gesellschaft den Eindruck hat, in Forschungsprozesse und wissenschaftspolitische Entscheidungen eingebunden zu sein. Um dieser Frage nachzugehen, wurde von der EUROPÄISCHEN KOMMISSION eine Umfrage in Auftrag gegeben, die sich mit den allgemeinen Ansichten europäischer Bürger zu Wissenschaft und Technologie auseinandersetzt [16].

Die über 27.000 Teilnehmer_innen aus allen EU Mitgliedsstaaten wurden unter anderem dazu befragt, wie gut sie sich über die Entwicklungen in Wissenschaft und Technologie

\footnotetext{
1 Zusätzlich zu den Strategie-Papieren zu Beginn jeder Legislaturperiode wurde im Jahr 2007 die Hightech-Strategie zum Klimaschutz veröffentlicht.
} 
informiert fühlen. Abbildung 1 zeigt, dass sich im europäischen Mittel $^{2}$ lediglich $40 \%$ der Befragten sehr gut bzw. ziemlich gut informiert fühlen. Einzig in fünf Ländern fühlen sich über $50 \%$ der Teilnehmer_innen informiert (Dänemark (65\%), Schweden (61\%), Luxemburg (58\%), Vereinigtes Königreich (56\%) und Frankreich (51\%)). Deutschland liegt mit 43 \% knapp über dem europäischen Durchschnitt.

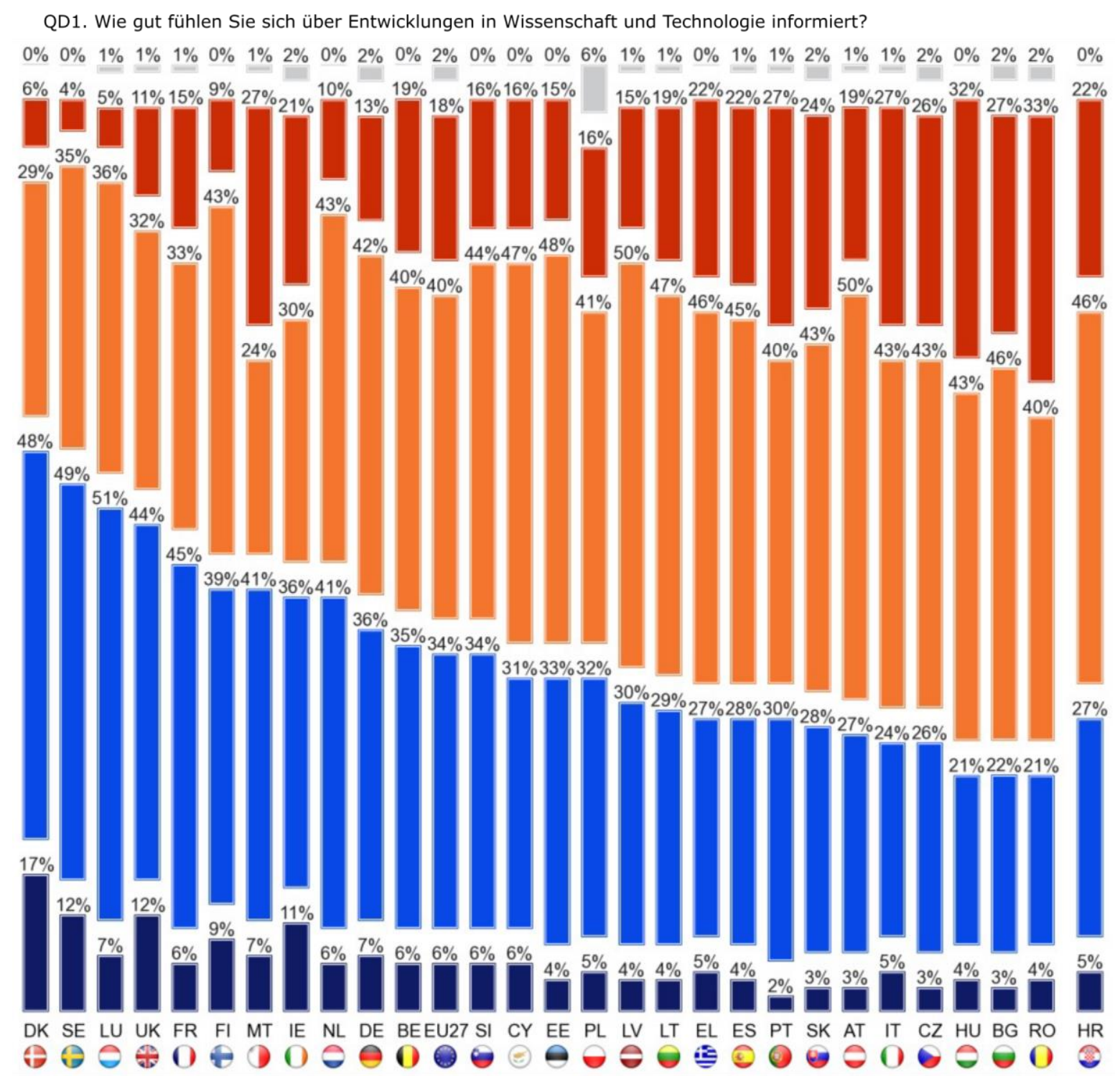

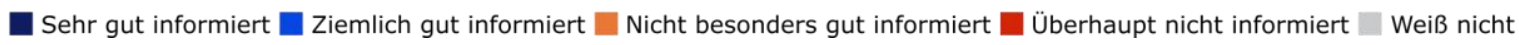

Abbildung 1: Wie gut fühlen sich europäische Bürger über Entwicklungen in Wissenschaft und Technologie informiert (aus dem Englischen übersetzt nach EUROPEAN CoMmISSION ${ }^{[17]}$ ).

Die Teilnehmer_innen wurden zusätzlich auch zu ihrem Interesse an Entwicklungen in Wissenschaft und Technologie befragt. Mit 53 \% liegt hier das europäische Mittel deutlich höher, wobei Deutschland mit 54 \% erneut nur knapp über dem Durchschnitt liegt [16]. Aus den Ergebnissen lässt sich sowohl für die EU als auch für Deutschland im Speziellen schließen, dass sich zahlreiche Menschen zwar gerne über wissenschaftliche Inhalte informieren würden, sich aus unterschiedlichsten Gründen jedoch nicht informiert fühlen.

2 Die Umfrage wurde in allen 28 Mitgliedsstaaten durchgeführt, da die Erhebung jedoch vor dem offiziellen Beitritts Kroatiens stattfand, beziehen die Ergebnisse der EU27 Kroatien nicht mit ein. 
Zudem zeigen knapp die Hälfte der europäischen sowie der deutschen Bevölkerung ein generelles Desinteresse an wissenschaftlichen Innovationen. Die Gründe für diese Diskrepanzen sollen im Folgenden näher erläutert werden.

\subsection{Naturwissenschaftliches Interesse in Gesellschaft und Schule}

\subsubsection{Naturwissenschaftliches Interesse der Gesellschaft}

Die Auseinandersetzung der Gesellschaft mit naturwissenschaftlichen Inhalten bedarf einem gewissen Maß an Allgemeinbildung, deren Grundstein bereits in der Schule gelegt werden muss. Diese wird im deutschsprachigen Raum als naturwissenschaftliche Grundbildung bezeichnet und zum Teil mit Scientific Literacy gleichgesetzt [18]. Der Begriff der Scientific Literacy ist dabei nicht eindeutig definiert; vielmehr existieren zahlreiche Definitionsvorschläge (vgl. u. a. LAUGKSCH ${ }^{[19]}$ und ANELLI ${ }^{[20]}$ ) und synonym genutzte länderspezifische Fachbegriffe, wie Public Understanding of Science in Großbritannien und La Culture Scientifique im französischsprachigen Raum [19, 21]. Eine unter anderem in einigen deutschen Kerncurricula der Länder verwendete Definition der naturwissenschaftlichen Grundbildung ist die des OECD/PISA-KonSORTIUMS:

„Naturwissenschaftliche Grundbildung ist die Fähigkeit, naturwissenschaftliches Wissen anzuwenden, naturwissenschaftliche Fragen $z u$ erkennen und aus Belegen Schlussfolgerungen zu ziehen, um Entscheidungen zu verstehen und zu treffen, die die natürliche Welt und die durch menschliches Handeln an ihr vorgenommen Veränderungen betreffen." [18]

Es soll also nicht Ziel des naturwissenschaftlichen Unterrichts sein, lediglich reines Faktenwissen zu präsentieren. Vielmehr soll ein Verständnis über naturwissenschaftliche Konzepte dazu beitragen, dass jede Person nach Beendigung der schulischen Ausbildung in der Lage ist, wissenschaftliche Fragestellungen kritisch zu reflektieren. Dazu gehören zum einen Fragen, die politische Relevanz besitzen, wie beispielsweise die Abschätzung der Ursachen und Folgen des Klimawandels, der Einsatz von Nanopartikeln in Alltagsmaterialien und Nahrungsmitteln oder das Einhalten europäischer Luftqualitätsstandards durch das Einführen von Dieselfahrverboten. Zudem soll die naturwissenschaftliche Grundbildung jedoch auch dazu beitragen, alltäglichen Fragestellungen zu begegnen, damit beispielsweise Zutatenlisten von Lebensmitteln gedeutet und Beipackzettel von Medikamenten verstanden werden können. Gleichzeitig soll sie es einem jedem Individuum ermöglichen, eigenmächtig Entscheidungen bezüglich des persönlichen Energieverbrauchs, der Müllvermeidung oder des Transports zu treffen [22].

Der Anspruch naturwissenschaftlicher Grundbildung ist hierbei nicht, dass wissenschaftliche Fortschritte in der Tiefe durchdrungen werden, sie soll jedoch dazu befähigen, Erkenntniswege nachzuvollziehen und sich über mögliche Auswirkungen auf das eigene Leben und die Umwelt klarzuwerden. Die Bedeutung einer solchen Grundbildung wird dabei noch einmal ersichtlicher, wenn man sich bewusst macht, dass viele naturwissenschaftliche Inhalte von Erwachsenen erst nach Beendigung ihrer Schullaufbahn erlernt werden können [23]. Als Beispiel sei hier die Nanotechnologie genannt, die - Stand 2016 bisher nur in wenigen Kerncurricula der deutschen Länder Erwähnung findet, jedoch in Forschung, Wirtschaft und Politik eine wichtige Rolle spielt [24]. Eine differenzierte Meinungsbildung bezüglich neuer Erkenntnisse und Technologien ist jedoch nur möglich, wenn Forschungsergebnisse kommuniziert und von der Öffentlichkeit auch wahrgenommen werden. 
Typischerweise werden neue Erkenntnisse aus der Forschung in wissenschaftlichen Journalen publiziert (zumeist peer-reviewed und mit möglichst hohem Impact Factor), ehe sie zudem auf Tagungen präsentiert werden. Sowohl die gewählten Journale als auch die Tagungen wenden sich dabei vor allem an eine spezifische Forschungsgemeinde mit ähnlichem wissenschaftlichen Schwerpunkt, so dass die Erkenntnisse vornehmlich innerhalb der Gemeinschaft wahrgenommen werden [25].

Die europäische und amerikanische Öffentlichkeit hingegen bezieht ihre Informationen bezüglich Wissenschaft und Technologie vor allem aus Fernsehen, Zeitungen und Internet. Weitere, seltener verwendete Quellen sind Zeitschriften, Radioprogramme, Bücher und Soziale Medien oder Blogs [16, 26]. Dabei deuten die vom US-amerikanischen NATIONAL SCIENCE BOARD veröffentlichten Zahlen von 2016 an, dass Online-Medien eine vermehrte Rolle spielen und beispielsweise auch Zeitungen immer öfter online abgerufen werden [26].

Um die allgemeine Öffentlichkeit zu erreichen, müssen wissenschaftliche Ergebnisse also zunächst journalistisch aufgearbeitet werden. Welche Erkenntnisse und Themen dabei ihren Weg in die journalistische Berichterstattung finden, wird zumeist von (kontroversen) Ereignissen bestimmt. CORBETT \& DURFEE ${ }^{[27]}$ fassen diesbezüglich verschiedene Studien zusammen die unter anderem zeigen, dass „menschliche Tragödien und andere Komponenten einer guten Geschichte“" eine Rolle bei der Auswahl naturwissenschaftlicher Themen spielen. Das führt jedoch unter anderem dazu, dass über kleinere und weniger spektakuläre Erkenntnisse wenig bis gar nicht berichtet wird. Im Zeitalter des Internets, in dem Nachrichten und Informationen rund um die Uhr abgerufen werden können, erhöht sich zudem der Druck auf Journalisten und Medienkanäle, möglichst vor der Konkurrenz wichtige Nachrichten zu verbreiten, so dass gründliche Recherche oftmals auf der Strecke bleibt [28].

Nicht zuletzt seitdem Begriffe wie „Fake News“ oder „Lügenpresse“ immer häufiger Verwendung finden, stellt sich außerdem die Frage, wie viel Vertrauen den Naturwissenschaften selbst, aber auch der naturwissenschaftlichen Berichterstattung, entgegengebracht wird. Im Zuge einer auf Publikumserfolg abzielenden Dramatisierung werden oftmals „Expertenmeinungen“ einander gegenübergestellt, ohne den Grad der wissenschaftlichen Akzeptanz beider Meinungen zu nennen, wodurch beiden eine ähnliche Gewichtung zugesprochen wird ${ }^{[29]}$. Diese augenscheinliche Unsicherheit innerhalb der Wissenschaftscommunity führt dazu, dass auch der Laie eine gewisse Verunsicherung verspürt [30].

Beispielhaft seien hier der Klimawandel und seine Folgen zu nennen: Obwohl der Klimawandel seit den späten 1980 er Jahren in der Öffentlichkeit diskutiert wird [31], stehen Wissenschaftler_innen, die den Klimawandel als fortschreitend und anthropogen ansehen, Klimawandelskeptikern_innen und -leugner_innen gegenüber, deren Argumente u.a. auch von einigen nationalen wie internationalen Politiker_innen aufgegriffen und verbreitet werden [32]. Aber auch Umweltkatastrophen (bspw. Tschernobyl [33]) oder Lebensmittelskandale (z. B. die Fipronil- und Dioxinbelastung von Eiern ${ }^{[34]}$ ) werfen kein gutes Bild auf die einzelnen naturwissenschaftlichen Disziplinen und lassen Zweifel an ihren Vertreter_innen zu [35].

Ob Fernsehen, Zeitung oder Internet, die wissenschaftlichen Themen, denen die allgemeine Öffentlichkeit tagtäglich begegnet, sind häufig medial aufgebläht. Zudem werden die Inhalte oftmals von verschiedenen Interessenvertretern aus Wirtschaft und Politik bestimmt [27]. Dadurch werden kleinere Errungenschaften zum Teil nicht wahrgenommen. Zwar nutzen Wissenschaftler_innen vermehrt auch die direkte Kommunikation mit 
der Gesellschaft, bspw. durch das Führen eines Blogs [28, 36], jedoch bleibt es für den Laien weiterhin schwierig, sich zu informieren, da die Vertrauenswürdigkeit eines Blogs und seiner Verfasser_in nicht an der Zahl der Klicks und Likes abgelesen werden kann [28]. Entsprechend verwundert es nicht, dass sich viele Europäer_innen nicht ausreichend informiert fühlen, oder wissenschaftlichen Themen sogar mit Desinteresse begegnen.

\subsubsection{Naturwissenschaftliches Interesse von Schülerinnen und Schülern}

Das Interesse von SuS an den verschiedenen naturwissenschaftlichen Disziplinen, insbesondere Biologie, Chemie und Physik, ist bereits seit über 40 Jahren ein wichtiger Forschungsgegenstand, der auch in der heutigen Zeit nicht an Aktualität verliert. Dabei stellen die meisten Forscher_innen immer wieder ernüchternd fest, dass das Interesse insbesondere an den Fächern Chemie und Physik sehr gering ist, wobei Biologie hier zumeist eine Sonderstellung einnimmt ${ }^{[1]}$.

Das erklärte Ziel der KULTUSMINISTERKONFERENZ (KMK), SuS eine naturwissenschaftliche Grundbildung an die Hand zu geben, die ihnen eine „aktive Teilhabe an gesellschaftlicher Kommunikation und Meinungsbildung über technische Entwicklungen und naturwissenschaftliche Forschung" [37] ermöglichen soll, kann jedoch nur erreicht werden, wenn diese ein allgemeines Interesse am Erwerb naturwissenschaftlicher Kompetenzen zeigen und die entsprechenden Schulfächer nicht frühzeitig abwählen. Zudem ist insbesondere mit Blick auf die globale Wettbewerbsfähigkeit Deutschlands als Wissens- und Wirtschaftsstandort das Interesse verschiedener Stakeholder aus Politik und Wirtschaft groß, möglichst viele Jugendliche für eine Ausbildung oder ein Studium im MINT-Bereich (Mathematik, Informatik, Naturwissenschaft und Technik) zu begeistern [11].

In der Literatur existieren zahlreiche Definitionen des Begriffs Interesse (vgl. u.a. SwARAT \& ORTONY [38]), der nicht in allen Publikationen zu diesem Thema trennscharf von anderen Konstrukten wie Motivation oder Einstellung abgegrenzt wird [39]. In der aktuellen Diskussion wird Interesse als ein Konstrukt verstanden, das aus der Interaktion einer Person mit seiner Umgebung bzw. einem Gegenstand aus dieser entsteht (Person-GegenstandsTheorie) [40, 41]. Dabei kann es sich um ein konkretes Objekt, ein Thema, einen Lerninhalt oder eine abstrakte Idee handeln [41].

Es wird zudem zwischen zwei Formen - dem situationalen und dem individuellen Interesse - unterschieden (vgl. Abbildung 2) [38, 40-44]. Situationales Interesse beschreibt einen temporären Zustand, bei dem die Person mit einem (Lern-) Gegenstand in Kontakt kommt und ihm seine Aufmerksamkeit widmet [38,43]. Dieser Gegenstand kann für die Person im Moment der Aktivität unterschiedlich interessant wirken und zu „fokussierter Aufmerksamkeit, erhöhter kognitiver Funktionsfähigkeit, Ausdauer und affektiver Beteiligung “ [41] führen. Dieses situationale Interesse kann sowohl vorübergehend sein als auch, wenn es über eine gewisse Zeit hinweg aufrechterhalten wird, in individuelles Interesse übergehen. Letzteres wird als dispositionale Eigenschaft angesehen, sich mit bestimmen Gegenständen bzw. Inhalten immer wieder freiwillig und ausdauernd zu beschäftigen [38, 43].

Viele Interessenstudien im naturwissenschaftlichen Bereich zeigen, dass das Interesse an naturwissenschaftlichen Fächern im Vergleich zu anderen Schulfächern verhältnismäßig gering ist $[1,45,46]$ und dass es mit steigender Klassenstufe kontinuierlich sinkt [1,47-49], Dieser Verlust nimmt besonders im Verlauf der Sekundarstufe I zu [1], konnte jedoch auch bereits in der Grundschule beobachtet werden [50, 51]. Dabei sind die Fächer Chemie und Physik deutlich weniger beliebt als das Fach Biologie [49, 52-54]. Jungen zeigen insgesamt ein größeres Interesse an den Naturwissenschaften, wobei die Verteilung bezüglich der Geschlechter noch einmal hinsichtlich der verschiedenen Fächer differenziert werden 
kann. Während das Fach Chemie keine signifikanten Geschlechterunterschiede aufweist, ist Physik für Mädchen häufig weniger von Interesse als für Jungen. Dahingegen zeigen Mädchen ein ausgeprägtes Interesse am Fach Biologie, das bei Jungen dennoch einen guten Stand hat [52, 55, 56]. Diese Unterschiede stimmen auch mit Befunden zum Selbstkonzept von Mädchen und Jungen überein, welches neben dem Interesse als Prädiktor für Lernerfolg gilt und das im Vergleich zur Biologie in den Fächern Chemie und Physik deutlich geringer ausfällt [53,57].

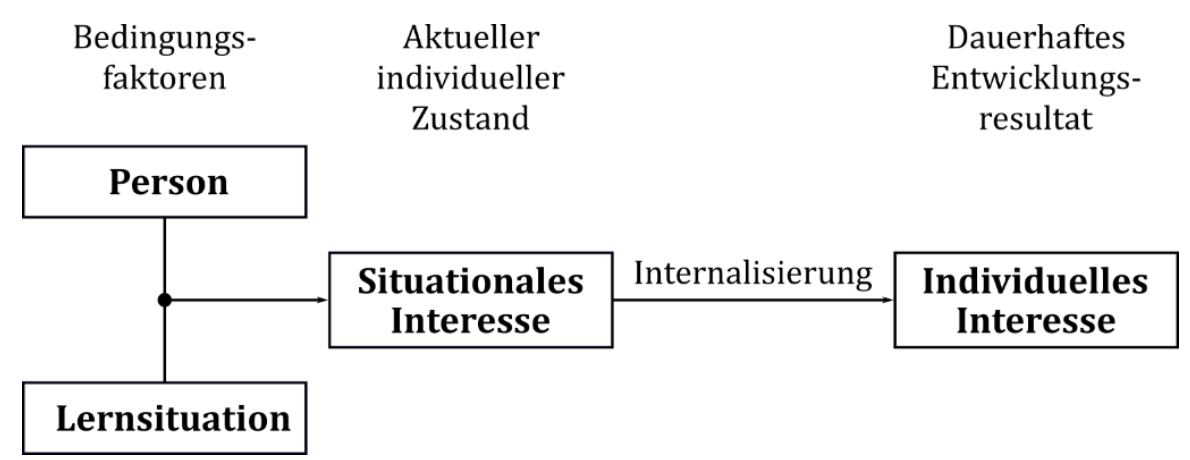

Abbildung 2: Rahmenmodell der Interessengenese nach KRAPP [58].

Obwohl der Interessenverlust in den naturwissenschaftlichen Fächern insgesamt als anerkannt gilt, darf er nicht verallgemeinert werden. Zum einen sinkt mit steigendem Alter das generelle Interesse der SuS an Schule, da sich mit zunehmender Identitätsentwicklung in der Adoleszenz auch individuelle Interessen ausschärfen, die oftmals außerhalb der Schule liegen [59, 60]. Zum anderen zeigen Studien, dass sich der Interessenverlust nicht generell auf das Sachinteresse der SuS, sondern vielmehr auf die Naturwissenschaften als Schulfächer bezieht. Dabei wird insbesondere bemängelt, dass die Relevanz des Schulstoffs für den eigenen Alltag sowie die Gesellschaft nicht deutlich wird [1, 61]. Zudem kommt hinzu, dass insbesondere Chemie und Physik als besonders schwere Fächer bewertet werden, was u.a. auf die zunehmende Mathematisierung der Inhalte (mathematische und chemische Formeln), das hohe Abstraktionsniveau (z.B. Wechsel zwischen makroskopischer, submikroskopischer und symbolischer Ebene ${ }^{[62]}$ ) und eine exakte Fachsprache zurückzuführen ist [49, 52, 54].

In zahlreichen Studien ist man deshalb dazu übergegangen, zusätzlich zum Fachinteresse auch das Sachinteresse zu untersuchen. Es zeigt sich, dass einzelne Themengebiete aus den verschiedenen Naturwissenschaften deutlich positiver bewertet werden, als die jeweiligen Schulfächer. Insgesamt schätzt eine deutliche Mehrheit der SuS die Naturwissenschaften als relevant für ihr eigenes Leben, die Gesellschaft sowie den allgemeinen technologischen Fortschritt ein ${ }^{[1,49,63]}$ und es sind gerade Inhalte aus diesen Bereichen, die für sie von großem Interesse sind. Hierzu gehören $u$. a. verschiedene Themen rund um den menschlichen Körper (z.B. Krankheiten, Wirkung von Medikamenten), Naturphänomene sowie die Ursachen und Folgen des Klimawandels [52, 55, 64].

Eine Übersichtsarbeit von CHRISTIDOU [61] zum Interesse und zur Haltung von SuS gegenüber den Naturwissenschaften zeigt jedoch, dass Inhalte von SuS oftmals als abstrakt und dekontextualisiert wahrgenommen und nur selten Bezüge zu Themen von individuellem Interesse hergestellt werden. Zwar wird versucht, mithilfe von Experimenten und alltagsnahen Themen situationales Interesse zu erzeugen, jedoch sinkt das entfachte Interesse oftmals spätestens während der Auswertung der Experimente [65]. 
Es scheint einleuchtend, dass ein fehlendes Interesse an den naturwissenschaftlichen Fächern einen Einfluss auf die Fächerwahl in der Oberstufe [66] und somit auch auf das Erlangen einer ausreichenden naturwissenschaftlichen Grundbildung hat. Zudem wirkt sich fehlendes Interesse auch negativ auf die Berufswahl im MINT-Bereich aus [38]. Das geringe Interesse vieler Jugendlicher, einen MINT-Beruf zu wählen, wird zusätzlich dadurch verschärft, dass naturwissenschaftliche Berufe sowie die in ihnen arbeitenden Wissenschaftler_innen mit unzähligen stereotypen Vorstellungen verknüpft sind.

Diesbezüglich ließen MEAD \& MÉTRAUX [67] bereits 1957 ca. 35.000 SuS aus US High Schools Aufsätze schreiben, in denen sie beschreiben sollten, was sie über die Naturwissenschaften sowie über Wissenschaftler_innen denken. Es zeigte sich, dass das vorherrschende Bild von Wissenschaftler_innen dem eines eher älteren Mannes mit Brille und weißem Kittel entspricht, der in einem Labor an Experimenten arbeitet. Um zu überprüfen, ab welchem Alter sich dieses stereotype Bild in Kindern manifestiert, entwickelte CHAMBERS [68] 1983 den Draw-a-Scientist-Test, kurz DAST, und ließ 4807 kanadische, US-amerikanische und australische Kinder der Vorschule bis Klasse 5 Bilder von Wissenschaftler_innen malen. Während die Bilder der SuS aus Vorschule und erster Klasse noch gar keine, bis wenige stereotype Merkmale aufwiesen, nahm ab Klasse 2 die Anzahl der stereotypen Merkmale stetig zu. Auch hier fanden sich zahlreiche Laborkittel, Brillen, Bärte und Laborgeräte in den Zeichnungen wieder. Besonders bezeichnend war zudem, dass lediglich $28 \mathrm{der}$ 4807 Bilder Wissenschaftlerinnen darstellten und dass diese Bilder allesamt von Mädchen gezeichnet wurden (die 49\% der Stichprobe ausmachten).

Insbesondere der DAST wurde seither in zahlreichen Ländern und mit verschieden Altersstufen wiederholt. Zwar zeigen sich zum Teil kleinere sozioökonomische und länderspezifische Unterschiede, jedoch hat sich das beschriebene stereotype Bild von Wissenschaftler_innen seit MEAD \& MÉTRAUX über die Jahre gehalten [69, 70]. Dieses, vornehmlich als Männerdomäne wahrgenommene, Bild der Wissenschaft beeinflusst vor allem junge Frauen in ihrem Selbstbild und ihrer Haltung gegenüber den Naturwissenschaften und entsprechend auch in ihrer Berufswahl [61, 71]. Dabei kommen Kinder und Jugendliche während ihrer Schulzeit typischerweise überhaupt nicht mit Wissenschaftler_innen in Kontakt. Sie wachsen jedoch mit Bildern von Wissenschaftler_innen aus Film, Fernsehen, Büchern, Comics und Videospielen auf, die diese Stereotype oftmals fördern und Wissenschaftler_innen zudem als außerordentlich schlau, dafür aber als sozial inkompetent, exzentrisch und distanziert darstellen [61, 71] - mit Charaktereigenschaften also, die eher abschreckend wirken.

\subsubsection{Naturwissenschaftliches Interesse im internationalen Vergleich}

Eine Vielzahl der im vorherigen Kapitel zitierten Studien zum Interesse von Kindern und Jugendlichen an den Naturwissenschaften stammen aus den USA, Großbritannien, Kanada, Australien, Deutschland oder Frankreich und stimmen in ihren Aussagen und Erkenntnissen weitgehend überein. Alle der genannten Länder sind Mitgliedsstaaten der ORGANISATION FOR ECONOMIC CO-OPERATION AND DEVELOPMENT (OECD), besitzen ein vergleichsweise hohes Bruttoinlandsprodukt (BIP) und verschreiben sich u.a. gemeinsamen Bildungszielen. Ein Blick auf die eingangs erwähnte Studie zum Interesse von EU-Bürgern an Wissenschaft und Technologie ${ }^{[16]}$ zeigt jedoch, dass das Interesse in Staaten mit eher hohem BIP über dem EU-Durchschnitt liegt und dass vor allem Staaten mit niedrigerem BIP das untere Ende der Skala dominieren. Wenn jedoch die zitierten Interessenstudien - die ein sinkendes Interesse an den Naturwissenschaften im Laufe der Schulzeit bescheinigen - Ländern mit einem vermeintlich hohen Interesse entstammen, stellt sich die 
Frage, wie es um das Interesse der SuS in wirtschaftlich weniger entwickelten Staaten steht.

Erste Aufschlüsse diesbezüglich liefert das internationale Vergleichsstudienprojekt Relevance of Science Education (ROSE), das sich mit Meinungen, Einstellungen, Interessen und Hoffnungen bezüglich Naturwissenschaften und Technik von SuS aus gut 40 Ländern im Alter von 15 Jahren, also vor Ende der Bildungspflicht der meisten Länder, auseinandersetzt [45]. Der ROSE-Fragebogen enthält rund 250 geschlossene Items mit vierstufiger Likert-Skala sowie eine offene Frage, die in Kategorien wie „Einstellungen gegenüber dem naturwissenschaftlichen Unterricht“ und „Meinungen zu Naturwissenschaft und Technik“ unterteilt sind [72].

Betrachtet man das Interesse der Teilnehmer_innen am naturwissenschaftlichen Unterricht, ist zunächst positiv zu vermerken, dass der Durchschnittswert fast aller Länder für die Aussage „Naturwissenschaftlicher Unterricht ist interessant"3 (School science is interesting) sowohl bei Mädchen als auch bei Jungen über dem Mittelwert liegt [73]. Jedoch ist auffällig, dass die Ergebnisse für die meisten der wirtschaftlich eher entwickelten Länder", wie England, Japan oder Finnland, nur knapp im positiven Bereich liegen, während die weniger entwickelten Länder, wie Uganda, die Philippinen oder Indien, deutlich positivere Werte aufweisen [73]. Dieser Trend zeigt sich noch deutlicher in Bezug auf die Aussage „Ich mag naturwissenschaftlichen Unterricht mehr als die meisten anderen Fächer“ (I like school science better than most other subjects) ${ }^{[45] . ~ H i e r ~ l i e g e n ~ d i e ~ W e r t e ~ d e r ~ m e i s t e n ~}$ entwickelten Länder wie in Abbildung 3 dargestellt unterhalb des Mittelwertes, was mit den Ergebnissen der Studien der oben genannten OECD-Länder übereinstimmt. Immerhin scheinen deutsche Jugendliche jedoch mit einem Wert um den Mittelwert die Naturwissenschaften im Vergleich zu anderen entwickelten Ländern deutlich öfter anderen Fächern vorzuziehen. Die Werte der weniger entwickelten Länder hingegen liegen vor allem auf der positiven Seite der Likert-Skala, der naturwissenschaftliche Unterricht scheint für die Teilnehmer_innen also von größerem Interesse zu sein als andere Fächer.

Eine weitere Erkenntnis aus dem vorherigen Kapitel ist zudem, dass SuS die Naturwissenschaften generell als wichtig für die Gesellschaft erachten, insgesamt jedoch wenige Ambitionen haben selbst in einem MINT-Beruf zu arbeiten. Diesbezüglich zeigen Ergebnisse für das Item "Naturwissenschaften und Technik sind wichtig für die Gesellschaft" (Science and technology are important for society), dass diese Aussage von SuS aller untersuchten Ländern deutlich positiv bewertet wird [45]. Zwar zeichnen sich auch hier kleinere Unterschiede zwischen entwickelten und weniger entwickelten Ländern ab, diese sind aber im Gegensatz zu den Ergebnissen der Aussage „Ich möchte Wissenschaftler_in werden" (I would like to become a scientist) nahezu vernachlässigbar klein [45]. Die Durchschnittswerte der Antworten für alle höher entwickelten Länder liegen hier deutlich auf der negativ konnotierten Seite der Likert-Skala. Zudem zeigen Mädchen eine noch deutlichere Abneigung gegenüber MINT-Berufen als Jungen. In den weniger entwickelten Ländern hingegen ist das Interesse am Beruf der Wissenschaftler_in groß, wobei auch hier ähnliche Unterschiede zwischen Jungen und Mädchen zu vermerken sind.

\footnotetext{
${ }^{3}$ Bezüglich dieser Frage sind in der Literatur lediglich Daten von 25 Ländern zu finden. Für Deutschland sind in diesem Fall keine Werte angegeben.

4 Der Entwicklungsstand eines Landes wurde bei ROSE anhand des Human Development Index (HDI) der VEREINTEN NATIONEN festgelegt, der sowohl das BIP pro Kopf, sowie Lebenserwartung und Dauer der Ausbildung berücksichtigt [74].
} 
Die eingangs erwähnten Befunde zum Interesse von SuS an den Naturwissenschaften können also nicht ohne weiteres auf alle Länder übertragen werden. Die Gründe für die aufgezeigten Unterschiede zwischen eher entwickelten und weniger entwickelten Ländern sind sicherlich vielfältig, jedoch noch nicht ausreichend empirisch belegt. Eine mögliche Erklärung ist, dass Bildung, vor allem im Alter von 15 Jahren, in vielen der weniger entwickelten Länder ein Luxusgut ist, das sich nicht jede Familie leisten kann. Eine Studie von SJøBERG [75] mit 13-jährigen Kindern aus 21 Ländern deutet darauf hin, dass Wissenschaftler_innen in ärmeren Ländern zudem als heldenhafte Vorbilder gelten, die daran arbeiten, die Lebensstandards in diesen Ländern zu verbessern. Gleichzeitig zeigt ein DAST auch in dieser Studie, dass Kinder aus entwickelten Ländern eher eine größere Anzahl an stereotypen Merkmalen zeichnen, die z. T. negativ konnotiert sind.

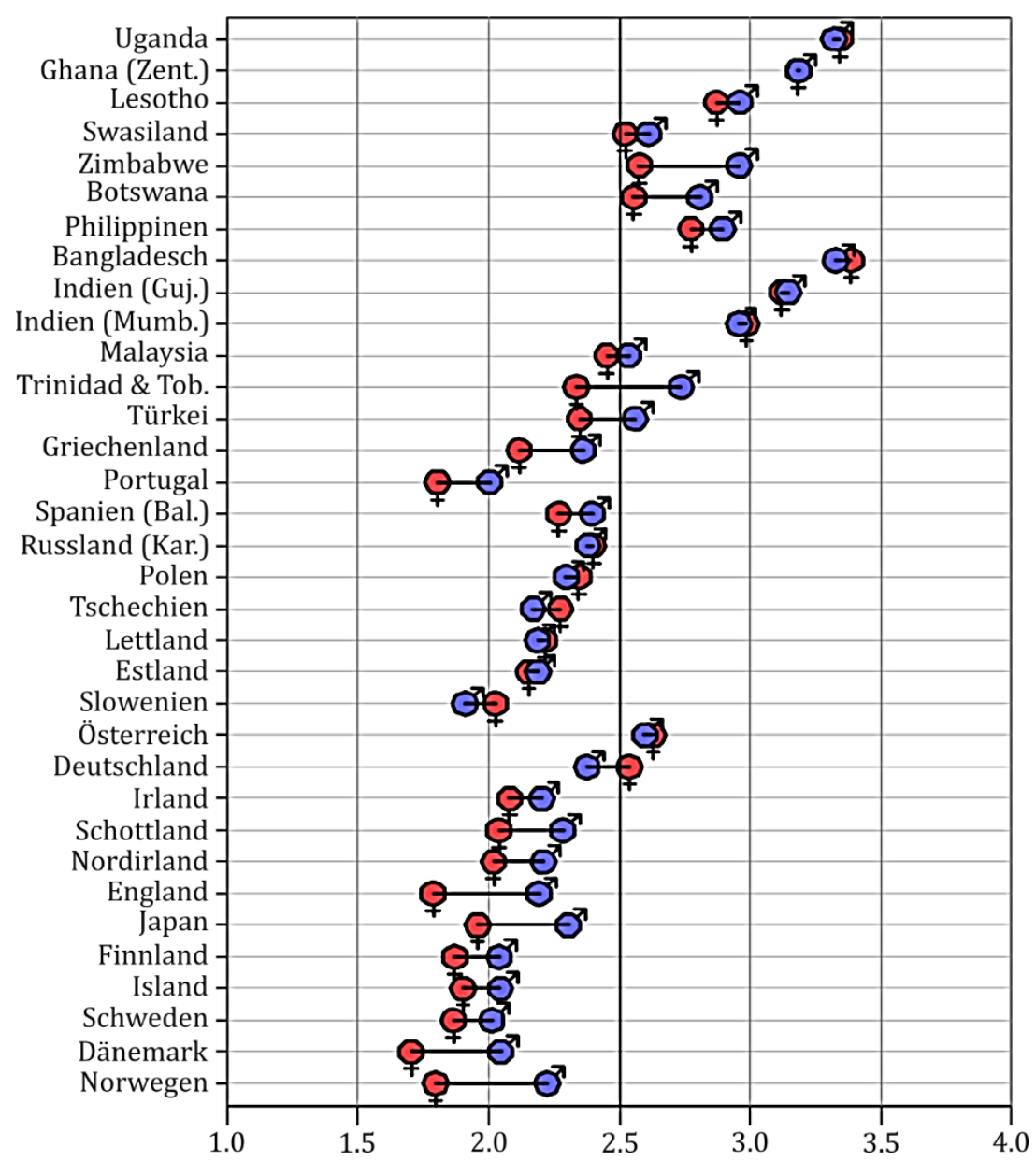

Abbildung 3: Länderspezifische Verteilung der Antworten von Jungen und Mädchen bezügliche der Frage „Ich mag naturwissenschaftlichen Unterricht mehr als die meisten anderen Fächer“ (aus dem Englischen übersetzt nach SJøBERG \& SCHREINER [76]).

\subsection{Konzepte zur Steigerung des naturwissenschaftlichen Interesses in der Schule}

Die Erkenntnisse zum Interesse von Kindern und Jugendlichen an den Naturwissenschaften und insbesondere an den naturwissenschaftlichen Schulfächern hat national wie international zum Hinterfragen traditioneller naturwissenschaftlicher Unterrichtskonzepte geführt. Dabei wird unter anderem bemängelt, dass naturwissenschaftliche Inhalte oftmals als isolierte Fakten dargestellt werden, so dass es SuS schwer fällt, verschiedene Konzepte mental zu verknüpfen und diese in anderen Situationen anzuwenden [77]. Zudem verdeutlicht die systematische Anordnung der Inhalte zumeist nicht die Relevanz des 
Erlernten für den Alltag der SuS. In den Fokus der Forschung rückten deshalb verschiedene Lehr-/Lernformate, innerhalb derer die Erarbeitung naturwissenschaftlicher Inhalte anhand lebensweltlicher und gesellschaftsrelevanter Kontexte erfolgt.

Das allgemeine Bestreben ist es dabei zum einen, das Relevanzempfinden und somit auch das Interesse und die Motivation von SuS durch die Einbettung der Inhalte in für sie relevante Kontexte zu steigern und zum anderen die Scientific Literacy der SuS zu fördern. Wie STUCKEY ET AL. feststellen, werden dem Begriff der Relevanz in der naturwissenschaftsdidaktischen Literatur jedoch verschiedene Bedeutungen zugeschrieben, weshalb sie drei Dimensionen des Relevanzbegriffes formulieren: (i) die persönliche Dimension, (ii) die gesellschaftliche Dimension und (iii) die berufliche Dimension [78]. Innerhalb dieser Dimensionen kann weiterhin zwischen intrinsischer und extrinsischer Zuschreibung der Relevanz, durch den Lernenden selbst oder durch das persönliche Umfeld bzw. die Gesellschaft, sowie zwischen gegenwärtiger und zukünftiger Relevanz unterschieden werden (siehe Abbildung 4) [79].
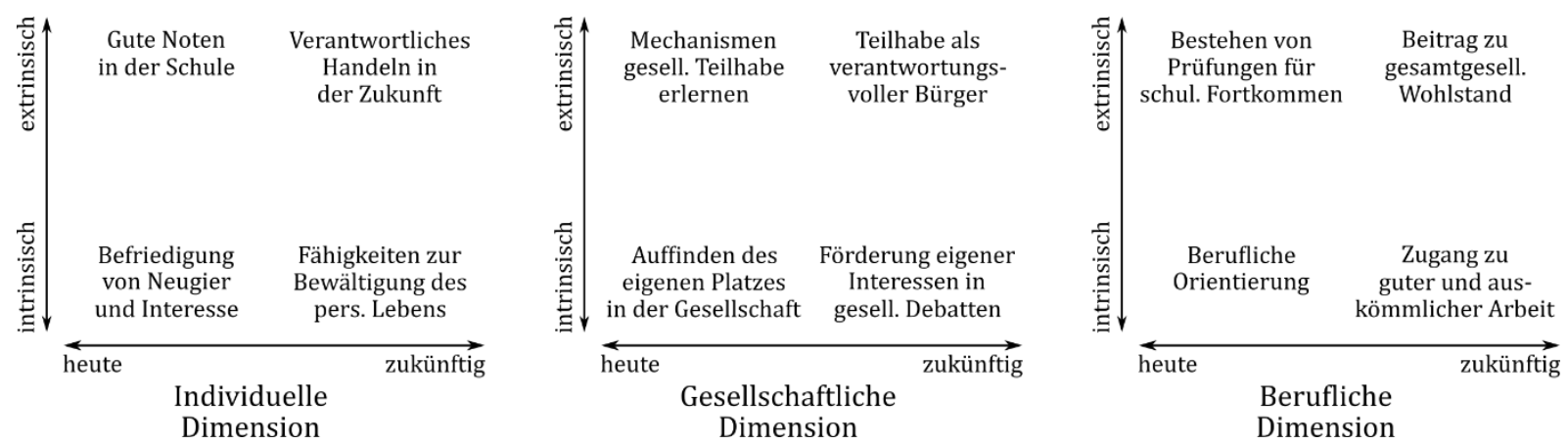

Abbildung 4: Modell der drei Dimension der Relevanz nach STUCKEY ET AL. [79].

Viele der kontextorientierten Unterrichtskonzepte, wie bspw. die britischen Salters Kurse [80] oder das deutsche vom BundESMINISTERIUM FÜR BILDUNG UND FoRSCHUNG (BMBF) geförderte Konzept Chemie im Kontext (ChiK) [81], nutzen Kontexte aus der Lebenswelt der SuS, um den fachwissenschaftlichen Inhalten aus Sicht der Lernenden Bedeutung zuzumessen und das Interesse sowie die Motivation der SuS zu steigern. Dabei bezieht sich der in diesen Projekten verwendete Relevanzbegriff vornehmlich auf die intrinsischen Aspekte der persönlichen und gesellschaftlichen Dimensionen. Ausgehend von Kontexten wie u. a. „Erwünschte Verbrennungen und unerwünschte Folgen“ “ ${ }^{82]}$ oder „Alkohol - zum Trinken viel zu schade" [83] werden fachliche Inhalte hierbei nach dem need-to-know-Prinzip vermittelt [84], anhand dessen (Basis-) Konzepte dann erarbeitet werden, wenn sie für das Verstehen der in den jeweiligen Kontext eingebetteten Inhalte notwendig sind ${ }^{855}$. Um zudem zu verhindern, dass das erworbene Wissen lediglich kontextspezifisch abgerufen wird, werden die Inhalte nachfolgender Kontexte, soweit möglich, wiederholt auf bereits erlernte Konzepte rekurriert.

Anderen Unterrichtskonzepten wie dem gesellschaftskritisch-problemorientierten Chemieunterricht nach EILKS [86] liegen sogenannte Socio-Scientific Issues (SSI), d.h. Kontexte mit einem „kontroverse[n], naturwissenschaftsbezogene[n] Anliegen mit gesellschaftlicher Bedeutung" [87] zugrunde. Hier stehen bei der Auswahl der Themen die fachwissenschaftlichen Inhalte zunächst im Hintergrund. Stattdessen stehen soziale, ökonomische und ökologische Interessen verschiedener Stakeholder im Fokus, deren Betrachtung offene Diskussionen ermöglicht und individuelle Entscheidungen von Seiten der SuS fordert [88]. Eine Relevanzzuschreibung erfolgt hier vornehmlich in Bezug auf die gesellschaftliche Dimension. Dabei spielen in Hinblick auf die Förderung der Scientific Literacy von SuS insbesondere zukünftig extrinsische Zuschreibungen eine Rolle. 
Unabhängig vom Relevanzbegriff werden in der Literatur zahlreiche wiederkehrende Merkmale für die Auswahl von geeigneten Kontexten wie Authentizität, Alltagsbezug, außergewöhnliche Phänomene und aktuelle Ereignisse genannt, auf Grundlage derer VAN VORST ET AL. ein Modell zur „Operationalisierung der Merkmale“ erarbeiteten, das sie in Bezug auf Merkmale auf Seiten der SuS bzw. des Kontextes sowie der Interaktion zwischen SuS und Kontext differenzieren (siehe Abbildung 5) [89]. Insbesondere die Authentizität und die Bekanntheit des Lerngegenstandes spielen dabei eine zentrale Rolle, die wiederum auf Seiten der SuS sowie der Kontexte von verschiedenen Aspekten beeinflusst werden. Um als authentisch zu gelten, muss die durch den Kontext gezeichnete Situation von SuS als glaubwürdig eingeschätzt werden. Auf Seiten des Kontextes wird dies laut der Autoren durch die geeignete Wahl einer Darstellungsform sowie einem gewissen Grad an Komplexität erreicht. Auch die Bekanntheit der Situation trägt prinzipiell zur Authentizität des Kontextes bei. Häufige und unmittelbare Erfahrungen mit dem Lerngegenstand unterstreichen dabei die Alltäglichkeit der Situation und tragen zur Einschätzung der Glaubwürdigkeit bei. Aber auch Gegenstände und Situationen, die SuS nicht alltäglich begegnen, bspw. jedoch aus Medien wie Fernsehen, Internet oder Büchern bekannt sind, können als authentisch wahrgenommen werden. Diese unter dem Begriff „Besonderheit“ zusammengefassten Situationen sind SuS zwar insgesamt weniger vertraut, eine erste Studie zum Einfluss der Kontextmerkmale zeigt jedoch, dass gerade das Merkmal Besonderheit interessenfördernd zu wirken scheint [89, 90].

\begin{tabular}{|c|c|c|}
\hline Schüler_in & & Kontext \\
\hline Glaubwürdigkeit & Authentizität & $\begin{array}{c}\text { Darstellungsform } \\
\text { Komplexität }\end{array}$ \\
\hline $\begin{array}{l}\text { Häufigkeit der Erfahrung } \\
\text { Umittelbarkeit der Erfahrung }\end{array}$ & Alltäglichkeit/Besonderheit & $\begin{array}{c}\text { Häufigkeit der Situation } \\
\text { Unmittelbarkeit der Situation }\end{array}$ \\
\hline Aktualität der Erfahrung & Bekanntheit & Aktualität der Situation \\
\hline
\end{tabular}

Abbildung 5: Modell zur Operationalisierung von Kontexten nach vAN VORST ET AL. [89].

Die genannten kontext- und SSI-basierten Unterrichtskonzepte unterscheiden sich folglich nicht nur in ihrem Relevanzbegriff, sondern zudem auch hinsichtlich ihrer primären Kontextmerkmale. Während Projekte wie ChiK einen deutlichen Fokus auf alltägliche Kontexte legen, finden bei Konzepten wie dem gesellschaftskritisch-problemorientierten Unterricht vornehmlich besondere Kontexte Anwendung. Weder die eine noch die andere Ausrichtung kann dabei jedoch als prinzipiell besser oder schlechter bewertet werden. Inwiefern die jeweiligen Kontexte von unterschiedlichen SuS als relevant eingeschätzt werden und ihr situationales Interesse fördern, lässt sich letztendlich nicht generalisieren.

\subsubsection{Relevanz aktueller Forschung im naturwissenschaftlichen Unterricht}

Neben dem wahrgenommenen Schwierigkeitsgrad des naturwissenschaftlichen Unterrichts werden von Seiten der SuS vor allem mangelnde Kontextualisierung, Aktualität und Relevanz der Themen beklagt. Dennoch werden aktuelle Forschungsinhalte im Unterricht häufig nicht zeitnah abgebildet. Beispielhaft kann dies anhand der Repräsentation von 
Membranmodellen in Biologiebüchern dargestellt werden, die auch bezüglich der Forschung des SFB eine Rolle spielen.

Obwohl das Lipid-Floß-Modell bereits 1997 von Simons \& IKONEN [91] publiziert wurde, zeigt eine Analyse sechs aktueller Biologiebücher der Sekundarstufe II ${ }^{5}$, die zwischen 2016 und 2019 erschienen sind, dass das Modell bisher lediglich in drei Lehrwerken Erwähnung findet. Dabei ist es keinesfalls so, dass das Modell vornehmlich in den neueren Publikationen zu finden ist. Im erst 2019 erschienen LINDER BIOLOGIE ${ }^{[92]}$ wird das FlüssigMosaik-Modell als noch heute gültig beschrieben. In einem Werk von Cornelsen ist zudem folgendes zu lesen:

„Das Flüssig-Mosaik-Modell stellt die heute allgemein akzeptierte Vorstellung vom Aufbau der Biomembran dar. Es dient als Grundlage der aktuellen zellbiologischen Forschung." [93]

Natürlich liegt es in der Natur von Modellen, dass sie als theoretische Rekonstruktionen von etwas ${ }^{[94]}$ weder als richtig noch falsch interpretiert, sondern höchstens als zur Beschreibung bzw. Untersuchung eines Sachverhaltes geeignet oder ungeeignet angesehen werden können. Entsprechend verliert das Flüssig-Mosaik-Modell auch nicht per se an Gültigkeit. Dennoch ist es bezeichnend, dass neue Erkenntnisse auch nach über 20 Jahren noch keinen Einzug in manche Schullehrwerke gehalten haben. Dies wird weiterhin auch dadurch forciert, dass das Lipid-Floß-Modell auch in keinem der Biologiecurricula Erwähnung findet ${ }^{6}$. Während in manchen lediglich der Vergleich von Membranmodellen gefordert wird, explizieren sieben von ihnen weiterhin das Flüssig-Mosaik-Modell. Insgesamt ist natürlich nicht auszuschließen, dass Lehrkräfte auch lehrbuchunabhängig Materialien zur Verfügung stellen, was jedoch voraussetzt, dass die Lehrkräfte sich hinsichtlich neuer Erkenntnisse kontinuierlich weiterbilden.

Dennoch soll naturwissenschaftliche Bildung laut den Bildungsstandards der KMK für die Fächer Chemie, Biologie und Physik eine „Meinungsbildung über technische Entwicklungen und naturwissenschaftliche Forschung" ermöglichen [37]. Ein Blick in die Rahmenlehrpläne der Chemie ${ }^{7}$ zeigt jedoch, dass bereits der Begriff „Forschung“ kaum genannt wird. Bereinigt man die Curricula um Begriffe wie Lernforschung oder Doppelungen in wiederkehrenden Überschriften, so zeigt sich die in Abbildung 6 dargestellte Verteilung der Häufigkeiten des Begriffs. In sechs Bundesländern wird Forschung gar nicht erwähnt, in vier weiteren nur jeweils ein oder zwei Mal. Die meisten Nennungen (neun) lassen sich im baden-württembergischen Kerncurriculum finden. Auch Hamburg zählt mit 6 Nennungen zu den Spitzenreitern, bezieht zu aktueller Forschung im Unterricht jedoch auch wie folgt Stellung:

„Der Versuch, fortlaufend aktuelle Forschungsergebnisse aufzunehmen und damit vermeintlich unverzichtbare Inhalte in einem verbindlichen Wissenskanon festzuschreiben, mündet in eine Überforderung von Lernenden und Lehrenden." [95]

Entsprechend scheint es notwendig auch außerhalb des naturwissenschaftlichen Unterrichts Konzepte zu erarbeiten, die durch Einbindung aktueller Forschung einen Beitrag zur Steigerung des Interesses an den Naturwissenschaften leisten.

\footnotetext{
5 Die analysierten Bücher und die für die Analyse relevanten Textstellen sind im Anhang zu finden.

${ }^{6}$ Eine Liste der analysierten Biologiecurricula ist dem Anhang zu entnehmen.

${ }^{7}$ Eine Liste der analysierten Chemiecurricula ist ebenfalls dem Anhang zu entnehmen.
} 


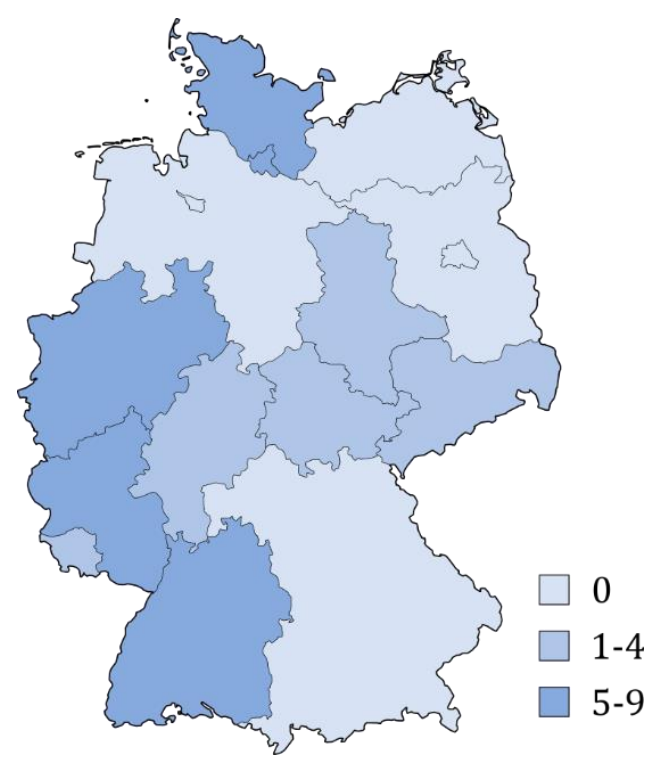

Abbildung 6: Häufigkeit des Wortes „Forschung“ in deutschen Chemie-Kerncurricula der Sekundarstufe II.

\subsection{Konzepte zur Steigerung des naturwissenschaftlichen Interesses in der Öffentlichkeit}

Das Interesse an den Naturwissenschaften wurde bisher vor allem im Kontext der schulischen Bildung betrachtet. Die ersten naturwissenschaftlichen Erfahrungen werden jedoch schon in frühster Kindheit gemacht, beispielsweise beim Fallenlassen eines Spielzeugs. Auch wenn das Kind sich keine expliziten Gedanken über die zugrundeliegenden physikalischen Gesetzmäßigkeiten macht, birgt das Phänomen des Fallens eine gewisse Faszination, das anhand von verschiedenen anderen Objekten weiter erforscht wird. Auch während und nach der Schulzeit begegnen einem jedem Menschen im Verlauf seines Lebens zahlreiche Situationen mit Bezug zu naturwissenschaftlichen Kontexten, mit denen er sich - mal mehr und mal weniger freiwillig - auseinandersetzt.

Um den Einfluss außerschulischer Faktoren auf das Interesse an den Naturwissenschaften zu analysieren, wurden in verschiedenen internationalen Studien sowohl Jugendliche als auch Erwachsene u. a. zu ihrem Interesse an den Naturwissenschaften und ihrer Teilnahme an außerschulischen naturwissenschaftlichen Aktivitäten befragt. Ergebnisse einer Studie mit 10- bis 14-jährigen SuS zeigen, dass neben Faktoren wie MINT-Kenntnissen, den Einstellungen der Eltern oder der Freude an den Naturwissenschaften auch außerschulische Aktivitäten einen Einfluss auf das Interesse der SuS haben [96]. Verschiedene Studien mit Erwachsenen zeigen zudem, dass vor allem Besuche von Science-Centern und naturwissenschaftlichen Museen in der Kindheit sowie das Schauen von wissenschaftlichen TV-Sendungen signifikante Korrelationen zum naturwissenschaftlichen Interesse aufweisen, während wissenschaftliche Magazine und Bücher sowie naturwissenschaftliche Erfahrungen in der Schule keine Signifikanz zeigen [97]. Zwar haben auch diese einen generellen Einfluss auf das Interesse, jedoch scheinen gerade Science-Center von besonderer Bedeutung [98-100].

Die (frühzeitige) Teilnahme an außerschulischen naturwissenschaftlichen Aktivitäten scheint also die Chance zu bergen, zunächst situationales Interesse zu schaffen, welches sich positiv auf das allgemeine aber auch das schulische naturwissenschaftliche Interesse auswirkt. Dabei beschränken sich mögliche Aktivitäten nicht nur auf die untersuchten Wissenschaftsmedien (Bücher, Magazine, TV-Sendungen), Science-Center und -Museen. 
Es existieren zudem zahlreiche Schülerlabore, Bildungsmessen, Science Slams oder Citizen Science-Projekte - nur um einige Aktivitäten zu nennen - die es sowohl SuS als auch der interessierten Allgemeinheit ermöglichen, sich auf zumeist freiwilliger Basis mit Themen aus verschiedenen naturwissenschaftlichen Disziplinen zu befassen. Solche interessenfördernden naturwissenschaftlichen Aktivitäten werden in der internationalen Literatur zumeist als Science Outreach (SO)-Aktivitäten bezeichnet. Um die vielen verschiedenen Arten und das insgesamt sehr große Feld des SO etwas zu systematisieren, wird im Folgenden den Fragen nachgegangen, was genau unter SO zu verstehen ist, welche unterschiedlichen Konzepte existieren, für welche Zielgruppen und von welchen Interessensgruppen und Personenkreisen diese entwickelt und wie diese Aktivitäten evaluiert werden.

\subsubsection{Naturwissenschaftliche Öffentlichkeitsarbeit - Science Outreach}

Als Differenzierung des Begriffs Public Outreach, der im Deutschen mit Öffentlichkeitsarbeit übersetzt wird, kann der Ausdruck Science Outreach als eine Öffentlichkeitsarbeit im Sinne der Naturwissenschaften, also als naturwissenschaftliche Öffentlichkeitsarbeit bezeichnet werden. In der Literatur finden sich keine expliziten Definitionen für SO, jedoch beschreiben PoliaKofF \& WEBB den Begriff Public Engagement, der in diesem Kontext unter anderem als Einbeziehung der Öffentlichkeit, jedoch ebenfalls auch als Öffentlichkeitsarbeit übersetzt werden kann, als „any scientific communication that engages an audience outside of academia“ [101]. Ein etwas weiterführender Begriff der Scientific Communication - also der naturwissenschaftlichen Kommunikation - wurde zudem von BURNS, O'CoNNOR \& STOCKLMAYER definiert:

„Science Communication [...] may be defined as the use of appropriate skills, media, activities, and dialogue to produce one or more of the following personal responses to science (the vowel analogy)

Awareness, including familiarity with new aspects of science

Enjoyment or other affective responses, e.g. appreciating science as entertainment or art

Interest, as evidenced by voluntary involvement with science or its communication

Opinions, the forming, reforming or confirming of science-related attitudes

Understanding of science, its content, processes and social factors" [102]

Insbesondere die beiden Aspekte Understanding of science - das Verstehen der Naturwissenschaften - und Opinions - das Formen einer Meinung -besitzen einen gewissen Bildungsanspruch, da ohne das Erlangen von fachlichen Kompetenzen weder die Inhalte und Prozesse der Naturwissenschaften verstanden werden können, noch eine differenzierte Meinungsbildung in Bezug auf naturwissenschaftliche Themen möglich ist.

Vor dem Hintergrund, dass Lernen ein lebenslanger Prozess ist der sich nicht auf die, im Vergleich zu unserer durchschnittlichen Lebensspanne, wenigen Jahre der schulpflichtigen Zeit beschränkt [103], werden allgemein drei Bildungsformen unterschieden: formale, non-formale und informelle Bildung. Diese werden in der Literatur zumeist anhand der Orte an denen Lernprozesse stattfinden, der Zielorientierung dieser Prozesse und Möglichkeiten der Zertifizierung definiert. Als formale Bildungsprozesse werden solche bezeichnet, die in Bildungsinstitutionen wie Schulen, Ausbildungsstätten und Universitäten stattfinden und mit einem Leistungszertifikat enden. Die zu erbringenden Leistungen unterliegen dabei gewissen Anforderungen, die u. a. in Curricula festgelegt werden, und das 
Lernen ist stets zielgerichtet. Orte non-formaler Bildung sind ebenfalls strukturierte Institutionen, wie bspw. Musikschulen und Vereine, die in den meisten Fällen jedoch freiwillig besucht werden. Zwar erfolgt auch hier das Lernen zielgerichtet, jedoch sind die Entfaltungsmöglichkeiten größer und es erfolgen keine landes- oder bundesweit vereinheitlichten Leistungsüberprüfungen. Informelles Lernen hingegen findet in der Familie, im Freundeskreis oder in der Freizeit statt. Es bezieht sich vor allem auf unterbewusste und unstrukturierte Lernprozesse, wie den Erwerb von Einstellungen, Handlungsweisen oder Werten, die nicht zertifiziert werden und insbesondere aus Sicht der Lernenden nicht zielgerichtet sind [104, 105].

Anhand dieser Unterscheidungen fällt es jedoch schwer, die zahlreichen und unterschiedlichen existierenden S0-Aktivitäten einer dieser Bildungsformen zuzuweisen. Oftmals werden außerschulische Lernorte pauschal als informell bezeichnet [61]. Wie verhält es sich jedoch mit Schülerlaboren, die zumeist institutionalisiert sind und deren Inhalte sich in der Regel an den Kerncurricula der Länder orientieren? Das zielgerichtete Lernen in diesen Institutionen spricht für eine Einordnung in die non-formale Bildung. Die meisten SuS besuchen Schülerlabore jedoch nicht freiwillig, sondern müssen im Zuge einer Exkursion daran teilnehmen. In Bezug zur Schule bemängeln BÄUMER ET AL. zudem, dass dort nicht nur formale Lernprozesse, sondern u. a. auch informelles Lernen, z. B. im Freundeskreis, stattfindet. Sie schlagen deshalb vor, an Stelle von verschiedenen Bildungsformen von formalen, non-formalen und informellen Bildungskontexten zu sprechen, innerhalb derer sowohl intentional, sowie inzidentell - also unbeabsichtigt - gelernt wird: „It is not learning itself that is formal, nonformal, or informal, but the context in which it takes place" [106].

In Anlehnung an dieses Konzept differenzieren MASCHKE \& STECHER zwischen der Kontextund Subjektperspektive auf das Lernen (siehe Tabelle 1) [107]. In Bezug auf die Kontextperspektive unterscheiden sie zwischen Kontexten mit Organisationsbezug, unter die formale und non-formale Bildungsangebote fallen, und Kontexten mit lebensweltlichen Bezügen (informell). Dabei variieren die drei Bildungsformen aus der Kontextperspektive, wie bereits oben beschrieben weiterhin bezüglich der Zielorientierung und Möglichkeiten der Zertifizierung an den jeweiligen Lernorten. Jedoch wird aus der Subjektperspektive zwischen intentionalem und inzidentellem Lernen differenziert.

Formale Kontexte sind beispielsweise vor allem von intentionalem Lernen geprägt. Der verpflichtende schulische Unterricht folgt einem Curriculum, dessen Inhalte in einer bestimmten Zeitspanne gelehrt werden. Der Abschluss der schulischen Laufbahn wird durch ein Zeugnis attestiert und ermöglicht die Teilnahme an weiteren (formalen) Bildungsangeboten. Neben dem Erwerb fachspezifischer Kompetenzen finden in der Schule jedoch auch weitere Lernprozesse statt, die zum einen die Vermittlung von Werten und Routinen in peer-to-peer Interaktionen beinhalten und zum anderen unter dem Begriff hidden curriculum zusammengefasst werden können [107]. Zinnecker beschreibt diesen „heimlichen Lehrplan“ als „die Werte und Routinen, die durch das Lehrpersonal und die Unterrichtsordnung den SuS übermittelt [werden und] den ,eigentlichen' kulturellen Lernprozess in der Schule bilden“ [108]. Diese Prozesse erfolgen jedoch vor allem aus Subjektperspektive nicht bewusst, weshalb sie dem inzidentellen Lernen zuzuschreiben sind. 
Tabelle 1: Lernprozesse aus Subjekt- und Kontextperspektive nach MASCHKE \& STECHER [107].

\begin{tabular}{|c|c|c|c|c|}
\hline & & & \multicolumn{2}{|c|}{ Subjektperspektive auf das Lernen } \\
\hline & & & $\begin{array}{c}\text { Intentionales Lernen } \\
\text { (explizit) }\end{array}$ & $\begin{array}{l}\text { Inzidentelles Lernen } \\
\text { (implizit) }\end{array}$ \\
\hline \multirow{3}{*}{ 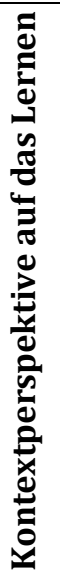 } & \multirow{2}{*}{$\begin{array}{l}\text { Kontexte mit } \\
\text { Organisations } \\
\text {-bezug }\end{array}$} & $\begin{array}{c}\text { Formal } \\
\text { (Bildungsangebot) }\end{array}$ & $\begin{array}{l}\text { (1) Schule, Berufsschule, } \\
\text { Universität, } \\
\text { (Ausbildung-) Betrieb }\end{array}$ & $\begin{array}{l}\text { (2) z. B. „hidden } \\
\text { curriculum“ }\end{array}$ \\
\hline & & $\begin{array}{c}\text { Non-Formal } \\
\text { (Bildungsangebot) }\end{array}$ & $\begin{array}{l}\text { (3) Kindergarten, } \\
\text { Musikschule, VHS, } \\
\text { Nachhilfe etc. }\end{array}$ & $\begin{array}{l}\text { (4) z. B. „hidden } \\
\text { curriculum“ }\end{array}$ \\
\hline & $\begin{array}{l}\text { Lebenswelt- } \\
\text { liche } \\
\text { Kontexte }\end{array}$ & Informell & $\begin{array}{l}\text { (5) Lernen in der Familie } \\
\text { (z. B. Hausaufgaben), } \\
\text { selbstorganisierte } \\
\text { Lerngruppen, } \\
\text { selbstgesteuertes } \\
\text { Lernen (z. B. mit } \\
\text { Medien) etc. }\end{array}$ & $\begin{array}{l}\text { (6) Beiläufiges Lernen im } \\
\text { Rahmen von } \\
\text { Mediennutzung, } \\
\text { Peerbeziehungen, } \\
\text { Familie etc. }\end{array}$ \\
\hline
\end{tabular}

\subsubsection{Systematische Gliederung von Science Outreach-Aktivitäten}

Die in Tabelle 1 dargestellte Differenzierung des Bildungsbegriffs anhand von Bildungskontexten soll im Folgenden als Grundlage für eine systematische Gliederung von SO-Aktivitäten dienen. Dazu muss jedoch zunächst der Aspekt der Freiwilligkeit thematisiert werden. Im vorangegangen Beispiel wurden Schülerlabore als in den meisten Fällen institutionell und zielorientiert definiert, die jedoch nicht zertifiziert werden, was für eine Einordnung in den non-formalen Bereich spricht. Jedoch werden non-formale Bildungsangebote in der Literatur oftmals mit einer freiwilligen Teilnahme in Verbindung gebracht [105, 107]. Da die Entscheidung ein Schülerlabor zu besuchen bei der Lehrkraft liegt, kann nicht pauschal von einer freiwilligen Teilnahme aller SuS ausgegangen werden. Ähnlich verhält es sich z. B. auch beim Besuch von Bildungsmessen, der prinzipiell auf freiwilliger Basis geschehen kann und bestenfalls auch soll, der aber oftmals durch Lehrkräfte (Exkursion) oder durch Familienmitglieder gesteuert wird. Da der Grad der Freiwilligkeit bei der Teilnahme an verschiedenen SO-Aktivitäten nur bedingt beurteilt werden kann, soll dieser Aspekt bei der Gliederung der Aktivitäten nur eine untergeordnete Rolle spielen.

Beim Blick in die Literatur wird deutlich, dass S0-Aktivitäten so zahlreich und vielfältig sind, wie die Zielgruppen, für die sie entwickelt wurden. Die Suche nach dem geschlossenen Ausdruck Science Outreach bei Google Scholar führt zu mehr als 7000 Ergebnissen. Eine Differenzierung der Ergebnisse nach Erscheinungsjahren zeigt, dass das Thema seit Beginn der 90er Jahre ( $<50$ Publikationen) deutlich an Bedeutung gewonnen hat, so dass in den letzten 5 Jahren kontinuierlich mehr als 500 Publikationen mit Bezug zu SO entstanden sind (vgl. Abbildung 7). Dabei sei zu bedenken, dass diese Zahlen sich vor allem auf englischsprachige Publikationen beschränken und dass Publikationen die andere Outreach Begriffe verwenden (z. B. Chemistry Outreach, Science Communication, Public Engagement, wissenschaftliche Öffentlichkeitsarbeit) zum größten Teil nicht miterfasst wurden. 


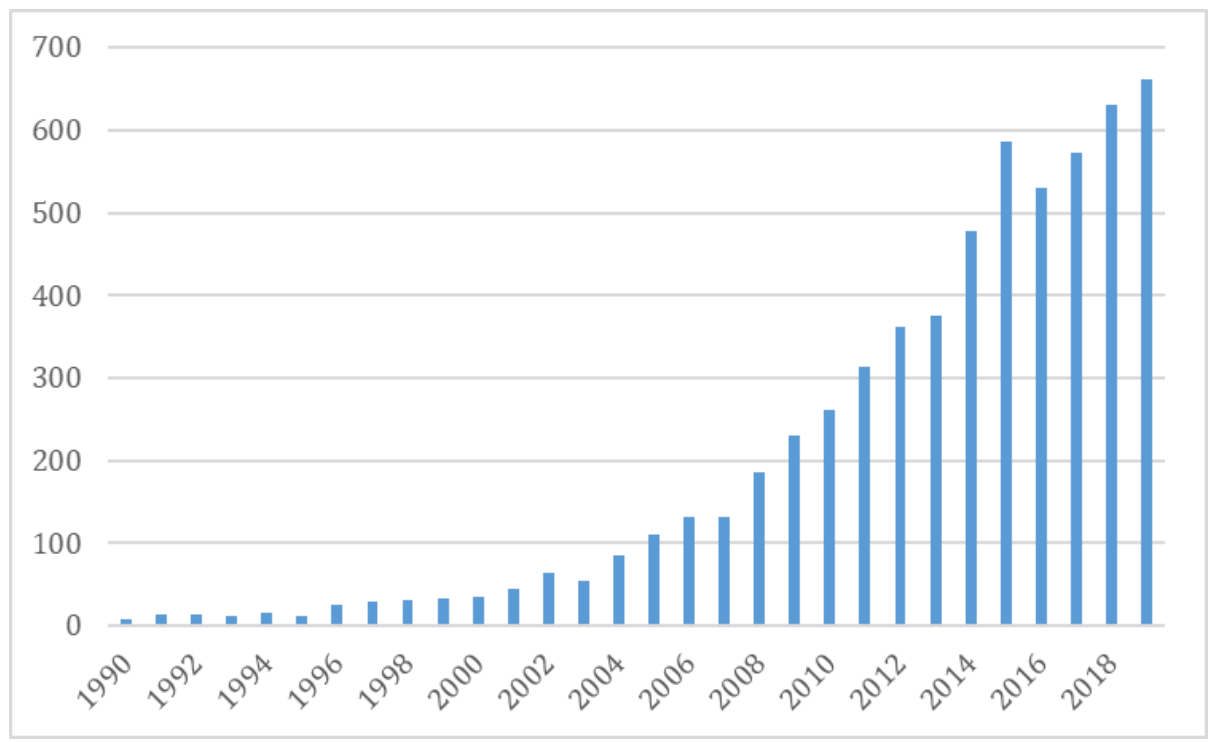

Abbildung 7: Anzahl der in Google Scholar gelisteten Publikationen mit dem zusammenhängenden Ausdruck „Science Outreach“ seit 1990.

Die Vielfältigkeit der Aktivitäten kann zudem am Beispiel des Schülerlabors verdeutlicht werden, das von HAUPT ET AL. bezüglich der Ausrichtung der verschiedenen Schülerlabore in sechs verschiedene Kategorien eingeteilt wird. Neben klassischen Schülerlaboren differenzieren die Autoren zwischen Schülerforschungszentren, Lehr-Lernlaboren sowie Schülerlaboren zur Wissenschaftskommunikation, mit Bezug zum Unternehmertum und mit Berufsorientierung [109]. Dabei werden weitere Mischformen der verschiedenen Laborkategorien nicht ausgeschlossen. Die in Tabelle 2 vorgenommene systematische Gliederung verschiedener SO-Aktivitäten wurde lediglich anhand übergeordneter Konzepte vorgenommen und erhebt keinen Anspruch auf Vollständigkeit. Da eine Einordnung der übergeordneten Konzepte aufgrund der Vielfalt ihrer Aktivitäten nicht immer eindeutig möglich ist, werden die sechs postulierten Kategorien und die ihnen zugeordneten Konzepte nachfolgend genauer erläutert.

Tabelle 2: Einteilung von SO-Aktivitäten in sechs SO-Bildungskontexte hinsichtlich (vorherrschenden) Lernprozessen aus Subjekt und Kontextperspektive

\begin{tabular}{|c|c|c|c|c|}
\hline & & & \multicolumn{2}{|c|}{ Subjektperspektive auf das Lernen } \\
\hline & & & Intentionales Lernen & Inzidentelles Lernen \\
\hline \multirow{3}{*}{ 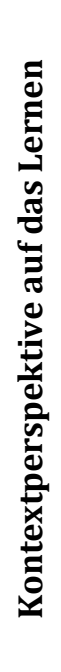 } & \multirow{2}{*}{$\begin{array}{l}\text { Kontexte mit } \\
\text { Organisations- } \\
\text { bezug }\end{array}$} & Formal & $\begin{array}{l}\text { (1) Unterrichtsmaterialien } \\
\text { Mobile Labore I } \\
\text { Experimentierkoffer } \\
\text { Nachmittagsangebote }\end{array}$ & $\begin{array}{l}\text { (2) Wissenschaftler_innen in } \\
\text { der Schule }\end{array}$ \\
\hline & & $\begin{array}{l}\text { Non- } \\
\text { Formal }\end{array}$ & $\begin{array}{l}\text { (3) Schülerlabore } \\
\text { Mobile Labore II } \\
\text { Science Camps }\end{array}$ & $\begin{array}{l}\text { (4) Science-Center \& -Museen } \\
\text { Universitäre Angebote } \\
\text { Angebote aus Industrie, } \\
\text { Wirtschaft und Politik } \\
\text { Wissenschaftliches Theater }\end{array}$ \\
\hline & $\begin{array}{l}\text { Lebenswelt- } \\
\text { liche Kontexte }\end{array}$ & Informell & $\begin{array}{l}\text { (5) Citizen Science } \\
\text { Websites I } \\
\text { Bücher, Magazine, TV I }\end{array}$ & $\begin{array}{l}\text { (6) Bildungsmessen } \\
\text { Science Slams } \\
\text { Science in der Community } \\
\text { Websites II } \\
\text { Bücher, Magazine, TV II }\end{array}$ \\
\hline
\end{tabular}




\section{(1) Intentionales Lernen in formalen Kontexten}

Projekte aus dieser Kategorie besitzen vor allem den Anspruch, den formalen naturwissenschaftlichen Unterricht zu bereichern und ihn unter anderem durch Kontextualisierung und Forschungsbezüge interessanter zu gestalten. Allen voran sei die Entwicklung von Unterrichtsmaterialien zu nennen. Im Fokus der Bemühungen steht hierbei insbesondere die Erarbeitung neuartiger Experimente zu aktuellen Themen aus der Forschung. Im deutschsprachigen Raum wurden beispielsweise von verschiedenen chemiedidaktischen Arbeitsgruppen Experimente mit Bezug zur Fotochemie [110-112], Elektrochemie [113, 114] und Nanotechnologie [115,116] entwickelt. Dabei wurde darauf geachtet, dass die fachlichen Hintergründe der z. T. in Schulbüchern wenig vertretenen, aber für unseren Alltag relevanten Themen wie organische Leuchtdioden (Organic Light Emitting Diode, OLED) mit Hilfe curricular relevanter Konzepte verstanden werden können, um eine Einbettung in den Regelunterricht zu erleichtern.

Neben Projekten zur Förderung der inhaltlichen Dimensionen des naturwissenschaftlichen Unterrichts existieren zudem mobile Labore ${ }^{8}$ und Experimentierkoffer, die vor allem darauf abzielen, das Experimentiererlebnis der SuS authentischer und forschungsnaher zu gestalten. Als ein Beispiel sei hier das Alabama SCIENCE IN Motion-Programm (ASIM) genannt [117]. Da Schulen in den USA - ähnlich wie in Deutschland - nur ein begrenztes Budget für Chemikalien, Gerätschaften etc. zur Verfügung steht, wurden an elf Standorten im US-Bundesstaat Alabama jeweils zwei Vans, einer für Chemie und einer für entweder Physik oder Biologie, mit modernen Geräten und Materialien im Wert von jeweils über \$100.000 ausgestattet. Diese können von Schulen angefordert und die Materialien zur Unterstützung der Lehre verwendet werden. Zudem bietet ASIM auch Kurse für Lehrkräfte an, in denen diese sowohl den Umgang mit den Gerätschaften lernen, als auch Anregungen für Experimente erhalten. In ähnlicher Weise bieten zudem Experimentierkoffer, die u. a. als Klassensätze gekauft oder ausgeliehen werden können, im kleineren Maßstab Materialien, Chemikalien und Experimentieranleitungen zu bestimmten Themenbereichen [118-120].

Im Rahmen naturwissenschaftlicher Nachmittagsangebote und Arbeitsgemeinschaften in der Schule haben SuS zudem die Möglichkeit, sich tiefergehend mit naturwissenschaftlichen Themen auseinanderzusetzen, die nicht unbedingt curricular relevant sein müssen [121]. Während in der Sekundarstufe I vor allem die Interessenförderung im Vordergrund steht und zumeist Phänomene auf der makroskopischen Ebene betrachtet werden, bieten Angebote für die Sekundarstufe II, wie z. B. JUGEND FORSCHT [122], die Möglichkeit, sich vertiefend mit aktuellen naturwissenschaftlichen Themen auseinanderzusetzen und verschiedene Problemstellungen zudem eigenständig zu bearbeiten.

Sowohl Unterrichtsmaterialien zu aktuellen Themen als auch mobile Labore und Experimentierkoffer dienen dazu, den Regelunterricht zu bereichern. Sie können also zum einen klar dem formalen Kontext zugerechnet werden, zum anderen erfolgt die Verwendung durch die Lehrkraft, so dass aus Sicht der SuS weiterhin von intentionalem Lernen geredet werden kann. Auch die genannten Nachmittagsangebote finden in der Schule, also im formalen Kontext statt. Obwohl die Angebote in Bezug auf die curriculare Anschlussfähigkeit

\footnotetext{
${ }^{8}$ Hier muss zwischen zwei Arten mobiler Labore unterschieden werden. Es existieren sowohl mobile Labore die Schulen die Möglichkeiten bieten moderne wissenschaftliche Geräte im Unterricht einzusetzen. Diese werden der Kategorie (1) zugeschrieben. Zudem existieren mobile Labore, die außerschulischen Schülerlaboren ähneln, und bei denen nicht die SuS zum Schülerlabor, sondern das Schülerlabor zu den SuS kommt (Kategorie (3)).
} 
nicht mit dem Regelunterricht zu vergleichen sind, entscheiden sich die teilnehmenden SuS trotz vielfältiger Angebote und oftmals freiwillig für die außerunterrichtliche Beschäftigung mit naturwissenschaftlichen Themen, so dass aus Sicht der SuS auch hier von intentionalem Lernen ausgegangen werden kann.

\section{(2) Inzidentelles Lernen in formalen Kontexten}

Eine weiteres S0-Konzept das im formalen Kontext Anwendung findet ist der Besuch von Wissenschaftler_innen in der Schule, der vielfältige Formen annehmen kann. Ein typisches Projekt ist diesbezüglich das SCIENCE SQUAD der Univiersity of Colorado in Boulder. Die Mitarbeiter, in diesem Fall Studierende aus Naturwissenschaften und Technik, besuchen verschiedene Schulen mit SuS aller Altersstufen und führen dort Demonstrationsund Mitmachexperimente durch [123]. Die Experimente müssen dabei nicht unbedingt einen Bezug zum Curriculum aufweisen und auch nicht inhaltlich aufgearbeitet werden. Vielmehr ist das Ziel, für die jeweiligen naturwissenschaftlichen Disziplinen zu begeistern. Auch im Zuge des Projektes Present Your PHD Thesis To A 12-YeAR-Old kommen junge Wissenschaftler_innen in die Schule und präsentieren den SuS die Ergebnisse ihres Dissertationsprojekts. Die publikumsgerechte Aufarbeitung ihrer Arbeit soll zum einen die Kommunikationskompetenzen der Doktoranden fördern und zum anderen SuS einen Einblick in aktuelle Forschung geben [124].

Beiden Konzepten ist gemein, dass es vordergründig nicht darum geht den SuS fachwissenschaftliche Inhalte zu vermitteln. Neben der Interessenförderung liegt der Nutzen beider Aktivitäten insbesondere darin, SuS den Kontakt mit Wissenschaftler_innen zu ermöglichen. Im Sinne inzidentellen Lernens sollen so zum einen Berufsperspektiven aufgezeigt werden und zum anderen soll sich die Interaktion mit den Wissenschaftler_innen positiv auf die prävalenten stereotypen Vorstellungen der SuS auswirken. Um zudem insbesondere Schülerinnen Vorbilder aus den Naturwissenschaften zu präsentieren, setzen einige SO-Programme gezielt junge Naturwissenschaftlerinnen ein [125].

\section{(3) Intentionales Lernen in non-formalen Kontexten}

Als Orte non-formalen Lernens werden in der Literatur u. a. strukturierte Institutionen und Organisationen wie Musikschulen und Vereine genannt. Mit Blick auf SO findet Lernen im non-formalen Kontext beispielsweise in Universitäten, Museen, Science-Centern, Forschungseinrichtungen oder der Industrie statt. Diese Orte sind es auch, die intentionales Lernen, z. B. in Schülerlaboren, fördern. Diesbezüglich legen HAUPT ET AL. Kriterien fest, die für alle MINT-Schülerlabore gelten: (i) es handelt sich um einen außerschulischen Lernort, (ii) das Schülerlabor wird dauerhaft, d.h. mindestens 20 Tage pro Jahr betrieben und (iii) das Hauptaugenmerkt liegt auf dem „eigene[n] Experimentieren mit dem Forschungsprozess als Schwerpunkt“. Allgemeine Ziele von Schülerlaboren sind dabei die „Steigerung von Wissenschaftsinteresse und Wissenschaftsverständnis“ und die „Nachwuchsförderung für MINT-Berufe und MINT-Studiengänge“ "109]. Wie bereits erwähnt, können Schülerlabore in ihrer Ausrichtung variieren. Die Ausrichtungen der jeweiligen Schülerlabore hängen dabei stark von den Institutionen ab, von denen sie angeboten werden. So werden an Universitäten beispielsweise Lehr-Lern-Labore in die fachdidaktische Ausbildung von Lehramtsstudierenden eingebunden. Dahingegen werden Schülerlabore zur Wissenschaftskommunikation sowie Schülerlabore mit Berufsorientierung oftmals von Forschungseinrichtungen oder der Industrie angeboten. Gleichzeitig ist es natürlich nicht ausgeschlossen, dass auch universitäre Schülerlabore Wissenschaftskommunikation betreiben und die Inhalte ihrer Forschung präsentieren [109].

Für Deutschland und den deutschsprachigen Raum zählt die Website LERNORTLABOR (LELA) ${ }^{[126]}$ alle Schülerlabore auf, die den genannten Kriterien entsprechen und ordnet 
sie zudem nach den von HAUPT ET AL ${ }^{[109]}$ postulierten Kategorien. Zurzeit führt die Seite 405 Schülerlabore (Stand 06/2020) in Deutschland, Österreich, Luxemburg, Italien und der Schweiz (siehe Abbildung 8), wobei es sich hierbei nicht ausschließlich um MINTSchülerlabore handelt und beispielsweise auch geisteswissenschaftliche Labore wie das Göttinger YLAB ${ }^{[127]}$ geführt werden.

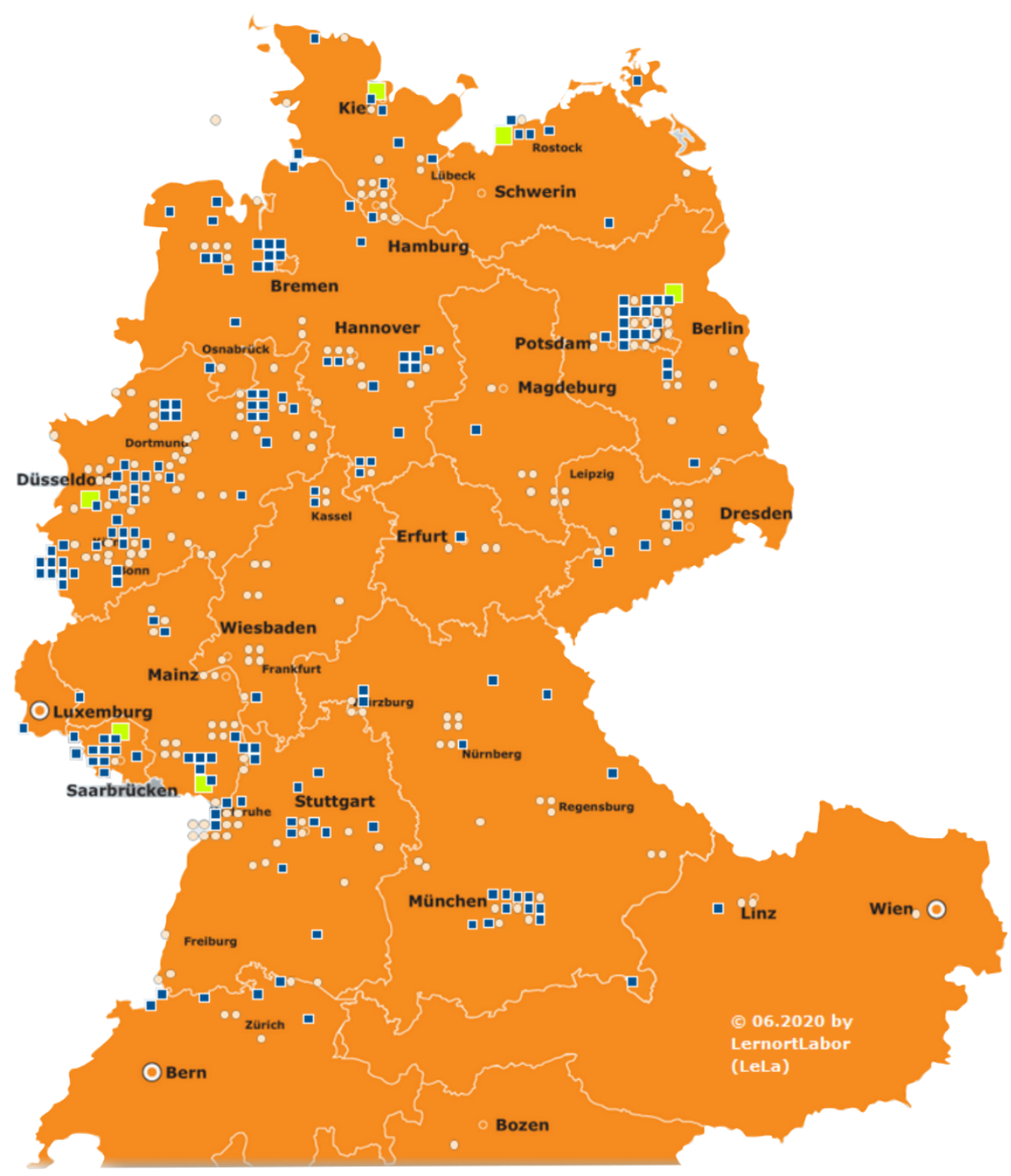

Abbildung 8: Verteilung der auf der Website LELA gelisteten Schülerlabore in Deutschland sowie im deutschsprachigen Raum [126].

Der Besuch von Schülerlaboren wird zumeist von der Lehrkraft im Rahmen einer Exkursion initiiert. Je nach Intention des Besuchs (Interessenförderung, Berufsorientierung, Abiturvorbereitung etc.) können die Themen der zumeist eintägig stattfindenden Schülerlabore variieren. Gemein ist ihnen jedoch in den meisten Fällen, dass die fachlichen Inhalte auf das Kerncurriculum des jeweiligen Landes rekurrieren und dass sie den Regelunterricht „in Bezug auf authentische, lebensweltbezogene, naturwissenschaftlich-technische Themenfelder und Arbeitsweisen" [128] bereichern.

Wie Abbildung 8 zeigt, sind die zahlreichen Labore jedoch nicht gleichmäßig verteilt, sondern sind vor allem in Ballungszentren mit Universitäts- und Industriestandorten vorzufinden. Der Besuch von Schülerlaboren ist je nach Schulstandort neben den Kosten für das Schülerlabor auch mit erhöhten Kosten für Transport und ggf. Unterkunft sowie einem zeitlichen und personellen Aufwand verbunden. Abhilfe schaffen sollen hier mobile La- 
bore. Diese können Adaptionen außerschulischer Schülerlaborangebote für den Unterricht an Schulen darstellen, bei denen Wissenschaftler_innen Experimente zu Themen des Schülerlabors im Klassenzimmer mit den SuS durchführen [129]. Zudem existieren Initiativen wie der vom BMBF geförderte INNOTRUCK, der als Labor- und Ausstellungsraum auf Rädern sowohl für öffentliche Veranstaltungen als auch von Schulen angefordert werden kann.

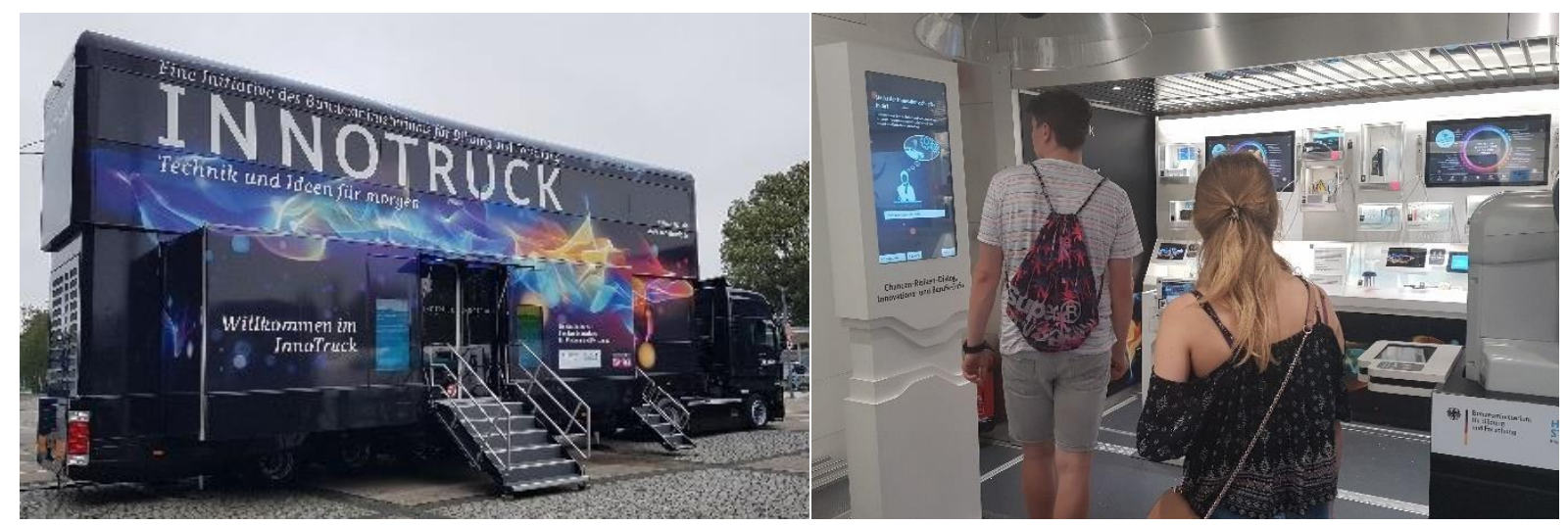

Abbildung 9: Der InNoTruck des BMBF am 22.05.2019 in Pankow, Berlin, beim 12. Ausbildungstag Pankow \& Lichtenberg.

Während der von der Lehrkraft oder Schule initiierte Besuch von außerschulischen Laboren aufgrund des Exkursionscharakters eindeutig dem intentionalen Lernen in non-formalen Kontexten zugeordnet werden kann, ist eine Einordnung der mobilen Labore hier nicht ohne weiteres möglich. Zwar lassen sich Angebote wie der INNOTRUCK - auch wenn er zur Schule kommt - problemlos als außerschulische Lernorte deuten, die Durchführung eines Schülerlabors im Klassenzimmer könnte jedoch ebenso gut der Wissenschaftler_in in der Schule aus Kategorie (2) zugeordnet werden. Für die Einordnung in den intentionalen non-formalen Kontext spricht jedoch, dass es sich bei den Schülerlaboren nicht um bloße Experimentalvorträge handelt, sondern dass, ähnlich wie an außerschulischen Standorten, das eigene Experimentieren im Vordergrund steht. Dennoch muss zugegeben werden, dass die Grenzen hier verschwimmen.

Neben Schülerlaboren bieten viele der genannten Organisationen auch Science Camps an, die den Laboren in ihrem Aufbau und ihren Zielen prinzipiell ähneln. Science Camps unterscheiden sich jedoch in ihrer Dauer - die Zeitspannen variieren zwischen einem Tag [130] und mehreren Wochen [131] - sowie der freiwilligen Teilnahme der SuS. Die an Wochenenden und in den Ferien stattfindenden Camps bedürfen einer separaten und schulunabhängigen Anmeldung. Die Intentionalität des Lernens liegt hier in der bewussten und freiwilligen Auseinandersetzung der zumeist wissenschaftsbegeisterten SuS mit MINT-Themen, die bereits bei der Anmeldung für das Science Camp bekannt sind oder die explizit gewählt werden können.

\section{(4) Inzidentelles Lernen in non-formalen Kontexten}

Auch inzidentelles Lernen ist in non-formalen Kontexten möglich. Beispiele solcher Bildungsorte stellen Science-Center und Science-Museen dar. Beide bieten interaktive Ausstellungen zu naturwissenschaftlichen Themen, die verschiedene Medientypen, reale Objekte sowie zahlreiche Mitmachexperimente beinhalten [132]. Sie unterscheiden sich vor allem darin, dass Museen eigene Sammlungen historischer Exponate besitzen. Besucher_innen haben die Möglichkeit, sich je nach Alter und Interesse mit verschiedenen Themen auseinanderzusetzen. Dabei entscheiden die Besucher_innen selbst, mit welchem 
Thema sie sich wie lange beschäftigen. Dies geschieht zumeist spontan und aus dem situationalen Interesse heraus, weshalb das Lernen vornehmlich inzidentell stattfindet, auch wenn die Besucher_innen sich zuvor aktiv dafür entschieden haben, einen der beiden Bildungsorte zu besuchen.

Auch Universitäten bieten zahlreiche Aktivitäten an, die von Besucher_innen aus ähnlichen Beweggründen wie Science-Center und -Museen wahrgenommen werden. Beispielsweise öffnen zahlreiche Universitäten zur NACHT DES WISSENS (auch LANGE NACHT DER WISSENSCHAFT) ihre Türen für die Öffentlichkeit, um anhand von Ausstellungen, Workshops und hands-on-Experimenten Einblicke in die Forschung an den verschiedenen Fakultäten zu gewähren. Zudem werden auch außerhalb dieser Nächte zahlreiche Aktivitäten wie Kinder-Universitäten, Workshops oder Science Shows angeboten, die vor allem der Interessenförderung, aber auch der Unterhaltung dienen. Ähnlich verhält es sich mit Angeboten aus Industrie und Wirtschaft. Beispielsweise ermöglichen Tage der offenen Tür einen Blick hinter die Kulissen verschiedener lokaler Labore und Firmen. Zudem bietet beispielsweise das OfFICE OF SPACE SCIENCE der NATIONAL AERONAUTICS AND SPACE AdMinisTRATION (NASA) verschiedene SO-Aktivitäten wie Ausstellungen, Workshops und Vorträge an, die u. a. in Science-Centern oder Planetarien stattfinden [133].

Ein eher unbekanntes aber nicht minder interessantes Projekt ist das FUSION SCIENCE THEATER des Madison Area Technical College in Wisconsin. In Anlehnung an Science Shows nutzen die Wissenschaftler_innen des Projektes theaterpädagogische Methoden um Kindern im Grundschulalter auf eine altersgerechte, spannende und inzidentell lehrreiche Art und Weise mit ersten naturwissenschaftlichen Konzepten in Kontakt zu bringen [134].

\section{(5) Intentionales Lernen in informellen Kontexten}

Auch in informellen Kontexten (zu Hause, in der Gemeinde, in der Umwelt etc.) kann intentionales Lernen stattfinden. Beispielsweise bieten Citizen Science-Projekte, die u. a. von der Bundesregierung im Zuge der eingangs erwähnten Hightech Strategie gefördert werden [11], verschiedene Möglichkeiten die Öffentlichkeit in wissenschaftliche Prozesse aktiv einzubeziehen. Die Rolle der Öffentlichkeit kann dabei von Projekt zu Projekt variieren. Teilnehmer_innen können selbst Daten sammeln, vorhandene Daten auswerten, Ergebnisse diskutieren oder gar für sie relevante Fragen generieren, die in die Forschung aufgenommen werden [135]. Projekte solcher Art existieren laut SILVERTOWN u. a. zu Klimawandel, invasiven Arten, Naturschutzbiologie, Renaturierungsökologie, Gewässermonitoring und Populationsökologie [136].

Im Zeitalter digitaler Medien spielen zudem Websites sowohl für SuS als auch für die Allgemeinheit eine wichtige Rolle für informelle Lernprozesse. Online-Tutorien ermöglichen es SuS, in der Schule behandelte Fachinhalte zu wiederholen und zu vertiefen, um bspw. die Bearbeitung von Hausaufgaben oder das Lernen für Klausuren zu unterstützen. Mithilfe von Suchmaschinen und Online-Enzyklopädien können zudem gezielt Informationen zu einem bestimmten Thema abgerufen werden. Ferner ermöglichen Blogs und Videoplattformen es Wissenschaftler_innen, ihre Forschung auch außerhalb ihrer Wissenschaftsgemeinde zu präsentieren und sich mit der Gesellschaft darüber auszutauschen [137]. Einige Websites wie TED-ED spezialisieren sich sogar darauf, Wissen intentional zu vermitteln, indem sie verschiedene naturwissenschaftliche und gesellschaftlich relevante Themen in kurzen Videosequenzen präsentieren und anhand von anschließenden geschlossenen und offenen Frageformaten sowie weiterführenden Informationen das Lernen weiter unterstützen [138]. Hier muss jedoch klar zwischen intentionalem und inzidentellem Lernen im Internet unterschieden werden. Natürlich ist es auch möglich. aus Versehen - z. B. kontrolliert durch Algorithmen auf Videoplattformen - auf Onlinemedien mit 
wissenschaftlichem Hintergrund zu stoßen, weshalb das informelle Lernen mithilfe von Websites sowohl als intentional als auch als inzidentell eingestuft wird.

Ähnliches gilt für weitere mediale Formate wie Bücher; Magazine und Fernsehen. Oftmals entscheiden sich Konsumenten gezielt für den Kauf wissenschaftlicher Magazine (z. B. SPEKTRUM DER WISSENSCHAFT, SCIENTIA) oder anhand des Fernsehprogramms für das Schauen einer speziellen Sendung. Entsprechend kann hier von intentionalem Lernen ausgegangen werden. Stoßen Leser aber bspw. beim Warten in einer Arztpraxis auf ein entsprechendes Magazin, besitzt das Lernen einen deutlich inzidentelleren Charakter.

\section{(6) Inzidentelles Lernen in informellen Kontexten}

Bildungsmessen bzw. Wissenschaftsfestivals, international auch als Science Festivals bezeichnet, sind zumeist regelmäßig stattfindende Wissenschaftsausstellungen im öffentlichen Raum, die auf den ersten Blick zahlreiche Ähnlichkeiten zu Science-Centern aufweisen $[139,140]$. An verschiedenen interaktiven Ständen können Besucher_innen sich über aktuelle Forschungsthemen aus Wissenschaft und Industrie informieren und mit Wissenschaftler_innen verschiedener Disziplinen ins Gespräch kommen. Zudem werden oftmals weitere Aktivitäten wie Vorträge, Workshops und Science Shows angeboten [140]. Die Ausmaße dieser Events variieren von lokalen eintägigen bis hin zu professionellen ein- bis mehrwöchigen Veranstaltungen, die - wie im Falle der alle zwei Jahre stattfindenden IDEENEXPO in Hannover - fast 400.000 Besucher_innen ${ }^{[141]}$ zählen können. Laut BuLTITUDE, McDonaldS \& CUSTEAD dienen Science Festivals dazu, Wissenschaft, Technologie und Ingenieurwesen zu „feiern“ und dabei die interessierte Allgemeinheit zu einer Auseinandersetzung mit wissenschaftlichen Inhalten zu motivieren [140]. Zudem geben sie Jugendlichen Einblicke in vielfältige Berufe, heben sich jedoch aufgrund ihres interaktiven Charakters und der Möglichkeit zum Wissenserwerb deutlich von Studien- und Ausbildungsmessen ab. Trotz der vornehmlich geplanten Teilnahme der Besucher_innen geschieht das Lernen hauptsächlich inzidentell, da vor allem situationales Interesse Einfluss auf das Stehenbleiben und Informieren an verschiedenen Ständen der Ausstellung hat.

Eine weitere SO-Aktivität, die sowohl bei Bildungsmessen aber auch in anderen informellen und z. T. non-formalen Kontexten angeboten wird, ist der sogenannte Science Slam, der 2006 zum ersten Mal in Darmstadt stattfand [142]. In Anlehnung an Poetry Slams präsentieren hierbei Wissenschaftler_innen mithilfe kurzer populärwissenschaftliche Vorträge ihre Forschung. HILL beschreibt Science Slams wie folgt:

„SlammerInnen verdeutlichen die Relevanz ihres Forschungsgebiets häufig mit Bezügen zur alltäglichen Lebenswelt, veranschaulichen komplexe Sachverhalte mit Bildern aus dem Internet, erklären schwer verständliche Inhalte anhand von Metaphern und Analogien, beziehen das Publikum mit in ihren Vortrag ein und versuchen, witzig und locker zu wirken." [142]

Dabei ist dem Publikum normalerweise zuvor nicht bekannt, welche Thematiken von den Slammern vermittelt werden.

Ähnliche Formate finden sich auch bei Science in the Community-Projekten wieder. Zu diesen zählen Aktivitäten, die die Allgemeinheit an verschiedenen öffentlichen Orten mit wissenschaftlichen Themen konfrontieren. Die Projekte PINT OF SCIENCE, SCIENCE AND CockTAILS und NERD NITE verbinden einen geselligen Abend in einer Bar oder Kneipe mit der Vermittlung von naturwissenschaftlichen Themen anhand kurzer Vorträge [143]. Der ExPERIMENTELLE LEE(H)RSTAND hingegen nutzt leerstehende Verkaufsflächen in frequentierten Innenstädten um diese kurzzeitig in Veranstaltungsräume zu konvertieren, in denen SuS anhand verschiedener Formate der Öffentlichkeit naturwissenschaftliche Experimente 
präsentieren [144]. Eine weitere Möglichkeit, die Öffentlichkeit mit wissenschaftlichen Themen zu konfrontieren, stellen Posterkampagnen dar. Um den 400. Jahrestag des Teleskops von GALILEO zu feiern wurden beim FROM EARTH TO THE UnIVERSE-Projekt weltweit astronomische Bilder mit kurzen Beschreibungen an öffentlichen Orten wie Parks, UBahnstationen, Krankenhäusern und sogar in Gefängnissen aufgehängt [145]. Menschen die zufällig auf die Poster stießen konnten so selbst entscheiden, ob und wie lange sie sich mit den Bildern und ihren Hintergründen beschäftigten. Zu guter Letzt bieten sich auch beim Surfen im Internet (hier vor allem vermehrt auf verschiedenen Social Media Plattformen), Blättern in Magazinen oder Herumschalten im Fernsehen immer wieder spontan Möglichkeiten, sich mit wissenschaftlichen Themen auseinanderzusetzen. Im Gegensatz zur aktiven Suche nach Inhalten bestimmter Themenbereiche, erfolgt das Lernen hier jedoch inzidentell.

\subsubsection{Zielgruppenorientierung von Science Outreach-Aktivitäten}

So vielfältig die existierenden SO-Aktivitäten sind, so divers sind auch die daran teilnehmenden Personen, die alle ihre eigenen Interessen, Bedürfnisse, Einstellungen und Wissensstände mitbringen [102]. Zu Beginn der Entwicklung von SO-Aktivitäten empfiehlt BuLTITUDE deshalb, zunächst anhand von Faktoren wie Alter, Bildungsstand, sozioökonomischem Status und Interesse zu reflektieren, welche Personenkreise an den jeweiligen Aktivitäten wahrscheinlich teilnehmen werden [30]. Im Zuge des hier vorgestellten SO-Projektes wurden, basierend auf eigenen Erfahrungswerten und Erkenntnissen aus der Literatur, drei Zielgruppen anhand des Alters der Teilnehmer_innen festgelegt:

1. Kinder im Vor- und Grundschulalter

2. Kinder und Jugendliche ab der Sekundarstufe I

3. Die interessierte Allgemeinheit

Wie in Kapitel 3.1.2 dargestellt zeigen einige Studien, dass das Interesse von Kindern an den Naturwissenschaften bereits im Grundschulalter zu sinken beginnt [50, 51]. Deshalb erscheint es besonders wichtig, bereits früh situationales Interesse im Bereich der Naturwissenschaften zu wecken. Projekte, die sich explizit dieser Zielgruppe zuwenden, versteifen sich deshalb nicht auf das Vermitteln fachlicher Inhalte, sondern machen sich vor allem die Begeisterungsfähigkeit von jungen SuS zunutze, um ihr Interesse zu entfachen und aufrecht zu erhalten [146].

Ab der Sekundarstufe I beginnt in vielen Ländern der formale Unterricht in den einzelnen naturwissenschaftlichen Disziplinen. Entsprechend rekurrieren Konzepte für die zweite Zielgruppe oftmals auf curriculare Inhalte und Konzepte. Hier steht neben der generellen Interessenförderung vor allem die Kontextualisierung naturwissenschaftlicher Lerninhalte im Vordergrund, um der wahrgenommenen Abstraktheit der Wissenschaften entgegenzuwirken [81, 86]. Zudem rückt vermehrt die Berufsorientierung und das damit einhergehende Aufzeigen von Rollenvorbildern in den Fokus der Bemühungen.

Gerade bei öffentlichen Veranstaltungen beschränken sich die Teilnehmer_innen jedoch nicht nur auf SuS, so dass gerade im Bereich non-formaler und informeller Kontexte auch die interessierte Allgemeinheit in den Blick gefasst werden muss. Dabei geht es vor allem darum zu informieren, ohne dabei belehrend zu wirken. Um Personen jeden Bildungsgrads, mit und ohne naturwissenschaftlichem Vorwissen, einbeziehen zu können, müssen Aktivitäten flexibel gestaltet werden. Eine besonders wichtige Rolle fällt hier den Wissenschaftler_innen zu, die zunächst mithilfe von geschickten Fragen, Menschenkenntnis und viel Feingefühl das Vorwissen und Interesse der Besucher_innen ausloten müssen, damit 
sie in der Lage sind das Interesse der jeweiligen Person zu halten, um im besten Fall zu ihrer naturwissenschaftlichen Allgemeinbildung beizutragen.

\subsubsection{Science Outreach-Akteure}

Die Kommunikation von Forschungsergebnissen außerhalb der Wissenschaftscommunity kann nur gelingen, wenn es Personen gibt, die sich im SO-Bereich engagieren. BOYETTE \& RAMSEY identifizieren beispielsweise „praktizierende Wissenschaftler_innen, Ingenieur_innen, MINT-Lehrkräfte, Fachleute für Wissenschaftskommunikation und wissenschaftsbasierte kommerzielle Anbieter" als Berufsgruppen, die auf Bildungsmessen als Aussteller anzutreffen sind [147]. In ihrer Studie zur Rolle von Wissenschaftler_innen bei Bildungsmessen zeigen sie zudem, dass die Interaktion mit Wissenschaftler_innen einen positiven Einfluss auf die Gesamtbewertung solcher Messen durch die Besucher_innen hat. Eine Studie von Woods-TownSEND ET AL. mit SuS in einem non-formal inzidentellen MEET THE SCIENTIST Projekt zeigt zudem, dass die zuvor stereotypen Vorstellungen der SuS bezüglich Wissenschaftler_innen dem Bild von gewöhnlichen und zugänglichen Menschen weichen [148].

Von Seiten der SO-Adressat_innen scheint der direkte Kontakt mit Wissenschaftler_innen also eine wichtige Rolle für das Gelingen solcher Aktivitäten zu spielen. Inwiefern sehen sich jedoch Wissenschaftler_innen in der Verantwortung, SO zu betreiben, und welche Hürden nehmen sie diesbezüglich wahr? Poliakoff \& WEBB zeigen in einer Studie zum Willen von Wissenschaftler_innen an SO-Aktivitäten teilzunehmen, dass vier Faktoren einen signifikanten Einfluss haben: (i) früheres Verhalten (Umfang der vorherigen Partizipation an SO), (ii) Einstellung (Wahrnehmung von SO als positiv oder negativ), (iii) wahrgenommene Verhaltensweisen (Wahrnehmung der eigenen Fähigkeiten zur Teilnahme an SO) und (iv) deskriptive Normen (inwiefern Wissenschaftler_innen wahrnehmen, dass ihre Kolleg_innen sich an SO beteiligen) [101]. Andere Studien zeigen zudem, dass ältere Wissenschaftler_innen eher SO betreiben als jüngere Wissenschaftler_innen [149, 150], wobei Online SO-Aktivitäten wiederum vor allem von jüngeren Wissenschaftlern_innen betrieben werden [151]. Zudem scheinen männliche Wissenschaftler eher gewillt, an SO teilzunehmen, wobei die Unterschiede hier zumeist nicht signifikant sind [151].

In einer Studie der RoYal SOCIETY identifizieren die teilnehmenden Wissenschaftler_innen jedoch auch einige Hürden in Bezug auf Wissenschaftskommunikation. So geben $64 \%$ der Befragten an, dass ihnen die Zeit für SO fehlt, da sie diese für ihre Forschung benötigen [149]. Zudem werden die „eigenen Kommunikationskompetenzen, mangelnde institutionelle Unterstützung und die Auffassung, dass SO eine wertlose Tätigkeit für die anspruchsvollsten Wissenschaftler_innen darstellt" von JOHNSON, ECKLUND \& LINCOLN als weitere hinderliche Faktoren zusammengefasst [152].

\subsubsection{Evaluierung von Science Outreach-Aktivitäten}

In der naturwissenschaftsdidaktischen Forschung ist es üblich, mithilfe qualitativer und quantitativer Methoden die Effekte verschiedener Interventionen, beispielsweise hinsichtlich Interessen- und Motivationsförderung oder Lernzuwachs, zu untersuchen. Auch die Evaluierung von SO-Aktivitäten ist wünschenswert. Die große Anzahl an Projekten, die trotz ähnlicher Ziele häufig nicht miteinander vergleichbar sind, macht es jedoch schwierig, Handlungsempfehlungen für die Untersuchung ihrer Effektivität auszusprechen. Dies ist weder für die Aktivitäten im Allgemeinen möglich, noch können einheitliche Empfehlungen für die in Kapitel 3.3.1.1 postulierten SO-Bildungskontexte formuliert werden, da sich auch innerhalb dieser Kategorien die Konzepte stark unterscheiden. Insbe- 
sondere der Einfluss eher unstrukturierter Aktivitäten, wie der Besuch von Science-Museen oder Bildungsmessen ist gegenüber strukturierteren Aktivitäten, wie dem Besuch eines Schülerlabors, schwierig zu messen. Dies ist besonders der Offenheit dieser Aktivitäten geschuldet.

Der Besuch eines Schülerlabors durch eine Schulklasse erfolgt beispielsweise im Rahmen eines bestimmten Themas. Im Zuge dieses Besuches ist es möglich, zunächst Informationen wie Schulform, Klassenstufe, Herkunft (in Bezug auf das Bundesland), Geschlechterverteilung und eine Einschätzung bezüglich des Lernstands der Klasse durch die Lehrkraft einzuholen. Zudem ermöglichen Pre-Tests eine Erhebung des Vorwissens und der Einstellungen der SuS hinsichtlich der Thematik des Schülerlabors. Mithilfe eines PostTests kann im Anschluss an die Aktivität der Erfolg des Labors bezüglich verschiedener Faktoren gemessen werden und Follow Up-Tests ermöglichen es zudem, Aussagen über die Nachhaltigkeit der Labore zu treffen.

Im Kontrast dazu beginnen die Schwierigkeiten in Bezug auf die Evaluation von Bildungsmessen bereits beim Erheben der Besucher_innenzahlen, insbesondere dann, wenn der Besuch kostenlos ist und dadurch keine Aussagen anhand von Ticketverkäufen gemacht werden können [140]. Zudem fällt es schwer, Faktoren wie Motivation, Interesse und Lernzuwachs zu messen, da sich die Teilnehmer_innen hinsichtlich Attributen wie Alter, Geschlecht, Vorwissen und sozioökonomischem Status unterscheiden. Zudem variieren die Zeit, die sie auf der Messe verbringen, die Themen und Aktivitäten, mit denen sich die Besucher_innen beschäftigen, und auch die Intensität der Auseinandersetzung mit den präsentierten Inhalten. Es kann also, im Gegensatz zum Schülerlabor, nicht davon ausgegangen werden, dass alle Teilnehmer_innen den gleichen Vortrag gehört und die gleichen Experimente durchgeführt haben - eine Vergleichbarkeit des Erlebnisses ist also nicht gegeben. Dies gilt insbesondere für alle eher unstrukturierten SO-Aktivitäten [153].

Der Vergleich von Evaluationen zu SO-Aktivitäten der verschiedenen Bildungskontexte zeigt, dass Fragebogenstudien am häufigsten verwendet werden, um die unterschiedlichen Projekte bezüglich verschiedener Aspekte zu untersuchen (siehe Tabelle 3). Dabei fällt auf, dass insbesondere Prozesse intentionalen Lernens in formalen und non-formalen Kontexten in Längsschnittstudien untersucht werden und dass die meisten Publikationen in diesem Bereich Evaluationsergebnisse enthalten. Aktivitäten inzidentellen Lernens und informelle Projekte werden hingegen deutlich seltener evaluiert und zumeist in Querschnittstudien untersucht. Während die Längsschnittstudien sich zudem oftmals nur der Untersuchung einer Variable widmen (z. B. Interesse), sind in Querschnittstudien häufig mehrere Variablen Gegenstand der Forschung, anhand derer sowohl Merkmale der Teilnehmer_innen (u. a. Interesse, Motivation, Relevanzempfinden, Lernzuwachs), als auch die Aktivität selber untersucht werden sollen. Diese Art der Evaluation dient vor allem der Legitimierung der SO-Projekte mit Blick auf weitere finanzielle Förderungen.

Aufgrund der großen Anzahl und Vielfalt der Aktivitäten ist es kaum möglich, allgemeine Aussagen über die Evaluationsergebnisse zu treffen. Jedoch werden SO-Projekte in der Regel vorwiegend positiv bewertet. Mit Blick auf die Evaluationsdesigns der verschiedenen Aktivitäten müssen diese Ergebnisse jedoch kritisch betrachtet werden. Die Neuartigkeit der Umgebung oder des Lerngegenstands kann das situationale Interesse durchaus beeinflussen, gerade einmalige Interventionen und entsprechend auch einmalige Evaluationen lassen jedoch keine Aussagen über die Nachhaltigkeit der SO-Aktivitäten zu [154]. Zwar existieren einige Studien zur Nachhaltigkeit von Schülerlaboren [155-157], jedoch fehlen diesbezüglich Daten hinsichtlich anderer SO-Bildungskontexte. 
Tabelle 3: Vergleich verschiedener Evaluationsformen hinsichtlich der unterschiedlichen SO-Bildungskontexte

\begin{tabular}{|c|c|c|c|c|c|}
\hline & Autoren & So-Aktivität & Übergeordnetes Forschungsinteresse & Teilnehmer_innen & Design \\
\hline \multirow{2}{*}{ 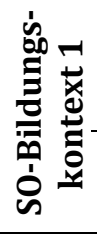 } & $\begin{array}{l}\text { Long et al. } \\
\text { (2012) [118] }\end{array}$ & $\begin{array}{l}\text { Mobiles Labor I, Ex- } \\
\text { perimentierkoffer }\end{array}$ & Wissenserwerb & $\begin{array}{l}\text { Lehrkräfte, SuS (im weitesten } \\
\text { Sinne) }\end{array}$ & $\begin{array}{l}\text { Beobachtungen, Inter- } \\
\text { views, Fokusgruppen, Fra- } \\
\text { gebogen (Post) }\end{array}$ \\
\hline & $\begin{array}{l}\text { Thomas } \\
\text { (2012) [158] }\end{array}$ & Mobiles Labor I & Wissenserwerb & SuS & Fragebogen (Pre, Post) \\
\hline \multirow{2}{*}{ 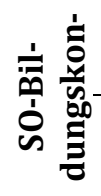 } & $\begin{array}{l}\text { Clark et al. } \\
(2016)^{[124]}\end{array}$ & $\begin{array}{l}\text { Wissenschaftler_in } \\
\text { in der Schule }\end{array}$ & Interesse & Wissenschaftler_innen, SuS & Fragebogen (Post) \\
\hline & $\begin{array}{l}\text { Laursen et al. } \\
(2007)^{[123]}\end{array}$ & $\begin{array}{l}\text { Wissenschaftler_in } \\
\text { in der Schule }\end{array}$ & Interesse, Wissenserwerb & Wissenschaftler_innen, Lehrkräfte & Interviews (Post) \\
\hline \multirow{9}{*}{ 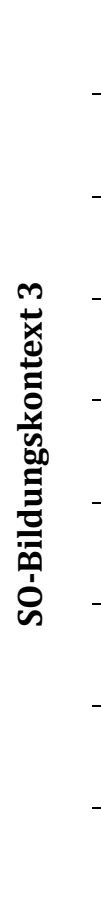 } & $\begin{array}{l}\text { Brand et al. } \\
\text { (2008) [159] }\end{array}$ & Schülerlabor & Selbstkonzept, Interesse & $\begin{array}{l}\text { SuS, } \\
\text { Kontrollgruppe }\end{array}$ & $\begin{array}{l}\text { Fragebogen (Pre, Post, } \\
\text { Follow Up) }\end{array}$ \\
\hline & $\begin{array}{l}\text { Budke et al. } \\
\text { (2017) [129] }\end{array}$ & $\begin{array}{l}\text { Schülerlabor, Mobi- } \\
\text { les Labor II }\end{array}$ & Interesse, Selbstkonzept & SuS & $\begin{array}{l}\text { Fragebogen (Pre, Post, } \\
\text { Follow Up) }\end{array}$ \\
\hline & $\begin{array}{l}\text { Glowinski } \\
(2007)^{[160]}\end{array}$ & Schülerlabor & Interesse & SuS & $\begin{array}{l}\text { Fragebogen (Post, Follow } \\
\text { Up) }\end{array}$ \\
\hline & $\begin{array}{l}\text { Guderian } \\
(2006)^{[156]}\end{array}$ & Schülerlabor & Interesse & SuS & Fragebogen (Pre, Post) \\
\hline & $\begin{array}{l}\text { Huwer } \\
(2015)[155]\end{array}$ & $\begin{array}{l}\text { Schülerlabor, Mobi- } \\
\text { les Labor II }\end{array}$ & Motivation, Wissenserwerb & $\begin{array}{l}\text { SuS, } \\
\text { Kontrollgruppe }\end{array}$ & $\begin{array}{l}\text { Fragebogen (Pre, Post, } \\
\text { Follow Up) }\end{array}$ \\
\hline & $\begin{array}{l}\text { Markowitz } \\
(2014)^{[161]}\end{array}$ & Science Camp & Interesse & Ehemalige SuS & Fragebogen (Follow Up) \\
\hline & $\begin{array}{l}\text { Pawek } \\
(2009)^{[162]}\end{array}$ & Schülerlabor & Interesse & SuS & $\begin{array}{l}\text { Fragebogen (Pre, Post, } \\
\text { Follow Up) }\end{array}$ \\
\hline & $\begin{array}{l}\text { Penn et al. } \\
(2007)[163]\end{array}$ & Science Camp & Konzeptverständnis & $\mathrm{SuS}$ & $\begin{array}{l}\text { Zeichnungen, Interviews } \\
\text { (Pre, Post) }\end{array}$ \\
\hline & $\begin{array}{l}\text { Priemer et al. } \\
\text { (2018) [164] }\end{array}$ & Schülerlabor & Interesse & SuS & Fragebogen (Post) \\
\hline
\end{tabular}




\begin{tabular}{|c|c|c|c|c|c|}
\hline & $\begin{array}{l}\text { Priemer et al. } \\
(2014)^{[165]}\end{array}$ & Schülerlabor & Interesse & $\mathrm{SuS}$ & Metaanalyse \\
\hline & $\begin{array}{l}\text { Simon } \\
(2019)^{[157]}\end{array}$ & Schülerlabor & Interesse, Selbstkonzept & Wissenschaftler_innen, SuS & Fragebogen (Pre, Post) \\
\hline & $\begin{array}{l}\text { Streller } \\
(2015)^{[166]}\end{array}$ & Schülerlabor & Interesse, Selbstkonzept & $\mathrm{SuS}$ & $\begin{array}{l}\text { Fragebogen (Pre, Post, } \\
\text { Follow Up) }\end{array}$ \\
\hline \multirow{3}{*}{ 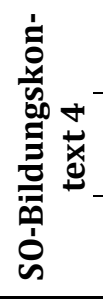 } & $\begin{array}{l}\text { Kerby et al. } \\
(2010)^{[134]}\end{array}$ & $\begin{array}{l}\text { Wissenschaftliches } \\
\text { Theater }\end{array}$ & Wissenserwerb & Kinder/SuS (4-12 Jahre) & Fragebogen (Pre, Post) \\
\hline & $\begin{array}{l}\text { Lewalter et al. } \\
\text { (2009) [132] }\end{array}$ & Science-Museum & Motivation, Relevanz, Interesse & Lehrkräfte, SuS & Fragebogen (Post) \\
\hline & $\begin{array}{l}\text { Neubauer } \\
(2015)^{[167]}\end{array}$ & Science-Museum & Interesse, Wissenserwerb & $\mathrm{SuS}$ & $\begin{array}{l}\text { Fragebogen (Pre, Zwi- } \\
\text { schen, Post) }\end{array}$ \\
\hline \multirow{3}{*}{ 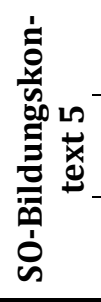 } & $\begin{array}{l}\text { Raddick et al. } \\
(2010)^{[168]}\end{array}$ & Citizen Science & Motivation, Demografie & Teilnehmer_innen & Interviews \\
\hline & $\begin{array}{l}\text { Ruiz-Mallén et } \\
\text { al. (2016) [169] }\end{array}$ & Citizen Science & Interesse, Wissenserwerb & SuS & Fokusgruppe \\
\hline & $\begin{array}{l}\text { Silva et al. } \\
\text { (2016) [135] }\end{array}$ & Citizen Science & Interesse, Motivation, Wissenserwerb & Lehrkräfte, SuS & $\begin{array}{l}\text { Fragebogen, Fokusgruppe } \\
\text { (Post) }\end{array}$ \\
\hline \multirow{3}{*}{ 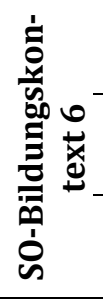 } & $\begin{array}{l}\text { Boyette et al. } \\
(2019)^{[147]}\end{array}$ & Bildungsmesse & Wissenserwerb, Demografie & Besucher_innen ab 14 Jahren & Fragebogen (Post) \\
\hline & $\begin{array}{l}\text { Illingworth et } \\
\text { al. (2015) }{ }^{[170]}\end{array}$ & Bildungsmesse & Interesse & Lehrkräfte, SuS & Fragebogen (Post) \\
\hline & $\begin{array}{l}\text { Jensen et al. } \\
(2014)^{[139]}\end{array}$ & Bildungsmesse & Interesse, Motivation, Demografie & Besucher_innen & $\begin{array}{l}\text { Fragebogen, Fokusgruppe } \\
\text { (Post, Follow Up) }\end{array}$ \\
\hline \multirow{3}{*}{ 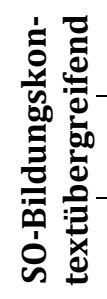 } & $\begin{array}{l}\text { Potvin et al. } \\
(2014)^{[39]}\end{array}$ & Versch. SO & Interesse, Motivation & $\mathrm{SuS}$ & Metaanalyse \\
\hline & $\begin{array}{l}\text { Vennix et al. } \\
\text { (2018) [171] }\end{array}$ & Versch. SO & Motivation, Interesse & $\mathrm{SuS}$ & Fragebogen (Post) \\
\hline & $\begin{array}{l}\text { Young et al. } \\
\text { (2017) [121] }\end{array}$ & Versch. SO & Interesse & SuS & Metaanalyse \\
\hline
\end{tabular}




\subsection{Fazit}

Innerhalb dieses Kapitels wurde dargelegt, dass von Seiten der Politik eine immer stärkere Beteiligung der Gesellschaft an wissenschaftlichen Prozessen eingefordert wird, ein Großteil der Gesellschaft sich diesbezüglich jedoch noch nicht ausreichend informiert fühlt. Das liegt u. a. auch daran, dass wissenschaftliche Erkenntnisse zumeist für den Laien oft unzugänglich und zudem nur schwer verständlich publiziert werden. Zudem fällt es bezüglich populärwissenschaftlich aufgearbeiteter Informationen oft schwer, die Glaubwürdigkeit der zahlreichen Quellen einzuschätzen.

Der Grundstein für eine gewünschte naturwissenschaftliche Grundbildung der Gesellschaft wird in der Schule gelegt. Zahlreiche Studien zeigen jedoch, dass das Interesse an den MINT-Fächern mit zunehmenden Alter sinkt. Dabei wird hinsichtlich der fachlichen Inhalte eine fehlende Kontextualisierung bemängelt, während der naturwissenschaftlichen Forschung gleichzeitig eine hohe Relevanz für die Gesellschaft zugeschrieben wird. Das mangelnde Interesse von SuS an naturwissenschaftlichen Berufsfeldern wird zudem auch durch vorherrschende stereotype Vorstellungen bezüglich Wissenschaftler_innen und der wissenschaftlichen Forschung bedingt.

Im Rahmen des schulischen Unterrichts existieren zwar zahlreiche didaktische Konzepte, die sich der Kontextualisierung des Unterrichts verschrieben haben, gleichzeitig zeigen die Analysen von Schulbüchern und Rahmenlehrplänen jedoch, dass es oft viele Jahre dauert, bis neue Erkenntnisse aus der Forschung in schulischen Lehrwerken verankert werden und dass aktuelle Forschungsergebnisse in der Schule kaum eine Rolle spielen.

Über den Unterricht hinaus können sich jedoch auch außerschulische Lernorte positiv auf die Interessengenese auswirken. Entsprechend bieten Projekte wissenschaftlicher Öffentlichkeitsarbeit, die in dieser Arbeit erstmalig hinsichtlich ihrer Kontext- und Subjektperspektiven systematisiert werden, die Möglichkeit, sowohl SuS als auch die Allgemeinheit bezüglich aktueller Forschungserkenntnisse zu informieren und für die Naturwissenschaften zu begeistern. Aufbauend auf diese Erkenntnisse soll nachfolgend am Beispiel des Sonderforschungsbereichs 803 der Universität Göttingen untersucht werde, welche Konzepte und Formate sich eigenen, um fachwissenschaftliche Inhalte aktueller Grundlagenforschung für SuS und die Öffentlichkeit zugänglich zu machen. 


\section{Sonderforschungsbereich 803}

Sonderforschungsbereiche sind von der DEUTSCHEN FORSCHUNGSGEMEINSCHAFT (DFG) geförderte „langfristig, auf die Dauer von bis zu zwölf Jahren angelegte Forschungseinrichtungen der Hochschulen, in denen Wissenschaftler[_innen] im Rahmen eines fächerübergreifenden Forschungsprogramms zusammenarbeiten“ [172]. Innerhalb solcher Forschungseinrichtungen arbeiten verschiedene Forschergruppen über die Grenzen ihrer jeweiligen Fächer, Institute, Fachbereiche und Fakultäten hinweg in zahlreichen Teilprojekten an einem wissenschaftlich exzellenten Forschungsprogramm. Die Länge der Förderperioden beträgt dabei jeweils vier Jahre, wobei die Projekte um weitere zwei Förderperioden verlängert werden können. Dadurch ist u. a. eine Beantragung weiterer Teilprojekte möglich, deren Ausrichtungen sich oftmals aus der vorangegangen Forschung ergeben. Zurzeit fördert die DFG 275 SFBs, von denen zehn der Universität Göttingen zugeordnet sind (Stand 6/2020) [173]. Zu diesen zählt auch der seit 2009 unter der Leitung von Prof. Dr. Claudia Steinem und Prof. Dr. Ulf Diederichsen geförderte Sonderforschungsbereich 803 (SFB 803).

\subsection{Forschung im SFB 803}

Der SFB 803 trägt den Titel „Funktionalität kontrolliert durch Organisation in und zwischen Membranen " (Functionality controlled by organization in and between membranes) und befindet sich zurzeit in der dritten und somit letzten Förderperiode. Innerhalb des Forschungsprogramms erfolgt die Grundlagenforschung an biomimetischen Membranen dabei interdisziplinär sowohl an verschiedenen Fakultäten (Chemie, Physik, Biologie und Psychologie, Mathematik und Informatik) sowie der UNIVERSITÄTSMEDIZIN der Universität Göttingen, als auch an der FAKULTÄT FÜR LEBENSWISSENSCHAFTEN der Technischen Universität Braunschweig und dem Göttinger MAX-PlanCK-InstiTUT FÜR BIOPHYSIKALISChE ChEMIE. Ziel des SFB ist es dabei, die Wechselwirkungen von Membranlipiden und -proteinen auf molekularer Ebene zu verstehen. Dazu soll sowohl der Einfluss der räumlichen als auch der zeitlichen Anordnung der Membranbestandteile untersucht werden [174].

Der Fokus der Forschung liegt auf zwei übergeordneten Themenkomplexen. Die dem Themenkomplex A zugeordneten Teilprojekte beschäftigen sich mit der „Organisation und Funktion von Peptiden und Proteinen in Lipidmembranen “ [174]. Von Interesse sind hier beispielsweise allgemeine Konzepte der Selbstorganisation von Transmembran-Peptidhelices als Modelle für die Organisation von Proteinen in Lipidmembranen (siehe Abbildung 10) sowie die Struktur-Funktionsbeziehungen von ionenleitenden Kanälen wie dem K2P K+ Kanal, der eine Rolle bei der Signalkaskade des menschlichen Tastsinns spielt. 

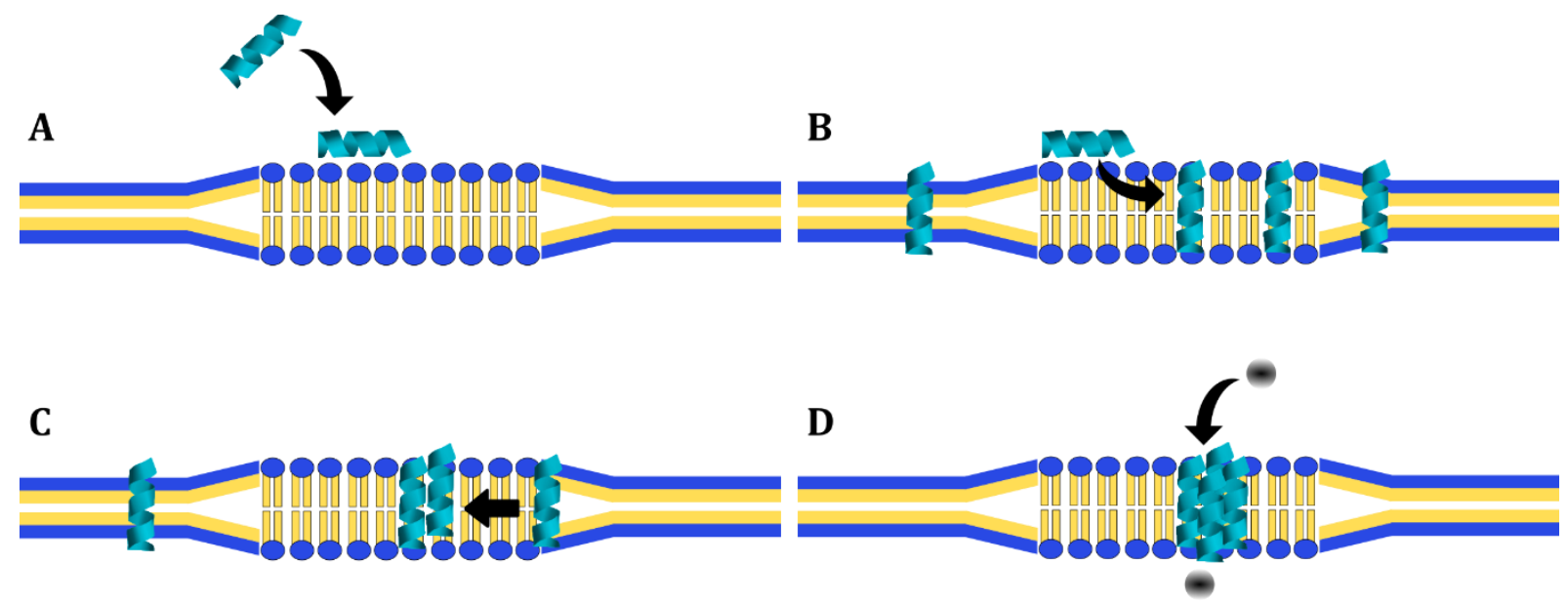

Abbildung 10: Schematische Darstellung der Interaktion von Peptiden mit Membranen (A). Weiterhin sind die Anreicherung und Insertion in die Membran (B), die Aggregation (C) und schließlich die Bildung von Kanälen (D) für den Transport von Stoffen über die Membran abgebildet [175].

Die Forschung der Teilprojekte des Themenkomplexes B widmet sich hingegen Molekülstrukturen, die im Zusammenhang mit Membran-Membran Interaktionen stehen. Das Ziel ist es hierbei vornehmlich, durch SNARE-Proteine gesteuerte Fusionsprozesse, wie die an den Synapsenenden ablaufende Fusion von neurotransmittergefüllten Vesikeln mit der synaptischen Membran (siehe Abbildung 11), zu entschlüsseln und eine Verbindung zwischen der molekularen Struktur, der Lipidzusammensetzung sowie der Membranmechanik herzustellen. Zur Klärung der übergeordneten Fragen werden in den Teilprojekten u. a. molekulardynamische Simulationen sowie biomimetische Membranen genutzt. Letztere bilden als Modelle biologischer Membranen ausgewählte Eigenschaften dieser ab und ermöglichen somit eine exakte Aufklärung einzelner Prozesse hinsichtlich komplexer biologischer Systeme.

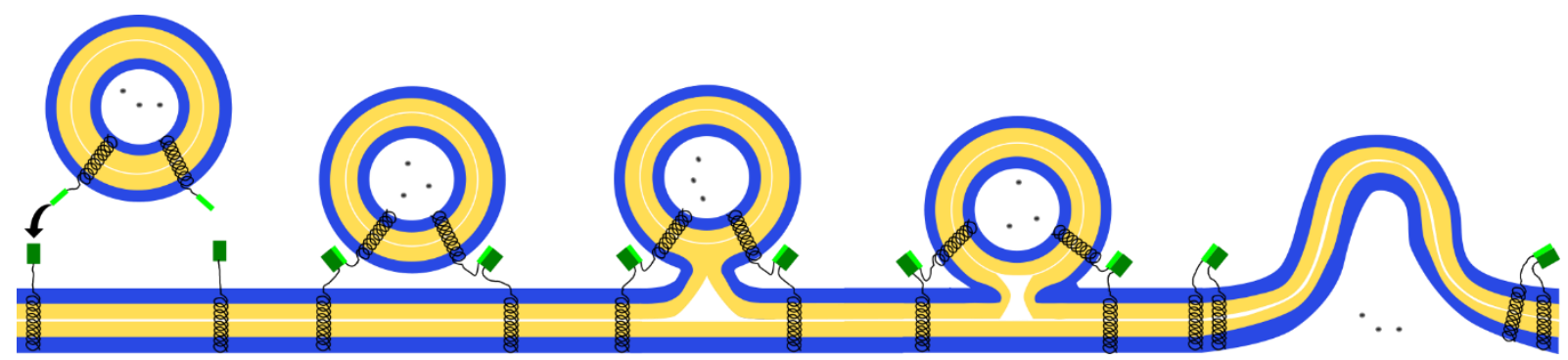

Abbildung 11: Schematische Darstellung der SNARE-vermittelten Fusion eines Vesikels mit einer Lipidmembran. Der im Vesikel eingeschlossene Inhalt kann nach Fusion der Membranen ausgeschüttet werden [175].

Insgesamt wird im SFB 803 während der dritten Förderperiode an neun Teilprojekten im Themenkomplex A (zwei weitere sind bereits abgeschlossen) und an acht Projekten im Komplex B geforscht, die zudem Unterstützung durch zwei Serviceprojekte erfahren. Während eines dieser Projekte vor allem administrative Aufgaben wahrnimmt, wirkt das wissenschaftliche Projekt Z02 unterstützend bei der mathematischen Datenanalyse, beispielsweise von Patch-Clamp-Aufnahmen. Seit der letzten Förderperiode wird zusätzlich ein Öffentlichkeitsarbeits-Projekt gefördert, das der Wissenschaftskommunikation in Schule und Gesellschaft dienen soll. 


\section{2 Öffentlichkeitsarbeits-Projekt}

Moderne Wissensgenerierung in der Grundlagenforschung, wie sie im SFB 803 erfolgt, wird bislang in der Öffentlichkeit und so auch im Schulkontext kaum wahrgenommen. Dies ist zum einen mit einer unzureichenden naturwissenschaftlichen Grundbildung der Gesellschaft sowie einer mangelnden Kommunikation von Forschungsergebnissen, die auch für Laien verständlich ist, zu begründen (vgl. Kapitel 3.1.1). Zum anderen dauert es oftmals sehr lange, bis Erkenntnisse aus der Grundlagenforschung, zumeist im Rahmen angewandter Forschung, zu Innovationen führen, die wiederum der Öffentlichkeit eindrücklicher präsentiert werden können [176].

Wie zu Beginn des Kapitels 3 dargelegt, gewinnt jedoch der Dialog zwischen Forschung und Gesellschaft (auch politisch motiviert) zunehmend an Bedeutung. Um die Forschung des SFB anhand verschiedener Projekte einer breiten Öffentlichkeit zugänglich zu machen, wurde das Öffentlichkeitsarbeits-Projekt (Ö-Projekt) mit dem Titel „Making Science Visible - Information, Motivation and Participation in Formal and Non-formal Settings"entwickelt. Ziel des Projektes ist es, die Forschungsinhalte des SFB zielgruppengerecht aufzuarbeiten und in verschiedene SO-Aktivitäten einzubetten. Dabei geht es nicht allein darum, über die Inhalte des SFB zu informieren. Vielmehr soll die Auseinandersetzung mit aktuellen wissenschaftlichen Themen das situationale Interesse bezüglich der Naturwissenschaften bzw. im Speziellen der Membranforschung gefördert werden, um vornehmlich SuS dazu zu motivieren, sich intensiver mit naturwissenschaftlichen Themen zu beschäftigen. Verschiedene Möglichkeiten der Partizipation, wie die aktive Teilhabe an der wissenschaftlichen Arbeit, z. B. in Form von Facharbeiten, aber auch der Teilhabe am wissenschaftlichen Diskurs, u. a. im Rahmen von Bildungsmessen, sollen diesen Prozess weiterhin unterstützen.

Mit Bezug auf die in Kapitel 3.3.1.2 genannten Zielgruppen von SO-Aktivitäten und den formulierten Zielen des Ö-Projektes wurde der Fokus auf die zweite Gruppe der Kinder und Jugendlichen ab der Sekundarstufe I gelegt, wobei dieser hinsichtlich SuS der Sekundarstufe II noch weiter ausdifferenziert wurde [177]. Zwar scheint mit Blick auf die Interessenentwicklung von SuS eine möglichst frühe Interessenförderung bereits im Grundschulalter sinnvoll, aufgrund der Spezifität des Themenbereiches des SFB und mögliche Anknüpfungspunkte an die Kerncurricula der Sekundarstufe II, sowohl in den Fächern Biologie als auch Chemie, fiel die Entscheidung jedoch zugunsten Gruppen älterer SuS. Dennoch wenden sich gewisse Aktivitäten wie Bildungsmessen auch an jüngere SuS sowie die interessierte Allgemeinheit. Des Weiteren wurde im Sinne der Nachhaltigkeit des ÖProjektes auch die sich als Teilmenge der interessierten Allgemeinheit ergebende Gruppe der Lehrkräfte in den Blick genommen. Diese können zum einen in ihrer Rolle als Gestalter von Lernprozessen als Multiplikatoren dienen und zum anderen anhand von Feedback wichtige Impulse für die Optimierung der entwickelten Materialien und Aktivitäten liefern.

Die allgemeine Idee des Ö-Projektes basiert auf dem Vorläuferprojekt SFB@ScHooL, im Zuge dessen eine Unterrichtseinheit mit dem Titel „Vom Tensid zur Biomembran “ ${ }^{[178]}$ sowie ein erstes korrespondierendes Bildungsmessekonzept entwickelt wurden. Anknüpfend an diese Vorarbeiten wurden Themen wie Medikamentenwirkung und -entwicklung, Ursachen von Krankheiten, Wirkung von Giften sowie die Arbeitsweisen von Wissenschaftler_innen als mögliche Themen der Öffentlichkeitsarbeit des SFB identifiziert [179], die in Anlehnung an das Modell der fachdidaktischen Transferforschung (vgl. Abbildung 12) in enger Zusammenarbeit von Fachdidaktik und Fachwissenschaft inhaltlich und didaktisch aufbereitet und in verschiedene Aktivitäten eingebettet wurden [175]. 


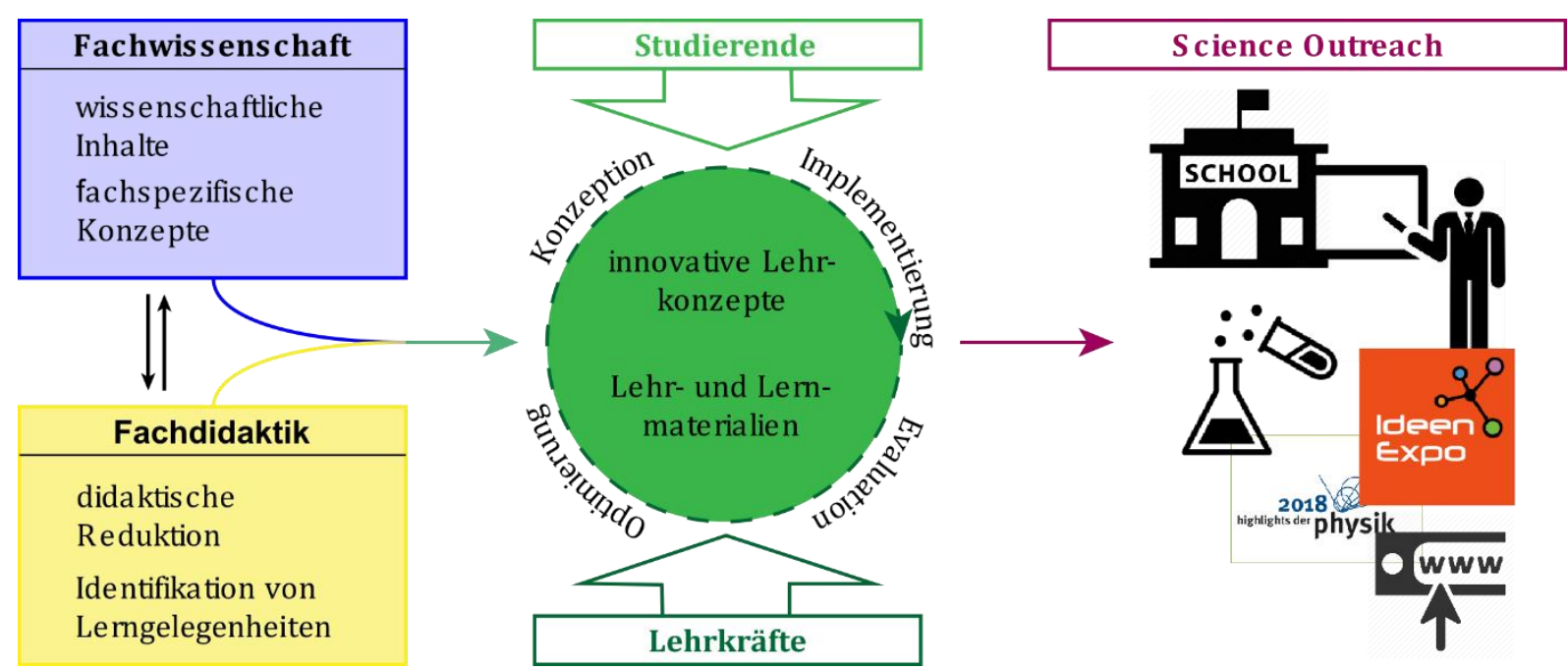

Abbildung 12: Modell der Fachdidaktischen Transferforschung in Bezug auf Science Outreach adaptiert nach WILKE, DEGE \& WAITZ [180].

Bis auf den informell-intentionalen Bildungskontext, der sich im Rahmen der Öffentlichkeitsarbeit von Forschungsgruppen wie dem SFB 803 höchstens in Form von Citizen Science-Projekten umsetzen ließe, die im Kontext der Membranforschung jedoch nicht sinnvoll erschienen, decken die innerhalb des Ö-Projekts entwickelten Aktivitäten alle anderen Bildungskontexte ab (vgl. Tabelle 4). Während sich Formate wie Unterrichtseinheiten, Schülerlabore und Science Camps, Facharbeiten, Adaptierte Primärliteratur und der $\mathrm{Zu}-$ kunftstag deutlich an die Zielgruppe der SuS wenden, bieten die Nacht des Wissens, Bildungsmessen und Social Media Angebote auf Instagram und Twitter sowie eine Website auch der Allgemeinheit die Möglichkeit, sich über die Forschung im SFB zu informieren. Auf Fortbildungen konnten sich zudem auch Lehrkräfte über die interdisziplinäre curriculare Einbindung von Forschungsthemen in den naturwissenschaftlichen Unterricht informieren.

Tabelle 4: SO-Projekte des SFB 803 differenziert hinsichtlich der sechs SO-Bildungskontexte.

\begin{tabular}{|c|c|c|c|c|}
\hline & & & \multicolumn{2}{|c|}{ Subjektperspektive auf das Lernen } \\
\hline & & & Intentionales Lernen & Inzidentelles Lernen \\
\hline \multirow{6}{*}{ 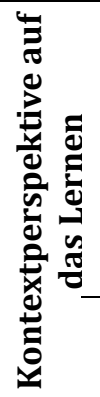 } & \multirow{5}{*}{$\begin{array}{l}\text { Kontexte mit } \\
\text { Organisations- } \\
\text { bezug }\end{array}$} & Formal & (1) Unterrichtsmaterialien & $\begin{array}{l}\text { (2) Adaptierte } \\
\text { Primärliteratur }\end{array}$ \\
\hline & & & (3) Schülerlabore & (4) Nacht des Wissens \\
\hline & & & Science Camps & Zukunftstag \\
\hline & & Non-rormal & Facharbeiten & \\
\hline & & & Lehrerfortbildungen & \\
\hline & $\begin{array}{l}\text { Lebensweltliche } \\
\text { Kontexte }\end{array}$ & Informell & (5) & $\begin{array}{l}\text { (6) Bildungsmessen } \\
\text { So-ial Media }\end{array}$ \\
\hline
\end{tabular}

Nachfolgend wird in Kapitel 5 zunächst die didaktische Aufarbeitung des Themas der Biomembranforschung präsentiert, bevor in Kapitel 6 die Einbettung dieser Inhalte in vier SO-Formate dargelegt und anhand entsprechender Evaluationsergebnisse hinsichtlich ihrer Eignung als Formate der Öffentlichkeitarbeit des SFB 803 reflektiert wird. 


\section{Didaktische Erschließung der Forschung des SFB 803}

Mit Blick auf Oberstufenschüler_innen als die primäre Zielgruppe der zu entwickelnden SO-Aktivitäten wurden zunächst die Kerncurricula ${ }^{9}$ der Sekundarstufe I und II für die Fächer Biologie und Chemie ${ }^{[181-183]}$ auf inhalts- und prozessbezogene Kompetenzen hin untersucht, die für eine interdisziplinäre Aufarbeitung der Forschungsinhalte des SFB 803 eine Rolle spielen. Dabei ging es zum einen darum zu erfassen, welche Kompetenzen in welchen Jahrgangsstufen vorausgesetzt werden können. Zum anderen sollten die Ergebnisse der Analyse aber auch hinsichtlich der interessierten Allgemeinheit als ein Indiz für das von der Öffentlichkeit zu erwartende Vorwissen dienen.

Im Biologieunterricht der Sekundarstufe I geht es vordergründig um den Bau und die Funktion von Zellen, die als Grundeinheiten verstanden werden sollen (vgl. Tabelle 5). Dabei werden pro- und eukariotische Zellen anhand ihrer Zellbestandteile unterschieden. Ihre Betrachtung beschränkt sich hierbei vornehmlich auf lichtmikroskopische Aufnahmen. In der Oberstufe kommt die Betrachtung elektronenmikroskopischer Aufnahmen hinzu und es rücken zellinterne und zellübergreifende Prozesse in den Vordergrund. Membranen und ihre Bestandteile werden in diesem Zuge zwar behandelt und auch Membranmodelle wie das Flüssig-Mosaik-Modell diskutiert, insgesamt werden sie aber modellhaft als kompartimentbildende Einheiten verstanden, die u. a. die Ausbildung von Gradienten ermöglichen, gleichzeitig aber auch Transportprozesse nötig machen. Zudem sollen die Struktur-Funktionsbeziehungen von Phospholipiden zwar behandelt werden, ihre Betrachtung erfolgt jedoch nur modellhaft.

Tabelle 5: Nach Kompetenzen geordnete Lernziele aus dem niedersächsischen Kerncurriculum Biologie [181, 182], die bezüglich der SO-Aktivitäten des SFB 803 von Relevanz sind ${ }^{10}$. EP = Einführungsphase, QP = Qualifikationsphase.

\begin{tabular}{|c|c|c|}
\hline Kompetenz & Lernziel & Phase \\
\hline \multirow{2}{*}{$\begin{array}{l}\text { Mit Modellen } \\
\text { arbeiten }\end{array}$} & ... erläutern biologische Sachverhalte mithilfe von Modellen & $\mathrm{EP}, \mathrm{QP}$ \\
\hline & $\begin{array}{l}\text {... wenden Modelle an, erweitern sie und beurteilen die Aussagekraft und Gül- } \\
\text { tigkeit (Flüssig-Mosaik-Modell (EP)) }\end{array}$ & $\mathrm{EP}, \mathrm{QP}$ \\
\hline \multirow[t]{2}{*}{$\begin{array}{l}\text { Struktur und } \\
\text { Funktion }\end{array}$} & $\begin{array}{l}\text {... beschreiben den Bau und die wesentlichen Eigenschaften biologisch bedeut- } \\
\text { samer Moleküle (Lipide, Proteine, Nucleinsäuren) }\end{array}$ & $\mathrm{EP}$ \\
\hline & $\begin{array}{l}\text {... erläutern Struktur-Funktionsbeziehungen auf der Ebene von Molekülen mo- } \\
\text { dellhaft (Phospholipide, komplementäre Basen der DNA) }\end{array}$ & $\mathrm{EP}$ \\
\hline \multirow[t]{2}{*}{$\begin{array}{l}\text { Komparti- } \\
\text { mentierung }\end{array}$} & $\begin{array}{l}\text {... erläutern modellhaft den Aufbau von Biomembranen (Flüssig-Mosaik-Mo- } \\
\text { dell) }\end{array}$ & $\mathrm{EP}$ \\
\hline & $\begin{array}{l}\text {... erläutern biologische Phänomene mithilfe verschiedener Arten von } \\
\text { Stofftransport zwischen Kompartimenten (passiver und aktiver Transport) }\end{array}$ & $\mathrm{QP}$ \\
\hline \multirow{2}{*}{$\begin{array}{l}\text { Information } \\
\text { und Kommu- } \\
\text { nikation }\end{array}$} & $\begin{array}{l}\text {... beschreiben den Weg vom adäquaten Reiz über die Auslösung der Erregung } \\
\text { und die Erregungsweiterleitung zum Gehirn }\end{array}$ & Sek I \\
\hline & 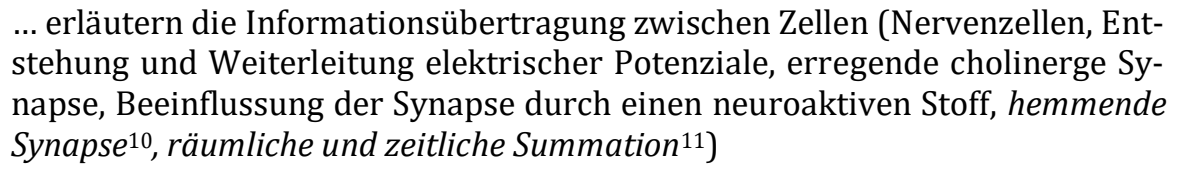 & $\mathrm{QP}$ \\
\hline
\end{tabular}

\footnotetext{
${ }^{9}$ Die Analyse beschränkt sich auf die Kerncurricula des Landes Niedersachsen.

${ }^{10}$ Die Tabellen 5 und 6 enthalten lediglich eine Auswahl der relevanten Lernziele. Die ungekürzten Tabellen sind im Anhang zu finden.

${ }^{11}$ Zusätzliche Lernziele auf erhöhtem Anforderungsniveau.
} 
Obwohl die Zellbiologie insgesamt zwar ein sehr prominentes Thema im Biologieunterricht darstellt, das über die Jahrgangsstufen hinweg immer wieder aufgegriffen und vertieft wird, werden Membranen sowie die an ihnen ablaufenden Prozesse oft nur modellhaft betrachtet. Für die Grundlagenforschung im Bereich der Biomembranen sind es aber gerade die Membranbestandteile, wie Lipide und Proteine, und ihre Interaktionen auf molekularer Ebene, die von großem Interesse sind. Dabei sollen z. B. Transportprozesse, die im Biologieunterricht lediglich modellhaft behandelt werden, mithilfe von Kenntnissen über die beteiligten Moleküle aus chemischer Sicht aufgeschlüsselt werden. Eine Betrachtung der Forschungsinhalte des SFB kann also nur interdisziplinär erfolgen - und muss dies auch - um dem allgemein interdisziplinären Charakter naturwissenschaftlicher Forschung Rechnung zu tragen. Das Kerncurriculum der Chemie bietet hier einige Anknüpfungspunkte für eine fächerübergreifende Erarbeitung der Thematik. So können bspw. neben den Struktur-Funktionsbeziehungen der Phospholipide anhand ihrer Molekülstruktur auch ihre Struktur-Eigenschaftsbeziehungen erarbeitet werden. In Tabelle 6 werden diesbezüglich einige Kompetenzen aufgezählt, die für die im Rahmen dieser Arbeit vorgestellten Experimente eine Rolle spielen.

Tabelle 6: Nach Kompetenzen geordnete Lernziele aus dem niedersächsischen Kerncurriculum Chemie [181, 183], die bezüglich der SO-Aktivitäten des SFB 803 von Relevanz sind. EP = Einführungsphase, QP = Qualifikationsphase.

\begin{tabular}{|c|c|c|}
\hline Kompetenz & Lernziel & Phase \\
\hline \multirow[t]{2}{*}{$\begin{array}{l}\text { Stoff-Teil- } \\
\text { chen }\end{array}$} & $\begin{array}{l}\text {... differenzieren zwischen polaren und unpolaren Atombindungen/Elektro- } \\
\text { nenpaarbindungen in Molekülen }\end{array}$ & $\begin{array}{l}\text { Sek I, } \\
\text { EP }\end{array}$ \\
\hline & $\begin{array}{l}\text {... beschreiben die Molekülstruktur von Aminosäuren, Proteinen, Kohlenhyd- } \\
\text { raten (Glucose, Fructose, Saccharose, Stärke) und Fetten. }\end{array}$ & $\mathrm{QP}$ \\
\hline \multirow[t]{2}{*}{$\begin{array}{l}\text { Struktur-Ei- } \\
\text { genschaft }\end{array}$} & $\begin{array}{l}\text {... erklären Stoffeigenschaften anhand ihrer Kenntnisse über zwischenmoleku- } \\
\text { lare Wechselwirkungen: Van-der-Waals-Kräfte, Dipol-Dipol, Wasserstoffbrü- } \\
\text { ckenbindungen }\end{array}$ & $\mathrm{EP}, \mathrm{QP}$ \\
\hline & ... unterscheiden zwischen Hydrophilie und Lipophilie & $\mathrm{EP}$ \\
\hline $\begin{array}{l}\text { Donator-Ak- } \\
\text { zeptor }\end{array}$ & $\begin{array}{l}\text {... nennen die charakteristischen Teilchen wässriger sauerer und alkalischer } \\
\text { Lösungen (Hydronium/Oxonium-Ion und Hydroxid-Ion) }\end{array}$ & $\mathrm{QP}$ \\
\hline Energie & $\begin{array}{l}\text {... beschreiben, dass Systeme bei chemischen Reaktionen Energie mit der Um- } \\
\text { gebung, z.B. in Form von Wärme, austauschen können und dadurch ihren Ener- } \\
\text { giegehalt verändern }\end{array}$ & Sek I \\
\hline
\end{tabular}

Insgesamt ist die Forschung des SFB jedoch so spezifisch und komplex, dass es aufgrund des benötigten Vorwissens nicht sinnvoll erschien, SO-Aktivitäten lediglich auf Basis der Forschungsinhalte zu kreieren. Stattdessen wurden drei Leitfragen formuliert, die mit Blick auf die Inhalte der Kerncurricula der Fächer Biologie und Chemie zu einer ganzheitlichen Betrachtung der Membranforschung beitragen sollen:

(1) Welche Struktur-Eigenschaftsbeziehungen sind für die Kompartimentbildung in Organismen verantwortlich?

(2) Woraus bestehen Biomembranen und wie können diese Grenzen überwunden werden?

(3) Woran wird im SFB 803 geforscht und welche Methoden werden dazu verwendet?

Anhand dieser Leitfragen wurden verschiedene Themenfelder abgeleitet, die experimentell aufgearbeitet wurden. 
Tabelle 7: Inhalte der anhand der Leitfragen abgeleiteten Themenfelder.

\begin{tabular}{llc}
\hline Themenfeld & Inhalte & Kapitel \\
\hline $\begin{array}{l}\text { Struktur-Eigenschafts- } \\
\text { beziehungen amphiphi- } \\
\text { ler Moleküle }\end{array}$ & $\begin{array}{l}\text { Eigenschaften (Oberflächenaktivität, benetzende Wirkung, Dis- } \\
\text { pergiervermögen) und Struktur von Tensiden, Hydrophobie, } \\
\text { Hydrophilie, Amphiphilie }\end{array}$ & 5.1 \\
\hline $\begin{array}{l}\text { Selbstorganisation am- } \\
\text { phiphiler Moleküle }\end{array}$ & $\begin{array}{l}\text { Selbstorganisation von Tensiden (Monoschichten, Mizellen, Vesi- } \\
\text { kel), geometrische Abhängigkeit der Aggregatbildung }\end{array}$ & 5.2 \\
\hline $\begin{array}{l}\text { Membranen und ihre } \\
\text { Bestandteile }\end{array}$ & $\begin{array}{l}\text { Membranen im Vergleich, Eigenschaften der Membranbestand- } \\
\text { teile }\end{array}$ & 5.3 \\
\hline Membrantransport & $\begin{array}{l}\text { Erleichterte Diffusion (in Abhängigkeit von Größe und Ladung, } \\
\text { carriervermittelt), Exocytose }\end{array}$ & 5.4 \\
\hline $\begin{array}{l}\text { Fluoreszenz } \\
\text { Bedingungen für Farbigkeit und Fluoreszenz, Fluoreszenzanre- }\end{array}$ & 5.5 \\
\hline $\begin{array}{l}\text { Untersuchungsmetho- } \\
\text { den in der Membran- } \\
\text { forschung }\end{array}$ & $\begin{array}{l}\text { Lipidextraktion, Vesikelpräparation, Fluoreszenzmikroskopie, } \\
\text { Fluoreszenzspektroskopie }\end{array}$ & 5.6 \\
\hline
\end{tabular}

Die Auswahl und Entwicklung der Experimente erfolgte in erster Linie vor dem Hintergrund einer Anwendung im Chemie- und Biologieunterricht. Entsprechend sollten die Experimente möglichst mit einem geringen zeitlichen, materiellen und finanziellen Aufwand einhergehen und ungefährliche Chemikalien enthalten. Da innerhalb des Ö-Projektes auch SO-Aktivitäten wie Schülerlabore und Summer Schools geplant sind, wurde zwar weitestgehend versucht, diese Anforderungen zu erfüllen, der Entwicklung insbesondere neuer Experimente wurden jedoch nur bedingt Grenzen gesetzt. Insgesamt wurden im Zuge dieser Arbeit zahlreiche neue didaktische Experimente entwickelt, es wurde jedoch auch auf einige bekannte und etablierte Experimente zurückgegriffen. Ziel der Ausgestaltung der verschiedenen Themenfelder war es also explizit nicht, diese mit ausschließlich neuartigen Experimenten zu füllen. Stattdessen ermöglicht gerade die Verwendung etablierte Experimente und ihre Einordnung in biologische Kontexte ein Aufzeigen fächerübergreifender Kongruenzen und eine Anknüpfung an bestehende Unterrichtspraktiken, was sich positiv auf eine unterrichtliche Implementation auswirken kann [184]. Die im Folgenden vorgenommen didaktischen Betrachtungen erfolgen mit Blick auf die primäre Zielgruppe vornehmlich auf Oberstufenniveau. Innerhalb der verschiedenen SO-Aktivitäten, die in Kapitel 6 näher beschrieben werden, müssen diese Betrachtungen je nach Bildungskontext und Vorwissen bzw. Alter der Teilnehmer_innen gegebenenfalls didaktisch noch weiter reduziert werden.

\subsection{Themenfeld 1 - Struktur-Eigenschaftsbeziehungen amphiphiler Moleküle}

In lebenden Organismen werden verschiedene Zellen durch Plasmamembranen voneinander abgegrenzt. Auch Organellen innerhalb der Zellen, wie der Zellkern, Mitochondrien oder Chloroplasten, sind von Membranen umgeben. Diese zusätzlichen Kompartimente innerhalb der Zelle besitzen charakteristische Proteine, die die strukturellen und funktionellen Eigenschaften der einzelnen Organellen bestimmen. Die Kompartimentierung innerhalb der Zellen ermöglicht es dabei, unterschiedliche Konzentrationsgradienten verschiedener Stoffe wie Enzymen und Ionen aufzubauen, um die in den Organellen ablaufenden biochemischen Prozesse zu optimieren [185]. 
Biomembranen bestehen zu einem großen Teil aus Lipiden, die zu einer Doppelschicht angeordnet sind. Die Anordnung von Lipiden zu Doppelschichten in einem wässrigen Medium liegt in der amphiphilen Natur der Lipidmoleküle begründet. Diese besitzen, wie in Abbildung 13A am Beispiel eines typischen Phospholipids dargestellt, eine hydrophile Kopfgruppe sowie zwei lange hydrophobe Kohlenwasserstoffketten. Um das Verständnis für die Selbstorganisation amphiphiler Moleküle zu übergeordneten Strukturen wie Lipiddoppelschichten zu fördern, können zunächst die Eigenschaften amphiphiler Moleküle erarbeitet werden. Hierzu bietet sich die Verwendung von Tensiden aus Spülmitteln an, da diese, bis auf eine Schwanzgruppe, den Lipiden in ihrer Struktur sehr ähneln (siehe Abbildung 13B). Zudem ermöglichen sie es, die Eigenschaften amphiphiler Moleküle wie Grenzflächenaktivität, benetzende Wirkung und Dispergiervermögen anhand von Alltagsphänomenen wie der Oberflächenspannung von Wasser sowie im Kontext von Waschprozessen im Haushalt zu untersuchen.

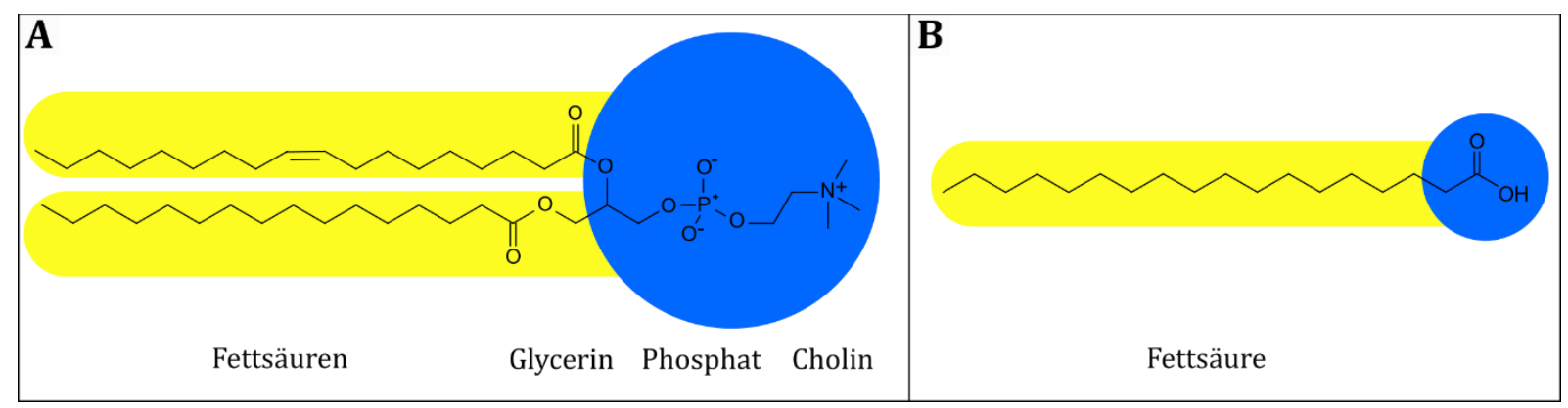

Abbildung 13: Skelettformel eines Phospholipids (A, Phosphatidylcholin, hier POPC) sowie eines Tensids (B, Stearinsäure) mit farblicher Unterlegung der hydrophoben Ketten (gelb) sowie der hydrophilen Kopfgruppen (blau).

\section{Experiment 1.1a - Grenzflächenspannung zwischen Wasser und Öl}

Im Sinne eines Problemexperiments kann dieser Versuch beispielsweise im Unterricht zum Einstieg in das Themenfeld genutzt werden, um anschließend hypothesengelenkt Eigenschaften von Tensiden zu erarbeiten [186].

Geräte und Chemikalien: Hohes Becherglas, kleines enghalsiges Gefäß, Pinzette, Speiseöl (angefärbt mit Paprikapulver), Wasser, Spülmittel.

Versuchsdurchführung: Angefärbtes Öl wird bis zum Rand in ein kleines enghalsiges Gefäß gefüllt. Das Gefäß wird vorsichtig in ein mit Wasser gefülltes Becherglas gestellt, so dass es vollständig mit Wasser bedeckt ist. Anschließend wird ein Tropfen Spülmittel zum Wasser hinzugegeben.

Beobachtung: Beim Platzieren des ölgefüllten Gefäßes im Wasser verbleibt das Öl zunächst im Gefäß. Nach der Zugabe von Spülmittel steigt das Öl nach kurzer Zeit an die Wasseroberfläche (siehe Abbildung 14). 


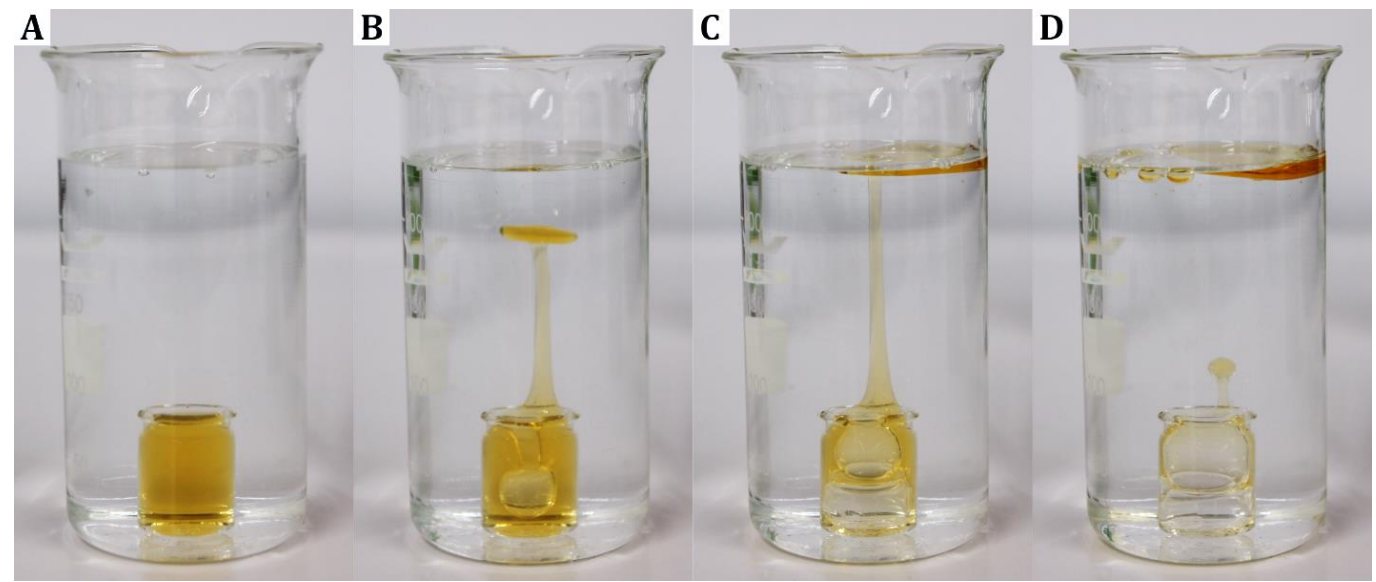

Abbildung 14: Vor Zugabe des Spülmittels zum Wasser verbleibt das angefärbte Öl im enghalsigen Gefäß (A). Nach der Zugabe des Tensids steigt das Öl aus dem Gefäß heraus an die Wasseroberfläche (B-D).

Deutung: Öl besitzt eine geringere Dichte als Wasser und müsste deshalb aus dem Gefäß heraus an die Oberfläche steigen. Die Oberflächenspannung von Wasser ist bei einer Temperatur von $20^{\circ} \mathrm{C}$ mit 72,75 $\mathrm{N} \cdot \mathrm{m}^{-1}[187]$ deutlich größer als die des hauptsächlich aus Triglyceriden bestehenden Öls $\left(\sim 30 \mathrm{~N} \cdot \mathrm{m}^{-1}{ }^{[188]}\right)$. Entsprechend verbleibt das Öl in seinem Gefäß. Die Zugabe von Tensidlösung setzt die Grenzflächenspannung zwischen Wasser und Öl herab, so dass das Öl aus dem Gefäß austreten kann.

\section{Experiment 1.1b -Tropfen auf einer Münze}

Aufgrund des geringen Material- und Zeitaufwands eignet sich dieses Experiment sowohl im Klassenraum als auch auf Bildungsmessen zur Erarbeitungen des Einflusses von Tensiden auf die Oberflächenspannung des Wassers.

Geräte und Chemikalien: Münzen, Pasteurpipetten, 2 Bechergläser, Glasrührstab, Wasser, Spülmittel.

Versuchsdurchführung: Mit einer Pasteurpipette wird tropfenweise Wasser auf eine entfettete Münze gegeben. Die Tropfen werden gezählt bis das Wasser überläuft. Im Anschluss wird der Vorgang mit einer Spülmittellösung wiederholt.

Beobachtung: Auf die Münzen können deutlich mehr Wassertropfen als Tropfen der Tensidlösung aufgetragen werden. Zudem liegt das Wasser gewölbt auf der Münze auf, während sich die Spülmittellösung deutliche flacher auf der Münze verteilt (siehe Abbildung 15).

A

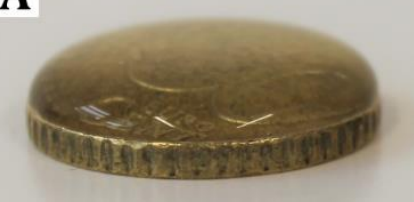

B

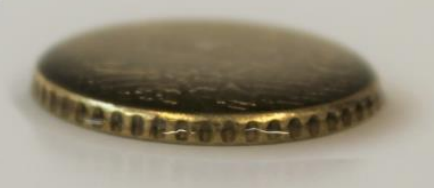

Abbildung 15: Wassertropfen (A) sowie Tropfen einer Tensidlösung (B) auf einer 50-Cent Münze.

Deutung: Wasser besitzt eine verhältnismäßig große Oberflächenspannung, die es dem Wasser erlaubt eine stark gewölbte Form auf der Münze anzunehmen, ohne von der Münze zu fließen. Erst wenn die Menge des Wassers und damit die Gewichtskraft, die von Innen auf die Oberfläche wirkt, größer als die Oberflächenspannung ist, fließt der Tropfen von der Münze. Die Zugabe einer Tensidlösung setzt die Oberflächenspannung des Wassers herab, so dass die Lösung bereits deutlich früher von der Münze fließt. 


\section{Experiment 1.1c - Schwimmende Büroklammer}

Dieses allseits bekannte Experiment zeigt aufmakroskopischer Ebene, welche Kräfte an der Wasseroberfläche wirken. Trotz der Möglichkeit, analog zu Experiment $1.1 b$ den Einfluss von Tensiden auf die Oberflächenspannung zu erarbeiten, eignet es sich jedoch nicht für eine sich wiederholende Anwendung auf Bildungsmessen [189].

Geräte und Chemikalien: Erlenmeyerkolben, Büroklammer, Wasser, Spülmittel.

Versuchsdurchführung: Ein Erlenmeyerkolben wird bis zum Rand mit Wasser befüllt, so dass sich das Wasser leicht über den Rand wölbt und eine Büroklammer wird vorsichtig auf die Wasseroberfläche gelegt. Anschließend wird das Wasser mit einem Tropfen Spülmittel versetzt.

Beobachtung: Die Büroklammer schwimmt auf der Wasseroberfläche, wobei sich das Wasser unter der Büroklammer nach unten wölbt (Abbildung 16). Bei Zugabe von Spülmittel sinkt die Büroklammer zu Boden.

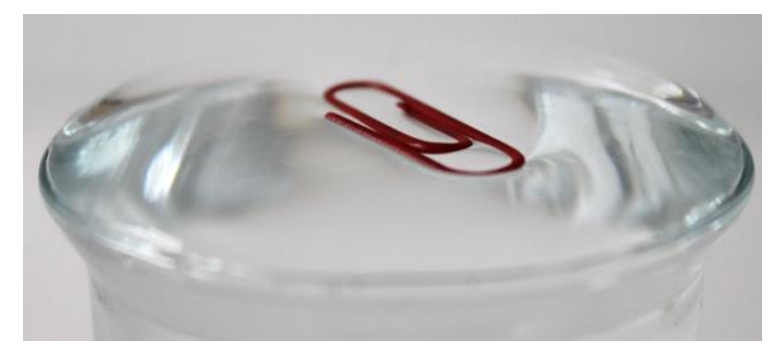

Abbildung 16: Eine Büroklammer liegt aufgrund der Oberflächenspannung trotz größerer Dichte auf der Oberfläche des Wassers auf.

Deutung: Aufgrund ihrer Form ist das Gewicht einer liegenden Büroklammer über eine relativ große Fläche verteilt. Die Kraft der Oberflächenspannung ist lokal größer als die Gewichtskraft der Büroklammer, so dass diese trotz höherer Dichte auf dem Wasser schwimmt. Die Zugabe der Tensidlösung setzt die Oberflächenspannung des Wassers herab, so dass die Büroklammer zu Boden sinkt.

Anmerkungen zur Deutung der Experimente: Die Experimente 1.1a bis 1.1c bedienen sich allesamt des Phänomens der Grenzflächenspannung zweier Phasen. Dabei werden sowohl die Eigenschaften der Oberflächen- bzw. Grenzflächenspannung des Wassers demonstriert, als auch der Einfluss von Tensiden auf diese thematisiert. Die Grenzflächenspannung zweier Phasen kann sowohl mechanisch als auch thermodynamisch definiert werden. Nach der mechanischen Definition ist die Grenzflächenspannung eine Kraft pro Länge, nach der thermodynamischen eine Energie pro Fläche. In beiden Fällen besitzt sie die Einheit $N \cdot \mathrm{m}^{-1}$. Im Chemieunterricht werden zumeist die intermolekularen Kräfte der beteiligten Moleküle der Wasser-zu-Luft-Grenze betrachtet, wobei die Moleküle der Luft hier vernachlässigt werden. Dabei wird die Grenzflächenspannung des Wassers oftmals als eine Kraft beschrieben, die senkrecht und nach innen gerichtet auf die an der Oberfläche liegenden Wassermoleküle wirkt. Jedoch werden hierbei lediglich die anziehenden intermolekularen Kräfte der Moleküle betrachtet. Eigentlich müssten jedoch zugleich auch abstoßende Kräfte berücksichtigt werden, da Teilchen in der flüssigen Phase aufgrund ihrer Teilchenbewegung ständig beide Kräfte erfahren.

Abbildung 17A zeigt Wassermoleküle sowohl inmitten der flüssigen Phase, als auch an ihrer Oberfläche. Auf Moleküle in der flüssigen Phase wirken anziehende und abstoßende Kräfte gleichermaßen aus allen Richtungen. Die Moleküle an der Oberfläche können jedoch, unter Vernachlässigung der Gasphase, nur mit Molekülen neben und unter ihnen wechselwirken. Unter der Annahme, dass die abstoßenden Kräfte als isotrop angesehen 
werden können, sie also in alle Richtungen gleichermaßen wirken, entsteht auf vertikaler Ebene ein Ungleichgewicht, da die abstoßenden Kräfte nur nach unten wirken können. Dieses Ungleichgewicht muss von den anisotropen anziehenden Kräften ausgeglichen werden. Auf horizontaler Ebene wirkt die abstoßende Kraft hingegen in alle Richtungen, sie befindet sich also im Gleichgewicht. Deshalb können die anziehenden Kräfte hier im Betrag größer sein als die abstoßenden, ohne das Kräftegleichgewicht des Teilchens zu stören [190]. Die Zugabe eines Tensids führt dazu, dass die Wassermoleküle an der Oberfläche mit den polaren Köpfen der Tenside wechselwirken, wodurch sich die Moleküle dem Gleichgewichtszustand der inneren Wassermoleküle annähern und die Oberflächenspannung abnimmt (siehe Abbildung 17B).

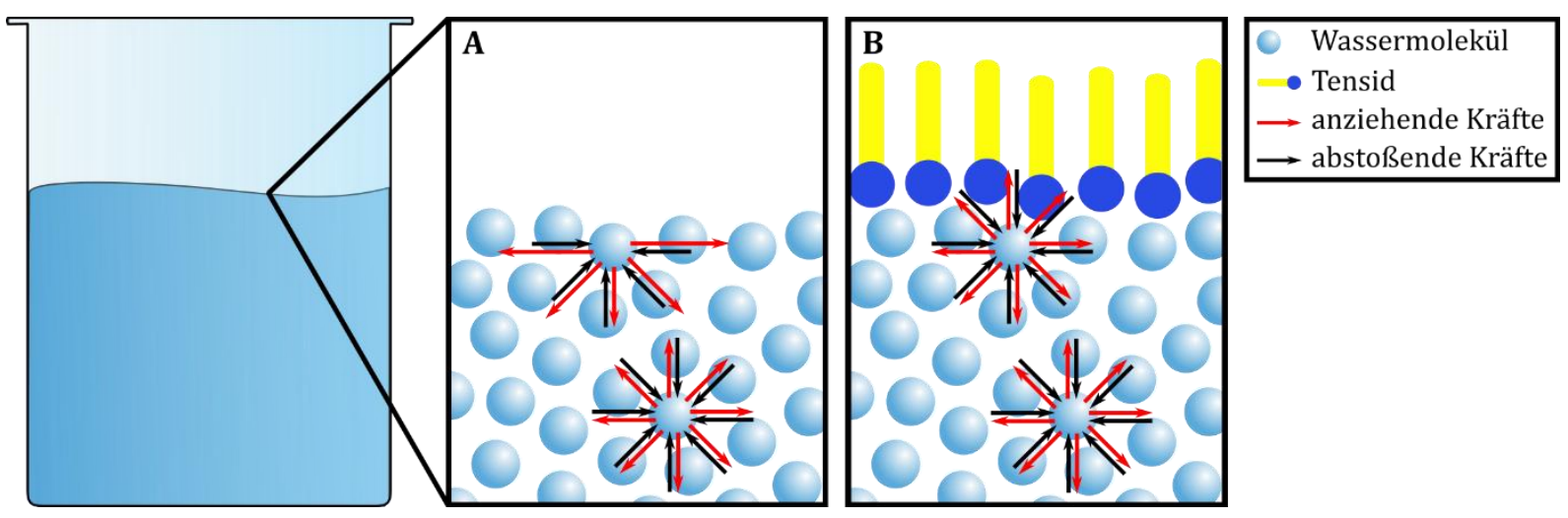

Abbildung 17: Schematische Darstellung der anziehenden und abstoßenden Kräfte die auf Wassermoleküle an der Oberfläche und im Inneren der Flüssigkeit wirken. (A) vor, (B) nach Zugabe von Tensidmolekülen.

Für die Erarbeitung der Eigenschaften von Tensiden reicht es aus, die Oberflächen- bzw. Grenzflächenspannung auf die Anziehungskräfte der Wassermoleküle und die fehlenden Nachbarmoleküle an der Oberfläche zurückzuführen. Die SuS sollten dabei erkennen, dass die Tenside Wechselwirkungen mit den Wassermolekülen an der Grenzfläche eingehen, die zur Herabsetzung der Grenzflächenspannung führen.

\section{Experiment 1.2a - Benetzung von Baumwolle}

Dieses Experiment ermöglicht die unterrichtliche Einführung bzw. Wiederholung der Begriffe hydrophil und hydrophob. Die Baumwolle kann dabei didaktisch reduziert als hydrophob betrachtet, oder alternativ die Benetzbarkeit glatter und rauer Oberflächen thematisiert werden [191].

Geräte und Chemikalien: Sauberes Baumwollstück (z. B. Geschirrtuch), 2 Bechergläser, Glasrührstab, 2 Pasteurpipetten, Wasser, Spülmittel.

Versuchsdurchführung: Auf einem Baumwollstück werden vorsichtig je ein Tropfen Wasser und ein Tropfen Spülmittellösung platziert und die Tropfenform beobachtet.

Beobachtung: Der Wassertropfen liegt nahezu rund auf dem Baumwolltuch auf, während der Tropfen der Spülmittellösung deutlich flacher aufliegt und schnell in das Tuch einzieht (vgl. Abbildung 18). 


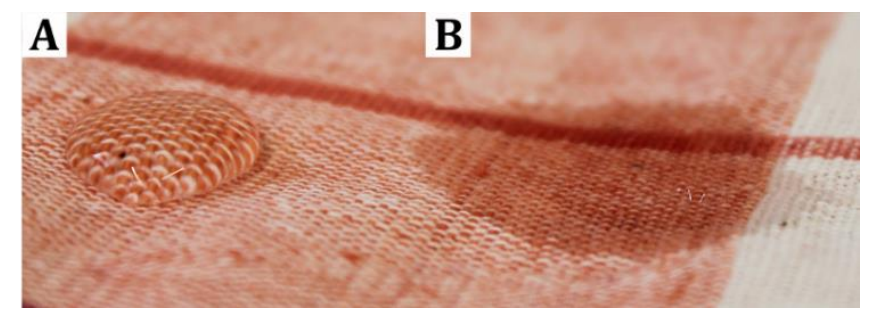

Abbildung 18: Ein Wassertropfen (A) sowie ein Tropfen einer Spülmittellösungen auf einem Baumwolltuch (B).

Deutung: Baumwollfasern bestehen zum größten Teil aus Celluloseketten, die aufgrund zahlreicher Hydroxylgruppen hydrophile Eigenschaften besitzt. Die unverzweigten Ketten bilden intermolekulare Wasserstoffbrückenbindungen aus, die für die Reißfestigkeit der Fasern verantwortlich sind. Die unregelmäßige Struktur der Fasern sorgt beim Auftragen eines Wassertropfens für Lufteinschlüsse zwischen Faser und Tropfen, die für eine schlechtere Benetzbarkeit der Baumwollfasern trotz ihrer prinzipiell hydrophilen Eigenschaften sorgen. Die Tensidmoleküle des zweiten Tropfens setzen die Grenzflächenspannung zwischen den Lufteinschlüssen und dem Wasser herab, so dass der Tropfen in die Baumwolle einziehen kann.

\section{Experiment 1.2b - Wollfaden auf der Wasseroberfläche}

Alternativ zu Experiment 1.2a kann dieser Versuch zusätzlich auch auf Bildungsmessen eingesetzt werden, da bei geringem Materialverbrauch die Benetzbarkeit von Wollfäden wiederholt betrachtet werden kann [186].

Geräte und Chemikalien: 2 Bechergläser, Glasrührstab, Wollfaden, Wasser, Spülmittel.

Versuchsdurchführung: Zwei Bechergläser werden zur Hälfte mit Wasser befüllt und eine Lösung mit etwas Spülmittel versetzt. Anschließend wird gleichzeitig jeweils ein kurzer Wollfaden auf den Oberflächen der Lösungen platziert.

Beobachtung: Die Wollfäden bleiben zunächst auf den Oberflächen der Lösungen liegen. Nach kurzer Zeit sinkt der Wollfaden auf der Spülmittellösung zu Boden, während der andere Faden auf der Wasseroberfläche verbleibt (Abbildung 19).

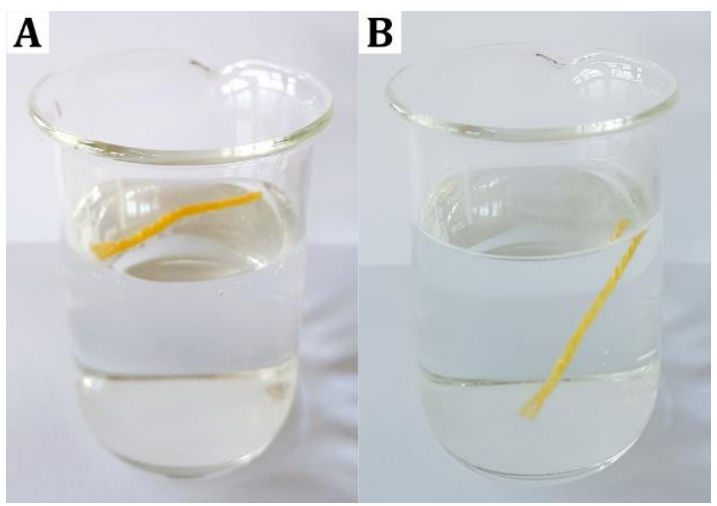

Abbildung 19: Ein Wollfaden bleibt auf einer Wasseroberfläche liegen (A), während er von einer Tensidlösung benetzt wird und langsam zu Boden sinkt (B).

Deutung: Ähnlich wie die Cellulose der Baumwolle besitzt das in Wolle enthaltene Keratin hydrophile Eigenschaften. Jedoch ist auch hier aufgrund der Lufteinschlüsse zwischen Faser und Wasser die Benetzung der Fasern durch das Wasser gering. Die Zugabe von Tensiden verringert die Grenzflächenspannung zwischen Wasser und Luft, so dass der Faden vollständig benetzt werden kann und zu Boden sinkt. 
Anmerkungen zur Deutung der Experimente: Im Vergleich zu Wassermolekülen innerhalb eines Tropfens befinden sich die Moleküle an der Oberfläche in einem energetisch ungünstigen Zustand, weshalb eine Minimierung der Oberfläche angestrebt wird. Da Kugeln bei gleichbleibender Masse die geometrische Form mit der geringsten Oberfläche darstellen, bilden sich in der Gasphase nahezu runde Wassertropfen. Kommen die Tropfen mit einer festen Phase in Kontakt, dann treten die Wassermoleküle neben der Gasphase auch mit der festen Phase in Wechselwirkung. Der Grad der Benetzung der festen Oberfläche, hängt dabei zum einen von der Art der Wechselwirkungen, zum anderen von der Beschaffenheit der Oberfläche ab. Starke anziehende Wechselwirkungen zwischen den Wassermolekülen und der festen Phase, wie beispielsweise die Ausbildung von Wasserstoffbrückenbindungen, führen zu einer flachen Tropfenform und einer stärkeren Benetzung der Oberfläche. Bei schwachen Wechselwirkungen ist es hingegen für die Wassermoleküle energetisch günstiger, die Kontaktfläche mit der festen Phase und gleichzeitig auch die Oberfläche des Tropfens möglichst gering zu halten (siehe Abbildung 20). Entsprechend ihrer Wechselwirkungen mit Wasser werden die Oberflächen als hydrophil oder hydrophob bezeichnet, wobei das Maß der Benetzung mithilfe des Kontaktwinkels $\theta$ beschrieben wird.

A

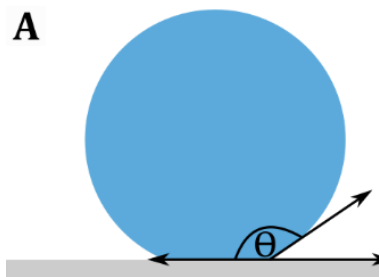

hydrophob
B

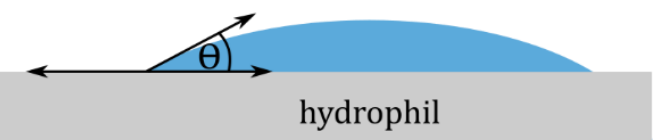

Abbildung 20: Schematische Darstellung der Benetzung einer hydrophoben (A) sowie einer hydrophilen Oberfläche (B) durch einen Wassertropfen.

Neben den Wechselwirkungen der festen Phase spielt auch die Beschaffenheit ihrer Oberfläche eine Rolle. Raue Oberflächen lassen Lufteinschlüsse zwischen der flüssigen und der festen Phase zu, so dass neben den flüssig-festen auch flüssig-gasförmige Phasengrenzen vorliegen (Abbildung 21A). Dadurch wird makroskopisch die hydrophile Eigenschaft der Oberfläche herabgesetzt. Tenside können die Benetzbarkeit von Oberflächen erhöhen, indem ihre hydrophilen Anteile Wechselwirkungen mit den Wassermolekülen und ihre hydrophoben Anteile Wechselwirkungen sowohl mit der festen (im Falle hydrophober glatter Oberflächen), als auch der gasförmigen Phase eingehen (siehe Abbildung 21B).

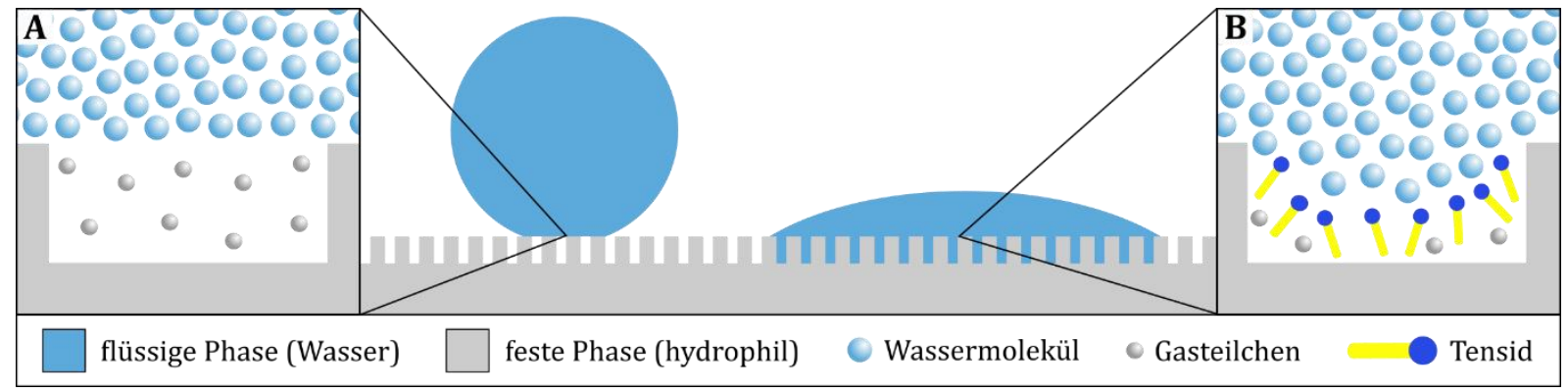

Abbildung 21: Die Benetzbarkeit einer rauen hydrophilen Oberfläche kann durch Lufteinschlüsse (A) herabgesetzt werden. Die Zugabe von Tensiden führt hingegen zu einer Erhöhung der Benetzbarkeit (B).

Die hier verwendeten Woll- und Baumwollfasern sind eigentlich nur bedingt für die Betrachtung der Netzwirkung von Tensiden geeignet, da die Fasern auf makroskopischer Ebene hydrophobe Eigenschaften besitzen, die allein mit der Molekülstruktur nicht erklärt werden können. Vor dem Hintergrund, dass die Netzwirkung der Tenside jedoch 
eine wichtige Rolle in Waschprozessen spielt, ermöglichen es die Fasern, einen relevanten Bezug zur Lebenswelt der SuS herzustellen. Entsprechend ist es möglich die Fasern als eher hydrophob zu betrachten und ggf. in Bezug auf ihre Molekülstruktur auf den Einfluss von Lufteinschlüssen hinzuweisen. In diesem Zusammenhang können Tenside von den SuS als Teilchen verstanden werden, die sowohl mit der festen hydrophoben, als auch mit der flüssigen hydrophilen Phase wechselwirken und somit eine vollständige Benetzung der Fasern ermöglichen.

\section{Experiment 1.3a - Suspensionsbildung}

Die Bildung von Suspensionen ermöglicht die Betrachtung der Wirkung von Tensiden, beispielsweise im Kontext des Waschens schmutzbehafteter Textilien [192].

Geräte und Chemikalien: 3 Bechergläser, Glasrührstab, Schnelllauftrichter, Filterpapier, Filtriergestell, Spatel, Mangan(IV)-oxid (GHS07, GHS08), Wasser, Spülmittel.

Versuchsdurchführung: In einen Trichter mit Filter wird eine Spatelspitze Mangan(IV)oxid gegeben. Einige Milliliter Wasser werden in den Trichter gefüllt und das Filtrat in einem Becherglas aufgefangen. Wenn das Wasser vollständig durchgelaufen ist, wird der Vorgang mit einer Spülmittellösung wiederholt.

Beobachtung: Nach Zugabe des Wassers wird ein klares Filtrat im Becherglas aufgefangen. Das Filtrat der Tensidlösung hingegen ist grau getrübt (Abbildung 22A, B).

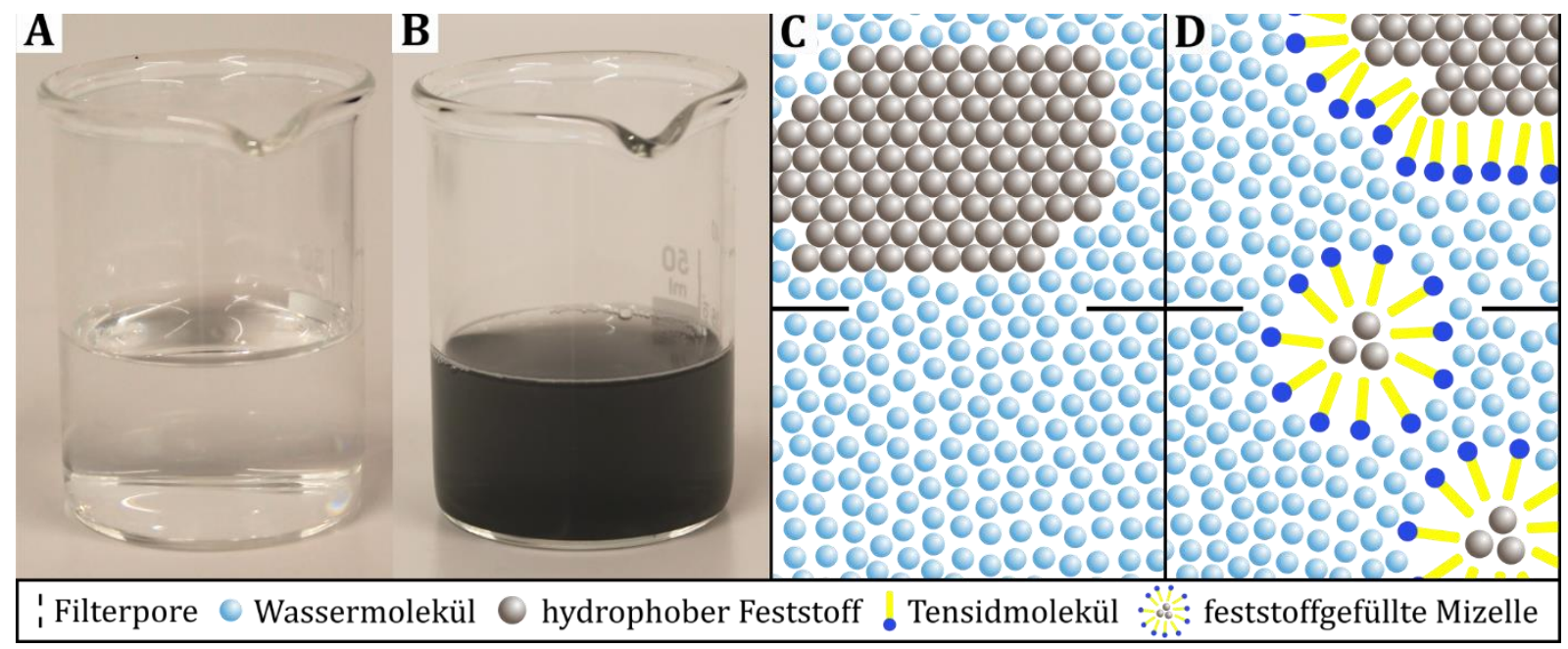

Abbildung 22: Filtrat nach Zugabe von Wasser (A) und nach Zugabe einer Tensidlösung (B), sowie schematische Darstellung des Filtrationsprozesses auf Teilchenebene (C, D)

Deutung: Die hydrophoben Partikel des Mangan(IV)-oxids sind in Wasser nicht löslich, so dass das Manganoxid im Filter verbleibt. Bei Zugabe der Spülmittellösung wechselwirken die hydrophoben Anteile der Tenside mit den hydrophoben Manganoxidteilchen. Zusätzliche Wechselwirkungen der hydrophilen Anteile der Tenside mit dem Wasser ermöglichen eine Suspendierung der Manganoxidteilchen im Wasser, die aufgrund ihrer geringen Größe somit durch den Filter treten können (vgl. Abbildung 22C, D).

\section{Experiment 1.3b - Emulsionsbildung}

Analog zu Experiment 1.3a kann die Eigenschaft von Tensiden zur Emulsionsbildung am Beispiel der Entfernung von Ölen beim Geschirrspülen untersucht werden [191].

Geräte und Chemikalien: 2 Reagenzgläser, 2 Stopfen, Wasser, Spülmittel, Speiseöl.

Versuchsdurchführung: Zwei Reagenzgläser werden jeweils zu einem Drittel mit Öl und zu einem weiteren Drittel mit Wasser befüllt. Zu einem der Gläser wird zusätzlich noch etwas 
Spülmittel gegeben. Anschließend werden beide Gläser mit einem Stopfen verschlossen und kräftig geschüttelt.

Beobachtung: Wasser und Öl liegen in den Reagenzgläsern in zwei getrennten Phasen vor, wobei das Öl die obere Phase bildet. Bei Zugabe des Spülmittels sinkt dieses zunächst zu Boden des Reagenzglases. Das Schütteln sorgt für eine Durchmischung der Phasen. Während sich im Reagenzglas ohne Spülmittel größere Öltropfen bilden, die sich schnell wieder von der wässrigen Phase trennen (Abbildung 23B), liegen die Öltröpfchen im Reagenzglas mit Spülmittel fein verteilt in der wässrigen Phase vor. Zudem ist eine Schaumbildung zu beobachten und die beiden Phasen liegen auch nach längerer Wartezeit zum größten Teil gemischt vor (Abbildung 23C).

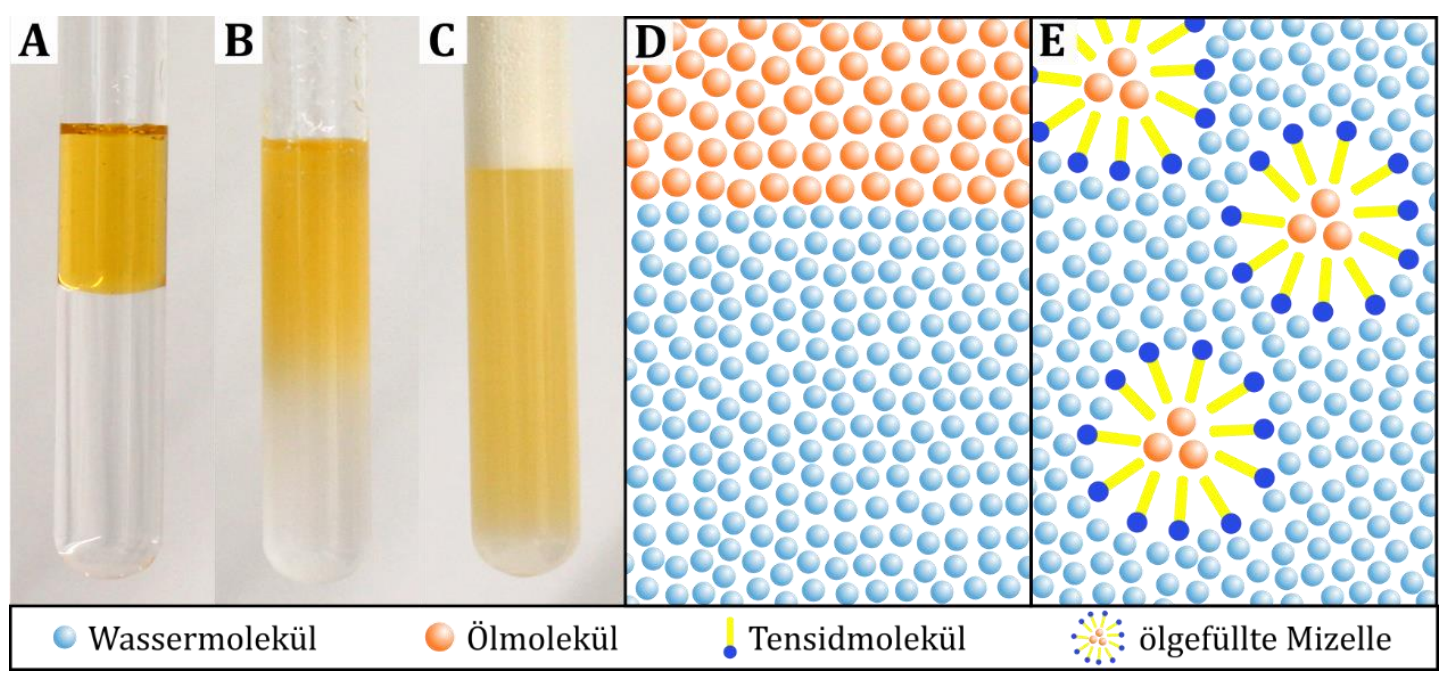

Abbildung 23: Wasser-Öl Gemisch vor dem Schütteln (A) bzw. nach dem Schütteln (B, C), Das Reagenzglas in (C) enthält zusätzlich Spülmittel. (D, E) Schematische Darstellung der Emulsion von Öl in Wasser.

Deutung: Wasser als hydrophile und Öl als hydrophobe Flüssigkeiten entmischen sich nach dem Schütteln schnell wieder. Wird die mit Tensidlösung versetzen Flüssigkeiten geschüttelt, so orientieren sich die hydrophoben Anteile der Tenside wie in Abbildung 23D dargestellt in Richtung der Ölmoleküle und die hydrophilen Anteile in Richtung der Wassermoleküle. Die Tensidmoleküle schirmen auf diese Weise die Ölmoleküle vom Wasser ab, wodurch die Grenzflächenspannung zwischen den Phasen herabgesetzt wird, die ohne Tensidzugabe zur Ausbildung energetisch günstigerer, größerer Öltröpfchen beitragen würde.

Anmerkungen zur Deutung der Experimente: Tenside sind gut in Wasser löslich, besitzen zudem aber auch die Eigenschaft, Stoffe, die sich aufgrund ihrer hydrophoben Eigenschaften weder in Wasser lösen noch mit Wasser mischen lassen - hier sowohl einen Feststoff als auch eine Flüssigkeit -in Wasser zu dispergieren. Das lässt darauf schließen, dass Tensidmoleküle in der Lage sind, sowohl mit hydrophilen als auch mit hydrophoben Stoffen zu wechselwirken. Auch bei diesen Experimenten ist es sinnvoll, sich der Analogie der Waschprozesse zu bedienen. Dabei stellt das Mangan(IV)-oxid feste Schmutzpartikel dar, während das Öl in Zusammenhang mit fettigem Geschirr betrachtet werden kann. Beim Übergießen des Feststoffes und dem Schütteln des Öls gehen Tensidmoleküle mit ihrem hydrophoben Anteil Wechselwirkungen mit dem Manganoxidteilchen bzw. den Ölmolekülen ein. Die hydrophilen Anteile zeigen dabei in Richtung der Wassermoleküle, die ebenfalls mit den Tensidmolekülen wechselwirken. Die Bewegungsenergie der fließenden Lösung sorgt im Falle der Manganoxidteilchen dafür, dass diese „weggewaschen“ werden können. Beim Öl sorgt der Schüttelprozess für eine Durchmischung der beiden Phasen und die Entstehung von in Wasser dispergierten Öltropfen. Ohne die Zugabe von 
Tensiden streben die Öltropfen durch Fusionierung mit anderen Tropfen die Ausbildung einer energetisch günstigeren, möglichst kleinen Grenzschicht an. Sind jedoch Tensidmoleküle vorhanden, setzen diese die Grenzflächenspannung zwischen den Öltropfen und dem Wasser herab, so dass diese fein verteilt in Wasser emulgiert vorliegen können.

\section{Einordnung der Experimente im Kontext der SO-Leitfragen}

Die in diesem Themenfeld beschriebenen Experimente zu den Struktur-Eigenschaftsbeziehungen der Tenside gehören zum Repertoire einer jeden Chemielehrkraft und sind somit keine Unbekannten. Die Betrachtung der Eigenschaften von Tensiden ermöglicht jedoch einen interdisziplinären Einstieg in das Thema der Biomembranen, das vornehmlich dem Biologieunterricht zugeschrieben wird. Dort werden vor allem die amphiphilen Membranlipide oft nur stiefmütterlich behandelt und stattdessen eher zellübergreifende Prozesse betrachtet. In der aktuellen Forschung steht jedoch die Untersuchung des $\mathrm{Zu}$ sammenspiels von Lipiden und Proteinen im Vordergrund, so dass eine interdisziplinäre Heranführung an das Thema der Biomembran, ihrer Bestandteile sowie deren Eigenschaften sinnvoll erscheint.

Anhand der Experimente zu den Eigenschaften der Tenside können somit zunächst sowohl hydrophile als auch hydrophobe Eigenschaften für diese abgeleitet und der Begriff der Amphiphilie definiert werden. Dieser ist zentral für die Erarbeitung der selbstständigen Organisation amphiphiler Moleküle zu übergeordneten Strukturen, die der Kompartimentbildung in Organismen zugrunde liegt. Die Experimente in diesem Abschnitt bilden also den Grundstein für einen Einblick in die Membranforschung im Sinne der ersten Leitfrage.

Insbesondere mit SuS der Oberstufe sollte deshalb eine Betrachtung der Molekülstruktur typischer Tenside erfolgen, aber auch mit anderen Zielgruppen können Tenside modellhaft als Teilchen mit einer wasserliebenden und einer wassermeidenden bzw. ölliebenden Seite erarbeitet werden. Aufgrund der Relevanz der Amphiphilie für das Verständnis der Selbstorganisation und Kompartimentbildung, sollte auch im Falle eines Auslassens dieses Abschnitts in höheren Klassenstufen noch einmal die Bedeutung der Amphiphilie in Zusammenhang mit dem Thema der Biomembranen hervorgehoben werden.

\subsection{Themenfeld 2 - Selbstorganisation amphiphiler Moleküle}

Die Selbstorganisation amphiphiler Teilchen zu verschiedenen supramolekularen Aggregaten beschreibt die Grundlage für zahlreiche Prozesse in lebenden Organismen. Beispielsweise ermöglichen Lipide durch Bildung von Lipiddoppelschichten eine Kompartimentierung zu Zellen und Organellen. Je nach Lipidklasse bilden sie zudem auch Mizellen, die u. a. an Fettspeicherprozessen im Darm beteiligt sind sowie Vesikel, die eine wichtige Rolle in Stofftransportprozessen spielen. Insbesondere Mizellen und Vesikel sind zudem von großer Bedeutung für die aktuelle biochemische Forschung, u. a. für Rekonstitutionsprozesse von Proteinen, oder, im Falle von Vesikeln, bezüglich der Untersuchung biologischer Transportphänomene [193,194].

Die Bildung supramolekularer Aggregate wie Mizellen hängt unter anderem von der Konzentration der amphiphilen Moleküle sowie ihres Packungsparameters ab. Bei Zugabe amphiphiler Teilchen zu Wasser liegen diese zunächst als isolierte Monomere sowohl in der Lösung als auch an ihrer Oberfläche vor. Mit zunehmender Zahl amphiphiler Teilchen steigt auch ihre Zahl an der Oberfläche, da die Interaktion der hydrophoben Anteile mit der Luft energetisch günstiger ist als mit den Wasserteilchen. Entsprechend sinkt zugleich die Oberflächenspannung des Wassers. Ab einem gewissen Punkt - wenn die Oberfläche 
mit amphiphilen Molekülen gesättigt ist - bleibt die Oberflächenspannung nahezu konstant. Hier liegt die kritische Mizellbildungskonzentration (critical micelle concentration, $\mathrm{CMC}$ ), bzw. im Falle anderer Aggregate die kritische Aggregatbildungskonzentration (critical aggregation concentration, CAC). Die CMC variiert dabei für verschiedene amphiphile Moleküle und ist zudem temperaturabhängig [195].

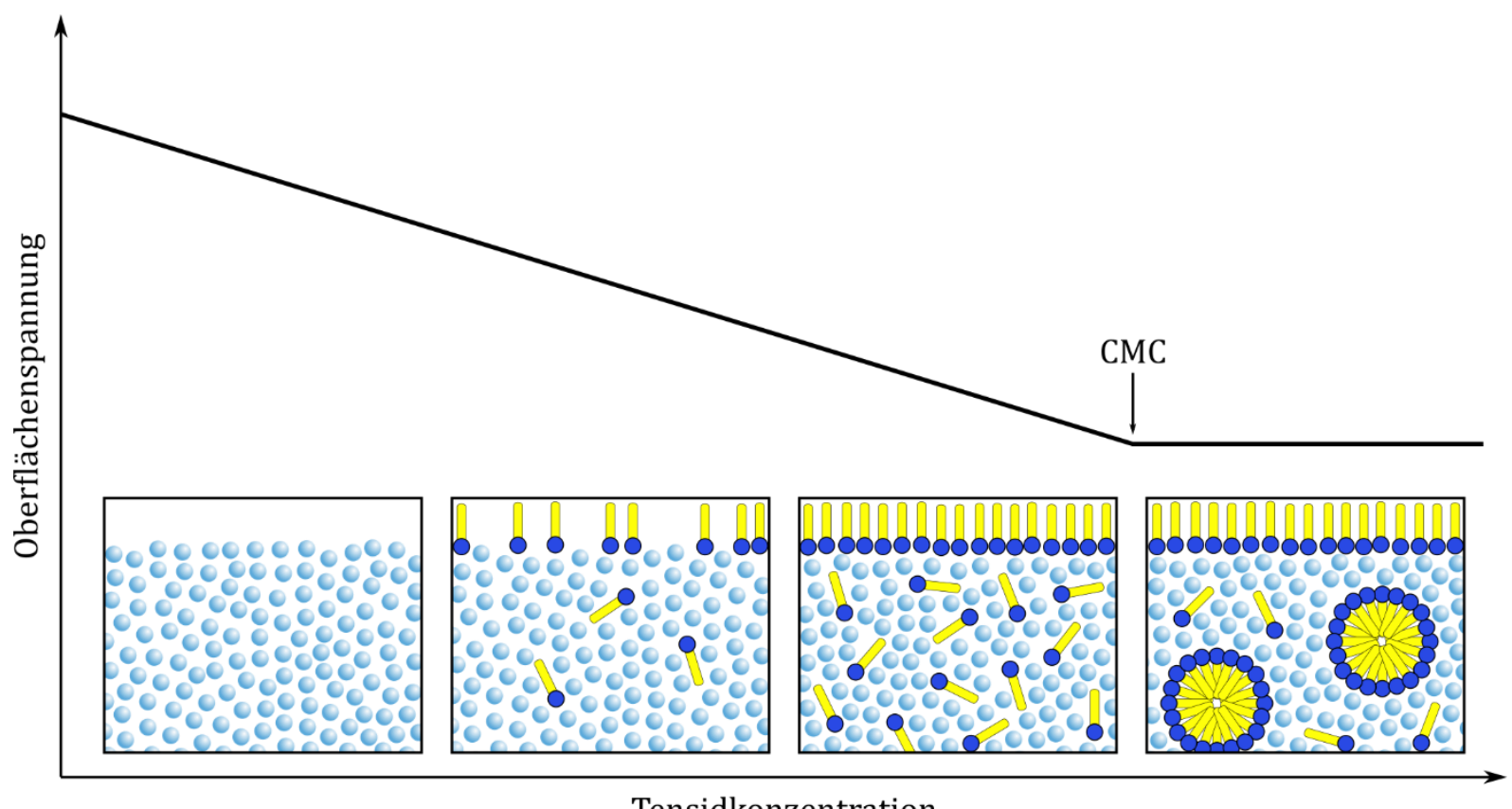

Tensidkonzentration

Abbildung 24: Änderung der Oberflächenspannung in Abhängigkeit der Tensidkonzentration.

Die Geometrie der Aggregate wird neben der Konzentration auch von der Geometrie und den Wechselwirkungen der beteiligten amphiphilen Teilchen bestimmt. Dabei spielen sowohl die Größe bzw. Länge der Kopf- und Schwanzgruppen der Teilchen eine Rolle, als auch ihre jeweiligen anziehenden und abstoßenden Kräfte. Die möglichen Aggregationsformen sind also vom Kettenvolumen $V$, der Kettenlänge $l$, und der Fläche der Kopfgruppe $A$ abhängig [195]. Hieraus kann der Packungsparameter $P$ bestimmt werden:

$$
P=\frac{V}{A \cdot l}
$$

Der Wert des Packungsparameters lässt dabei Aussagen über die geometrische Form des Aggregats zu. Einige der Aggregatformen, die amphiphile Moleküle in Abhängigkeit ihres Packungsparameters annehmen können, sind in Abbildung 25 dargestellt.

Mithilfe der Experimente in diesem Abschnitt kann sowohl die Bildung von Monoschichten amphiphiler Moleküle an der Wasseroberfläche, als auch die Bildung mizellarer Strukturen erarbeitet werden. Zudem wird die Abhängigkeit der Bildung verschiedener supramolekularer Aggregate von der Geometrie der zugrundeliegenden amphiphilen Teilchen thematisiert. Ein Modellexperiment mit 3D-Modellen verschiedener geometrischer Strukturen amphiphiler Teilchen bietet zudem die Möglichkeit, den Einfluss der Geometrie der Teilchen auf die Form des Aggregats auch auf makroskopischer Ebene erfahrbar zu machen. 


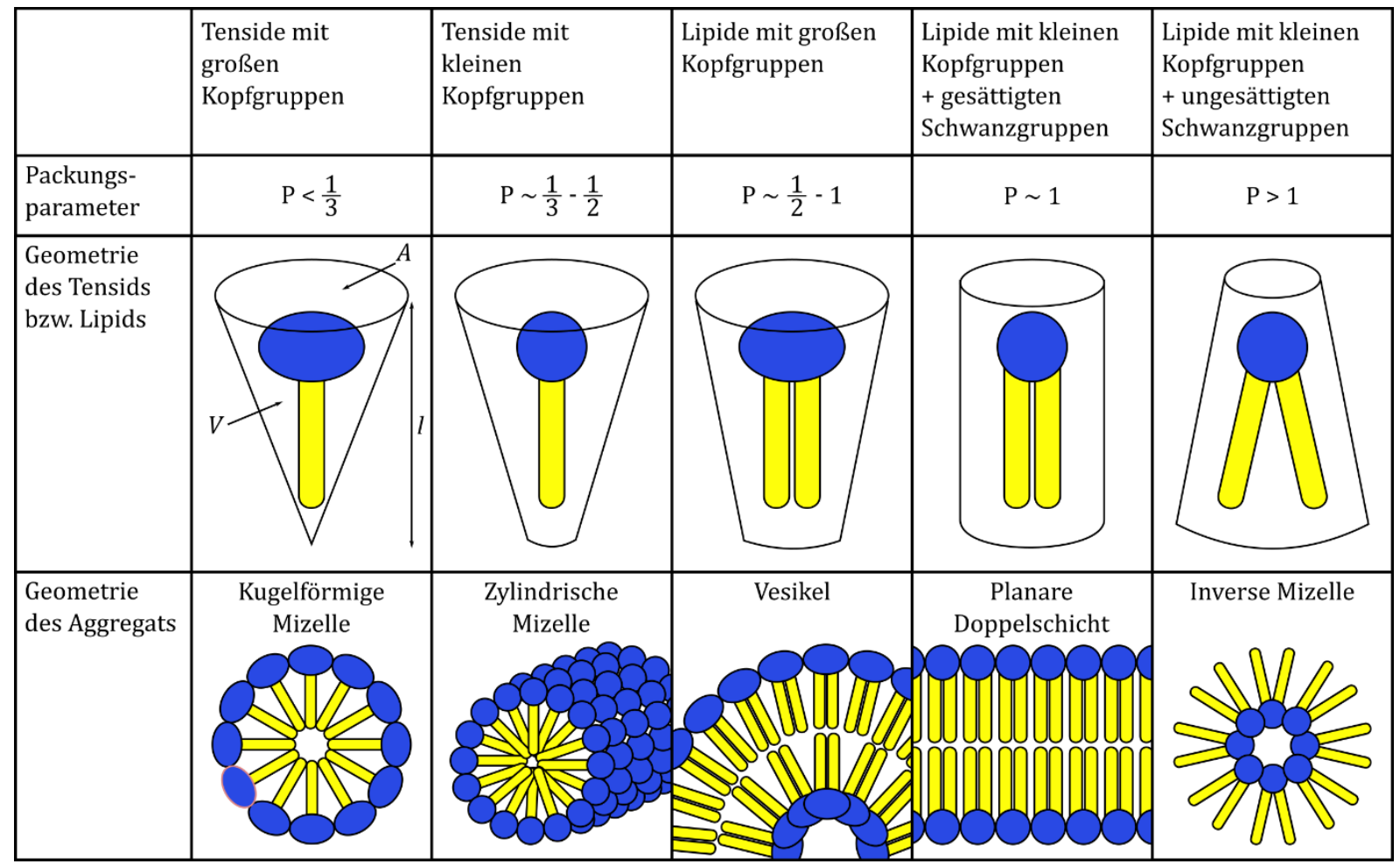

Abbildung 25: Lipidgeometrien in Abhängigkeit von ihrem Packungsparameter nach ISRAELACHVILI [195].

\section{Experiment 2.1a - Selbstorganisation von Tensiden zu Monoschichten}

Anhand dieses Experiments lässt sich im Unterricht bei Kenntnis über den amphiphilen Charakter von Tensiden die Selbstorganisation zu einer Tensid-Monoschicht auf der Wasseroberfläche ableiten.

Geräte und Chemikalien: Kristallisierschale, Becherglas, Glasrührstab, Wasser, Spülmittel, Speiseöl (angefärbt mit Paprikapulver).

Versuchsdurchführung: Eine Kristallisierschale wird einige Zentimeter hoch mit Wasser gefüllt und etwas angefärbtes Öl hinzugegeben. Anschließend werden einige Tropfen Spülmittel in die Mitte des Ölfilms getropft.

Beobachtung: Bei Zugabe des angefärbten Öls zum Wasser bilden sich Öltropfen, die nach kurzer Zeit einen zusammenhängenden Ölfilm auf der Oberfläche des Wassers bilden. Werden einige Tropfen Spülmittel hinzugegeben bewegt sich das Öl schlagartig an den Rand der Kristallisierschale.

Deutung: Die Bildung eines zusammenhängenden Ölfilms sorgt für eine möglichst kleine Phasengrenze und ist somit energetisch begünstigt. Bei Zugabe von Spülmittel in die Mitte des Ölfilms ordnen sich die Tensidteilchen mit der hydrophilen Seite zum Wasser und der hydrophoben Seite zur Luft zeigend an der Wasseroberfläche an. Dabei verdrängen sie den Ölfilm an den Rand der Kristallisierschale und setzen zudem die Oberflächenspannung herab (Abbildung 26). 


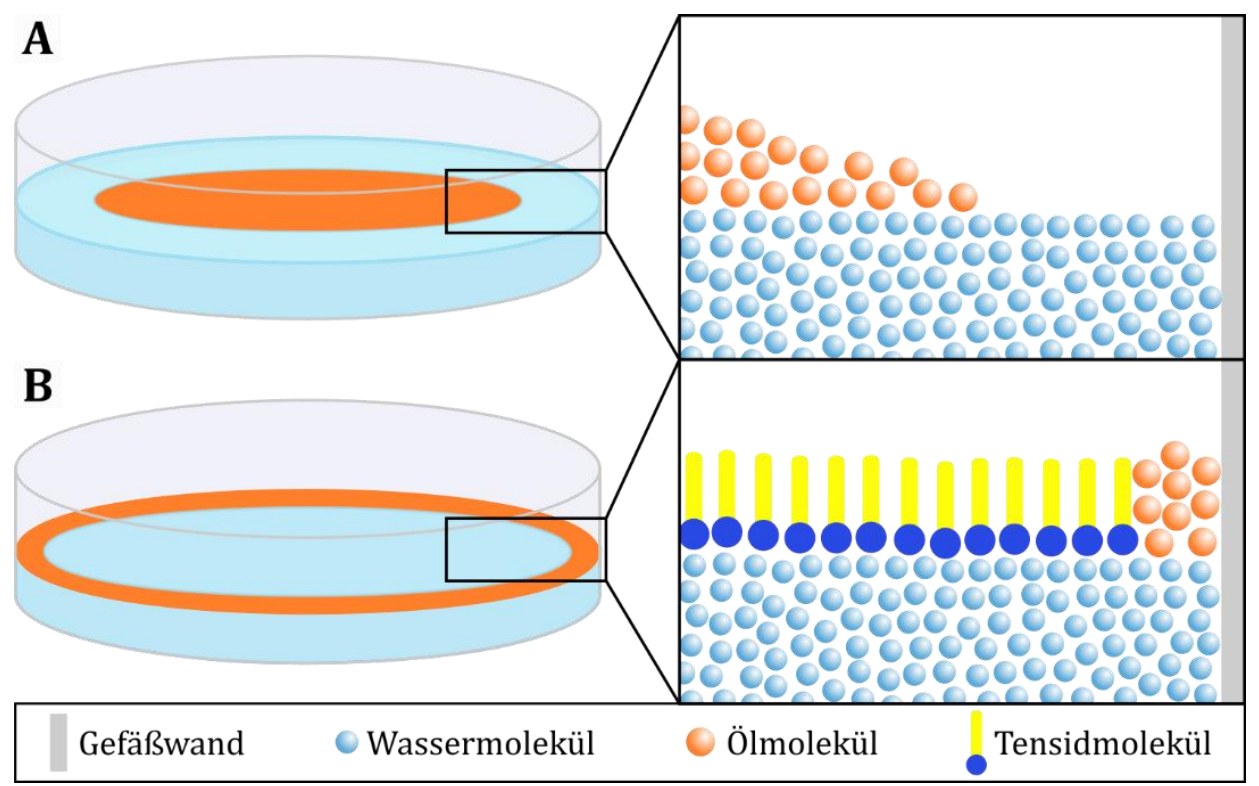

Abbildung 26: Schematische Darstellung einer Ölschicht auf der Wasseroberfläche auf makroskopischer und submikroskopischer Ebene vor (A) und nach der Zugabe eines Tensids (B).

\section{Experiment 2.1b - Selbstorganisation von Stearinsäuremolekülen}

Wurde der amphiphile Charakter von Tensiden im Unterricht noch nicht erarbeitet, kann dies am Beispiel der Selbstorganisation von Stearinsäuremolekülen an den Phasengrenzen zu Wasser bzw. zu Luft erfolgen [192].

Geräte und Chemikalien: 2 Bechergläser (100 mL und $250 \mathrm{~mL}$ ), Magnetrührer, Spatel, Glasrührstab, Pasteurpipetten, Wasserbad, Wasser, Stearinsäure (Octadecansäure), Methylenblau (GHS07).

Versuchsdurchführung: Ein Spatel feste Stearinsäure wird in ein zur Hälfte mit Wasser gefülltes Becherglas ( $250 \mathrm{~mL}$ ) gegeben und dieses solange erhitzt, bis die Stearinsäure vollständig geschmolzen ist. Die Lösung wird in einem Wasserbad abgekühlt, so dass die Stearinsäure erstarrt. Die feste Stearinsäure wird vorsichtig aus dem Becherglas entnommen, wobei sie ggf. vom Rand gelöst werden muss. In einem zweiten Becherglas werden einige Milliliter Wasser mit wenigen Kristallen Methylenblau angefärbt. Anschließend wird je ein Tropfen der Methylenblaulösung auf die Ober- und Unterseite der Stearinsäure gegeben.

Beobachtung: Beim Erhitzen des Wassers löst sich die feste Stearinsäure und bildet eine farblose flüssige Phase über dem Wasser. Beim Abkühlen kristallisiert die Stearinsäure zu einer festen weißen Scheibe aus. Wird ein angefärbter Wassertropfen auf die Unterseite der Scheibe gegeben, so zerfließt er und besitzt einen kleinen Kontaktwinkel. Der Wassertropfen auf der Oberseite hingegen besitzt einen deutlichen größeren Kontaktwinkel (Abbildung 27A, B).

Deutung: Bei Stearinsäure handelt es sich um eine gesättigte Fettsäure mit 18 Kohlenstoffatomen, die aufgrund ihrer Struktur amphiphile Eigenschaften besitzt. Beim Erhitzen des Wassers schmilzt die Stearinsäure und bildet eine zweite Phase oberhalb des Wassers. Die Stearinsäuremoleküle ordnen sich dabei, wie in Abbildung 27D dargestellt, an der Phasengrenze zu Wasser so an, dass ihre hydrophilen Anteile in Richtung des Wassers zeigen. An der Phasengrenze zu Luft weisen hingegen die hydrophoben Anteile nach au- 
ßen. Werden die Flüssigkeiten langsam abgekühlt, erstarrt die Stearinsäure wieder, wobei die Moleküle ihre Ausrichtung beibehalten. Entsprechend weist die Unterseite hydrophile, die Oberseite hingegen hydrophobe Eigenschaften auf.

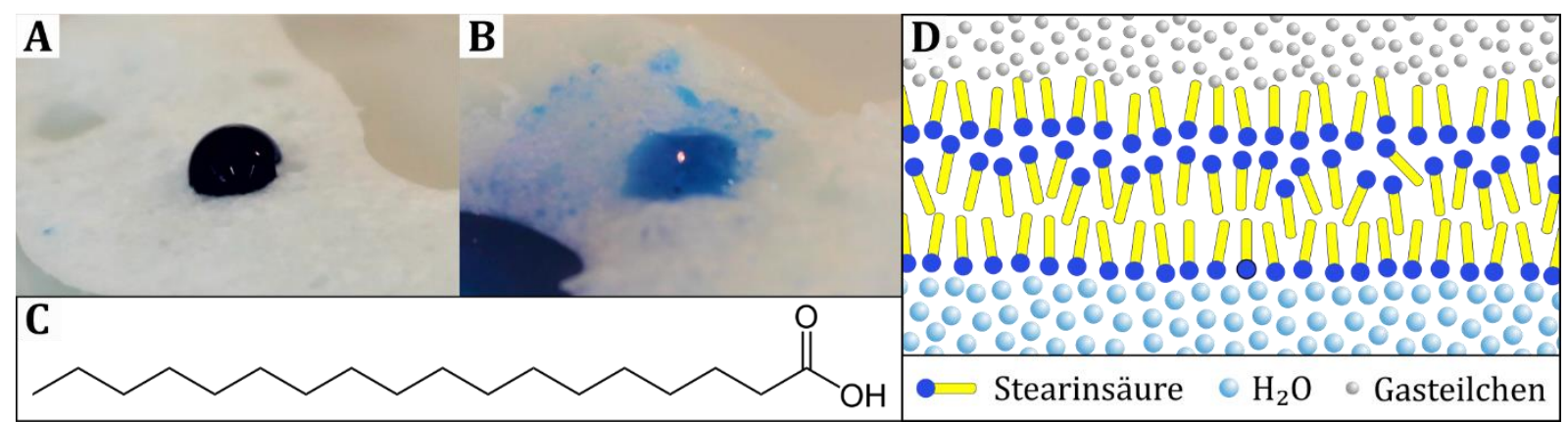

Abbildung 27: Angefärbter Wassertropfen auf der hydrophoben (A) bzw. hydrophilen Oberfläche der Stearinsäure (B). (C) Strukturformel der Stearinsäure, (D) Deutung auf Teilchenebene.

Anmerkungen zur Deutung der Experimente: Anhand der Erkenntnisse bezüglich der hydrophilen und hydrophoben Eigenschaften amphiphiler Moleküle können Hypothesen bezüglich des Verhaltens der Moleküle an Phasengrenzen wie der Wasser-zu-Luft Grenze aufgestellt und überprüft werden. Die Zugabe eines Tensids zu einem auf der Wasseroberfläche schwimmenden Ölfilm weist auf eine sich ausbreitende Tensidschicht hin, die das Öl an den Rand des Gefäßes verdrängt. Dass die Tensidmoleküle sich dabei mit ihren hydrophilen Köpfen zum Wasser und mit ihren hydrophoben Schwänzen zur Luft hin orientieren, kann mithilfe der Stearinsäure noch einmal überprüft werden. Zwar besteht die erstarrte Stearinsäureplatte aus mehr als einer Monoschicht an Stearinsäuremolekülen, jedoch orientieren sich die Moleküle sowohl an der Grenzfläche zum Wasser als auch an der Grenzfläche zur Luft mit dem Kopfende nach unten, so dass die hydrophilen Köpfe zum Wasser und die hydrophoben Schwanzteile zur Luft hin ausgerichtet sind, was wiederum die unterschiedlichen Benetzungseigenschaften der Ober- und Unterseite erklärt.

\section{Experiment 2.2 - Lichtstreuung in Spülmittellösungen}

Die Betrachtung Lichtstreuung in Spülmittellösungen ermöglicht einen qualitativen Nachweis der Bildung von Mizellen in Wasser. Aufgrund seiner Reproduzierbarkeit eignet sich der Versuch insbesondere auch für die Anwendung bei Bildungsmessen [196].

Geräte und Chemikalien: 3 Bechergläser, Laserpointer, Glasrührstab, Spatel, demin. Wasser, Natriumchlorid, Spülmittel.

Versuchsdurchführung: Drei Bechergläser werden zu zwei Dritteln mit demineralisiertem Wasser gefüllt. Eines davon wird mit etwas Natriumchlorid, ein weiteres mit einigen Tropfen Spülmittel versetzt. Anschließend wird jede der drei Lösungen horizontal mit dem Licht eines Laserpointers durchstrahlt.

Beobachtung: Beim Durchstrahlen der Spülmittellösung wird der Strahlengang durch die Lösung sichtbar. Weder im Wasser noch in der Natriumchloridlösung ist dies ebenfalls zu beobachten (Abbildung 28).

Deutung: Bei Zugabe von Spülmittel zu Wasser bilden sich neben einer Monotensidschicht an der Wasseroberfläche auch runde Aggregate - sogenannte Mizellen - die die hydrophoben Bestandteile der Tenside von den polaren Wassermolekülen abschirmen. Wird die Lösung mit einem Laser durchstrahlt, dann wird das Licht an den in der Lösung dispergierten Aggregaten gestreut, so dass der Laserstrahl für das menschliche Auge auch 
von der Seite zu sehen ist. Die in der Natriumchloridlösung vorliegenden Ionen sind hingegen zu klein, um das Licht des Lasers zu streuen.

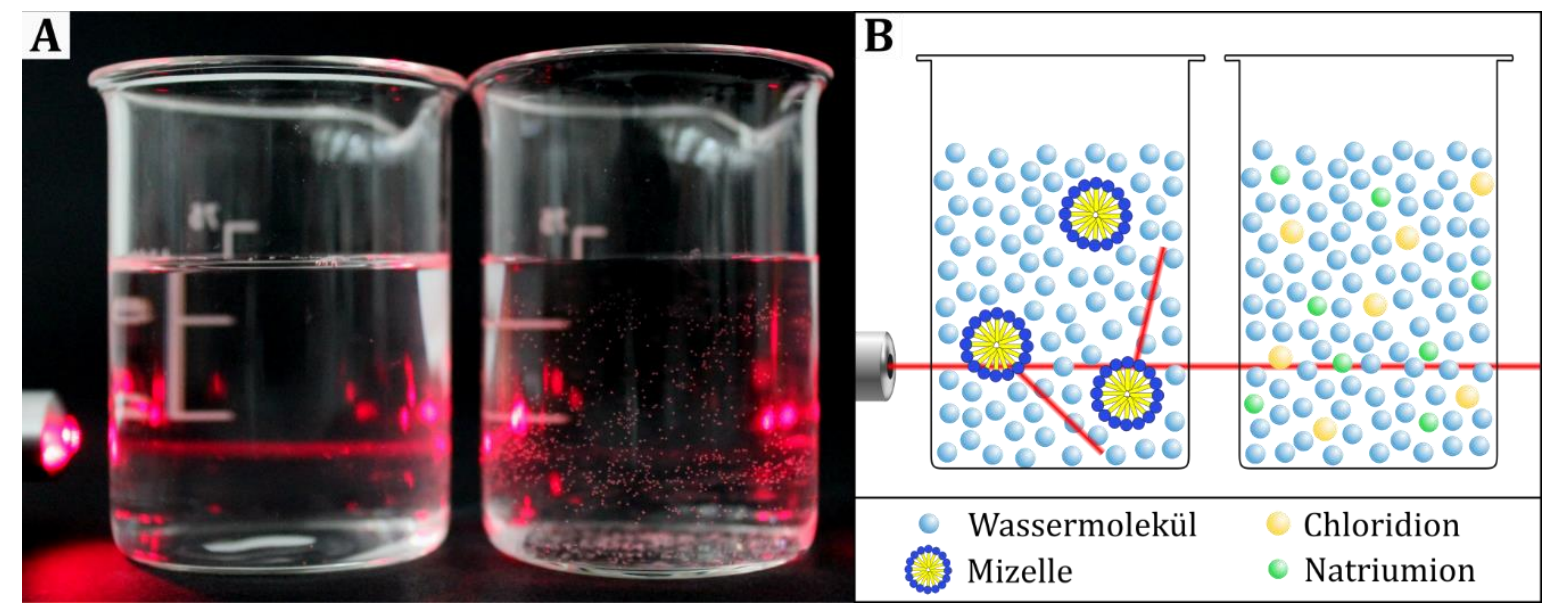

Abbildung 28: (A) Lichtstreuung in einer Tensidlösung (links). In einer Natriumchloridlösung (rechts) kann keine Lichtstreuung beobachtet werden. (B) Schematische Darstellung der Streuung des Lichts an Mizellen.

Anmerkungen zur Deutung des Experiments: Je nach Molekülstruktur des verwendeten Tensids bewegen sich die Durchmesser von Mizellen im Bereich weniger Nanometer. Solche kolloiden Partikel können mithilfe des sogenannten Tyndall-Effekts, der auf der MieStreuung von Licht an Partikeln im ungefähren Größenbereich $(d \geq \lambda / 20)^{[197]}$ des einfallenden Lichtes beruht, nachgewiesen werden. Entsprechend kann anhand des Vorhandenseins des Tyndall-Effekts auf eine Bildung kolloider Teilchen im Nanometerbereich geschlossen werden. Wie genau diese Aggregate aussehen, lässt sich im Zuge dieses Experiments zunächst lediglich anhand der bereits erworbenen Erkenntnisse bezüglich der Eigenschaften von Tensiden modellieren. Diesbezüglich sind u. a. sowohl mizellare als auch vesikuläre Geometrien denkbar.

Das Auftreten einer Lichtstreuung bei Vorhandensein kolloider Teilchen in Lösung wird in der Forschung im Zuge von Messungen der dynamischen Lichtstreuung (Dynamic Light Scattering, DLS) genutzt, um Aussagen über die Größe der gelösten Moleküle oder Aggregate zu treffen. Auf diese Weise können unter anderem in ähnlichen experimentellen Ansätzen wie im nachfolgenden Versuch die durchschnittlichen Radien von Vesikeln bestimmt werden [198].

\section{Experiment 2.3 - Selbstaggregation amphiphiler Moleküle in Abhängigkeit ihrer geometrischen Form}

In Anlehnung an DEL BIANCO, TORINO \& MANSY [199], kann anhand dieses Versuchs die Selbstaggregation zu Mizellen und Vesikeln in Abhängigkeit der geometrischen Form der verwendeten Tenside und Lipide untersucht werden. Die Verwendung von Plastikspritzen als Chromatographiesäulen ermöglicht eine Anwendung sowohl im Unterricht als auch im Schülerlabor [200].

Geräte und Chemikalien: Größenausschluss-Chromatographiesäulen (Sephadex G25, VWR), 11 Bechergläser (10x $50 \mathrm{~mL}$ und 1x $500 \mathrm{~mL}$ ), Spatel, Pasteurpipetten, Stativmaterial, pH-Meter, UV-Lampe, Ultraschallgerät, Magnetrührer, demin. Wasser, Fettsäure (Linolen-, Palmitin- oder Ölsäure), Lecithin, Pyranin (GHS07), Natriumhydroxidlösung (0,1 M; GHS05), Salzsäurelösung (0,1 M; GHS05), Spülmittel.

Versuchsdurchführung: Unter Rühren werden 4 Spatelspitzen Pyranin in $500 \mathrm{~mL}$ Wasser gelöst. Der pH-Wert der Lösung wird gemessen und wenn nötig unter Verwendung von 
Natriumhydroxid- oder Salzsäurelösung auf $\mathrm{pH}=8$ eingestellt. In 5 Bechergläsern (50 mL) werden jeweils $40 \mathrm{~mL}$ der Lösung vorgelegt und die folgenden Chemikalien hinzugegeben: (A) eine Spatelspitze Spülmittel, (B) eine Spatelspitze Lecithin, (C) eine Spatelspitze einer Fettsäure, (D) eine Spatelspitze einer Fettsäure sowie etwas Natriumhydroxidlösung, um den $\mathrm{pH}-$ Wert auf $\mathrm{pH}=11$ einzustellen. Der Lösung im fünften Becherglas (E) wird nichts hinzugefügt. Alle fünf Lösungen werden für 5 Minuten in ein Ultraschallbad gestellt (10 Minuten, falls ein haushaltsübliches Brillenultraschallbad verwendet wird) und im Anschluss für weitere 5 Minuten ruhen gelassen.

Während der Größenauschlusschromatographie werden alle Schritte unter UV-Licht durchgeführt, um die Sichtbarkeit des Pyranins zu erhöhen. Für jede der Lösungen wird eine Säule solange geöffnet, bis die Oberfläche des Säulenmaterials nahezu trocken liegt. Zwei Tropfen der jeweiligen Lösungen werden vorsichtig auf das Säulenmaterial gegeben. Sobald die Lösungen in das Material eingesunken sind, werden einige Tropfen demineralisiertes Wasser hinzugegeben, bis sich eine farbige Bande in der obersten Schicht des Säulenmaterials gebildet hat. Anschließend wird die Säule vorsichtig bis zum Rand mit Wasser gefüllt. Die aus der Öffnung der Säule tropfende Lösung wird aufgefangen, bis die beobachtbare Fluoreszenz ungefähr in der Mitte der Säule angekommen ist. Um den Prozess zu stoppen, wird die Säule an ihrem unteren Ende wieder verschlossen.

Hinweis: Es ist möglich, eine Säule für alle Lösungen zu nutzen (Spülen notwendig!). Die Verwendung unterschiedlicher Säulen ermöglicht es jedoch, die Beobachtungen zu vergleichen.

Beobachtung: Die leicht gelbe und transparente Pyraninlösung leuchtet gelb bis grün unter UV-Licht. Nach der Behandlung im Ultraschallbad liegen in den Bechergläsern A bis D Suspensionen vor, während die Lösung in Glas E transparent bleibt. Für die verschiedenen Lösungen formen sich in den Säulen unterschiedliche fluoreszierende Banden: (A) eine Bande, (B) zwei Banden, (C), zwei Banden, (D), eine Bande, (E) eine Bande (Abbildung 29).
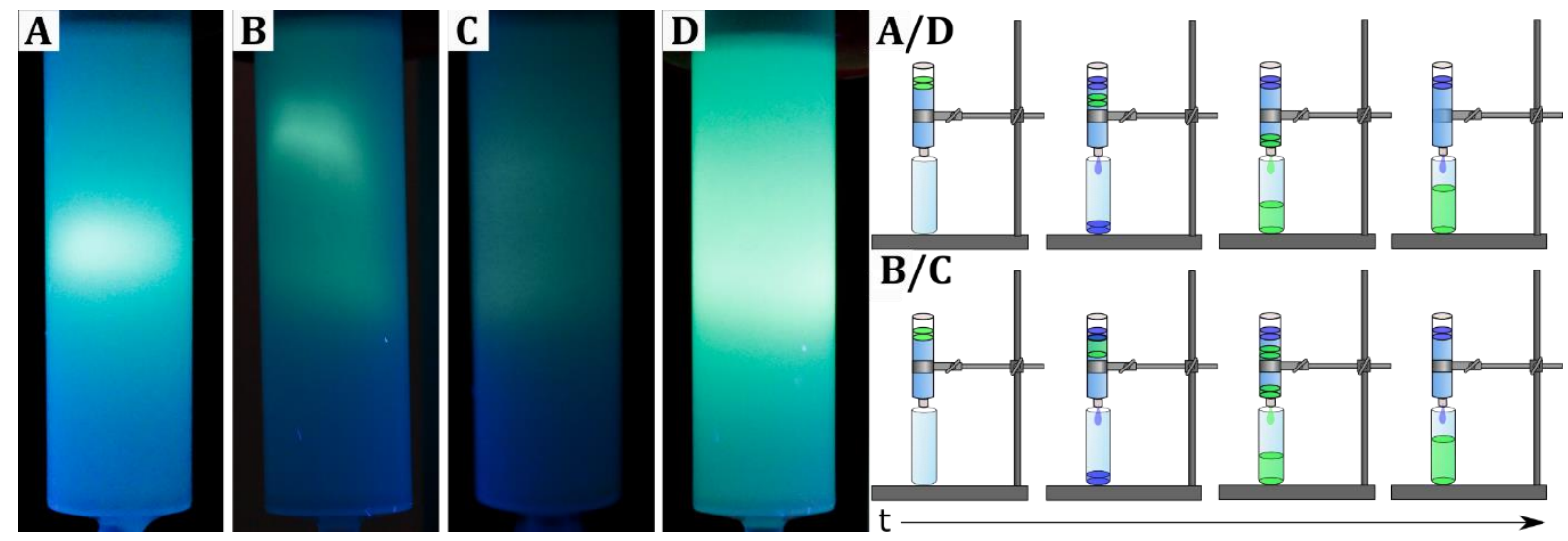

Abbildung 29: Größenausschluss-Chromatographiesäulen (Sephadex 25) unter UV-Licht von A) Pyraninlösung + Spülmittel, B) Pyraninlösung + Lecithin, C), Pyraninlösung + Ölsäure (pH = 8) und D) Pyraninlösung + Ölsäure $(\mathrm{pH}=11)[200]$.

Deutung: Beim Lösen von Spülmittel, Lecithin und Fettsäuren in den angefärbten Lösungen entstehen supramolekulare Aggregate mit Größen im Nanometerbereich. Dabei kann die Bildung der Aggregate auf die amphiphilen Eigenschaften der jeweiligen Moleküle zurückgeführt werden. Während der Säulenchromatografie werden die verschiedenen Bestandteile der Lösung vor allem aufgrund ihrer Größe aufgetrennt. Die erste Säule mit der Tensidlösung zeigt nur eine fluoreszierende Bande. Tensidmoleküle in Wasser bilden bekanntermaßen Mizellen, die auf der Säule nicht sichtbar von anderen Bestandteilen der 
Lösung getrennt werden können. Entsprechend ist nur eine fluoreszierende Bande zu sehen, die auf die enthaltenen Pyraninmoleküle zurückgeführt werden kann. Lecithin besitzt zwei hydrophobe Ketten und ist damit zu voluminös, um Mizellen zu formen, so dass stattdessen Vesikel entstehen. Während der Aggregation zu Vesikeln wird wässrige Pyraninlösung im Inneren der Hohlkugeln eingeschlossen, was dazu führt, dass zwei fluoreszierende Banden entstehen - die untere enthält die deutlich größeren Vesikel, die obere die frei vorliegenden Pyraninmoleküle (siehe Abbildung 30).
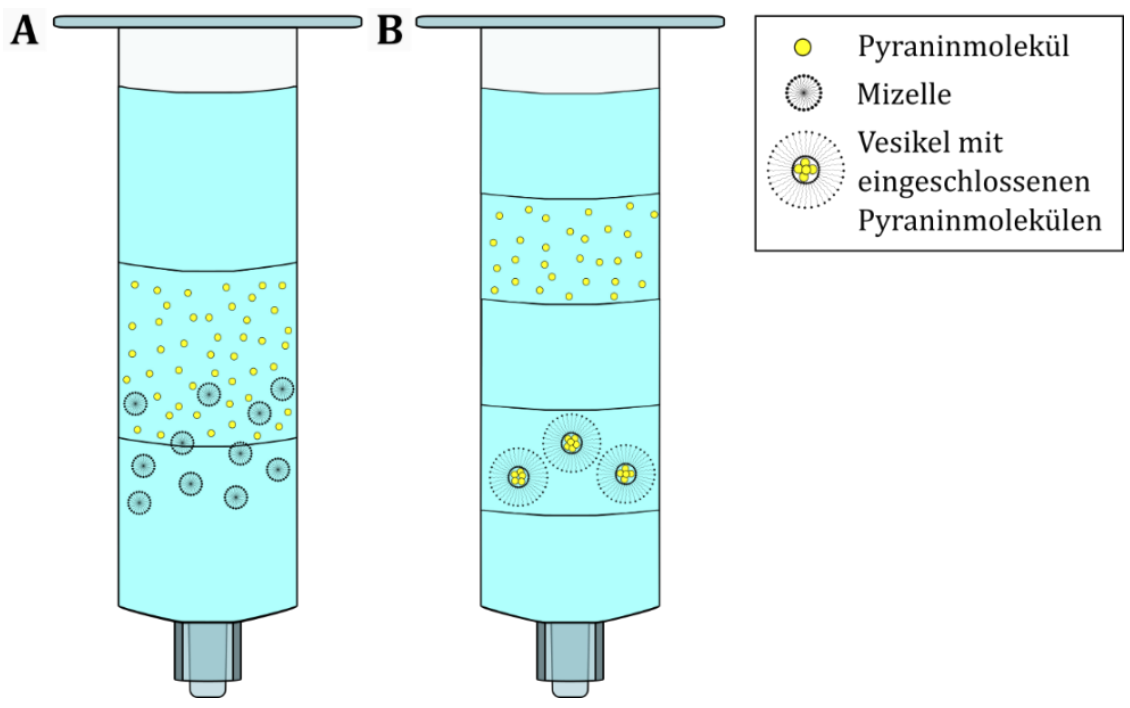

Abbildung 30: Schematische Darstellung der Auftrennung der Moleküle in den jeweiligen Säulen. (A) Lösungen A und D, (B) Lösungen B und C [200].

In Abhängigkeit vom pH-Wert der Fettsäurelösungen treten sowohl eine $(\mathrm{pH}=11)$ als auch zwei Banden ( $\mathrm{pH}=8$ ) auf. Das lässt darauf schließen, dass der pH-Wert einen Einfluss auf die Aggregation der Moleküle hat, was zur Bildung von sowohl Mizellen als auch Vesikeln führt. Die letzte Lösung ohne weitere Zusätze zeigt nur eine Bande, da hier lediglich der Farbstoff enthalten ist.

Anmerkungen zur Deutung des Experiments: Die geometrische Form der Aggregate amphiphiler Moleküle hängt stark vom Packungsparameter der Monomere ab. Fettsäuren besitzen im Gegensatz zu Lipiden wie den Lecithinen nur eine hydrophobe Kette, was ihnen eine eher konische Geometrie verschafft. Dennoch zeigen die hier genannten Fettsäuren in Abhängigkeit vom pH-Wert unterschiedliche Aggregate. Bei einem pH-Wert >10 liegen die Fettsäuren vollständig deprotoniert vor. Die geladenen Kopfgruppen sorgen dabei für eine stark konische Form und somit für die Bildung von Mizellen. Im pH-Bereich von 710 liegen die Fettsäuren nur partiell deprotoniert vor, was die Ausbildung von Wasserstoffbrückenbindungen zwischen zwei Fettsäuremolekülen ermöglicht (siehe Abbildung 31A). Die entstehenden Dimere ähneln geometrisch Lipiden mit zwei hydrophoben Ketten, die insgesamt eine eher zylindrische Geometrie besitzen, was wiederum die Bildung von Vesikeln erklärt.

Typischerweise wird die Bildung amphiphiler Aggregate lediglich in Bezug auf Tenside und Waschprozesse im Chemieunterricht behandelt. Dieselben Konzepte spielen jedoch auch in biologischen Kontexten wie bei der Bildung von Lipiddoppelschichten eine wichtige Rolle, so dass interdisziplinäre Bezüge bspw. zum vesikulären Transport von Neurotransmittern am synaptischen Spalt hergestellt werden können. Zudem bietet die Verwendung herkömmlicher Plastikspritzen als Chromatographiesäulen die Möglichkeit, das Verfahren der Größenausschluss-Chromatographie, das in der biochemischen Forschung 
häufig zur Aufreinigung von Proteinen sowie hier beschrieben zur Trennung von Vesikeln verwendet wird, auch in schulischen Kontexten anzuwenden.

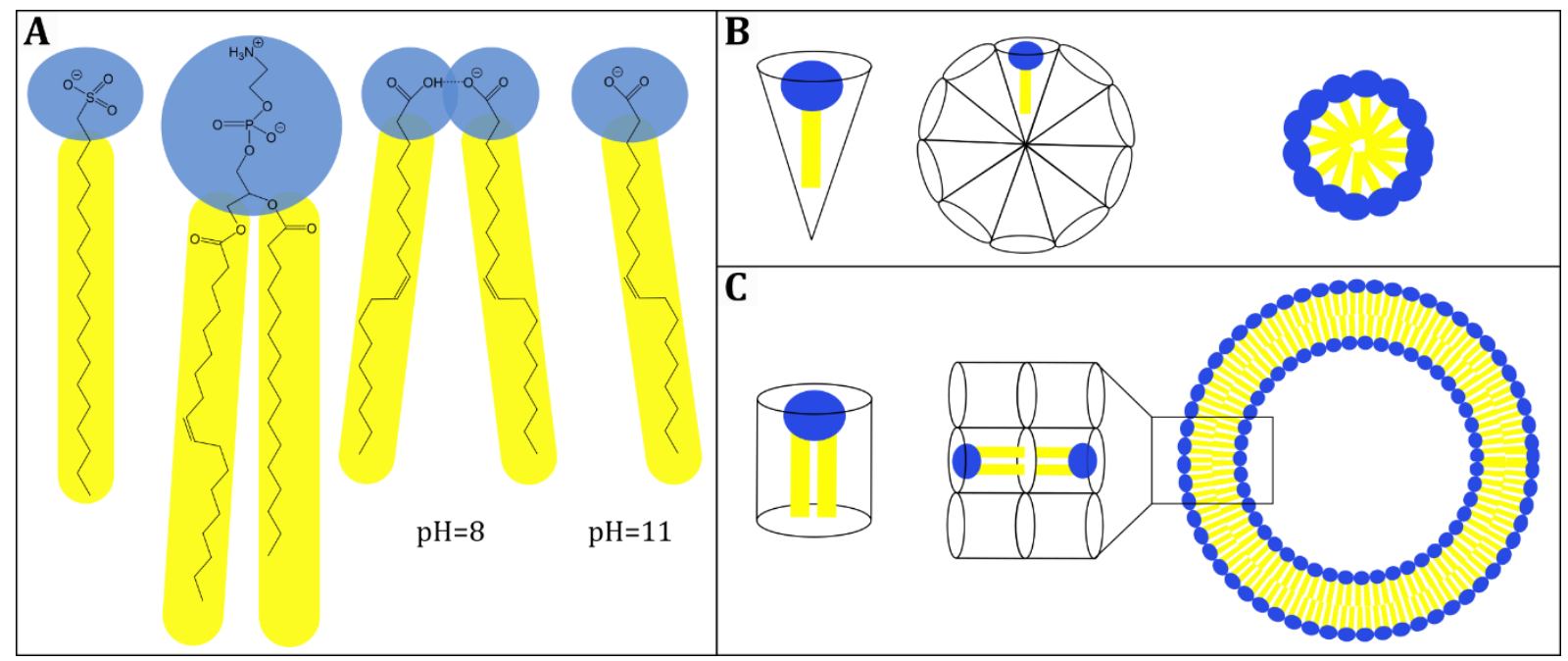

Abbildung 31: (A, von links nach rechts) Typisches Sulfonat-Tensid, Phosphocholin-Lipid, Ölsäuredimer bei $\mathrm{pH}=8$, deprotoniertes Ölsäuremonomer bei $\mathrm{pH}=11$. (B) Darstellung eines Tensids mit konischer Geometrie das Mizellen bildet. (C) Darstellung eines zylindrischen Lipids, das Vesikel bildet [200].

\section{Experiment 2.4 - 3D Modelle verschiedener Lipidgeometrien}

Die Verwendung gedruckter 3D-Modelle ermöglicht zusätzlich haptische Erfahrungen auf makroskopischer Ebene bezüglich des Einflusses von Lipidgeometrien auf die Selbstaggregation amphiphiler Moleküle [175].

Geräte und Chemikalien: 3D-gedruckte Lipidmodelle mit eingelassenen Magneten, großes Glasgefäß, Wasser.

Versuchsdurchführung: In ein mit Wasser gefülltes Glasgefäß werden 3D-gedruckte Lipidmodelle gleicher Geometrien (zylindrisch, konisch) gegeben.

Beobachtung: Die zylindrischen Lipidmodelle schwimmen an der Wasseroberfläche. Dabei zeigen die blau markierten Enden der Modelle ins Wasser und die gelb markierten Enden ragen teilweise heraus. Zudem ziehen sich die blauen Enden der Modelle gegenseitig an. Bei den konischen Lipidmodellen ziehen sich hingegen die gelben Spitzen der Modelle gegenseitig an. Dabei entsteht ein eher rundes Gebilde, bei dem die gelben spitzen Enden nach innen und die blauen flachen Enden nach außen zeigen.

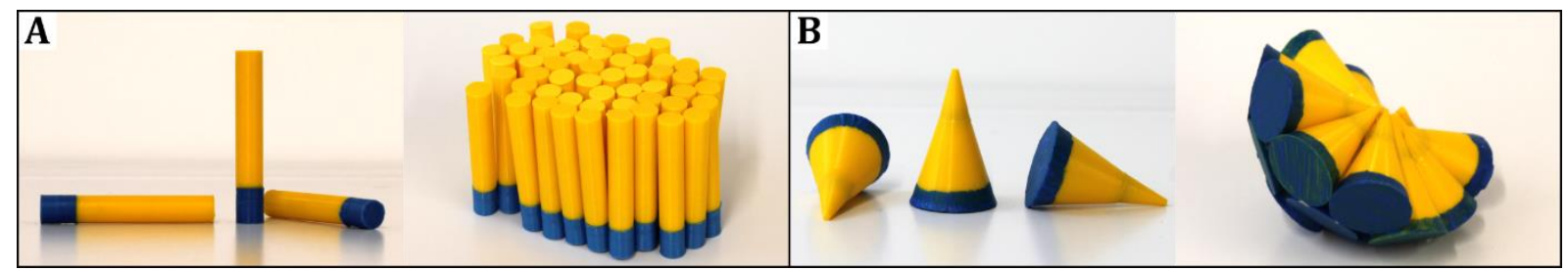

Abbildung 32: 3D-gedruckte Tensid- bzw. Lipidmodelle mit zylindrischer (A) und konischer Geometrie (B).

Deutung: Die Anordnung der Modelle ist auf ihre Dichte sowie die Anziehungskräfte der eingelassenen Magneten zurückzuführen. Die zylindrischen Modelle bilden an der Wasseroberfläche eine Monoschicht, während die konischen Modelle sich zu einer mizellaren Struktur anordnen (vgl. Abbildung 32).

Anmerkungen zur Deutung des Experiments: Die gedruckten 3D Modelle amphiphiler Moleküle ermöglichen es, auf makroskopischer Ebene eine räumliche Vorstellung bezüglich 
des Einflusses verschiedener Molekülgeometrien auf die Ausbildung unterschiedlicher Aggregate zu gewinnen. Die zylindrischen Modelle bilden planare Strukturen aus, während die konischen Modelle kugelförmige Aggregate ergeben. In Wasser kann so sowohl die Ausbildung von Tensidmonoschichten an der Wasseroberfläche als auch die Aggregation zu Mizellen dargestellt werden, wobei die Modelle dabei nicht ohne ausführliche Modellkritik verwendet werden können. Obwohl beide Strukturen in Lösung von Tensiden des gleichen Packungsparameters herrühren, können sie im Modellversuch nur mithilfe unterschiedlicher geometrischer Körper dargestellt werden. Zudem können die magnetischen Anziehungskräfte nicht ohne weiteres mit zwischenmolekularen Wechselwirkungen gleichgesetzt werden. Außerhalb des wässrigen Mediums können die zylindrischen Modelle alternativ auch zu Lipiddoppelschichten arrangiert werden. Hierdurch ist eine deutlichere Abgrenzung der konischen Körper als Modelle für Tensidmoleküle zu den zylindrischen Körpern als Modelle für Lipidmoleküle möglich.

\section{Exkurs: 3D Druck}

Additive Fertigungstechniken (Additive Manufacturing), die heute vor allem unter dem Ausdruck 3D Druck bekannt sind, wurden erstmals in den achtziger Jahren entwickelt, um die Produktion von Prototypen zu beschleunigen. Im Gegensatz zu subtraktiven Fertigungsprozessen, bei denen ein Produkt durch das Entfernen von Substrat erhalten wird, werden bei der additiven Fertigung dreidimensionale Objekte erstellt, indem verschiedene Materialien Schicht für Schicht aufgetragen werden. Die Technik eignet sich insbesondere dann, wenn das Design eines Objektes wie bei der Erstellung eines Prototyps oftmals wechselt, das zu erstellende Objekt ein komplexes Design besitzt, oder das benötigte Produktionsvolumen sehr gering ist. Mittlerweile ist es möglich 3D Objekte aus Materialien wie Keramiken, Metallen oder Kunststoffen herzustellen. Entsprechend dieser Vielzahl an Ausgangssubstraten, existieren auch verschiedene Drucktechniken, auf die hier jedoch nicht näher eingegangen wird[201, 202].

Die Einsatzgebiete des 3D Drucks sind vielfältig und reichen von der Luftfahrt, über die Automobilindustrie, Architektur, Kunst, Robotik und Modewelt bis hin zu pharmakologischen und medizinischen Anwendungen [202]. Beispielsweise wird das Verfahren in der Zahnmedizin genutzt, um Implantate zu erstellen, und Unternehmen wie die Deutsche Bahn verwenden es, um Einzelteile wie Mantelhaken, Kopfstützen, aber auch individualisierte Schilder mit Blindenschrift zu drucken [203]. In der Medizin wird zudem seit gut 20 Jahren Forschung im Bereich des Bioprinting betrieben [204], bei dem biologisches Material sowie (Stamm-) Zellen als Ausgangsmaterialien verwendet werden. Insbesondere mit Blick auf die allgemeine Knappheit an Spenderorganen ist es von Interesse, mithilfe additiver Fertigungsprozesse Gewebe und Organe zu produzieren, die aufgrund der Verwendung patienteneigener Zellen keine Abstoßungsreaktionen erfahren. Obwohl die Forschung noch einige Hürden nehmen muss, u. a. stellt die Vaskularisierung des Gewebes also die Ausbildung von Blutgefäßen - noch ein Problem dar, ist es beispielsweise bereits gelungen, kontraktierendes Herzmuskelgewebe sowie ein Miniaturmodell eines Herzens aus menschlichen Stammzellen zu drucken [205, 206]. Auch wenn die Verwendung am Menschen in diesem Falle noch nicht möglich ist, wird das erstellte Gewebe beispielsweise genutzt, um medizinische Wirkstoffe zu testen [207]. Des Weiteren werden zudem allgemeine additive Fertigungstechniken in der Medizin eingesetzt, um komplizierte Operationen im Vorfeld anhand von Modellen des Patienten zu üben, was insbesondere auch Eingang in die Ausbildung von Medizinstudenten findet [201]. 
Spätestens seit dem Auslaufen einiger Patente für additive Fertigungstechniken existieren 3D-Drucker, die auch für den häuslichen Gebrauch erschwinglich sind. Einfache Geräte können bei einschlägigen Onlinehändlern bereits für unter $200 €$ erstanden werden, und auch gängige Filamente wie das biologisch abbaubare PLA (Polymilchsäure), das auch für den Druck der Tensid- und Lipidmodelle verwendet wurde, sind bereits für gut $20 €$ pro Kilo erhältlich. Dieser Umstand macht 3D-Drucker auch für den schulischen Gebrauch zunehmend interessanter, zumal das Erstellen von 3D Objekten mit der z. T. kostenfrei erhältlichen Software nicht zuletzt auch mediale Kompetenzen der SuS fördert. Auch für den Chemieunterricht lassen sich in der Literatur bereits einige Anwendungsbeispiele finden. Allen ist dabei gemein, dass sie, ähnlich wie bei einem Molekülbaukasten, genutzt werden, um die Anordnung von Atomen innerhalb eines Molekülverbundes darzustellen. Der Druck von 3D Modellen ermöglicht es jedoch auch, auf einfache Weise Modelle größerer Strukturen wie DNA [208, 209], Proteinen [210-212] oder Komplexverbindungen [208] zu erstellen. Die zusätzliche Darstellung von Orbitalen ermöglicht es zudem, schwer zu fassende quantenmechanische Zustände zu visualisieren [213-215].

\section{Einordnung der Experimente im Kontext der SO-Leitfragen}

Im Anschluss an das erste Themenfeld, in dem der Begriff der Amphiphilie erarbeitet wurde, kann diese Eigenschaft der Tensidmoleküle im Rahmen des zweiten Abschnitts zur Selbstorganisation amphiphiler Tenside genauer untersucht werden. Dabei können erste Vermutungen zur Ausbildung einer Monotensidschicht auf der Wasseroberfläche aus Experiment 2.1a am Beispiel der Selbstorganisation von Stearinsäuremolekülen überprüft werden. Das Sichtbarwerden eines Laserstrahls in wässriger Lösung durch Zugabe eines Tensids (Exp. 2.2) kann zum Anlass genommen werden, die SuS selbstständig Modelle sowohl der Monotensidschicht an der Wasseroberfläche als auch für die in wässriger Lösung entstehenden Aggregate entwickeln zu lassen. Neben der Bildung von Mizellen ist ohne weitere Untersuchungen auch durchaus die Bildung weiterer Aggregate wie bspw. von Vesikeln denkbar. So kann das Experiment zur Selbstaggregation amphiphiler Moleküle in Abhängigkeit von ihrer geometrischen Form gleich in zweierlei Hinsicht genutzt werden. Zum einen gibt es Hinweise auf die Existenz verschiedener Aggregate, zum anderen zeigt es die Relevanz der Molekülgeometrie für die Selbstassemblierung zu unterschiedlichen Aggregaten auf. Mit der Auswertung des Experiments kann somit indirekt die Bildung von Mizellen im Experiment zur Lichtstreuung nachgewiesen werden. Gleichzeitig erfolgt mit der Bildung der Vesikel eine erste Kompartimentierung, die lediglich durch die Verwendung von Lipiden sowie die Ausbildung lipidähnlicher Geometrien möglich ist.

Die in diesem Themenfeld vorgestellten Experimente bieten somit die Möglichkeit, sowohl die Selbstorganisation im Allgemeinen als auch Eigenschaften kompartimentbildender Moleküle zu erarbeiten, wodurch inhaltlich ein Brückenschlag von der ersten zur zweiten Leitfrage erfolgt. Die 3D-gedruckten Modelle aus Experiment 2.4 können innerhalb des Themenfelds variabel eingesetzt werden, um die Entwicklung einer Modellvorstellung der submikroskopischen Ebene zu unterstützen. Dabei können die Modelle den SuS bereits gedruckt zur Verfügung gestellt werden. Prinzipiell bietet eine Auseinander- 
setzung mit der Methode des 3D-Drucks jedoch auch die Möglichkeit, durch die Erarbeitung eigener Modelle ${ }^{12}$ und die Diskussion ihrer Vorteile und Grenzen die Modellkompetenz der SuS weiter zu fördern und durch die Thematisierung verschiedener Facetten des 3D-Drucks u. a. auch auf Anwendungsgebiete in der Membranforschung hinzuweisen.

\subsection{Themenfeld 3 - Membranen und ihre Bestandteile}

Membranen sind an einer Reihe von biochemischen Prozessen beteiligt bzw. für sie verantwortlich. $\mathrm{Zu}$ diesen Prozessen gehören $u$. a. Fotosynthese, Zellatmung, Reizweiterleitung sowie der Aufbau elektrochemischer Gradienten [216]. Entsprechend beschäftigt sich die Wissenschaft bereits seit über 100 Jahren mit der Erforschung von Membranen und ihren Eigenschaften. Dabei wurden über die Zeit verschiedene Membranmodelle entwickelt, die den jeweiligen Stand der Forschung widerspiegeln. Eines der ersten Modelle stammt von GORTER \& GRENDEL (1925), die feststellten, dass die Anzahl an Lipiden in roten Blutkörperchen gerade so groß ist, dass sie deren Oberfläche zwei Mal bedecken können. Basierend auf früheren Erkenntnissen zu der Anordnung von Lipiden an der Wasseroberfläche schlossen sie, dass Membranen aus einer Lipiddoppelschicht bestehen müssen, bei denen die polaren Anteile der Lipide jeweils zu den wässrigen Innen- und Außenmedien hin ausgerichtet sind [217]. Die Theorie der Lipiddoppelschicht wurde 1935 von DANIELLI \& DAVson durch das Sandwich-Modell erweitert. Basierend auf den Erkenntnissen, dass Membranen neben Lipiden auch aus Proteinen bestehen und diese Proteine an der Oberfläche der Membranen aktiv sind, gingen sie davon aus, dass die beiden Seiten der Lipiddoppelschicht - ähnlich einem Sandwich - jeweils mit einer Proteinschicht bedeckt sind [218].

Mit der Entwicklung der Elektronenmikroskopie erkannte man, dass Membranen nicht nur einzelne Zellen voneinander abgrenzen, sondern dass z. B. auch Zellorganellen von Membranen umgeben sind. Die Entwicklung neuer Techniken führte zudem zu zahlreichen Erkenntnissen, die mithilfe des Sandwich-Modells nicht mehr hinreichend erklärt werde konnten. Beispielsweise existieren Proteine, die sich über relative milde Verfahren wie die Zugabe von Chelatbildnern von der Membran lösen lassen. Gleichzeitig lassen sich über $70 \%$ der Proteine jedoch kaum oder lediglich über invasive Verfahren wie Denaturierung aus der Membran entnehmen. Um diese und weitere Erkenntnisse in Einklang zu bringen, postulierten SINGER \& NICOLSON 1972 das Flüssig-Mosaik-Modell, das auch heute noch in zahlreichen Schulbüchern und sogar im Kerncurriculum der Biologie zu finden ist (vgl. Kapitel 3.2.1) [182]. Danach bestehen Biomembranen aus einer wie in Abbildung 33 dargestellten Lipiddoppelschicht mit darin eingebetteten Proteinen, die sowohl durch die Membran durchreichen als auch nur peripher mit ihr assoziiert sein können, so dass letztere lediglich von einer Seite der Membran zugänglich sind. Zudem handelt es sich bei Membranen aufgrund der nichtkovalenten hydrophoben und hydrophilen Wechselwirkungen ihrer Bestandteile um eher fluide als kristalline Systeme, in denen sich die Lipide und Proteine frei bewegen können [219].

\footnotetext{
12 In diesem Fall sollten der Einfachheit halber keine Magnete in die Modelle eingesetzt werden. Alternativ ist es möglich, die einzelnen geometrischen Formen mit einer Heißklebepistole zusammenzufügen.
} 


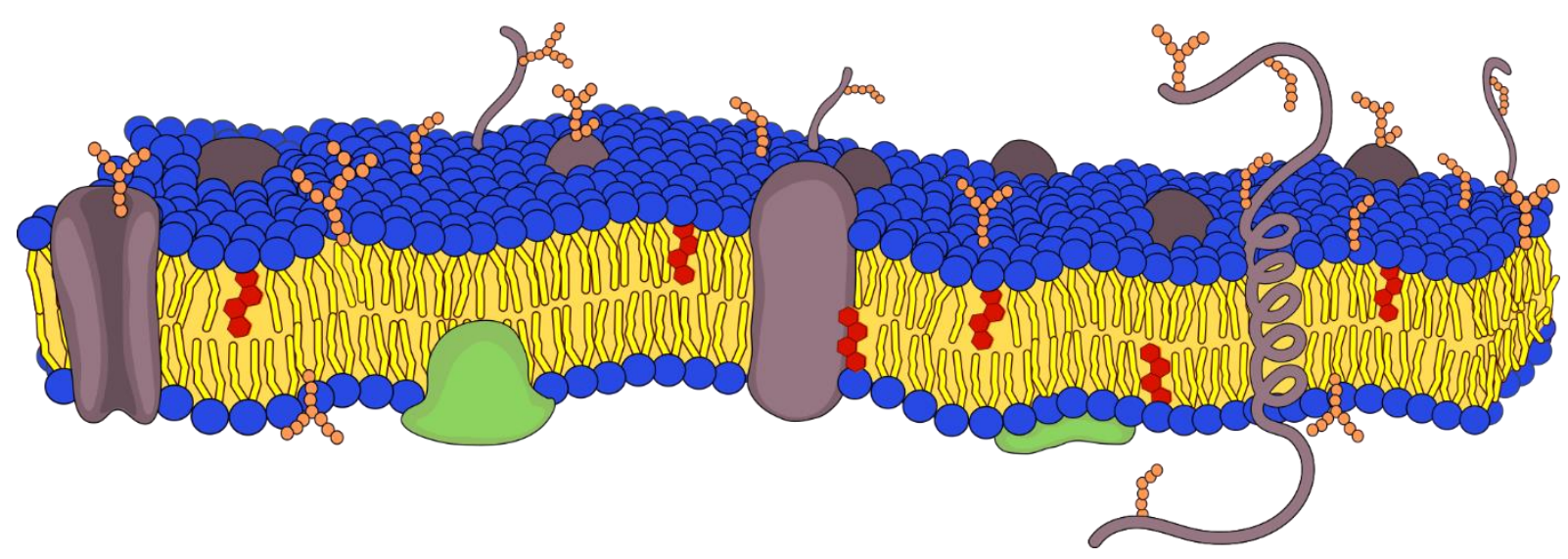

Abbildung 33: Typische Darstellung einer Membran nach dem Flüssig-Mosaik-Modell bestehend aus Lipiden (blau-gelb), Cholesterinen (blau-rot) sowie Transmembranproteinen (braun), die durch die Membran durchreichen und Kanäle bilden können, und peripheren Proteinen (grün), die nur an einen Lipidmonolayer assoziiert sind. In orange sind Polysaccharide dargestellt, die sowohl an Lipide als auch Proteine gebunden sein können.

Mithilfe verschiedener Techniken, wie bspw. der Fluoreszenzmikroskopie, konnte festgestellt werden, dass sich Lipide vor allem durch laterale Diffusion innerhalb ihrer Lipidmonoschicht bewegen, sich aber auch um ihre eigene vertikale Achse drehen und zudem die hydrophoben Ketten ständig in Bewegung sind. Lipidmoleküle sind außerdem in der Lage von einer Monoschicht in die andere zu wechseln (Flip-Flop). Dieser Vorgang zieht sich jedoch über Stunden hin, während die Teilchen z. B. einen lateralen Diffusionskoeffizienten von ca. $10^{-8} \mathrm{~cm}^{2} \cdot \mathrm{s}^{-1}$ besitzen (Abbildung 34) [185].

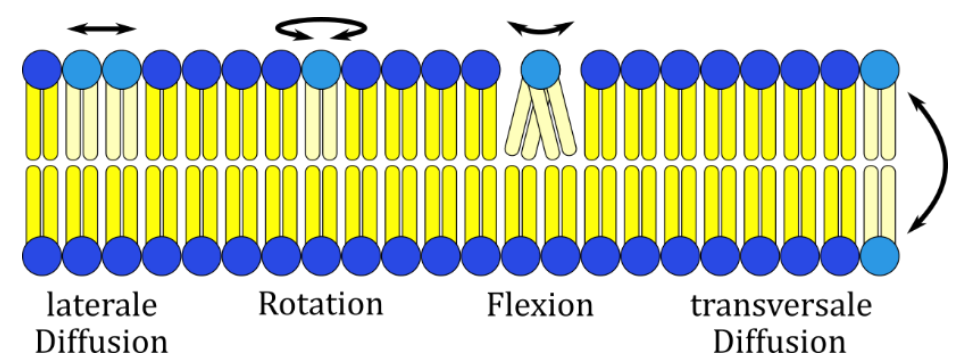

Abbildung 34: Schematische Darstellung der Membranfluidität.

Lipiddoppelschichten bestehen zu einem großen Teil aus Phospholipiden, die sich grob in Phosphoglyceride, mit einem Glycerin-Rückgrat, und Sphingolipide, mit einem Sphingosin- statt Glycerin-Rückgrat, unterteilen lassen. Zudem enthalten Membranen auch Glycolipide, die anstatt phosphatgebundener Kopfgruppen Zuckerreste binden, sowie Cholesterin (siehe Abbildung 35). Im Gegensatz zu den Phospholipiden besitzen Cholesterine ein Steroidgerüst an dem eine kurze Kohlenwasserstoffkette und eine einzelne Hydroxylgruppe hängen, wodurch die Moleküle insgesamt sehr starr sind [185].

Sphingolipid

Abbildung 35: Strukturformeln typischer Membranlipide sowie von Cholesterin. Die Glycerin- (blau) und Sphingosinbestandteile (rot) sind farbig hervorgehoben. 
Obwohl das Flüssig-Mosaik-Modell von einer allgemeinen Fluidität aller Lipide und Proteine ausgeht, zeigen Studien, dass sich innerhalb der Membranen Cluster aus vornehmlich Sphingolipiden und Cholesterinen bilden, deren Fluidität deutlich geringer ist als im Rest der Membran. Um diese Erkenntnis mit dem Flüssig-Mosaik-Modell zu vereinbaren, entwickelten SIMONS \& IKONEN 1997 das Lipid-Floß-Modell, das davon ausgeht, dass es innerhalb der fluiden Membran relativ geordnete Bereiche gibt (Flöße, liquid ordered phase, $\mathrm{l}_{\mathrm{o}}$ ), die innerhalb einer ungeordneteren, fluideren Phase treiben (liquid disordered phase, $l_{d}$ ) [91]. Den verhältnismäßig kleinen und starren Cholesterinen wird dabei eine stabilisierende Wirkung zugeschrieben, da sie die Zwischenräume der Sphingolipide besetzen und die Moleküle somit dichter gepackt sind. Mittlerweile geht man davon aus, dass Lipidflöße mit verschiedenen Proteinen assoziiert sind, die im Zusammenspiel wichtige Aufgaben bezüglich der Signalübertragung und des Transmembrantransports übernehmen [220].

Anhand der nachfolgenden Experimente können die Bestandteile von Membranen sowie deren Eigenschaften genauer untersucht werden. Dazu wird zunächst noch einmal auf Tenside rekurriert, da Seifenhäute es ermöglichen, die Bildung von Membranen auf makroskopischer Ebene zu zeigen und diese auf einige ihrer Eigenschaften hin zu untersuchen. Anschließend werden zwei Möglichkeiten zur Untersuchung von biologischen Membranen anhand von roten Zwiebelzellen beschrieben, die eine Erarbeitung einiger Membranbestandteile sowohl auf makroskopischer als auch auf mikroskopischer Ebene zulassen.

\section{Experiment 3.1 - Seifenhäute als Membranen}

Die submikroskopische Betrachtung von Seifenhäuten als makroskopisch wahrnehmbare Membranen ermöglicht bei Vergleich der umgebenden Medien eine eigenständige Erarbeitung des submikroskopischen Aufbaus von Biomembranen. Auf Bildungsmessen eigenen sich Seifenhäute ungewöhnlicher Geometrien zudem, um ein situationales Interesse bei Besucher_innen zu wecken [221].

Geräte und Chemikalien: Becherglas (1 L), Messzylinder, Glasrührstab, Spatellöffel, Gestelle geometrischer Formen (z. B. 3D-gedruckt), Wasser, Spülmittel, Tapetenkleister.

Versuchsdurchführung: In $900 \mathrm{~mL}$ warmen Wasser werden $60 \mathrm{~mL}$ Spülmittel sowie ein gehäufter Spatellöffel Tapetenkleister gelöst und die Lösung für einige Stunden ruhen gelassen. Anschließend werden verschiedene Gestelle geometrischer Formen (z. B. Tetraeder, Würfel) in eine Seifenblasenlösung eingetaucht und langsam wieder herausgezogen.

Beobachtung: Es bilden sich Seifenhäute mit unterschiedlichen Geometrien, die von den Kanten der Gestelle ausgehend in der Mitte zusammenlaufen (Abbildung 36).
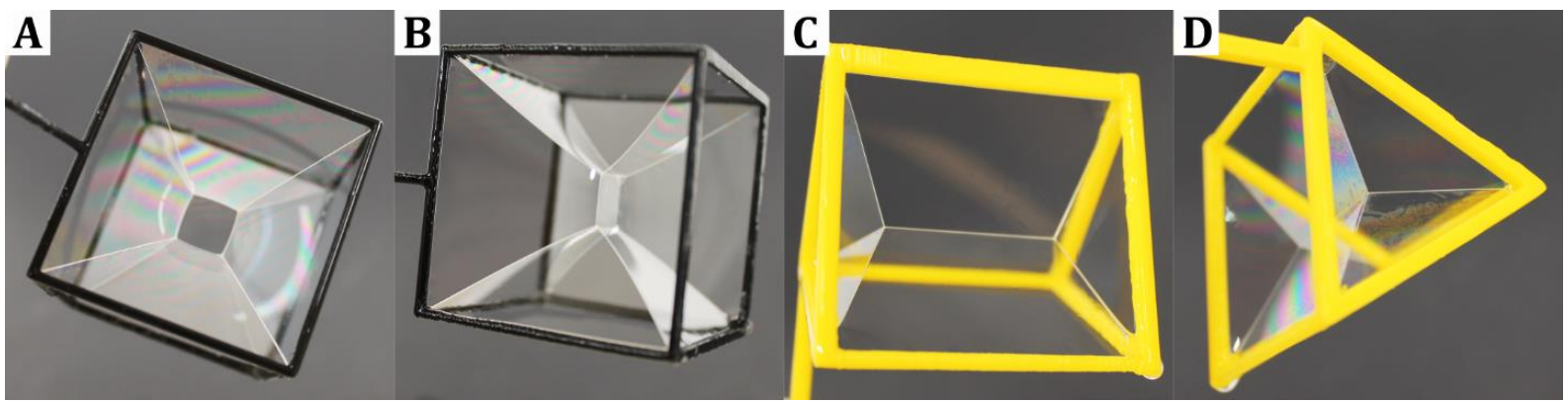

Abbildung 36: Seifenhäute in 3D-gedruckten geometrischen Formen.

Deutung: Beim Herausziehen der geometrischen Formen aus der Seifenblasenlösung bildet sich eine Seifenhaut, die submikroskopisch aus einer Tensiddoppelschicht besteht. Dabei schließen die hydrophilen Köpfe der Tenside Wassermoleküle in der Doppelschicht 
ein, während die hydrophoben Enden der Tenside zur Luft zeigen. Da keine Luft durch die Gestelle gepustet wird, wie es bei Seifenblasen der Fall ist, bilden sich lediglich Seifenhäute innerhalb der geometrischen Formen, die jeweils von den Kanten der Gestelle ausgehen. Ein Lufteinschluss bleibt dabei aus. Die zu beobachtenden Geometrien der Seifenhäute im Inneren der Gestelle sind jederzeit reproduzierbar und auf ein Bestreben zur Oberflächenminimierung zurückzuführen.

Anmerkungen zur Deutung des Experiments: Seifenblasen sind SuS zwar allgemein bekannt, sie werden jedoch selten als Membranen wahrgenommen. Dabei ähneln sie auf molekularer Ebene, wie in Abbildung 37 dargestellt, strukturell den Biomembranen. Seifenhäute bestehen aus zwei Tensidmonoschichten, die eine dünne Schicht Wasser umschließen. Dabei sind ihre hydrophilen Köpfe jeweils zum Wasser hin ausgerichtet, während die hydrophoben Ketten zur Luft zeigen. Die Lipidmoleküle in biologischen Membranen liegen hingegen invertiert vor - ihre hydrophilen Köpfe zeigen jeweils nach Außen in Richtung der wässrigen Zellinnen- und -außenmedien und die hydrophoben Schwänze sind ins Innere der Lipiddoppelschicht gerichtet. Anhand der Erkenntnisse zu den StrukturEigenschaftsbeziehungen und der Selbstassemblierung von Tensiden ist es SuS möglich, Aussagen über den Aufbau von Seifenhäuten zu treffen und diese mit der Struktur biologischer Membranen zu vergleichen.

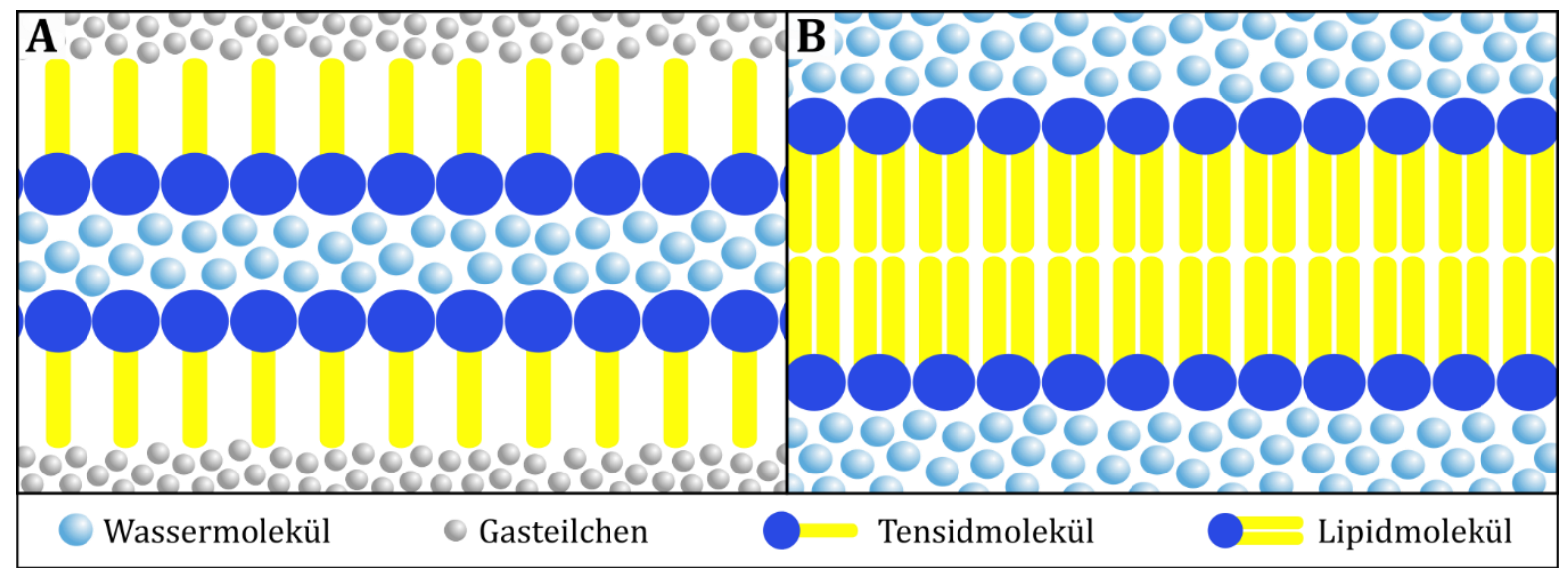

Abbildung 37: Vergleich der submikroskopischen Struktur von Seifenblasen (A) mit derer von Lipiddoppelschichten (B).

Die für die meisten SuS ungewöhnliche Anordnung der Seifenhäute innerhalb der geometrischen Formen bietet zudem die Möglichkeit, die Thematik der Oberflächenminimierung aufzugreifen, mithilfe derer sowohl die runden Formen von Seifenblasen als auch die sechseckigen Geometrien von Bienenwaben erklärt werden können. Beide Formen wurden lange als die optimalen Geometrien minimaler Oberflächen für den Einschluss eines bestimmten Volumens angenommen, der Beweis dieser Theorien hat Mathematiker jedoch lange beschäftigt [222]. Auch in Bezug auf Biomembranen spielen solche Minimalflächen eine wichtige Rolle. So nehmen beispielsweise auch Vesikel nach der Knospung eine bevorzugt runde Form an und erst vor wenigen Jahren konnte zudem gezeigt werden, dass das Membrannetzwerk des Endoplasmatischen Retikulums rampenartig verzweigt ist und trotz einer insgesamt großen Oberfläche dabei den Gesetzmäßigkeiten der Minimalflächen folgt [223, 224]. 


\section{Experiment 3.2a - Eigenschaften von Bestandteilen einer Biomembran - Makro- skopische Untersuchung}

Die Untersuchung der Eigenschaften roter Zwiebelhäute in verschiedenen Reagenzien ermöglicht es, im zeitlichen Rahmen einer Schulstunde auf verschiedene Bestandteile von Biomembranen zu schließen [175].

Geräte und Chemikalien: 11 Reagenzgläser mit Stopfen, Reagenzglasständer, Skalpell, Pinzette, Ethanol (GHS02, GHS07), demin. Wasser, Spülmittellösung, Öl, Essigessenz, Eiklar, Magainin 2, rote Zwiebel.

Versuchsdurchführung: Zunächst wird ein ca. $1 \mathrm{~cm}^{2}$ großes Stück der Haut einer roten Zwiebel mit einer Pinzette abgezogen. Die Zwiebelhaut wird mit einem Skalpell in fünf Streifen geschnitten und zum Auswaschen des roten Zwiebelsafts an den Schnittkanten in ein Reagenzglas mit Wasser gegeben. Dieses wird anschließend eine Minute lang geschüttelt, das Wasser wird getauscht und der Vorgang nochmals wiederholt. Anschließend werden zehn Proben, fünf Reagenzgläser mit Zwiebelhaut und fünf ohne, entsprechend Tabelle 8 vorbereitet.

Tabelle 8: Ansätze für die Versuchsreihe mit und ohne Zugabe von Zwiebelhaut.

\begin{tabular}{ll}
\hline Reagenzgläser mit Zwiebelhaut & Reagenzgläser mit Vergleichsproben \\
\hline (1) 4 mL Leitungswasser & (6) $4 \mathrm{~mL}$ Wasser + $1 \mathrm{~mL}$ Öl \\
(2) 4 mL Spülmittellösung & (7) $4 \mathrm{~mL}$ Seifenlösung + $1 \mathrm{~mL}$ Öl \\
(3) $4 \mathrm{~mL}$ Ethanol & (8) $4 \mathrm{~mL}$ Ethanol + ca. $1 \mathrm{~mL}$ Eiklar \\
(4) $4 \mathrm{~mL}$ Essigessenz & (9) $4 \mathrm{~mL}$ Essigessenz + ca. $1 \mathrm{~mL}$ Eiklar \\
(5) $4 \mathrm{~mL}$ Magainin-2-lösung (4 $4 \mathrm{M}$; optional) & (10) $4 \mathrm{~mL}$ Essigessenz + $1 \mathrm{~mL}$ Öl \\
\hline
\end{tabular}

Beobachtung: Reagenzglas (1) zeigt keine Veränderung, die flüssige Phase bleibt farblos, die Zwiebelhaut behält die lila-rötliche Farbe. Die Lösungen in den Reagenzgläsern (2) bis (5) färben sich lila-rötlich, die Zwiebelhäute entfärben sich. Die Flüssigkeiten in den Reagenzgläsern (6) und (10) zeigen eine klare Phasengrenze. In Reagenzglas (7) bilden sich ebenfalls zwei Phasen, wobei die untere Phase kleine gelbliche Tröpfchen beinhaltet. In den Reagenzgläsern (8) und (9) ist zudem jeweils die Bildung eines weißen Feststoffs zu beobachten (Abbildung 38).

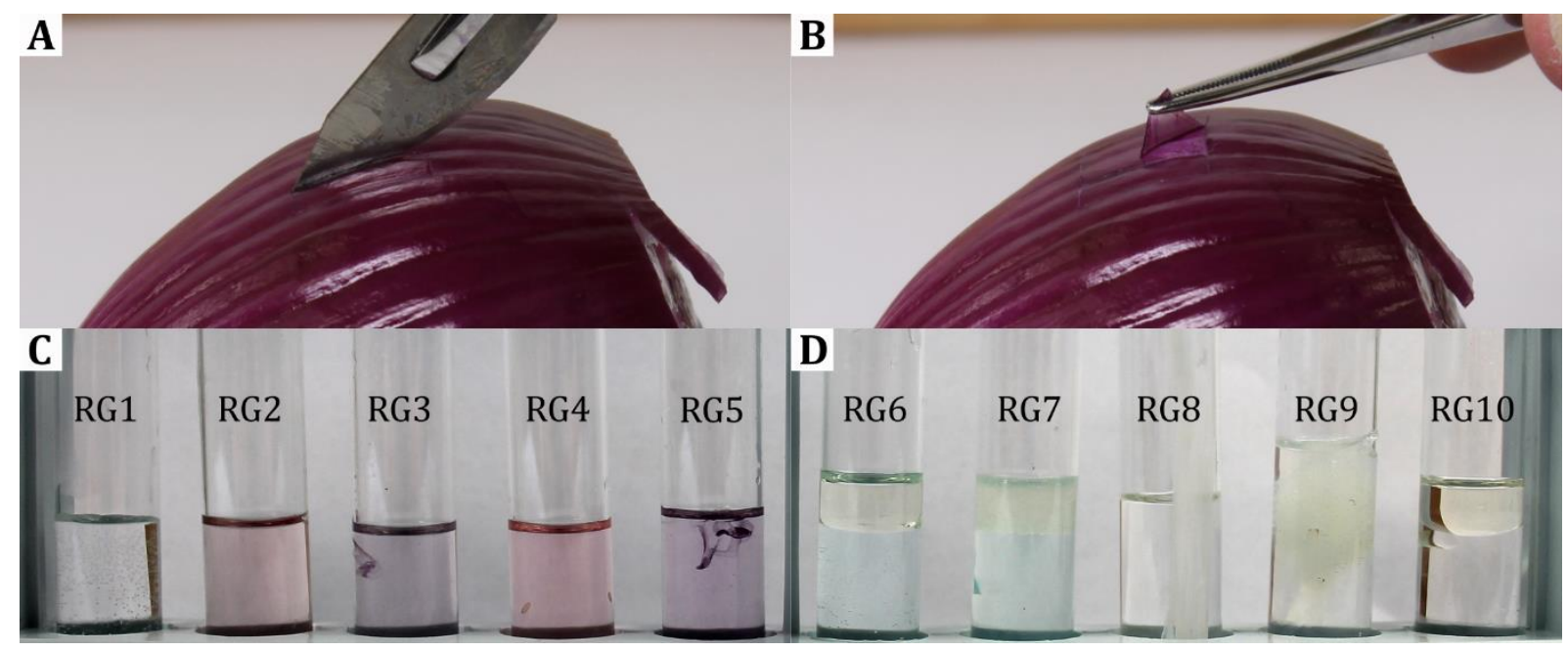

Abbildung 38: (A, B) Präparation von Zwiebelhäuten. (C, D) Lösungen entsprechend der Tabelle, links Proben mit Zwiebelhäuten, rechts Vergleichslösungen. 
Deutung: Zellmembranen bestehen aus Lipiden und Proteinen und wirken als Barriere, welche das Zellinnere von der Umgebung separiert. Eine Permeabilisierung der Vakuolenmembran kann durch Reaktionen bzw. Wechselwirkungen der Lipide oder Proteine mit verschiedenen Substanzen erfolgen. (RG1) zeigt keine Veränderung da die Membran intakt bleibt und nichts aus dem Zellinneren austreten kann. In (RG2) bis (RG5) zeigt die deutliche Färbung der Lösung, sowie die Entfärbung der Zwiebelhäute, dass die Vakuolenmembran permeabilisiert oder zerstört wurde und der Zellsaft in die umgebende wässrige Phase austreten kann.

Tabelle 9: Schlussfolgerungen bezüglich der Wirkung verschiedener Substanzen auf Lipidmembranen.

\begin{tabular}{|c|c|c|}
\hline Probe & Referenz & Schlussfolgerung \\
\hline RG1 & RG6 & $\begin{array}{l}\text { Die Lipidmembran kompartimentiert das rote Zellinnere durch hydrophile und } \\
\text { hydrophobe Wechselwirkungen, wie es durch die Phasengrenze in RG6 verdeut- } \\
\text { licht wird. }\end{array}$ \\
\hline RG2 & RG7 & $\begin{array}{l}\text { RG7 zeigt, dass Tenside Öl in wässriger Umgebung emulgieren können. Durch die } \\
\text { Wechselwirkung des Tensids mit den öl-ähnlichen, hydrophoben Ketten der Lip- } \\
\text { ide in der Vakuolenmembran werden diese aus der Membran herausgelöst und } \\
\text { die Membran hierdurch zerstört. In der Folge tritt der Zellsaft aus der Vakuole } \\
\text { aus. }\end{array}$ \\
\hline RG3 & RG8 & $\begin{array}{l}\text { Durch Vergleich mit RG8 wird gezeigt, dass Ethanol die Proteine der Membran } \\
\text { denaturiert, daher tritt in RG3 der Zellsaft ebenfalls aus. }\end{array}$ \\
\hline RG4 & RG10 & $\begin{array}{l}\text { Die Reaktion von Essigessenz mit Eiklar in RG9 zeigt, dass denaturiertes Protein } \\
\text { ausfällt. Schlussfolgernd reagieren in RG4 die Proteine in der Vakuolenmembran } \\
\text { mit der Essigessenz und werden denaturiert. Hierdurch entstehen Fehlstellen in } \\
\text { der Membran, in deren Folge der Zellsaft in die umgebende wässrige Phase aus- } \\
\text { treten kann. Der Vergleich mit RG } 10 \text { zeigt zudem, dass Essigessenz im Gegensatz } \\
\text { zu Tensiden die Lipidbestandteile der Vakuolenmembran nicht aus der Membran } \\
\text { löst. }\end{array}$ \\
\hline RG5 & & $\begin{array}{l}\text { Bei Magainin } 2 \text { handelt es sich um ein antimikrobielles Peptid, das Kanäle in der } \\
\text { Lipidmatrix der Vakuolenmembran bildet }[225,226] \text {. Diese Kanäle sind nicht spezi- } \\
\text { fisch, weshalb der Vakuolensaft durch sie austreten kann und chemische Gradien- } \\
\text { ten über die Membran hinweg zusammenbrechen. Dieser Mechanismus ist eine } \\
\text { Möglichkeit für die Wirkung moderner Antibiotika auf Basis von Oligopeptiden. }\end{array}$ \\
\hline
\end{tabular}

\section{Experiment 3.2b - Eigenschaften von Bestandteilen einer Biomembran - Mikrosko- pische Untersuchung}

Die makroskopischen Untersuchungen aus Experiment 3.2a können anhand dieses Versuchs um mikroskopische Untersuchungen ergänzt werden. Aufgrund des zusätzlichen zeitlichen Aufwandes ist dies jedoch eher im Rahmen eines Schülerlabors sinnvoll [175].

Geräte und Chemikalien: Reagenzglas mit Stopfen, Reagenzglasständer, Skalpell, Pinzette, Pasteurpipetten, Lichtmikroskop, Objektträger Ethanol (GHS02, GHS07), demin. Wasser, Spülmittellösung, Essigessenz, Magainin 2, rote Zwiebel.

Versuchsdurchführung: Vier mind. 1x1 cm² große Bereiche roter Zwiebelhaut werden mit einer Pinzette von einer roten Zwiebel abgezogen. Zum Auswaschen des roten Zwiebelsafts an den Schnittkanten werden die Zwiebelhäute in ein Reagenzglas mit Wasser gegeben. Dieses wird anschließend eine Minute lang geschüttelt, das Wasser wird getauscht und der Vorgang nochmals wiederholt. Anschließend werden die Zwiebelhäute jeweils auf einem Objektträger für die Mikroskopie präpariert. Es wird eine Probe unter das Mikroskop gelegt und fokussiert, anschließend wird vorsichtig mit einer Pipette eine 
der folgenden Lösungen zugegeben und die Beobachtung notiert: (1) 1 mL Leitungswasser, (2) $1 \mathrm{~mL}$ Seifenlösung, (3) $1 \mathrm{~mL}$ Ethanol, (4) $1 \mathrm{~mL}$ Essigessenz und (5) $1 \mathrm{~mL}$ Magainin2-lösung $(4 \mu \mathrm{M})$.

Beobachtung: Probe (1) mit Leitungswasser besitzt nur an den Schnittkanten ungefärbte Vakuolen, der Zellsaft bleibt in den intakten Zellen eingeschlossen. Bei den Proben (2) bis (5) wird je eine Entfärbung aller Zellen beobachtet (Abbildung 39).

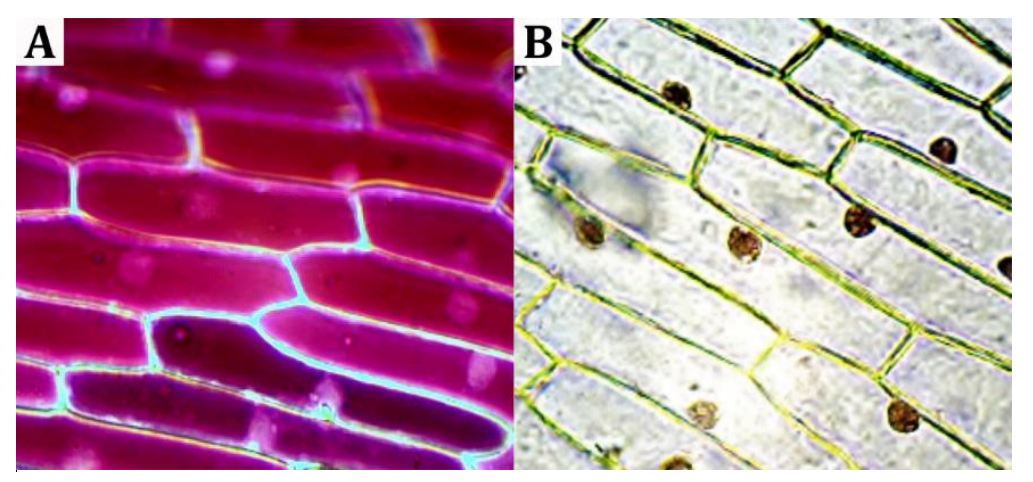

Abbildung 39: Durchlichtmikroskopische Aufnahmen einer Zwiebelhaut vor (A) bzw. nach der Behandlung mit Magainin 2 (B).

Deutung: In Analogie zu Versuch 3.2a zeigen die Proben in Leitungswasser keine Veränderung. Die Vakuolenmembran bleibt intakt und behält ihre Wirkung als Barriere bei. Durch Behandlung der Zwiebelhäute mit Spülmittellösung, Ethanol oder Essigessenz werden die Zellen entfärbt. Bei Zugabe von Spülmittellösung werden einzelne Lipidmoleküle emulgiert, bei Zugabe von Essigessenz werden hingegen die Proteine denaturiert, wodurch in beiden Fällen die kompartimentierende Eigenschaft der Vakuolenmembran aufgehoben wird und der Zellsaft austreten kann. Magainin 2 hingegen bildet Kanäle in der Membran, welche das austreten des Zellsaftes ermöglichen, was im Falle eines Bakteriums zum Zusammenbrechen der Gradienten an der Membran und somit zur Inaktivierung des Bakteriums führt $[225,226]$.

Anmerkungen zur Deutung der Experimente: Bei Zugabe verschiedener Substanzen wie Spülmittellösung, Ethanol, Essigessenz und Magainin 2 zu roten Zwiebelzellen kann bereits auf makroskopischer Ebene ein Austreten des farbigen Zellsafts beobachtet werden. Die Verwendung von verschiedenen Vergleichsproben ermöglicht es, Gründe für das Austreten des Zellsafts zu erarbeiten und in diesem Zusammenhang Aussagen über die Bestandteile von Membranen zu treffen. Die Zugabe der genannten Substanzen zu Öl und Eiklar dient der Veranschaulichung der den SuS bekannten Prozessen der Emulsion und Denaturierung. Öle, die zur Klasse der Lipide gehören, werden von Tensiden emulgiert, was darauf schließen lässt, dass auch in Biomembranen Lipide vorliegen könnten, deren Emulgation zur Permeabilisierung der Membran führt. Alkohole und Säuren hingegen denaturieren die Proteine im Eiklar indem sie die Ausbildung von Wasserstoffbrückenbindungen innerhalb der Proteine unterbinden. Auch diese irreversible Veränderung der Sekundär- und Tertiärstruktur der Proteine führt zum Verlust der Integrität der Zellmembran, was zum Austritt des Zellsafts führt.

Die Denaturierung von Zellproteinen findet auch im Alltag der SuS Anwendung. Alkohole wie Ethanol und Isopropanol werden u. a. als Desinfektionsmittel eingesetzt [227]. Die antibakterielle und fungizide Wirkung basiert dabei auf der Denaturierung von Membranproteinen, die zur Bildung von Fehlstellen und somit einer Inaktivierung von Bakterien und Pilzen führt. Ähnliche Prozesse machen sich auch einige Tierarten zum Schutz vor 
Fressfeinden zunutze. Beim innerhalb der Experimente verwendete Magainin 2, Bestandteil des Gifts des Afrikanischen Klauenfroschs, handelt es sich um ein antibakterielles Peptid, das zwar weder Lipide aus der Membran herauslöst, noch Proteine denaturiert, stattdessen jedoch unspezifische Membrankanäle bildet, die ein Austreten des Zellsafts ermöglichen (vgl. Abbildung 10, Kapitel 4.1). Da Magainin 2 nicht zu den typischen Schulchemikalien gehört, eignet sich dieser Versuchsteil eher weniger für den schulischen Gebrauch, ermöglicht jedoch mit Blick auf universitäre Aktivitäten zur Öffentlichkeitsarbeit, wie bspw. Science Camps, einen Einblick in die aktuelle Forschung des SFB 803.

\section{Einordnung der Experimente im Kontext der SO-Leitfragen}

Anhand des Experiments zur Oberflächenminimierung von Seifenhäuten wird in Bezug auf die vorherigen Themenfelder noch einmal auf Tenside rekurriert. Dies ermöglicht es, Oberflächenminimierungsprozesse auf makroskopischer Ebene zu betrachten und zugleich aus dem Alltag bekannte Phänomene (runde Seifenblasen, sechseckige Bienenwaben) zu thematisieren. Der Wechsel zur submikroskopischen Ebene bezüglich des Aufbaus von Biomembranen wird durch die vorherige Betrachtung des molekularen Aufbaus von Seifenhäuten erleichtert. Ein Vergleich der Innen- und Außenmedien von Seifenblasen und biologischen Membranen ermöglicht somit eine selbstständige Erarbeitung eines Modells der Anordnung von Lipiden zu einfachen Lipiddoppelschichten.

Die anschließenden Experimente zu den Eigenschaften von Membranbestandteilen lassen zudem induktiv Schlüsse bezüglich der Bestandteile von Biomembranen zu. Während die Versuche in Einklang mit den Erkenntnissen aus Experiment 2.3 weitere Indizien für die Existenz von Lipiden als wichtige Bausteine von Membranen liefern, deuten sie zudem das Vorhandensein von Proteinen an. Gleichzeitig führt jedoch die Zugabe von Essigessenz zu Öl zu keiner Veränderung der Lipide. Das von den SuS erarbeitete Modell der Lipiddoppelschicht reicht an diesem Punkt nicht mehr aus, um die Beobachtungen zu erklären. Neben den Lipiden müssen also noch weitere Substanzen in die Membran eingebettet sein. An dieser Stelle lohnt es sich, die Entwicklung der in der Einleitung dieses Abschnitts genannten Membranmodelle historisch zu betrachten und die Erkenntniswege der Wissenschaftler_innen in Ansätzen nachzuvollziehen. Diesbezüglich können die SuS ihr Wissen über das im Kerncurriculum verankerte Flüssig-Mosaik-Modell um Erkenntnisse aus der aktuellen Membranforschung hinsichtlich des Lipid-Floß-Modells erweitern.

Während die Vergleichsproben im Experiment 3.2a befriedigende Erklärungen für die Färbung der Lösungen in RG 2 - 4 liefern, reichen sie nicht aus, um sich auch die porenbildende Wirkung des Magainin 2 zu erarbeiten. Anhand dieses Versuchsteils lohnt sich jedoch ein Blick in die Forschung des SFB 803. Im Rahmen des Themenkomplex A beschäftigen sich gleich mehrere Teilprojekte mit der Insertion und Organisation von Peptidhelices in Lipidmembranen [174]. Dabei wurden unter anderem verschiedene Faktoren der Permeabilisierung von künstlichen Membranen durch Porenbildung mithilfe der antimikrobiellen Peptide Magainin 2 und Melittin (Hauptbestandteil des Bienengifts) untersucht [226]. Die Relevanz daraus resultierender Erkenntnisse kann den SuS beispielsweise in Bezug auf zunehmende Antibiotikaresistenzen verdeutlicht werden, da antimikrobielle Peptide bereits als alternative therapeutische Wirkstoffe eingesetzt werden [228].

Weiterhin kann anhand des Beispiels des Magainin 2 auch eine Überleitung zum Thema des Membrantransports erfolgen. Obwohl die von Magainin 2 gebildeten Poren aufgrund ihrer fehlenden Selektivität die kompartimentierende Wirkung der Membran aufheben, können anhand dieses Beispiels Hypothesen über die Eigenschaften von Membrankanälen aufgestellt werden, die sowohl eine Aufrechterhaltung der Kompartimentierung, als auch einen selektiven Transport gewährleisten. 


\subsection{Themenfeld 4 - Membrantransport}

Lipiddoppelschichten dienen in erster Linie der Kompartimentierung verschiedener Reaktionsräume, wodurch sie die Ausbildung von Konzentrationsgradienten und elektrischen Potentialdifferenzen ermöglichen. Sie sind jedoch nicht gänzlich undurchlässig. Kleinen lipophilen und ungeladenen Teilchen, wie beispielsweise $0_{2}, \mathrm{~N}_{2}$ und $\mathrm{CO}_{2}$, ist es möglich, durch die Membran zu diffundieren. Dieser als einfache Diffusion bezeichnete Transportweg verläuft immer mit dem Konzentrationsgradienten. Auch kleinen polaren Molekülen wie Wasser ist es prinzipiell möglich durch die Membran zu diffundieren, jedoch sind ihre Durchflussgeschwindigkeiten aufgrund der hydrophoben Innenseiten von Membranen deutlich langsamer als die unpolarer Moleküle[229].

Um die unterschiedlichen Funktionen verschiedener Zellen und Organellen zu gewährleisten, bedarf es neben Lipiden zahlreicher Proteine, die verschiedene Aufgaben erfüllen. Viele Transmembranproteine - Proteine, die komplett durch die Membran durchreichen - sind bspw. an Membrantransportprozessen beteiligt. Solche Proteine reichen gleich mehrfach durch die Membran, was u. a. die Ausbildung von Kanälen ermöglicht. Die Art und Weise, wie diese Membranproteine Ionen und Moleküle transportieren, und auf welche Teilchen sie sich spezialisiert haben, hängt dabei von ihren Strukturen ab. Es kann jedoch vereinfacht zwischen wenigen Transportformen unterschieden werden.

In Abhängigkeit vom Konzentrationsgradienten und der elektrischen Potenzialdifferenz wird übergeordnet zwischen passivem und aktivem Transport differenziert. Ähnlich wie die einfache Diffusion über die Lipidmembran verläuft der passive Transport entlang des Konzentrationsgradienten. Die schnelle Diffusion erfolgt über Kanalproteine, die durchgängige Poren in der Lipiddoppelschicht bilden und es vor allem anorganischen Ionen und kleinen Molekülen wie Wasser oder Ammoniak ermöglichen, entlang des Gradienten ungehindert von einer Membranseite zur anderen zu diffundieren. Neben Kanälen existieren zudem Carrierproteine, die keine offenen Poren bilden, sondern immer nur in eine Richtung geöffnet vorliegen. Durch Bindung einer oder mehrerer Moleküle an spezifischen Bindungsstellen kommt es zu einer Konformationsänderung, bei der sich das Protein in die zunächst offene Richtung schließt und anschließend in die andere Richtung öffnet. Diese Arten des Transports werden auch als erleichterte Diffusion bezeichnet (siehe Abbildung 40A - D). Unter anderem zur Aufrechterhaltung von Gradienten bedarf es neben dem passiven Transport auch eines gerichteten Transports gegen den Konzentrationsgradienten bzw. die elektrische Potenzialdifferenz. Dieser aktive Transport wird ebenfalls von Carrierproteinen gesteuert, bedarf jedoch der Zufuhr von Energie (z. B. in Form von ATP) [229].

Neben dem kanal- und carriervermittelten Transport können zudem Membrankomponenten wie Lipide, Proteine oder Kohlenhydrate in eine Membran eingefügt oder aus ihr entfernt werden. Diese als Exo- und Endocytose bezeichneten Prozesse (Abbildung 40E, F) ermöglichen u. a. auch den schnellen Transport von Neurotransmittern an den Synapsen von Nervenzellen. Bei der Exocytose fusioniert ein sogenanntes Vesikel (rundes Membrankompartiment) mit einer anderen Membran. Dabei vereinen sich die in der Membran des Vesikels enthaltenen Lipide, Proteine und Kohlenhydrate mit der Zielmembran und die im Vesikel eingelagerten Teilchen werden in das Zielkompartiment ausgeschüttet. Der umgekehrte Prozess - die Abschnürung eines Membranabschnittes zu einem Vesikel - wird als Endocytose bezeichnet [230]. 


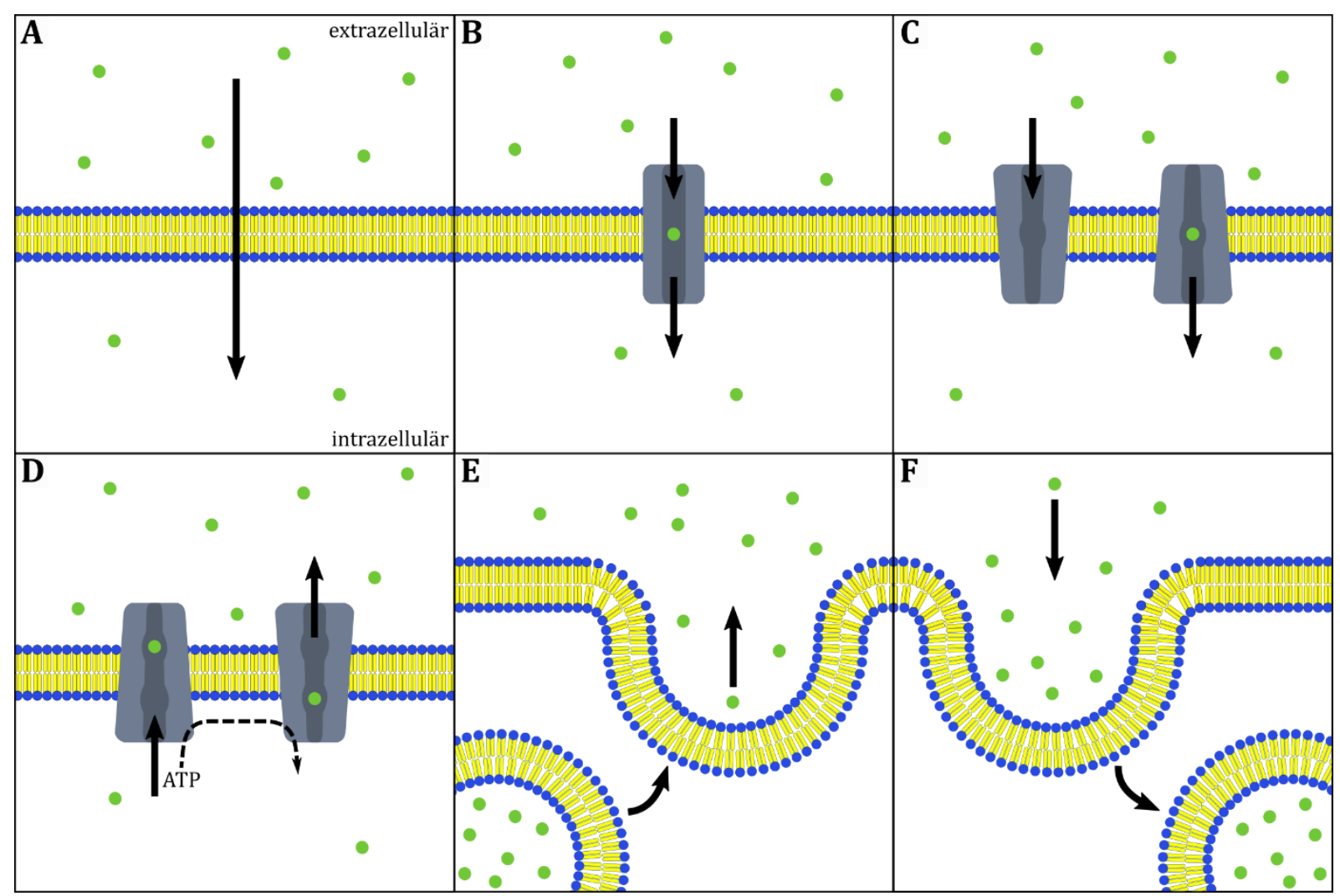

Abbildung 40: Schematische Darstellung verschiedener Membrantransportarten. (A) einfache Diffusion, (B) schnelle Diffusion durch ein Kanalprotein, (C) carriervermittelter passiver Transport, (D) carriervermittelter aktiver Transport, (E) Exocytose, (F) Endocytose.

Innerhalb dieses Abschnitts werden einige Modellexperimente bezüglich des kanal- und des carriervermittelten passiven Transports sowie des Prozesses der Exocytose aufgezeigt. Die Experimente ermöglichen es dabei, sowohl die Größen- sowie die Ladungsselektivität der Kanäle zu thematisieren als auch den passiven und aktiven carriervermittelten Transport hinsichtlich Uniport, Symport und Antiport weiter auszudifferenzieren. Mit Blick auf das Kerncurriculum der Biologie bietet es sich an, den Prozess der Exocytose im Kontext der Erregungsübertragung im Nervensystem zu behandeln. Anhand der Wirkweise des Nervengifts Botulinumtoxin kann zudem die Bedeutung von SNARE-Proteinen für die Vesikelfusion mit der Membran erarbeitet werden, die insbesondere auch in der Forschung des SFB 803 eine wichtige Rolle spielen.

\section{Experiment 4.1a - Erleichterte Diffusion großer Moleküle}

Mithilfe dieses Experiments kann die Diffusion unterschiedlich großer Moleküle im wässrigen Medium anhand ihrer Fluoreszenzeigenschaften untersucht werden. Aufgrund der verwendeten Chemikalien bietet sich eine Anwendung vor allem im Schülerlabor an.

Geräte und Chemikalien: Becherglas (250 mL), 2 Schnappdeckelgläser (30 mL), Spatel, Dialyseschlauch $(14 \mathrm{kD}, 10 \mathrm{~cm})$, Frischhalteklammern oder Gummibänder, Laserpointer (grün, violett), demin. Wasser, dextrangebundenes Texas $\operatorname{Red}^{13}$ (70 kD), Pyranin (GHS07).

Versuchsdurchführung: In zwei Schnappdeckelgläsern werden eine Spatelspitze Texas Red und eine Spatelspitze Pyranin jeweils in $10 \mathrm{~mL}$ Wasser gelöst. Ein Dialyseschlauch

13 Texas Red ${ }^{\circledR}$ ist ein Trademark von Molecular Probes, Inc. 
wird von innen und außen gründlich mit Wasser gespült, eine Öffnung mit einer Frischhalteklammer verschlossen und der Schlauch so in ein mit Wasser gefülltes Becherglas gelegt, dass das obere Ende herausragt. Der Schlauch wird jeweils mit ca. $10 \mathrm{~mL}$ beider Lösungen befüllt und mit einer zweiten Klammer verschlossen. Anschließend werden die beiden übrigen Lösungen in den Schnappdeckelgläsern sowie der Inhalt des Becherglases und des Dialyseschlauches mittels eines violetten und eines grünen Laserpointers untersucht.

Beobachtung: Die beiden Lösungen in den Schnappdeckelgläsern können mithilfe des violetten Lasers im Falle von Pyraninlösung zu grüner und im Falle von Texas Red-Lösung zu roter Fluoreszenzemission angeregt werden (siehe Abbildung 41A). Der grüne Laser regt hingegen lediglich die Texas Red-Lösung zur Fluoreszenz an. Bei Bestrahlung des Inhalts des Becherglases mit dem violetten Laser ist zunehmend auch eine grüne Fluoreszenz außerhalb des Dialyseschlauchs zu beobachten. Eine durch den grünen Laser angeregte rote Fluoreszenz ist hingegen lediglich innerhalb des Dialyseschlauchs wahrnehmbar.

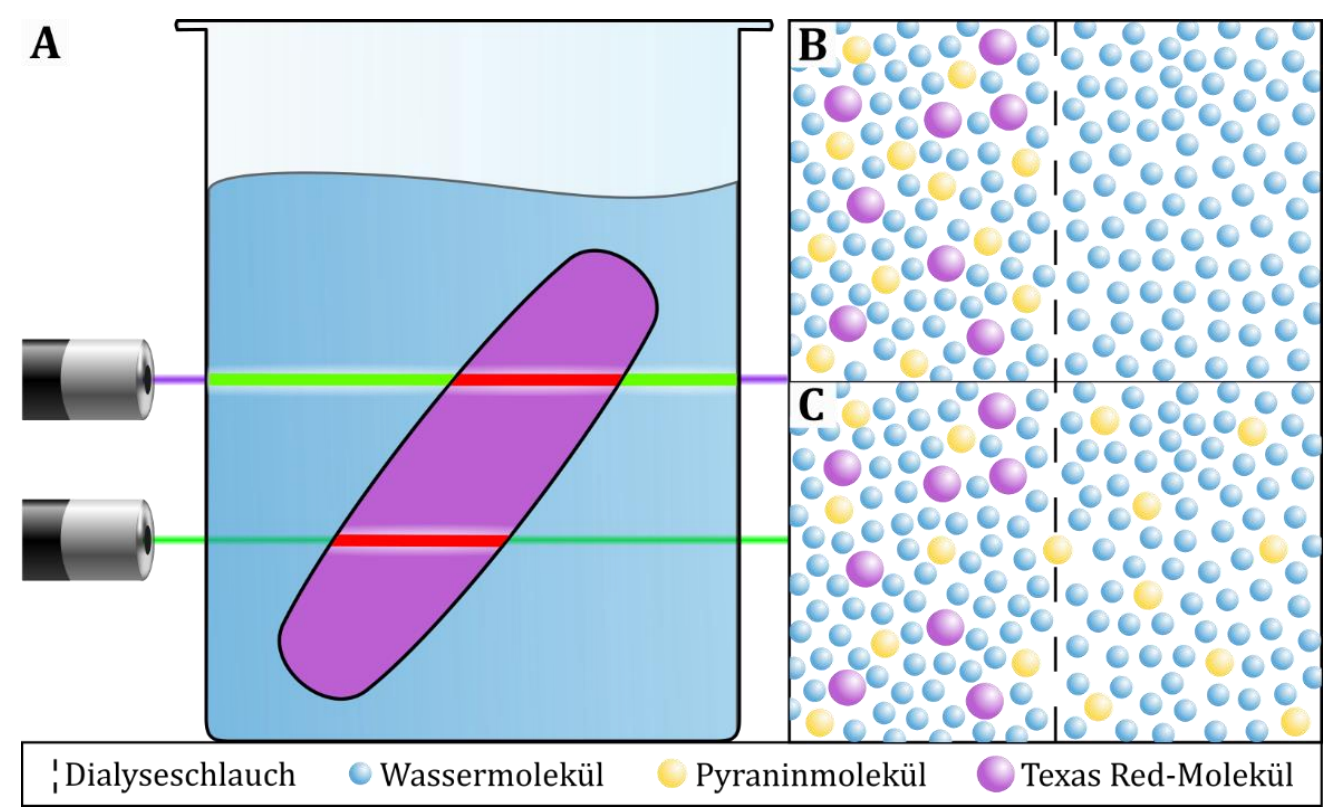

Abbildung 41: (A) Schematische Darstellung der Anregung zur Fluoreszenz auf makroskopischer Ebene. (B, C) Submikroskopische Darstellung der Diffusion der Pyraninmoleküle durch den Dialyseschlauch.

Deutung: Der Dialyseschlauch weist eine Porengröße von $14 \mathrm{kD}$ auf. Er fungiert somit als semipermeable Membran, durch die lediglich Moleküle durchtreten können, die in ihrem Durchmesser kleiner als die Porengröße sind. Mithilfe des UV-Lichts kann der Durchtritt der Pyraninmoleküle, die sich in ihrer Größe deutlich von Ionen oder kleinen Molekülen wie Wasser unterscheiden, entlang des Konzentrationsgefälles in das wässrige Außenmedium nachgewiesen werden. Die Dextran-gebundenen Texas Red-Moleküle sind hingegen mit einer Größe von 70 kD zu groß, um durch die Poren durchzutreten (vgl. die Strukturformeln der Moleküle in Abbildung 42). Entsprechend verbleiben sie im Dialyseschlauch, was anhand der laserinduzierten roten Fluoreszenz nachgewiesen werden kann. 


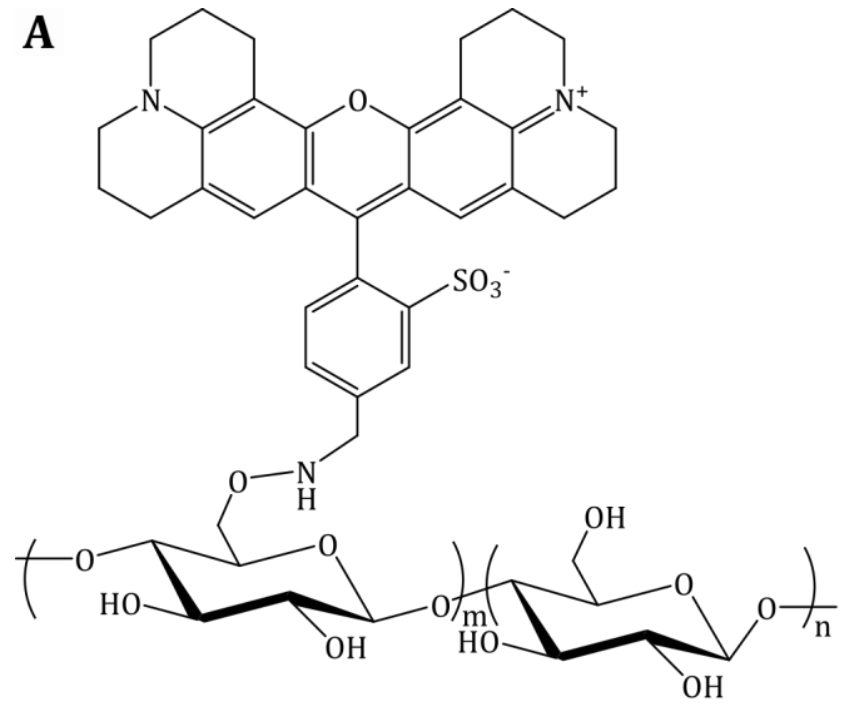

Dextrangebundenes Texas Red
B

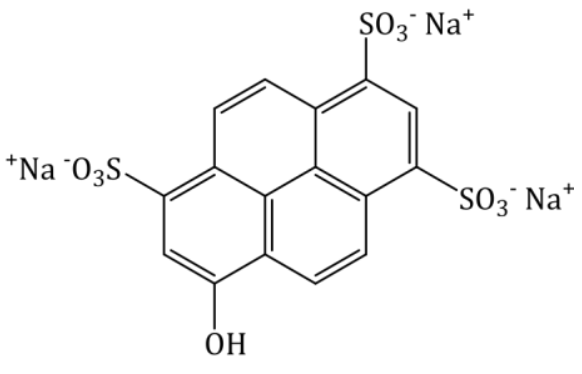

Pyranin

Abbildung 42: Strukturformeln der Moleküle von dextrangebundenem Texas Red sowie Pyranin. Die Glucoseeinheiten können $\alpha$-1,4- oder $\alpha$-1,6-glycosidisch verbunden sein, so dass verzweigte Dextrannetzwerke entstehen.

\section{Experiment 4.1b - Erleichterte Diffusion geladener Teilchen}

Ausgehend vom Dissoziationsgleichgewicht von Kohlensäure mit Kohlenstoffdioxid kann u. a. im Unterricht die Diffusion von Protonen anhand der Farbveränderung eines Indikators beobachtet werden.

Geräte und Chemikalien: Becherglas (250 mL), Dialyseschlauch (10 cm), Frischhalteklammern oder Gummibänder, demin. Wasser, Phenolphthaleinlösung ( $w=0,5 \%$; GHS02, GHS07), Natriumhydroxidlösung (0,1 M, GHS05), Mineralwasser (kohlensäurehaltig).

Versuchsdurchführung: In einem Becherglas werden ca. 200 mL Wasser mit einigen Tropfen Phenolphthaleinlösung versetzt und es wird solange Natriumhydroxidlösung hinzugetropft, bis die Lösung leicht violett gefärbt ist. Ein Dialyseschlauch wird von innen und außen gründlich mit Wasser gespült, eine Öffnung mit einer Frischhalteklammer verschlossen und der Schlauch so in ein mit Wasser gefülltes Becherglas gelegt, dass das obere Ende herausragt. Anschließend wird der Schlauch mit Mineralwasser befüllt und das obere Ende mit einer weiteren Klammer verschlossen.

Beobachtung: In der violetten Lösung bilden sich ausgehend vom Dialyseschlauch farblose Schlieren. Nach ca. 15 Minuten ist die gesamte Lösung entfärbt (Abbildung 43A, B).

Deutung: Kohlensäure liegt in einem mehrstufigen Dissoziationsgleichgewicht mit Kohlenstoffdioxid vor, bei dem auch Protonen gebildet werden. Die Diffusion der Protonen aus dem Dialyseschlauch heraus sorgt für eine Änderung des pH-Werts des umgebenden wässrigen Mediums, die anhand der Entfärbung der Phenolphthaleinlösung beobachtet werden kann. 


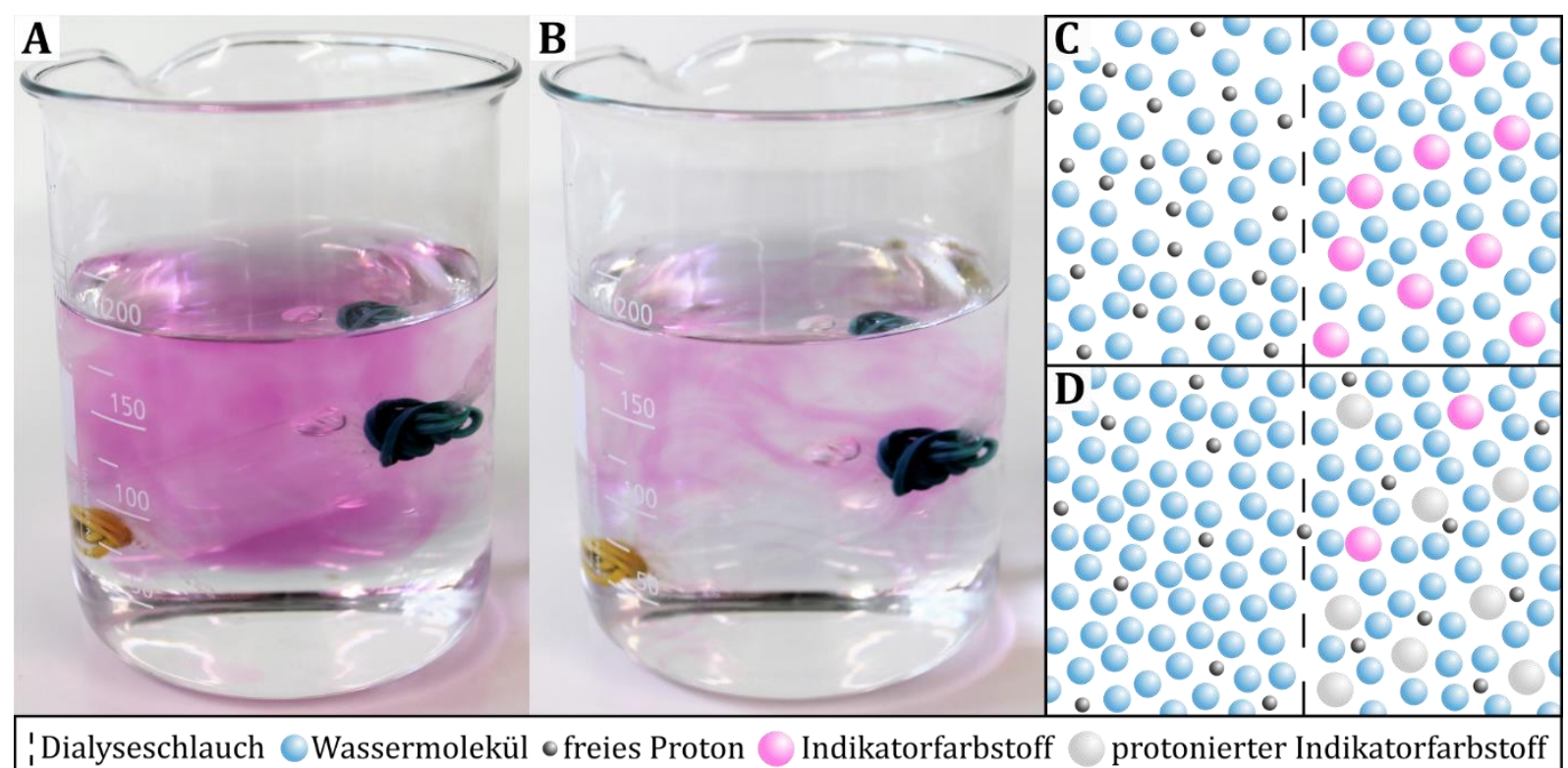

Abbildung 43: (A, B) Die Phenolphthaleinlösung außerhalb des Dialyseschlauch entfärbt sich. (C, D) Schematische Darstellung der Diffusion von Protonen durch den Dialyseschlauch, die zur Protonierung und dadurch Entfärbung des Indikatorfarbstoffs führt.

Anmerkungen zur Deutung der Experimente: Je größer, polarer und geladener ein Molekül ist, desto langsamer verläuft die einfache Diffusion durch die Lipiddoppelschicht. Transportproteine die Kanäle bilden ermöglichen es auch solchen Teilchen, mit dem Konzentrationsgefälle schnell von einer Seite der Membran zur anderen zu diffundieren. Dabei bilden die Kanäle nur schwache Wechselwirkungen mit den zu transportierenden Teilchen aus. Eine Vielzahl der Kanäle ist dabei selektiv auf bestimmte Moleküle oder Molekülsorten spezialisiert. Ähnlich wie in Experiment 4.1a bestimmt u. a. die Porengröße, welche Moleküle durch den Kanal durchtreten können. Es existieren jedoch auch zahlreiche Kanäle, wie bspw. die auf Wassermoleküle spezialisierten Aquaporine, die nicht nur größenselektiv sind, sondern auch deutlich kleinere geladene Teilchen wie Protonen nicht passieren lassen.

Für den Transport von Ionen existieren deshalb spezielle Ionenkanäle, die oftmals spezifisch eine bestimmte Ionensorte wie Kalium- oder Natriumionen transportieren. Der Transport der geladenen Teilchen hängt dabei nicht nur vom Konzentrationsgradienten $\mathrm{ab}$, sondern auch von der Potenzialdifferenz der beiden membranumgebenden Medien, die zusammen als elektrochemischer Gradient bezeichnet werden. So wird die Durchlässigkeit spannungsabhängiger Kanäle beispielsweise über Änderungen des Membranpotenzials gesteuert [231]. Auch in Experiment 4.1b hängt die Diffusion der Protonen aus dem Dialyseschlauch in die Umgebung vom elektrochemischen Gradienten ab. Die Diffusion der Protonen aus dem Dialyseschlauch heraus sorgt jedoch für das Einstellen eines Gleichgewichts, was in Zellen vermieden wird, da die Ausbildung von Gradienten eine wichtige Rolle in zahlreichen Prozessen wie bspw. der Erregungsübertragung an Nervenzellen spielt. Für den Transport von Teilchen gegen den elektrochemischen Gradienten werden deshalb aktive Transportprozesse benötigt, die nur unter Energiezufuhr ablaufen.

\section{Experiment 4.2 - Erleichterte carriervermittelte Diffusion}

Dieser Versuch dient als Modellexperiment für den carriervermittelten Membrantransport. Im Unterricht ist er insbesondere als Lehrerdemonstrationsexperiment denkbar [232].

Geräte und Chemikalien: Becherglas, U-Rohr, Stativmaterial, 2 Messpipetten $10 \mathrm{~mL}$ und $20 \mathrm{~mL}$ ), Pasteurpipette, Glasrührstab, Magnetrührer mit kleinem Rührfisch, UV-Lampe, 
demin. Wasser, Kronenether ([18]Krone-6; GHS07), Dichlormethan (GHS07, GHS08), Kaliumpermanganatlösung (0,1 M; GHS07, GHS09).

Versuchsdurchführung: Ein U-Rohr mit Rührfisch wird in ein Stativ eingespannt und mithilfe einer Pipette mit 20 mL Dichlormethan befüllt. Zu einem Schenkel des U-Rohrs werden vorsichtig $5 \mathrm{~mL}$ Wasser hinzugegeben, zum anderen $5 \mathrm{~mL}$ Kaliumpermanganatlösung. In einem Becherglas wird eine 0,4 M Kronenetherlösung in Dichlormethan hergestellt. $1 \mathrm{~mL}$ der Lösung wird mittels einer Pasteurpipette in die Dichlormethanphase eingebracht. Anschließend wird der Magnetrührer auf höchster Stufe eingestellt.

Beobachtung: In der farblosen Dichlormethanphase sind nach Zugabe des Kronenethers violette Schlieren zu beobachten. Nach einiger Zeit (ca. $10 \mathrm{~min}$ ) zeigt auch das Wasser eine violette Färbung.

Deutung: Aufgrund ihrer Polarität können die Kalium- und Permanganationen nicht ohne Weiteres durch die unpolare Dichlormethanphase diffundieren. Der zugegebene Kronenether besitzt einen Hohlraum, der gerade groß genug ist, um ein Kaliumion einzulagern (siehe Abbildung 44). Um die Elektroneutralität zu wahren, wird das Permanganation ebenfalls an den Kronenether assoziiert. In diesem Verbund werden die Ionen durch die Dichlormethanphase transportiert, was anhand der violetten Färbung zu beobachten ist.

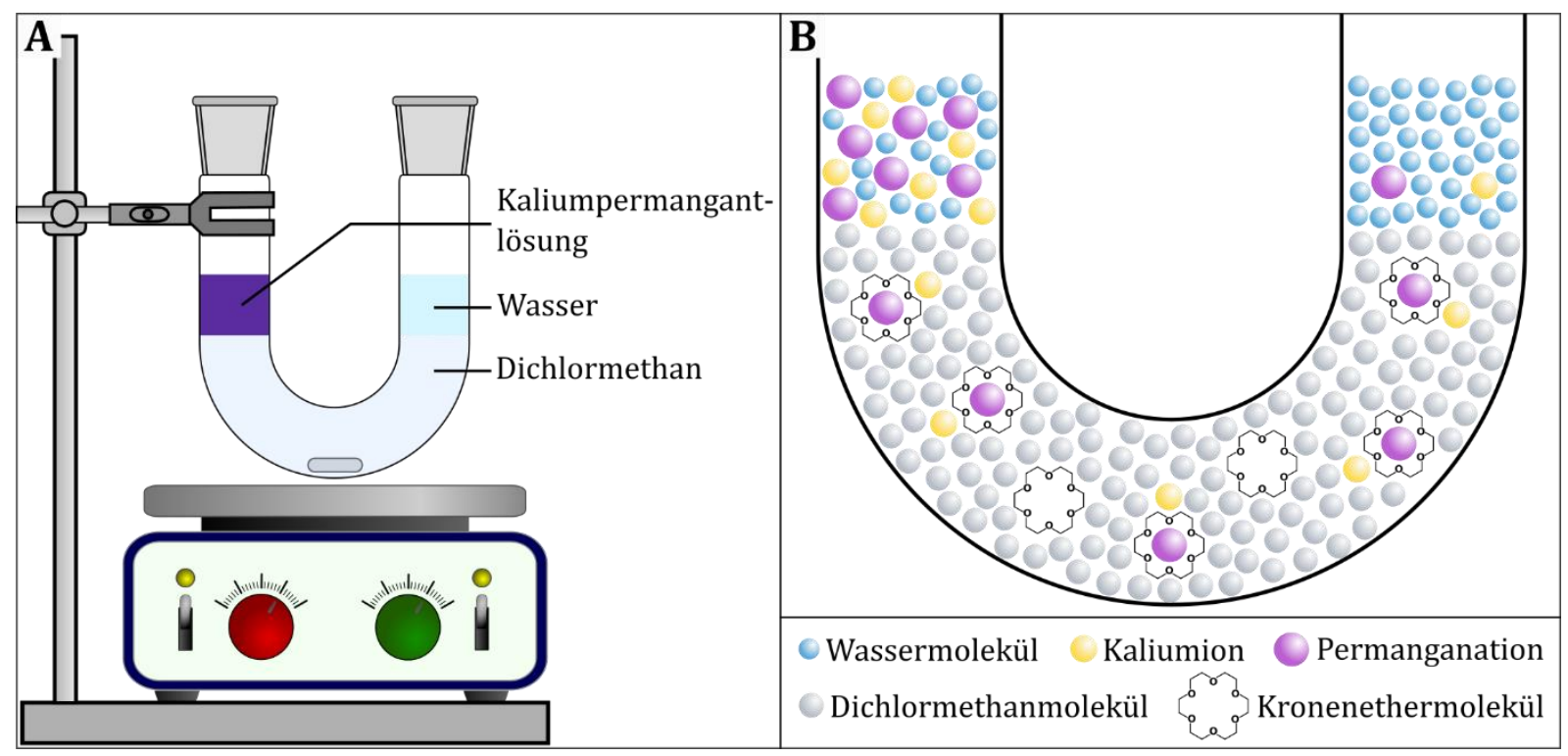

Abbildung 44: (A) Versuchsaufbau für das Modellexperiment zur erleichterten carriervermittelten Diffusion. (B) Schematische Darstellung der ablaufenden Prozesse auf Teilchenebene nach FRIEDRICH \& OETKEN [232].

Anmerkungen zur Deutung des Experiments: Im Gegensatz zur schnellen Diffusion, bei der die Kanalproteine nur wenig mit den zu transportierenden Teilchen wechselwirken, besitzen Carrierproteine Bindungsstellen für spezifische Teilchen. Beim carriervermittelten Transport sind die Transportproteine nur in eine Richtung geöffnet. Bindet ein entsprechendes Molekül an die Bindungsstelle, so geht dies mit einer Konformationsänderung des Proteins einher, die zu einer Schließung des Proteins in die zunächst offene und zur Öffnung in die entgegengesetzte Richtung führt. Die Carrierproteine können dabei als einfache Transporter fungieren, die nur eine einzige Teilchensorte transportieren (Uniport), es sind jedoch auch gekoppelte Transporte mehrere Teilchenarten möglich. Beim Symport werden zwei Teilchen gleichzeitig von der einen zur anderen Seite transportiert, wobei der Transport erst stattfindet, wenn beide Teilchen an die Bindungsstellen gebunden 
sind (siehe Abbildung 45). Zudem können beim Antiport auch zwei Teilchen aus entgegengesetzten Richtungen auf die jeweils andere Membranseite befördert werden [229].

Sowohl beim Sym- als auch beim Antiport kann der Transport mit dem Konzentrationsgradienten und somit passiv erfolgen. Einige Carrierproteine nutzen jedoch auch die aus dem elektrochemischen Gradienten resultierende Energie des einen Teilchen aus, um ein weiteres Teilchen aktiv zu befördern. Diese Art des Transports wird als sekundär aktiver Transport bezeichnet [233]. In Bezug auf das Modellexperiment entspricht der Transport der Kalium- und Permanganationen durch die Dichlormethanphase mittels Kronenether als Carrier am ehesten dem passiven Symport, da beide Ionen mit dem Konzentrationsgefälle transportiert werden.

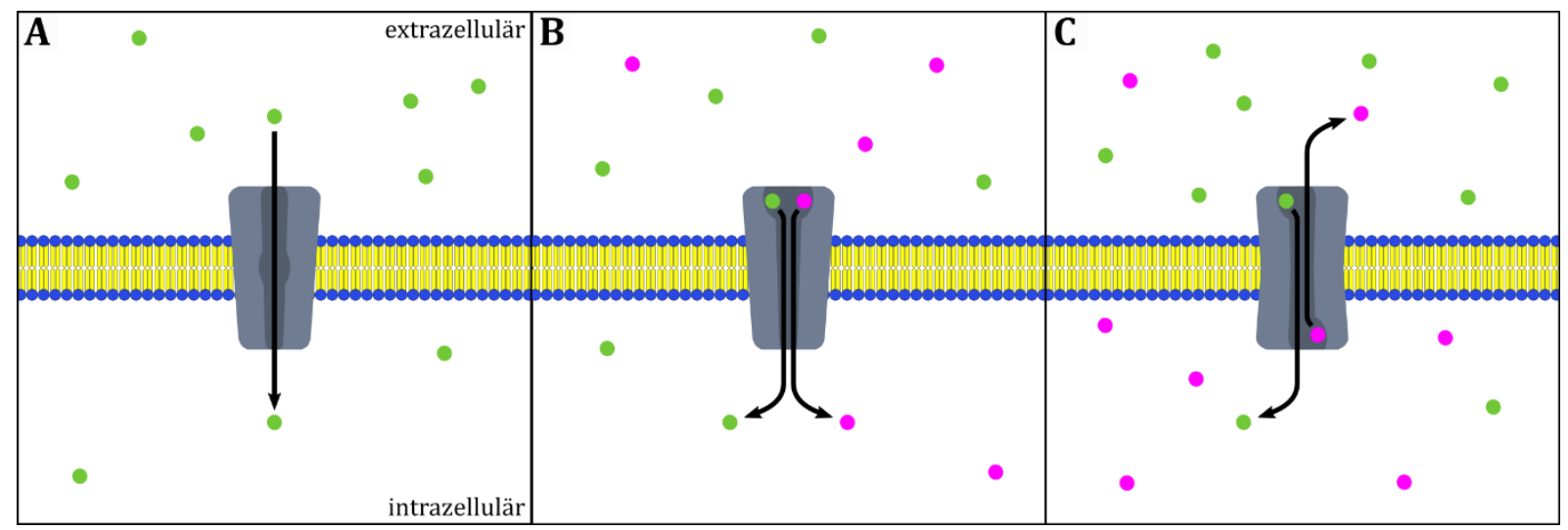

Abbildung 45: Schematische Darstellung verschiedener carriervermittelter Membrantransportmechanismen. (A) Uniport, (B) Symport, (C) Antiport (passiv).

\section{Experiment 4.3a - Exocytose}

Als Modellexperiment zur Darstellung der Vesikelfusion am synaptischen Spalt kann dieser Versuch sowohl im Unterricht als auch auf Bildungsmessen eingesetzt werden [175].

Geräte und Chemikalien: 4 Bechergläser (2x $250 \mathrm{~mL}, 500 \mathrm{~mL}$ und $1 \mathrm{~L}$ ), großes Reagenzglas ( $\varnothing 20-30 \mathrm{~mm})$, Stativmaterial, Einwegspritze $(10 \mathrm{~mL}), 2$ Vollpipetten $(20 \mathrm{~mL}$ und $100 \mathrm{~mL})$, Glasrührstab, Spatel, Wasser, Spülmittel, Pyranin (GHS07), Glucose, Methylenblau (GHS07).

Versuchsdurchführung: Zunächst werden die folgenden Lösungen unter vorsichtigem Rühren angesetzt: (1) in $1 \mathrm{~L}$ Wasser wird etwa $1 \mathrm{~g}$ Spülmittel gelöst, (2) $100 \mathrm{~mL}$ dieser Spülmittellösung werden in ein $250 \mathrm{~mL}$ Becherglas überführt und mit einer Spatelspitze Methylenblau sowie $6 \mathrm{~g}$ Glucose versetzt, (3) in einem $500 \mathrm{~mL}$ Becherglas werden $32 \mathrm{~g}$ Glucose in $400 \mathrm{~mL}$ der Lösung 1 gelöst, (4) eine Hälfte der Lösung 3 wird mit einer Spatelspitze Pyranin versetzt.

Ein großes Reagenzglas wird in ein Stativ eingespannt und anschließend die Lösungen 1 bis 3 übereinandergeschichtet. Dazu werden zunächst ca. $40 \mathrm{~mL}$ von Lösung 1 in das Reagenzglas gefüllt und diese vorsichtig und langsam mit $10 \mathrm{~mL}$ der Lösung 2 unterschichtet. Anschließend werden beide Lösungen mit etwa $40 \mathrm{~mL}$ der Lösung 3 unterschichtet. Lösung 4 wird mit einer Spritze aufgezogen. Die Spritze wird ca. $1 \mathrm{~cm}$ über der Oberfläche der Lösungen im Reagenzglas positioniert und es werden sukzessive einzelne Tropfen in das Reagenzglas getropft. Die Fallhöhe der Tropfen wird so lange variiert, bis sich Antiblasen (invertierte Seifenblasen) in der Lösung bilden. Das Reagenzglas kann zur besseren Visualisierung zudem mit einer UV-Lampe bestrahlt werden. 
Beobachtung: Die Lösungen 1 bis 3 liegen im Reagenzglas als drei getrennte Phasen vor. Beim Zutropfen der Lösung 4 (Pyraninlösung) bilden sich kleine mit Pyraninlösung gefüllte Blasen, die durch die oberste Schicht an die Phasengrenze zwischen der mittleren und der unteren Schicht sinken. Dort platzen die Blasen nach einiger Zeit und geben ihren Inhalt an die unterste Phase ab. Pyraninlösung die keine Blasen bildet verbleibt in der obersten Schicht und färbt diese gelb (Abbildung 46A - C).

Deutung: Beim Zutropfen einer Glucose-Tensidlösung mit hoher Dichte orientieren sich die hydrophoben Bereiche der Tensidmoleküle in Richtung der Luft. Sobald der Tensidtropfen auf die Tensidschicht des Becherglases trifft wird ein dünner Luftfilm (in Abbildung 46F hellblau gezeigt) durch eine weitere Tensidschicht eingeschlossen. Dabei bildet sich eine doppelte Tensidschicht, die analog einer Lipiddoppelschicht aufgebaut ist (siehe Abbildung 46F). Aufgrund der hohen Dichte der eingeschlossenen farbigen Tensidlösung sinkt diese sogenannte Antiblase [234], bis an die Grenzfläche der untersten Schicht, da die Dichte in der Blase etwa der Dichte der untersten Phase entspricht. An dieser Grenzfläche platzt die Antiblase und der Farbstoff wird in die untere Phase abgegeben.

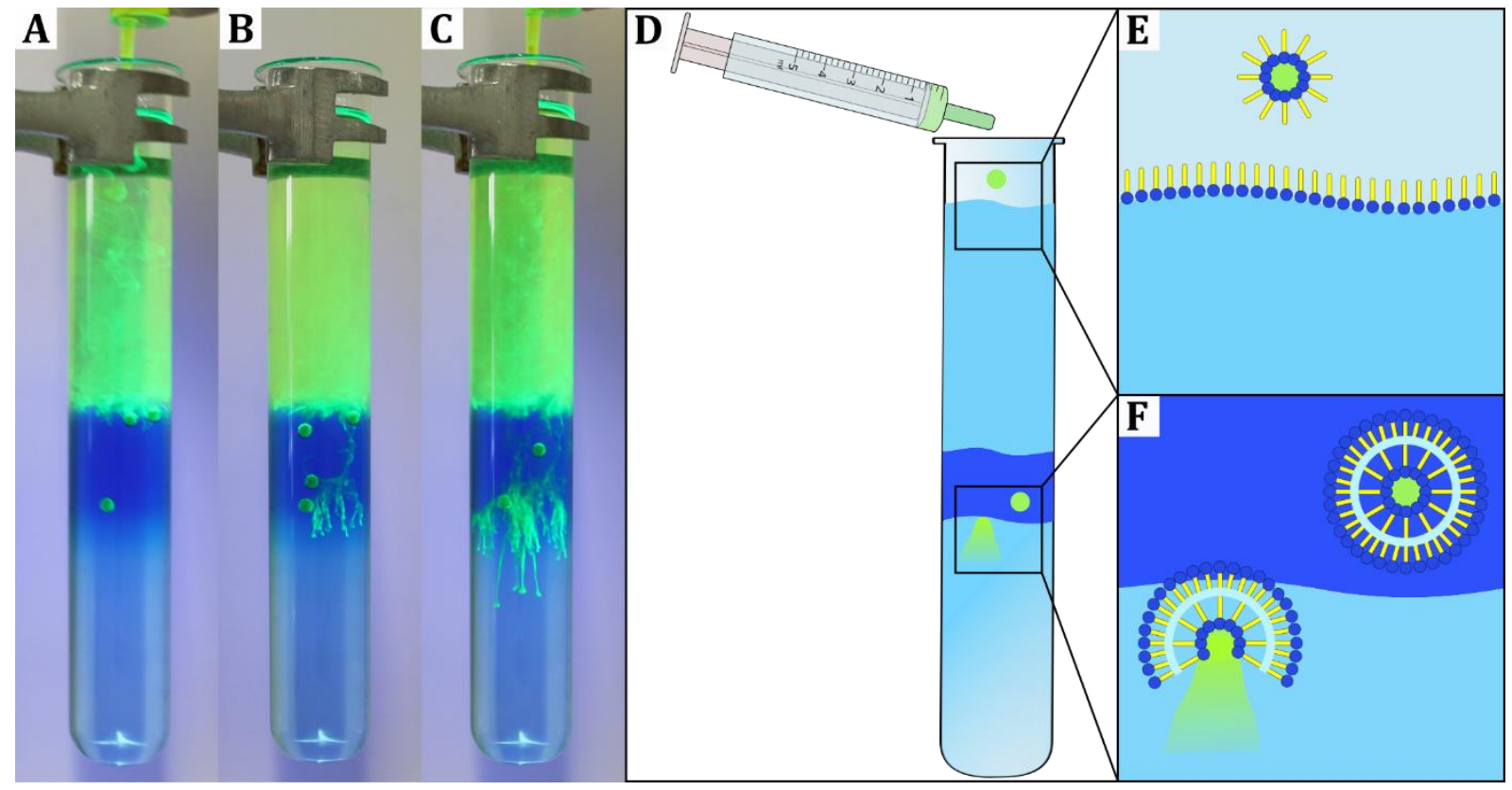

Abbildung 46: (A-C) Eine farbstoffgefüllte Antiblase sinkt durch die zwei oberen Lösungen mit geringerer Dichte, verbleibt kurz an der Grenzschicht zur untersten Lösung und gibt beim Platzen den eingeschlossen Farbstoff in die unterste Phase ab. (D-F) Schematische Darstellung des Modellexperiments.

\section{Experiment 4.3b - Gehinderte Exocytose}

Analog zu Experiment 4.3 stellt dieser Versuch ein Modell für die gehinderte Exocytose dar und kann beispielsweise im Kontext des Wirkmechanismus von Botulinumtoxinen eingesetzt werden. Da die verwendeten Natriumalginatblasen jedoch nicht platzen, eignet sich dieser Versuch nicht für eine Verwendung auf Bildungsmessen.

Geräte und Chemikalien: 4 Bechergläser $(100 \mathrm{~mL}, 250 \mathrm{~mL}, 500 \mathrm{~mL}$ und $1 \mathrm{~L})$, großes Reagenzglas ( $\varnothing$ 20-30 mm), Stativmaterial, Eppendorf-Pipettenspitze (5 mL), 2 Vollpipetten (20 mL und $100 \mathrm{~mL}$ ), Glasrührstab, Spatel, Magnetrührer mit Rührfisch, Waage, Schere, Pasteurpipette, Wasser, Spülmittel, Pyranin (GHS07), Glucose, Methylenblau (GHS07), Natriumalginat, Calciumchlorid (GHS07).

Versuchsdurchführung: Ähnlich wie in Versuch 4.3a werden 4 Lösungen angesetzt: (1) in $1 \mathrm{~L}$ Wasser werden $15 \mathrm{~g}$ Calciumchlorid mithilfe eines Magnetrührers und im Anschluss 
zudem vorsichtig etwa $1 \mathrm{~g}$ Spülmittel mittels eines Glasrührstabs gelöst, (2) $100 \mathrm{~mL}$ dieser Lösung werden in ein $250 \mathrm{~mL}$ Becherglas überführt und mit einer Spatelspitze Methylenblau sowie $2 \mathrm{~g}$ Glucose versetzt, (3) in einem $500 \mathrm{~mL}$ Becherglas werden $32 \mathrm{~g}$ Glucose in $400 \mathrm{~mL}$ der Lösung 1 gelöst, (4) in einem $100 \mathrm{~mL}$ Becherglas werden 0,5 g Natriumalginat in $40 \mathrm{~mL}$ Wasser mithilfe eines Magnetrührers gelöst. Anschließend wird die Lösung mit einer Spatelspitze Pyranin versetzt.

Ein großes Reagenzglas wird in ein Stativ eingespannt und anschließend die Lösungen 1 bis 3 übereinandergeschichtet. Dazu werden zunächst ca. $40 \mathrm{~mL}$ von Lösung 1 in das Reagenzglas gefüllt und diese vorsichtig und langsam mit $10 \mathrm{~mL}$ der Lösung 2 unterschichtet. Anschließend werden beide Lösungen mit etwa $40 \mathrm{~mL}$ der Lösung 3 unterschichtet. Mit einer Schere wird die Öffnung der Pipettenspitze etwas vergrößert und diese etwa $4 \mathrm{~cm}$ über dem Duranglas eingespannt. Die Pipettenspitze wird mit wenigen Millilitern der Lösung 4 befüllt, so dass die Lösung in das Reagenzglas tropft und kleine Kugeln entstehen. Sollten sich stattdessen lange Fäden bilden, muss die Fallhöhe variiert werden.

Beobachtung: Die Lösungen 1 bis 3 liegen im Reagenzglas als drei getrennte Phasen vor. Beim Zutropfen der Lösung 4 (Alginatlösung) bilden sich kleine mit Pyraninfarbstoff gefüllte Kugeln, die durch die oberste Schicht an die Phasengrenze zwischen der mittleren und der unteren Schicht sinken. Dort verweilen die Kugeln und lediglich die oberste Schicht weist eine leichte gelbe Färbung auf (Abbildung 47A - C).

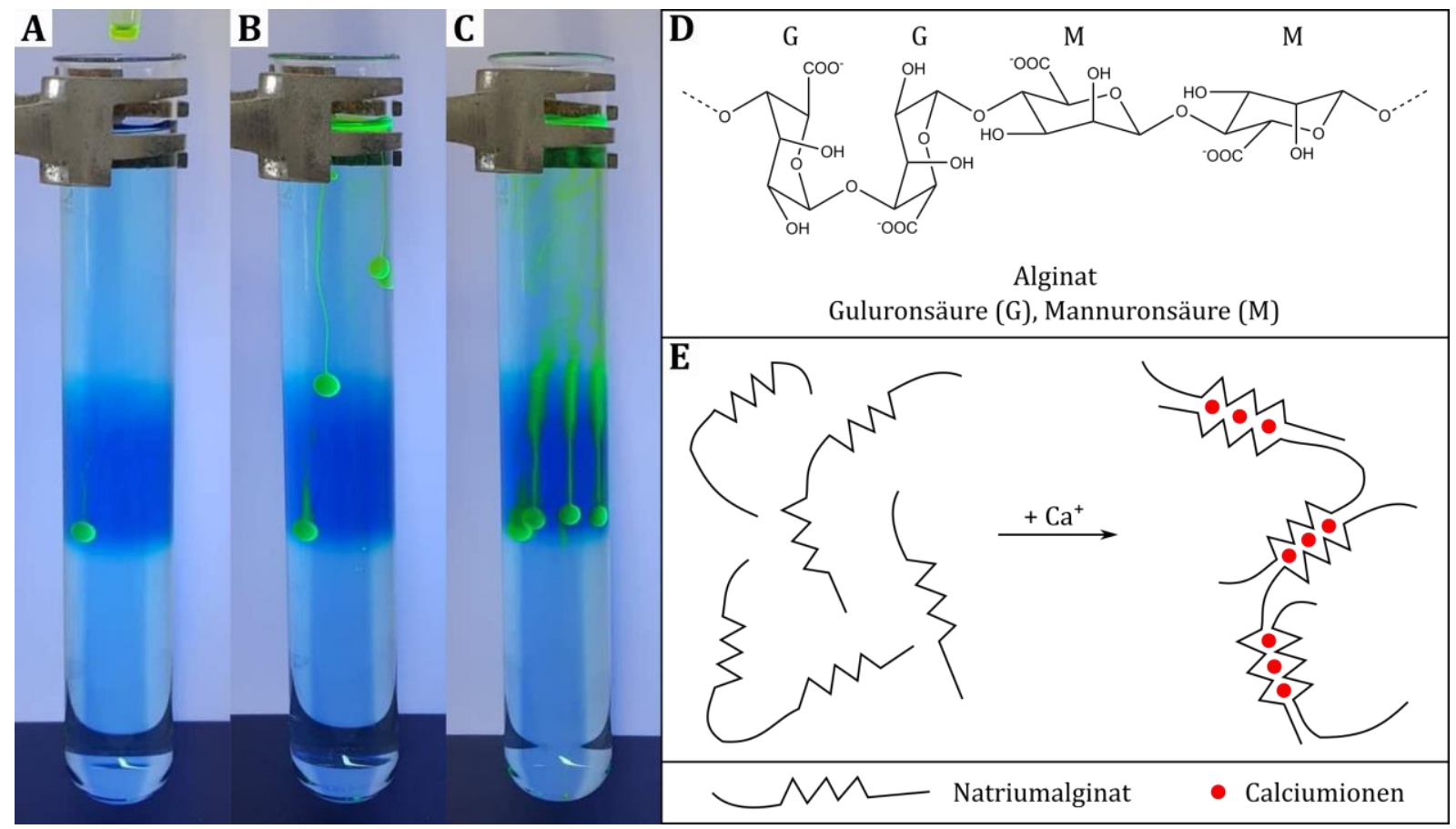

Abbildung 47: (A-C) Eine farbstoffgefüllte Natriumalginatblase sinkt durch die zwei oberen Lösungen mit geringerer Dichte und verbleibt auch nach längerer Zeit an der Grenzschicht zur untersten Lösung derselben Dichte. (D) Strukturformel von Alginat, (E) schematische Darstellung des Alginatkomplexes [235].

Deutung: Natriumalginat ist ein kettenförmiges Polysaccharid, das aus den Monomeren $\alpha$-L-Guluronsäure und $\beta$-D-Mannuronsäure besteht. Während verknüpfte $\beta$-D-Mannuronsäuremoleküle geradlinige Ketten ausbilden, bilden $\alpha$-L-Guluronsäuremoleküle geknickte Ketten aus (siehe Abbildung 47D). Beim Zutropfen der Algniatlösung zu einer Calciumchloridlösung lagern sich verschiedene Kettenabschnitte mit mehreren Guluronsäuremolekülen zusammen, wobei in den entstehenden Hohlräumen Calciumionen in einem 
Chelatkomplex gebunden werden (siehe Abbildung 47E) [236]. Um die Tropfen bildet sich so eine Gelschicht, in die die übrige Alginatlösung eingebettet ist.

Die in diesem Fall auch mit dem Farbstoff Pyranin gefüllten Alginatbällchen besitzen eine höhere Dichte als die Lösungen 1 und 2, weshalb sie an die Grenzfläche der zweiten und dritten Lösung sinken und dort verweilen. Die membranartige Gelschicht ist dabei nicht gänzlich undurchlässig für den Farbstoff, der aufgrund seiner geringeren Dichte aus den Alginatbällchen heraus in die erste Lösung diffundiert.

Anmerkungen zur Deutung der Experimente: Bei der Erregungsübertragung im Nervensystem spielt insbesondere der Transportmechanismus der Exocytose eine wichtige Rolle. Nervenzellen bestehen aus einem System weitverzweigter Dendriten, von denen aus Aktionspotenziale über das Axon zu den präsynaptischen Endigungen geleitet werden. Diese Endigungen bilden wiederum mit den Dendriten anderer Nervenzellen sogenannte Synapsen, über die die Potentiale von einer Nervenzelle zur nächsten übertragen werden.

An chemischen Synapsen erfolgt die Weiterleitung solcher Signale über die Ausschüttung von Neurotransmittern, wie z. B. Acetylcholin, die in kleinen Membranbläschen, den Vesikeln, aufbewahrt sind. Erreicht ein Aktionspotential eine präsynaptische Endigung, werden spannungsabhängige Calciumkanäle geöffnet, so dass sich die Calciumionenkonzentration im Inneren der Endigung erhöht. Diese erhöhte Konzentration fördert die Bildung sogenannter SNARE-Komplexe - Proteinkomplexe zwischen Membranproteinen der präsynaptischen Endigung und der neurotransmittergefüllten Vesikel - die zu einer Fusion der beiden Membranen und der Ausschüttung der Neurotransmitter führen. Diese Neurotransmitter gelangen über den synaptischen Spalt zur postsynaptischen Region der Dendriten weiterer Nervenzellen, wo sie als Rezeptoren für Ionenkanäle dienen, deren Öffnung ein Aktionspotential in der nächsten Nervenzelle auslöst (siehe Abbildung 48B).

A

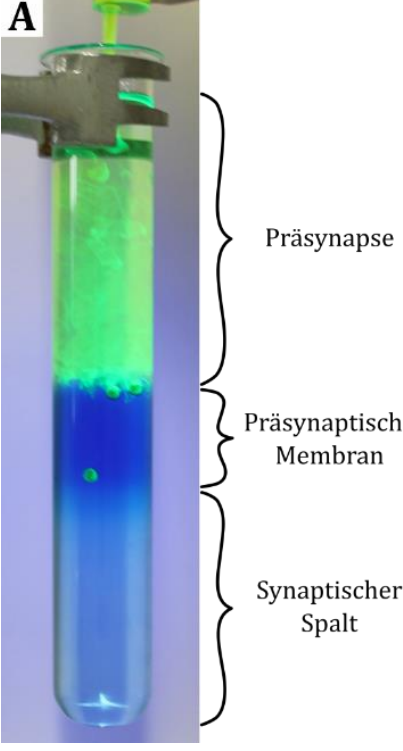

B

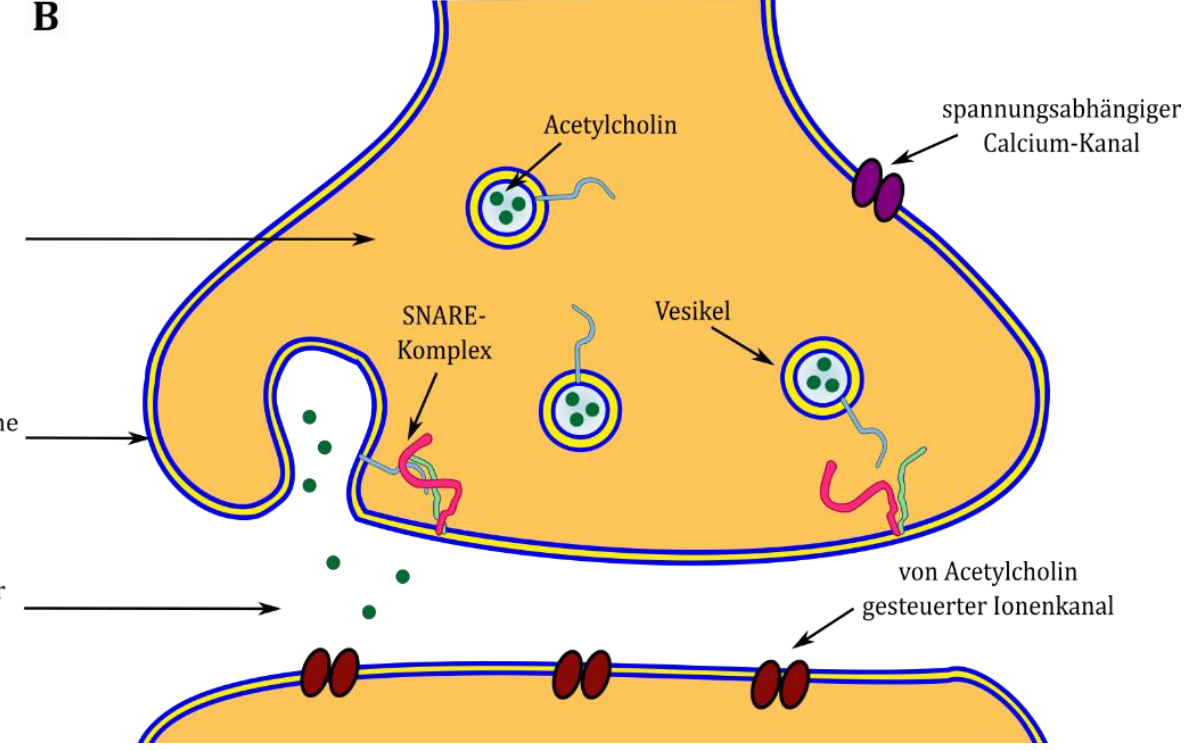

Abbildung 48: Vergleich des Modellexperiments zur Exocytose (A) mit der schematische Darstellung der Ausschüttung von Neurotransmittern durch Exocytose am synaptischen Spalt (B).

Das Experiment 4.4a stellt ein Modell für die Ausschüttung von Neurotransmittern in den synaptischen Spalt dar. Dabei stellen die verschiedenen Phasen von oben nach unten gesehen die Präsynapse, die präsynaptische Membran und den synaptischen Spalt dar. Die Antiblasen dienen als Modelle für Vesikel, die mit einer gefärbten Lösung (= Neurotransmitter) gefüllt sind. Nur wenn die Antiblasen sich beim Platzen an der unteren Grenze der 
blau angefärbten Phase befinden, geben sie ihren Inhalt in die unterste Phase, also in den synaptischen Spalt ab. Lediglich die Anlagerung an die Membran der präsynaptischen Endigung ermöglicht also eine Ausschüttung des Neurotransmitters in den synaptischen Spalt.

In der aktuellen Forschung, u. a. auch des SFB 803, ist insbesondere die Aufschlüsselung verschiedener Prozesse der Membranfusion, die durch SNARE-Proteine gesteuert werden, von großem Interesse. Bereits bekannt ist bspw., dass die Proteine Angriffspunkte verschiedener Toxine wie Tetanus- und Botulinumtoxin sind [237]. Letzteres wird umgangssprachlich auch als Botox ${ }^{14}$ bezeichnet. Insbesondere vor dem Hintergrund, dass SuS ein großes Interesse an medizinischen Themen zeigen ${ }^{[55,238]}$ bietet sich eine Betrachtung der Wirkweisen von Botox auf die SNARE-Proteine sowie damit einhergehend den Prozess der Exocytose an, um das aktuelle Forschungsinteresse bezüglich der SNAREs zu verdeutlichen. Das Experiment 4.3b bietet durch die Verwendung von farbstoffgefüllten Alginatbällchen zudem zusätzlich die Möglichkeit, das Modellexperiment zur Exocytose in Bezug auf die Wirkung von Botox zu erweitern.

\section{Exkurs: Forschung an SNARE-Komplexen}

Zahlreiche wichtige zelluläre Prozesse wie bspw. die Ausschüttung von Neurotransmittern, die Befruchtung von Eizellen und der Eintritt von Viren in Wirtszellen beruhen auf der Fusion von Membranen [239]. Entsprechend besteht in der Membranforschung ein großes Interesse an der Aufklärung solcher Fusionsprozesse. Ein beliebter Forschungsgegenstand sind synaptische Vesikel, da diese mit einem Durchmesser von rund $40 \mathrm{~nm}$ nur eine begrenzte Anzahl von Proteinen besitzen [240]. Für die Membranfusion maßgeblich verantwortlich sind sogenannte SNARE-Proteine (soluble N-ethylmaleimide-sensitive-factor attachment receptor). Ihre Beteiligung am Fusionsprozess - in diesem Fall von synaptischen Vesikeln - konnte erstmalig für die Proteine SNAP-25, Synaptobrevin und Syntaxin nachgewiesen werden, da diese an verschiedenen Stellen von Tetanus- bzw. unterschiedlichen Botulinumtoxinen gespalten werden (vgl. Abbildung 49B und Abbildung 50) und eine Membranfusion durch den jeweiligen Ausfall eines dieser drei Proteine unterbunden wird [241-243].

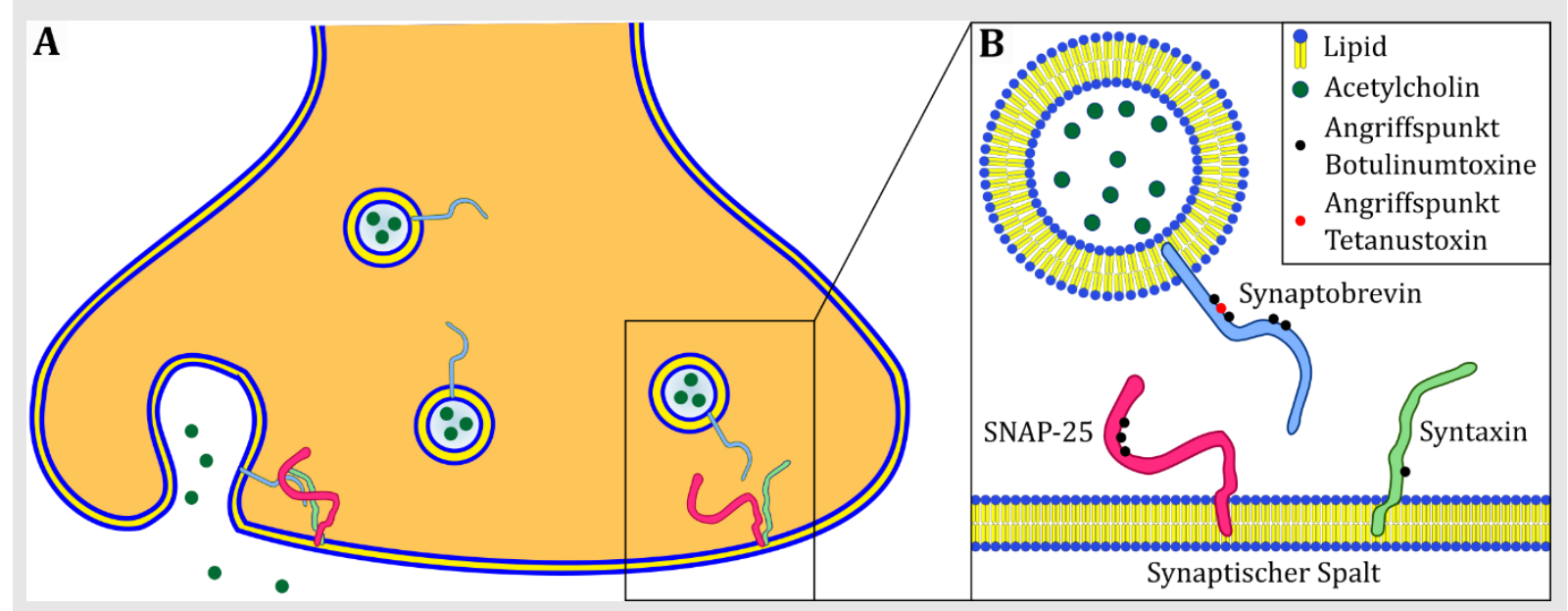

Abbildung 49: Schematische Darstellung der am Fusionsprozess beteiligten Proteine SNAP-25, Synaptobrevin und Syntaxin samt der Angriffspunkte für das Tetanus- und verschiedene Botulinumtoxine.

${ }^{14}$ Botox $^{\circledR}$ ist ein Trademark von Allergan Inc. 
Neben diesen in den synaptischen Vesikeln (Synaptobrevin) und in der synaptischen Membran (SNAP-25 und Syntaxin) angesiedelten SNARE-Proteinen existieren noch zahlreiche weitere SNAREs, im Kontext dieser Arbeit beschränken sich die weiteren Betrachtungen jedoch auf die drei genannten und besonders ausgiebig untersuchten Proteine.

Im Prozess der Membranfusion muss Energie aufgebracht werden, um repulsive ionische Kräfte sowie die Hydratisierung der beiden Membranen zu überwinden [244]. Diese Energie wird durch die Ausbildung eines SNARE-Komplexes aufgebracht, in dem sich die drei genannten Proteine zu einem helikalen viersträngigen (coiled-coil) Komplex zusammenlagern, wobei SNAP-25 zwei $\alpha$-Helices zur Verfügung stellt.

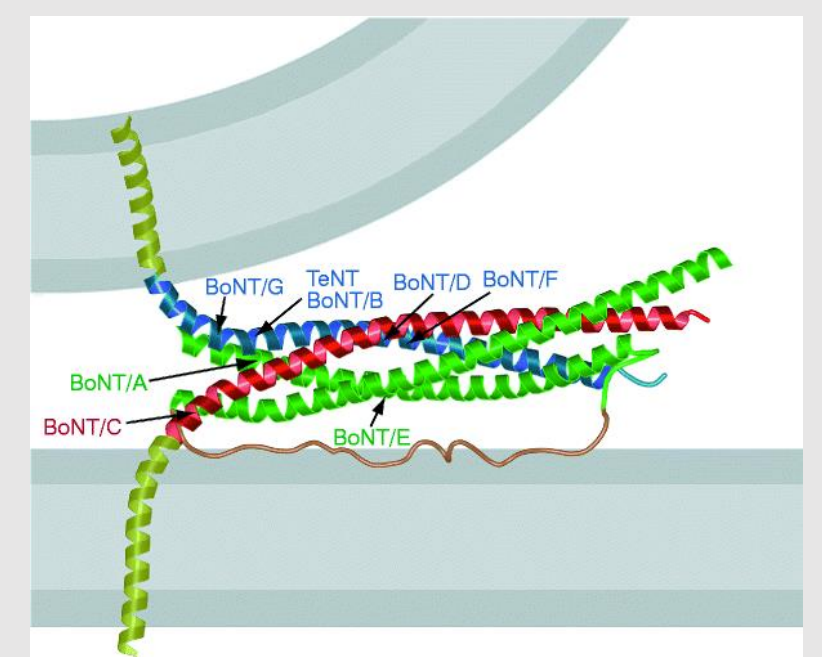

Abbildung 50: Modell des coiled-coil SNARE-Komplexes [237]. Markiert sind die Angriffspunkte für verschiedene Tetanus- (TeNT) und Botulinumtoxine (BoNT).

Dieser Prozess der Zusammenlagerung (Zippering), der an den N-terminalen Enden der Proteine beginnt und in Richtung der C-terminalen membranverankerten Enden verläuft, wird durch das Einströmen von Calciumionen durch spannungsabhängige Kanäle an der Synapsenendigung bewirkt. Bei der sich anschließenden Fusion der Membranen entstehen verschiedene Intermediate. Dabei kommt es zunächst zur Hemifusion, bei der jeweils eine Lipidschicht des Vesikels bzw. der synaptischen Membran fusionieren, so dass ein uhrglasförmiger sogenannter fusion stalk entsteht. Diesem Zustand folgt der Kontakt der beiden weiteren Lipidschichten, die durch Fusion eine Pore ausbilden [245, 246].

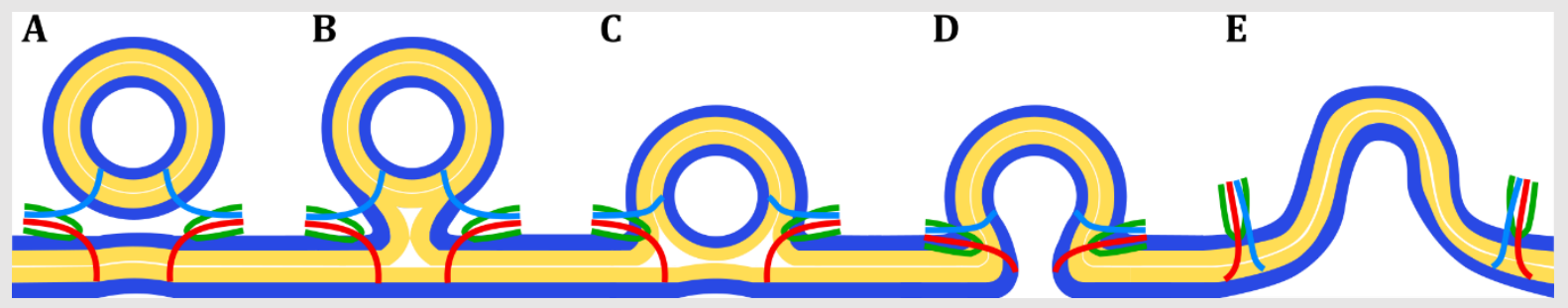

Abbildung 51: Schematische Darstellung der SNARE-vermittelten Membranfusion nach JAHN \& SCHELLER [246]. (A) Ausbildung von SNARE-Komplexen, (B) Hemifusion (Fusion Stalk), (C) Hemifusion (Transmembrankontakt), (D) Öffnung der Fusionspore, (E) Weitung der Fusionspore.

Diese Metaprozesse der SNARE-vermittelten Fusion bedürfen teilweise noch weiterer fachlicher Klärung und sind deshalb in der Forschung des SFB von besonderem Interesse. In Kooperation experimenteller und theoretischer Forschungsgruppen des Themenkomplex B, die sich u. a. molekulardynamische Simulationen zunutze machen [247], werden beispielsweise die Prozesse der Stalk-Bildung und der Porenvergrößerung genauer unter- 
sucht. Von Interesse sind hierbei u. a. die Veränderungen der Energielandschaft, die Geometrien während der Fusion sowie der Einfluss der Lipidzusammensetzung. Ergänzend spielt zudem auch die Entwicklung von neuen Untersuchungsmethoden zur Beobachtung von Fusionsprozessen eine wichtige Rolle, von denen einige in der Einleitung zu Kapitel 5.6 näher erläutert werden [174].

\section{Einordnung der Experimente im Kontext der SO-Leitfragen}

Im Rahmen des Themas der Zellbiologie werden im Biologieunterricht der Oberstufe verschiedene Membrantransportprozesse behandelt. Neben aktiven und passiven Transportprozessen werden auch die Prozesse der Endo- und Exocytose thematisiert. Letztere wird im Themenschwerpunkt Neurologie zudem im Zuge der Erregungsweiterleitung an den Synapsen erneut aufgegriffen. Die verschiedenen Stofftransportwege werden dabei oftmals anhand von Informationstexten und Abbildungen erarbeitet.

Die in diesem Abschnitt vorgestellten Experimente bieten diesbezüglich die Möglichkeit, Erkenntnisse hinsichtlich (chemischer) Bedingungen für den Membrantransport, wie die Teilchengröße oder die Ladung der Teilchen, anhand von Modellsystemen zu erarbeiten. Die in Kapitel 6.3.1 vorgestellte Adaptierte Primärliteratur zum Ionenkanal VDAC-1 kann dabei ergänzend verwendet werden, um auch spannungsabhängige Kanäle zu betrachten und Einblicke in die diesbezügliche Forschung des SFB 803 zu geben.

Eine weitere Möglichkeit zur Verquickung von schulischen mit Forschungsinhalten bieten die Modellexperimente zur Exocytose bzw. gehinderten Exocytose, die allgemein den Prozess der Membranfusion veranschaulichen sollen [248]. Die Betrachtung der Wirkweise von Botox im Kontext der Neurologie ermöglicht die Thematisierung der Relevanz von SNARE-Proteinen für den Fusionsprozess, die wiederum wichtiger Forschungsgegenstand im SFB sind. Zudem können auch endocytotische Prozesse erneut aufgegriffen werden, da Botulinumtoxin, ähnlich wie andere Krankheitserreger, den Vorgang der Endocytose nutzt, um in Zellen einzudringen.

Für die Untersuchung von SNARE-Proteinen werden u. a. Selbstassemblierungsprozesse ausgenutzt, um Vesikel mit gewünschten Lipid- und Proteinmischungen zu erhalten. Um die Auswirkungen spezifischer Mischungsverhältnisse beispielsweise auf die SNARE-vermittelte Fusion beobachten zu können, bedarf es entsprechender wissenschaftlicher Techniken. Neben molekularen Simulationen spielen hierbei Verfahren eine Rolle, in denen Fluoreszenzmarker genutzt werden, um auch kleinste Strukturen und Prozesse abbilden zu können. Vor dem Hintergrund, dass das Thema der Fluoreszenz weder in den niedersächsischen Kerncurricula der Chemie [183] noch der Physik [249] aufgeführt wird, wurde es im Sinne der dritten Leitfrage aus Kapitel 5 als wichtige Thematik für das Verständnis der Forschung und der verwendeten Methoden des SFB 803 identifiziert.

\subsection{Themenfeld 5 - Fluoreszenz}

Für die Betrachtung von Membranen spielt u. a. die Mikroskopie eine wichtige Rolle, da sie es ermöglicht, auch Strukturen unter der Auflösungsgrenze des menschlichen Auges sichtbar zu machen. Das Auflösungsvermögen von typischen Lichtmikroskopen ist jedoch durch das ABBE-Limit begrenzt, das maßgeblich von der Beugung bestimmt wird [250], so dass mithilfe dieser Technik lediglich Strukturen im Größenbereich bis ca. 200 nm untersucht werden können. Eine Möglichkeit, das ABBE-Limit zwar nicht zu umgehen, aber dennoch Strukturen unterhalb dieser Grenze nachzuweisen und zu beobachten bietet die Fluoreszenzmikroskopie. Durch Ausnutzung der Autofluoreszenz verschiedener Moleküle 
(bspw. Chlorophyll, beta-Carotine, Kollagen) oder der gezielten Markierung von Molekülen mittels Fluorochromen können diese nach Anregung anhand des emittierten Fluoreszenzlichts in der Membran lokalisiert werden (siehe Abbildung 52). Methoden wie die konfokale Fluoreszenzmikroskopie ermöglichen es dabei, den Kontrast der gewonnen Bilder noch weiter zu erhöhen [251]. Zudem erlauben es neuere superauflösende Mikroskopietechniken, wie die STED-Mikroskopie (Stimulated Emission Depletion) - für die der Göttinger Forscher STEFAN HELL 2014 mit dem Nobelpreis der Chemie ausgezeichnet wurde - und die MinFlux-Mikroskopie, Auflösungen auch deutlich unterhalb des ABBELimits im einstelligen Nanometerbereich zu erzielen [252, 253]. Neben den verschiedenen fluoreszenzmikroskopischen Techniken existieren dabei noch weitere Verfahren, die das Phänomen der Fluoreszenz nutzen, wie u.a. die Fluoreszenzspektroskopie und -thomographie. Da bezüglich der Forschung des SFB 803 eine möglichst präzise Auflösung submikroskopischer Membranprozesse vonnöten ist, gehören sowohl die Fluoreszenzmikroskopie als auch die -spektroskopie zu den wichtigsten Forschungsmethoden des SFB.

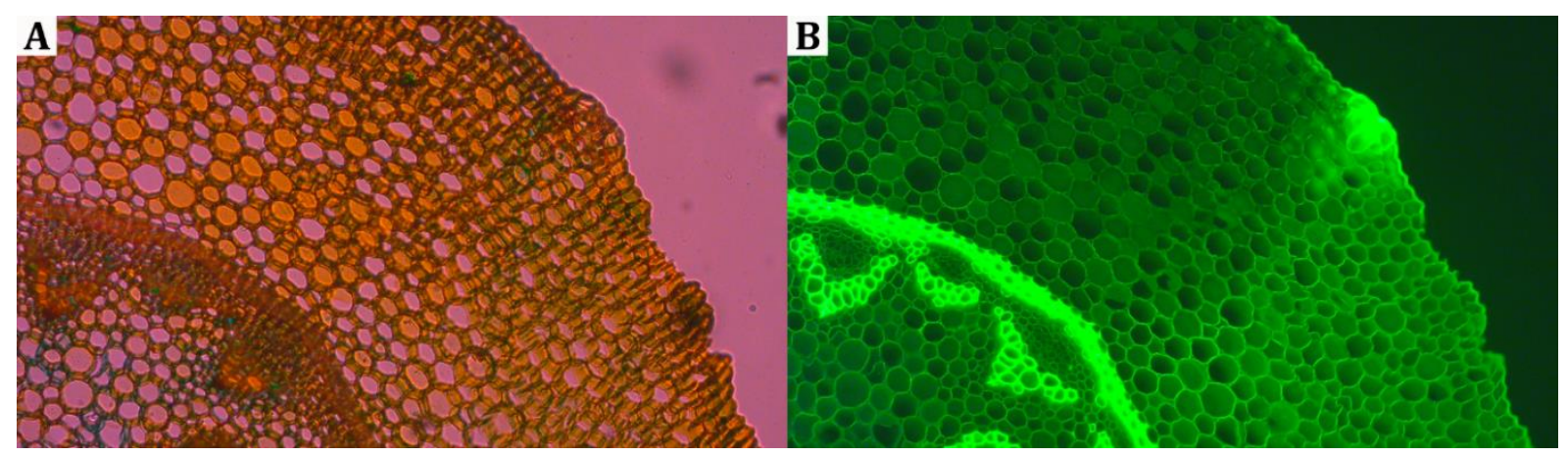

Abbildung 52: (A) Mikroskopische Aufnahmen des Sprossquerschnitts eines Maiglöckchens (Convallaria majalis), (B) Eigenfluoreszenz nach Anregung mit UV-Licht.

Die Fluoreszenz basiert auf der Anregung eines Moleküls von einem niedrigen in ein höheres Energieniveau und der strahlenden Desaktivierung in das Ursprungsniveau durch Emission eines Photons. Hierbei wird zunächst durch Schwingungsrelaxation ein Teil der Energie strahlungslos abgegeben, bevor die strahlende Desaktivierung erfolgt. Entsprechend ist der Energiebetrag der emittierten Strahlung geringer als der absorbierte Energiebetrag. Aufgrund der antiproportionalen Beziehung der Energie zur Wellenlänge, ist die absorbierte Wellenlänge kürzer als die emittierte, was als Stokes-Shift, bzw. mit Blick auf das elektromagnetische Spektrum auch als Rotverschiebung, bezeichnet wird [254, 255]. Zur Veranschaulichung der verschiedenen Prozesse können diese wie in Abbildung 53 in einem vereinfachten JABŁOŃSKI-Diagramm dargestellt werden. 


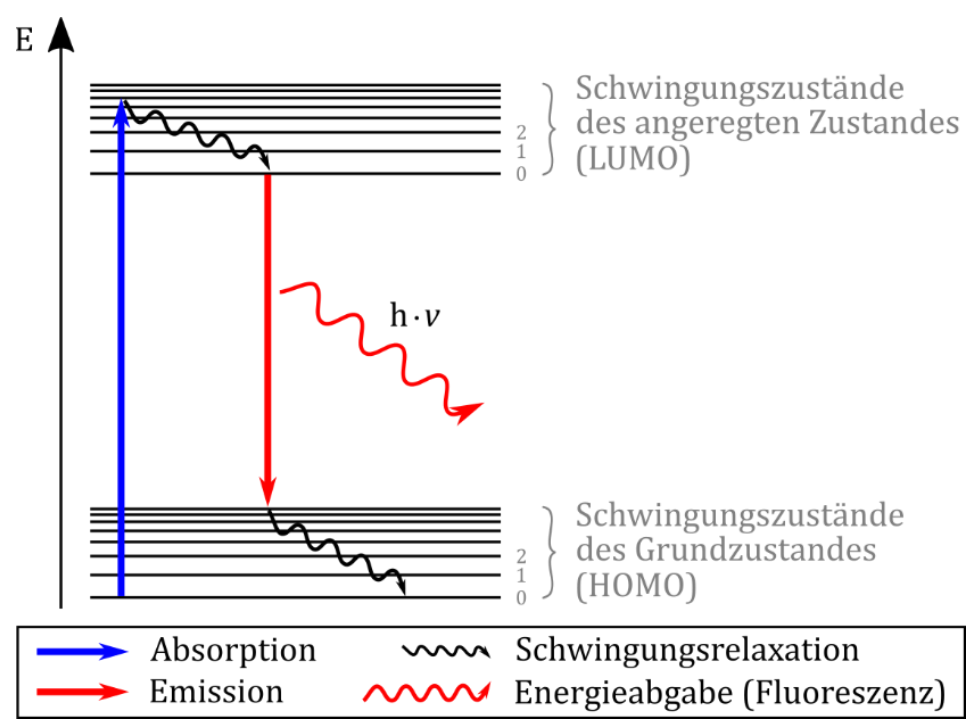

Abbildung 53: Schematische Darstellung der Anregung zur Fluoreszenz in einem vereinfachten JABŁOŃSKIDiagramm.

Ob ein Molekül strahlend oder strahlungslos desaktiviert, hängt unter anderem von seiner Molekülstruktur ab. Strahlungslose Desaktivierung erfolgt vor allem durch Schwingungsrelaxation, d. h. durch Translations- und Rotationsbewegungen innerhalb des Moleküls. Abbildung 54 zeigt die Strukturformeln einer nicht fluoreszierenden und einer fluoreszierenden Verbindung (Phenolphthalein und Fluorescein), die bis auf ein Sauerstoffatom, das wie eine Brücke zwischen der Phenolat- und der Cyclohexadienongruppe wirkt, die gleiche Struktur besitzen. Beide absorbieren Licht im sichtbaren Bereich $\left(\lambda_{P}=558\right.$, $\lambda_{F}=496$ ), jedoch fluoresziert lediglich Fluorescein, was vor allem mit der verminderten Rotationsfähigkeit der Phenolatgruppe aufgrund der Sauerstoffbrücke zu erklären ist [256].<smiles>COc1ccc(C(=C2C=CC(=O)C=C2)c2ccccc2C(=O)[O-])cc1</smiles><smiles>COc1ccc2c(c1)OCCc1c(-c3ccccc3C(=O)[O-])ccc-2c1CCCO</smiles>

Abbildung 54: Strukturformeln von Phenolphthalein (links, $\mathrm{pH}>$ 8,2) und Fluorescein (rechts).

Die nachfolgenden Experimente bieten die Möglichkeit anhand erster Erkenntnisse bezüglich der Farbigkeit organischer Moleküle verschiedene Eigenschaften fluoreszierender Farbstoffe zu erarbeiten. Dabei werden neben Faktoren die eine Fluoreszenzemission beeinflussen auch FRET- (Förster-Resonanzenergietransfer) und Quenchingeffekte thematisiert, die in der Forschung z. T. gezielt eingesetzt werden, um z. B. Fusionsmessungen durchführen zu können.

\section{Experiment 5.1 - Blue Bottle}

Obwohl dieser Versuch typischerweise nicht verwendet wird, um den Begriff der Farbigkeit auf submikroskopischer Ebene zu erklären, eignet er sich insbesondere aufgrund seiner Wiederholbarkeit für den Einsatz auf Bildungsmessen [257].

Geräte und Chemikalien: 2 Bechergläser, Schnappdeckelglas, Waage, Spatel, Pasteurpipetten, demin. Wasser, D-Glucose, Methylenblaulösung ( $w=0,2 \%$; GHS07), Natriumhydroxidlösung ( $w=10 \%$; GHS05). 
Versuchsdurchführung: In einem Schnappdeckelglas wird $1 \mathrm{~g}$ D-Glucose in ca. $10 \mathrm{~mL}$ Wasser gelöst. Anschließend werden wenige Milliliter Natriumhydroxid- sowie 1-2 Tropfen Methylenblaulösung hinzugegeben. Das Schnappdeckelglas wird verschlossen und die Lösung kurz ruhen gelassen. Schließlich wird das Gläschen kräftig geschüttelt und wieder für einige Minuten stehen gelassen.

Beobachtung: Die Lösung erscheint zunächst blau, entfärbt sich aber nach kurzer Zeit. Das Schütteln der Lösung führt zu einer erneuten Blaufärbung. Nach kurzer Zeit liegt die Lösung erneut farblos vor (Abbildung 55).

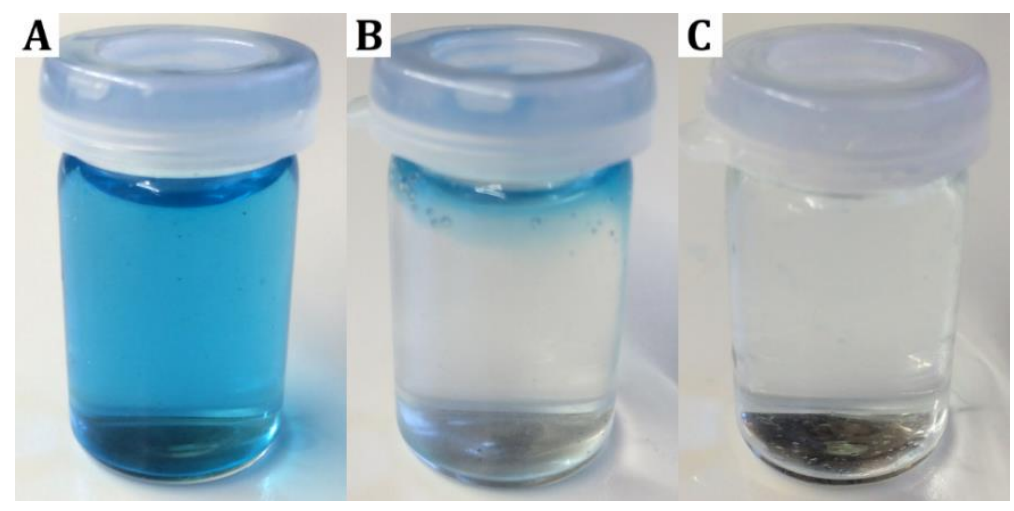

Abbildung 55: Die nach dem Schütteln blaue Lösung (A) liegt nach kurzer Zeit wieder entfärbt vor (B, C).

Deutung: Methylenblau besitzt ein ausgedehntes konjugiertes $\pi$-Elektronensystem, das ausschlaggebend für die blaue Färbung der Lösung ist. In diesem Versuch dient es als Oxidationsmittel für die Oxidation der Glucose zu Gluconsäure, wobei das Methylenblau zu Leukomethylenblau reduziert wird (vgl. Abbildung 56). Dabei wird das konjugierte $\pi$-System unterbrochen, weshalb die Lösung farblos erscheint. Durch das Schütteln wird Sauerstoffgas in die Lösung eingetragen, das als Oxidationsmittel für das Leukomethylenblau zurück zum Methylenblau dient, welches wieder für eine Oxidation der Glucose bereitsteht.

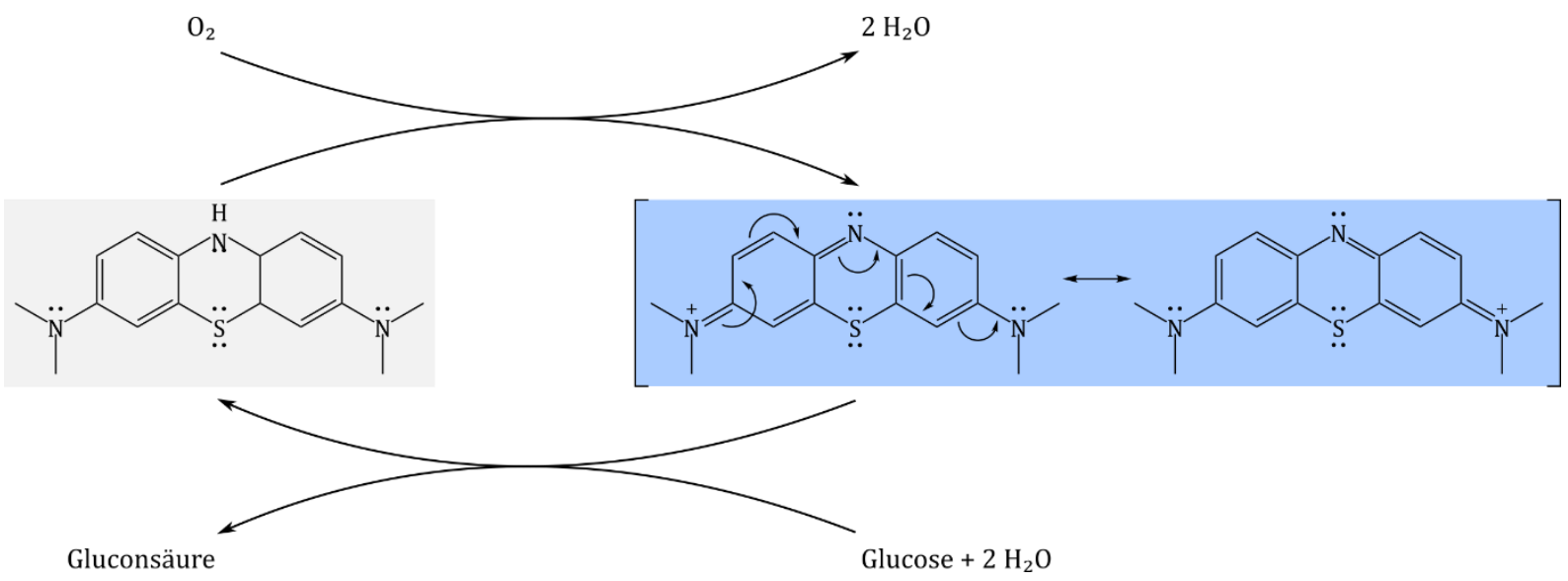

Abbildung 56: Reaktionsschema der reversiblen Reaktion von Leukomethylenblau (links, farblos) zu Methylenblau (rechts, blau).

Anmerkungen zur Deutung des Experiments: Die Farbigkeit eines Stoffes beruht auf seiner Fähigkeit, elektromagnetische Strahlung des sichtbaren Bereichs zu absorbieren. Bei organischen Verbindungen hängt diese vor allem von der Länge des konjugierten Doppelbindungssystems ab. Wird ein Molekül, das Doppelbindungen besitzt, durch Energie einer bestimmten Wellenlänge angeregt, so wird ein Elektron aus dem im Grundzustand höchsten besetzen $\pi$-Orbital (Highest Occupied Molecular Orbital, HOMO) in das niedrigste unbesetzte $\pi$-Orbital angehoben (Lowest Unoccupied Molecular Orbital, LUMO). Umso mehr 
konjugierte Doppelbindungen in einem System vorhanden sind, umso näher liegen die Orbitale zusammen, so dass die Energie die aufgewendet werden muss, um ein Elektron vom HOMO ins LUMO zu befördern, geringer und die Wellenlänge entsprechend größer wird (siehe Abbildung 57A). Liegt die absorbierte Energie im Wellenlängenbereich des sichtbaren Lichts (ca. 380 - $750 \mathrm{~nm}$ ), nehmen wir die Substanz als farbig wahr. So ist $\beta$ Carotin mit einem System von elf konjugierten Doppelbindungen beispielsweise für die orangene Farbe von Karotten verantwortlich (Abbildung 57B).
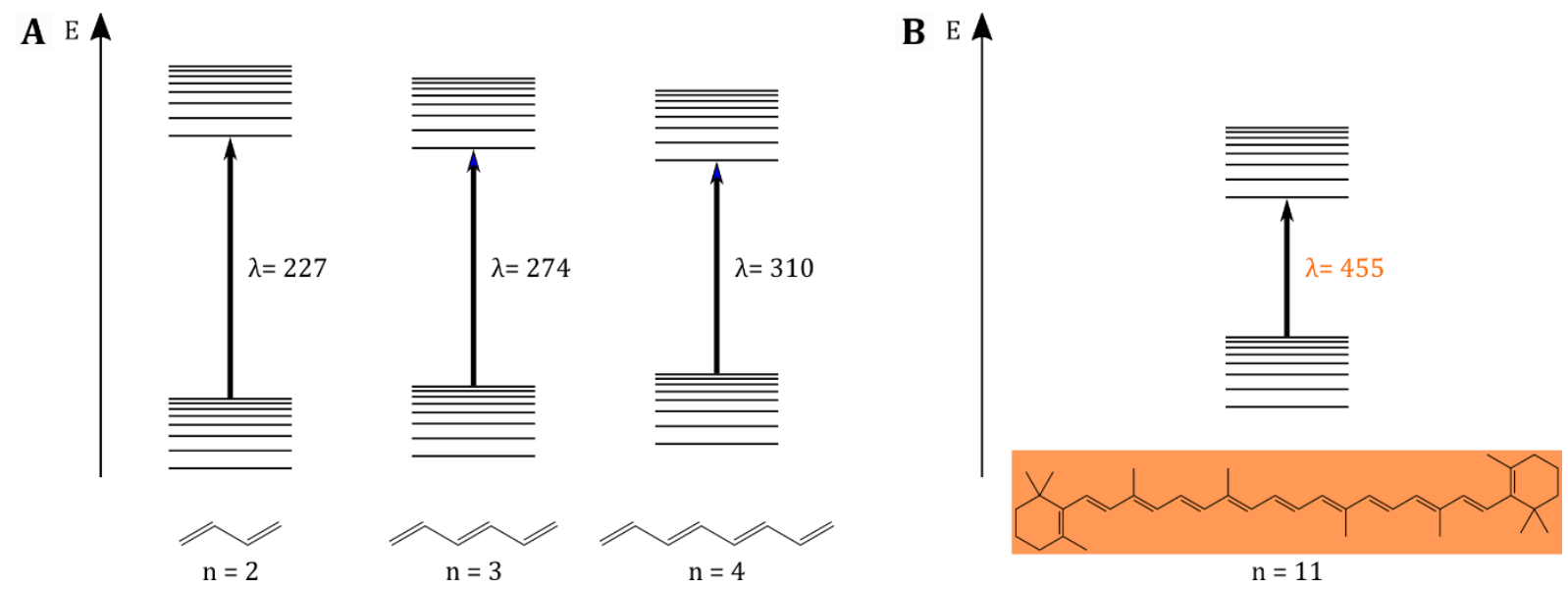

Abbildung 57: (A) Mit größer werdenden konjugierten $\pi$-System nimmt die absorbierte Wellenlänge zu. (B) $\beta$-Carotin besitzt ein konjugiertes $\pi$-System mit 11 Doppelbindungen, das für eine orangene Farbwahrnehmung verantwortlich ist.

Die wahrgenommene Farbe entspricht dabei der Komplementärfarbe der absorbierten Wellenlänge (subtraktive Farbmischung). Die Abregung auf ein energetisch niedrigeres Niveau erfolgt zumeist über strahlungslose Desaktivierung. Dabei wird die Energie durch Schwingungsrelaxation in Form von Wärme an die Umgebung abgegeben. Neben der $\pi \rightarrow \pi^{*}$-Übergänge bestehen noch weitere Möglichkeiten der Anregung, wie bspw. Charge-Transfer-Übergänge in Komplexverbindungen, diese werden hier jedoch der Einfachheit halber vernachlässigt [254, 258].

Bei der Oxidation der Glucose durch das Methylenblau wird eines der Stickstoffatome des Methylenblaus protoniert, was eine Unterbrechung des über das Molekül verteilten konjugierten Doppelbindungssystem zur Folge hat, wodurch die Lösung farblos erscheint. Die Reaktion des Leukomethylenblaus mit Sauerstoff zu Methylenblau sorgt für die Wiederausbildung des konjugierten Systems, so dass die Energie, die zur Anregung eins Elektrons aufgebracht werden muss, wieder im Bereich der Wellenlängen des sichtbaren Lichts liegt.

\section{Experiment 5.2 - Strukturelle Abhängigkeit der Fluoreszenz}

Dieses Experiment kann u. a. im Unterricht eingesetzt werden, um die auftretende Fluoreszenz didaktisch reduziert auf die eingeschränkten Rotationsbewegungen innerhalb des Morinmoleküls zurückzuführen [259].

Geräte und Chemikalien: 3 Bechergläser (50 mL), Tüpfelplatte, Pasteurpipetten, Spatel, UV-Lampe, demin. Wasser, Aluminiumchlorid Hexahydrat (GHS05), Morin (GHS09), Ethanol (GHS02, GHS07), Essigsäurelösung (1 M; GHS05).

Versuchsdurchführung: In einem Becherglas wird eine Spatelspitze Morin in $10 \mathrm{~mL}$ Ethanol gelöst. Auf eine Tüpfelplatte werden in je ein Feld die Morinlösung, eine Aluminiumchloridlösung sowie Ethanol gegeben und alle drei mit einem Tropfen Essigsäurelösung 
angesäuert. Anschließend werden sowohl die Aluminiumchloridlösung als auch das Ethanol mit etwas Morinlösung versetzt. Schließlich werden die Lösungen unter UV-Licht betrachtet.

Beobachtung: Bei Zugabe der Morinlösung intensiviert sich die gelbe Färbung der Aluminiumchloridlösung. Unter UV-Licht ist eine blau-grüne Fluoreszenz zu beobachten, wobei alle anderen Lösungen keine Fluoreszenz zeigen (Abbildung 58).

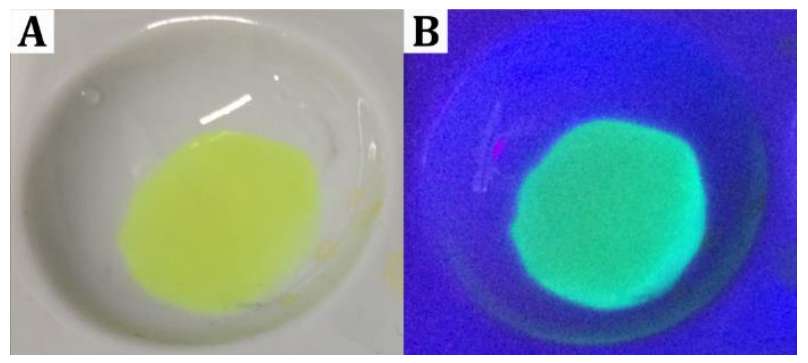

Abbildung 58: (A) Gelbe Färbung der Lösung bei Tageslicht, (B) blau-grüne Fluoreszenz unter UV-Licht.

Deutung: Morin bildet in Anwesenheit von Aluminiumionen einen Chelatkomplex, an dem u. a. auch die Resorcingruppe beteiligt ist. Aufgrund der Chelatierung wird die Rotationsfähigkeit des Resorcins eingeschränkt (siehe Abbildung 59B, rot markierte Bindungsstellen), so dass eine strahlungslose Desaktivierung nur eingeschränkt möglich ist. Entsprechend erfolgt die Energieabgabe in Form von strahlender Desaktivierung, die als Fluoreszenz wahrgenommen werden kann.

A $\mathrm{E}$

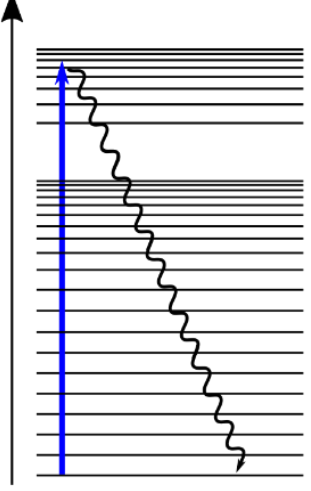

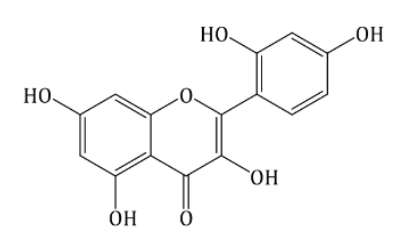

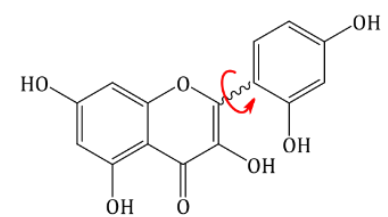

B E

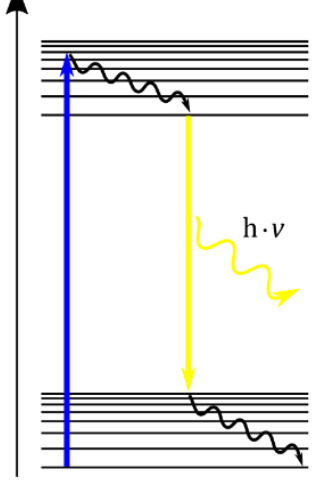

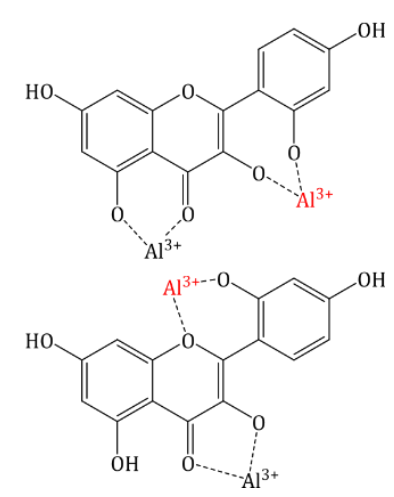

Abbildung 59: (A) Das Morinmolekül ist zunächst sterisch nicht gehindert, so dass eine Abregung über Schwingungsrelaxation möglich ist. (B) Bzgl. der Chelatierung mit Aluminiumionen werden verschiedene Bindungsstellen vorgeschlagen. Die rot markierten Bindungsstellen verhindern eine Rotation und verringern die Schwingungszustände, so dass eine Abregung mittels Fluoreszenzemission erfolgt.

Anmerkungen zur Deutung des Experiments: Morin gehört zur Gruppe der Flavonoide, die in der Natur eine wichtige Rolle als Blütenfarbstoffe spielen. Es bildet in Anwesenheit von Aluminiumionen aber auch anderen Zentralteilchen wie Zink-, Kupfer-, Eisen- oder Cadmiumionen Chelatkomplexe aus, die z. T. charakteristisch fluoreszieren. Die Fluoreszenz wird dabei in der Literatur auf intramolekulare Protonentransfers im elektronisch angeregten Zustand (Excited State Intramolecular Proton Transfer, ESIPT) zurückgeführt [260]. Für die schulische Verwendung ist jedoch eine didaktische Reduzierung auf die Einschränkung von Rotationsbewegungen innerhalb des Morinmoleküls aufgrund der Chelatierung ausreichend. Diesbezüglich lassen sich in der Literatur verschiedene Vorschläge für die Bindungsstellen sowie die Stöchiometrie der Komplexe in Abhängigkeit vom Lösungsmittel, pH-Wert und dem Zentralatom finden [261-264]. Anhand dieser möglichen Bindungsstellen können sterische Hinderungen bezüglich Rotationsbewegungen diskutiert 
und im Zusammenspiel mit dem oben gezeigten Beispiel von Phenolphthalein und Fluorescein die Bedeutung des Rotationsvermögens hinsichtlich der Fluoreszenzeigenschaft von Molekülen erarbeitet werden.

\section{Experiment 5.3 - Temperaturabhängigkeit der Lumineszenz}

Aufgrund der Verwendung von flüssigem Stickstoff eignet sich dieser Versuch zur Temperaturabhängigkeit der Lumineszenz vornehmlich für eine Verwendung in Schülerlaboren, kann jedoch auch bei Formaten wie der Langen Nacht der Wissenschaft Anwendung finden [265].

Geräte und Chemikalien: Rundkolben mit Schliff und Stopfen (100 mL), Spatel, Pasteurpipette, UV-Lampe, Dewargefäß, Stativmaterial, Handschuhe, Kupfer(I)-iodid (GHS05, GHS07, GHS09), Pyridin (GHS02, GHS07), Stickstoff (flüssig, GHS04).

Versuchsdurchführung: Unter dem Abzug wird in einem Rundkolben eine Spatelspitze Kupfer(I)-iodid mit wenigen Millilitern Pyridin versetzt, so dass das Salz vollständig gelöst vorliegt. Der verschlossene Kolben wird, in ein Stativ eingespannt, für ca. 30 Sekunden in flüssigen Stickstoff gehalten und dabei mit UV-Licht bestrahlt.

Beobachtung: Bei Raumtemperatur zeigt die gelbe Lösung keine Lumineszenz. Wird der Kolben in flüssigen Stickstoff getaucht, erstarrt die Lösung und beginnt orange zu lumineszieren. Wird das Gefäß dem flüssigen Stickstoff entnommen, erlischt die Lumineszenz bei Raumtemperatur nach kurzer Zeit wieder (Abbildung 60A, B).

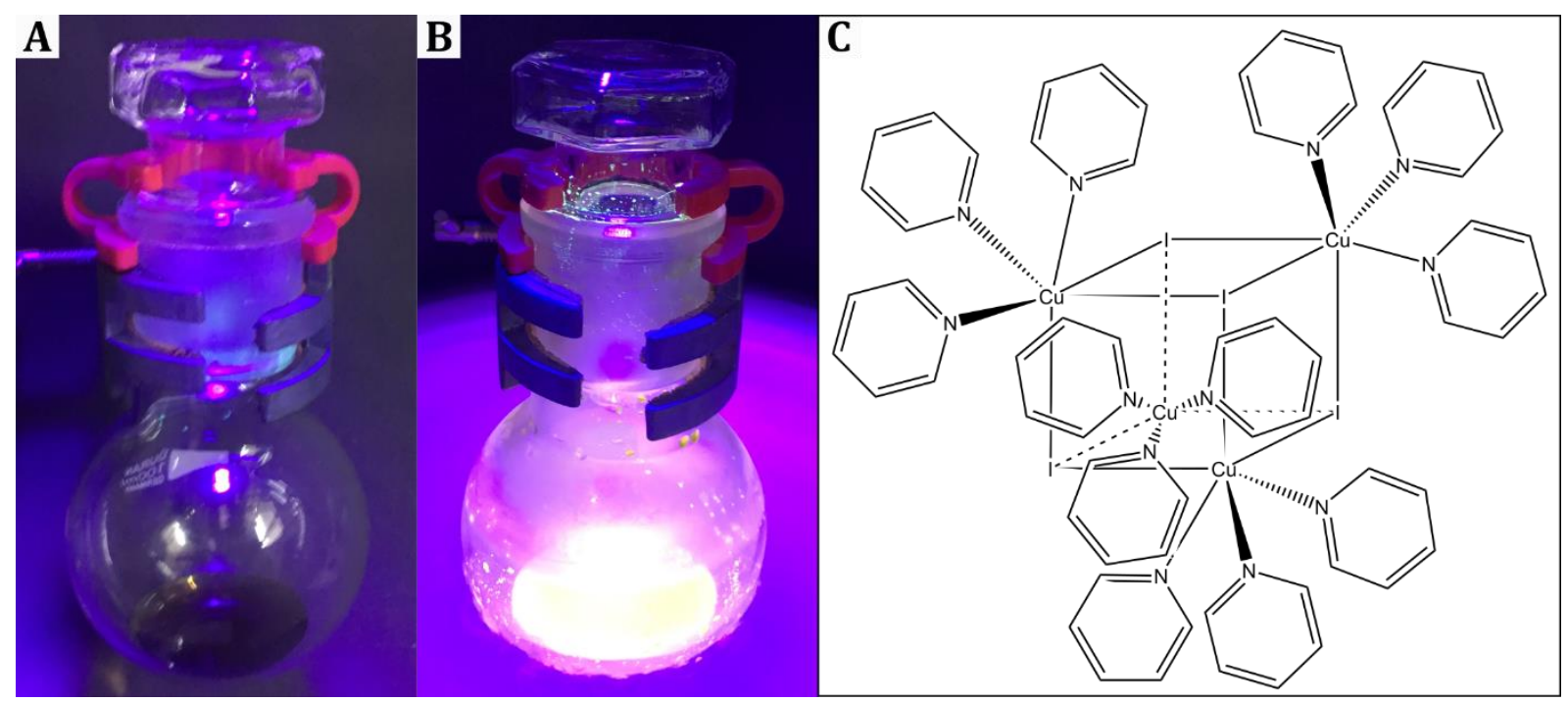

Abbildung 60: Trispyridinkomplexes unter UV-Licht bei Raumtemperatur (A), sowie unter Kühlung in flüssigem Stickstoff (B). (C) Vorgeschlagene Komplexstruktur des Trispiridinkomplexes [265].

Deutung: Bei Zugabe von Pyridin zu Kupfer(I)-iodid entsteht ein Trispyridinkupfer(I)-iodid-Komplex (siehe Abbildung 60C) [266].

$$
4 \mathrm{CuI}+12 \mathrm{C}_{5} \mathrm{H}_{5} \mathrm{~N} \rightarrow \mathrm{Cu}_{4} \mathrm{I}_{4}\left(\mathrm{C}_{5} \mathrm{H}_{5} \mathrm{~N}\right)_{12}
$$

Die gelbe Lösung zeigt zunächst bei Raumtemperatur keine Lumineszenz, da die absorbierte Energie durch Schwingungsrelaxation strahlungslos emittiert wird. Wird die Lösung mithilfe des flüssigen Stickstoffs abgekühlt, ändert sich auch der Aggregatzustand der Lösung von flüssig zu fest. Die Abkühlung mindert dabei die Fähigkeit der Moleküle zu Translations- und Rotationsbewegungen, weshalb die absorbierte Energie in Form von Licht emittiert wird. 
Anmerkungen zur Deutung des Experiments: Beim Trispyridinkupfer(I)-iodid-Komplex handelt es sich um einen Charge-Transfer-Komplex, für den zwei mögliche angeregte $\mathrm{Zu}$ stände vorgeschlagen werden, der ${ }^{3}$ XLCT- sowie der ${ }^{3}$ CC-Zustand (Abbildung 61A) [267]. Dabei erfolgt die Anregung beim ${ }^{3}$ XLCT-Zustand (triplet halide-to-ligand charge transfer) vom Halogenid zum Liganden, während die Anregung beim ${ }^{3} \mathrm{CC}-Z u s t a n d$ (tripled cluster centered) vom Halogenid zum Metall erfolgt. Die Abregung erfolgt an dieser Stelle also aus einem Tripletzustand heraus, weshalb es sich bei der Lumineszenz streng genommen um Phosphoreszenz handelt. Auf phänomenologischer Ebene ist jedoch kein für die Phosphoreszenz typisches Nachleuchten wahrnehmbar. Didaktisch reduziert sollte an dieser Stelle deshalb von Lumineszenz gesprochen werden. Dabei können die unterschiedlichen Übergänge wie in (Abbildung 61B, C) vereinfacht dargestellt werden. Die Auftretenswahrscheinlichkeit der jeweiligen Zustände ist abhängig von der Temperatur, wobei der ${ }^{3} \mathrm{CC}$ eher bei höheren, der ${ }^{3}$ XLCT-Zustand eher bei niedrigeren Temperaturen bevorzugt besetzt wird.
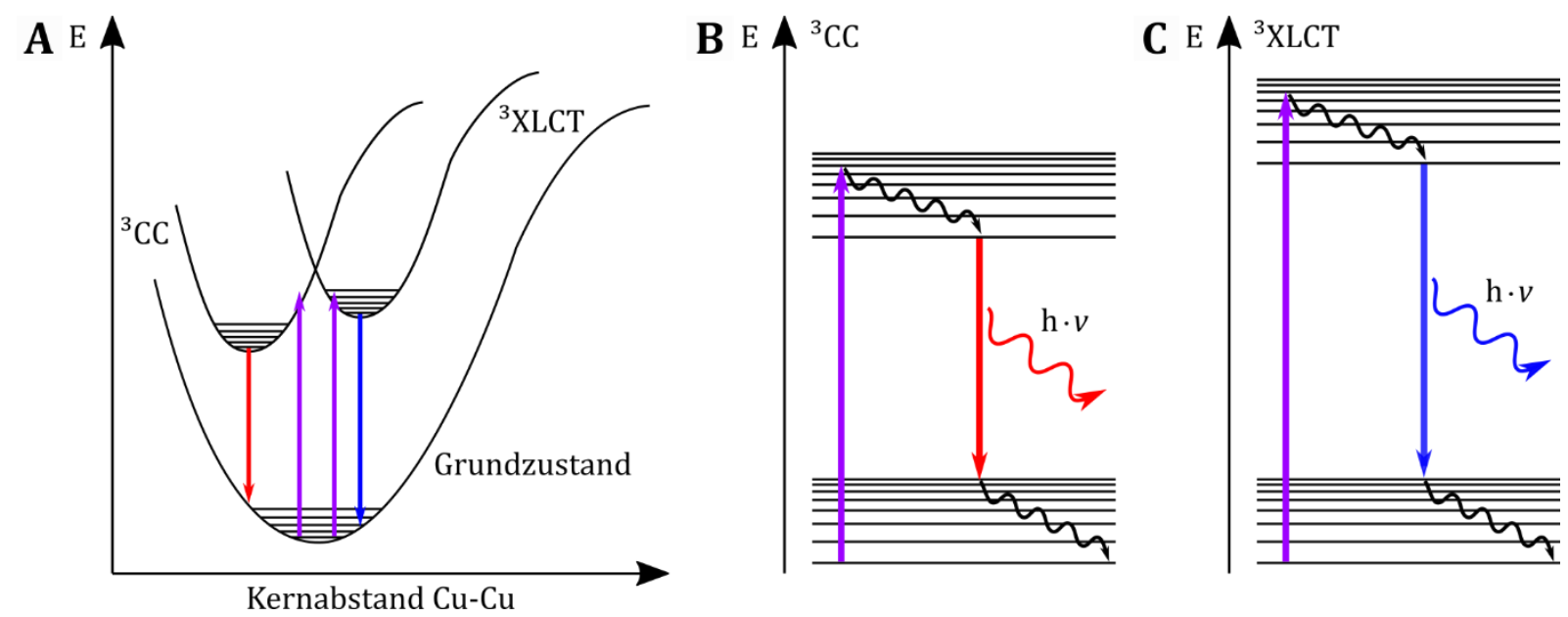

Abbildung 61: (A) Energiediagramm für die möglichen Potentialverläufe der angeregten Zustände ${ }^{3} \mathrm{CC}$ und ${ }_{3}^{3}$ XLCT des Trispyridinkupfer(I)-iodid-Komplexes. (B, C) Darstellung der Anregung in korrespondierende Jabłoński-Diagrammen [265].

Allgemein lässt sich hinsichtlich der fachlichen Deutung des Experiments ein QuenchingEffekt durch die Pyraninmoleküle bezüglich der strahlungslosen Emission des Komplexes bei Raumtemperatur nicht ausschließen, der nach Durchführung des Experiments 5.7b auch rückblickend noch einmal thematisiert werden kann. An dieser Stelle reicht es jedoch, analog zu Experiment 5.2 die auftretende Lumineszenz beim Abkühlen der Lösung auf eine verminderte Schwingungsrelaxation zurückzuführen.

Durch Trocknung bzw. Erwärmung der Lösung kann zusätzlich eine Abspaltung von Pyridinmolekülen aus dem Komplex erreicht werden, die zu farblichen Veränderungen der beobachtbaren Lumineszenz führen. Anhand dieser Beispiele ist eine tiefergehende Auseinandersetzung mit der Thematik der Lumineszenzthermochromie möglich [265].

\section{Experiment 5.4 - Anregung von Fluoreszenzfarbstoffen}

Die Anregung von Fluoreszenzfarbstoffen mit Laserstrahlen verschiedener Wellenlängen ist aufgrund ihrer Reproduzierbarkeit nicht nur im Unterricht, sondern auch auf Bildungsmessen flexibel anwendbar. Jedoch muss in beiden Fällen ein sorgsamer Umgang mit den Laserpointern gewährleistet sein.

Geräte und Chemikalien: 4 Schnappdeckelgläser, UV-Lampe, 3 Laserpointer (violett, grün, rot), 4 Lösungen mit blauer, grüner, gelber und roter Fluoreszenz (z. B. Textmarkerlösungen, Chlorophylllösung) 
Versuchsdurchführung: Vier Lösungen die blau, grün, gelb bzw. rot fluoreszieren werden nacheinander jeweils mit UV-Licht sowie einem violetten, grünen und roten Laserstrahl durchleuchtet.

Beobachtung: Unter UV-Licht fluoreszieren alle Lösungen mit einer bestimmten Farbe. Werden die Lösungen mit einem violetten Laserstrahl durchleuchtet, ist bei allen vier Lösungen entsprechend ihrer Farbe unter UV-Licht der Strahlengang als Fluoreszenz wahrzunehmen. Wird ein grüner Laserstrahl verwendet, ist lediglich bei den Lösungen die gelb und rot fluoreszieren eine Fluoreszenz zu sehen. Unter Bestrahlung mit einem roten Laserstrahl ist in keiner der Lösungen eine Fluoreszenz wahrnehmbar (vgl. Abbildung 62).

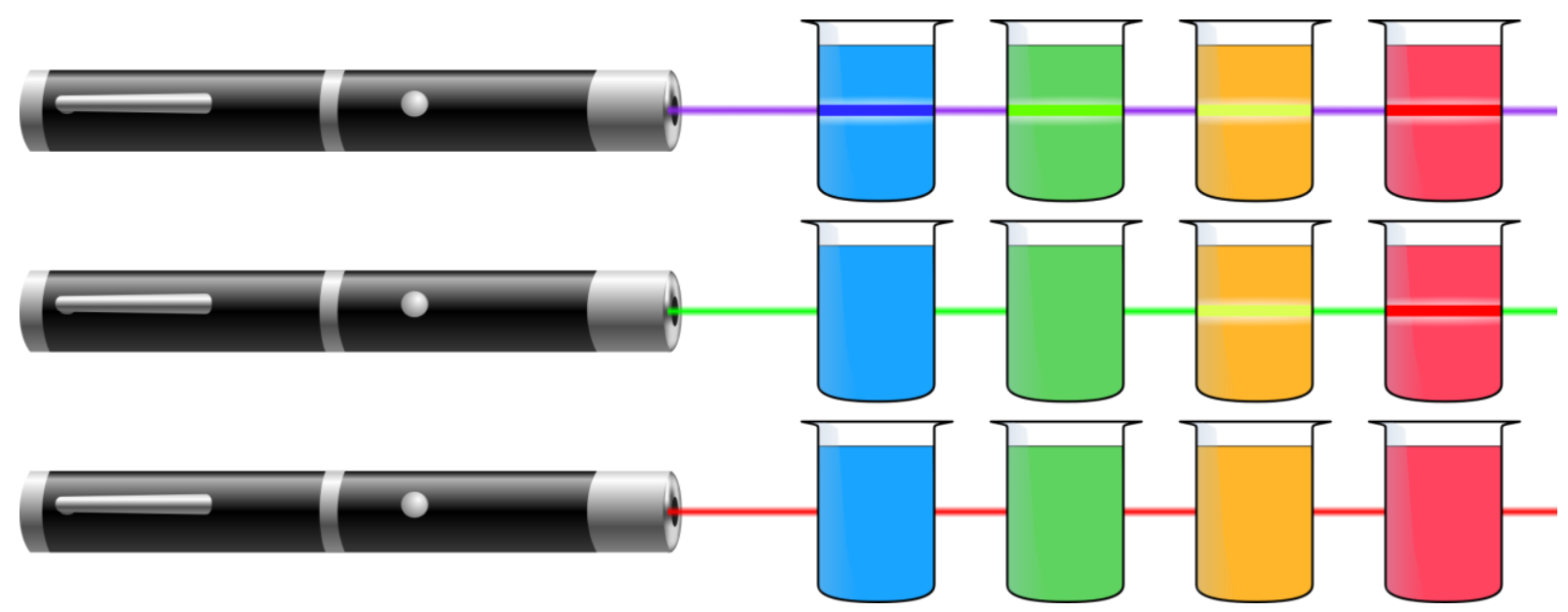

Abbildung 62: Beobachtete Fluoreszenz verschiedenfarbig fluoreszierender Lösungen nach Anregung mit Laserstrahlen verschiedener Wellenlängen.

Deutung: Laser strahlen Licht bestimmter Wellenlängenbereiche aus, wobei die Länge der Wellen vom violetten zum roten Licht zunimmt (siehe Abbildung 63). Gleichzeitig sinkt jedoch der Energiegehalt der Photonen, so dass das violette Licht deutlich energiereicher ist als das rote. Unter UV-Licht fluoreszieren alle Lösungen in einer charakteristischen Farbe und emittieren somit Licht einer bestimmten Wellenlänge. Während der energiereichste Laser (violett) alle Lösungen zur Fluoreszenz anregt, ist der grüne Laserstrahl mittlerer Energie lediglich in der Lage, zwei Lösungen zum Leuchten zu bringen, während beim energieärmsten roten Laserstrahl keine der Lösungen fluoresziert. Da weder der grüne noch der rote Laser in der Lage sind, eine grüne bzw. rote Fluoreszenz hervorzurufen, lässt sich folgern, dass die Energie des eingestrahlten Lichts für die Emission von Fluoreszenzlicht größer sein muss, als das emittierte Licht.

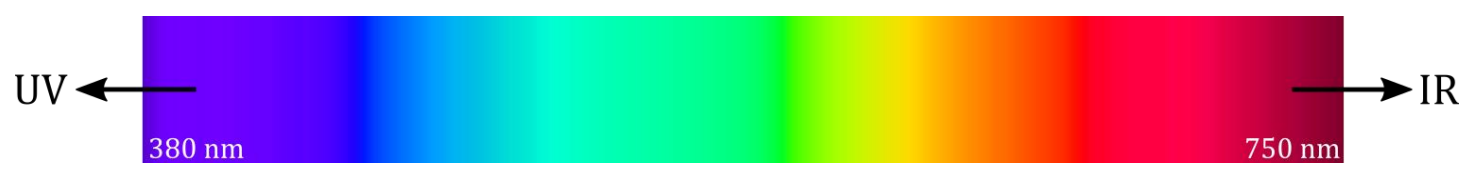

Abbildung 63: Elektromagnetisches Spektrum des sichtbaren Lichts. Links an das Spektrum schließt sich der Bereich der ultravioletten Strahlung an (UV), rechts des Spektrums folgt der Bereich der Infrarotstrahlung (IR).

Anmerkungen zur Deutung des Experiments: Im Gegensatz zu herkömmlichen „weißen“ Lichtquellen strahlen Laser annähernd monochromatisches Licht einer bestimmten Wellenlänge bzw. eines bestimmten Wellenlängenbereichs aus (violett $\sim 405 \mathrm{~nm}$, grün $\sim 532 \mathrm{~nm}$, rot $\sim 650 \mathrm{~nm}$ ). Entsprechend der antiproportionalen Beziehung zwischen der Wellenlänge des Lichts und der Energie eines Photons sinkt mit zunehmender Wellenlänge der Energiegehalt der Photonen, so dass das Licht des violetten Lasers energiereicher als das des roten Lasers ist. Für die Anregung verschiedener Fluorophore bedarf es 
bedingt durch ihre Molekülstruktur eines bestimmten Mindestenergiegehalts. Ähnlich wie bei Molekülen farbiger Substanzen wird nach Absorption einer bestimmten Energiemenge ein Teil der Energie strahlungslos über Translations- und Rotationsbewegungen abgegeben - wobei dieser Anteil gering ist - so dass das emittierte Licht einen geringen Energiegehalt und somit eine größere Wellenlänge als das absorbierte Licht besitzt, was auch als STOKES-Shift bezeichnet wird. Dieser auch als Rotverschiebung bezeichnete Effekt erklärt auch, warum z. B. die Energie eines grünen Laserstrahls nicht ausreicht, um einen im grünen Bereich fluoreszierenden Fluorophor zur Fluoreszenzemission anzuregen.
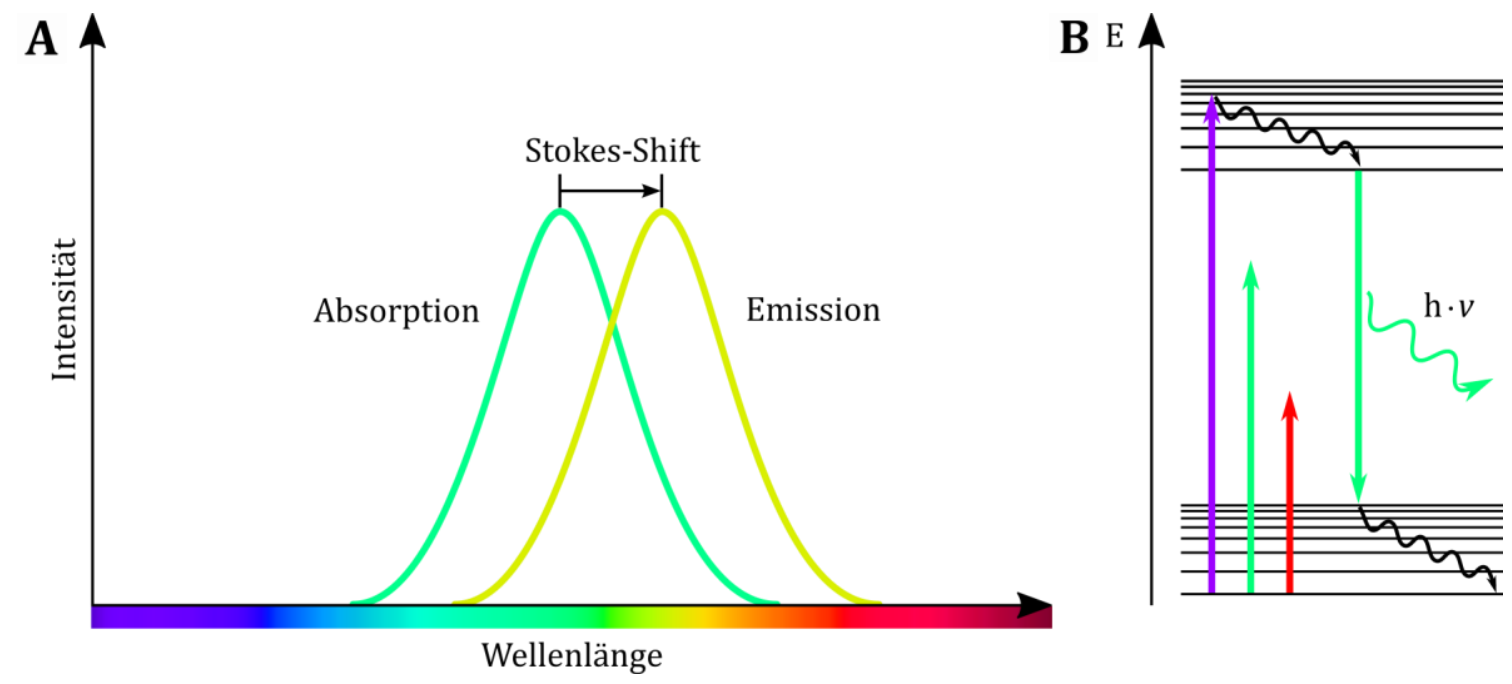

Abbildung 64: (A) Das Emissionsspektrum ist im Vergleich zum Absorptionsspektrum deutlich rotverschoben. (B) Aufgrund des STOKES-Shift reichen die Energien grüner bzw. roter Wellenlängenbereiche nicht aus, um eine grün fluoreszierende Substanz zur Fluoreszenz anzuregen.

\section{Experiment 5.5 - Knicklichter - alternative Anregungsmöglichkeiten von Fluores- zenzfarbstoffen}

Obwohl Knicklichter allseits bekannt sind, ist ihre Funktionsweise den meisten Menschen nicht bewusst. Vor allem auf Bildungsmessen können sie dazu dienen, das situationale Interesse der Besucher_innen zu wecken [268].

Geräte und Chemikalien: Knicklichter, UV-Lampe.

Versuchsdurchführung: In einem abgedunkelten Raum werden ungeknickte Knicklichter mit UV-Licht beleuchtet. Anschließend wird ein Knicklicht ohne UV-Bestrahlung an einer einzelnen Stelle geknickt.

Beobachtung: Auch ohne die Knicklichter zu knicken fluoreszieren sie unter UV-Beleuchtung in verschiedenen Farben. Dabei ist die Fluoreszenz nicht im gesamten Knicklicht zu beobachten, sondern auf ein zweites Röhrchen beschränkt, dass im Inneren des Knicklichts liegt (siehe Abbildung 65A). Beim Knicken bricht das innere Röhrchen sicht- und hörbar und an der Bruchstelle ist Fluoreszenzlicht wahrzunehmen, das sich langsam im Knicklicht verteilt.

Deutung: Knicklichter bestehen aus einem biegsamen Kunststoffröhrchen mit einem darin eingelassenen Glasröhrchen (siehe Abbildung 65B), die eine Wasserstoffperoxidlösung räumlich von einer Oxalsäureesterlösung (z. B. Bis(2,4,6-trichlorphenyl)oxalat) trennen. Zudem enthält eines der Röhrchen einen Fluoreszenzfarbstoff, der je nach gewünschter Leuchtfarbe des Knicklichts variiert. 

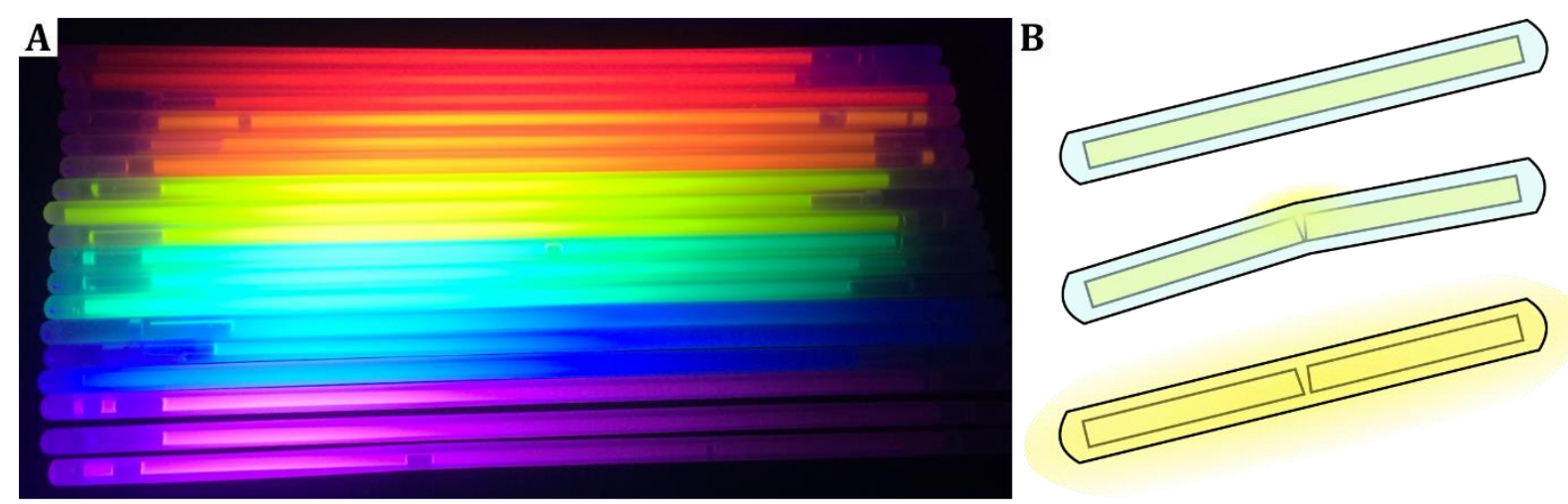

Abbildung 65: (A) Verschiedenfarbige Knicklichter im ungeknickten Zustand unter UV-Licht. (B) Schematische Darstellung der Funktion von Knicklichtern.

Beim Knicken des Knicklichts bricht das Glas und die beiden Flüssigkeiten mischen sich. Das Wasserstoffperoxid reagiert mit dem Oxalsäureester zu 1,2-Dioxetandion, das hochreaktiv ist und zu zwei Kohlenstoffdioxidmolekülen zerfällt (siehe Abbildung 66). Dabei liegt eines der Moleküle in einem angeregten Singulettzustand vor, dass seine Energie auf die in der Mischung vorhandenen Fluoreszenzfarbstoffe übertragen kann. Bei der Abregung der Farbstoffmoleküle wird diese Energie wiederum in Form von Photonen abgegeben, so dass ein Leuchten zu beobachten ist. Der Fluoreszenzfarbstoff liegt nach der Reaktion unverändert vor, so dass er, auch nachdem das Knicklicht nicht mehr leuchtet, von UV-Licht zur Fluoreszenz angeregt werden kann.

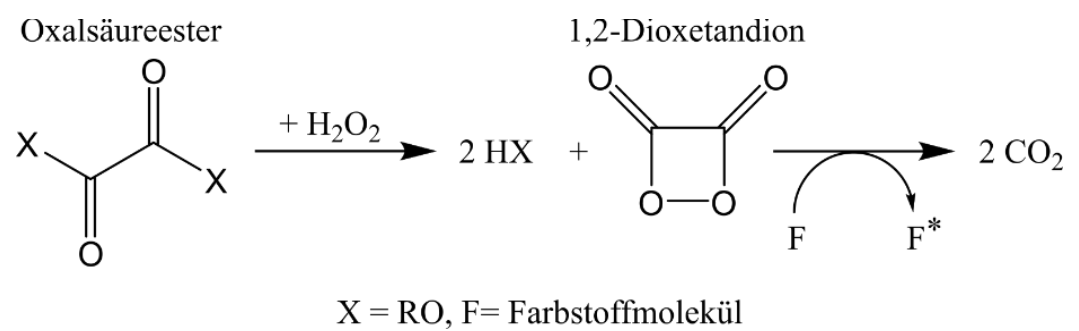

Abbildung 66: Allgemeiner Reaktionsmechanismus für die Reaktion eines Oxalsäureesters mit Wasserstoffperoxid in einem Knicklicht.

Anmerkungen zur Deutung des Experiments: In den bisherigen Experimenten erfolgte eine Anregung von Fluoreszenzfarbstoffen immer mithilfe von energiereichem Licht. Es bestehen jedoch weitere Möglichkeiten, dem System die benötigte Energie zuzuführen. Wird ein Molekül infolge einer chemischen Reaktion zur Emission eines Photons angeregt, so spricht man von Chemolumineszenz. Dabei gibt entweder eines der Reaktionsprodukte, das sich in einem angeregten Zustand befindet, die Energie selbst unter Emission eines Photons ab, oder überträgt sie - wie in diesem Fall - auf einen an der Reaktion unbeteiligten Fluoreszenzfarbstoff [255].

Die Behandlung der Chemilumineszenz bietet sich in vielerlei Hinsicht an. Zum einen kann das Phänomen der Fluoreszenz noch einmal an einem für die SuS bekannten Alltagsgegenstand betrachtet werden. Zudem kann in diesem Zuge auch auf die Relevanz der Chemielumineszenz für biolumineszente Prozesse in der Natur, z. B. bei Tieren und Einzellern, verwiesen werden. Die Verwendung von Knicklichtern bietet sich zusätzlich auch zur Ausbildung eines situationalen Interesses an, das insbesondere bei Bildungsmessen eine wichtige Rolle spielen kann (vgl. Kapitel 6.4). Zudem ist die strahlungslose Energieübertragung von einem Molekül auf ein anderes auch von elementarer Bedeutung für einige der nachfolgenden Experimente. 


\section{Experiment 5.6 - Sensitivität von Fluoreszenzfarbstoffen}

Insbesondere bei Betrachtung verschiedener Forschungsmethoden wie der Fluoreszenzmikroskopie und -spektroskopie, bspw. in Schülerlaboren, eignet sich dieser Versuch, um die Sensitivität von Fluoreszenzfarbstoffen zu verdeutlichen.

Geräte und Chemikalien: 6 Schnappdeckelgläser, fluoreszierende Lösung (z. B. Textmarkerlösung), UV-Lampe, Laserpointer (violett oder grün)

Versuchsdurchführung: Mithilfe einer fluoreszierenden Lösung wird eine Verdünnungsreihe erstellt, indem $1 \mathrm{~mL}$ der farbigen Lösung mit $10 \mathrm{~mL}$ Wasser versetzt wird. Wiederum $1 \mathrm{~mL}$ dieser neuen Lösung wird mit weiteren $10 \mathrm{~mL}$ Wasser versetzt. Auf diese Weise werden 5-6 verdünnte Lösungen erstellt und alle nacheinander mit einem Laserstrahl durchleuchtet.

Beobachtung: Die farbigen Textmarkerlösungen fluoreszieren unter UV-Licht. Die Lösungen der Verdünnungsreihe erscheinen ab der dritten Verdünnungsstufe farblos. Beim Durchstrahlen der Lösungen mit einem Laserpointer ist der Strahlengang als Fluoreszenzlicht wahrnehmbar. Die Fluoreszenz liegt auch bei Farblosigkeit der Lösungen noch vor, verliert aber mit zunehmender Verdünnung an Intensität (Abbildung 67).

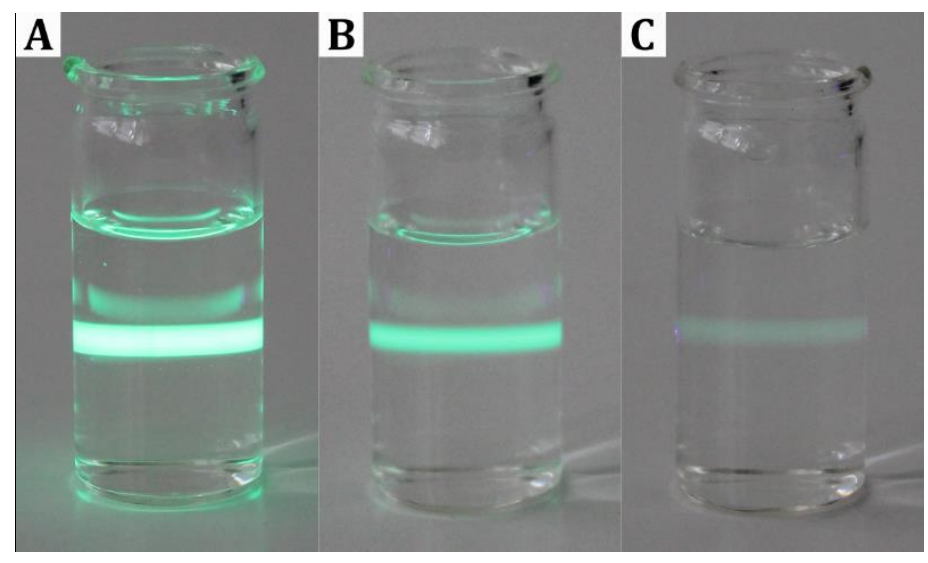

Abbildung 67: Fluoreszenz in vormals farbigen Textmarkerlösungen nach mehrmaliger Verdünnung.

Deutung: Die Farbintensität einer Lösung hängt u. a. von der Konzentration der Farbstoffmoleküle ab, weshalb eine Verdünnung schnell zur makroskopischen Entfärbung der Lösung führt. Fluoreszenzfarbstoffe besitzen eine hohe Sensitivität, weshalb eine Fluoreszenz auch noch bei starker Verdünnung wahrnehmbar ist.

Anmerkungen zur Deutung des Experiments: Die Fluoreszenzintensität eines Fluoreszenzfarbstoffs hängt von seiner jeweiligen Quantenausbeute ab. Diese beschreibt das Verhältnis zwischen der Anzahl absorbierter Photonen und der daraus resultierenden Emissionsintensität und kann beispielsweise durch strahlungslose Übertragung der Energie auf ein anderes Molekül verringert werden (vgl. Experiment 5.7a) [251]. Allgemein unterliegt die wahrgenommene Fluoreszenzintensität einer Lösung, ähnlich wie die Farbintensität, dem Verdünnungseffekt, jedoch ist auch noch bei sehr geringen Fluorophorkonzentrationen, insbesondere bei einer hohen Quantenausbeute, eine Fluoreszenz wahrnehmbar. Diese hohe Sensitivität wird beispielsweise in der Fluoreszenzspektroskopie ausgenutzt (Experiment 6.3b) und ermöglicht es gerade bei den neueren fluoreszenzmikroskopischen Verfahren, auch bei Auflösungen im Bereich weniger Nanometer Strukturen über die Fluoreszenz einiger weniger Moleküle weiter aufklären zu können. 


\section{Experiment 5.7a - Quenching-Effekt}

Der Quenching-Effekt spielt insbesondere in Experiment 6.3b eine wichtige Rolle, weshalb es sich in Verbindung mit diesem Experiment lohnt, den Effekt zunächst experimentell anhand dieses Versuchs zu erarbeiten [269].

Geräte und Chemikalien: 2 Schnappdeckelgläser, Pasteurpipette, Spatel, UV-Lampe, chininhaltige Lösung (z. B. Tonic Water), Natriumchlorid.

Versuchsdurchführung: Wenige Milliliter einer chininhaltigen Lösung werden in zwei Schnappdeckelgläser gefüllt. Unter Bestrahlung mit UV-Licht wird zu einer der Lösungen eine Spatelspitze Natriumchlorid hinzugegeben.

Beobachtung: Beide Lösungen fluoreszieren zunächst. Nach Zugabe des Natriumchlorids $\mathrm{zu}$ einer der Lösungen ist für diese keine Fluoreszenz mehr zu beobachten (Abbildung 68).

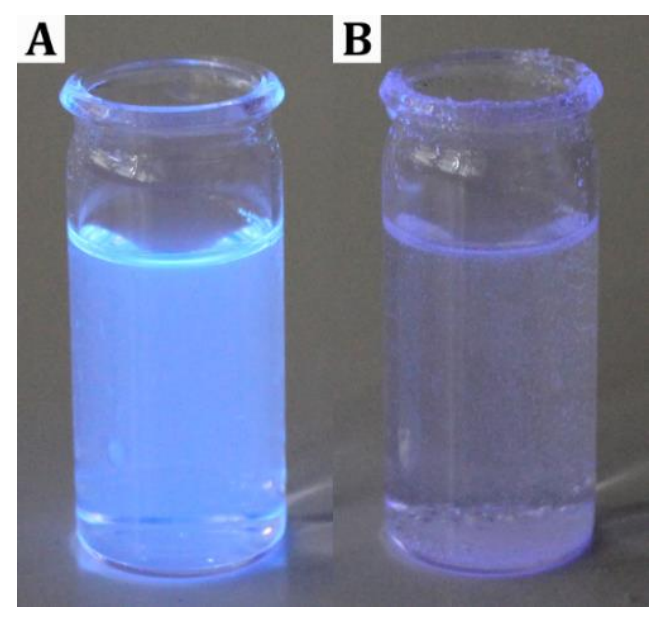

Abbildung 68: Chininhaltige Lösung unter UV-Licht vor (A) und nach Zugabe von Natriumchlorid (B).

Deutung: Zusätzliche Teilchen in Lösung können für eine Löschung (engl:: Quenching) der Fluoreszenz bzw. zu einer Minderung ihrer Intensität führen. In diesem Experiment dienen die Chloridionen als Stoßpartner, auf die die Energie des angeregten Fluorophors übertragen wird. Dieser kehrt anschließend durch strahlungslose Desaktivierung in den Grundzustand zurück.

\section{Experiment 5.7b - Self-quenching von Pyranin}

Auch der Self-quenching-Effekt findet in Experiment 6.3b Anwendung. Entsprechend kann der Effekt zuvor ebenfalls anhand dieses Versuchs erarbeitet werden.

Geräte und Chemikalien: Schnappdeckelglas, Messpipette (5 mL), Spatel, Glasrührstab, demin. Wasser, Pyranin (GHS07).

Versuchsdurchführung: In ein Schnappdeckelglas werden unter UV-Licht nach und nach jeweils wenige Kristalle Pyranin in $1 \mathrm{~mL}$ Wasser gelöst.

Beobachtung: Nach Zugabe erster weniger Kristalle Pyranin zu Wasser fluoresziert die Lösung leicht grün. Die stetige Zugabe weiterer Kristalle sorgt zunächst für eine Intensivierung der Fluoreszenz, die bei weiterer Zugabe wieder abnimmt (Abbildung 69). 


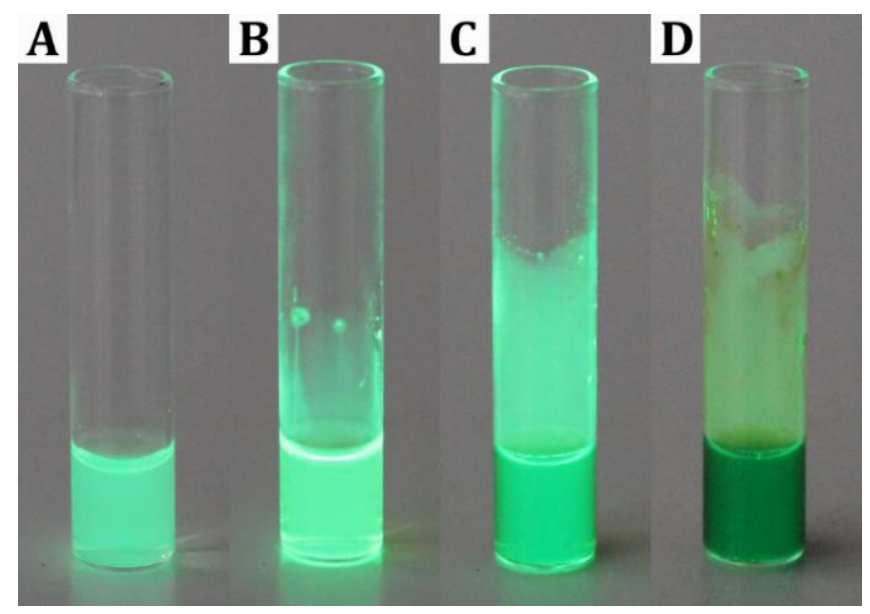

Abbildung 69: Pyraninlösung unter UV-Licht bei sukzessiver Zugabe von Pyraninkristallen.

Deutung: Die Intensität der Fluoreszenz der Pyraninlösung hängt von der Konzentration der Pyraninmoleküle ab. Mit steigender Konzentration steigt zunächst auch die Fluoreszenzintensität, was mit der zunehmenden gleichzeitigen Anregung der Fluorophore erklärt werden kann. Ab einer gewissen Konzentration sinkt jedoch die Intensität wieder. Analog zu Experiment 5.7a kommt es bei steigender Konzentration vermehrt zu Stoßprozessen der Moleküle, bei denen die Energie von einem Fluorophormolekül auf ein anderes übertragen wird. In Abwesenheit von nicht-fluoreszierenden Stoßpartnern wie Halogenidionen kommt es bei Erhöhung der Fluorophorkonzentration zusätzlich zur Ausbildung nicht-fluoreszierender Dimere der Farbstoffmoleküle, die zusätzlich für die Energieübertragung zur Verfügung stehen.

Anmerkungen zur Deutung der Experimente: Das Phänomen des Quenchings von Fluoreszenz wird in der Forschung gezielt angewendet, um bspw. die Fusion von Lipidmembranen [270] (vgl. Experiment 6.3b) oder die Faltung von Proteinen beobachten zu können [271]. Dabei wird ausgenutzt, dass bei räumlicher Nähe von Fluorophoren eine Energieübertragung stattfindet, die für eine Löschung der Fluoreszenz sorgt. Obwohl das Phänomen seit über einem Jahrhundert untersucht wird, konnte der dahinterliegende Mechanismus noch immer nicht gänzlich geklärt werden. In der Literatur lassen sich einige Theorien finden, die auf ein Zusammenspiel verschiedener physikalischer Effekte hindeuten. Während die Theorie der Energieübertragung auf nicht-fluorophore Stoßpartner für die Deutung des Versuchs 5.7a in schulischen Kontexten ausreichend ist, vermag diese nicht zufriedenstellend zu erklären, wieso Pyraninmoleküle bei hoher Konzentration dem Self-quenching-Effekt unterliegen. Eine mögliche Erklärung dieses Phänomens ist die Bildung nicht-fluoreszierender Dimere, die mit einer Veränderung des Energiestufenprofils einhergeht ${ }^{[272]}$. Anhand dieser Theorie kann die Energieübertragung beider Versuchsteile im Sinne einer didaktischen Reduktion auf das Energiestufendiagramm in Abbildung $70 \mathrm{~B}$ reduziert werden. 


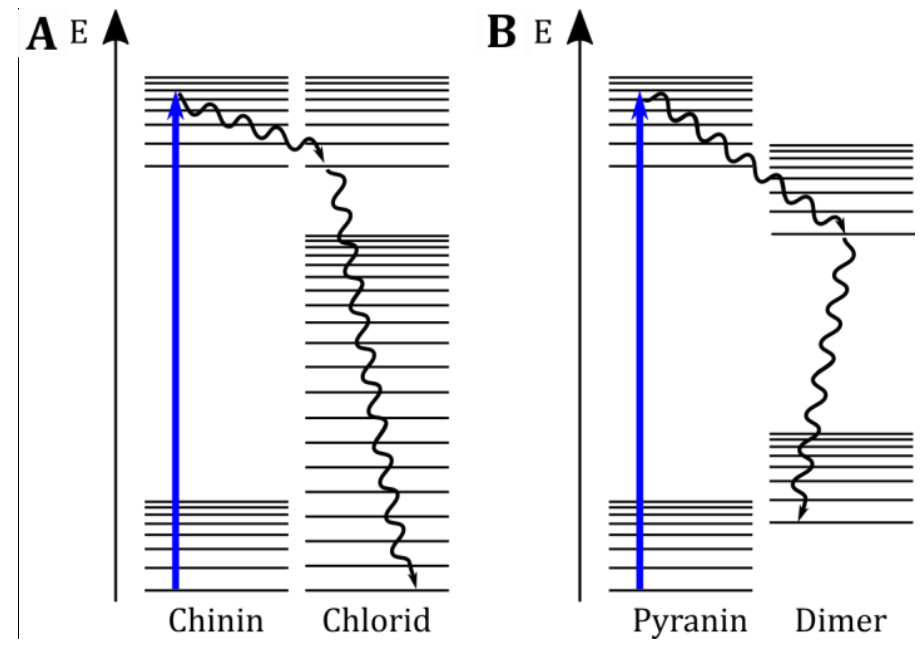

Abbildung 70: (A) JABŁOŃSKI-Diagramm für die strahlungslose Energieübertagung auf einen Stoßpartner mit unterschiedlichem Energieprofil. (B) Veranschaulichung einer möglichen Erklärung für das Phänomen des Self-quenching-Effekts.

\section{Experiment 5.8 - Förster-Resonanztransfer (FRET)}

FRET-Effekte werden in der Forschung zum Teil explizit ausgenutzt, an anderer Stelle sollen sie vermieden werden. Unter anderem spielt er auch in der Adaptierten Primärliteratur aus Kapitel 6.3.2 eine Rolle, weshalb dieser Versuch beispielsweise im Rahmen der Erarbeitung dieses Artikels im Unterricht oder im Schülerlabor eingesetzt werden kann.

Geräte und Chemikalien: 3 Schnappdeckelgläser, Laserpointer (violett, grün), 2 Textmarkerlösungen (grüne und gelbe Fluoreszenz).

Versuchsdurchführung: In zwei Schnappdeckelgläser werden je eine grün bzw. gelb fluoreszierende Textmarkerlösung nacheinander mit einem violetten und einem grünen Laserstrahl durchleuchtet. Anschließend wird jeweils eine Hälfte der beiden Lösungen in ein drittes Schnappdeckelglas überführt. Das Gemisch wird ebenfalls nacheinander mit dem Licht der zwei Laser durchstrahlt.

Beobachtung: Die Fluoreszenz der gelb fluoreszierenden Lösung ist sowohl bei Bestrahlung mit einem violetten als auch einem grünen Laserstrahl zu beobachten. Die Fluoreszenz der grün fluoreszierenden Lösung ist hingegen nur bei Bestrahlung mittels des violetten Laserstrahls zu sehen. Das Gemisch zeigt beim Durchleuchten mit beiden Lasern jeweils eine gelbe Fluoreszenz (Abbildung 71).

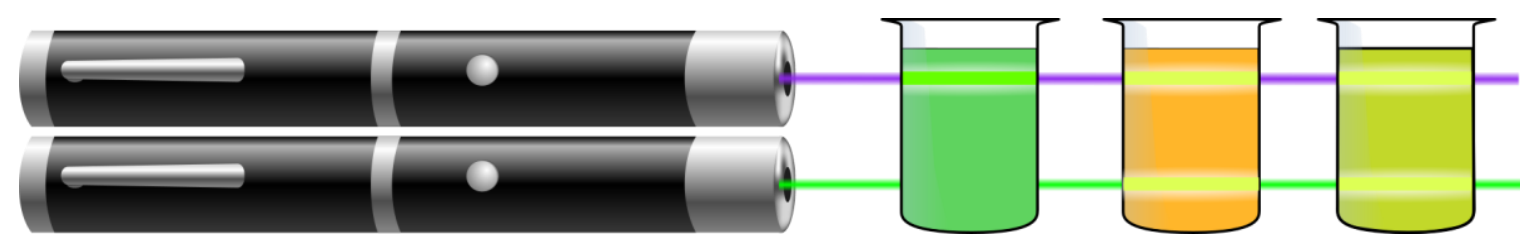

Abbildung 71: Beobachtete Fluoreszenz bei Anregung einer grün fluoreszierenden (links), einer gelb fluoreszierenden (Mitte) und einer Mischung aus den ersten beiden Lösungen (rechts) mit einem violetten und einem grünen Laserstrahl.

Deutung: Das Licht des violetten Lasers ist energiereich genug, um die Moleküle der beiden Lösungen sowohl zur grünen als auch zur gelben Fluoreszenzemission anzuregen. Die Energie des grünen Laserstrahls reicht hingegen lediglich aus, um den gelb emittierenden Farbstoff anzuregen. Werden beide Lösungen gemischt, liegen beide Farbstoffe nebeneinander in Lösung vor. Das Licht des grünen Lasers wird weiterhin nur vom gelb emittie- 
renden Fluoreszenzfarbstoff absorbiert. Mithilfe des violetten Laserstrahls können hingegen beide Farbstoffe angeregt werden. Überlappt dabei das Emissionsspektrum des einen Fluorophors (grüne Emission, energiereicher) mit dem Absorptionsspektrum des anderen Fluorophors, dann wird die Emissionsenergie auf diesen übertragen, so dass die energieärmere Fluoreszenzfarbe wahrnehmbar ist (vgl. Abbildung 72). Vereinfacht kann die Emission dabei als Aneinanderreihung von STOKES-Verschiebungen verstanden werden.

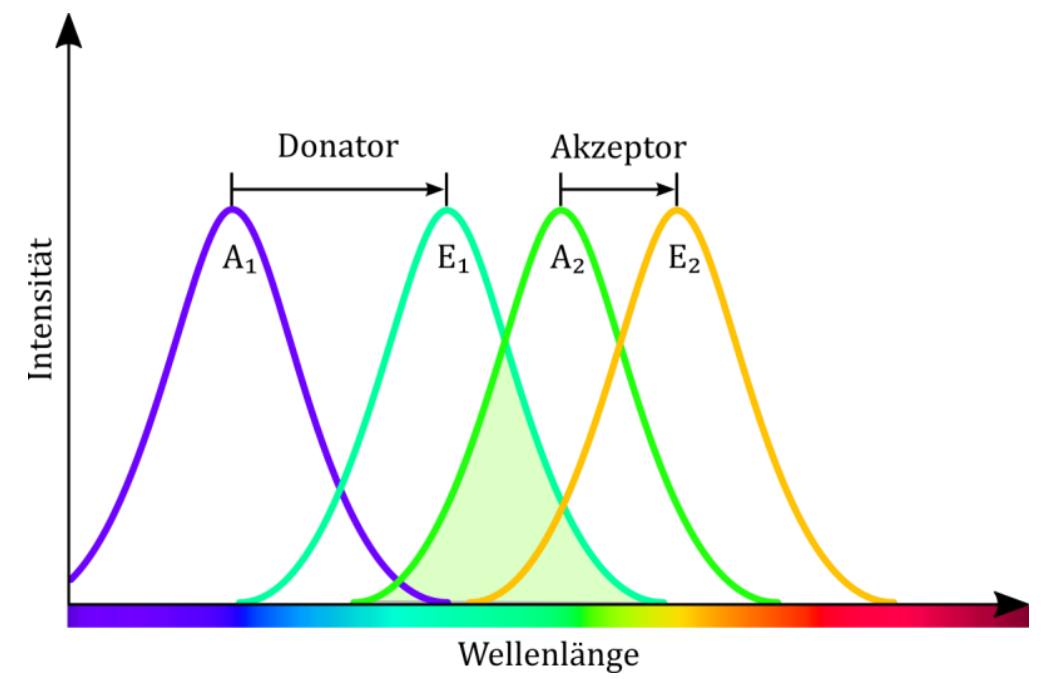

Abbildung 72: Das Emissionsspektrum des ersten Farbstoffs ( $E_{1}$ ) überlappt mit dem Absorptionsspektrum des zweiten Farbstoffs $\left(A_{2}\right)$. Dies ermöglicht eine Energieübertragung vom ersten auf den zweiten Farbstoff, so dass es bei der Farbstoffmischung bei Anregung mit beiden Lasern zu einer gelben Fluoreszenz kommt.

Anmerkungen zur Deutung des Experiments: Das Modellexperiment zum FöRSTER-Resonanztransfer (FRET) ermöglicht es, die strahlungslose Energieübertragung von einem angeregten Farbstoff auf einen zweiten Farbstoff phänomenologisch zu betrachten. Eine strahlungslose Energieübertragung nach FÖRSTER [273] ist dann möglich, wenn sich zwei unterschiedliche Farbstoffmoleküle in einem Abstand von ca. 5 bis $10 \mathrm{~nm}$ befinden und das Emissionsspektrum des Donatorfarbstoffs mit dem Absorptionsspektrum des Akzeptorfarbstoffs überlappt [255]. Ist dies der Fall, wird die Energie des angeregten Donators basierend auf Dipol-Dipol-Wechselwirkungen strahlungslos an das Akzeptormolekül abgegeben, welches dadurch in einen angeregten Zustand versetzt wird. Unter Abgabe eines Photons kehrt der Akzeptorfarbstoff schließlich in seinen Grundzustand zurück [274]. Der FRET-Effekt kann sowohl mithilfe von vereinfachten JABŁOŃSKI-Diagrammen veranschaulicht werden (Abbildung 73) als auch anhand des Vergleichs von Emissionsspektren der Farbstoffmischungen (vgl. Abbildung 74) verdeutlicht werden.

Die Bedingung, dass sich die Farbstoffmoleküle für einen FRET bis auf wenige Nanometer nahekommen müssen, wird auch in der Membranforschung ausgenutzt, um bspw. Proteine in einer Membran zu lokalisieren und Protein-Protein-Interaktionen abzubilden [275, 276]. Ein ungewolltes Auftreten des FRET-Effekts kann jedoch auch dazu führen, dass Fluoreszenzfarbstoffe für bestimmte experimentelle Arbeiten nicht geeignet sind, wie es in der in Kapitel 6.3.2 vorgestellten Adaptierten Primärliteratur zur Synthese von Glycosphingolipiden mit fluoreszierenden Fettsäuren der Fall ist. Auch die Natur nutzt zudem den FRET-Effekt, um den Prozess der Photosynthese effektiver zu gestalten. Dazu absorbieren Pigmente des Lichtsammelkomplexes die Energie einfallenden Lichts und transferieren diese z. T. mehrere hundert Ångström weit zum Reaktionszentrum [277]. 

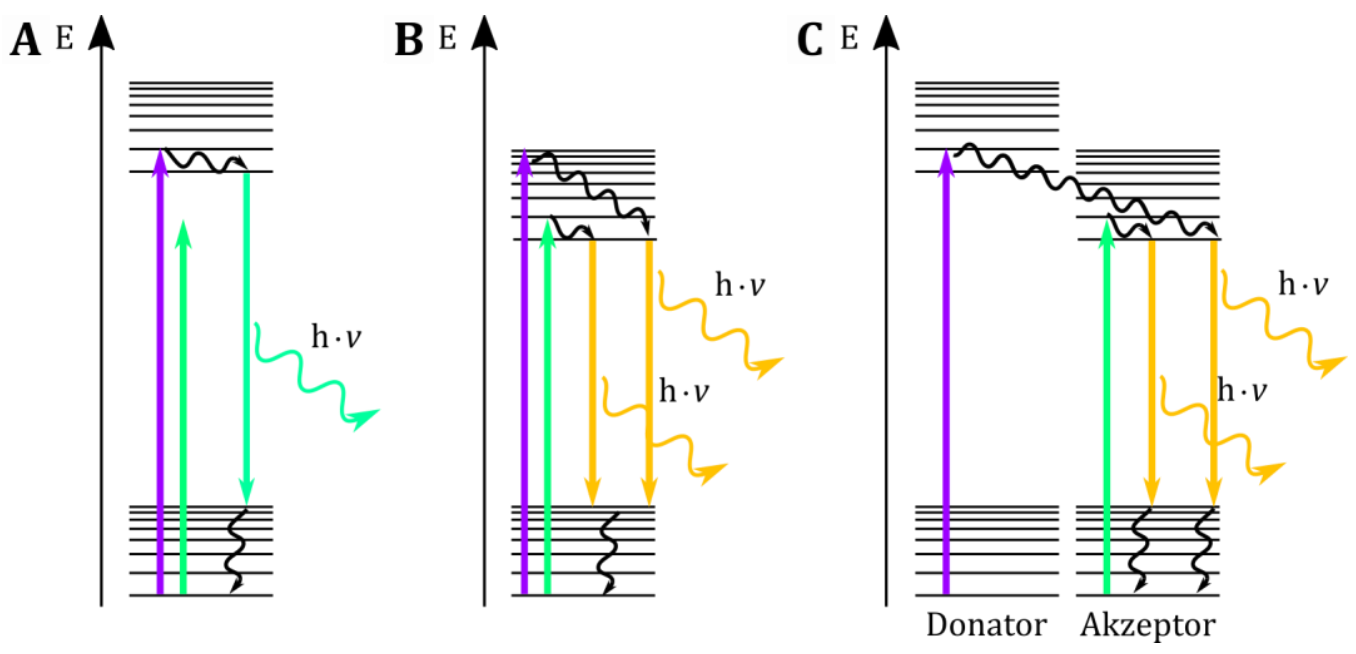

Abbildung 73: Darstellung des FRET-Effektes im JABŁOŃSKI-Diagramm. (A) der grün fluoreszierende Farbstoff kann nur mithilfe des violetten Lasers zur Fluoreszenz angeregt werden. (B) Der gelb fluoreszierende Farbstoff kann von beiden Lasern zu Fluoreszenz angeregt werden. (C) Bei Anregung der Farbmischung mit einem violetten Laser kommt es zur Energieübertragung auf das zweite Fluorophor, so dass eine Emission gelben Lichts folgt.

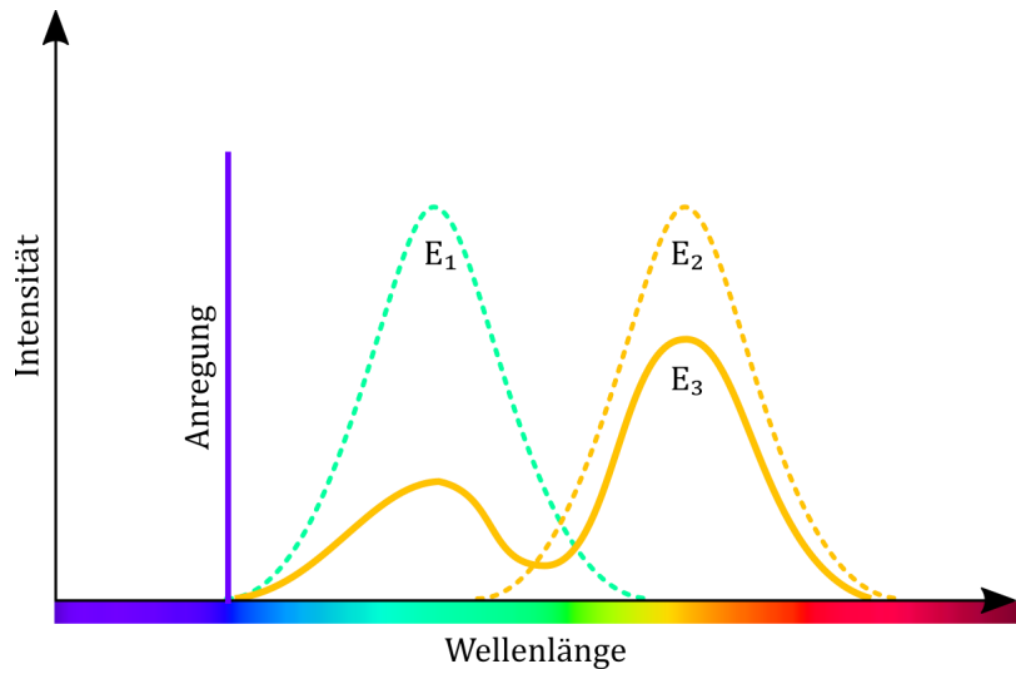

Abbildung 74: Emissionsspektren der Farbstoffmischungen nach Anregung mit einem violetten Laser nach HochreITER ET AL. [278]. $E_{1}$ und $E_{2}$ sind theoretische Emissionsspektren für die einzelnen Farbstoffe. $E_{3}$ verdeutlicht den FRET-Effekt. Das Integral von $\mathrm{E}_{3}$ entspricht dem von $\mathrm{E}_{1}$, der Peak bei $\mathrm{E}_{1}$ ist jedoch abgeschwächt, dafür bei $\mathrm{E}_{2}$ deutlich höher.

\section{Einordnung der Experimente im Kontext der SO-Leitfragen}

Die Betrachtung des Themas der Fluoreszenz bedarf zunächst einer Einführung des Begriffs der Farbigkeit. Hierbei ist es sinnvoll, die zum Teil komplizierten physikalischen Hintergründe auf wenige Konzepte zu reduzieren und diese mithilfe einfacher JABŁOŃSKIDiagramme soweit wie möglich zu visualisieren. Entsprechend werden die Farb- und Fluoreszenzerscheinungen der hier vorgestellten Experimente hinsichtlich der Deutung allesamt auf Systeme konjugierter Doppelbindungen zurückgeführt.

Dass ein ausgedehntes delokalisiertes $\pi$-Elektronensystem eine Grundbedingung für die Farbigkeit von Substanzen darstellt, kann von SuS anhand des Blue Bottle-Experiments erarbeitet werden. Um den entstehenden Farbeindruck erklären zu können, sollten in diesem Zuge die Begriffe der Absorption und Emission sowie der subtraktiven und additiven Farbmischung wiederholt werden, die bereits aus dem Physikunterricht bekannt sein 
sollten. Anhand der zwei sich anschließenden Experimente strukturellen und zur Temperaturabhängigkeit der Fluoreszenz kann darauf geschlossen werden, dass die verminderte Fähigkeit der Moleküle zur Rotation und Schwingung Einfluss auf die Art der Emission hat. Sowohl das Phänomen der Farbigkeit, als auch das der Fluoreszenz werden also auf einfache Struktur-Eigenschaftsbeziehungen reduziert, für die kein tiefgründiges Vorwissen nötig ist.

Der Versuch zur Anregung von Fluoreszenzfarbstoffen ermöglicht es SuS unter Einbezug des elektromagnetischen Spektrums Aussagen über den Energiegehalt von Licht unterschiedlicher Wellenlängen zu treffen. Dabei wird zunächst die Zufuhr von Energie in Form von Licht als eine wichtige Bedingung für die Fluoreszenz erkannt. Anhand des Experiments zu alternativen Anregungsmöglichkeiten kann anschließend gezeigt werden, dass die Energiezufuhr zum System nicht notwendigerweise auf elektromagnetische Strahlung beschränkt ist. In diesem Zug sollte auch auf die Gründe für die unterschiedlichen Energiegehalte von Absorption und Emission eingegangen werden, anhand derer die Rotverschiebung (Stokes-Shift) erklärt werden kann.

Aufbauend auf diesen grundlegenden Erkenntnissen zur Fluoreszenz können in den nachfolgenden Experimenten verschiedene Eigenschaften erarbeitet werden, die sich in der Membranforschung zunutze gemacht werden. Diesbezüglich ermöglicht es die hohe Sensitivität von Fluoreszenzfarbstoffen, dass insbesondere mithilfe neuerer Techniken wie der STED- und der MinFlux-Mikroskopie Strukturen auch deutlich unterhalb des ABBELimits abgebildet und unterschieden werden können. Effekte wie das Self-quenching und Dequenching von Fluoreszenz sowie der FRET werden zudem gezielt eingesetzt, um beispielsweise die Fusion von Vesikeln beobachten oder Aussagen bezüglich der Interaktion von Proteinen und Lipiden treffen zu können [239, 278, 279]. Beide Effekte können dabei von den SuS anhand des Experiments 6.3b oder der Adaptieren Primärliteratur aus Kapitel 6.3.2 noch einmal im Kontext der Forschung des SFB vertiefend behandelt werden.

\subsection{Themenfeld 6 - Untersuchungsmethoden in der Membranfor- schung}

Für die Untersuchung der Eigenschaften und Funktionen von Membranen und ihren Bestandteilen werden Modellmembransysteme mit definierten Lipid- und Proteinzusammensetzungen genutzt, um anschließend anhand der Erkenntnisse Vorhersagen über komplexere Systeme wie Zellen treffen zu können [280]. Es existieren verschiedene Modellmembrantypen, die je nach Forschungsschwerpunkt variiert werden können. Übergeordnet können diese hinsichtlich kugelförmiger und planarer Membransysteme differenziert werden ${ }^{[281] . ~ Z u ~ d e n ~ k u g e l f o ̈ r m i g e n ~ M o d e l l m e m b r a n e n ~ g e h o ̈ r e n ~ d i e ~ V e s i k e l, ~ d i e ~ b a s i e r e n d ~}$ auf ihrer Größe in Small Unilamellar Vesicles (SUV, < $100 \mathrm{~nm}$ ), Large Unilamellar Vesicles (LUV, $100 \mathrm{~nm}-1 \mu \mathrm{m}$ ) und Giant Unilamellar Vesicles (GUV, > $1 \mu \mathrm{m}$ ) eingeteilt werden. Während synaptische Vesikel bspw. im Größenbereich der SUVs liegen [282], werden aufgrund ihrer optisch gut auflösbaren Größe bevorzugt GUVs als Modellmembranen verwendet. Zur Herstellung der GUVs werden zumeist zwei Techniken verwendet - das Quellen und die Elektroformation - zu denen wiederum verschiedene methodische Ansätze existieren [281, 283, 284].

Prinzipiell eignen sich Vesikel zwar, um z. B. Transportprozesse oder die Verteilung verschiedener Lipide und Proteine über die Membran hinweg zu bestimmen, jedoch liegen sie, im Gegensatz zu biologischen Membranen, die u. a. über die Anbindung an das Cytoskelett eine Spannung erfahren, eher ungespannt vor [280]. Abhilfe schaffen hier planare Membransysteme, die in verschiedenen Variationen existieren und jeweils ihre eigenen 
Vor- und Nachteile mit sich bringen. Bei festkörperunterstützen Membranen werden Lipidschichten aus Substraten wie Glas, Indiumzinnoxid (Indium Tin Oxide, ITO) oder Gold präpariert. Sie besitzen eine hohe Langzeit- und mechanische Stabilität, jedoch ist das Eintragen von Proteinen und Peptiden in die Membranen aufgrund der Nähe der Lipiddoppelschicht zum Substrat schwierig.

Bei sogenannten schwarzen Membranen, die über ein kleines Loch in einer Plastikwand gespannt sind und dadurch zwei wässrige Kompartimente voneinander trennen, sind beide Seiten der Lipiddoppelschicht zugänglich. Jedoch ist hier die Stabilität der Membran deutlich geringer als bei den festkörperüberspannenden Membranen. Eine Alternative, die die Vorteile beider Methoden verbindet und auch im SFB 803 oft Anwendung findet, sind die porenüberspannenden Membranen (siehe Abbildung 75) [285-287]. Hierbei werden Lipiddoppelschichten auf poröse Substrate aufgebracht, die Porendurchmesser von $50 \mathrm{~nm}$ bis $5 \mu \mathrm{m}$ aufweisen. Das Substrat zwischen den Poren sorgt dabei für die Stabilität der Membran, während die Lipiddoppelschicht über den Poren an zwei wässrige Kompartimente grenzt [216, 280,288].
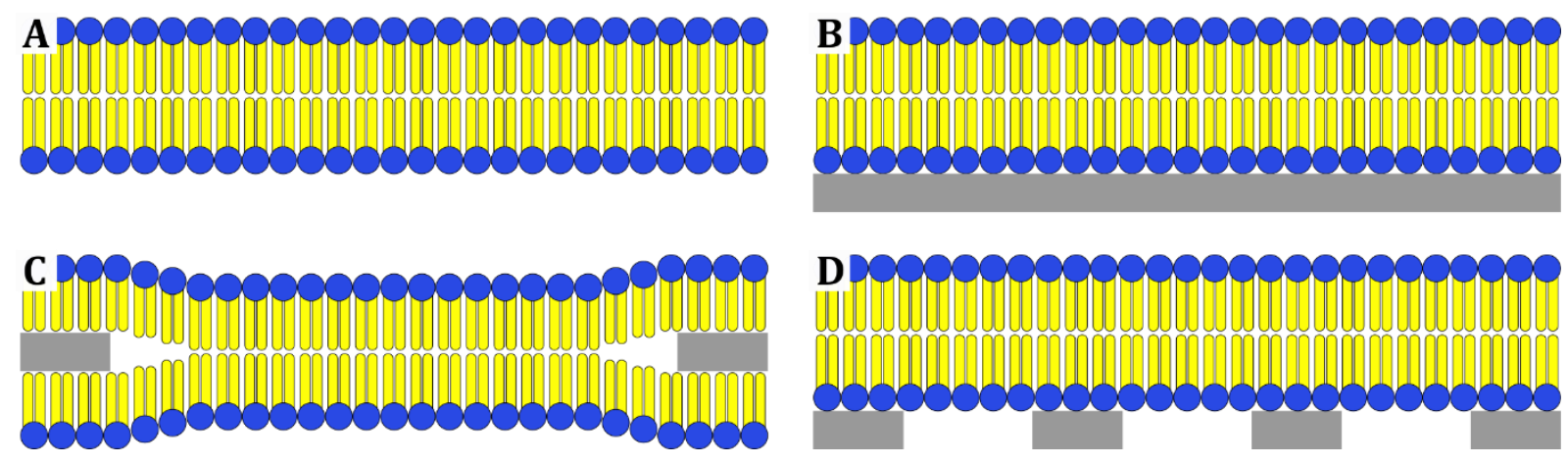

Abbildung 75: (A) freistehende Membran, (B) festkörperunterstützte Membran, (C) schwarze Membran, (D) porenüberspannende Membran (nach STEIN ET AL. [281]).

Untersucht werden diese künstlichen Membranen mit verschiedenen Methoden, wie u. a. der Fluoreszenzmikroskopie und -spektroskopie, der Patch-Clamp-Technik, Rasterkraftmikroskopie (AFM), Kernspinresonanzspektroskopie (NMR) oder Molekulardynamischen Simulationen [174]. Nachfolgenden werden sowohl Experimente zur Herstellung von Vesikeln, als auch zur fluoreszenzmikroskopischen und -spektroskopischen Untersuchung dieser vorgestellt.

\section{Experiment 6.1a - Lipidextraktion aus Eigelb - Isolation von Lecithin}

Dieser Versuch ermöglicht die Extraktion von Lipiden aus Eigelb, die zur Herstellung von Vesikeln in Experiment 6.1b und 6.1c verwendet werden können. Aufgrund des hohen Zeitaufwands eignet sich die Extraktion jedoch lediglich für die Anwendung in Schülerlaboren $[175,289]$.

Geräte und Chemikalien: 2 Bechergläser, 4 Reagenzgläser, Reagenzglasständer, Schnappdeckelglas, Glasrührstab, Standzylinder (10 mL), Trichter mit Filter, Filtriergestell, Stativmaterial, Wasserbad, Heizplatte, Thermometer, Ethanol (GHS02, GHS07), n-Heptan (GHS02, GHS07, GHS08, GHS09), Aceton (GHS02, GHS07), Stickstoffgas (GHS 04).

Versuchsdurchführung: Unter dem Abzug wird Eigelb (vom Eiklar getrennt) in einem Becherglas mit $10 \mathrm{~mL}$ Ethanol versetzt und beides mit einem Glasstab homogenisiert (siehe Abbildung 76A). Das Gemisch wird mit 12,5 mL n-Heptan versetzt und der entstehende Niederschlag abfiltriert. Das Filtrat wird in einem $30{ }^{\circ} \mathrm{C}$ warmen Wasserbad eingedampft, bis sich eine Emulsion bildet. Die entstandene Emulsion wird mit $5 \mathrm{~mL}$ Aceton versetzt, gut durchmischt und das überstehende Aceton abgegossen. Der Vorgang wird mit dem 
Rückstand drei Mal wiederholt. Anschließend wird der Rückstand in 5 mL n-Heptan aufgenommen und kann so in einem Schnappdeckelglas kühl gelagert werden. Für die weitere Verwendung kann das Lösungsmittel abgedampft werden

Beobachtung: Nach Zugabe des n-Heptans fällt ein Feststoff aus. Sowohl das Filtrat als auch das Acetongemisch besitzen eine gelbe Farbe. Nach dem Abdampfen des n-Heptans bleibt ein wachsartiger, hellgelber Feststoff zurück (Abbildung 76).

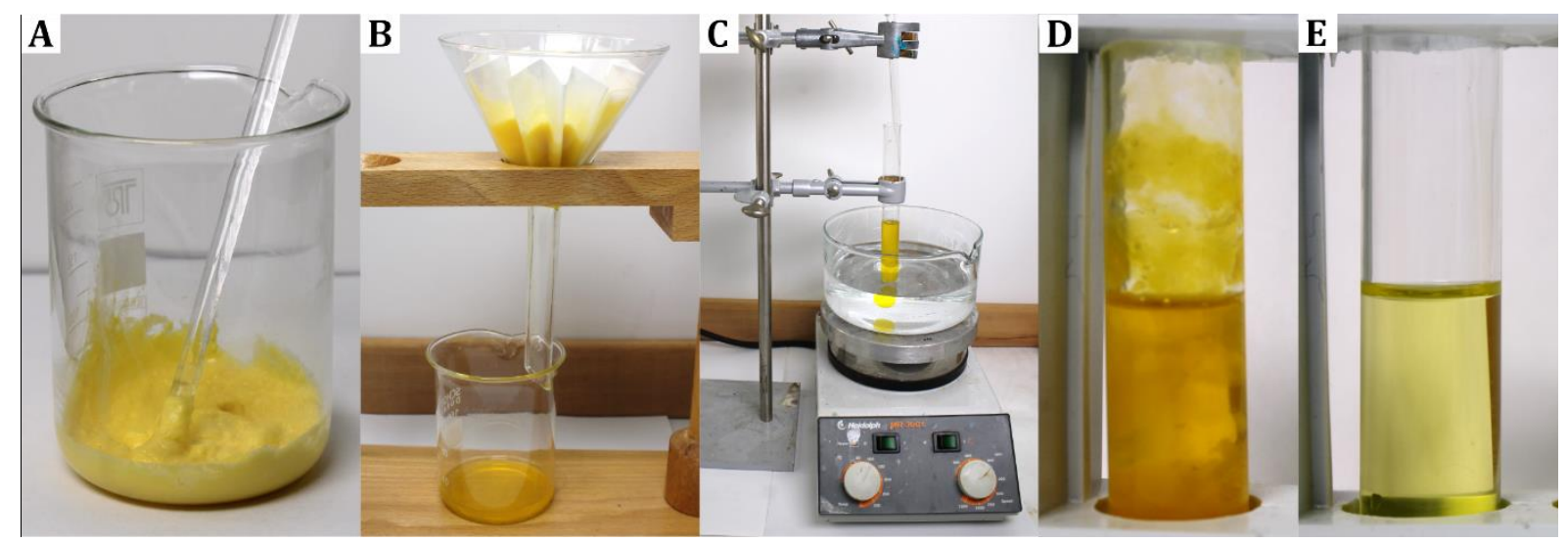

Abbildung 76: Eigelb wird zunächst mit Ethanol homogenisiert (A). Nach Zugabe von n-Heptan wird abfiltriert (B) und das Lösemittel eingedampft (C). Die entstandene Emulsion (D) nach dreimaligen Waschen mit Aceton (E).

Deutung: Bei Zugabe des n-Heptans fallen die in unpolaren Lösungsmitteln unlöslichen Bestandteile des Eigelbs, wie Proteine und Kohlenhydrate, aus. Dahingegen lösen sich Lecithin und andere Lipide in n-Heptan. Durch Zugabe von Aceton werden Fette sowie die Farbstoffe des Eigelbs gelöst, wobei Lecithin als Feststoff ausfällt. Die gewonnenen Lecithine gehören zur Gruppe der Phosphatidylcholine (vgl. Abbildung 13, S. 39), die zu den Phospholipiden gehören und in tierischen und pflanzlichen Zellmembranen vorkommen.

\section{Experiment 6.1b - Herstellung von Lipidfilmen durch Quellen}

Bei dieser für SuS adaptierten Forschungsmethode zur Herstellung von Vesikeln können entweder die Lipide aus Experiment 6.1 a oder käuflich erworbenes Egg PC verwendet werden. Eine Durchführung der Synthese ist grundsätzlich auch im Unterricht denkbar, eignet sich aufgrund der hohen Wartezeiten jedoch eher für ein Schülerlabor [175, 283].

Geräte und Chemikalien: 3 Bechergläser, Reagenzglas, Reagenzglasständer, Glasrührstab, Stativmaterial, Pasteurpipette, Kolbenhubpipette, Wasserbad, Heizplatte, Thermometer, Objektträger mit Vertiefung, Licht- oder Fluoreszenzmikroskop, demin. Wasser, n-Heptan (GHS02, GHS07, GHS08, GHS09), Lecithin (Experiment 6.1a oder Egg PC), Saccharoselösung (1 M), Glucoselösung (1 M), Texas Red DHPE (1,2-Dihexadecanoyl-sn-Glycero-3Phosphoethanolamin; optional).

Versuchsdurchführung: Unter dem Abzug werden in einem Becherglas 0,2 g Lecithin in $3 \mathrm{~mL}$-Heptan gelöst. 2,75 mL der Lösung werden in ein Reagenzglas überführt und in einem Wasserbad langsam auf $30{ }^{\circ} \mathrm{C}$ erhitzt. Mithilfe einer Pasteurpipette wird vorsichtig ein Stickstoffgasstrom auf die Lösung gerichtet, um das Lösungsmittel zu verdampfen. Nach dem Verblasen des Lösungsmittels werden 5 mL Saccharoselösung in das Reagenzglas gegeben und dieses für 60 Minuten ruhen gelassen. Daraufhin werden $15 \mu \mathrm{L}$ Glucoselösung in die Vertiefung eines Objektträgers gegeben und weitere $15 \mu \mathrm{L}$ der Lecithinlösung hinzupipettiert. Anschließend wird die Probe unter dem Mikroskop betrachtet. 
Optional: Für die Fluoreszenzmarkierung des Lipidfilms kann eine 0,7 M Texas Red-Lösung in n-Heptan hergestellt und $38 \mu \mathrm{L}$ der Texas Red-Lösung zu Beginn mit den 2,75 mL der Lecithinlösung gemischt werden. Anschließend wird in gleicher Weise wie mit der reinen Lecithinlösung verfahren und die Probe schließlich unter einem Fluoreszenzmikroskop betrachtet.

Beobachtung: Im Falle der Zugabe von Texas Red-Lösung bleibt nach dem Verdampfen des Lösungsmittels ein leicht rötlicher Feststoff an der Gefäßwand zurück. Der Rückstand löst sich nach Zugabe der Saccharoselösung vom Gefäßrand ab und die Lösung trübt sich leicht. Unter dem Mikroskop sind kleine, blasenartige runde Strukturen zu erkennen, die bei Anregung mit grünem Licht rot fluoreszieren (siehe Abbildung 77).

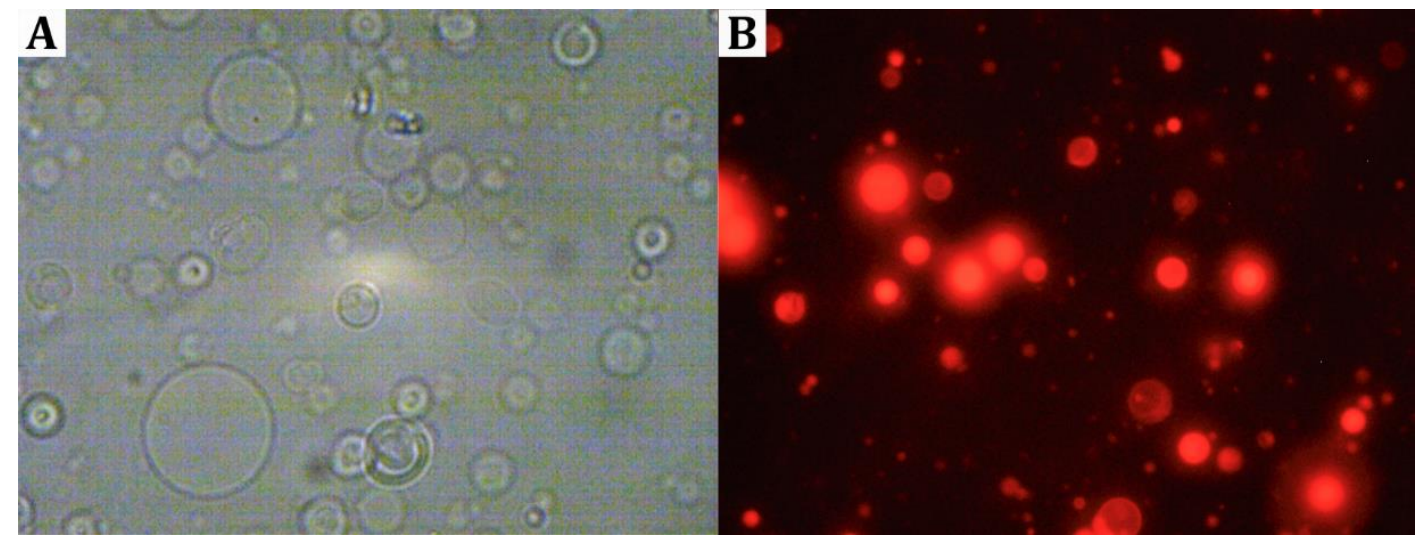

Abbildung 77: (A) Durchlichtmikroskopische Aufnahmen von Vesikeln. (B) Fluoreszenzmikroskopische Aufnahmen Texas Red-markierter Vesikel.

Deutung: Lecithin löst sich in n-Heptan und bei Zugabe von Texas Red verteilt sich dieses homogen in der Lösung. Nach dem Verblasen des Lösungsmittels bleiben geordnete Lipidfilme an der Reagenzglaswand zurück. Bei Zugabe der Saccharoselösung lösen sich einzelne Lipidschichten von der Gefäßwand ab und lagern sich aufgrund des hydrophoben Effekts zu Vesikeln zusammen. Die Vesikel sind dabei so groß, dass sie unter dem Mikroskop betrachtet werden können. Die verschiedenen Brechungsindizes der Saccharoselösung im Inneren der Vesikel und der Glucoselösung als Außenmedium erleichtern die Betrachtung der Vesikel insbesondere unter dem Lichtmikroskop [281].

\section{Experiment 6.1c - Die Barrierewirkung von Lipidmembranen}

Anhand dieses Versuchs kann die Barrierewirkung von Lipidmembranen gegenüber Protonen untersucht werden. Bei vorheriger Synthese der Vesikel durch die Lehrkraft, ist u. a. auch eine Verwendung im Unterricht denkbar.

Geräte und Chemikalien: 3 Bechergläser, Reagenzglas, Reagenzglasständer, Glasrührstab, Stativmaterial, Pasteurpipette, Kolbenhubpipette, Wasserbad, Heizplatte, Thermometer, Objektträger mit Vertiefung, Trichter mit Filter, Filtriergestell Licht- oder Fluoreszenzmikroskop, demin. Wasser, n-Heptan (GHS02, GHS07, GHS08, GHS09), Lecithin (Experiment 6.1a oder Egg PC), konzentrierter Rotkohlsaft, Salzsäure (1 M; GHS05).

Versuchsdurchführung: Für die Herstellung des konzentrierten Rotkohlsafts wird ein viertel eines Rotkohls in $600 \mathrm{~mL}$ Wasser mit einem Stabmixer zerkleinert und das Gemisch filtriert. Zur Erhöhung der Konzentration wird die Lösung durch Sieden auf etwa $100 \mathrm{~mL}$ reduziert. Für die Präparation der Vesikel wird bis zum Verblasen der Lösung analog zu Experiment $6.1 \mathrm{~b}$ vorgegangen. Im Anschluss werden $5 \mathrm{~mL}$ konzentrierte Rotkohllösung in das Reagenzglas gegeben und dieses für 60 Minuten ruhen gelassen. Daraufhin werden $15 \mu \mathrm{L}$ der Lösung in die Vertiefung eines Objektträgers gegeben und mit dem Mikroskop 
untersucht. Schließlich werden einige Tropfen Salzsäurelösung hinzu pipettiert und die Lösungen erneut unter dem Mikroskop betrachtet.

Beobachtung: Unter dem Mikroskop sind kleine blasenartige Strukturen zu erkennen, die etwas intensiver rosa gefärbt sind als das sie umgebende Medium. Bei Zugabe der Salzsäurelösung färbt sich das Umgebungsmedium rot, während die runden Strukturen ihre Farbe beibehalten (Abbildung 78A, B).

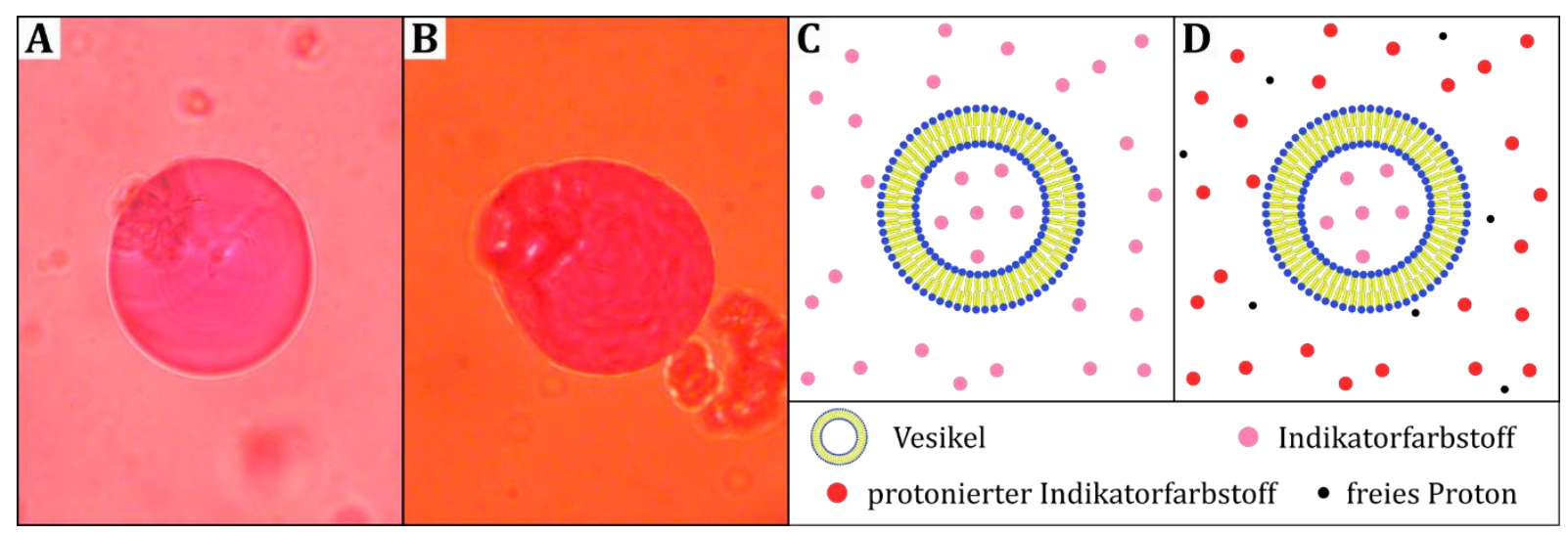

Abbildung 78: Mikroskopische Aufnahmen eines farbstoffgefüllten Vesikels vor (A) und nach Zugabe von Salzsäurelösung (B). (C) und (D) zeigen korrespondierende schematische Darstellungen des Versuchs auf Teilchenebene.

Deutung: Wie bereits in Experiment 6.1b entstehen Vesikel, die in diesem Fall mit konzentriertem Rotkohlsaft gefüllt sind. Bei Zugabe der Salzsäurelösung schlägt die Farbe des als Indikator bekannten Rotkohlsafts außerhalb der Vesikel zu Rot um. Innerhalb der Vesikel bleibt die rosa Farbe der Lösung erhalten, da die geladenen Protonen nicht durch die Lipiddoppelschicht der Vesikel diffundieren können.

Anmerkungen zur Deutung der Experimente: In der Membranforschung existieren zur Herstellung von Vesikeln im allgemeinen zwei Methoden - das Quellen und die Elektroformation [281]. Beide Methoden ermöglichen es GUVs mit spezifischen Zusammensetzungen zu synthetisieren, die anschließend mithilfe verschiedener Techniken untersucht werden können.

Beim Quellen werden Lipidmoleküle zunächst in einem organischen Lösungsmittel gelöst. Anschließend wird das Lösungsmittel durch Verblasen verdampft. Dabei ordnen sich die Lipidmoleküle zu mehreren Lipiddoppelschichten zusammen, die sich an der Gefäßwand anlagern. Die Zugabe einer Pufferlösung sorgt dafür, dass diese auch zwischen die jeweiligen Doppelschichten gelangt. Um verschiedenen Kräften wie dem osmotischen Druck, elektrostatischer Abstoßung zwischen den Lipidköpfen und der Interaktion der hydrophoben Lipidbestandteile an den Seiten der Membranen mit dem wässrigen Medium weniger ausgesetzt zu sein, quellen die Lipidschichten auf und bilden Vesikel aus (siehe Abbildung 79). Dieser recht langsame Prozess kann beispielsweise durch die gezielte Wahl von ionischen Kopfgruppen oder der Zugabe von Saccharose beschleunigt werden [281]. 


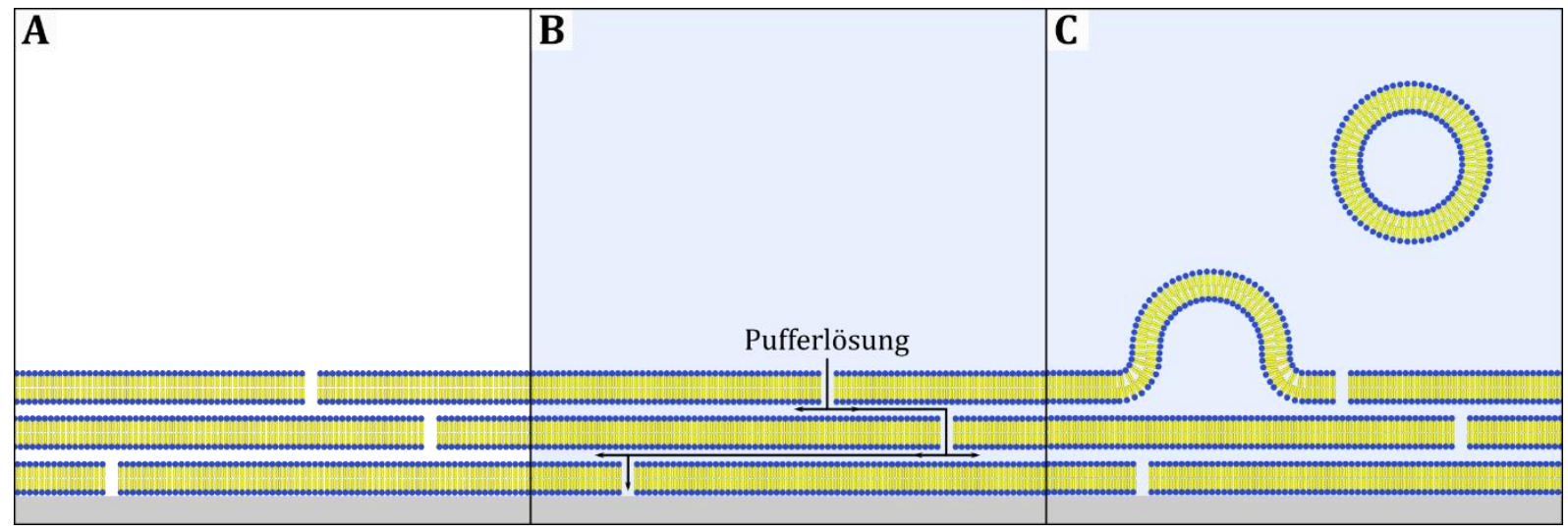

Abbildung 79: Schematische Darstellung der Vesikelpräparation durch Quellen nach STEIN ET AL. [281]. (A) Lipiddoppelschichten nach Verblasen des Lösungsmittels, (B) Zugabe einer Pufferlösung, (C) Aufquellen der Lipidschichten zu Vesikeln.

Bei der Elektroformation wird zunächst ähnlich vorgegangen wie beim Quellen, nach der Zugabe des Puffers wird jedoch eine Spannung angelegt, die die Formierung von Vesikeln durch u.a. elektrostatische Wechselwirkungen zwischen dem elektrischen Feld und den Lipidladungen sowie der Veränderung der Intermembrankräfte durch Umverteilung der

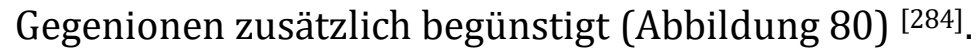

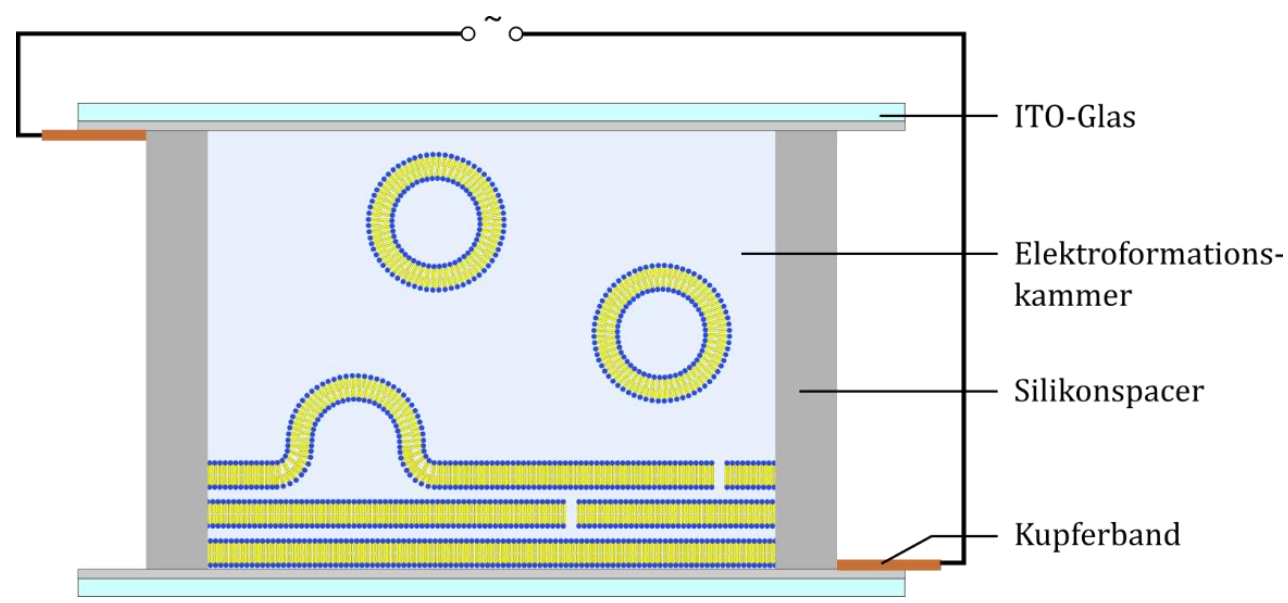

Abbildung 80: Schematische Darstellung der Vesikelpräparation in einer Elektroformationskammer nach STEIN ET AL. [281]).

Die für die Synthese der Vesikel benötigen Lipide können entweder in Form von Egg PC käuflich erworben, oder anhand der Versuchsanleitung von 6.1a aus Eigelb extrahiert werden. Sowohl die Extraktion als auch das Quellen der Lipidfilme ist jedoch sehr zeitintensiv, weshalb sich die Versuche eher für die Verwendung in Schülerlaboren eignen, zumal die meisten Schulen keinen Zugang zu einem Fluoreszenzmikroskop besitzen. Der Einschluss von konzentriertem Rotkohlsaft in den Vesikeln ermöglicht es allerdings, die Barrierewirkung der Membranen zu demonstrieren, wodurch ein Rückbezug zu den Versuchen des vierten Themenfelds zum Membrantransport möglich ist.

\section{Experiment 6.2a - Fluorochromierung von Pflanzenbestandteilen}

Dieser Versuch bedarf dem vorherigen Einlegen von Pflanzenbestandteilen in Textmarkerlösung, eignet sich im Anschluss jedoch für makro- und mikroskopische Betrachtungen unter UV-Licht im Unterricht und auf Bildungsmessen. 
Geräte und Chemikalien: Schnappdeckelgläschen, Topfpflanze mit weißen Blüten (Begonien und Petunien sind besonders geeignet), Objektträger, Mikroskopierbesteck, Lichtmikroskop und UV-Lampe oder Fluoreszenzmikroskop, gelbe oder grüne Textmarkerlösungen.

Versuchsdurchführung: Mehrere weiße Blüten werden so abgeschnitten, dass ihnen 1$2 \mathrm{~cm}$ ihres Stängels bleiben. Über Nacht werden die Stängel der Blüten in einer Fluoreszenzfarbstofflösung eingelegt und die Blüten schließlich in einem abgedunkelten Raum unter UV-Licht untersucht. Einige der Blüten und Querschnitte der Stängel werden zudem auf einem Objektträger präpariert und unter dem Fluoreszenzmikroskop untersucht. Alternativ können die Proben auch unter einem ausgeschalteten Lichtmikroskop untersucht werden, während die Proben von der Seite mit einer UV-Lampe beleuchtet werden.

Beobachtung: Unter UV-Licht sind zelluläre Strukturen der Blüten zu erkennen, die bei Tageslicht nicht zu sehen waren (siehe Abbildung 81). Die Betrachtung der Proben unter dem Fluoreszenzmikroskop ermöglicht es zudem, noch feinere Strukturen zu erkennen, die bei normaler Lichteinstrahlung nicht oder nur kaum zu unterscheiden sind.

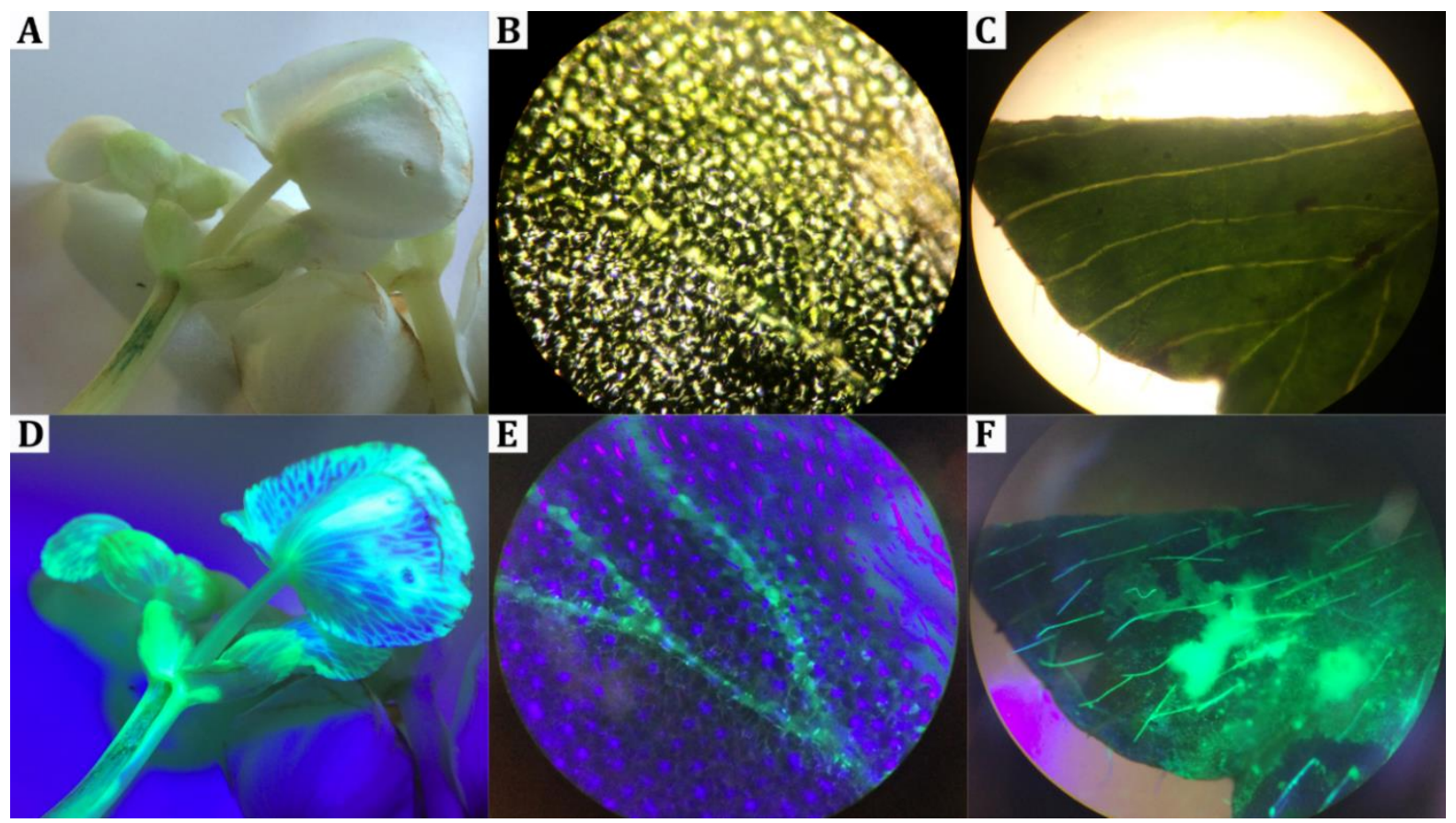

Abbildung 81: Vergleich fluoreszenzmarkierter Pflanzenbestandteile bei Tageslicht (oben) bzw. unter UVLicht (unten). In (D) und (E) ist das Leitgewebe anhand der Fluoreszenz deutlich zu erkennen. In (F) fluoreszieren hydrophile Trichome.

Deutung: Die Pflanzen nehmen über Nacht die Farbstofflösung auf. Dabei ist es den Farbstoffmolekülen vor allem aufgrund ihrer Größe nicht möglich, ungehindert in alle Zellen einzudringen. Bei Bestrahlung mit UV-Licht wird deshalb vor allem das Leitgewebe sichtbar, das innerhalb der Pflanze für den Transport von Wasser zuständig ist. Zudem treten die Pflanzenhaare (Trichome) deutlich hervor, da hydrophile Trichome von der Pflanze u. a. als Wasserspeicher genutzt werden.

\section{Experiment 6.2b - Konfokale Fluoreszenzmikroskopie von Fibroblasten}

Da die verwendeten Fibroblasten präpariert und ein konfokales Fluoreszenzmikroskop vorhanden sein muss, eignet sich dieser Versuch vornehmlich für die Verwendung in Schülerlaboren. Bei Vorhandensein eines mobilen Auflicht-Fluoreszenzmikroskops ist jedoch auch eine Anwendung auf Bildungsmessen möglich [290]. 
Geräte und Chemikalien: Petrischale ( $\mu$-dish low, $35 \mathrm{~mm}$ ), Kolbenhubpipette, Mikroreaktionsgefäß, Brutschrank, Orbitalschüttler, Konfokales Laser Scanning Mikroskop (CLSM), NIH-3T3-Zelllinie (Fibroblasten), Zellkulturmedium (450 mL Dulbecco's Modified Eagle's Medium (DMEM), 4,5 g $\cdot \mathrm{L}^{-1}$ Glucose mit $4 \mathrm{mM}$ L-Glutamin, $50 \mathrm{~mL}$ Foetal Bovine Serum (FBS)), Paraformaldehyd-Lösung (PFA, $w=4 \%$; GHS02, GHS05, GHS07, GHS08) in Phosphat-gepufferter Salzlösung (PBS), CellMask ${ }^{\mathrm{TM}}$ Orange Plasma Membrane Stains (GHS07), Rinderserumalbumin-Blockierungslösung (Rinderserumalbumin (BSA, $w=5 \%$ ) und Triton X-100 ( $w=0,3 \%$; GHS05, GHS07, GHS09) in PBS-Lösung), 4',6-Diamidin-2-phenylindol (DAPI, $500 \mathrm{ng} \cdot \mathrm{L}^{-1}$ in Methanol; GHS02, GHS06, GHS07, GHS08), Phalloidin Alexa Fluor 488 (GHS06), Immersionsöl.

Versuchsdurchführung: a) Fluoreszenzmarkierung verschiedener Zellbestandteile: Zur Markierung der Zellmembranen werden Fibroblasten in speziellen Petrischalen mit einem Zellkulturmedium überschichtet, bis zur gewünschten Konfluenz im Brutschrank bei $37^{\circ} \mathrm{C}$ kultiviert und einmal mit $37^{\circ} \mathrm{C}$ warmen Medium gewaschen. Anschließend wird $1 \mathrm{~mL}$ Reagenzlösung ( $1 \mu \mathrm{L}$ Cellmask in $999 \mu \mathrm{L}$ Medium) in die Petrischale gegeben und die Zellen für 10 Minuten bei $37^{\circ} \mathrm{C}$ im Brutschrank inkubiert. Zur Fixierung, Blockierung und Permeabilisierung der Fibroblasten wird die Probe drei Mal mit $1 \mathrm{~mL}$ PBS-Lösung gewaschen, mit $1 \mathrm{~mL}$ PFA-Lösung versetzt und 20 Minuten bei Raumtemperatur inkubiert. Nach weiterem dreimaligen Waschen mit $1 \mathrm{~mL}$ PBS-Lösung wird die Probe mit $1 \mathrm{~mL}$ Blockierungslösung versetzt und für 30 Minuten bei Raumtemperatur inkubiert. Anschließend wird weitere drei Mal mit 1 mL PBS-Lösung gewaschen. Für die Markierung des Zellkerns und des Aktin-Zytoskeletts wird das fluoreszenzgelabelte Phalloidin steril mit PBS auf $165 \mathrm{nmol} \cdot \mathrm{L}^{-1}$ und die DAPI-Lösung mit PBS auf $50 \mathrm{ng} \cdot \mathrm{mL}^{-1}$ verdünnt und beide Lösungen zur Probe gegeben. Anschließend wird die Probe 60 Minuten bei Raumtemperatur inkubiert. Nach dreimaligem Waschen mit 1 mL PBS-Lösung, bei dem die Proben jeweils 5 Minuten auf einen Orbitalschüttler gestellt werden, wird die Probe erneut mit 1 mL PBS-Lösung befüllt und kann mikroskopiert werden.

b) Konfokale Fluoreszenzmikroskopie: Eine Probe wird in $1 \mathrm{~mL}$ PBS-Lösung mittels CLSM mikroskopiert. Dazu wird Immersionsöl zwischen das Hochleistungsobjektiv und das Präparat gegeben, um Streulicht zu minimieren. Die verschiedenen Fluoreszenzfarbstoffe werden separat angeregt und die entstandenen Bilder im Anschluss mithilfe einer Bildbearbeitungssoftware (z. B. Fiji [291]) eingefärbt und überlagert.

Beobachtung: Auf den konfokalmikroskopischen Aufnahmen sind je nach Anregungswellenlänge verschiedene Strukturen zu erkennen. Werden die Aufnahmen am Computer angefärbt und überlagert, wird deutlich, dass die Strukturen jeweils zusammengehören (Abbildung 82). 


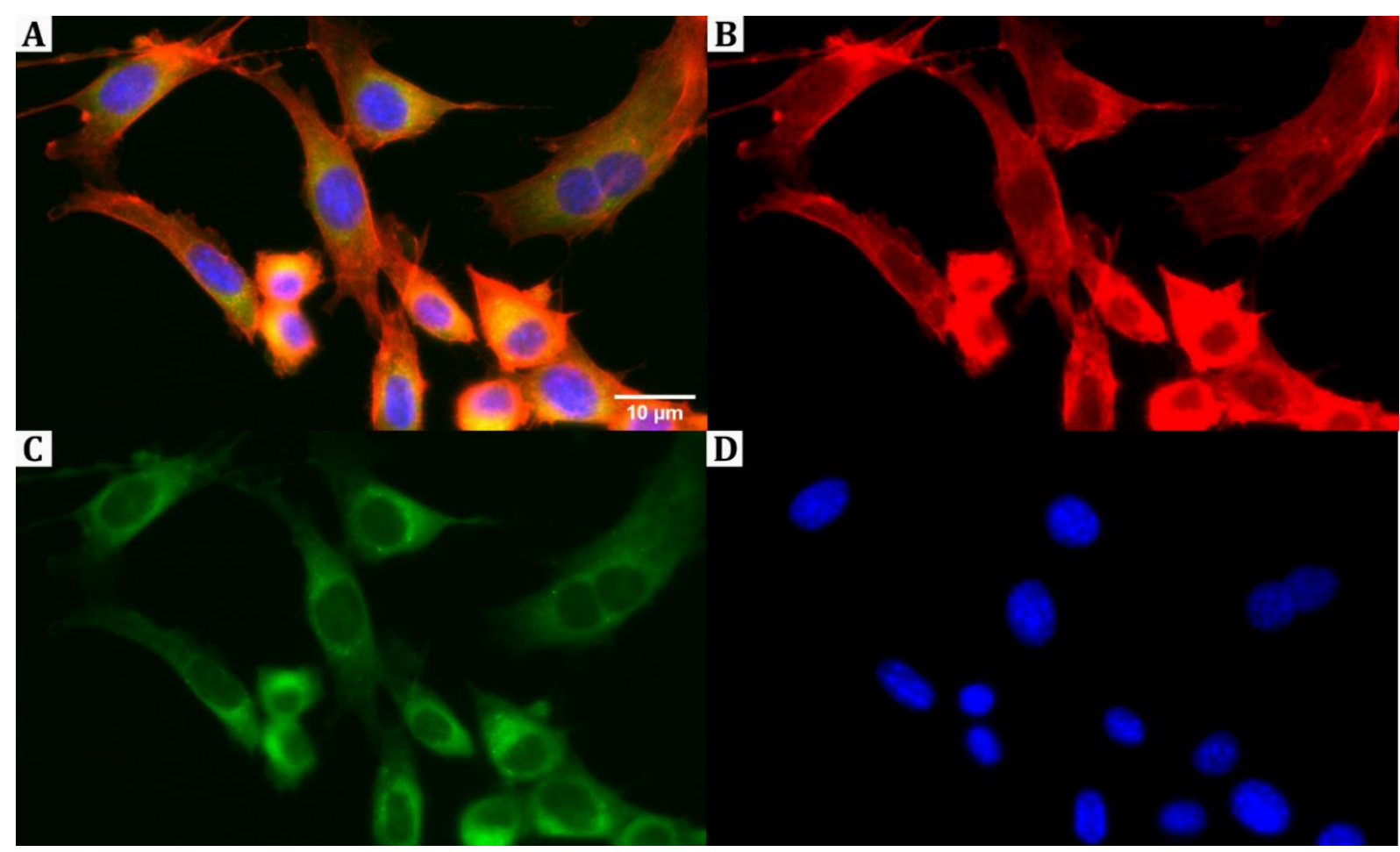

Abbildung 82: Konfokalmikroskopische Aufnahmen von Fibroblasten. In (A) wurden die Aufnahmen des rot markierten Aktin-Zytoskeletts (B), der grün markierten Membran (C) und des blau markierten Zellkerns (D) überlagert.

Deutung: Die Markierung von Zellen mit unterschiedlichen Fluorophoren, wie den hier verwendeten Fibroblasten, ermöglicht es, verschiedene Zellbestandteile wie das Zytoskelett, die Zellmembran und den Zellkern deutlich voneinander unterscheiden zu können. Die Fluorophore binden dabei an unterschiedliche Proteine, die spezifisch für die jeweiligen Zellbestandteile sind. Die Verwendung verschiedener Anregungswellenlängen ermöglicht so eine gezielte Visualisierung zellulärer Strukturen unterhalb der Auflösungsgrenze klassischer Lichtmikroskope, so dass Aussagen über die biologischen Funktionen der jeweiligen Zellen und Zellbestandteile möglich sind.

Anmerkungen zur Deutung der Experimente: Im Gegensatz zur herkömmlichen Mikroskopie, die SuS aus dem Biologieunterricht bekannt sein dürfte, ermöglicht es die konfokale Laserscanning-Mikroskopie (Confocal Laser Scanning Microscopy, CLSM) fluoreszenzmarkierte Präparate dreidimensional aufzulösen. Dazu werden die Präparate punktförmig abgerastert, wobei lediglich das Licht vom Punktlichtdetektor registriert wird, das sich in der Brennebene befindet (vgl. Abbildung 83B). Fluoreszenzlicht das ober- und unterhalb der Brennebene liegt und bei nicht-konfokaler Mikroskopie zu Unschärfen führen würde, wird hingegen nicht detektiert [292]. Da das punktförmige Abrastern der verschiedenen Ebenen des Präparats kein Gesamtbild liefert, müssen die einzelnen detektierten Lichtintensitäten mithilfe von Bildbearbeitungsprogrammen zunächst noch zu einer einheitlichen Darstellung zusammengefügt werden. So lassen sich die Präparate sowohl dreidimensional darstellen als auch die einzelnen Ebenen in z-Richtung abbilden. Wurden mehrere Fluoreszenzmarker verwendet und diese mit verschiedenen Anregungswellenlängen detektiert, können diese Aufnahmen zudem übereinandergelegt werden, um ein ganzheitliches Abbild des Präparats zu liefern. 

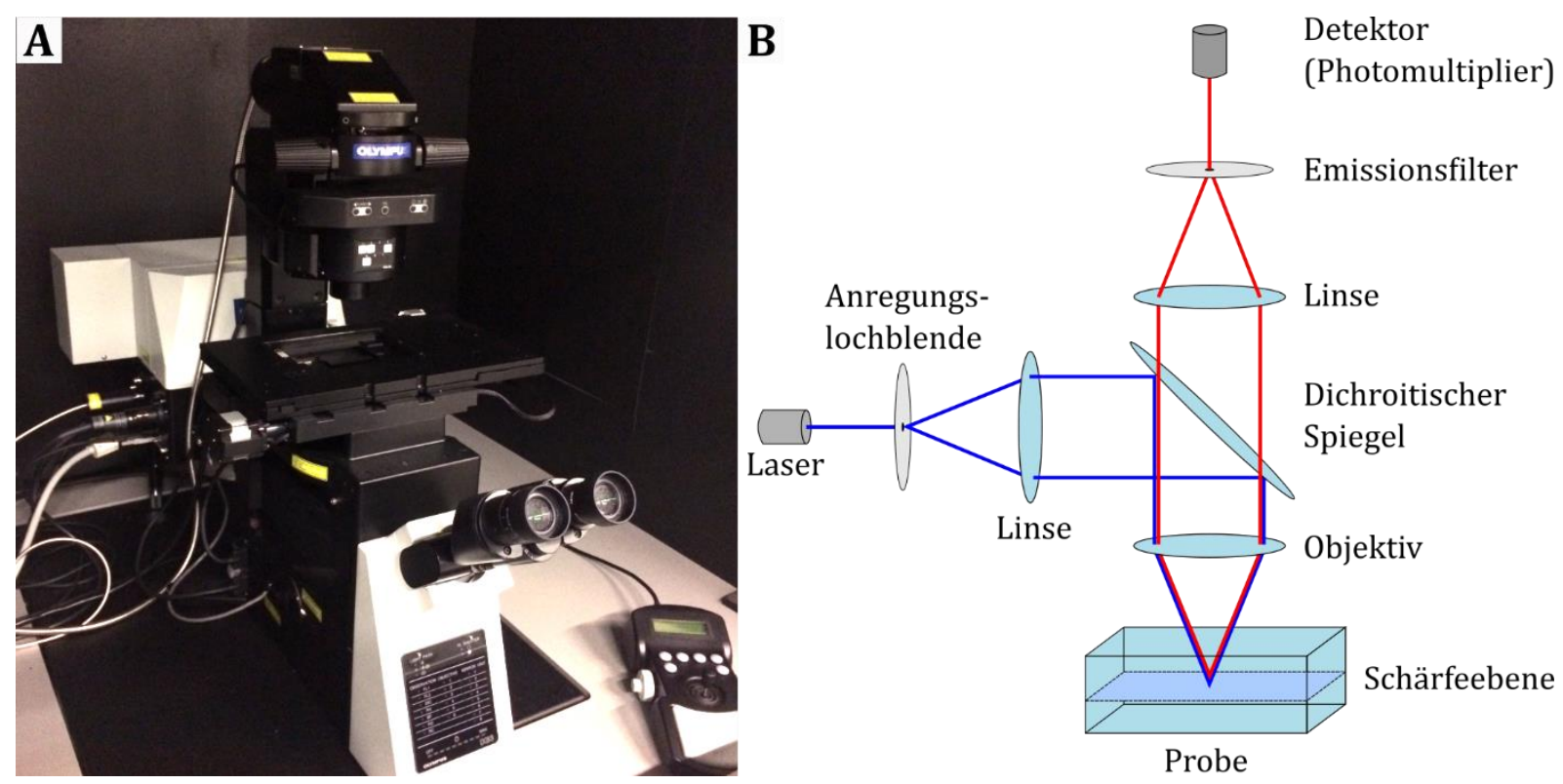

Abbildung 83: (A) Konfokal-Fluoreszenzmikroskop (CLSM; FluoView1200; Olympus, Tokyo, Japan), Hochleistungsobjektiv (UPLFLN 100xO2PH, NA=1,3; Olympus, Tokyo, Japan). (B) schematische Darstellung des Aufbaus eines Fluoreszenzmikroskops.

Die in $6.2 \mathrm{~b}$ beschriebene Präparation von fluoreszenzmarkierten Zellen und die anschließende Untersuchung mit einem CLSM eignet sich im Sinne einer Öffentlichkeitsarbeit aufgrund des präparativen Aufwands und der Kosten für ein CLSM vornehmlich in Bezug auf Schülerlabore, wobei die Präparation der Zellen bereits im Voraus stattfinden kann. Die Anschaffung eines nicht-konfokalen Fluoreszenzmikroskops für die Verwendung auf Bildungsmessen durch den SFB ermöglicht es jedoch, fluoreszenzmarkierte Zellen zudem auch auf Bildungsmessen zu präsentieren.

Um die Bedeutung der Fluoreszenzmikroskopie auch in schulischen Kontexten verdeutlichen zu können ist es möglich, Blüten und andere Pflanzenbestandteile mit fluoreszierender Textmarkerlösung anzufärben. Während das Anfärben von Blüten mit blauer Tinte im Biologieunterricht häufig Anwendung findet, ermöglicht die Verwendung von fluoreszierenden Farbstofflösungen es dieses Experiment insofern zu erweitern, als eine Betrachtung des Leitgewebes, ähnlich wie in der Forschung, erst durch Bestrahlung mit UV-Licht möglich ist. Vergleichende mikroskopische Betrachtungen mithilfe der originären Lichtquelle bzw. einer sekundären UV-Lichtquelle verdeutlichen zudem, dass die Fluoreszenzmarkierung auch auf mikroskopischer Ebene eine Unterscheidung verschiedener Bestandteile des Präparats ermöglicht.

\section{Experiment 6.3a - Fluoreszenzmarkierte Fusion von Seifenblasen}

Dieses Modellexperiment zur Fusion von Vesikeln ist u. a. auf Bildungsmessen einsetzbar und kann auch von jüngeren Besucher_innen durchgeführt werden.

Geräte und Chemikalien: 2 Bechergläser (100 mL), Messzylinder, Glasrührstab, 2 Seifenblasendosen mit Ringen, UV-Lampe, Wasser, Puderzucker, Glycerin, Spülmittel.

Versuchsdurchführung: In zwei Bechergläsern werden jeweils in $50 \mathrm{~mL}$ Wasser $25 \mathrm{~mL}$ Spülmittel, $10 \mathrm{~mL}$ Glycerin und $25 \mathrm{~g}$ Puderzucker vermischt, wobei zu einer der Lösungen noch eine Spatelspitze Pyranin hinzugegeben wird. Die beiden Lösungen werden einige Stunden ruhen gelassen und anschließend vorsichtig gerührt. Mit beiden Seifenblasenlösungen wird möglichst gleichzeitig eine kleine Seifenblase erzeugt und diese auf dem jeweiligen Seifenblasenring aufgefangen. Die Seifenblasen werden mit UV-Licht bestrahlt 
und die Pyranin enthaltende Blase von oben vorsichtig auf die Seifenblase ohne Pyranin gelegt, so dass eine gemeinsame Seifenhaut entsteht.

Beobachtung: Die Seifenblase mit Pyranin fluoresziert grün unter UV-Licht. Werden die beiden Seifenblasen aufeinandergelegt, bildet sich eine gemeinsame Grenzflächenschicht, die ebenfalls fluoresziert. Zudem ziehen sich nach kurzer Zeit auch fluoreszierende Schlieren durch die Membran der zuvor nicht fluoreszierenden Seifenblase (Abbildung 84).

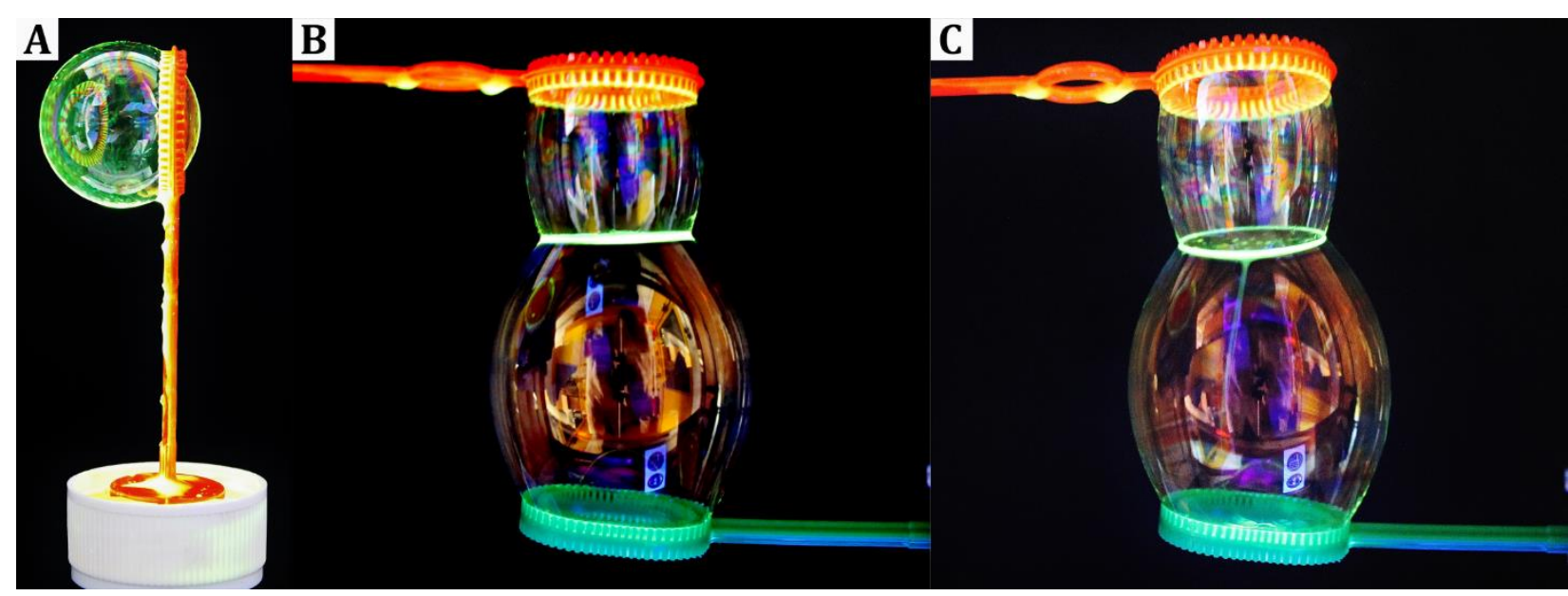

Abbildung 84: (A) Mit Pyranin versetze Seifenblase. (B) Beim Aufeinanderlegen zweier Seifenblasen, wird der fluoreszierende Farbstoff der oberen Seifenblase auf die untere Seifenblase übertragen (C).

Deutung: Zwischen den Tensidschichten der Seifenblasen befinden sich sowohl die Wassermoleküle, als auch - im Falle der Pyraninlösung - die Farbstoffmoleküle. Werden die beiden Blasen aufeinandergelegt, bildet sich eine gemeinsame Grenzschicht, die lediglich aus einer Tensiddoppelschicht besteht und beide Seifenblasen miteinander verbindet. Die zwischen den Tensidschichten eingebetten Moleküle können somit ungehindert von einer Seifenblase zur anderen wechseln, wodurch, der Schwerkraft folgend, deutlich fluoreszierende Schlieren in der unteren Seifenblase zu beobachten sind.

\section{Experiment 6.3b - Fluoreszenzspektroskopische Fusionsmessungen}

Fluoreszenzspektroskopische Untersuchungen sind lediglich in Schülerlaboren möglich, anhand der Fluoreszenzspektren können prinzipiell jedoch auch im Unterricht Aussagen über die Fusion von Vesikeln getroffen werden [239, 279].

Geräte und Chemikalien: 2 Reagenzgläser, Becherglas (500 mL), Kolbenhubpipette, Wasserbad, Heizplatte, Trockenschrank, Vortexer, LiposoFast Extruder, Küvetten, Fluorimeter, demin. Wasser, Kaliumchloridlösung (2,5 M)DOPE (1,2-dioleoyl-sn-glycero-3-phosphpethanolamin; GHS06, GHS08), DOTAP (1,2-dioleoyl-3-trimethylammonium-propan), Oregon Green 488 DHPE (1,2-Dihexadecanoyl-sn-Glycero-3-Phosphoethanolamin; GHS07, GHS08), POPC (1-palmitoyl-2-oleoyl-sn-glycero-3-phosphocholin), POPG (1-Palmitoyl-2-Oleoyl-sn-Glycero-3-Phosphoglycerol), Chloroform (GHS6, GHS8), Stickstoff (GHS04), HEPES (2-(4-/2-Hydroxyethyl)-1-piperazinyl)-ethansulfonsäure; GHS06, GHS08), Polycarbonatmembran $(100 \mathrm{~nm})$.

Versuchsdurchführung: a) Herstellung der Lipidfilme: Die Lipide werden entsprechend Tabelle 10 in zwei Reagenzgläser gegeben und jeweils in $400 \mu \mathrm{L}$ Chloroform gelöst. Das Lösungsmittel wird jeweils in einem Wasserbad bei $30{ }^{\circ} \mathrm{C}$ vorsichtig unter Einfluss eines Stickstoffgasstroms verdampft. Anschließend werden die verbleibenden Lipidfilme im Trockenschrank für 3 Stunden bei Raumtemperatur getrocknet. In einem Becherglas werden 2,38 g HEPES (20 mM) in $500 \mathrm{~mL}$ demin. Wasser gelöst und ein $\mathrm{pH}$-Wert von $\mathrm{pH}=7,5$ 
eingestellt. Der Puffer wird vor der Verwendung filtriert und unter Anlegen eines Vakuums entgast. Die Lipidfilme werden zum Quellen mit je $500 \mu$ L HEPES-Pufferlösung versetzt und 30 Minuten ruhen gelassen. Anschließend werden die Lösungen drei Mal für je 30 Sekunden gevortext, wobei zwischendurch jeweils Pausen von 5 Minuten eingelegt werden. Die Lösungen werden - jeweils getrennt - mithilfe des LiposoFast Extruders durch eine $100 \mathrm{~nm}$ Polycarbonatmembran extrudiert, um SUVs mit Durchmessern $\leq 100 \mathrm{~nm}$ zu erhalten.

Tabelle 10: Mischungsverhältnisse zur Herstellung zweier unterschiedlicher Vesikelpopulationen

\begin{tabular}{l|lll|ll}
\hline \multicolumn{3}{|l}{ Kationische Lipidmischung } & \multicolumn{2}{c}{ Anionische Lipidmischung } \\
\hline Lipid & DOPE & DOTAP & OG DHPE & POPC & POPG \\
mol\% & 48 & 50 & 2 & 50 & 50 \\
$\mu \mathrm{L}$ & 29,65 & 33,50 & 14,92 & 40,10 & 65,06 \\
\hline
\end{tabular}

b) Fluoreszenzspektroskopische Messung: Eine Küvette wird mit $750 \mu \mathrm{L}$ HEPES-Pufferlösung gefüllt und an ihr eine erste Referenzmessung bei einer Anregungswellenlänge von $\lambda=508 \mathrm{~nm}$ durchgeführt. Für die Fusionsmessung werden zunächst $100 \mu \mathrm{L}$ der kationischen Vesikellösung in die Küvette gegeben und die Messung mit derselben Anregungswellenlänge gestartet. Nach Einstellen eines konstanten Werts werden $100 \mu \mathrm{L}$ der anionischen Vesikellösung in dieselbe Küvette überführt und die Messung für $1000 \mathrm{~s}$ durchgeführt. Im Anschluss wird analog eine zweite Küvette mit $750 \mu \mathrm{L}$ HEPES-Pufferlösung $100 \mu \mathrm{L}$ der kationischen Vesikellösung sowie $200 \mu \mathrm{L}$ einer Kaliumchloridlösung gefüllt. Nach Einstellen eines konstanten Werts werden abermals $100 \mu \mathrm{L}$ der anionischen Vesikellösung hinzugegeben und die Messung gestartet.

Beobachtung: Die zuvor auf den Wert 1 normierte Fluoreszenzintensität der ersten Messung steigt nach Zugabe der anionischen Vesikellösung zunächst schnell an, erreicht nach ca. 300 Sekunden ihr Maximum und nimmt im Anschluss langsam ab(Abbildung 85, schwarz). Die gemessene Intensität der zweiten Mischung bleibt nach Zugabe der anionischen Vesikellösung zunächst für kurze Zeit konstant und nimmt im Anschluss nahezu linear ab (Abbildung 85, rot).

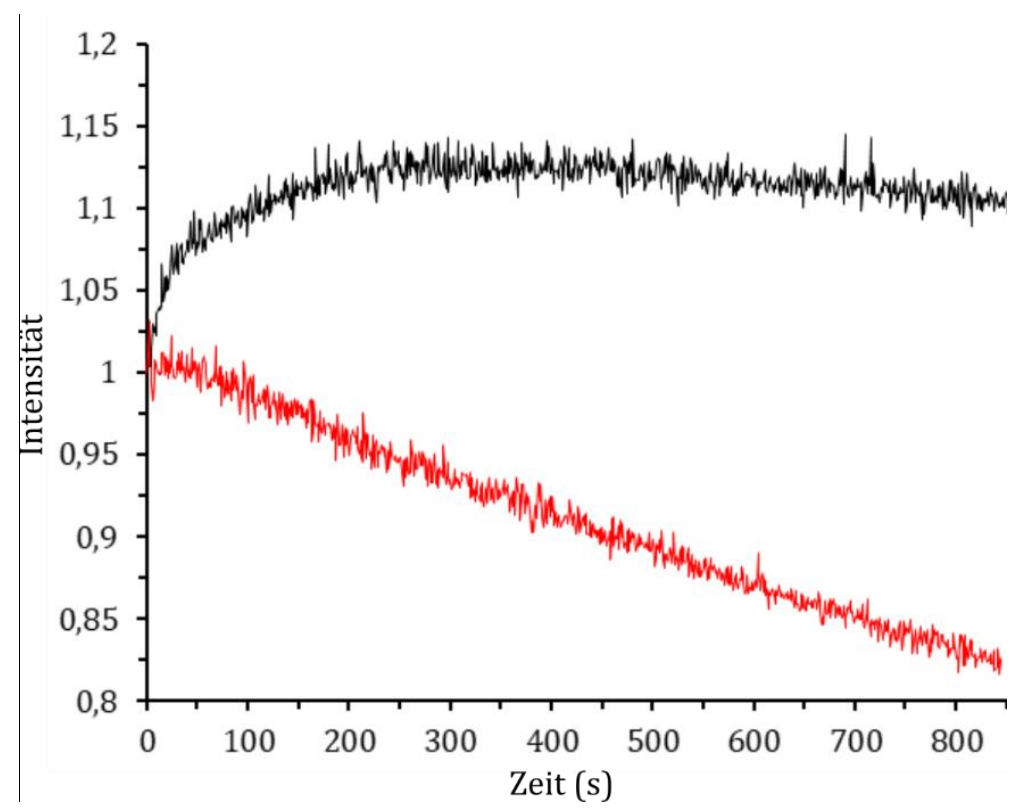

Abbildung 85: Zeitabhängige fluoreszenzspektroskopische Messung der Intensität in Mischungen kationischer und anionischer Vesikellösungen. Dabei entspricht der schwarze Graph der ersten, der rote der zweiten Messung. 
Deutung: Da die Lipide der ersten Lipidmischung positiv und die Lipide der zweiten Lipidmischung negativ geladen sind, ziehen sich die beiden Vesikelpopulationen nach Zusammenführung der Lösungen aufgrund elektrostatischer Wechselwirkungen gegenseitig an, so dass es zu einer Membranfusion kommt. Aufgrund der hohen Fluorophorkonzentration innerhalb der kationischen Vesikelmembranen unterliegen die Moleküle dem Self-quenching-Effekt, weshalb die Fluoreszenzintensität vor Zugabe der anionischen Vesikellösung relativ gering ist. Da sich die Lipide innerhalb der Membran gegeneinander bewegen, verteilen sich die fluoreszenzmarkierten Lipide nach der Fusion der beiden Vesikelpopulationen innerhalb der Membran, die nun eine höhere Oberfläche aufweist. Dadurch nimmt der Abstand zwischen den markierten Lipiden ab, so dass auch das Selfquenching abnimmt (vgl. Abbildung 86). Entsprechend wird diese Art der Fusionsmessung auch als Dequenching Assay bezeichnet.

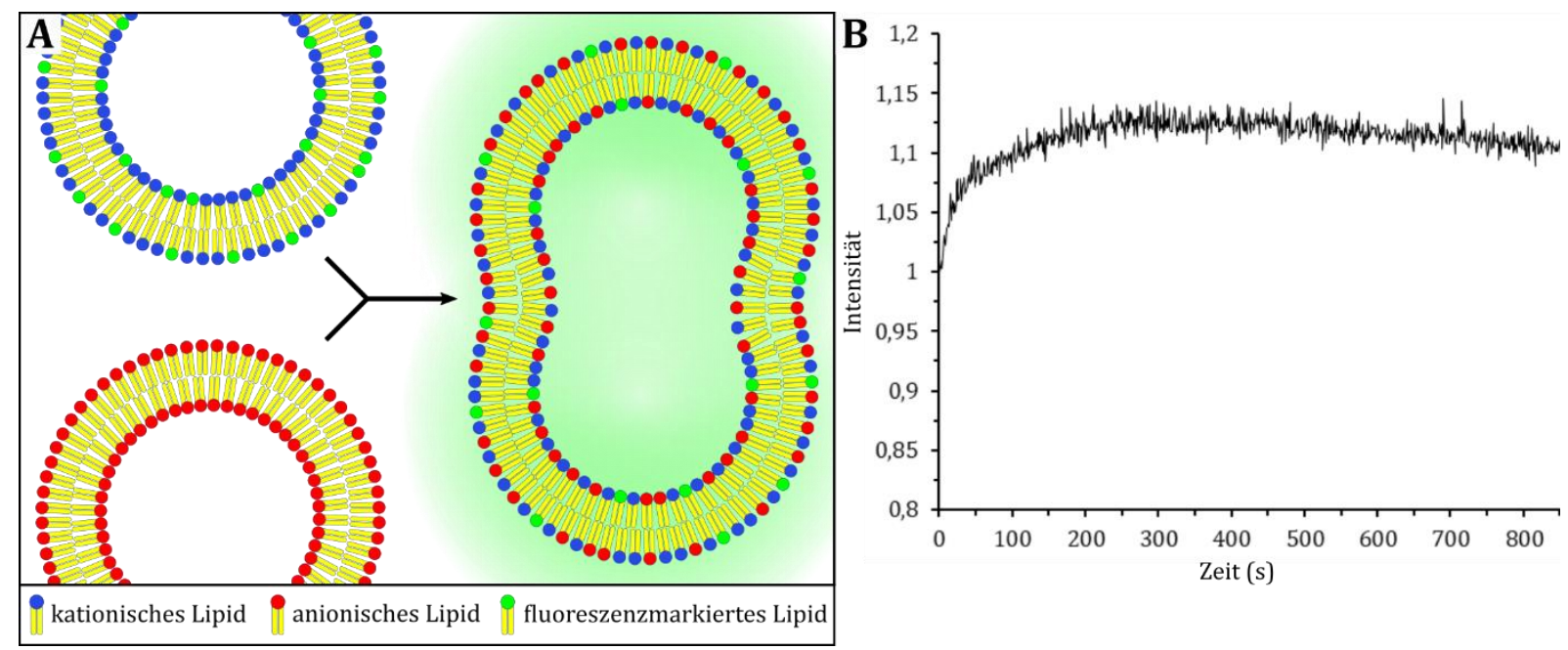

Abbildung 86: (A) Gequenchte Vesikelpopulationen mit kationischen und fluoreszenzmarkierten Lipiden fusionieren mit Vesikelpopulationen anionischer Vesikel, wodurch es zum Dequenching kommt. Entsprechend ist spektroskopisch ein Anstieg der Intensität zu beobachten (B).

Wird bei der zweiten Messung zunächst Kaliumchlorid zur Lösung der ersten Vesikelpopulation hinzugegeben, so wechselwirken die positiv geladenen Lipide der Vesikel mit den in Lösung vorliegenden Chloridionen. Bei Zugabe der negativ geladenen Vesikelpopulation gehen auch diese Wechselwirkungen mit den gelösten Kaliumionen ein. Die Entstehung elektrochemischer Doppelschichten an den Phasengrenzen zwischen Vesikeln und umgebendem Medium verhindern dabei, dass es zur nötigen Annäherung der Vesikel kommt, so dass die Wahrscheinlichkeit für Fusionen sinkt (vgl. Abbildung 87). Die Abnahme der Fluoreszenzintensität sowohl im zweiten als auch zum Ende des ersten Teilversuchs ist zudem damit zu begründen, dass die Fluorophore dem Prozess des Photobleachings unterliegen. 

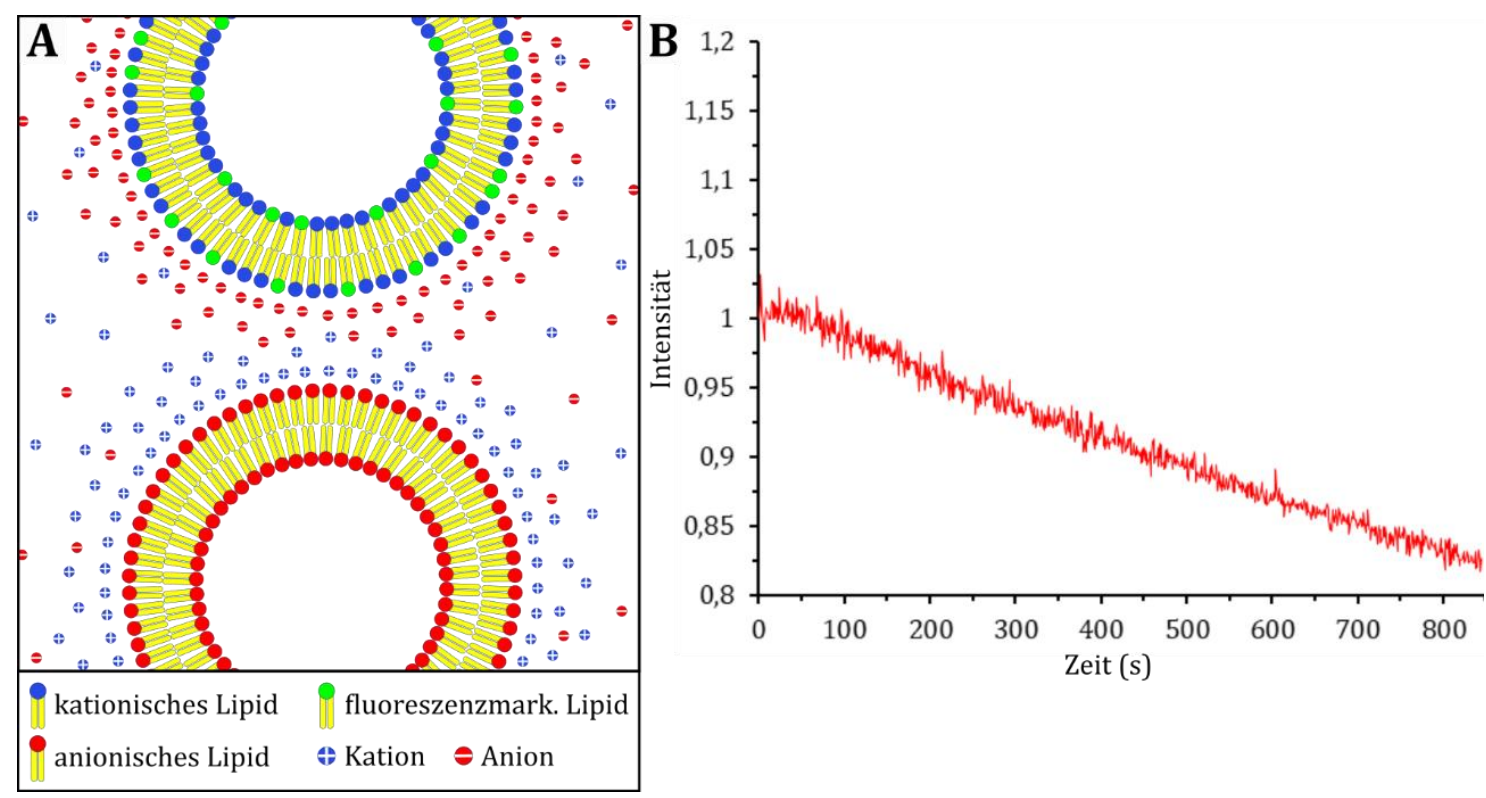

Abbildung 87: (A) In ionischen Lösungen bilden sich elektrochemische Doppelschichten an den Phasengrenzen der Vesikelpopulationen, so dass die Wahrscheinlichkeit für Fusionen sinkt. Die Intensität während der spektroskopischen Messung nimmt wegen Bleaching-Effekten kontinuierlich ab.

Anmerkungen zur Deutung der Experimente: Die Fluorimetrie ähnelt vom Prinzip her der Photometrie, wobei anstelle der Transmission die Intensität der auftretenden Fluoreszenz einer Lösung gemessen wird. Die Fluoreszenzemission wird dabei in einem Winkel von $90^{\circ}$ zur Anregungsstrahlung erfasst, um Interferenzen durch diese zu vermeiden. Die Deutung des ersten Teilversuchs kann anhand der Erkenntnisse bezüglich des Self-quenching-Effekts aus Versuch 5.7b erfolgen. Für die Betrachtungen reicht es dabei aus, wenn die SuS das Flüssig-Mosaik-Modell heranziehen, um die Lipidwanderung innerhalb der fusionierten Vesikelmembran und das damit verbundene Dequenching der Fluoreszenz zu erklären. Der Versuch zur Fluoreszenzmarkierung von Seifenblasen kann zudem verwendet werden, um die Fusion der Vesikel und die damit einhergehende Verteilung der Fluorophore innerhalb der Membran auf makroskopischer Ebene zu modellieren.

Die fluoreszenzspektroskopischen Messungen eigenen sich abermals vor allem für die Verwendung in Schülerlaboren, wobei die spektroskopischen Messungen, eingebettet in einen entsprechenden Forschungskontext, theoriegeleitet auch im regulären Unterricht ausgewertet werden können. Diesbezüglich existieren sogar gleich mehrere Anknüpfungspunkte zur Membranforschung des SFB, da zum einen aufgrund elektrostatischer Wechselwirkungen der jeweiligen Lipidmischungen selektive Fusionen möglich sind, mithilfe derer bspw. eine gezielte Einbettung von Proteinen in die Membran erfolgt [293, 294]. Zudem ermöglicht es eine simultane Fluoreszenzmarkierung der Vesikelmembranen und des Vesikelinhalts mit verschiedenen Fluorophoren, die zunächst eintretende Lipiddurchmischung während der Hemifusion zeitlich von der Öffnung der Fusionspore durch das jeweilige Dequenching der Fluorophore abzugrenzen [270].

Im Kontext der Erregungsübertragung am synaptischen Spalt kann zudem noch einmal auf die Rolle der SNARE-vermittelten Fusion Bezug genommen werden. Diesbezüglich zeigt der zweite Versuchsteil am Beispiel der Ionenzugabe, inwiefern mithilfe von Methoden wie dem Dequenching Assay eine Untersuchung der Wirkung von Botulinumtoxin auf die Membranfusion möglich ist. 


\section{Einordnung der Experimente im Kontext der SO-Leitfragen}

Die Experimente des letzten Themenfelds bieten eine Reihe von Einblicken in verschiedene Aspekte der Membranforschung des SFB. Da die Forschung auf der Untersuchung biomimetischer Membranen basiert, bietet die Präparation von Vesikeln, als eine von vielen Methoden zur Herstellung künstlicher Membransysteme, die Möglichkeit, eine der grundlegenden Techniken der Forschung des SFB kennenzulernen. Anhand der gewonnenen Vesikel können zudem weitere Untersuchungen, u. a. zur Barrierefunktion der Membran, aber auch zur Vesikelfusion, durchgeführt werden. Durch das Ersetzen von Chloroform durch n-Heptan konnte die Methode des Quellens dabei insofern angepasst werden, als sie nun auch von SuS durchgeführt werden kann. Jedoch geht mit der Substitution auch ein erhöhter Zeitaufwand für das Verblasen des Lösungsmittels einher.

Die Experimente zur Fluoreszenzmikroskopie und -spektroskopie bilden zwei wichtige Forschungsmethoden ab, mit deren Hilfe biomimetische Membransysteme untersucht werden können. Sie legitimieren zudem noch einmal die inhaltliche Aufarbeitung des Themas der Fluoreszenz im Zuge der hier vorgenommenen didaktischen Erschließung der Biomembranforschung, da die in Kapitel 5.5 erarbeiteten Konzepte benötigt werden, um die Forschungsergebnisse zu interpretieren. Leider bieten sich diese forschungsnahen Experimente jedoch lediglich für eine Öffentlichkeitsarbeit im universitären Kontext an, wie bspw. Schülerlabore oder Tage der offenen Tür. Die Modellexperimente zur Fluorochromierung von Blüten sowie zur Fluoreszenzmarkierung von Seifenblasen ermöglichen jedoch zumindest einen experimentellen Zugang zu den Methoden, so dass diese trotzdem im Unterricht behandelt werden können. Weitere Einblicke in die explizite Forschung des SFB bieten zudem die beiden in Kapitel 6.3 vorgestellten Adaptierten Primärliteraturen. Während die erste Publikation zu spannungsabhängigen Konformationsänderungen des Ionenkanals VDAC-1 vornehmlich molekulardynamische Simulationen beschreibt, beinhaltet die zweite Publikation zur Synthese von Glycosphingolipiden mit fluoreszenzmarkierten Fettsäuren sowohl fluoreszenzspektroskopische als auch -mikroskopische Untersuchungen. 


\section{Entwicklung und Evaluation verschiedener Science Outreach-Formate}

Neben der möglichst ganzheitlichen experimentellen Aufarbeitung der Thematik der Biomembranen und damit einhergehend der aktuellen Membranforschung, stand die Einbettung der Experimente in verschiedene SO-Formate im Vordergrund dieser Arbeit. Dazu wurden nach ausführlicher Sichtung der existierenden SO-Formate verschiedene Aktivitäten ausgewählt, die mit Blick auf die verschiedenen SO-Bildungskontexte unterschiedliche Formen der thematischen Auseinandersetzung mit den Inhalten des SFB 803 und der naturwissenschaftlichen Forschung im Allgemeinen ermöglichen sollten. Ähnlich wie bei der experimentellen Ausgestaltung der Themenfelder orientieren sich dabei auch die verschiedenen Formate an den in Kapitel 5 formulierten Leitfragen, wobei hier je nach Aktivität und teilnehmenden Personenkreisen unterschiedliche Schwerpunkte gesetzt wurden.

Von den neun in Kapitel 4.2 erwähnten Formaten, die im Rahmen der Öffentlichkeitsarbeit des SFB 803 umgesetzt wurden oder noch umgesetzt werden sollen, wurden vier im Rahmen dieses Dissertationsprojektes entwickelt und zum Großteil evaluiert (siehe Tabelle 11). Viele der Konzepte für weitere Formate, wie bspw. die Nacht des Wissens, die sich am Bildungsmessekonzept (vgl. Kapitel 6.4)orientiert, oder die Gestaltung des Zukunftstags in der Niedersächsischen Staatskanzlei, gingen dabei aus diesen grundlegenden Formaten hervor.

Tabelle 11: S0-Projekte des SFB 803 differenziert hinsichtlich der sechs SO-Bildungskontexte. Die hervorgehobenen Projekte werden nachfolgend vorgestellt.

\begin{tabular}{ccccc}
\hline & & \multicolumn{2}{c}{ Subjektperspektive auf das Lernen } \\
\cline { 3 - 4 } & & Intentionales Lernen & Inzidentelles Lernen \\
\cline { 3 - 5 } & & Formal & (1) Unterrichtsmaterialien & $\begin{array}{c}\text { (2) Adaptierte } \\
\text { Primärliteratur }\end{array}$ \\
\cline { 3 - 5 } & & (3) Schülerlabore & (4) Nacht des Wissens \\
Kontexte mit & Science Camps & Zukunftstag \\
bezug & Non-Formal & Facharbeiten & \\
\hline
\end{tabular}

Vor dem Hintergrund, dass SuS der Sekundarstufe II als Hauptzielgruppe für die SO-Aktivitäten des SFB definiert wurden, lag die Entwicklung von Unterrichtsmaterialien und hier insbesondere einer Unterrichtseinheit nahe. Aufgrund des interdisziplinären Charakters der Forschung an Biomembranen erfolgte die Entwicklung der Einheit mit Blick auf die Schnittmenge curricular relevanter Inhalte aus den Fächern Biologie und Chemie, so dass innerhalb dieser Einheit typische biologische Grundlagen anhand zugrundeliegender chemischer Aspekte erarbeitet werden können. Zwar ist es hierbei prinzipiell möglich, Bezüge zur aktuellen Forschung im Sinne der dritten Leitfrage herzustellen, jedoch fällt es schwer, authentische Einblicke in die Methoden und den Forschungsalltag zu geben. Eine tiefergreifendere Auseinandersetzung mit forschungsrelevanten Inhalten bietet diesbezüglich die Entwicklung von Schülerlaboren, da die Nähe zur Universität eine Einbindung von wissenschaftlichen Methoden sowie von Wissenschaftler_innen ermöglicht. Der Natur der Grundlagenforschung ist es jedoch geschuldet, dass die Forschung des SFB 
zunächst in einen für die SuS interessanten Kontext eingebettet werden muss, der die Relevanz der Forschung verdeutlicht. Mit Blick auf die Forschung an SNARE-Proteinen fiel die Wahl hierbei auf die Wirkweise von Botulinumtoxin (Botox), das SuS aus der Boulevardpresse bekannt ist.

Zusätzlich zu der Frage woran geforscht wird, ist vor allem in Hinblick auf existierende stereotype Vorstellungen und die Berufsorientierung von SuS auch die Frage wie geforscht wird, also u. a. in welchen Sozialformen oder mit welchen Methoden, von Interesse. Adaptierte Primärliteratur bietet hier die Möglichkeit, durch Auseinandersetzung mit einem bestimmten Forschungsgegenstand Einblicke in die Erkenntnisprozesse von Wissenschaftler_innen zu geben, wobei die Erarbeitung fachlicher Inhalte eher in den Hintergrund rückt. Obwohl für Adaptierte Primärliteratur prinzipiell auch andere Anwendungsmöglichkeiten denkbar sind - einige werden im Verlaufe dieses Kapitels genannt wird sie hier deshalb eher dem formal inzidentellen SO-Bildungskontext zugeschrieben (vgl. Tabelle 11). Die Entwicklung von Konzepten für interaktive Ausstellungen auf Bildungsmessen rundet die Auswahl der SO-Aktivitäten dahingehend ab, dass neben dem Ziel, einen Beitrag zur Berufsorientierung von SuS zu leisten, die Forschung des SFB einem größeren Publikum präsentiert werden kann, so dass trotz gleichbleibendem Fokus auf SuS der Oberstufe auch der interessierten Allgemeinheit die Möglichkeit gegeben wird, sich mit aktuellen Forschungsthemen auseinanderzusetzen.

\subsection{Formal Intentional - Unterrichtseinheiten}

Wie bereits eingangs erwähnt, basiert das SO-Projekt des SFB 803 auf dem Pilotprojekt SFB@SCHOOL. Innerhalb dieses Projektes wurde in Kooperation zwischen Forschung, Chemiedidaktik und Schule eine kompetenzorientierte Unterrichtseinheit zum Thema Biomembranen entwickelt und im Chemieunterricht einer 11. Klasse eines Gymnasiums durchgeführt und evaluiert [178]. Die Entwicklung der Unterrichtseinheit mit dem Titel „Vom Tensid zur Biomembran“ hatte zum Ziel, die typischerweise im Biologieunterricht angesiedelten Inhalte des Themas der Biomembranen im Sinne der stark interdisziplinär ausgerichteten Forschung des SFB 803 mit curricular relevanten chemischen Aspekten zu verknüpfen. Ausgehend von den Struktur-Eigenschaftsbeziehungen von Tensiden, die den Lipiden strukturell ähneln, sollten die Struktur-Funktionsbeziehungen von Membranen und ihren Bestandteilen erarbeitet und anschließend Einblicke in die Forschung des SFB 803 gegeben werden. Die Erarbeitung der fachlichen Inhalte sollte zudem fest mit einer Förderung der Modellkompetenz der SuS verknüpft werden, da Modelle sowohl in der naturwissenschaftlichen Lehre als auch in der Forschung selbst eine wichtige Rolle spielen.

\section{Exkurs: Die Relevanz von Modellen in Forschung und Lehre}

Modelle werden in den Naturwissenschaften vornehmlich verwendet, um Gegenstände und Phänomene, die nicht direkt beobachtbar sind, sowie deren Eigenschaften (intellektuell) zugänglich zu machen [295]. In der Chemie dienen Modelle typischerweise dazu, Phänomene auf submikroskopischer Ebene zu erklären, z. B. anhand eines Teilchenmodells oder mithilfe der verschiedenen Atommodelle, sie können jedoch auch zur Beschreibung großtechnischer Anlagen oder wissenschaftlicher Untersuchungsmethoden verwendet werden. In der Biologie reicht der Einsatz von der Nachahmung biologischer Originale (z. B. originalgroße Herzmodelle), über die Darstellung komplexer Systeme wie dem Oköoder Immunsystem, bis hin zur Erklärung submikroskopischer Strukturen und Prozesse, wie der helikalen Struktur der DNA oder den verschiedenen Membranmodellen und den 
an ihnen ablaufenden Transportprozessen [296]. Ihnen gemein ist, dass erst die Darstellung von wissenschaftlichen Ergebnissen in Form von Denkmodellen und ggf. die Verbildlichung dieser in Anschauungsmodellen eine Kommunikation ihrer Implikationen ermöglicht. Insbesondere in Bezug auf die submikroskopische Ebene dienen sie dabei als „Interpretation eines empirischen Phänomens" [295], da sie nicht, wie im Falle des Herzmodells, mit einem realen Objekt verglichen werden können. Gerade diese Interpretation von Forschungsergebnissen anhand bereits existierender Modelle oder durch die (Weiter-)Entwicklung von Modellen ermöglicht es jedoch, neue Erkenntnisse und Theorien zu generieren.

Auch innerhalb der Bildungsstandards für die Fächer Chemie, Biologie und Physik wird neben dem Experimentieren vor allem der Verwendung von Modellen eine wichtige Rolle im Prozess der Erkenntnisgewinnung zugeschrieben [37, 297, 298]. HARRISON \& TREAGUST gehen sogar so weit zu sagen, dass es nahezu unmöglich ist, die Naturwissenschaften ohne die Verwendung von Modellen zu lehren bzw. zu erlernen [299]. Obwohl die Visualisierung von abstrakten Lerngegenständen prinzipiell lernförderlich wirken kann, sei es u. a. in Form von maßstabsgetreuen, ikonisch-symbolischen oder theoretischen Modellen, bergen sie auch das Potential der Ausbildung von Fehlvorstellungen. Weit verbreitet ist bspw. die Meinung, dass Modelle exakte Abbilder der Realität darstellen [300]. Gerade maßstabsgetreue biologische Modelle, die oftmals spielzeugartig anmuten, lassen diesen Schluss zu, selbst wenn auch sie nur gewisse Aspekte des Originals widerspiegeln. Entsprechend werden z. T. jedoch auch submikroskopischen Objekten wie Atomen makroskopisch wahrnehmbare oder im Anschauungsmodell dargestellte Eigenschaften wie Farbe und Form zugeschrieben.

Die Verwendung von Modellen im Unterricht erfordert also auch immer eine Thematisierung der Eigenschaften und Grenzen von Modellen und die Ausbildung einer Modellkompetenz auf Seiten der SuS. UPMEIER ZU BELZEN \& KRÜGER entwickelten diesbezüglich - zunächst für den Biologieunterricht - ein Kompetenzmodell, dass sich aufgrund seines epistemologischen Charakters auch auf den Unterricht in anderen naturwissenschaftlichen Fächern übertragen lässt. In diesem unterscheiden sie zwischen fünf Teilkompetenzen, die sie wiederum in drei Niveaustufen differenzieren (vgl. Tabelle 12) [94, 301]. Die Niveaustufen beschreiben dabei, inwiefern die Reflexion von Modellen sich lediglich auf das Modellobjekt selbst, bzw. auf seine Funktion und das zugrundeliegende Objekt oder Phänomen bezieht. 
Tabelle 12: Verschiedene Niveaus der naturwissenschaftlichen Modellkompetenz unterteilt in fünf Teilkompetenzen UPMEIER ZU BELZEN \& KRÜGER [94].

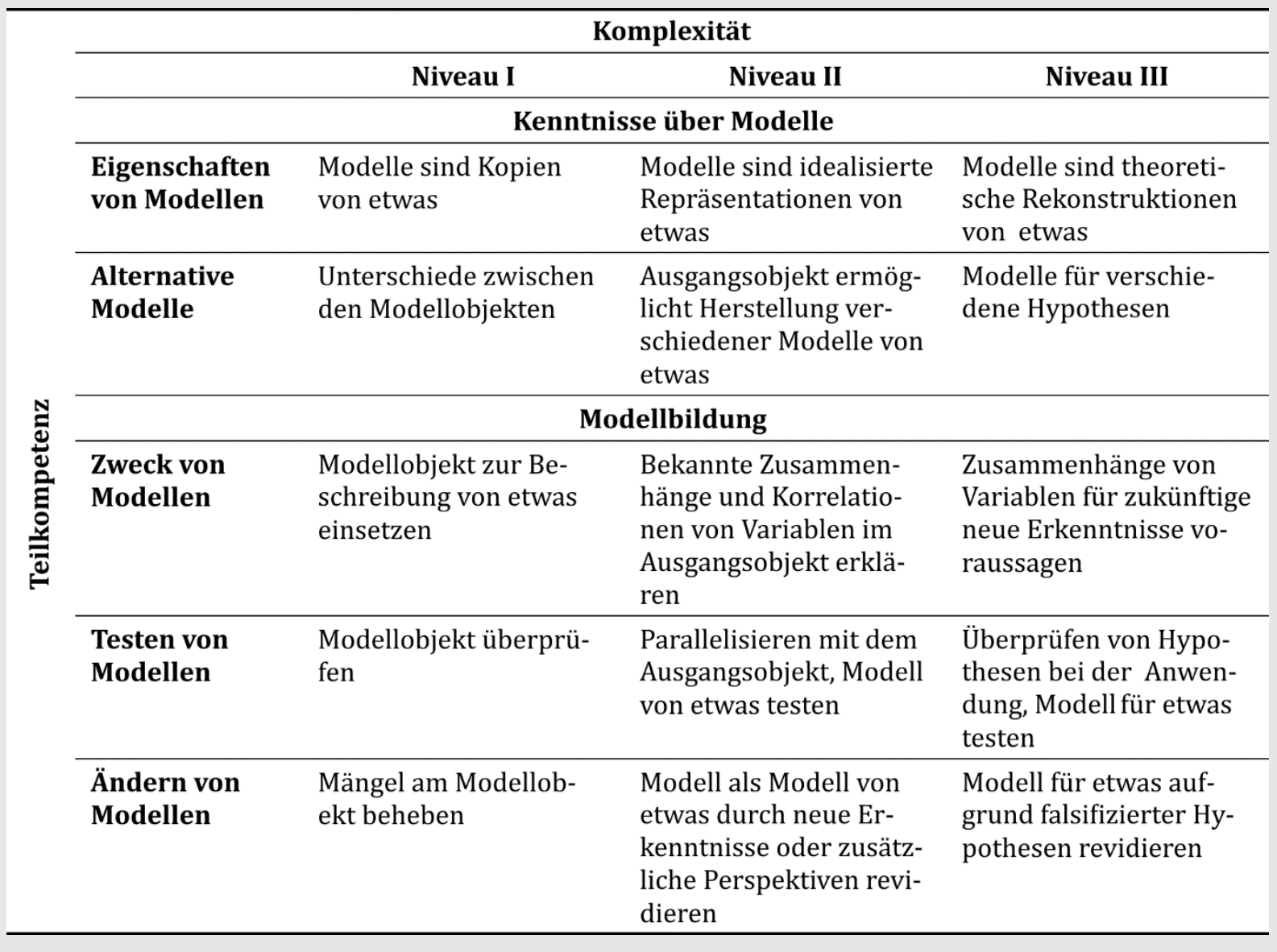

\subsubsection{Ursprüngliche Unterrichtseinheit „Vom Tensid zur Biomembran“}

Unterrichtskonzepte zur Förderung der Modellkompetenz bedienen sich oftmals der historischen Betrachtung von Modellen [302]. Während die Eigenschaften und Zwecke von Modellen auch anhand einzelner Beispiele erarbeitet werden können, vermitteln gerade historische Beispiele, wie die Entwicklung der verschiedenen Atommodelle, die Bedeutung des Testens und des sich ggf. anschließenden Änderns von Modellen, und lassen zudem Schlüsse über die Existenzberechtigung alternativer Modelle zu. Das Thema der Biomembranen bietet hier die Möglichkeit, die Modellkompetenz von SuS mit Blick auf die Entwicklung von Membranmodellen in einem vergleichsweise jungen historischen Kontext zu fördern. Zudem ermöglicht der Einstieg in das Thema der Biomembranen über die Struktur-Eigenschaftsbeziehungen von Tensiden, die Entwicklung von Modellen amphiphiler Moleküle auf Seiten der SuS und in Konsequenz eine Reflexion dieser Modelle mit Blick auf die Selbstorganisation amphiphiler Moleküle sowie Lipidmembranen. Die diesbezüglich entwickelte Struktur der Einheit sowie die korrespondierenden Inhalte und Experimente sind in Tabelle 13 aufgeführt. 
Tabelle 13: Inhalte und korrespondierende Experimente der verschiedenen Sequenzen der Unterrichtseinheit Vom Tensid zur Biomembran.

\begin{tabular}{lll}
\hline Sequenz & Inhalte & Experimente \\
\hline $\begin{array}{l}\text { Struktur-Eigen- } \\
\text { schaftsbeziehun- } \\
\text { gen von Tensiden }\end{array}$ & $\begin{array}{l}\text { Eigenschaften (Oberflächenaktivität, benetzende Wirkung, Dis- } \\
\text { pergiervermögen) und Struktur von Tensiden, Hydrophobie, }\end{array}$ & $\begin{array}{l}1.1 \mathrm{a} / \mathrm{b} / \mathrm{c}, \\
\text { Hydrophilie, Amphiphilie }\end{array}$ \\
\hline $\begin{array}{l}\text { Selbstorganisation } \\
\text { zu Mizellen und }\end{array}$ & $\begin{array}{l}\text { Modelle in den Naturwissenschaften, Entwicklung von Modellen } \\
\text { Monolayern }\end{array}$ & 2.1a/b, 2.2 \\
\hline $\begin{array}{l}\text { Von der Seifen- } \\
\text { blase zur Membran }\end{array}$ & $\begin{array}{l}\text { Vergleich Seifenhäute und Biomembranen auf submikroskopi- } \\
\text { scher Ebene, Membranmodelle im Wandel der Zeit }\end{array}$ & \\
\hline $\begin{array}{l}\text { Stofftransport } \\
\text { durch Ionenkanäle }\end{array}$ & $\begin{array}{l}\text { Stofftransport zwischen Kompartimenten, Untersuchung von } \\
\text { Ionenkanälen in der aktuellen Forschung (Patch-Clamp-Tech- }\end{array}$ & 4.2 \\
\hline
\end{tabular}

Die Unterrichtseinheit umfasst fünf thematische Sequenzen die jeweils für den zeitlichen Rahmen einer Doppelstunde konzipiert wurden. Die fachlichen Inhalte der einzelnen Abschnitte beziehen sich vornehmlich auf chemische und biologische Kompetenzen der Qualifikationsphase, weshalb sich die Einheit insbesondere für einen fächerübergreifenden Chemie- oder Biologieunterricht in der 10. bzw. 11. Klasse eignet. Die einzelnen Sequenzen sind dabei insoweit in sich geschlossen, als z. B. bei bereits vorhandenem Vorwissen zu den Struktur-Eigenschaftsbeziehungen der Tenside auch ein Einstieg in die Thematik anhand der zweiten Sequenz möglich ist.

Mit Blick auf die (selektiv) kompartimentierende Wirkung von Biomembranen beginnt die Unterrichtseinheit mit der Betrachtung der Struktur-Eigenschaftsbeziehung von Tensiden, die eine Erarbeitung der Eigenschaften amphiphiler Moleküle anhand alltäglicher Phänomene ermöglichen. Dazu führen die SuS in Gruppen verschiedene Experimente zur Oberflächenaktivität, benetzenden Wirkung sowie zum Dispergiervermögen von Tensiden durch und stellen im Anschluss anhand ihrer Erkenntnisse aus den Versuchen und ihrem Vorwissen zur Polarität der verwendeten Chemikalien und Materialien Hypothesen bezüglich der allgemeine Struktur der Tenside auf. Ohne explizite Kenntnisse über die chemische Zusammensetzung bzw. die Strukturformel der Tenside, erfolgt hier bereits eine erste Modellierung auf Seiten der SuS anhand der gewonnen Erkenntnisse, die in Form von Anschauungsmodellen skizziert werden. Die anschließende Betrachtung verschiedener Strukturformeln amphiphiler und nicht-amphiphiler Moleküle ermöglicht zudem einen Vergleich der hypothetisierten mit existierenden Strukturen und eine Zuordnung einiger der Moleküle zu den erstellten Modellen. Die entwickelten Modelle dienen dabei zunächst nur der Veranschaulichung bzw. Beschreibung des Modellobjekts (Niveau I) ohne dass von den SuS bereits eine weitere Nutzung zur Erklärung von bekannten Zusammenhängen ins Auge gefasst wird [94].

Der zweite Abschnitt ist thematisch der Selbstorganisation amphiphiler Moleküle zu Mizellen und Monoschichten gewidmet, fokussiert jedoch zudem die Förderung der Modellkompetenz der SuS. Im Laufe der Sequenz sollen die zuvor gewonnenen Erkenntnisse über die amphiphilen Eigenschaften der Tenside genutzt werden, um Vorhersagen über die Selbstaggregation der Moleküle in Wasser zu treffen und diese anhand verschiedener Experimente zu überprüfen. Dazu sollen die SuS ihre mentalen Modelle mithilfe von Streichhölzern als Anschauungsmodelle verbildlichen, um die Kommunikation über die Modelle zwischen den SuS zu unterstützen. Um eine bewusste Nutzung der Modelle zur Erkenntnisgewinnung zu fördern, erfolgt zunächst eine Explikation des Modellbegriffs, 
indem verschiedene Alltags- und wissenschaftliche Modelle vorgestellt werden und der Frage „Was ist ein Modell?“ nachgegangen wird. In einem Think-Pair-Share-Ansatz sollen die SuS zunächst ihre eigene Modellvorstellung definieren, bevor sie diese mit derer anderer vergleichen. Im Plenum wird schließlich ein gemeinsamer Modellbegriff definiert, der die Anforderungen an (wissenschaftliche) Modelle herausarbeitet. Die Definition entspricht dabei der momentanen Vorstellung der Lernenden und muss sich zu diesem Zeitpunkt nicht mit dem diesbezüglichen Wissenschaftsverständnis decken. Ausgehend vom Spülvorgang öligen Geschirrs, der als Modellobjekt dient, werden von den SuS Modelle entwickelt die diesen abbilden. Basierend auf Erkenntnissen, die von den SuS aus weiteren Experimenten gewonnen werden, werden diese Modelle überprüft und ggf. geändert (testen und ändern von Modelle, Niveau II) [94]. Anschließend werden die Verwendung sowie das Testen und Ändern der Modelle noch einmal mit Blick auf den postulierten Modellbegriff reflektiert.

Im dritten Abschnitt erfolgt der Übergang von der chemischen Betrachtung von Tensiden zur interdisziplinären Betrachtung von Biomembranen in der Schnittstelle zwischen Chemie und Biologie. Anhand der Kenntnisse über die Selbstaggregation von Tensiden werden zunächst Hypothesen bezüglich des Aufbaus von Seifenblasenhäuten aufgestellt. Der anschließende Vergleich von Molekülstrukturen typischer Tenside und Phospholipide mit Blick auf die Innen- und Außenmedien von Seifenhäuten und Biomembranen ermöglicht zudem die Entwicklung eines simplen Membranmodells. Um die (historische) Entstehung wissenschaftlicher Membranmodelle nachzuvollziehen, erstellen die SuS in Gruppen mithilfe verschiedener Materialien 3D-Modelle des Membranmodells von GoRTER \& GRENDEL [217], des Sandwich-Modells von DANIELLI \& DAVSON [218], des in Schulbüchern vornehmlich vertretenen Flüssig-Mosaik-Modells von SINGER \& NICHOLSON [219], sowie - mit Bezug zur aktuellen Forschung - des Lipid-Floß-Modells von SimONS \& IKONEN [91]. In diesem Zuge wird diskutiert, welche Umstände und Erkenntnisse die Entwicklung neuer Modelle nötig machten, welche Vorteile und Grenzen die jeweiligen Modelle besitzen und welche Rolle Modelle im wissenschaftlichen Erkenntnisprozess spielen. Dabei wird unter anderem die Bedeutung alternativer Modelle für verschiedene Hypothesen thematisiert, sowie ein Perspektivwechsel in Bezug auf die Modelle, weg von einer Herstellungs- hin zu einer Anwendungsperspektive, vorgenommen (Niveau III) [94]. Die anschließende theoretische Betrachtung der FRAP-Methode (Fluorescence Recovery After Photobleaching) [303], die Hinweise auf die Fluidität von Membranbestandteilen liefert, ermöglicht zudem erste Einblicke in aktuelle Forschungsmethoden.

Anhand der Erkenntnisse zum Aufbau der Membranen und der chemischen Eigenschaften ihrer Bestandteile sowie der Teilchen, die sowohl in die Zellen hinein- als auch heraustransportiert werden müssen, lässt sich im vierten Abschnitt die Notwendigkeit für Stofftransportprozesse herleiten. Dazu stellen die SuS Theorien zum Membrantransport verschiedener Teilchen anhand ihrer Polaritäten auf und planen ein Modellexperiment zur Überprüfung ihrer Hypothesen. Dabei nutzen sie Informationen zu den Eigenschaften verschiedener zur Verfügung stehender Chemikalien, um diese modellhaft als Analogien zu am Stofftransport beteiligten Elementen zu definieren. Mit Blick auf die Anforderungen des Kerncurriculums der Biologie werden das Modellexperiment und der zugrundeliegende Mechanismus anschließend im Kontext der verschiedenen aktiven und passiven Transportprozesse besprochen und eingeordnet. Zudem wird das Experiment in Bezug auf den erarbeiteten Modellbegriff der SuS analysiert. In diesem Zuge ist es möglich, bewusst zwischen Modellexperimenten zu unterscheiden, bei denen zum einen ein Sachverhalt selbst modelliert wird - in diesem Falle dient das hydrophile Innere des im Versuch verwendeten Kronenethers als Modell für Ionenkanäle - und bei denen zum anderen mit 
und an Modellen bzw. Modellsubstanzen experimentiert wird [248]. Letzteres lässt einen Brückenschlag zur aktuellen Membranforschung zu, in der an biomimetischen Membranen geforscht wird, die als Modelle für echte Biomembranen dienen. Einen weiteren Bezug zur Forschung ermöglicht zudem die theoretische Betrachtung der Patch-ClampTechnik, die zur Untersuchung von Ionenkanälen eingesetzt wird und für die mit ERWIN NEHER und BERT SAKMANN zwei weitere Göttinger Naturwissenschaftler 1991 mit dem Nobelpreis - in diesem Falle für Physiologie oder Medizin - ausgezeichnet wurden [304]. Anhand dieser Technik lassen sich zudem weitere curricular relevante Themen wie der elektrochemische Gradient und das Ruhepotential thematisieren.

Im letzten Abschnitt der Einheit zur aktuellen Forschung erfahren die SuS zunächst im Zuge eines Vortrags der Lehrkraft oder einer eingeladenen Wissenschaftler_in und anschließend anhand eines Films zum SFB 803 mehr über die Ziele, Inhalte und Methoden der aktuellen Membranforschung. Dabei wird insbesondere die Interdisziplinarität der Forschung betont. Zudem wird in Bezug auf die Forschungsfelder des SFB die gesellschaftliche Relevanz der anhaltenden Forschung hervorgehoben und ihre Bedeutung in für SuS interessanten Kontexten wie der Entwicklung neuer Medikamente diskutiert. Falls in der vorherigen Sequenz noch nicht geschehen, kann hier noch einmal erläutert werden, warum Membranforschung vorzugsweise an Modellmembranen stattfindet und es bietet sich an, den entwickelten Modellbegriff noch einmal zu überprüfen.

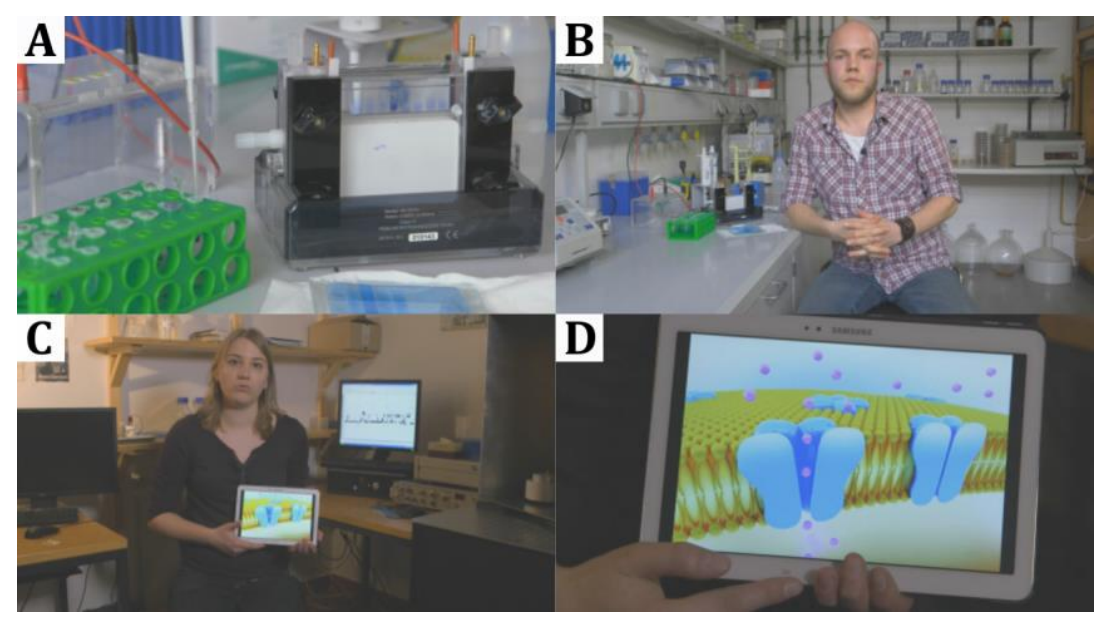

Abbildung 88: Ausschnitte aus dem in Kooperation mit BEILSTEIN TV produzierten Film zur Forschung des SFB 803.

\subsubsection{Evaluation}

Die Unterrichtseinheit wurde im Zuge des Projektes SFB@School in fünf aufeinanderfolgenden Wochen in einem Ergänzungs- und Wahlfachkurs Chemie einer 11. Klasse eines Gymnasiums durchgeführt. Die Evaluation erfolgte im Anschluss an die Einheit sowohl anhand eines kurzen Schülerfragebogens mit sieben geschlossenen Fragen sowie einem offenen Kommentarfeld als auch anhand eines abschließenden Gesprächs zwischen der die Einheit durchführenden Studentin und der Lehrkraft. Die geschlossenen Fragen des Fragebogens bezogen sich dabei auf mögliche Einblicke in die Membranforschung, das Interesse sowohl an der aktuellen Membranforschung als auch an einem naturwissenschaftlichen Studium, die verwendeten Methoden, Materialien und Experimente sowie das Modellverständnis der 14 teilnehmenden SuS.

Die die Unterrichtseinheit und ihre Lernformen sowie -materialien betreffenden Fragen wurden von den SuS auf einer fünfstufigen Likert-Skala im Durchschnitt als eher lernför- 
derlich und motivierend bewertet. Zudem gaben bis auf zwei SuS, die eine neutrale Haltung einnahmen, alle an, dass die Inhalte der Einheit ihnen einen Einblick in die Membranforschung vermittelt hätten. Bezüglich eines erweiterten Verständnisses über den Einsatz und die Funktion von Modellen äußerten sich die meisten zudem neutral bis positiv. Insgesamt konnte die Unterrichtseinheit jedoch weder dazu beitragen, das Interesse an einem naturwissenschaftlichen Studium, noch an der aktuellen Membranforschung zu fördern. Die wenigen freiwilligen Kommentare der SuS bestätigen dieses Bild noch einmal. Während die Struktur der Einheit und auch die Auswahl der Methoden und Experimente gelobt wurden, wurde vor allem der zu theoretische Bezug zur Forschung negativ angemerkt. Im Gespräch mit der Lehrkraft hob diese sowohl die gute Strukturierung der Einheit, als auch die Möglichkeit zur Förderung der Modellkompetenz positiv hervor. Zudem hatte sie den Eindruck, dass die SuS aufgrund des interdisziplinären Ansatzes ein besseres Verständnis für die Rolle chemischer Aspekte in der Biologie entwickelt hätten.

Dass eine einmalige Intervention nicht zu einem gesteigerten Interesse an einem naturwissenschaftlichen Studium oder der Membranforschung führt, ist mit Blick auf die in Kapitel 3.1.2 dargestellten Interessentheorien nicht verwunderlich. Zudem ermöglicht eine Fragebogenstudie im Post-Design im besten Fall eine Erhebung des Ist-Zustands, lässt jedoch keine Aussagen über eine Veränderung des Interesses zu. Trotz insgesamt relativ kleiner Stichprobe ist die ansonsten positive Einschätzung der Unterrichtseinheit durch die SuS sowie die Lehrkraft durchaus erfreulich. Gerade für einen Ergänzungs- und Wahlfachkurs, der zwar inhaltlich an curriculare Vorgaben, jedoch nicht an zentrale Prüfungen gebunden ist, stellt eine solche Einheit eine gute Möglichkeit dar, curriculare Inhalte in einem interdisziplinären Kontext mit Blick auf die aktuelle Forschung zu vermitteln. Eine Einbettung der Unterrichtseinheit in den (abiturrelevanten) Regelunterricht, sei es im Fach Chemie oder Biologie, wäre nach den niedersächsischen Kerncurricula (G9) [182, 183] in der Einführungsphase, d. h. der 11. Klassenstufe sinnvoll. Insbesondere die fünfte Sequenz zur aktuellen Forschung, erfordert jedoch entweder ausgeprägte Kenntnisse der Lehrkraft in Bezug auf die aktuelle Membranforschung, oder eine Kooperation mit einer Forschungseinrichtung aus diesem Bereich. Während in Göttingen und Umgebung, aber auch in anderen größeren Städten, sicherlich die Möglichkeit bestünde, Wissenschaftler_innen in die Schule einzuladen, ist dies jedoch nicht immer und überall der Fall.

\subsubsection{Erweiterter Unterrichtsmaterialienpool „Vom Tensid zur Biomembran“}

Das generelle Ziel des Ö-Projekts des SFB 803 ist es zwar, Einblicke in die aktuelle Membranforschung zu geben, mit Blick in Richtung einer Implementierung einer solchen Einheit in den Regelunterricht muss jedoch kritisch hinterfragt werden, in welcher Tiefe dieses Ziel tatsächlich erreicht werden kann. Hierbei muss auch die spezifische Rolle des SFB 803 reflektiert werden: Ist es das Ziel der Öffentlichkeitsarbeit, die Zielgruppe der SuS explizit für die Membranforschung zu begeistern, um somit auf lange Sicht Nachwuchs in diesem spezifischen Bereich zu generieren? Oder sollte der Anspruch nicht vielmehr ein Beitrag zur Förderung des situationalen Interesses anhand des Beispiels der Membranforschung sein, das der generellen Förderung des naturwissenschaftlichen Interesses dient?

Wird die beschriebene Unterrichtseinheit vor diesem Hintergrund noch einmal kritisch reflektiert, fällt auf, dass zwar mehrere Methoden der Membranforschung beschrieben werden, dass deren Betrachtung jedoch jederzeit nur theoretisch erfolgt. Die eigentliche Stärke der Einheit liegt vielmehr darin, dass sie implizit zwei wichtige Aspekte der Forschung vermittelt. Diese sind zum einen die Relevanz naturwissenschaftlicher Modelle für 
den Erkenntnisprozess und zum anderen die Interdisziplinarität naturwissenschaftlicher Forschung.

Eine Weiterentwicklung der Unterrichtseinheit erfolgte somit nicht in Hinblick auf die Forschung, sondern vielmehr in Bezug auf curriculare Anforderungen der Unterrichtsfächer Chemie und Biologie. In Anlehnung an die fünf Sequenzen des SFB@SCHOOL-Projekts wurden - mit Blick auf die Schule aber auch auf andere SO-Aktivitäten - die in der Einleitung zu Kapitel 5 genannten Leitfragen und daraus hervorgehend die sechs Themenfelder des experimentellen Teils dieser Arbeit abgeleitet. Insbesondere die ersten vier Themenfelder, die sich bereits in den Sequenzen der Unterrichtseinheit wiederfinden, wurden diesbezüglich um einige zum größten Teil neu entwickelte Experimente erweitert. Der daraus entstandene Pool an Unterrichtsmaterialien, der u. a. auch Vorschläge für mögliche Arbeitsaufträge enthält, deckt zahlreiche inhaltliche Aspekte des Biologiecurriculums der Oberstufe in Bezug auf Biomembranen und Membranprozesse ab, die mithilfe chemischer (Modell-)Experimente erarbeitet oder veranschaulicht werden können. Aufgrund seines Umfanges sollte er jedoch nicht als fertige Unterrichtseinheit, sondern vielmehr als Materialsammlung verstanden werden, deren Inhalte von Lehrkräften je nach Bedarf verwendet werden können. Die entsprechenden Inhalte und Experimente der einzelnen Sequenzen können der nachfolgenden Tabelle entnommen werden.

Tabelle 14: Inhalte und korrespondierende Experimente der verschiedenen Sequenzen des Materialienpools mit dem Titel Vom Tensid zur Biomembran.

\begin{tabular}{lll}
\hline Sequenz & Inhalte & Experimente \\
\hline $\begin{array}{l}\text { Struktur-Eigen- } \\
\text { schaftsbeziehun- } \\
\text { gen von Tensiden }\end{array}$ & $\begin{array}{l}\text { Eigenschaften (Oberflächenaktivität, benetzende Wirkung, Dis- } \\
\text { pergiervermögen) und Struktur von Tensiden, Hydrophilie, Lip- } \\
\text { ophilie, Amphiphilie }\end{array}$ & $\begin{array}{l}1.1 \mathrm{a} / \mathrm{b} / \mathrm{c}, \\
1.2 \mathrm{a} / \mathrm{b}, 1.3 \mathrm{a} / \mathrm{b}\end{array}$ \\
\hline $\begin{array}{l}\text { Selbstorganisation } \\
\text { zu Mizellen und } \\
\text { Monolayern }\end{array}$ & $\begin{array}{l}\text { Modelle in den Naturwissenschaften, Entwicklung von Modellen } \\
\text { zur Selbstorganisation von Tensiden }\end{array}$ & $2.1 \mathrm{a} / \mathrm{b}, 2.2,2.4$ \\
\hline $\begin{array}{l}\text { Von der Seifen- } \\
\text { blase zur Membran }\end{array}$ & $\begin{array}{l}\text { Vergleich Seifenhäute und Biomembranen auf submikroskopi- } \\
\text { scher Ebene, Membranmodelle im Wandel der Zeit, Membran- } \\
\text { bestandteile }\end{array}$ & $3.2 \mathrm{a} / \mathrm{b}$ \\
\hline Membrantransport & $\begin{array}{l}\text { Stofftransport zwischen Kompartimenten, erleichterte Diffu- } \\
\text { sion, Exocytose }\end{array}$ & $4.1 \mathrm{~b}, 4.2,4.3 \mathrm{a}$ \\
\hline
\end{tabular}

\subsubsection{Evaluation}

Die Unterrichtsmaterialien wurden im Rahmen einer Lehrerfortbildung präsentiert und im Anschluss an die Veranstaltung von einer Lehrkraft aus dem Raum Göttingen angefordert. Die Materialien wurden dabei von ihr und zwei weiteren Lehrkräften verwendet, um das Thema Biomembranen im Biologieunterricht in drei elften Klassen (Einführungsphase) parallel zu unterrichten. Den Lehrenden war es dabei freigestellt, in welchem Maße sie die bereitgestellten Materialien nutzen, verändern oder durch eigene Unterrichtsmaterialien ergänzen.

\section{Forschungsinteresse}

Obwohl die Unterrichtsmaterialien vor dem Hintergrund der Interessenförderung von SuS entwickelt wurde, bezog sich das Forschungsinteresse bezüglich der Evaluation der Materialien vornehmlich auf die Gruppe der Lehrkräfte. Zwar können Lehrkräfte das Interesse von SuS bezüglich der Thematik höchstens subjektiv einschätzen, eine Erhebung der interessenfördernden Wirkung der Materialien hätte jedoch bedeutet, dass die Mate- 
rialien erneut zu einer Unterrichtseinheit hätten zusammengefasst, die von allen teilnehmenden Lehrkräften gleichermaßen hätte durchgeführt werden müssen, was gegen die Idee eines Materialienpools sprach. Vielmehr war es von Interesse, in welchem Maße die Lehrkräfte die Materialien in ihren bestehenden Unterricht einbinden, woraus sich die folgenden Forschungsfragen ergeben:

F1 Inwiefern verwenden oder erweitern die Lehrkräfte die bereitgestellten Materialien für die Gestaltung ihres Unterrichts zum Thema Biomembranen?

F2 Welchen Nutzen sehen die Lehrkräfte in der interdisziplinären Erarbeitung des Themas der Biomembranen?

F3 Inwiefern planen die Lehrkräfte, die von ihnen verwendeten Materialien auch weiterhin in ihren Unterricht zum Thema Biomembranen zu integrieren?

\section{Evaluationsdesign}

Hinsichtlich des Evaluationsdesigns fiel die Entscheidung auf die Durchführung eines Leitfadeninterviews. Da die Lehrkräfte bereits im Voraus äußerten, dass sie die von ihnen durchgeführte Unterrichtseinheit zum Thema Biomembranen unter Einbezug der zur Verfügung gestellten Materialien gemeinsam erarbeitet hatten, wurde auch das Interview mit allen drei Lehrkräften gleichzeitig geführt. Dabei wurde versucht, das Interview mithilfe von Erzählaufforderungen zunächst so offen wie möglich zu gestalten [305]. Um im Sinne der ersten Fragestellung in Erfahrung zu bringen, wie die von ihnen erarbeitete Unterrichtseinheit aussah, wurden die Lehrkräfte deshalb gebeten, diese so ausführlich wie möglich zu beschreiben. Erst im Nachhinein wurden den Lehrkräften weitere konkretere Fragen bezüglich der Fragestellungen F2 und F3 gestellt.

\section{Evaluationsergebnisse}

F1 Inwiefern verwenden oder erweitern die Lehrkräfte die bereitgestellten Materialien für die Gestaltung ihres Unterrichts zum Thema Biomembranen?

Zum Einstieg in die Unterrichtssequenz folgten die Lehrkräfte zunächst insgesamt den zur Verfügung gestellten Materialien zu den Struktur-Eigenschaftsbeziehungen von Tensiden. Zur Wiederholung wichtiger chemischer Konzepte erweiterten sie diese um eine Videosequenz zur Polarität von Molekülen und der Ausbildung von Wasserstoffbrückenbindungen. Im zweiten Abschnitt nutzen die Lehrkräfte sowohl Streichholzmodelle als auch die beigelegten konischen und zylindrischen 3D-Modelle um die Selbstorganisation amphiphiler Teilchen mit den SuS nachzuvollziehen. Sie verzichteten an dieser Stelle auf weitere Experimente und gingen mithilfe des Biologiebuchs dazu über, die Bestandteile von Biomembranen zu erarbeiten. Im Anschluss nutzten sie den in der dritten Sequenz vorgeschlagenen Bau von Membranmodellen im Wandel der Zeit, die von Expertengruppen erarbeitet und vorgestellt wurden. Für das sich anschließende Thema des Membrantransports gingen alle drei Lehrkräfte dazu über, das Biologiebuch zu verwenden.

\section{F2 Welchen Nutzen sehen die Lehrkräfte in der interdisziplinären Erarbeitung des Themas} der Biomembranen?

Die Lehrkräfte erachteten vor allem die Erarbeitung der Thematik über den amphiphilen Charakter der Tenside und ihrer Selbstorganisation als hilfreich für das Verständnis der Biomembranen, da diese den Übergang zu Lipiden und ihrer Anordnung in Membranen erleichterten. Eine Lehrkraft fasste die Vorzüge der Unterrichtssequenz in Bezug auf die Forschungsfrage wie folgt zusammen: 
„Also es ist einiges auf der Mikroebene klargeworden, was vorher einfach nur auf der Modellebene klar wurde. [...] Das ist absolut genial, dass die Schüler jetzt ne Vorstellung haben, wie Leben sich hat [...] entwickeln können, weil sie sehen, ne Seifenblase entsteht von alleine, ohne dass es lebendig ist, es können Kompartimente durch Selbstorganisation von amphiphilen Teilchen entstehen. [...] Und das ist wirklich deutlich geworden. Grundlegend deutlich. Viel deutlicher als sonst und [...] jetzt haben sie [...] ne ganz andere existenzielle Vorstellung davon wie Leben entstanden sein kann. Und wir haben das an ganz vielen Stellen in Schule [...], dass die auf der Modellebene einsteigen und dann denken sie wissen alles, aber auf [...] ner Teilchenebene zum Beispiel überhaupt gar nichts erklären können."

F3 Inwiefern planen die Lehrkräfte, die von ihnen verwendeten Materialien auch weiterhin in ihren Unterricht zum Thema Biomembranen zu integrieren?

Trotz aller positiver Erfahrungen mit den Materialien und dem Gefühl eines tieferen Verständnisses der SuS bereitete die Länge der Unterrichtssequenz Probleme, für die die Lehrkräfte mit fünf bis sechs Doppelstunden deutlich mehr Zeit aufbrachten als gewöhnlich. Diesbezüglich sehen sie vor allem die großen Abstände zwischen den einzelnen, nur einmal wöchentlich stattfindenden Doppelstunden kritisch, da es den SuS schwerfiel, die Erkenntnisse bezüglich der Tenside nach mehreren Wochen auf den Kontext der Biomembranen zu übertragen, zumal zusätzlich auch die Weihnachtsferien in die Sequenz fielen. Eine der Lehrkräfte erklärte das Problem wie folgt:

\section{„Und das liegt daran, dass diese vielen Versuche und Auswertungen eben und der suk- zessive Aufbau, so charmant er ist, einfach in seiner Quantität nicht in die Stundenvor- gabe reinpasst."}

Dass sei auch der Grund dafür, warum diese Lehrkraft die Experimente zu den StrukturEigenschaften der Tenside beim nächsten Mal eher weglassen würde. Alle drei Lehrkräfte gaben jedoch an, in Zukunft die 3D-Modelle in den Unterricht einbinden zu wollen, um die Selbstassemblierung der Lipide zu verdeutlichen. Insgesamt standen die Lehrkräfte zudem auch dem Format des Unterrichtsmaterialienpools zwiespältig gegenüber. Während die Möglichkeit der freien Wahl der Experimente und Materialien prinzipiell als positiv empfunden wurde, wurde dennoch der Wunsch nach mehr Lenkung, bspw. in Form von Kurzentwürfen, geäußert.

\subsubsection{Reflexion des Formats}

Die bereits im SFB@SCHOOL-Projekt entwickelte Unterrichtseinheit diente als Grundlage für die Entwicklung von weiteren Experimenten zur Erarbeitung relevanter naturwissenschaftlicher Konzepte, die für ein Verständnis der Forschung des SFB eine Rolle spielen. Durch Anknüpfung an Alltagserfahrungen der SuS sowie interdisziplinär curricular relevante Inhalte, sollten Einblicke in die Forschung des SFB ermöglicht werden. Während die Eigenschaften von Biomembranen durch die SuS experimentell erarbeitet werden konnten, erfolgte der Blick in die Forschung jedoch lediglich auf theoretischer Ebene.

Eine tatsächliche authentische experimentelle Auseinandersetzung mit Forschungsmethoden scheint auch nach Entwicklung neuer Experimente nur in sehr geringem Maße möglich, da vor allem zeitliche Restriktionen eine Durchführung forschungsnaher Experimente im schulischen Rahmen erschweren. Um die Unterrichtseinheit in ihrer originalen Fassung im naturwissenschaftlichen Unterricht dennoch verankern zu können, bedarf es auf Seiten der Lehrkräfte einer tiefergreifenden Auseinandersetzung mit den Inhalten der Membranforschung, was angesichts der Fülle ihrer Aufgaben nicht zwingend von ihnen erwartet werden kann. Für eine explizite Einbindung von Forschungsinhalten in 
den naturwissenschaftlichen Unterricht eignet sich eine solche Unterrichtseinheit deshalb nur bedingt. Hier scheint es sinnvoller, Einblicke in die Forschung und den Alltag von Wissenschaftler_innen im Rahmen anderer SO-Formate wie Schülerlaboren anzubieten, für die geschultes Personal zur Verfügung steht.

Dies soll jedoch nicht implizieren, dass die Entwicklung von Unterrichtsmaterialien hinsichtlich einer projektgebundenen Öffentlichkeitsarbeit keinen Mehrwert bietet. Vielmehr scheint es sinnvoll, Lehrkräften Experimente sowie Vorschläge zur Einbindung dieser in den Fachunterricht an die Hand zu geben, die durch eine Verknüpfung curricular relevanter Inhalte verschiedener naturwissenschaftlicher Disziplinen implizit authentische Einblicke in die Interdisziplinarität naturwissenschaftlicher Forschung ermöglichen, sodass die einzelnen Fachrichtungen von SuS nicht mehr als strikt getrennt voneinander wahrgenommen werden. Die Äußerungen der Lehrkräfte deuten diesbezüglich zudem an, dass die fächerübergreifende Betrachtung der Membranen zu einem tiefergreifenderen Verständnis bei den SuS geführt hat. Gerade im Zuge eines länger währenden Projekts, in dessen Verlauf kontinuierlich weitere Experimente und Materialien entwickelt werden, scheint ein solcher Pool an Unterrichtsmaterialien, der jederzeit erweitert werden kann, deshalb sinnvoll.

Das Interview mit den Lehrkräften zeigte zudem, dass sie sich, trotz der freien Wählbarkeit der Materialien, eine deutlichere Darstellung möglicher Verknüpfungen der Experimente sowie der Anbindung an die jeweiligen Kerncurricula wünschen, was insbesondere in den jeweiligen Abschnitten zur „Einordnung der Experimente im Kontext der SO-Leitfragen" noch einmal expliziert wurde. Hier finden sich darüber hinaus auch weiterführende Vorschläge für die Anknüpfungen an die Lebenswelt der SuS sowie an die Forschung des SFB.

Insgesamt kann zusammengefasst werden, dass sich die Entwicklung von Unterrichtsmaterialien zur Einbindung von Forschungsinhalten in den MINT-Unterricht, zumindest hinsichtlich der Forschung des SFB 803, nur bedingt als SO-Format eignet. Obwohl die Forschung an Biomembranen auf den ersten Blick zahlreiche Anknüpfungspunkte an die Inhalte insbesondere des Biologieunterrichts der Sekundarstufe II bietet, ist gerade die Grundlagenforschung im Bereich der Biomembranen so speziell, dass die benötigten Kenntnisse zum Verstehen der Forschungsinhalte schnell die curricularen Vorgaben übersteigen. Entsprechend bieten sich diesbezüglich non-formale und informelle SO-Bildungskontexte eher an, um Einblicke in die explizite Forschung zu ermöglichen.

\subsection{Non-Formal Intentional - Schülerlabore}

Das non-formal intentionale Setting eines Schülerlabors bietet im Rahmen von SO die Möglichkeit, die zwar gesellschafts- aber nicht unbedingt curriculumsrelevanten Inhalte aktueller Forschung an curriculare Inhalte anzuknüpfen und außerhalb der Restriktionen formaler Settings sowohl Einblicke in aktuelle Forschungsthemen und -methoden als auch in den wissenschaftlichen Alltag zu geben. Entsprechend wurde bei der Entwicklung des hier vorgestellten Schülerlabors der Transfer von Forschungsinhalten in einen für SuS interessanten und relevanten Kontext fokussiert.

Hinsichtlich der thematischen Ausgestaltung des Schülerlabors fiel die Wahl auf die aktuelle Forschung an SNARE-Komplexen, da diese eine fundamentale Rolle bei der Erregungsweiterleitung im Nervensystem spielen, die auch in der Qualifikationsphase des Biologieunterrichts thematisiert wird. Da konkrete Forschungsinhalte des SFB, wie die Selbstorganisation von Transmembran-Peptidhelices in Lipidmembranen oder die 
SNARE-vermittelte Membranfusion, sowie der Nutzen der daraus abgeleiteten Erkenntnisse auch im Rahmen eines Schülerlabors noch zu abstrakt sind, mussten diese zunächst in einen für SuS relevanten Kontext eingebettet werden. Gerade im Bereich der Biomembranforschung liegen hier medizinische Kontexte nahe, da SNARE-Proteine u. a. Angriffspunkte für Tetanus- und Botulinumtoxine sind (vgl. Exkurs zu SNARE-Komplexen in Kapitel 5.4). Obwohl beide Toxine keine explizite Rolle in der Forschung des SFB spielen, bietet sich gerade Botulinumtoxin als thematischer Aufhänger für ein Schülerlabor an, da es zum einen maßgeblich zu Erkenntnissen bezüglich SNARE-Proteinen und Komplexen beigetragen hat [241-243] und zudem auch vielfältige gesellschaftliche Anknüpfungspunkte besitzt.

\section{Exkurs: Botulinumtoxin in Gesellschaft und Wissenschaft}

Das Nervengift Botulinumtoxin ist insbesondere unter dem Handelsnamen Botox bekannt, das vornehmlich im Bereich der ästhetisch-plastischen Chirurgie zur Glättung von Falten eingesetzt wird. Während Menschen älterer Generationen oftmals in Boulevardzeitschriften mit Vorher/Nachher-Vergleichen prominenter AnwenderInnen von Botox in Berührung kommen, ist das Thema in gewissem Sinne auch für Jugendliche präsent. Denn in Zeiten von Social Media spielen das Erscheinungsbild und die eigene sowie fremde Körperwahrnehmung eine zumeist unterschätzte Rolle im Leben von SuS. Obwohl (z. T. unrealistische) Schönheitsideale schon immer von den Medien transportiert und geprägt wurden [306], begegnen Nutzer_innen von vor allem bildbasierten Social Media Kanälen wie Instagram ganztägig perfekt inszenierten Fotos. Dabei werden nicht selten Filter und andere Apps zur Bildbearbeitung eingesetzt, um z. B. das Gesicht porenfreier, glatter und dünner zu gestalten [307]. Eine Übersichtsarbeit zu den Auswirkungen der Nutzung von Social Media auf das Selbstbild zeigt jedoch, dass sich die Nutzung insbesondere bildbasierter Plattformen negativ auf die eigene Körperwahrnehmung und das Essverhalten (im Sinne von Essstörungen) auswirkt [308]. Weitere Studien mit Frauen zwischen 18 und 29 Jahren zeigen zudem, dass die Nutzung von Social Media-Kanälen den Wunsch nach schönheitschirurgischen Eingriffen steigern kann [309]. Die Tatsachen, dass im Jahr 2018 bereits $48 \%$ der deutschen Jugendlichen zwischen 14 und 19 Jahren Instagram täglich und sogar $62 \%$ mindestens einmal wöchentlich nutzten [310], dass Botulinum- und Fillerbehandlung zu den beiden beliebtesten ästhetischen Behandlungen zählen [311] und dass über zwei Drittel der Chirurgen in einer Befragung der DEUTSCHEN GESELLSCHAFT FÜR ÄSTHETISCH-PlastisCHE CHIRURGIE (DGÄPC) angaben, dass Patienten (vornehmlich zwischen 18 und 25 Jahren) ihr eigenes, mit Filtern bearbeitetes Selbstportrait als Vorlage für die Behandlung mitgebracht haben [312], unterstreichen die Relevanz des Themas auch für die persönlich Dimension von SuS (nach STUCKEY ET AL. ${ }^{[78]}$ ) - wenngleich eher aus extrinsischer Perspektive.

Beim Nervengift Botulinumtoxin (BoNT) handelt es sich um vom Bakterium Clostridium botulinum unter anaerobischen Bedingungen produzierte Proteine, die in acht Serotypen (A-H) vorkommen [313, 314]. Die Toxine bestehen aus einer leichten und einer schweren Kette und können in verschiedene Domänen mit unterschiedlichen Funktionen differenziert werden. Während die Bindungsdomäne der schweren Kette für die Bindung an und die Endocytose in präsynaptische Nervenendigungen zuständig ist, sorgt die membranüberspannende Domäne für die Translokation der katalytischen Domäne (leichte Kette) in das Zytosol der Zelle, wo diese je nach Serotyp eines der SNARE-Proteine Synaptobrevin, SNAP-25 oder Syntaxin spaltet (siehe Abbildung 89) [313]. Um bei oraler Einnahme widrige $\mathrm{pH}$-Umgebungen im Gastrointestinaltrakt zu überwinden, sind die beiden Ketten zudem mit Hämaglutininen und weiteren nicht-toxischen Proteinen komplexiert [315] 

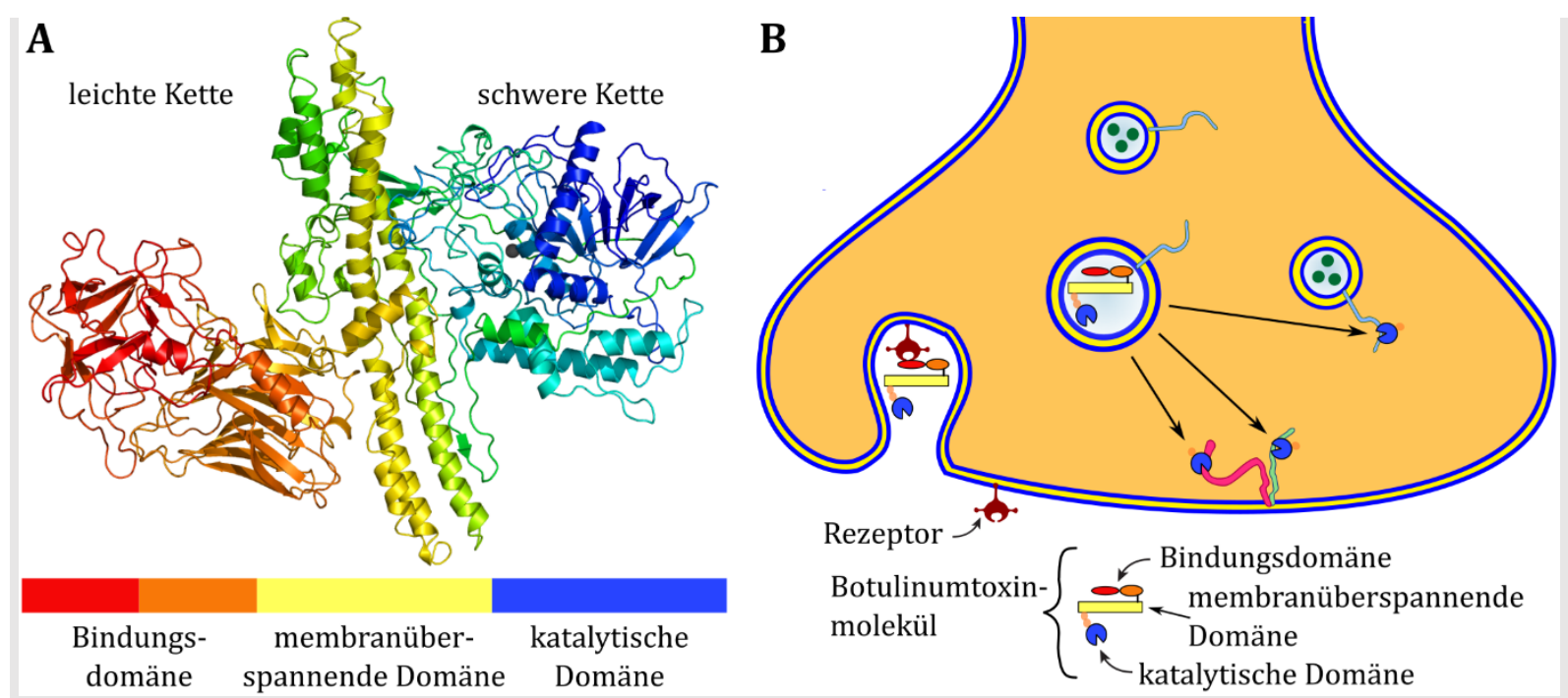

Abbildung 89: (A) Bändermodell des Botulinumtoxins (BoNT/A1). (B) Schematische Darstellung des Wirkmechanismus von Botulinumtoxin (in Anlehnung an RossetTo, PIrAZZinI \& MonTECUCCO [313]).

Die mit der Einnahme von BoNT einhergehende Krankheit Botulismus (lt. Botulus $=$ Wurst), die sich durch Lähmungserscheinungen mit häufiger Todesfolge äußert, wurde erstmals im 18. Jahrhundert nach dem Genuss von verdorbenen Würsten beobachtet [316]. Diese Lähmungserscheinungen sind auf die Spaltung der SNARE-Proteine und die dadurch unterdrückte Bildung des SNARE-Komplexes bzw. der Exocytose der synaptischen Vesikel und die fehlende Ausschüttung von Neurotransmittern in den synaptischen Spalt zu erklären. Obwohl es sich bei BoNT um das stärkste bekannte biologische Gift handelt (wenige Nanogramm reichen für eine Vergiftung aus) [316], findet das Toxin dennoch Anwendung in zahlreichen medizinischen Kontexten. Neben der kosmetischen Verwendung zur Faltenglättung wird BoNT u. a. auch bei Strabismus (Schielen), zur Behandlung von Dystonien (Bewegungsstörungen), bei Migräne sowie bei übermäßiger Schweißsekretion eingesetzt. Dabei handelt es sich bei zahlreichen Verwendungen um Off-Label-Indikationen, die nur bedingt in Deutschland zugelassen sind, bzw. von Krankenkassen übernommen werden [317, 318].

Trotz vieler medizinisch sinnvoller Anwendungen muss der Nutzen in Bezug zu möglichen Schäden und Missbräuchen bewertet werden. Aufgrund der hohen Toxizität von BoNT muss jede neue Charge einem $\mathrm{LD}_{50}$-Test unterzogen werden, da bereits kleinste Schwankungen in der Verdünnung fatale Folgen haben können. Obwohl erste tierfreie Alternativen entwickelt wurden, werden Schätzungen zufolge allein in Europa rund 400.000 Mäuse für $\mathrm{LD}_{50}$-Tests verwendet, die zumeist nach drei bis vier Tagen qualvoll ersticken [319]. Anfang 2018 machte zudem eine eindeutig fragwürdige tierische Nutzung von Botox Schlagzeilen. In einem Schönheitswettbewerb zur „Miss Kamel“ in Saudi-Arabien wurden demnach zwölf Kamele wegen der Verwendung von Botox disqualifiziert [320]. Zudem fällt BoNT unter das deutsche Kriegswaffenkontrollgesetz [321] und wurde seit dem zweiten Weltkrieg immer wieder in kriegerischen Auseinandersetzungen angewendet [322]. Mehrere Szenarien deuten zudem auf die Potentiale von bereits $1 \mathrm{~g}$ BoNT als bioterroristische Waffe mit möglichen Todesfällen in der Größenordnung von bis zu 1,5 Millionen Menschen hin [323,324].

Aufgrund seiner Relevanz im Sinne der persönlichen, aber vor allem der gesellschaftlichen Dimension wurde das Thema Botox als geeignet für das gesellschaftskritisch-problemorientierte Unterrichtsverfahren identifiziert. Dieses Unterrichtsverfahren, das wie in Kapitel 2.2 erwähnt eher die gesellschaftliche Relevanzdimension anspricht, orientiert sich am Ansatz der Education through Science von HoLBROOK \& RANNIKMAE [325], das statt 
dem alleinigen Vermitteln von naturwissenschaftlichen Inhalten durch Unterricht (Science through Education), das Erlangen einer für die SuS relevanten Allgemeinbildung durch den naturwissenschaftlichen Unterricht vorsieht, die zur Scientific Literacy der SuS beitragen soll [87]. Gerade die Auswahl kontroverser Themen soll hierbei zudem eine gezielte Förderung der Kommunikations- sowie der Bewertungskompetenz ermöglichen. Wie in Tabelle 15 dargestellt basiert das Konzept des gesellschaftskritisch-problemorientierten Chemieunterrichts auf einem Vier-Säulen-Modell [88], das sowohl die Ziele, die Kriterien für die Themenwahl, die methodischen Konsequenzen als auch die Struktur der Einheit definiert.

Tabelle 15: Vier-Säulen-Modell des gesellschaftskritisch-problemorientierten Chemieunterrichts nach EILKS [326],

\begin{tabular}{|c|c|c|c|}
\hline Ziele & $\begin{array}{l}\text { Kriterien für die } \\
\text { Themenwahl }\end{array}$ & $\begin{array}{l}\text { Methodische } \\
\text { Konsequenzen für die } \\
\text { Umsetzung }\end{array}$ & $\begin{array}{l}\text { Struktur des } \\
\text { Unterrichts }\end{array}$ \\
\hline $\begin{array}{l}\text { Allgemeinbildung/ } \\
\text { „education through sci- } \\
\text { ence“ }\end{array}$ & Authentizität & $\begin{array}{l}\text { Authentische Alltags- } \\
\text { medien }\end{array}$ & $\begin{array}{l}\text { 1. Zugang und Analyse } \\
\text { der Kontroverse }\end{array}$ \\
\hline $\begin{array}{l}\text { (Multidimensional) Sci- } \\
\text { entific Literacy }\end{array}$ & Relevanz & $\begin{array}{l}\text { Schülerorientiertes und } \\
\text { experimentelles Lernen } \\
\text { von Chemie }\end{array}$ & $\begin{array}{l}\text { 2. Fachliche Klärung un- } \\
\text { ter Einbezug experi- } \\
\text { menteller Arbeit }\end{array}$ \\
\hline $\begin{array}{l}\text { Förderung von Bewer- } \\
\text { tungskompetenz }\end{array}$ & $\begin{array}{l}\text { Bewertungslage offen } \\
\text { bzgl. gesellschaftlich re- } \\
\text { levanter Fragen }\end{array}$ & $\begin{array}{l}\text { Schülerzentrierte und } \\
\text { kooperative Lernfor- } \\
\text { men }\end{array}$ & $\begin{array}{l}\text { 3. Wiederaufgreifen der } \\
\text { kontroversen Prob- } \\
\text { lemlage }\end{array}$ \\
\hline $\begin{array}{l}\text { Förderung von Kom- } \\
\text { munikationskompetenz }\end{array}$ & Offene Diskutierbarkeit & $\begin{array}{l}\text { Methoden zur Struktu- } \\
\text { rierung kontroverser } \\
\text { Debatten }\end{array}$ & $\begin{array}{l}\text { 4. Erarbeitung und Dis- } \\
\text { kussion verschiede- } \\
\text { ner Perspektiven }\end{array}$ \\
\hline $\begin{array}{l}\text { Naturwissenschaftliche } \\
\text { Kenntnisse und Fähig- } \\
\text { keiten erlernen }\end{array}$ & $\begin{array}{l}\text { Fragestellung mit Be- } \\
\text { zug zu Chemie und } \\
\text { Technik }\end{array}$ & $\begin{array}{l}\text { Methoden zur Provoka- } \\
\text { tion und Explikation in- } \\
\text { dividueller Meinung }\end{array}$ & 5. Metareflexion \\
\hline
\end{tabular}

\subsubsection{Schülerlabor „Gifte für die Schönheit“}

Insbesondere mit Blick auf die kontroverse Nutzung von BoNT und dessen Bezug zur aktuellen Forschung an SNARE-Proteinen, erfüllt Botox in ausreichendem Maße die Kriterien zur Themenwahl. Anhand der letzten beiden Säulen zur Methodik und Struktur der Unterrichtseinheit wurde deshalb ein Schülerlabor mit dem Titel Gifte für die Schönheit Ein Schülerlabor zu Biomembranen am Beispiel des Nervengifts Botulinumtoxin entwickelt, das sich in die vier in Tabelle 16 dargestellten Abschnitte aufteilt.

Zum Einstieg in die Kontroverse werden zunächst Vorher/Nachher-Fotos von Prominenten präsentiert, die sich Botoxbehandlungen unterzogen haben, sowie kontroverse Zeitungsausschnitte und Videosequenzen gezeigt, die sich mit der Verwendung von Botox auseinandersetzen (u. a. bezüglich Botoxbehandlungen bei einem achtjährigen Mädchen). Anhand dieser Materialien werden von den SuS im Plenum sowohl Fragen formuliert (z. B. „Was ist Botox?", „Welche Gefahren gehen von Botox aus?", „Wie leicht lässt sich Botox erwerben?") als auch Hypothesen zur Wirkweise von Botox aufgestellt. 
Tabelle 16: Inhalte und korrespondierende Experimente der verschiedenen Sequenzen des Schülerlabors Gifte für die Schönheit.

\begin{tabular}{|c|c|c|}
\hline Sequenz & Inhalte & Experimente \\
\hline Biomembranen & $\begin{array}{l}\text { Einführung in die Kontroverse, Bestandteile von Biomembra- } \\
\text { nen, Selbstassemblierung, Transportmechanismen (erleichterte } \\
\text { Diffusion, Exocytose), Erregungsübertragung im Nervensystem, } \\
\text { Rolle von SNARE-Proteinen }\end{array}$ & $\begin{array}{l}3.2 \mathrm{a}, 2.4,4.1 \mathrm{a}, \\
4.3 \mathrm{a}\end{array}$ \\
\hline Botulinumtoxin & $\begin{array}{l}\text { Entdeckung, Struktur, Proteinstabilität im Gastrointestinaltrakt, } \\
\text { Wirkmechanismus, fluoreszenzspektroskopische Fusionsmes- } \\
\text { sungen, Laborführung }\end{array}$ & $\begin{array}{l}4.3 \mathrm{~b}, 5.7 \mathrm{a} / \mathrm{b} \\
6.3 \mathrm{~b}\end{array}$ \\
\hline Planspiel & $\begin{array}{l}\text { Diskussion der Frage „Soll Botox verboten werden?“, Metarefle- } \\
\text { xion }\end{array}$ & \\
\hline Ask a Scientist & $\begin{array}{l}\text { Kurzvortrag einer Wissenschaftler_in zum (Forschungs-) Alltag } \\
\text { im SFB 803, Klärung offener Fragen }\end{array}$ & \\
\hline
\end{tabular}

Zur fachlichen Klärung erhalten die SuS zunächst eine kurze Einführung in die Zellbiologie, die für SuS der Oberstufe eine Wiederholung fachwissenschaftlicher Inhalte aus der Sekundarstufe I darstellen sollte. Ein besonderes Augenmerk wird dabei auf die Bedeutung der Zellmembran gelegt, deren Bestandteile und Eigenschaften im Anschluss von den SuS anhand verschiedener Experimente erarbeitet werden. Neben der Selbstassemblierung von Lipiden zu planaren und kugelförmigen Strukturen, werden dabei auch Modellexperimente zu verschiedenen Transportprozessen wie der schnellen Diffusion und der Exocytose durchgeführt. Da die Erregungsübertragung im Nervensystem erst in der Qualifikationsphase thematisiert wird, muss deren Bedeutung für Prozesse wie die Muskelkontraktion ggf. erläutert werden. An diesem Punkt wird auch die Rolle von SNAREProteinen im Prozess der synaptischen Exocytose expliziert, so dass ein erster Brückenschlag zur Forschung des SFB 803 möglich ist. In Absprache mit der Lehrkraft sind in dieser Sequenz, insbesondere bei einem zeitlich eingeschränkten Laborbesuch, entsprechend des Vorwissens der SuS Kürzungen möglich.

Im Anschluss an eine Mittagspause beschäftigen sich die SuS näher mit dem Nervengift Botulinumtoxin. Dazu werden in einem Kurzvortrag die Historie des Botulismus, seine Symptome und die Wirkweise des Toxins dargelegt, bevor sie experimentell der Frage nachgehen, wie Botulinumtoxin bei oraler Aufnahme den Gastrointestinaltrakt überwindet. Im weiteren Verlauf werden die SuS in zwei Gruppen aufgeteilt. Während eine Gruppe (Modell-) Experimente zur Wirkweise von Botox sowie zu Selfquenching- und Dequenching-Effekten durchführt, erhält die andere Gruppe eine Führung durch die Labore des SFB 803 - angeleitet durch eine dort arbeitende Wissenschaftler_in - und führt mithilfe eines Fluorimeters Messungen zur Fusion zweier Vesikelpopulationen durch. Nach dem Wechsel der beiden Gruppen werden die Graphen der Fusionsmessungen mithilfe der Erkenntnisse zu Self- und Dequenching im Plenum gedeutet. Die Sequenz zu Botulinumtoxin schließt mit der Bewertung der Realitätsnähe der Tatortfolge „Feierstunde“ ab, in der einer der Hauptdarsteller nach Einnahme von Botox zunächst in Lebensgefahr schwebt, nach wenigen Tagen jedoch wieder bei der Arbeit anzutreffen ist.

Während der fachlichen Betrachtungen zu Botulinumtoxin wird lediglich dessen Wirkung bei oraler Aufnahme betrachtet. Um sowohl die Verwendung von Botox als ästhetisches Mittel als auch weitere kontroverse Aspekte in Bezug auf das Toxin aufzugreifen, ist der dritte Abschnitt der Schülerlabors von einem Planspiel geprägt. Zu Beginn dieses Planspiels lesen die SuS einen Zeitungsartikel zu sogenannten Botox-Parties, bei denen - in Anlehnung an klassische Tupperparties - Botoxbehandlungen in privaten Settings gleich 
bei mehreren Personen durchgeführt werden. Die Informationen im Text, die unter anderem auch auf den medizinischen Einsatz, sowie eine mögliche Bedrohung durch Botox als Waffe hinweisen, dienen dabei als Aufhänger für die während des Planspiels zu diskutierende „Frage Soll Botox verboten werden?“ Um, abgesehen von den fachlichen Informationen zu Botulinumtoxin, für die SuS eine Diskussionsgrundlage zu schaffen, wurden neun Rollenkarten entwickelt (Schönheitschirurg_in, Neurologe_in, Psychologe-In, Wissenschaftler_in, Tierschützer_in, Beautyredakteur_in, Patient_innen (zufrieden sowie unzufrieden) und Moderator_in), die anhand von Ausschnitten aus fachwissenschaftlichen Artikeln, Zeitungen, Webseiten und Blogs verschiedene Perspektiven zu Botox beleuchten. Dem Moderator, der eine Sonderrolle einnimmt und sowohl das Gespräch leitet, als auch für eine angenehme Diskussionsatmosphäre sorgt, stehen auf der Rollenkarte einige Schlagzeilen aus Zeitungsartikeln zur Verfügung, die er bei stockendem Gesprächsverlauf einwerfen kann. Die auf den anderen Rollenkarten zur Verfügung gestellten Informationen sollen von den SuS genutzt werden, um im Sinne einer Talkshow ihren Standpunkt in Bezug zur Ausgangsfrage zu vertreten. Die verschiedenen Rollen beschreiben dabei Positionen für und gegen ein Verbot von Botox, so dass die Bewertungslage insgesamt offen ist. In einer Metareflexion haben die SuS im Anschluss an die Diskussion, die nicht zwingend zu einem eindeutigen Ergebnis führen muss, noch einmal die Chance, unabhängig von ihrer jeweiligen Rolle Stellung zum Nutzen und zu den Gefahren von Botox zu nehmen.

Zu guter Letzt schließt das Schülerlabor mit einem Kurzvortrag einer Wissenschaftler_in aus dem SFB 803 ab, die in Kürze sowohl ihren Alltag als Wissenschaftler_in, als auch ihre Forschung beleuchtet. Dabei können die SuS eventuelle offene Fragen sowohl zu Botox als auch bezüglich der aktuellen Forschung klären. Im Zusammenspiel mit der Laborführung und den Fusionsexperimenten aus dem zweiten Abschnitt des Schülerlabors bietet dieses Ask-a-Scientist-Format die Möglichkeit, auch die berufliche Relevanzdimension, insbesondere in Bezug auf die berufliche Orientierung der SuS, anzusprechen.

Insgesamt ist das Schülerlabor als sechsstündiger Tageskurs (plus Mittagspause) konzipiert, konnte jedoch im Zuge dieser Arbeit in dieser Form nicht mehr evaluiert werden. Das im Rahmen dieses Projekts ausgearbeitete Thema „Gifte für die Schönheit“ wurde jedoch in das Ausstellungskonzept für Bildungsmessen integriert, das in Kapitel 6.4.1 näher erläutert wird.

\subsubsection{Reflexion des Formats}

Das non-formal intentionale Format eines Schülerlabors bietet die Möglichkeit, anknüpfend an das schulische Vorwissen der SuS, Einblicke in aktuelle naturwissenschaftliche Forschung zu geben. Um dabei die Relevanz der Grundlagenforschung des SFB im Bereich der Biomembranen für SuS zu explizieren, bedarf es jedoch einer Kontextualisierung der Forschungsinhalte. Die Wahl fiel diesbezüglich auf das Thema Botulinumtoxin, da das Toxin den Grundstein für die Forschung an SNARE-Proteinen legte und zudem die Möglichkeit zu einer gesellschaftskritischen Auseinandersetzung bietet.

Als Format projektgebundener Öffentlichkeitsarbeit bietet sich ein Schülerlabor zudem insbesondere deshalb an, da der Besuch einer Forschungseinrichtung das Experimentieren in einer authentischen Umgebung mithilfe originaler Forschungsmethoden ermöglicht. Der Austausch mit Wissenschaftler_innen kann zudem dazu beitragen, dass stereotype Vorstellungen bezüglich dem Beruf und der Person der Wissenschaftler_in anhand der eigenen Erfahrungen revidiert werden. 
Gerade eintägige Schülerlabore, wie das hier vorgestellte Konzept, unterliegen jedoch immer noch zeitlichen Einschränkungen, so dass gerade vor dem Hintergrund der benötigten Kontextualisierung der Forschung die Vermittlung der tatsächlichen Forschungsinhalte auf der Strecke bleibt. Diesbezüglich bieten einwöchige Science Camps, die ein- bis zweimal im Jahr in den Ferien angeboten werden, die Möglichkeit, deutlich tiefere Einblicke in die Membranforschung zu geben. Jedoch erreicht man mit solchen Science Camps zumeist nur gerade die SuS, die bereits ein ausgeprägtes Interesse und somit bereits auch ein ausgeprägtes Relevanzempfinden bezüglich der Naturwissenschaften besitzen. Entsprechend verfolgen die beiden Formate, trotz ähnlicher Ausrichtungen, unterschiedliche Ziele der Interessenförderung.

\subsection{Formal Inzidentell - Adaptierte Primärliteratur}

Neben der Entwicklung von Experimenten und Materialien, die es SuS ermöglichen sich mit den Forschungsinhalten des SFB 803 auseinanderzusetzen, sollen im Rahmen des Öffentlichkeitsprojekts zudem Einblicke in die Arbeitsweisen sowie in den Alltag von Wissenschaftler_innen gegeben werden. Mit Blick auf die Berufsorientierung von SuS soll dabei sowohl das Interesse an den Naturwissenschaften geweckt, als auch ein authentisches Bild der Wissenschaft vermittelt werden. Der Einsatz Adaptierter Primärliteratur (APL) 15 ist dabei primär im formalen Bildungskontext vorgesehen. Da im Sinne einer wissenschaftlichen Öffentlichkeitsarbeit jedoch nicht die reine Vermittlung von Fachwissen, sondern vielmehr der Einblick in die Wissenschaft eine wichtige Rolle spielt - der vornehmlich inzidentell erfolgt - wurde das Format der APL in den formal-inzidentellen SO-Bildungskontext eingeordnet.

Originäre Vorstellungen bezüglich naturwissenschaftlicher Berufe werden von SuS aus ihren eigenen Erfahrungen abgleitet. Wenn jedoch im familiären Umfeld keine Wissenschaftler_innen anzutreffen sind, beziehen SuS ihre Informationen vornehmlich aus verschiedenen Medien, wie TV, Büchern oder Comics, sowie dem naturwissenschaftlichen Unterricht. Während erstere oftmals stereotype Bilder von Wissenschaftler_innen fördern, vermitteln Fachlehrkräfte, neben fachlichen Inhalten, auch ein gewisses Bild ihres Faches. In Ermangelung anderer Vorbilder werden die Lehrkräfte im Sinne eines Prototypen-Abgleichs von SuS genutzt, um zu eruieren, ob der Beruf der Wissenschaftler_in zu ihnen passt [328]. Der naturwissenschaftliche Unterricht spiegelt jedoch - beginnend mit der oftmals strengen Trennung der Disziplinen Biologie, Chemie und Physik - nur bedingt das Wesen der Naturwissenschaften wider, das in der Literatur als Natur der Naturwissenschaften (Nature of Science, NoS) bezeichnet wird [329]. Zudem zeigen Studien in diesem Bereich, dass Studienanfänger der Fachwissenschaften sowie des Lehramts nur unzureichende Vorstellungen über die Tätigkeitsfelder von Wissenschaftler_innen besitzen [330]. In der Lehramtsausbildung mangelt es jedoch oftmals an Möglichkeiten, Einblicke in die aktuelle Forschung zu erhalten, so dass sich die Frage stellt, inwiefern Lehrkräfte in der Lage sind, ein authentisches Bild der Wissenschaft zu vermitteln.

Obwohl in der Literatur kein Zweifel daran besteht, dass ein Verständnis für die NoS wesentlicher Bestandteil der naturwissenschaftlichen Grundbildung ist, existiert zwischen Philosophen, Historikern und Didaktikern keine einheitliche Meinung bzgl. einer entsprechenden Definition. Laut LEDERMAN lassen sich jedoch trotz zahlreicher Definitionen genügend allgemeingültige Aussagen treffen, die für den schulischen Gebrauch ausreichend

\footnotetext{
15 Die hier beschriebenen Erfahrungen zur Verwendung von APL wurden zum Teil bereits im Journal CHEMKON publiziert [327].
} 
sind [331]. Beispielhaft für den deutschsprachigen Raum können hier die von URHAHNE ET AL. [332], in Anlehnung an MCCOMAS \& OLSON [333] sowie OSBORNE ET AL. [334], postulierten zehn Kerndimensionen der NoS genannt werden, die in Abbildung 90 dargestellt sind. Dabei unterscheiden die Autoren zusätzlich zwischen Vorstellungen über naturwissenschaftliches Wissen, naturwissenschaftliche Methoden sowie über Institutionen und soziale Handlungen.

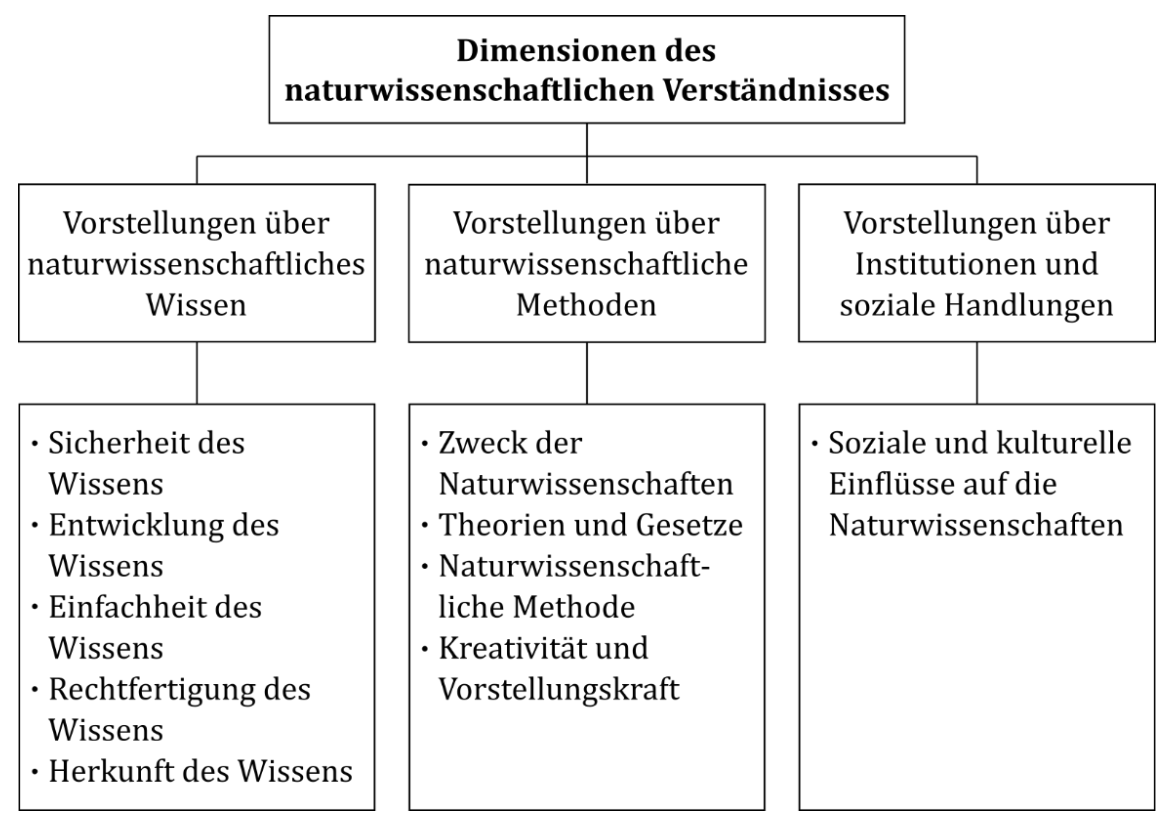

Abbildung 90: Dimensionen des naturwissenschaftlichen Verständnisses, in Anlehnung an URHAHNE, KREMER \& MAYER [332], SOWIe WERNER \& KREMER [335].

Während die Dimensionen der ersten Kategorie unter anderem die Vorläufigkeit und Subjektivität des Wissens beschreiben und in der letzten Kategorie soziale und kulturelle Einflüsse auf die Naturwissenschaften berücksichtigt werden, beziehen sich die Dimensionen zu den naturwissenschaftlichen Methoden auf (Fehl-)Vorstellungen bezüglich wissenschaftlicher Prozesse der Erkenntnisgewinnung. WENTORF ET AL. ${ }^{[336]}$ geht diese auf die Erkenntnisgewinnung beschränkte Betrachtung, insbesondere mit Blick auf die Erhebung der NoS-Vorstellungen von SuS, nicht weit genug, weshalb sie - in Anlehnung an das RIASEC-Modell von HoLLAND [337] (Realistic, Investigative, Artistic, Social, Enterprising, Conventional) - ein Erhebungsinstrument zur Erfassung der Vorstellungen bezüglich der Tätigkeitsbereiche von Naturwissenschaftler_innen (Nature of Scientists, NoSt) entwickelten. Die Items des NoSt-Fragebogens spiegeln dabei Aspekte wissenschaftlicher Tätigkeiten wie z. B. der Betreuung von Lehrveranstaltungen (Social), der Akquise von Forschungsgeldern (Enterprising), sowie das Lesen von Literatur (Investigative) wider.

Dass das Lesen, Sprechen und Schreiben einen großen Anteil der Arbeit von Wissenschaftler_innen ausmacht, zeigten TENOPIR \& KING [338]. Sie baten Naturwissenschaftler_innen, Ingenieur_innen und Mediziner_innen ihre Arbeitszeit hinsichtlich kommunikativer Aktivitäten zu analysieren. Dabei stellten sie fest, dass alle drei Gruppen gut $60 \%$ ihrer Zeit mit kommunikativen Tätigkeiten verbringen, wobei die Arten der Aktivitäten wie das Lesen bzw. Schreiben von Artikeln oder der direkten Kommunikation mit Kolleg_innen innerhalb der Gruppen zeitlich stark variieren. Obwohl die Kommunikation im naturwissenschaftlichen Unterricht zu den prozessbezogenen Kompetenzen zählt, spielt sie insbesondere bei Erkenntnisgewinnungsprozessen eher eine untergeordnete Rolle. Dabei werden in der Wissenschaft gerade beim Lesen wichtige Erkenntnisse gewonnen, wie PHIL- 
LIPS \& NORRIS feststellen: „When scientists read, they are doing inquiry“ [339]. Laut einer Studie zum Einsatz von Texten im Chemieunterricht lesen SuS jedoch nur selten Texte während des Unterrichts. Etwas über 40 \% der befragten Lehrkräfte setzen Texte höchstens alle drei Monate oder seltener in ihrem Unterricht ein und lediglich 11,4 \% verwenden Texte wenigstens einmal pro Woche oder öfter. Dabei werden die Texte häufig nur zur Wiederholung genutzt, jedoch deutlich seltener für die Erarbeitung von Sachverhalten oder zur Einführung in neue Themen verwendet [340]. Ähnliche Ergebnisse zeigen auch ältere Studien zum Einsatz von Physikschulbüchern [341]. Im naturwissenschaftlichen Unterricht stehen stattdessen Unterrichtskonzeptionen wie der forschend-entwickelnde Unterricht im Vordergrund, bei denen die Erkenntnisgewinnung anhand von (Schüler-)Experimenten einen hohen Stellenwert hat. Das fördert jedoch unter anderem die prominente Schülervorstellung, dass Wissenschaftler_innen die meiste Zeit ihres Arbeitstages im Labor verbringen [342].

Mit der Entwicklung von Adaptierter Primärliteratur lieferten YARDEN ET AL. [343] ein Konzept, dass das Potenzial besitzt, Erkenntnisgewinnung anhand wissenschaftlicher Texte zu fördern, sowie aktuelle Forschungsergebnisse in den Unterricht zu integrieren. Diese Form der Literatur, in der wissenschaftliche Originalartikel didaktisch aufgearbeitet werden, ermöglicht es zudem die Scientific Literacy der SuS sowohl im fundamental sense, d. h. des Verstehens von Texten, als auch im derived sense zu fördern, zu dem u. a. das Erlangen eines Verständnisses für die Natur der Naturwissenschaften gehört [344].

\section{Exkurs: Was versteht man unter Adaptierter Primärliteratur?}

Das Textgenre der APL stellt ein Bindeglied zwischen Schulbüchern und naturwissenschaftlicher Primärliteratur dar (vgl. Abbildung 91). Schulbücher orientieren sich zumeist an curricularen Vorgaben und geben das Wissen in einer an den Lerner angepassten Struktur wider [345]. Dabei greifen sie nur Konzepte auf, die in der Wissenschaft etabliert sind und zum Wissenschaftskonsens gehören [346]. Diesbezüglich zeigen vornehmlich amerikanische Studien, dass wissenschaftliche Aussagen fast immer als Wahrheiten dargestellt und nur selten Beweise für diese erbracht werden [347,348]. Daraus folgt ein erklärender und narrativer Textstil, der oftmals keine argumentativen Elemente beinhaltet. Diese fehlende Argumentationsstruktur und das starre Konstrukt der curricularen Vorgaben wirken sich jedoch nachteilig auf die Einbettung hochaktueller Themen aus, da Schulbücher dem rapiden Wissenszuwachs und der Wiedergabe fragiler Wissenskonstrukte nicht gerecht werden können [349].

Wissenschaftliche Primärliteratur wie Fachartikel hingegen spiegeln die Erkenntniswege und Denkprozesse der Wissenschaft wider, beschreiben den Prozess der Planung und Durchführung von Forschung und präsentieren deren Ergebnisse [25]. Sie richtet sich dabei primär an Forscher aus der eigenen Wissenschaftsgemeinde und besitzt eine charakteristische Organisationsstruktur, die zumeist eine Einleitung, einen Experimentalteil mit Methoden und Ergebnissen, sowie eine anschließende Diskussion beinhaltet [350]. Zudem zeichnet sie sich durch eine argumentative und logische Textstruktur aus. Eigene und fremde Ergebnisse und Theorien werden dabei nicht als absolut gesichert dargestellt, sondern anhand von Forschungsergebnissen untermauert oder widerlegt [339, 345].

Trotz ihrer Aktualität und der Möglichkeit, Einblicke in wissenschaftliche Arbeits- und Denkprozesse zu geben, eignet sich wissenschaftliche Primärliteratur aufgrund ihrer Komplexität eher nicht als alternatives oder unterstützendes Medium für den schulischen Gebrauch. Die Einführung von APL als neue Form der Wissenschaftskommunikation bietet jedoch die Chance, aktuelle Forschungsinhalte und den wissenschaftlichen Diskurs 
nah an einer Originalpublikation in den naturwissenschaftlichen Unterricht zu integrieren. Um dies zu ermöglichen, wird eine wissenschaftliche Originalpublikation adressatengerecht angepasst, ohne dabei wesentliche Strukturmerkmale des Originaltextes zu verändern [343, 351].

APL zielt dabei nicht nur auf die Vermittlung von Fachwissen ab, sondern zeigt zudem Prozesse moderner Wissensgenerierung auf. Damit unterscheidet sich APL von Sekundärliteratur wie Schulbüchern, bei denen das Fördern des Inhalts- und Konzeptverständnisses im Vordergrund steht, während bei APL das Augenmerk auf der Vermittlung von Forschungsprozessen und der syntaktischen Struktur wissenschaftlicher Kommunikation liegt. Zudem bleibt das Element der Unsicherheit des Wissens erhalten, die durch die argumentative Absicherung des Autors deutlich wird [346,352], in Schulbüchern jedoch aufgrund der Darstellung gesicherten Wissens zumeist nicht nötig ist.

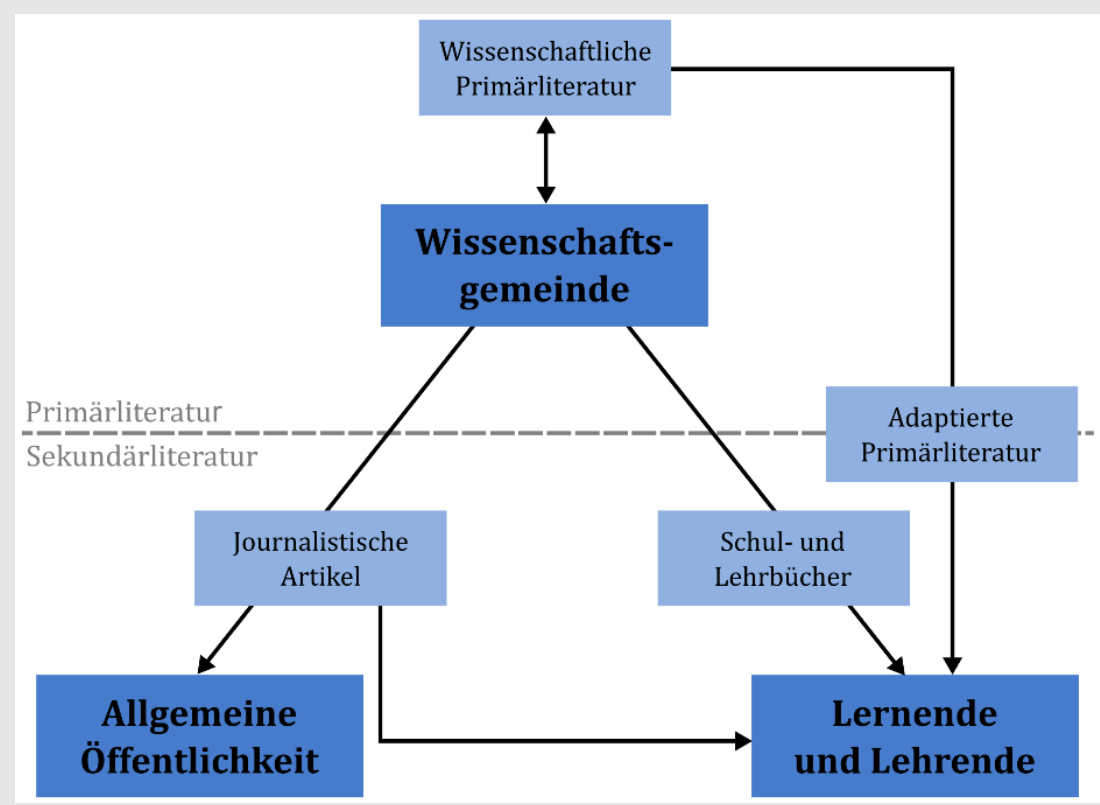

Abbildung 91: Übersicht über die verschiedenen Arten naturwissenschaftlicher Literatur und ihrer Zielgruppen [327].

Nach YARDEN, NORRIS \& PHILLIPS [345] reicht es bei der Erstellung von APL nicht aus, die zumeist englischsprachige Literatur zu übersetzen. Stattdessen sollte eine gezielte Anpassung der Literatur an die Zielgruppe erfolgen, bei der insbesondere die Struktur des Originaltextes (Einleitung, Experimentalteil mit Methoden und Ergebnissen, Diskussion) sowie der Argumentationsstil erhalten bleiben. Da die Adaptierung aller weiteren Abschnitte stark von der Auswahl der beizubehaltenden Ergebnisse abhängt, erfolgt der Adaptierungsprozess schrittweise und in mehr oder weniger umgekehrter Reihenfolge (siehe Tabelle 17). 
Tabelle 17: Ausgewählte Schritte des Adaptierungsprozesses von Originaltexten zu APL 16.

\begin{tabular}{cl}
\hline Schritt & \multicolumn{1}{c}{ Maßnahme } \\
\hline 1 & Auswahl der Primärliteratur \\
2 & Adaptierung der Ergebnisse \\
3 & Adaptierung der Methoden \\
4 & Adaptierung der Einleitung \\
5 & Adaptierung der Diskussion \\
6 & Auswahl eines Titel \\
7 & Schreiben einer Zusammenfassung \\
\hline
\end{tabular}

Um die Primärliteratur zielgruppengerecht umzuschreiben, sollte zunächst das Vorwissen der SuS berücksichtigt werden. Dazu ist es sinnvoll, die Einleitung um Erklärungen zu fachwissenschaftlichen Hintergründen zu erweitern, um eine fachliche Einordnung der Forschungsfragen zu ermöglichen. Das Vorwissen der SuS sollte auch eine Rolle bei der Auswahl der Ergebnisse spielen. Diese sind oft vielfältig und sehr detailreich und können auf wesentliche Erkenntnisse reduziert werden, solange sie nicht in ihrer generellen Aussage verändert werden.

Die Methodenbeschreibung in Fachartikeln fällt je nach Autor und Zeitschrift unterschiedlich detailliert aus. Da vor allem in der jeweiligen Wissenschaftsgemeinde viele der Methoden bekannt sind, fallen sie im Original oft kürzer aus, sollten aber in der APL genauer erklärt und ggf. visualisiert werden. Dahingegen sind detaillierte Experimentieranleitungen mit bspw. Informationen über die Hersteller von Chemikalien für die SuS nicht relevant und können ausgelassen werden.

Der Diskussionsteil kann als Herzstück sowohl des Originalartikels als auch der APL betrachtet werden. Der Schreibstil wechselt hier von zunächst erklärend zu argumentativ, um mithilfe der zuvor genannten Ergebnisse Theorien und Erklärungsmodelle des Autors zu stützen. Entsprechend ist der Abschnitt von Rechtfertigungen und Beweisen gekennzeichnet, die den SuS so aus Schulbüchern noch nicht bekannt sind. Ähnlich wie in der Einleitung ist es gegebenenfalls notwendig, einige Schlussfolgerungen und Annahmen des Autors näher zu erläutern, falls die Argumentation auf Vorwissen zurückgreift, welches die SuS nicht besitzen. Zudem darf konsequenterweise nur eine Diskussion von Ergebnissen erfolgen, die im Ergebnisteil der APL präsentiert wurden.

Für die unterrichtliche Anwendung finden sich in der ausschließlich israelischen und kanadischen Literatur zu APL drei verschiedene Modelle: Das Konversations-, das Problemlöse- und das Scientific Literacy-Modell[343, 353, 354]. Im Unterricht nach dem Konversationsmodell lesen die SuS die APL im Plenum, wobei nach jedem Abschnitt zunächst eine Pause gemacht wird, in der die SuS Fragen zum Text stellen können. Die Fragen können dabei sowohl inhaltlicher Natur als auch Forschungsfragen sein und werden von der Lehrkraft notiert. Die SuS formulieren zudem Hypothesen bezüglich der Beantwortung der Fragen bzw. möglicher verwendeter Forschungsmethoden. Im Anschluss wird der nächste Abschnitt abermals gemeinsam gelesen. Anhand der neuen Informationen können einige der Fragen bereits beantworten und Hypothesen verifiziert oder falsifiziert werden. Die SuS erschließen sich auf diese Weise gemeinsam den Inhalt des Textes, wobei die Rolle der Lehrkraft von einer zunächst anleitenden hin zu einer moderierenden Funktion wechselt [343].

\footnotetext{
${ }^{16}$ Eine konkrete Anleitung für die Erstellung von APL ist im Anhang dieser Arbeit zu finden.
} 
Beim Problemlösemodell werden die SuS zunächst mit einem Problem konfrontiert, das der von den Wissenschaftler_innen im Artikel untersuchten Fragestellung ähnelt. Falls das präsentierte Problem soziale Relevanz hat, werden zunächst die Auswirkungen auf die Gesellschaft diskutiert, bevor die SuS verschiedene Lösungsstrategien für das Problem entwickeln. Die Lehrkraft wirkt dabei unterstützend, indem sie weitere relevante Informationen beisteuert sowie entwickelte Lösungsstrategien kritisch hinterfragt. Nachdem ein ausreichender Lösungsansatz gefunden wurde, wobei dieser nicht dem Forschungsvorhaben des Artikels entsprechen muss, erhalten die SuS die APL, lesen diese Aufmerksam und vergleichen ihre Vorschläge mit den Forschungsmethoden und Ergebnissen der Wissenschaftler_innen [353].

Das Scientific Literacy-Modell bedient zunächst eher den fundamental sense [344] des Verstehens von Texten. Dazu wird neben der APL auch ein entsprechender journalistischer Artikel der Originalliteratur erstellt, oder ein bereits vorhandener Artikel, der sich auf das Original bezieht, herausgesucht. Die beiden Textarten können dabei auf verschiedene Weisen im Unterricht eingesetzt werden. Zum einen ist eine komplementäre Verwendung denkbar: Während der journalistische Artikel die Inhalte des Originals auf verständliche Weise präsentiert, bietet die APL tiefergreifende Informationen bezüglich der Methoden und der Erkenntnisse. Der journalistische Artikel könnte also als Einführung in die Thematik verwendet werden, um im Anschluss einige Fragen an den Artikel mithilfe der APL zu klären. Des Weiteren ist es möglich, die beiden Artikel zur Binnendifferenzierung zu nutzen. Dabei kann entweder nur der journalistische Artikel gelesen werden, der journalistische Artikel als Verständnishilfe für die APL eingesetzt oder die APL direkt verwendet werden. Zudem werden journalistische Artikel in der APL-Forschung verwendet, um in Vergleichsstudien $u$. a. Informationen bezüglich des Textverständnisses von SuS zu gewinnen [345,354].

Während jedes der genannten Modelle seine Vorzüge besitzt, gehen jedoch auch Nachteile mit ihnen einher, die FALK \& YARDEN tabellarisch zusammenfassen (vgl. Tabelle 18). Zu letzteren zählen u. a. der zeitliche Aufwand des Konversationsmodells und das zusätzliche Schreiben oder Finden passender journalistischer Artikel für das Scientific Literacy-Modell.

Tabelle 18: Differenzierung verschiedener Lehr-/Lernmodelle zur Einführung von APL hinsichtlich ihrer ihre Vorteile und Grenzen (aus dem Englischen übersetzt nach FALK \& YARDEN [353]).

\begin{tabular}{llll}
\hline Modell & Vorteile & Grenzen & Weitere Überlegungen \\
\hline $\begin{array}{l}\text { Konversations- } \\
\text { modell }\end{array}$ & $\begin{array}{l}\text { Fördert das Fragenstellen } \\
\text { der SuS und ermutigt zu } \\
\text { einer forschenden Heran- } \\
\text { gehensweise durch akti- } \\
\text { ves Lernen }\end{array}$ & $\begin{array}{l}\text { Zeitaufwendig und } \\
\text { manchmal mühselig we- } \\
\text { gen seiner iterativen Pha- } \\
\text { sen }\end{array}$ & $\begin{array}{l}\text { Konzentriert sich sowohl } \\
\text { auf den Inhalt als auch } \\
\text { die Merkmale der wis- } \\
\text { senschaftlichen Kommu- } \\
\text { nikation }\end{array}$ \\
\hline $\begin{array}{l}\text { Problemlöse- } \\
\text { modell }\end{array}$ & $\begin{array}{l}\text { Fördert eine forschende } \\
\text { Herangehensweise beim }\end{array}$ & $\begin{array}{l}\text { Die Lehrkraft muss ver- } \\
\text { stärkt fachlichen Input } \\
\text { liefern und als Vermittler } \\
\text { Lesen wissenschaftlicher }\end{array}$ & $\begin{array}{l}\text { Setzt inhaltliche Vor- } \\
\text { kenntnisse der SuS vor- } \\
\text { aus }\end{array}$ \\
\hline $\begin{array}{l}\text { Scientific } \\
\text { Literacy } \\
\text { Modell }\end{array}$ & $\begin{array}{l}\text { Fördert die Untersu- } \\
\text { chung verschiedener Art- } \\
\text { en von Artikeln bezüglich }\end{array}$ & $\begin{array}{l}\text { Es kann schwierig sein, } \\
\text { entsprechende Artikel } \\
\text { verschiedener Textgen- } \\
\text { res zu demselben Thema }\end{array}$ & $\begin{array}{l}\text { Konzentriert sich sowohl } \\
\text { auf den Inhalt als auch } \\
\text { die Merkmale der wis- } \\
\text { senschaftlichen Kommu- } \\
\text { nikation }\end{array}$ \\
& pe & zu finden & \\
\hline
\end{tabular}


Der Einsatz von APL bietet sich insbesondere für den naturwissenschaftlichen Oberstufenunterricht sowie die Studieneingangsphase an Universitäten an. Sowohl in der Oberstufe als auch in der Studieneingangsphase dominieren theoriebasierte Unterrichts- bzw. Vorlesungssequenzen und Experimente die naturwissenschaftliche Lehre. Sie spiegeln jedoch nur bedingt den wissenschaftlichen Alltag wider, der zum großen Teil von Kommunikation geprägt ist [338]. Gerade in der Oberstufe ist es daher im Sinne einer Berufsvorbereitung zweckmäßig, SuS einen authentischen Einblick in wissenschaftliche Arbeitsweisen zu vermitteln. Zudem können Studierende der Eingangsphase, die zumeist noch nicht an Forschungsprozessen beteiligt sind, in Vorbereitung auf die Erstellung von Qualifikationsarbeiten erste Erfahrungen mit Primärliteratur sammeln.

Bei der Verwendung von APL im schulischen Kontext ist es sinnvoll, zusammen mit den SuS die Unterschiede zwischen typischer Schulliteratur, wie Schulbüchern aber auch journalistischen Artikeln, und Primärliteratur herauszuarbeiten. Vor allem die argumentative Struktur der Primärliteratur steht in starkem Kontrast zu den Informationen in Schulbüchern, die zumeist als Fakten präsentiert werden. Gerade diese Unsicherheit des Wissens, die in der Primärliteratur zum Ausdruck kommt, ermöglicht einen Einblick in die verschiedenen Wege der naturwissenschaftlichen Erkenntnisgewinnung, zu denen auch das Widerlegen von Annahmen, das Erweitern von bestehenden Modellen und das Lernen aus fehlgeschlagenen Experimenten gehört.

Anhand von APL können zudem aktuelle und ggf. sogar kontroverse Themen, die noch nicht zum Wissenschaftskonsens gehören und in der Schulliteratur fehlen, im Unterricht thematisiert werden. Des Weiteren bietet APL die Möglichkeit, die Interdisziplinarität der naturwissenschaftlichen Forschung zu verdeutlichen und im Sinne einer Berufsorientierung verschiedene interdisziplinäre Tätigkeitsfelder aufzuzeigen. Diese spiegeln sich unter anderem auch in den verwendeten Methoden wider, die in der Schule oftmals unbekannt sind, es jedoch ermöglichen, ein authentischeres Bild von naturwissenschaftlicher Forschung und deren Arbeitsweisen zu vermitteln. Prinzipiell ist der Einsatz von APL jedoch nicht auf den schulischen oder universitären Kontext beschränkt. Gerade im Sinne einer erweiterten Öffentlichkeitsarbeit ermöglicht er das Publizieren von Forschungsergebnissen in einem verständlichen aber dennoch anspruchsvollen Format, so dass aktuelle Erkenntnisse zeitnah einer interessierten Öffentlichkeit zugänglich gemacht werden können.

Im Rahmen dieser Arbeit wurden zwei aus der Forschung des SFB entsprungene wissenschaftliche Artikel ausgewählt, um vorrangig für eine Nutzung im naturwissenschaftlichen Unterricht in das APL-Format übertragen zu werden. Im ersten Artikel von BRIONES ET AL. [231] mit dem Titel Voltage Dependence of Conformational Dynamics and Subconducting States of VDAC-1, der 2016 im BIOPHYSICAL JOURNAL publiziert wurde, werden aus Simulationen gewonnene Erkenntnisse mit experimentellen Befunden verglichen. Beim zweiten Artikel hingegen, der den Titel $\mathrm{Gb}_{3}$ Glycosphingolipids with Fluorescent Oligoene Fatty Acids: Synthethis and Phase Behaviour in Model Membranes trägt und 2017 von PATALAG ET AL. [355] im Journal CHEMBIOCHEM veröffentlicht wurde, handelt es sich um eine für synthetische Arbeiten typische Veröffentlichungen ${ }^{17}$.

\footnotetext{
17 Beide APL sind samt Zusatzmaterialien sind im Anhang dieser Arbeit zu finden.
} 


\subsubsection{Adaptierte Primärliteratur „Spannungsabhängige Konformationsänderun- gen des Ionenkanals VDAC-1: Ein Vergleich zwischen Simulation und Experi- ment"}

\subsubsection{Darstellung des Adaptionsprozesses}

Die Auswahl der ersten Publikation von BRIONES ET AL. erfolgte anhand mehrerer Kriterien. Zunächst sollte der Artikel Forschungsergebnisse des SFB widerspiegeln und möglichst einen interdisziplinären Charakter besitzen. Zudem sollte die Thematik Anknüpfungspunkte an die Kerncurricula der Oberstufe der Fächer Chemie und Biologie bieten, so dass auf das Vorwissen von SuS aufgebaut werden kann, sowie einen lebensweltlichen Bezug ermöglichen. Aufgrund des stark biochemischen Charakters der Forschung des SFB fiel die Wahl auf die oben genannte Publikation, da der in die äußere Mitochondrienmembran eingebettete VDAC-1-Kanal zwar sehr spezifische Eigenschaften besitzt, dessen allgemeine Funktionsweisen SuS aber bereits aus dem Biologieunterricht bekannt sind. Ferner beinhaltet der Artikel drei für die SuS typischerweise unbekannte Forschungsmethoden, wie unter anderem die Computerchemie (in silico), die ihnen einen Einblick in die vielfältigen Arbeitsweisen von Wissenschaftler_innen ermöglichen. Der Originalartikel umfasst zwölf Seiten, von denen zwei Seiten als Literaturverzeichnis dienen.

Die Adaptierung der Publikation und die Entwicklung eines begleitenden Arbeitsblatts erfolgten mit dem Ziel einer Verwendung der Materialien im Chemie- oder Biologieunterricht der Oberstufe. In Anlehnung an die Adaptierungsschritte aus Tabelle 17 erfolgte nach der Wahl der Primärliteratur zunächst eine Adaptierung des Ergebnisteils. Dieser umfasst im Originalartikel gut viereinhalb Seiten und ist damit für den schulischen Gebrauch zu ausführlich und lang. Die Ergebnisse der Studien beziehen sich auf die zwei VDAC-1 Kanäle des Menschen (mVDAC-1) und der Maus (hVDAC-1). Diese ähneln sich, wie im Originalartikel erwähnt, strukturell zu $98 \%$, weshalb in der APL auf eine Differenzierung zwischen den beiden Kanälen verzichtet wurde. Zudem wurde das Membransystem mit zwei unterschiedlichen Lipiden simuliert (1-Palmitoyl-2-oleoyl-sn-glycero-3phosphocholin (POPC) und 1-Palmitoyl-2-oleoyl-sn-glycero-2-phosphatidylethanolamin (POPE)), auf deren Unterscheidung zu Kürzungszwecken ebenfalls verzichtet wurde. Diese selektive inhaltliche Kürzung des Ergebnisteils wirkt sich auch auf die Adaptierungsschritte drei bis fünf aus, was noch einmal die Notwendigkeit eines umgekehrten Adaptierungsprozesses verdeutlicht.

Während im Ergebnisteil im Großen und Ganzen lediglich einige Kürzungen vorgenommen wurden, so dass die vorhandene Struktur im Allgemeinen erhalten bleibt, ändert sich der Methodenteil im Vergleich zur Primärliteratur deutlich stärker. Im Originalartikel wird zwischen vier Methoden unterschieden: (1) der molekulardynamischen Simulation, (2) der NMR-Spektroskopie, (3) der hVDAC-1-Rekonstitution sowie (4) den elektrophysiologischen Experimenten (hier Variationen des Patch-Clamp-Verfahrens). Da die Rekonstitution des hVDAC-1-Kanals lediglich die Methodik der Vesikelpräparation samt der Einbettung der Proteine in die Membran beschreibt, diese jedoch nicht zum Verständnis des restlichen Textes beiträgt, wurde sie in der APL ausgelassen. Die anderen drei Methoden sind SuS in der Regel unbekannt. Da es sich jedoch bei allen drei Methoden um gängige Verfahren handelt, werden im Originalartikel lediglich Randbedingungen wie Gerätespezifikationen sowie die verwendeten Chemikalien und ihre jeweiligen Mengen genannt. Diese Informationen sind für SuS jedoch irrelevant und werden stattdessen durch Erläuterungen der Methoden ersetzt. Zur visuellen Unterstützung des Lernprozesses wurden zudem zu jeder Methode Abbildungen erstellt, die sich typischer Symbolik bzgl. 
Biomembranen und Kanälen aus Schulbüchern bedienen, da vertraute Darstellungsformen oftmals als authentisch eingeschätzt werden [89], und wie in Abbildung 92 dargestellt z. T. Abbildungen aus der Originalpublikation ersetzen.

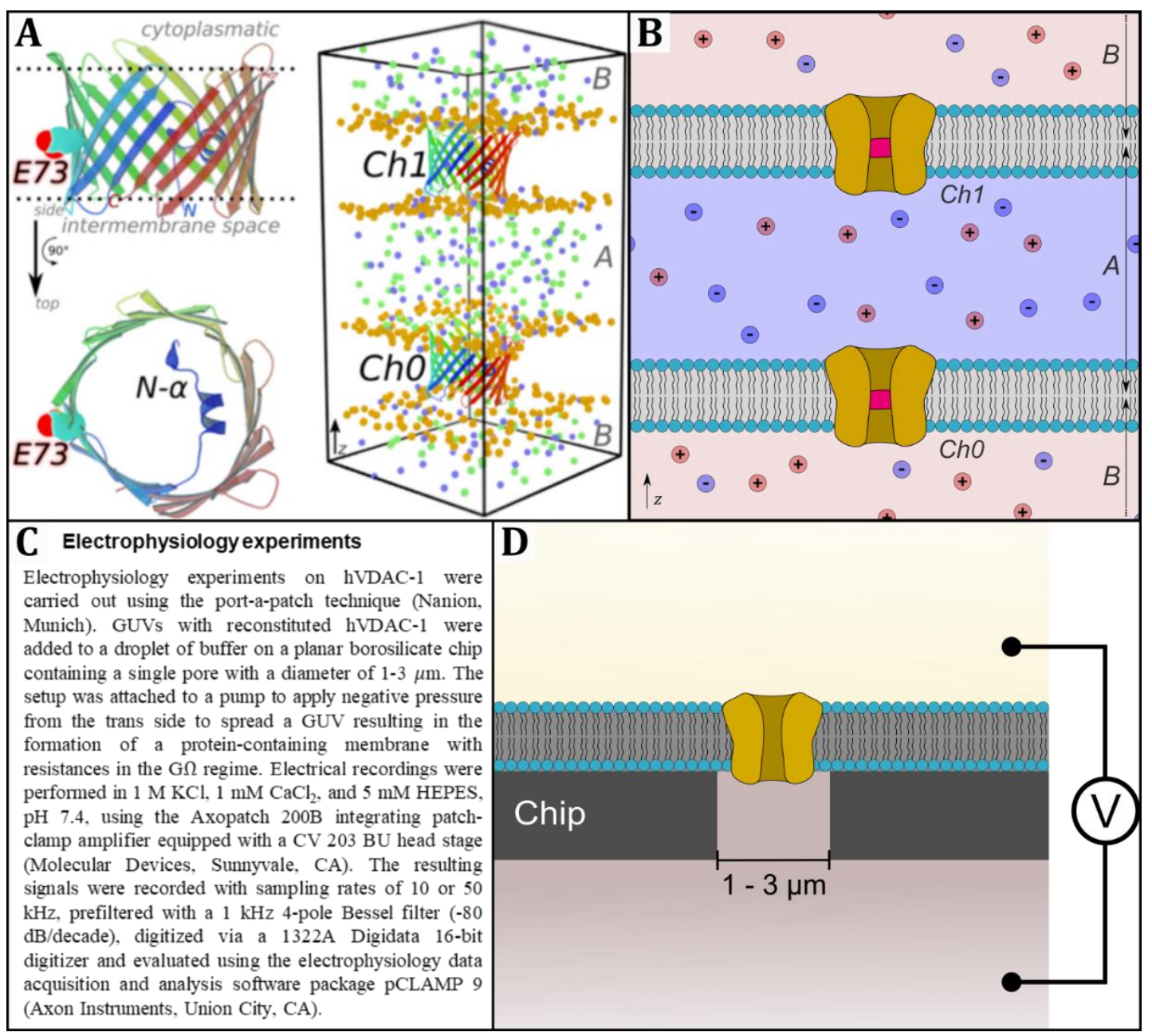

Abbildung 92: Beispiele zur Adaptierung von Abbildungen (oben) sowie Textausschnitten (unten) aus dem Originalartikel. Abbildung (A) [231] zeigt sowohl die Struktur des Kanals VDAC-1 sowie den verwendeten Simulationsaufbau. In der APL werden Struktur und Aufbau schematisiert dargestellt (B). Der Textausschnitt (C) ${ }^{[231]}$ zum Patch-Clamp-Verfahren enthält zahlreiche, für das Grundverständnis irrelevante, technische Angaben. In (D) wird das Verfahren in einer didaktisch reduzierten Form zur Messung eines Ionenstroms in Abhängigkeit eines angelegten Potentials dargestellt [327].

Im Originalartikel wie auch in der APL dient die Einleitung dazu, den Leser von einer allgemeinen Ausgangslage zu einer konkreten Problemstellung zu führen. Die einführenden Sätze können dabei, wie im Falle der Originalquelle, für Wissenschaftler_innen relativ trivial wirken: „Ion channels are membrane proteins important for controlling the passage of solutes between cellular compartments" [231]. Sie dienen aber gerade in Journalen mit breitem inhaltlichen Spektrum einem schnellen Einstieg in die Thematik des jeweiligen Artikels. In der APL wurde diese Einleitung anhand der curricularen Vorgaben mit Blick auf das Vorwissen der SuS noch erweitert.

„Ionenkanäle sind in Biomembranen befindliche Proteine und bestehen, wie alle Proteine, aus definierten Aminosäureketten. Sie erfüllen dabei die wichtige Funktion eines kontrollierten Durchlasses bestimmter Stoffe durch die Membran in ein anderes Kompartiment, da die Biomembran durch den hydrophoben Innenraum für wasserlösliche Stoffe undurchlässig ist." 
Das Rekurrieren auf das Vorwissen der SuS innerhalb der ersten zwei Sätze ermöglicht diesen eine Einordnung des Textes und seiner Inhalte in Bezug auf vorangegangene Unterrichtsinhalte. Bei der anschließenden Beschreibung des eigentlichen Objekts der Studie - des VDAC-1-Kanals - wurden bewusst Fachbegriffe wie „Apoptose“ verwendet, da diese typische Merkmale wissenschaftlicher Publikationen darstellen. Sie werden jedoch stets im Text erläutert, oder lassen sich aus dem Textzusammenhang herleiten. Ähnlich wie im Originalartikel enthält die Einleitung des Weiteren eine Zusammenfassung vorheriger Studienergebnisse sowie eine Aufzählung aktueller Hypothesen. Diese zeigen zum einen den aktuellen Forschungsstand auf und dienen in der APL zudem implizit dazu, die Bedeutung der Betrachtung vorheriger Ergebnisse - und somit die Bedeutung des Lesens von Primärliteratur für den Forschungsprozess - zu verdeutlichen. Das Aufzählen aktueller Hypothesen bezüglich des Schließmechanismus des Kanals stellt zudem noch einmal die Unsicherheit des Wissens in diesem Kontext heraus. Die Einleitung schließt mit der Zielsetzung der Arbeit ab, die die Forschungsfragen der Publikation expliziert.

Während der Leser im Abschnitt der Einleitung von einem allgemeinen Phänomen zu einem konkreten Problem geleitet wird, dient der Diskussionsteil dazu, die konkreten (Mess-)Ergebnisse in einen allgemeineren Kontext zu rücken. Dazu werden die Ergebnisse der Simulationen zunächst mit den Ergebnissen der Experimente verglichen. Anschließend werden die daraus abgeleiteten Schlussfolgerungen, insbesondere auch mit Blick auf die in der Einleitung erwähnten Erkenntnisse vorangegangener Studien, gerechtfertigt und in Bezug zu allgemeinen Phänomenen wie der Apoptose gesetzt, um den allgemeinen Anspruch der Forschung und deren Tragweite hervorzuheben. Zudem werden auch die Hinweise der Autoren darauf, dass der Schließmechanismus des Kanals trotz neuer Erkenntnisse immer noch nicht vollständig geklärt ist, in die APL übernommen, da sie noch einmal die Unsicherheit und die Entwicklung des Wissens verdeutlichen.

Zum Abschluss des Adaptionsprozesses werden der Titel „Spannungsabhängige Konformationsänderung des Ionenkanals VDAC-1: Ein Vergleich zwischen Simulation und Experiment" und die Zusammenfassung noch an die Inhalte der APL angepasst. Da die in der Überschrift erwähnten subconducting states in der APL keine Rolle mehr spielen, wurden sie der deutschen Übersetzung des Titels nicht mehr beigefügt. Stattdessen wurde ein Untertitel hinzugefügt, der die Aufmerksamkeit der SuS auf die für sie unbekannte Forschungsmethode der Simulation richten soll. In der APL wurden zudem die Literaturangaben bewusst weggelassen, da sie den Lesefluss stören, schwer zugänglich und zudem für Laien auch kaum verständlich sind. Dennoch bietet es sich bei der Verwendung von APL im Unterricht an, die Funktion von Literaturangaben zu diskutieren. Der auf sieben Seiten reduzierten APL ist stattdessen ein Abkürzungsverzeichnis beigefügt, dass jedoch ausdrücklich nicht als Teil der APL verstanden, sondern vielmehr als Möglichkeit zur Differenzierung genutzt werden soll. Für das in diesem Zusammenhang ebenfalls entwickelte Arbeitsblatt wurde der Fokus weniger auf fachwissenschaftlichen Inhalte als auf die Förderung prozessbezogener Kompetenzen gelegt. Zwar werden in den ersten beiden Aufgaben auch einige Verständnisfragen gestellt, Ziel des Arbeitsblattes ist es jedoch insgesamt, auf einer Metaebene Erkenntnisse über die Kommunikations- und Erkenntnisprozesse von Wissenschaftler_innen zu erlangen.

\subsubsection{Evaluation}

Wie zuvor beschrieben finden sich in der Literatur zu APL verschiedene Vorschläge für eine Einbettung des Genres in den naturwissenschaftlichen Unterricht. Sowohl BARAMTSABARI \& YARDEN [354], als auch NORRIS, STELNICKI \& DE VRIES [356], die SuS nach dem Scientific Literacy-Modell jeweils wissenschaftliche Texte als APL oder populärwissenschaftliche 
Artikel lesen ließen, nutzen dabei zur Evaluierung des Textverständnisses und der Haltung gegenüber dem jeweiligen Textgenre einen von BARAM-TSABARI \& YARDEN entwickelten Fragebogen, den die SuS im Anschluss an die Auseinandersetzung mit den ihnen zugeordneten Texten bearbeiteten. Bevor im Rahmen der in Kapitel 6.3.2 vorgestellten zweiten APL auch eine mögliche Verwendung im schulischen Kontext evaluiert wurde, sollte anhand dieser ersten APL zunächst überprüft werden, inwiefern sich das Genre der APL als ein Format der Öffentlichkeitsarbeit des SFB 803 eignet, um im Sinne einer NoS Einblicke in die naturwissenschaftliche Forschung zu geben.

\section{Forschungsinteresse}

Das primäre Forschungsinteresse bestand darin, erste Einblicke zu gewinnen, inwiefern die Arbeit mit APL das Verständnis der SuS von Prozessen naturwissenschaftlicher Erkenntnisgewinnung beeinflusst. Ausgehend von diesem Interesse wurden die folgenden Forschungsfragen abgeleitet:

F1 Wie stellen sich SuS den Alltag einer Naturwissenschaftler_in vor?

F2 Wie stellen sich SuS naturwissenschaftliche Forschungsprozesse vor?

F3 Welche Vorstellungen besitzen SuS bezüglich der Verwendung von Ergebnissen naturwissenschaftlicher Forschung?

Zudem sollte neben dem Verständnis für die naturwissenschaftliche Forschung auch die allgemeine Haltung der SuS gegenüber dem Textgenre der APL eruiert werden.

\section{Evaluationsdesign}

Die Beantwortung der Forschungsfragen erfolgte anhand eines leitfadengestützten Interviews erfolgen, das in einem Pre-/Post-Design eine Woche vor, sowie direkt nach dem Lesen der APL und der Bearbeitung des komplementären Arbeitsblatts durchgeführt wurde. Neben Erzählaufforderungen [305], die die SuS u. a. dazu ermuntern sollten, einen typischen Arbeitstag einer Naturwissenschaftler_in zu beschreiben, beinhaltete der Leitfaden des Post-Interviews zusätzlich explizite Fragen zu den Haltungen der SuS bezüglich des Textgenres, die sich an den Attitude Questions des Fragebogens von BARAM-TSABARI \& YARDEN orientierten.

Zur Auswertung der Interviews wurden die Tonaufnahmen der Interviews transkribiert und im Sinne einer strukturierenden qualitativen Inhaltsanalyse nach MAYRING ${ }^{[357]}$ untersucht. Diesbezüglich konnten fünf Kategorien identifiziert werden, in denen eine Beeinflussung des Verständnisses der SuS im Hinblick auf naturwissenschaftliche Erkenntnisprozesse vom Pre- zum Postinterview festgestellt werden konnten. Diese umfassen die Rolle (1)der Wissenschaftler_in, (2) der Fragestellung, (3) des Experimentes, (4) der Hypothesen sowie (5) der Kommunikation von Ergebnissen.

An der Evaluation nahmen insgesamt fünf SuS der Oberstufe im Alter von 16 und 17 Jahren teil $(\mathrm{w}=2, \mathrm{~m}=3)$, die zum Zeitpunkt der Interviews die 11. Klasse zweier Gymnasien aus dem Umkreis Göttingen besuchten. Dabei erklärten sich die SuS freiwillig bereit, außerhalb des Unterrichts an dem Projekt teilzunehmen.

\section{Evaluationsergebnisse}

Bei der Beschreibung des Alltags einer Wissenschaftler_in während der Pre-Interviews war besonders auffällig, dass dieser alleine - also als eine Art Einzelgänger_in - zu agieren schien. Der Fokus der Erzählungen lag bei allen fünf Teilnehmer_innen auf den Arbeitsweisen der Wissenschaftler_innen, es wurde jedoch nicht genannt, in welchen sozialen Kontexten diese gewonnen werden. Ähnliche Vorstellungen zeigen auch die in Kapitel 
3.1.2 erwähnten Draw-a-Scientist-Tests sowie andere Studien [68, 342]. Die Wissenschaftler_innen werden hier zumeist alleine und umgeben von wissenschaftlichen Gerätschaften in einem Labor gezeigt. Die Bearbeitung der APL durch die SuS führte jedoch dazu, dass unter anderem von Forschungsteams die Rede war. Dabei wurden Aspekte wie die Verteilung von Aufgaben im Team, die Absprache mit anderen Wissenschaftler_innen, sowie deren abschließende Meinung zu den Ergebnissen genannt. Das Bild von Wissenschaftler_innen scheint sich also von einer Einzelgänger_in hin zu einer Teamplayer_in gewandelt zu haben. Zwar arbeitetete die Wissenschaftler_in immer noch an einem eigenen Projekt, alle SuS erkannten jedoch, dass der Austausch mit den Kolleg_innen während des Forschungsprozesses eine wichtigere Rolle spielt.

Bezüglich der Herkunft der Fragestellung gaben alle SuS während des Pre-Interviews an, dass diese von der Wissenschaftler_in selbst generiert wird und sie bei der Auswahl Ihres Forschungsinteresses generell freie Hand hat. In einem zweiten Gedankenschritt erwähnten zudem einige der SuS, dass die Forschungsfrage gegebenenfalls auch vom Vorgesetzten (u.a. ranghöhere Professor_in, Universität, Staat) vorgegeben wird. Insgesamt existierte jedoch die Vorstellung, dass wissenschaftliche Fragestellungen zumeist aus dem persönlichen Interesse der Wissenschaftler_innen heraus generiert werden. Dabei scheinen insbesondere wissenschaftliche Vorarbeiten keine Rolle zu spielen. Dies wird insbesondere dadurch deutlich, dass keiner der SuS diese während der Pre-Interviews explizit erwähnte noch deren Rolle erkennbar umschrieb. Die Vorstellung, dass es Wissenschaftler_innen vornehmlich darum geht einen persönlichen Wissensdrang zu befriedigen, teilten bereits 1987 gut $60 \%$ von fast 12.000 Probanden in einer kanadischen Studie von AikenheAd, FlEming \& RYAN ${ }^{[358]}$. Auch im Post-Interview der APL blieben die Meinungen, dass die Frage von der Wissenschaftler_in selbst entwickelt oder vom Vorgesetzten vorgegeben wird, erhalten. Jedoch war eine gedankliche Veränderung aller SuS bezüglich des Prozesses der Fragengenerierung zu erkennen. Die Fragestellung ergab sich nun nicht mehr nur aus dem Eigeninteresse heraus, sondern basierte auf ausgiebigen Recherchen und den eigenen oder den Vorarbeiten von anderen. Es wurde von allen SuS erkannt, dass es also des Lesens wissenschaftlicher Artikel - hier spielen insbesondere die Ergebnisund Diskussionsteile eine wichtige Rolle - sowie der Kommunikation mit anderen Wissenschaftler_innen und Mitarbeiter_innen bedarf.

Das Experimentieren wurde von allen SuS vor dem Lesen der APL gleichermaßen als dominierende Tätigkeit von Wissenschaftler_innen genannt. Dabei standen insbesondere experimentelle Handlungen wie das „Ansetzen von Lösungen“ oder das „Kontrollieren des Drucks“ im Vordergrund. Zudem besaß die Fehleranalyse einen hohen Stellenwert. Auch in anderen Studien dominieren experimentelle Tätigkeiten das Handeln in der Forschung [336, 342]. Dabei stellen sich SuS das „Experimentieren nicht als zielgerichtetes Handeln vor. Vielmehr vertreten sie die Ansicht, Experimentieren bedeute etwas herauszufinden, etwas auszuprobieren und Entdeckungen zu machen“ [342], was sich zum Teil auch in den Antworten der interviewten SuS widerspiegelt. Nach dem Lesen der APL rückten klassische, aus dem Schulunterricht bekannte experimentelle Handlungen deutlich in den Hintergrund. Stattdessen ließ sich aus der Analyse aller Interviews ableiten, dass Wissenschaftler_innen im Sinne der Fragestellung aus verschiedenen Methoden auswählen und vermehrt am Computer arbeiten. Dabei wurde insbesondere die Simulation als eine wichtige Forschungsmethode bewertet und der iterative Charakter von Forschung sowie die Wichtigkeit einer sorgfältigen Dokumentation von Ergebnissen betont.

Bei der Beschreibung wissenschaftlicher Forschungsprozesse war besonders auffällig, dass es allen SuS schwerfiel, wissenschaftliche Fragestellungen von Hypothesen abzugrenzen. Dennoch galt das Beantworten bzw. Beweisen beider als ein wichtiger Teil des 
Forschungsprozesses. In den Erzählungen der SuS erfolgte dabei das Aufstellen der Hypothesen sowohl induktiv als auch deduktiv, wobei das generelle Ziel das Beweisen der Hypothese durch ausgewählte Experimente war. Hier spiegelt sich der unterrichtliche Einsatz von Experimenten zur Erkenntnisgewinnung wieder, für die von der Lehrkraft in der Regel Experimente ausgewählt werden, die zuvor aufgestellte Hypothesen belegen. Auch nach der Bearbeitung der APL gelang den SuS keine konkrete Unterscheidung zwischen Fragestellung und Hypothese. Jedoch betrachteten sie das Aufstellen von Hypothesen im Anschluss an die APL als einen iterativen Prozess, der durch das Beweisen, vor allem aber auch das Widerlegen der Hypothesen geprägt ist. Einer der SuS beschrieb diese Erkenntnis wie folgt: „Hauptsache es ist ein Fortschritt da. Auch wenn der Fortschritt ist, dass seine Hypothese, die er aufstellt, falsch ist, ist es ein Fortschritt, weil einfach wied er etwas entdeckt wurde, was jetzt nicht mehr entdeckt werden muss".

Bezüglich der Kommunikation der Ergebnisse bestand bei einigen SuS bereits die Vorstellung, dass Ergebnisse in Zeitschriften publiziert oder auf Kongressen vorgestellt werden. Andere hingegen äußerten auf die Frage hin, was mit den Forschungsergebnissen passiert, lediglich, dass diese genauen Fehlerbetrachtungen unterzogen werden müssten. Gerade diese SuS stellten im Nachhinein auch die Wichtigkeit des Kommunizierens der Ergebnisse heraus und sahen diese gar als „Sinn der Forschung, da so der Fortschritt nicht auf eine Person begrenzt ist".

Hinsichtlich der Haltung der SuS gegenüber dem Textgenre der APL waren sie allgemein der Auffassung, dass der Text insgesamt sehr anspruchsvoll aber dennoch informativ und interessant war. Dabei wurde der Schreibstil als einfach empfunden, vor allem die Fülle an Fachwörtern und Informationen, insbesondere im Experimentalteil, bereitete jedoch Schwierigkeiten. Neben der Einleitung des Textes und Vorkenntnissen aus dem Chemieund Biologieunterricht gaben einige der SuS an, dass vor allem die durch das Arbeitsblatt geforderte erneute Auseinandersetzung mit dem Text hilfreich für das Verständnis war.

Bei der Bewertung des Textgenres herrscht ein heterogenes Meinungsbild. Während zwei der SuS mit naturwissenschaftlichen Vorerfahrungen (Verwendung von Primärliteratur in naturwissenschaftlicher Facharbeit, Familienmitglied im Bereich naturwissenschaftlicher Forschung tätig) die APL nur mit Einschränkungen interessant fanden, erachteten die drei SuS ohne Vorerfahrungen die APL als sehr interessant. Ähnlich verhielten sich die Aussagen bezüglich der Frage, ob sie es als sinnvoll erachten, APL im Schulunterricht einzusetzen. Die zwei SuS mit Vorkenntnissen äußerten sich dabei eher kritisch und hinterfragten unter anderem, ob die Untersuchung eines speziellen Ionenkanals nicht zu spezifisch für den schulischen Gebrauch sei. Dahingehen erachteten die restlichen SuS den Einsatz von APL als sinnvoll und sogar wichtig. Dabei schlugen sie vor, APL in den Naturwissenschaften oder dem Seminarfach vor der Ausarbeitung der Facharbeit einzusetzen, um unter anderem die Angst vor der Arbeit mit Primärliteratur in diesem Kontext zu nehmen und Hilfestellungen für die Erarbeitung solcher Texte zu geben. Zudem äußerten diese drei SuS, dass sie den Eindruck hätten, einen besseren Einblick in den Alltag und die Arbeitsweisen von Wissenschaftler_innen erhalten zu haben.

Anhand der Schüleraussagen lässt sich ableiten, dass die Arbeit mit der verwendeten APL einen Einfluss auf das Verständnis der befragten SuS von Prozessen naturwissenschaftlicher Erkenntnisgewinnung hatte. Diesbezüglich konnten im Untersuchungszeitraum Veränderungen unterschiedlicher Ausprägung innerhalb der fünf identifizierten naturwissenschaftlichen Aspekte aufgezeigt werden. Neben der aus der Primärliteratur übernommenen argumentativen Textstruktur, scheint vor allem auch der Inhalt der APL eine Veränderung innerhalb dieser beeinflusst zu haben. Dabei erkannten die SuS insbesondere, 
dass das Zusammenspiel zwischen Simulation und Experiment ein wichtiges Werkzeug der Erkenntnisgewinnung ist.

Mit Blick auf die herausgearbeiteten Veränderungen, wie bspw. die Erkenntnis, dass Hypothesen nicht zwangsweise bewiesen werden müssen, sondern dass auch das Widerlegen dieser einen Fortschritt darstellt, liegt die Frage nahe, inwiefern die Arbeit mit APL einen Beitrag zum Verständnis der Natur der Naturwissenschaften [359] leisten kann. Da einige Studien zur Arbeit mit Primärliteratur mit Studierenden positive Effekte bezüglich der NoS zeigen [360,361], liegt der Gedanke nahe, dass das Lesen von APL ähnliche Effekte bei SuS zeigt. Die aus den Schülerantworten der Pre-Interviews abgeleiteten Vorstellungen der SuS beschreiben einige typische Fehlvorstellungen bezüglich der NoS, die u. a. auch in der Übersichtsarbeit zum diesbezüglichen Forschungsstand von HöTTECKE ${ }^{\text {[342] }}$ genannt werden. Betrachtet man die zehn Kerndimension der NoS von URHAHNE ET AL. [332] sowie die RIASEC-Dimension der NoSt nach WENTORF, HÖFFLER \& PARCHMANN [336], deuten sich bezüglich einiger dieser Dimensionen, wie der Sicherheit, der Entwicklung und der Rechtfertigung des Wissens sowie der sozialen und der investigativen Dimension, Veränderungen in den Schülerantworten an.

Insgesamt muss jedoch bedacht werden, dass die hier beschriebenen Ergebnisse nicht verallgemeinerbar sind, da sich diese lediglich auf eine APL beziehen und die Anzahl der befragten SuS sehr gering war. Bezüglich der Beantwortung des Fragebogens sind zudem Verzerrungseffekte nicht auszuschließen. Des Weiteren nahmen hinsichtlich dieser APL Simulationen einen hohen Stellenwert in den Erzählungen der SuS ein, weshalb es sich anbietet, in weiteren Studien auch die zweite APL hinzuzuziehen und die Wirkung von APL auf das Wissenschaftsverständnis auch im Regelunterricht und mit größeren Stichproben zu untersuchen.

\subsubsection{Adaptierte Primärliteratur „Synthese von Glycosphingolipiden mit fluores- zenzmarkierten Fettsäuren für die quantiative Untersuchung der Anbindung von Shiga-Toxin an Lipidmembranen“}

\subsubsection{Darstellung des Adaptionsprozesses}

Unter Berücksichtigung der Schülerkritik zur ersten APL wurde eine zweite Primärliteratur des SFB 803 ausgewählt, um für den schulischen Gebrauch adaptiert zu werden. Insgesamt fiel es dabei schwer einen Artikel des SFB zu finden, der einen eindeutigen Bezug zum Kerncurriculum ermöglicht. Obwohl der VDAC-1-Kanal als Ionenkanal zumindest Anknüpfungspunkte zu den im Biologiecurriculum verankerten Membrantransportprozessen bietet, ist die Kritik der SuS an der Spezifizität des Kanals und der Erforschung des Schließmechanismus berechtigt. Mit dem Artikel Gb3 Glycosphingolipids with Fluorescent Oligoene Fatty Acids: Synthethis and Phase Behaviour in Model Membranes von PATALAG ET AL. ${ }^{[355]}$ wurde gezielt eine Veröffentlichung gewählt, deren Format typisch für die Darstellung von Syntheseprozessen ist. Der Beitrag beschäftigt sich mit der Synthese und Fluoreszenzmarkierung von Glycosphingolipiden, die als Rezeptoren für eine Vielzahl von bakteriellen Toxinen und Viren fungieren, und ihrem Membranverhalten unter Bindung von Shiga-Toxinen. Während der Forschungsgegenstand und auch die verwendeten Methoden für SuS weiterhin vornehmlich unbekannt sind - was sich mit Blick auf die Grundlagenforschung des SFB kaum vermeiden lässt - bietet die Untersuchung des für Krankheitserreger rezeptiven Lipids einen alltags- und gesellschaftsrelevanten Kontext. Zudem sind die Teilreaktionen der zur Synthese des Fluoreszenzmarkers angewendeten WittigReaktion (nucleophile Addition, Reduktion, Oxidation) allesamt curricular relevant, so 
dass die Betrachtung der Synthese sowohl als Wiederholung einzelner Reaktionsarten genutzt werden kann als auch Einblicke in das Zusammenspiel der zumeist separat gelehrten Reaktionen ermöglicht. Ein weiterer für die Auswahl dieser Publikation maßgeblich entscheidender Faktor war die Tatsache, dass der Artikel einen Forschungsprozess beschreibt, der nicht zu dem erwarteten Ergebnis geführt hat. Er zeigt also, dass wissenschaftliche Forschung nicht immer glückt und zu einem „Heureka“-Moment führt, dass aber auch solche Erkenntnisse wichtig sind und zum wissenschaftlichen Alltag gehören.

Der Inhalt der acht Seiten langen Originalpublikation ist in die Abschnitte Einleitung, Ergebnisse und Diskussion, Schlussfolgerungen sowie in einen experimentellen Abschnitt unterteilt. Er enthält also keinen ausgewiesenen Methodenteil, weshalb auch in der APL darauf verzichtet wurde. Zwar finden sich im experimentellen Abschnitt sowie in der Supporting Information einige methodische Angaben u. a. zu den Reaktionsbedingungen und den NMR-Spektren, diese sind jedoch für das Verständnis der SuS nicht relevant, weshalb sie keine Erwähnung finden. Des Weiteren wurde auf einen separaten Diskussionsteil verzichtet, da die sofortige Diskussion der Teilergebnisse gleichzeitig als Rechtfertigung für weitere Forschungsschritte dient.

Der Ergebnisteil gliedert sich im Originaltext in fünf Abschnitte. Während im ersten Abschnitt die Synthese der mit zwei verschiedenen Fluoreszenzmarkern versehenen $\mathrm{Gb}_{3}-$ Moleküle beschrieben wird, beinhalten die anderen Abschnitte verschiedene (fluoreszenz-)spektroskopische und -mikroskopische Untersuchungen. Für die Synthese der fluoreszenzgelabelten $\mathrm{Gb}_{3}$-Moleküle wurden zwei verschiedene Fettsäuren synthetisiert. Da eine Unterscheidung zwischen diesen beiden Fettsäuren für SuS keinen inhaltlichen Mehrwert darstellt, erfolgte in der APL eine Reduzierung auf die einfachere Struktur einer Pentaenfettsäure mit einem endständigen Phenylrest. Zur Veranschaulichung der unterschiedlichen Reaktionsmechanismen der Wittig-Reaktion, wurden diese in mehrere Teilreaktionen aufgeteilt und $\mathrm{u}$. a. nucleophile Angriffe durch zusätzliche Pfeile verdeutlicht.

Da SuS die Verwendung von Schutzgruppen in den meisten Fällen nicht geläufig ist, wurde diese sowohl im Reaktionsschema als auch im Text ausführlicher dargestellt. Im Anschluss an die Synthese des markierten $\mathrm{Gb}_{3}$-Moleküls wurden die verschiedenen Fluoreszenzanalysen der Moleküle in einem zweiten Ergebnisteil zusammengefügt, wobei die spektroskopische Untersuchung der beiden Moleküle aus dem Originalartikel vernachlässigt wurde. Da der FRET-Effekt SuS nicht bekannt ist, wurde ein einfaches Energiestufendiagramm erstellt, das die strahlungsfreie Energieübertragung von einem Fluorophor auf den nächsten erklären soll. Alle weiteren Ergebnisse wurden schließlich hinsichtlich der Kürzung der Gb3-Synthese angepasst.

Die Einleitung des Originalartikels beginnt mit einer kurzen Beschreibung des Aufbaus von Lipidmembranen, die in der APL um verschiedene Informationen wie der Funktion der Membran und die Beschreibung verschiedener Membranmodelle erweitert wurde. Diese Informationen ermöglichen eine Einordnung des Artikels in bekannte Zusammenhänge aus dem Biologieunterricht und erweitern zudem die Modellvorstellung vom Flüssig-Mosaik-Modell um das neuere Lipid-Floß-Modell, welches der Deutung der Ergebnisse zugrunde liegt. Um die Relevanz des untersuchten $\mathrm{Gb}_{3}$-Moleküls zu verdeutlichen, wurde das Beispiel des Shiga-Toxins auch in die APL übernommen, wobei dieses um Informationen zur EHEC-Epidemie aus dem Jahre 2011 erweitert wurde. Um die Notwenigkeit einer Fluoreszenzmarkierung der synthetischen $\mathrm{Gb}_{3}$-Moleküle zu erläutern, wurden zudem die Grenzen der zur Verfügung stehenden Abbildungstechniken kurz referiert. Im Original wie auch in der APL schließt die Einleitung mit der Hypothese ab, dass sich die fluoreszenzmarkierten $\mathrm{Gb}_{3}$-Moleküle wie ihre natürlichen Gegenstücke in der geordneten 
$l_{0}$-Phase der Lipidschicht verteilen und zudem in der Lage sind, die Shiga-Toxin-Untereinheit STxB zu binden.

Diese Hypothese wird in beiden Artikeln in der Schlussfolgerung wieder aufgegriffen und die Befunde entsprechend analysiert. Obwohl das natürlich vorkommende $\mathrm{Gb}_{3}$-Molekül unter Bindung des STxB nur in der geordneten $\mathrm{l}_{0}$-Phase vorkommt, fand man das synthetisierte Molekül nach Bindung des Toxins bevorzugt in der ungeordneten $l_{d}$-Phase vor. Das lässt darauf schließen, dass die synthetisierten Fluoreszenzmarker sich nicht eignen, um das Verhalten natürlicher $\mathrm{Gb}_{3}$-Moleküle zu simulieren.

Die Zusammenfassung des Originalartikels wurde schließlich lediglich an die nach der Kürzung verbleibenden Inhalte der APL angepasst und der Titel „Synthese von Glycosphingolipiden mit fluoreszenzmarkierten Fettsäuren für die quantitative Untersuchung der Anbindung von Shiga-Toxin an Lipidmembranen“ insofern verändert, als das Shiga-Toxin, das die gesellschaftliche Relevanz der Forschung hervorhebt, in den Titel mit aufgenommen wurde. Da das Phänomen der Fluoreszenz sowohl im Original als auch in der APL eine wichtige Rolle spielt, in den Kerncurricula der Chemie und Physik jedoch keine Erwähnung findet [183, 249], wurden als Begleitmaterial auf einer DIN A4-Seite zusätzliche Informationen zur Fluoreszenz bereitgestellt, anhand derer die Wahl des Pentaensystems für die Fettsäure erarbeitet werden kann.

\subsubsection{Evaluation}

Während bei der Evaluation der ersten APL die Frage im Vordergrund stand, ob sich dieses für den deutschsprachigen Raum neuartige Genre eignet, um das Verständnis der SuS für die NoS zu fördern, stand im Rahmen der zweiten APL eine Einbindung des Textformats in den naturwissenschaftlichen Unterricht im Vordergrund. Aufgrund der sehr positiven Einschätzung des begleitenden Arbeitsblatts der ersten APL durch die Studienteilnehmer_innen, insbesondere als Hilfestellung zum Erlangen eines inhaltlichen Verständnisses, wurde erneut ein Arbeitsblatt als Begleitmaterial ausgearbeitet um eine aufgabenbasierte Bearbeitung der APL zu ermöglichen.

Während die ersten drei Aufgaben zum inhaltlichen Verständnis in Anlehnung an die Verständnisfragen aus dem Fragebogen von BARAM-TSABARI \& YARDEN [354] formuliert wurden, sollten die weiteren Aufgaben einen deutlichen Bezug zum niedersächsischen Kerncurriculum der Oberstufe im Fach Chemie aufweisen. Dazu wurde die APL hinsichtlich curricularer Überschneidungen überprüft. Anhand verschiedener aus der Synthese entnommener Molekülstrukturen und Reaktionsmechanismen wurde ein modularer Aufgabenkatalog entworfen, der es Lehrkräften ermöglicht, je nach Vorwissen der Lerngruppe Aufgaben auszuwählen, die verschiedene Lernzielen der Einführungs- und Qualifikationsphase im grundlegenden und erhöhtem Anforderungsniveau bedienen. Dies sollte mit Blick auf die Lehrkräfte eine variable Anwendung, sowohl im Sinne unterschiedlicher Lerngruppen als auch verschiedene Verwendungsarten im Unterricht (z. B. zur Überprüfung des bereits Gelernten während der Abiturvorbereitung), ermöglichen. Zudem sollte es SuS die Notwendigkeit vernetzten Wissens in der Forschung aufzeigen. Eine Übersicht der den Aufgaben zugeordneten Lernziele ist in Tabelle 19 dargestellt. 
Tabelle 19: Verknüpfung des modularen Fragenkatalogs mit dem niedersächsischen Kerncurriculum der gymnasialen Oberstufe im Fach Chemie [183]. Die kursiv gedruckten Lernziele entsprechen dem erhöhten Anforderungsniveau. S-T = Stoff-Teilchen, CR = Chemische Reaktion, S-E = Struktur-Eigenschaft, E = Energie, EP = Einführungsphase, $\mathrm{QP}=$ Qualifikationsphase.

\begin{tabular}{|c|c|c|}
\hline Aufgabe & Lernziel & Konzept/Phase \\
\hline 1 & $\begin{array}{l}\text {... unterscheiden die Stoffklassen der }[\ldots] \text { Alkanole, Alkanale, }[\ldots], \text { Ester } \\
{[\ldots] \text { anhand ihrer Molekülstruktur und ihrer Funktionellen Gruppen. }}\end{array}$ & $\mathrm{S}-\mathrm{T} / \mathrm{EP}$ \\
\hline 2 & $\begin{array}{l}\text {... wenden die IUPAC-Nomenklatur zur Benennung organischer Moleküle } \\
\text { an. }\end{array}$ & $\mathrm{S}-\mathrm{T} / \mathrm{EP}$ \\
\hline 3 & $\begin{array}{l}\text {... stellen Redoxreaktionen [...] mithilfe der formalen Größe der Oxidati- } \\
\text { onszahlen dar. }\end{array}$ & $\mathrm{CR} / \mathrm{EP}$ \\
\hline 4 & $\begin{array}{l}\text {... nutzen ihre Kenntnisse zur Erklärung von Siedetemperaturen und Lös- } \\
\text { lichkeiten. }\end{array}$ & $\mathrm{S}-\mathrm{E} / \mathrm{EP}$ \\
\hline 5 & $\begin{array}{l}\text {... beschreiben die Oxidierbarkeit primärer, sekundärer und tertiärer Al- } \\
\text { kanole }\end{array}$ & $\mathrm{CR} / \mathrm{EP}$ \\
\hline 6 & $\begin{array}{l}\text {... beschreiben die Reaktion mit Brom als Nachweis für Doppelbindungen } \\
\text { in Molekülen. }\end{array}$ & $\mathrm{S}-\mathrm{T} / \mathrm{QP}$ \\
\hline \multirow[t]{2}{*}{7} & ... beschreiben die Fehling-Reaktion. & $\mathrm{S}-\mathrm{T} / \mathrm{QP}$ \\
\hline & $\begin{array}{l}\text {... begründen anhand funktioneller Gruppen die Reaktionsmöglichkeiten } \\
\text { organischer Moleküle. }\end{array}$ & $\mathrm{S}-\mathrm{E} / \mathrm{QP}$ \\
\hline 8 & $\begin{array}{l}\text {... benennen die funktionellen Gruppen: Doppelbindung, Hydroxy-, Car- } \\
\text { bonyl, [...]. }\end{array}$ & $\mathrm{S}-\mathrm{T} / \mathrm{QP}$ \\
\hline 9 & ... unterscheiden die Konstitutionsisomerie und die cis-trans-Isomerie. & $\mathrm{S}-\mathrm{T} / \mathrm{QP}$ \\
\hline $10 / 11$ & ... erklären induktive Effekte. & $\mathrm{S}-\mathrm{E} / \mathrm{QP}$ \\
\hline 12 & $\begin{array}{l}\text {... planen [...] Synthesewege zur Überführung einer Stoffklasse in eine an- } \\
\text { dere }\end{array}$ & S-E/QP \\
\hline 13 & ... beschreiben die Mesomerieenergie des Benzols. & $\mathrm{E} / \mathrm{QP}$ \\
\hline 14 & ... beschreiben den Reaktionsmechanismus der elektrophilen Addition [...]. & $\mathrm{S}-\mathrm{E} / \mathrm{QP}$ \\
\hline
\end{tabular}

\section{Forschungsinteresse}

Da aus der Literatur bekannt ist, dass Lehrkräfte dem Konzept der APL zu Beginn oftmals eher ablehnend gegenüberstehen [345], und die im Zuge der ersten APL interviewten SuS bereits die fehlende Nähe zu den Unterrichtsinhalten der Sekundarstufe II bemängelten, wurde vorerst keine explizite Implementierung der APL in den Unterricht ins Auge gefasst. Stattdessen sollte das Konzept zunächst Lehrkräften präsentiert und eine Einschätzung ihrerseits bezüglich der Schultauglichkeit und möglicher Anwendungsbereiche eingeholt werden, da diese als Gestalter naturwissenschaftlichen Unterrichts für die Auswahl der zu verwendenden Lernmaterialien verantwortlich sind und ein Gespür für die Einsatzmöglichkeiten und mögliche Hürden neuer Materialien mitbringen, was wiederum bei der Formulierung der Forschungsfragen Berücksichtigung fand.

F1 Welche Haltung nehmen Lehrkräfte gegenüber dem Textgenre der APL ein?

F2 Welche Einsatzmöglichkeiten bzw. Hürden sehen Lehrkräfte bezüglich der Verwendung von APL im Unterricht?

\section{Evaluationsdesign}

Das Konzept der APL wurde im Rahmen einer Lehrerfortbildung 25 Lehrkräften der Naturwissenschaften präsentiert, von denen 15 um die Zusendung der APL und weiterer Materialien baten. In Zuge der Aushändigung der Materialien per E-Mail bot es sich an, die interessierten Lehrkräfte um ein konstruktives Feedback bezüglich des Textgenres 
anhand verschiedener Leitfragen zu bitten. Entsprechend wurden im Anschluss an die Veranstaltung die APL, das Zusatzmaterial zur Fluoreszenz, der modulare Fragenkatalog sowie ein Informationsblatt zum neuen Genre APL an insgesamt 26 Lehrkräfte, u. a. auch von Partnerschulen, mit mindestens je einem der Fächer Chemie oder Biologie versandt.

Die Rücklaufquote fiel mit vier Rückmeldungen relativ gering aus, wobei eine Lehrkraft das gesammelte Feedback des gesamten Chemiekollegiums ihrer Schule zurückmeldete. Aufgrund dieser Tatsache erschien eine kategoriengestützte Analyse des Feedbacks nicht sinnvoll, weshalb vornehmlich nur Einzelmeinungen in die Beantwortung der oben genannten Fragestellungen einfließen können.

\section{Evaluationsergebnisse}

Das Feedback der Lehrkräfte fiel insgesamt eher heterogen aus. Die Grundidee der Adaptierung von Primärliteratur wurde von allen Lehrkräften im Allgemeinen positiv bewertet. Gleiches galt zudem für den modularen Fragenkatalog, bei dem die Anpassung an das Chemiecurriculum als gelungen hervorgehoben wurde. Jedoch bewerteten die Lehrkräfte sowohl den Umfang als auch die inhaltliche Tiefe der APL eher negativ. Insbesondere aufgrund der zahlreichen Fremd- und Fachwörter sei die Lese- und Bearbeitungszeit vor allem für schwächere SuS sehr lang, weshalb sich die APL höchstens für die Erarbeitung durch leistungsstärkere eignet. Neben der Länge und Komplexität des Textes, bemängelten die Lehrkräfte zudem eine zu geringe inhaltliche Anknüpfung des Textes an die Kerncurricula. Obwohl der begleitende modulare Fragenkatalog diesbezüglich gelobt wurde, scheint ein Aufzeigen der curricular relevanten Inhalte im Rahmen der Bearbeitung der Aufgaben aus dem Fragenkatalog den Lehrkräften nicht auszureichen.

Hinsichtlich einer Optimierung der APL wurde u. a. eine Kürzung, insbesondere im Ergebnisteil der spektroskopischen und mikroskopischen Untersuchungen, empfohlen. Als methodischer Ansatz für die Arbeit mit APL wurde außerdem im Sinne des Scientific LiteracyModells eine langsame Heranführung an das Genre anhand kürzerer Texte und Beigabe zusätzlicher Hilfsmittel, wie eine Übersicht über Syntheseverfahren der organischen Chemie, vorgeschlagen. Eine Lehrkraft sah jedoch durchaus Entwicklungspotential in der Verwendung von APL, wenn diese im Rahmen eines Wahlfachs, bspw. als naturwissenschaftliche Alternative zur zweiten oder dritten Fremdsprache, eingesetzt würde.

Die Kritikpunkte der Lehrkräfte sind stellenweise sicherlich angebracht und somit auch nicht ganz unerwartet. Die Länge dieser zweiten entspricht mit sieben Seiten der Länge der ersten APL. Mit einer Lesezeit von durchschnittlich 60 Minuten sowie einer Bearbeitung des Arbeitsblatts von weiteren 30 Minuten lag die Bearbeitungszeit durch die Evaluationsteilnehmer_innen der ersten APL insgesamt im Rahmen einer Doppelstunde. Wird die Auswertung der Aufgaben hinzugenommen, müssen also mindestens zwei Doppelstunden für die Arbeit mit dieser APL im Unterricht eingeplant werden, was mit Blick auf bereits sehr volle Stundenpläne sicherlich nur dann von Lehrkräften in Betracht gezogen wird, wenn sie einen Mehrwert darin sehen. Eine weitere Kürzung der APL würde zwar zu einer angenehmeren Textlänge führen, dies würde jedoch zwangsläufig bedeuten, dass entweder zusätzliche Informationen, die mit Blick auf das Schülerverständnis hinzugefügt wurden, weichen müssten, oder dass die APL inhaltlich weiter gekürzt werden müsste. Insbesondere eine weitere Kürzung im Ergebnisteil würde dazu führen, dass der wissenschaftliche Charakter der zugrundliegenden Primärliteratur verloren ginge, so dass der Text näher an journalistische Artikel oder Schulbuchliteratur rücken würde. Gerade das würde jedoch die Entwicklung und Verwendung von APL obsolet machen. Auch ein Herausstreichen der Fremd- und Fachwörter, die in der APL in vielen Fällen sowieso 
bereits fehlen oder im Text erklärt werden, würde zu einer Einbuße der Authentizität führen. Jedoch wäre es hier denkbar, ähnlich wie in der ersten APL, als differenzierende Maßnahme ein Glossar zu erstellen.

Die inhaltliche Anknüpfung des Textes an das Kerncurriculum stellt weiterhin eine deutliche Hürde dar, die auch mit der Auswahl der zweiten APL nicht behoben werden konnte. Hier muss gerade der Nutzen von APL im Rahmen einer Öffentlichkeitsarbeit, zumindest hinsichtlich einer Anwendung im schulischen Kontext, in Frage gestellt werden. Sicherlich besteht bei aktueller Forschung immer das Problem, dass es lange dauert, bis neue Erkenntnisse Einzug in die Schulliteratur finden. Jedoch fehlt gerade mit Blick auf die Grundlagenforschung des SFB oftmals ein schulrelevanter Kontext und durch die Anwendung vernetzten Wissens fällt es schwer, gezielt einzelne Basiskonzepte zu fördern. Der Vorschlag der Lehrkräfte, einfachere kürzere Texte zu verwenden, könnte hier zumindest teilweise zu einer Lösung beitragen. Um bspw. beim Thema der Biomembranen zu bleiben, könnten (ältere) Artikel, die bahnbrechende oder gesellschaftsrelevante Entwicklungen zeigen, im Sinne einer Scientific Literacy erst anhand von journalistischen Artikeln und anschließend in Form von kürzeren adaptierten Publikationen erarbeitet werden. Dies würde den Übergang zu längeren Texten zumindest vereinfachen, so dass auch aktuellere Forschung betrachtet werden kann.

\subsubsection{Reflexion des Formats}

Mit APL wird hier ein für den deutschen Sprachraum neues Textgenre für den naturwissenschaftlichen Unterricht vorgestellt. Die Verwendung von APL bietet die Möglichkeit, anhand eines adaptierten wissenschaftlichen Artikels authentische Einblicke in naturwissenschaftliche Erkenntnisprozesse und Arbeitsweisen zu geben. Gerade im Hinblick auf die Berufsorientierung von SuS stellt sich die Frage, inwiefern das Lesen von APL einen Beitrag zum Verständnis der NoS auf Seiten der SuS leisten kann.

Die im Rahmen der zwei entwickelten APL durchgeführten Befragungen von SuS sowie Lehrkräften zeigen, dass beide Gruppen dem neuen Textgenre prinzipiell zumeist positiv gegenüberstehen. Dennoch äußerten sich sowohl Lehrkräfte als auch SuS auch kritisch. Dabei waren Kritikpunkte die von allen geäußert wurden die Länge und die inhaltliche Fülle bzw. Komplexität der Texte. Während die Lehrkräfte vor allem mit Blick auf die straffen Lehrpläne, aber auch die Leistungsfähigkeit ihrer SuS vor der Verwendung der APL eher zurückschreckten, sahen einige der SuS das Lesen der APL als eine willkommene Herausforderung und wichtige Kompetenz an. Diesbezüglich wurde von den SuS vorgeschlagen, APL im Seminarfach in Vorbereitung auf die Anfertigung der Facharbeit zu lesen, um z. B. Lesestrategien für die Recherche mit Primärliteratur zu entwickeln. Das scheint gerade insofern sinnvoll, als in den Hinweisen und Empfehlungen zum Seminarfach des Niedersächsischen Kultusministeriums unter anderem das „Lernen am Original“ gefordert wird. „Damit ist forschendes und entdeckendes Lernen und Experimentieren auch an neuen, innovativen Fragestellungen - ebenso gemeint wie die Synopsis und Deutung vorhandenen Wissens" [362].

Einige der SuS hinterfragten jedoch auch, inwiefern ein Text zu einem speziellen Ionenkanal wie dem VDAC-1 relevant für den Unterricht oder auch sie persönlich sei. Ähnliche Einwände äußerten auch die Lehrkräfte, die gerade inhaltlich die fehlende Nähe zum Kerncurriculum monierten. Hier muss die Frage nach der Zielsetzung der Verwendung von APL diskutiert werden. Das Feedback der Lehrkräfte lässt vermuten, dass für sie das vollständige inhaltliche Verstehen der APL durch die SuS im Vordergrund steht. Rückt man jedoch von dieser Zielsetzung etwas ab und betrachtet mögliche implizite Lernprozesse, wie die Förderung eines NoS-Verständnisses sowie Lesekompetenzen bezüglich 
primärer Literatur zur Förderung u. a. der Studierfähigkeit, bieten sich Möglichkeiten, die gegebenenfalls bei den Überlegungen der Lehrkräfte bisher keine Rolle gespielt haben. Bei einer Anwendung von APL in diesem Sinne muss SuS jedoch deutlich gemacht werden, dass das inhaltliche Verständnis, im Gegensatz zum Lesen gewöhnlicher Texte, nicht im Vordergrund steht. Eine Einführung in das Genre könnte hierbei, wie bereits erwähnt, langsam und stetig erfolgen, indem in Anlehnung an das Scientific Literacy-Modell zunächst journalistische Artikel und kürzere APL gelesen werden [354].

In Bezug auf ein besseres inhaltliches Verständnis könnte zudem die Wahl der zu adaptierenden Primärliteratur überdacht werden. Die bisherige Auswahl von Publikationen aus dem SFB 803 erfolgte mit Blick auf eine inzidentelle Form der Öffentlichkeitsarbeit. In Hinblick auf eine unterrichtliche Verwendung würden sich ggf. Publikationen der angewandten Forschung eher eignen als solche aus der Grundlagenforschung. Obwohl die Ergebnisse nicht minder spannend oder wichtig sind, lassen sich die Ergebnisse angewandter Forschung eher kontextualisieren, so dass die gesellschaftliche Relevanz der Ergebnisse leichter zu erfassen ist. Dennoch ist die Adaptierung von Primärliteratur kein ungeeignetes Format für eine gezielte Öffentlichkeitsarbeit, da sie es dem interessierten Leser ermöglicht Einblicke in Forschungsgebiete zu erhalten, die nicht primär in wissenschaftsjournalistischen Artikeln abgebildet werden.

\subsection{Informell Inzidentell - Bildungsmessen}

Die bisher aufgeführten Formate bedienen alle spezielle Zielgruppen, wodurch die Reichweite der Projekte automatisch eingeschränkt wird. Zwar bestehen hinsichtlich aller Projekte Bemühungen, diese möglichst weitläufig zu disseminieren (u. a. im Zuge von Publikationen in wissenschaftlichen Journalen, Präsentationen auf nationalen und internationalen Tagungen, Lehrerfortbildungen und einer Website), jedoch wird auf diese Weise dennoch nur ein ausgewähltes Publikum erreicht. Naturwissenschaftliche Bildungsmessen bieten dagegen die Möglichkeit, ein sehr breites Publikum anzusprechen. Obwohl solche Messen auch für die interessierte allgemeine Öffentlichkeit zugänglich sind, werden sie von zahlreichen Schulklassen und Lehrkräften besucht, so dass auch hier eine zielgruppenorientierte Arbeit möglich ist.

Die bekannteste deutsche Bildungsmesse ist die IDEENEXPO, die seit 2007 alle zwei Jahre neun Tage lang in Hannover stattfindet und 2019 in der siebten Auflage durchgeführt wurde. Mit zuletzt 395.000 Besucher_innen, 270 Aussteller_innen und einer Ausstellungsfläche von $110.000 \mathrm{~m}^{2}{ }^{[141]}$ zählt sie zu den besucherstärksten deutschen Messeveranstaltungen, zu denen unter anderem bekannte Technik- und Automobilmessen gehören. Obwohl sie als lokale Veranstaltung begann, reisen mittlerweile $53 \%$ der Besucher_innen aus Bundesländern außerhalb Niedersachsens an [363]. Unter den Ausstellern finden sich Unternehmen, Forschungseinrichtungen, Hochschulen und Schulen aus ganz Deutschland. Finanziert wird die kostenlose Messe dabei sowohl aus Ausstellerbeiträgen als auch aus Mitteln des Landes Niedersachsen. Die Zielsetzung der Messe wird auf der Website der IDEENEXPO wie folgt beschrieben:

„Ziel der IdeenExpo ist es, fundierte Wissensvermittlung mit einem hohen Spaßfaktor $z u$ verbinden. Gerade junge Besucher können spannende Entdeckungen machen und bekommen so die Gelegenheit, Naturwissenschaften und Technik hautnah aus einer neuen Perspektive zu entdecken. Das breite Angebot an Themen auf der IdeenExpo bietet jungen Menschen einen Kompass zur persönlichen Berufsorientierung. " [141]

Die Förderung des naturwissenschaftlichen Interesses vornehmlich junger Menschen, aber auch der allgemeinen Gesellschaft, steht also im Vordergrund. Entsprechend bietet 
die Messe zahlreiche Mitmachstationen und -experimente, Workshops, die z. T. von SuS für SuS angeboten werden, sowie Bühnenshows und Science Slams. Die Vielfalt der Aussteller bildet dabei zahlreiche berufliche MINT-Perspektiven ab, so dass sich die Besucher_innen aktiv sowohl über Ausbildungsmöglichkeiten, als auch über Studiengänge informieren können. Die 670 Exponate, die 2019 auf der IDEENEXPO zu besuchen waren, wurden dabei vornehmlich von Auszubildenden oder Studierenden der Unternehmen und Hochschulen entwickelt. Zudem stellten 25 Schülergruppen, die sich beim Schülerwettbewerb „Ideenfang“ durchgesetzt hatten, verschiedene kreative Projekte vor. Diese jungen Menschen sind es auch in den meisten Fällen, die die Exponate präsentieren und so ein Gespräch auf Augenhöhe ermöglichen [364].

Mit $400 \mathrm{~m}^{2}$ Ausstellungsfläche gehörte das BUNDESMINISTERIUM FÜR BILDUNG UND FORSCHUNG 2019 zu den größten Ausstellern der IDEENEXPO [364]. Mit seiner Präsenz unterstreicht das Ministerium die in seinem MINT-Aktionsplan beschriebene Förderung von Projekten im Handlungsfeld „MINT in der Gesellschaft“, die auch Bildungsmessen beinhaltet [365]. Als Beispiel wird hier das vom BMBF und der Deutschen PhysiKAlischen GeselLSCHAFT (DPG) geförderte Wissenschaftsfestival HIGHLIGHTS DER PHYSIK (HDP) genannt, das 2001 in Anlehnung an Veranstaltungen zum weltweiten Jahr der Physik (2000) initiiert wurde. Die HDP finden jährlich wechselnd in unterschiedlichen (Universitäts-)Städten Deutschlands in Kooperation mit den dort ansässigen Universitäten und weiteren lokalen Partnern statt. Im öffentlichen Raum, zumeist auf Marktplätzen bzw. in der Innenstadt, beherbergen Veranstaltungszelte fünf Tage lang eine Ausstellung zu einer ebenfalls jährlich wechselnden physikalischen Thematik mit bis zu 50 Exponaten. Des Weiteren werden über die Stadt verteilt Wissenschaftsshows, Workshops, Vorträge und Science Slams angeboten, die allesamt kostenfrei besucht werden können. Zuletzt wurden die verschiedenen Angebote in der 19. Auflage in Bonn von rund 60.000 Besucher_innen wahrgenommen [366]. Im Gegensatz zur IDEENEXPO, die ein sehr breites Spektrum an MINT-Aspekten abdeckt, fokussieren sich die HDP vornehmlich auf physikalische Themen, die jedoch, wie zuletzt mit Überschriften wie Mikrokosmos (2016) oder Herzrasen (2018) [367], auch Bezüge zu anderen MINT-Disziplinen sowie der Medizin zulassen. Durch ihre zentrale Lage wird die Veranstaltung nicht nur von Schulen der Region und interessierten Menschen, die sich explizit mit physikalischen Themen auseinandersetzen wollen, wahrgenommen, sondern in besonderem Maße auch spontan von „Innenstadtbummlern“ besucht. Obwohl viele Universitätsstädte mittlerweile Formate wie die NACHT DES WISSENS oder LANGE NACHT DER WISSENSCHAFT anbieten, bei denen vor allem universitäre und zum Teil auch weitere Forschungseinrichtungen der Städte für eine Nacht ihre Türen öffnen und sich der Öffentlichkeit präsentieren, macht gerade die Möglichkeit der eher zufälligen Teilnahme durch die zentrale Positionierung in der Innenstadt den Charme der HDP aus.

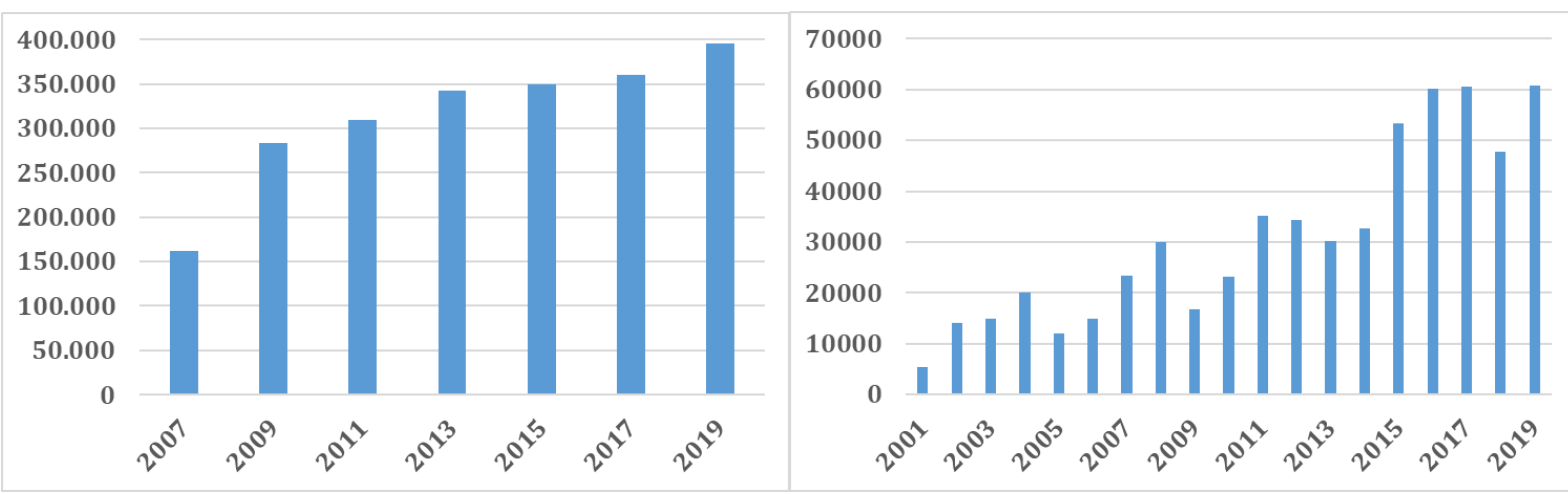

Abbildung 93: Besucher_innenzahlen der IDEENEXPO[141] (links) und der HIGHLIGHTS DER PHYSIK [366] (rechts). 
Die Vielfalt der MINT-Perspektiven, die Bildungsmessen wie die IDEENEXPO und auch die HDP abbilden, ermöglicht es, naturwissenschaftliche Öffentlichkeitsarbeit - in diesem Fall bezogen auf universitäre Einrichtungen - sowohl in allgemeiner Form, d. h. als Werbung für einen ganzen Fachbereich oder eine Fakultät, als auch für spezifische Forschungsgruppen oder -projekte, wie die Forschung des SFB 803, zu gestalten. Einschränkende Faktoren der Dissemination von Forschungsaspekten, wie die oben genannte Spezifität der Zielgruppe aber auch die Komplexität der Forschungsinhalte, spielen hier nur eine untergeordnete Rolle. Die große Auswahl an Exponaten ermöglicht es den Besucher_innen, selbst zu entscheiden, ob sie sich mit den in Bezug zum SFB präsentierten biochemischen Aspekten auseinandersetzten möchten oder ob sie sich lieber einem anderen Thema widmen. Ihnen ist es dabei zudem selbst überlassen, wie viel Zeit sie an verschiedenen Exponaten verbringen und wie tief sie in die jeweilige Materie einsteigen möchten. Daraus ergibt sich wiederum, dass gerade bei einem großen Angebot an Aktivitäten davon ausgegangen werden kann, dass die Besucher_innen eines bestimmten Exponats ein gewisses Interesse mitbringen, wobei die Art des Interesses hierbei natürlich variieren kann und von verschiedenen Aspekten wie bspw. der Thematik, der Attraktivität des Exponats auf den ersten Blick sowie weiteren intrinsischen Faktoren abhängt.

Insbesondere die Besucher_innenstruktur macht Bildungsmessen $\mathrm{zu}$ einem wichtigen Baustein der Öffentlichkeitsarbeit des SFB 803. Obwohl das Alter der Besucher_innen insgesamt sehr heterogen ist, ist durch die allgemeine Ausrichtung der Veranstaltungen auf die Zielgruppe der SuS ab der Sekundarstufe I die Erarbeitung eines Messekonzepts mit Fokus auf die Sekundarstufe II möglich und im Sinne des Ö-Projektes auch sinnvoll. Hier bietet es sich an, die bereits erarbeiteten Materialien und Experimente der anderen Projekte auf ihre Messetauglichkeit zu überprüfen, um insbesondere auch im Gespräch mit Lehrkräften auf diese verweisen zu können. Bildungsmessen ermöglichen es zudem auch die für den SFB eher zweitrangige Zielgruppe der interessierten Allgemeinheit zu erreichen und somit auch fernab des schulischen Alltags über die gesellschaftliche Bedeutung von Forschung zu informieren und für diese zu sensibilisieren.

\subsubsection{Das Messekonzept des SFB 803}

Ähnlich wie die Unterrichtsmaterialien baut auch das Messekonzept auf Vorarbeiten aus dem Pilotprojekt SFB@SCHOOL auf. Basierend auf der Unterrichtseinheit „Vom Tensid zur Biomembran" wurde im Zuge des Projektes ein Ausstellungsregal entwickelt, das sich am EXPOneer-Konzept orientiert und erstmalig auf der IDEENEXPO 2015 ausgestellt wurde.

\section{Exkurs: EXPOneer-Ausstellungsregale}

Das EXPOneer-Konzept beschreibt eine Reihe von Regalkonstruktionen, die als modulares Ausstellungssystem verstanden werden können und am LEIBNIZ-INSTITUT FÜR DIE PÄDAGOGIK DER NATURWISSENSCHAFTEN UND MATHEMATIK (IPN) in Kiel entwickelt wurden. Das Konzept entstand im Rahmen des von der EU geförderten IRRESISTIBLE-Projekts, innerhalb dessen Aktivitäten zur Sensibilisierung von SuS zum verantwortungsvollen Umgang mit Forschung und Innovation im Sinne des Konzeptes der Responsible Research and Innovation entwickelt wurden [368]. Dabei sollte SuS u. a. die Möglichkeit gegeben werden, auf möglichst professionelle Weise wissenschaftliche Ausstellungsstücke zu entwerfen. Um dabei schulischen Ansprüchen wie bspw. zeitlichen und monetären Restriktionen zu entsprechen sollten sie schnell und unkompliziert aufzubauen, dabei möglichst flexibel zu handhaben und wiederverwendbar sein. Zusätzlich sollten alle Materialien möglichst einfach erhältlich sein und zudem sollten die Kosten insgesamt möglichst gering ausfallen. 
Basierend auf diesen Kriterien wurde als Grundlage für die Exponate das Kallax-Regalsytem von IKEA gewählt, welches insbesondere deshalb für ein modulares Ausstellungssystem geeignet scheint, weil die Regale zum einen quadratische Schrankfächer besitzen, und zum anderen mit verschiedenen Fächeranzahlen und -ausrichtungen zu erwerben sind. Die quadratischen Fächer können als eigenständige Elemente mit Informationstafeln oder Bildschirmen ausgestattet werden oder als Vitrinen oder Hörstationen dienen. Ein Ausstellungshandbuch bietet zudem zahlreiche Bauanleitungen und Layoutvorlagen, die SuS eine eigenständige Planung und Erarbeitung ermöglichen [368, 369].

Obwohl das EXPOneer-Konzept ursprünglich für von SuS entwickelte Ausstellungen gedacht war, eignet es sich aufgrund der zugrundeliegenden Kriterien ebenso gut für Exponate im Bereich der Öffentlichkeitsarbeit. Zum einen lohnen sie sich wegen ihrer Flexibilität und kostengünstigen Anschaffung, um ggf. lediglich auf einer einzigen Veranstaltung eingesetzt zu werden, zum anderen sind sie gleichzeitig aber auch robust und transportabel, so dass sie auch auf mehreren Messen Verwendung finden können.

Das im Rahmen des SFB@School Projekts entwickelte EXPOneer zu Tensiden und Biomembranen dient auch nach einigen Jahren der Verwendung noch als Grundstein für viele Gespräche mit Messebesucher_innen (siehe Abbildung 94). Gerade Tenside sind vielen Besucher_innen - wenn auch nicht unter diesem Begriff - bekannt, so dass sogar mit SuS im Grundschulalter mithilfe der Abbildungen und Experimente Vorgänge bei Spülprozessen thematisiert werden können. Auch Seifenblasen sind allen Teilnehmer_innen ein Begriff, so dass der Übergang zum Aufbau von Biomembranen den meisten verständlich dargelegt werden kann. Zwei push-the-button-Experimente zu hydrophoben Textiloberflächen und zur Lichtstreuung verdeutlichen die Eigenschaften der Teilchen sowie die Selbstorganisation zu Mizellen. Vertiefende Einblicke in die Forschung des SFB ermöglichen das bereits in Kapitel 6.1.1 erwähnte Video sowie ein Informationstext. In den Vitrinen finden sich zudem weitere Einblicke ins Labor sowie Beispiele zu Tensiden und Membranen im Alltag.

Die Infotafeln des Regals enthalten vornehmlich erklärende Abbildungen und kurze, optisch voneinander getrennte Texte, die selten 60 Wörter übersteigen. Im Zusammenspiel mit den anderen Elementen des EXPOneers soll es den Besucher_innen so ermöglicht werden, sich selbstständig über Biomembranen zu informieren. Diese Möglichkeit wird jedoch nur von wenigen Menschen auch tatsächlich wahrgenommen. Stattdessen dient das Regal in erste Linie als Referenz für die Erläuterungen der Standbetreuer, die vor allem die Abbildungen nutzen, um ihre Erklärungen zu visualisieren. Somit fungiert das EXPOneer dennoch als wichtige Informationsquelle für die Besucher_innen. Auf die allgemeinen Erfahrungen mit den EXPOneer-Regalen wird jedoch im Zuge der Evaluation des Messekonzepts noch genauer eingegangen. 


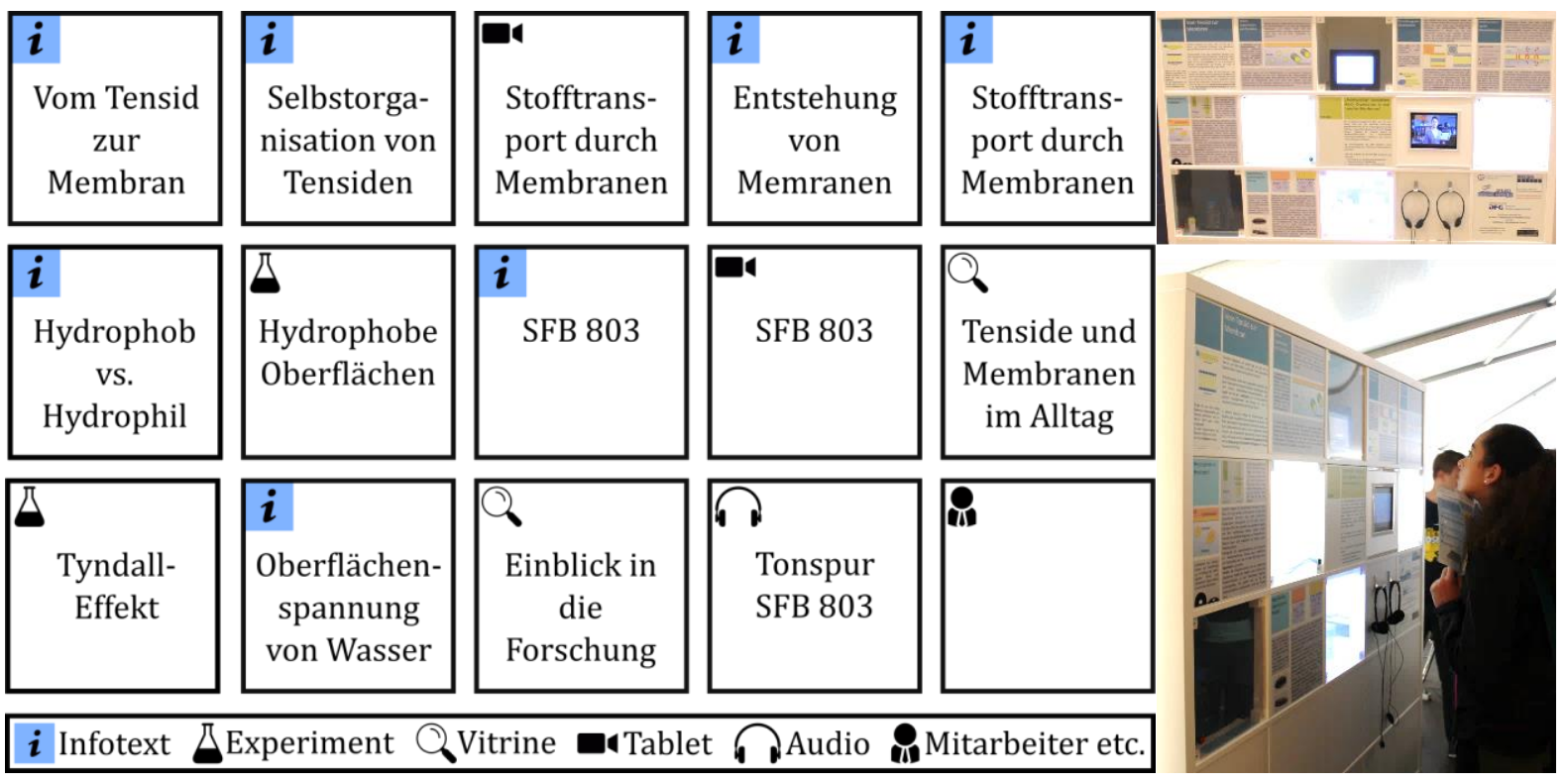

Abbildung 94: Schematische Darstellung der Inhalte der einzelnen Fächer des EXPOneers „Vom Tensid zur Biomembran“ (links), sowie dessen Einsatz auf Bildungsmessen (rechts).

Die Weiterentwicklung des Messekonzepts geht einher mit der Entwicklung der oben genannten anderen Projekte, die sich wiederum an den in Kapitel 5 formulierten Leitfragen orientieren. Das erste EXPOneer ermöglicht es diesbezüglich, Antworten auf die Fragen zu finden, welche Struktur-Eigenschaftsbeziehungen für die Kompartimentbildung verantwortlich sind und woraus Biomembranen bestehen. Zudem liefert es erste Informationen hinsichtlich verschiedener Membrantransportwege. In Anlehnung an das Schülerlaborkonzept zum Thema Botox widmet sich das zweite EXPOneer mit dem Titel „Gifte für die Schönheit" am Beispiel der Exocytose am synaptischen Spalt der Frage, wie Grenzen überwunden werden können (siehe Abbildung 95). Die Thematik schien dabei für die Öffentlichkeit gerade deshalb als geeignet, weil Botox den meisten Menschen aus den Medien ein Begriff ist und insbesondere aufgrund der zahlreichen medizinischen Anwendungsbereiche eine große Alltagsrelevanz aufweist. Gleichzeitig ermöglicht die Betrachtung von Botulinumtoxin aus wissenschaftlicher Sicht die Thematisierung der SNAREKomplexe und ihrer Funktionen, die wiederum einen Bezug zur Forschung des SFB im Sinne der dritten Leitfrage zulassen.

Um auch unabhängig vom ersten EXPOneer als geschlossene Einheit fungieren zu können, werden in einem kurzen Überblickstext sowie einer Animation zunächst Aufbau und Funktion von Membranen wiederholt. Diese dienen als Grundlage für die auf den nachfolgenden Infotafeln beschriebene SNARE-vermittelte Exocytose an der Präsynapse, die zusätzlich anhand eines push-the-button-Modellexperiments nachvollzogen werden kann. Zwei weitere Infotafeln sowie eine zweite Animation vermitteln zudem Inhalte zum als Botox bekannten Gift Botulinumtoxin sowie seiner Wirkweise am synaptischen Spalt. Die Informationen zum SFB 803 nehmen erneut Bezug zur SNARE-vermittelten Fusionen von Lipidmembranen und verdeutlichen, mithilfe welcher Forschungstechniken diese untersucht werden können. Auf einem weiteren Touchscreen können schließlich zusätzlich Animationen und kurze Präsentationen angewählt werden, die u. a. weiterführende Informationen zur Fluoreszenzmikroskopie enthalten. 


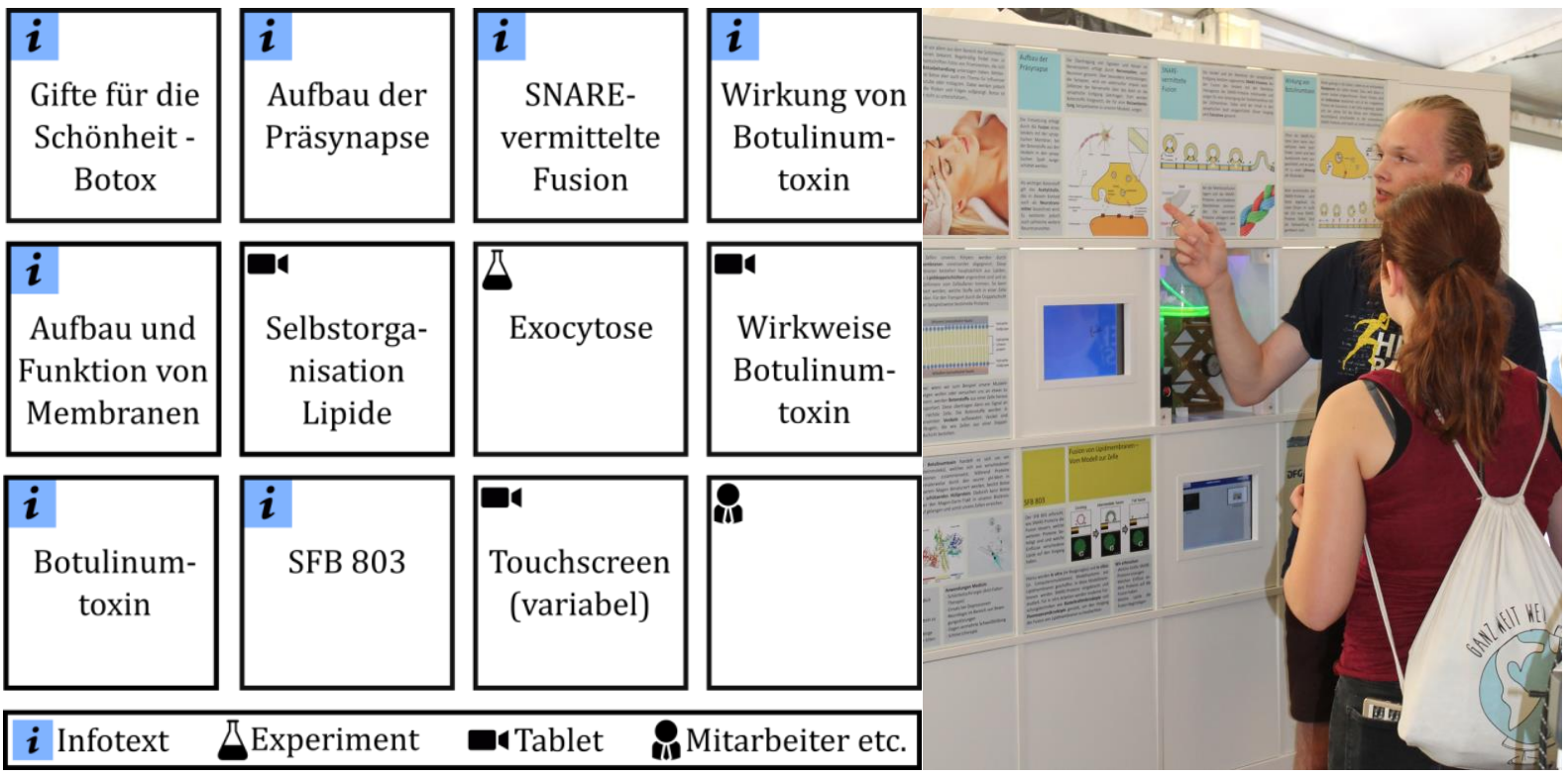

Abbildung 95: Schematische Darstellung der Inhalte der einzelnen Fächer des EXPOneers „Gifte für die Schönheit" (links) sowie dessen Einsatz auf Bildungsmessen (rechts).

Um schließlich im Sinne der dritten Leitfrage zudem auch verschiedene Forschungsmethoden genauer betrachten zu können, wurde ein drittes EXPOneer-Regal zur Rasterkraftmikroskopie (Atomic Force Microscopy, AFM) entwickelt (siehe Abbildung 96). In zwei Informationstexten werden Aufbau und Funktionsweise des AFM beschrieben. Einem weiteren Textelement können zudem Informationen über die Bedeutung der Rasterkraftmikroskopie für die Membranforschung entnommen werden. Zusätzlich stellt ein Animationsvideo die Funktion des Mikroskops noch einmal modellhaft dar und versucht zudem, anhand eines Größenvergleichs die Nanodimension begreifbar zu machen.
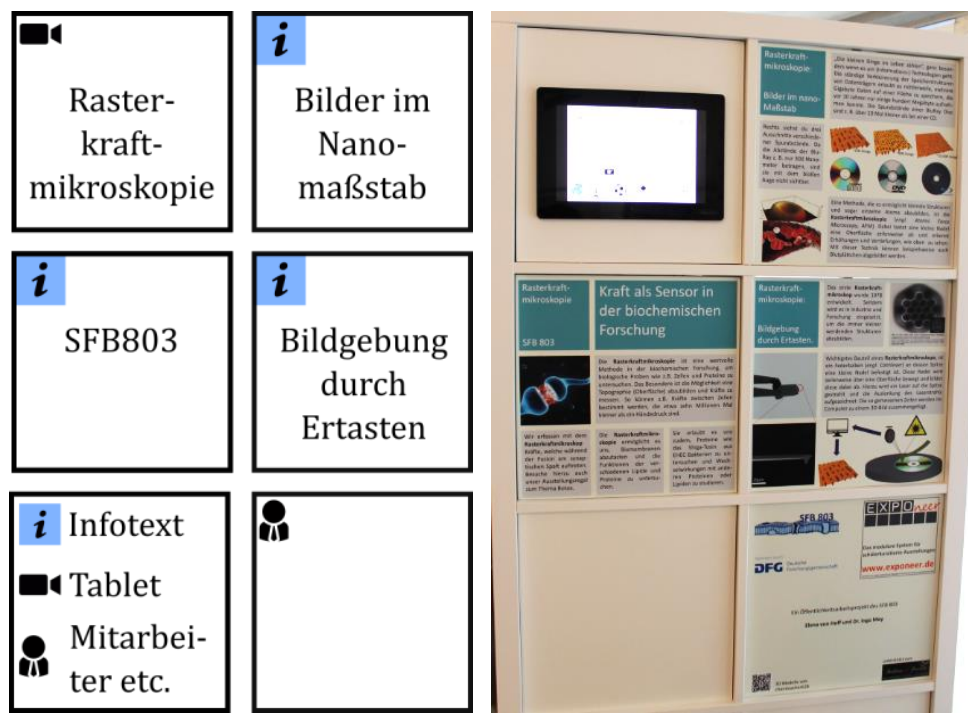

Abbildung 96: Schematische Darstellung der Inhalte der einzelnen Fächer des EXPOneers „Rasterkraftmikroskopie" (links), sowie dessen Einsatz auf Bildungsmessen (rechts).

Während die EXPOneer-Regale vor allem aufgrund der Visualisierung verschiedener wissenschaftlicher Inhalte die Möglichkeit bieten, Besucher_innen jeden Alters und unterschiedlichen Vorwissens an das Thema der Biomembranen heranzuführen, zeigen Beobachtungen, dass sie trotz interaktiver Module als Exponate nicht ausreichen, um Besucher_innen zur Teilnahme zu motivieren. Obwohl diese von Wissenschaftsfestivals naturgemäß wissenschaftliche Inhalte erwarten, scheint für die meisten Teilnehmer_innen 
dennoch der Festivalcharakter der Veranstaltung im Vordergrund zu stehen. Entsprechend erfolgt ein Wissenserwerb inzidentell, zumal die Teilnehmer_innen vor dem Besuch der Messe oft nicht wissen, welche Inhalte sie erwarten werden. Das Messekonzept für den SFB beinhaltet deshalb zahlreiche hands-on-Experimente. Die Experimente erfüllen dabei ähnliche Funktionen wie im Chemieunterricht. Neben der fachlichen Dimension, die u. a. das Vertrautwerden mit Phänomenen und die Vermittlung von Fachwissen beinhaltet, dienen sie im Fall von Bildungsmessen vor allem der Interessenentwicklung und förderung sowie der Motivationssteigerung, die wiederum der pädagogischen Dimension entsprechen ${ }^{[370] .}$

Der Unübersichtlichkeit und Schnelllebigkeit großer Messen geschuldet, müssen die angebotenen Experimente bestimmte Kriterien erfüllen. Da es selbst bei ausreichend Standpersonal zu Situationen kommen kann, in denen alle Betreuer in Gespräche verwickelt sind, und die Übersicht über den Ausstellungsstand kurzzeitig fehlen kann, müssen die eingesetzten Chemikalien möglichst ungefährlich sein. Zudem sollten die Experimente schnell durchzuführen sein und wenig Vorbereitungszeit benötigen. Im günstigsten Fall können die Versuche dabei ohne großen Aufwand sogar mehrfach wiederholt werden.

Da sich das Messekonzept zunächst am EXPOneer „Vom Tensid zur Biomembran“ orientierte, boten sich Experimente im Kontext von Tensiden, wie das Experiment 3.1 zur Oberflächenminimierung, sowie mikroskopische Betrachtungen von Pflanzenzellen (in Anlehnung an Experiment 3.2b) an. Mit der Entwicklung der weiteren EXPOneer-Regale traten Experimente zur Membranfusion (6.3a) und zur Exocytose (4.3a) in den Vordergrund. Zudem wurden verschiedene 3D-Modelle sowohl zu den Lipidgeometrien (2.4) als auch zur Rasterkraftmikroskopie dazu genutzt, um submikroskopische Vorgänge auch auf makroskopischer Ebene erfahrbar zu machen. Die Anschaffung eines einfachen Fluoreszenzmikroskops ${ }^{18}$ durch das Öffentlichkeitsprojekt des SFB 803, das vornehmlich für die Verwendung auf Bildungsmessen vorgesehen ist, ermöglichte es zudem, fluoreszenzmarkierte Zellen ${ }^{19}$ (u. a. Fibroblasten) zu untersuchen und somit eine weitere wichtige Forschungsmethode des SFB 803 zu beleuchten. Da das Phänomen der Fluoreszenz, wie bereits zuvor erwähnt, weder im niedersächsischen Kerncurriculum der Physik noch der Chemie Erwähnung findet [183, 249], wurden weitere Experimente, wie verschiedene Anregungsmöglichkeiten von Fluoreszenzfarbstoffen $(5.4,5.6)$ sowie die Fluorochromierung von Blüten (6.2a), in das Messekonzept integriert. Die Berücksichtigung neuer Experimente führte mitunter dazu, dass entsprechend der thematischen Fokussierung der Messe (wie im Fall der HDP) eine Auswahl geeigneter Experimente möglich ist, was je nach Platzangebot auf den Messen ebenso für die Mitnahme der EXPOneer-Regale gilt. In die kontinuierliche Weiterentwicklung des Messekonzepts flossen neben Erfahrungswerten auch Ergebnisse aus Evaluationen ein, die während der IDEENEXPO 2017 und der HDP 2018 durchgeführt wurden.

\footnotetext{
18 LED-Auflicht-Fluoreszenzmikroskop Axio Lab.A1, ZEISs Deutschland

${ }^{19}$ Bereitgestellt durch die Abteilung für Zellkultur des InSTITUTS FÜr ORGANISCHE Und BIOMOLEKULARE ChEMIE der UNIVERSITÄT GÖTTINGEN.
} 


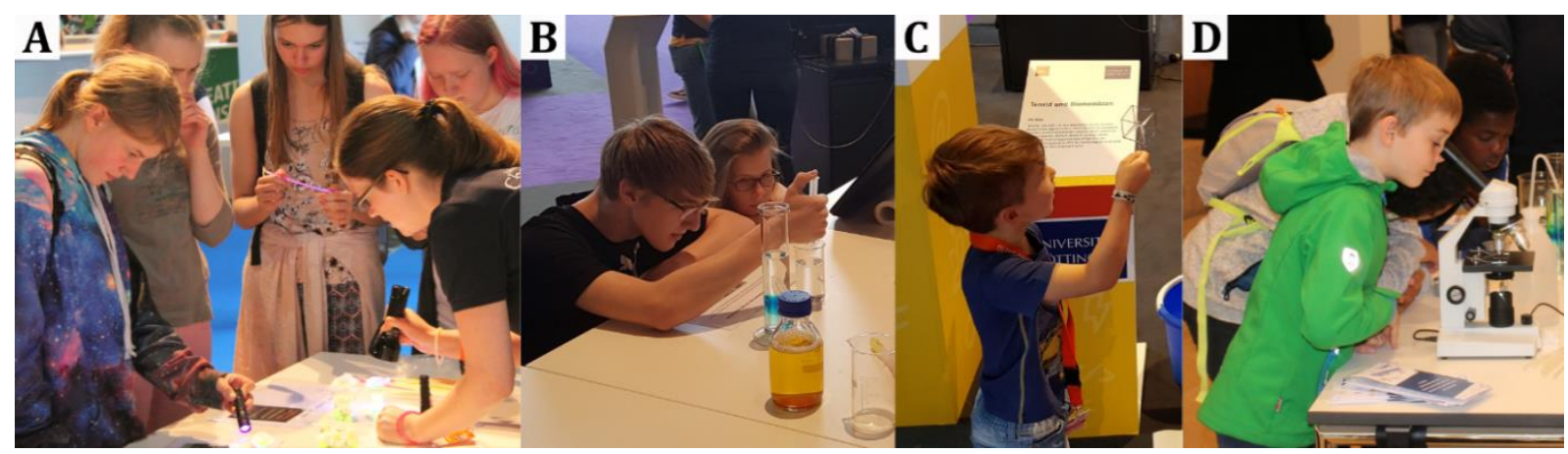

Abbildung 97: SuS unterschiedlicher Altersgruppen führen unter Anleitung Experimente zur Fluorochromierung von Blüten (A), zur Exocytose (B), sowie zur Oberflächenminimierung durch (C) und betrachten Pflanzenzellen unter dem Mikroskop (D).

\subsubsection{IdeenExpo 2017}

Wie der Tabelle 3 in Kapitel 3.3.3 zu entnehmen ist, lassen sich in der Literatur nur wenige Erhebungen in Bezug auf Bildungsmessen (SO-Bildungskontext 6) finden. Ihnen allen ist gemein, dass sich das Forschungsinteresse auf generelle Effekte des Messebesuchs bezieht, wie die Demographie, das Interesse oder die Motivation der Teilnehmer_innen. Evaluationen hinsichtlich der Wirksamkeit einzelner Projekte scheinen in der Literatur jedoch bislang keine Rolle zu spielen. Dieser Umstand lässt sich vermutlich mit der Schnelllebigkeit auf Messen, aber auch der von Veranstaltung zu Veranstaltung variierenden Randbedingungen erklären. Solche Schwierigkeiten, aber auch Aspekte wie u. a. personelle und technische Ausstattungen, flossen entsprechend in die Vorüberlegungen bzgl. des Evaluationsdesigns mit ein.

\section{Forschungsinteresse}

Hinsichtlich der Hauptzielgruppe des Ö-Projektes des SFB 803 galt das allgemeine Forschungsinteresse vornehmlich SuS, insbesondere der Sekundarstufe II. Die heterogene Besucher_innenstruktur ermöglichte es jedoch, auch Daten bezüglich sekundärer Zielgruppen wie MINT-Lehrkräften und der interessierten Öffentlichkeit zu erheben. Da die Vermittlungsschwerpunkte hinsichtlich der verschiedenen Zielgruppen variieren, ergeben sich auch unterschiedliche Forschungsfragen für die jeweiligen Gruppen. Da sich die Forschungsfragen für die Evaluationen der IDEENEXPO 2017 und der HDP 2018 zu großen Teilen überschneiden, werden sie gemeinsam aufgeführt.

\section{Schülerinnen und Schüler}

Hinsichtlich der Gruppe der SuS sind auf den ersten Blick vor allem Fragestellungen in Bezug auf motivations- und interessenfördernde Effekte des Messekonzepts interessant. Diese Aspekte lassen sich jedoch in einer Querschnittstudie nicht objektiv erheben, zumal zahlreiche Störfaktoren, wie motivations- und interessenfördernde Effekte des allgemeinen Messebesuchs, die Ergebnisse weiter verzerren würden. Entsprechend beziehen sich die Fragestellungen auf (mit Einschränkungen) messbare Effekte, wie den Erwerb von Fachwissen bezüglich dem Thema Biomembranen, sowie der Membranforschung im Allgemeinen. 
S1 Ist es mithilfe des Messekonzepts möglich, fachwissenschaftliche Inhalte zu vermitteln?

S2 Werden mithilfe des Messekonzepts Einblicke in die Membranforschung gegeben?

S3 Sind SuS daran interessiert, im Unterricht Einblicke in die Membranforschung zu erhalten?

\section{MINT-Lehrkräfte}

Da das Thema der Biomembranen explizit nur im Kerncurriculum der Biologie Erwähnung findet, die Membranforschung jedoch interdisziplinärer Natur ist und auch die innerhalb dieser Arbeit entwickelten Experimente und Projekte diese Interdisziplinarität aufgreifen, ist es hinsichtlich der Gruppe der Lehrkräfte von Interesse, inwiefern das Thema auch in der Schule bereits interdisziplinär unterrichtet wird. Zudem stellt sich mit Blick auf die oft fehlende Erwähnung des Forschungsbegriffs in den Kerncurricula fast aller Länder die Frage, inwiefern Bestrebungen bestehen, im MINT-Unterricht dennoch Einblicke in die Forschung zu geben.

L1 Wird das Thema der Biomembranen interdisziplinär auch außerhalb des Biologieunterrichts unterrichtet?

L2 Sind MINT-Lehrkräfte an weiterführenden Informationen zu Biomembranen und zur Membranforschung interessiert?

L3 Inwiefern werden im MINT-Unterricht bereits Einblicke in die naturwissenschaftliche Forschung gegeben?

\section{Interessierte Allgemeinheit}

In Bezug zur Gesellschaft wurde bereits Eingangs das Ergebnis einer Umfrage der EuROPÄISCHEN KOMMISSION ${ }^{[17]}$ hinsichtlich der Ansichten europäischer Bürger zu Wissenschaft und Technologie vorgestellt. An diese Thematik knüpfen auch die Fragestellungen für die Gruppe der interessierten Allgemeinheit an.

A1 Welcher Stellenwert wird der Forschung für die Gesellschaft zugeschrieben?

A2 Welcher Stellenwert wird der Forschung für die persönliche Zukunft zugeschrieben?

A3 Welchen Effekt haben das Alter bzw. das naturwissenschaftliche Verständnis auf den Stellenwert der Forschung in Bezug auf die Gesellschaft und die persönliche Zukunft?

\section{Allgemeine Fragestellungen}

Vor allem mit Blick auf das hier vorgestellte Messekonzept ergeben sich noch weitere Fragestellungen, die nicht spezifisch einer der genannten Zielgruppen zugeschrieben werden können.

F1 Welche Aspekte des Messekonzepts bewegen Besucher_innen dazu, am Ausstellungsstand stehenzubleiben?

F2 Welche Fragen haben Besucher_innen in Bezug auf das Thema der Biomembranen im Allgemeinen, oder der Biomembranforschung im Speziellen?

F3 Wie lange halten sich Besucher_innen im Schnitt am Ausstellungsstand auf? 


\section{Evaluationsdesign}

Zu Beginn des Disserationsvorhabens wurde basierend auf den Vorarbeiten des Projekts SFB@SCHOOL ein erstes Messenkonzept entworfen, das neben dem ersten EXPOneer verschiedene Experimente zur Oberflächenminimierung sowie Pflanzenzellen unter einem Auflichtmikroskop präsentierte. Zur Evaluation des Konzepts wurde ein Paper-PencilTest entworfen, anhand dessen mithilfe sechs geschlossener und vier offener Items das Schülerinteresse hinsichtlich des Themas des Ausstellungsstandes sowie motivationale Effekte des EXPOneer-Regals erfragt werden sollten. Es zeigte sich jedoch schnell, dass die Schnelllebigkeit der Messe und damit die hohe Fluktuation der Teilnehmer_innen und deren kurze Verweildauer in Bezug auf das Erhebungsdesign unterschätzt wurden. Die gewonnenen Erfahrungen wurden jedoch hinsichtlich der darauffolgenden Messen genutzt, um die Zielgruppen der Aktivitäten um MINT-Lehrkräfte und die interessierte Allgemeinheit zu erweitern, entsprechend die Fragestellungen für alle Gruppen auszuschärfen sowie das Erhebungsdesign auf die Gegebenheiten der Messen anzupassen.

Aufgrund der unterschiedlichen Fragestellungen fiel die Entscheidung bezüglich des Designs auf einen Methodenmix aus qualitativen und quantitativen Forschungsmethoden [371]. Für das Konzept der zweiten Bildungsmesse (2017), das weiterhin auf dem ersten EXPOneer basierte, jedoch um Experimente zur Molekülgeometrie und Exocytose erweitert wurde, wurden im Sinne von Paper-Pencil-Tests drei Kurzfragebögen für die jeweiligen Zielgruppen entwickelt. Da Fragebögen auf Papier personell wie logistisch nicht sinnvoll erschienen, wurde die Umfragenapplikation LimeSurvey ${ }^{\left[{ }^{372}\right]}$ verwendet, um die Evaluation auf einem Touchscreen durchführen zu können. Dies hatte u. a. den Vorteil, dass mithilfe einer eingangs gestellten Filterfrage eine automatische Zuordnung zur jeweiligen Zielgruppe und damit den entsprechenden Kurzfragebögen möglich war. Zum anderen schien die Benutzung des Touchscreens den (subjektiven) Effekt zu haben, dass die Umfrage bereitwilliger durchgeführt und zudem als deutlich kürzer wahrgenommen wurde. Zusätzlich ermöglichte die Einbindung von Abbildungen eine größere Variabilität der Fragen.

Neben der initialen Zuordnung wurden für jede Gruppe drei bis vier geschlossene und z. T. auch halboffene inhaltliche sowie jeweils zwei soziodemographische Fragen formuliert (bspw. zur Klassenstufe, zu den Unterrichtsfächern oder zum Alter). Je nach Inhalt der Fragen wurden sowohl nominale (dichotom, polynom) als auch ordinale Skalen (Lickert) gewählt und es waren Einfach- sowie Mehrfachnennungen möglich. Lickert-Skalen enthielten dabei immer jeweils vier Antwortmöglichkeiten, um eine Tendenz zur Mitte auszuschließen. Zudem wurde fachlichen nominalen Fragen die Antwortmöglichkeit „Ich weiß es nicht" hinzugefügt, um die Ratetendenz zu verringern [373].

Die Fragebögen wurden aufgrund der bei der ersten Bildungsmesse beobachteten geringen Verweildauer der Teilnehmer_innen bewusst kurzgehalten, um ein vorzeitiges Abbrechen der Fragenbeantwortung zu minimieren. Eine Überprüfung der Fragenauswahl und -formulierung im Sinne eines Pretests konnte nur durch eine informelle Begutachtung ${ }^{[374]}$ durch Kolleg_innen erfolgen, da eine Einschätzung der Fragen durch eine Kontrollgruppe nur im Zusammenhang mit dem Ausstellungsstand zielführend gewesen wäre.

Zusätzlich zu den Touchscreenumfragen wurde ein Leitfadeninterview für die Zielgruppe der SuS entwickelt. Da die Vorerfahrungen der ersten Bildungsmesse zeigten, dass die offenen Fragen des verwendeten Fragebogens oft ausgelassen wurden, sollten die leitfadengelenkten Gespräche mit SuS möglichst offene Antworten zulassen. Entsprechend wurde nur ein grobes Fragenkonzept festgelegt, um das Gespräch, wenn nötig, zu lenken. Die 
Fragen wurden diesbezüglich so gewählt, dass sie sowohl spezifische Schülerfragestellungen (S2, S3) als auch allgemeine Fragen (F1) abdeckten. Aufgrund der aktiven Einbindung des Interviewers in die Standbetreuung wurden Tonaufnahmen der Interviews erstellt, um diese im Nachhinein auszuwerten zu können.

\section{Evaluationsergebnisse}

Während der IDEENEXPO 2017 wurde der Ausstellungsstand (siehe Abbildung 98) täglich von zwei bis drei Mitarbeiter_innen betreut. Diese setzten sich aus Lehramtsstudierenden der Chemie, die (fach-)didaktische Vorkenntnisse besaßen, sowie Chemiestudierenden zusammen, die aktiv an der Forschung des SFB 803 beteiligt waren. Das Messekonzept wurde an den neun Ausstellungstagen von vielen Interessierten genutzt, wobei die Vormittage unter der Woche, sowie die Nachmittage an den Wochenenden deutlich besucherstärker waren als die restlichen Zeiten. Lehrkräfte und SuS ab ca. der 7. Klassenstufe waren vornehmlich unter der Woche am Vormittag zugegen (erstere konnten anhand von Ausweisen als Lehrkräfte identifiziert werden), während die anderen Zeiten von Familien und jüngeren Kindern geprägt waren. Die Verweildauer am Stand (ohne die Teilnahme an den Evaluationen) betrug normalerweise unter zehn, in den meisten Fällen sogar unter fünf Minuten, wobei hier zu erwähnen ist, dass diese Einschätzung auf Beobachtungen beruht.

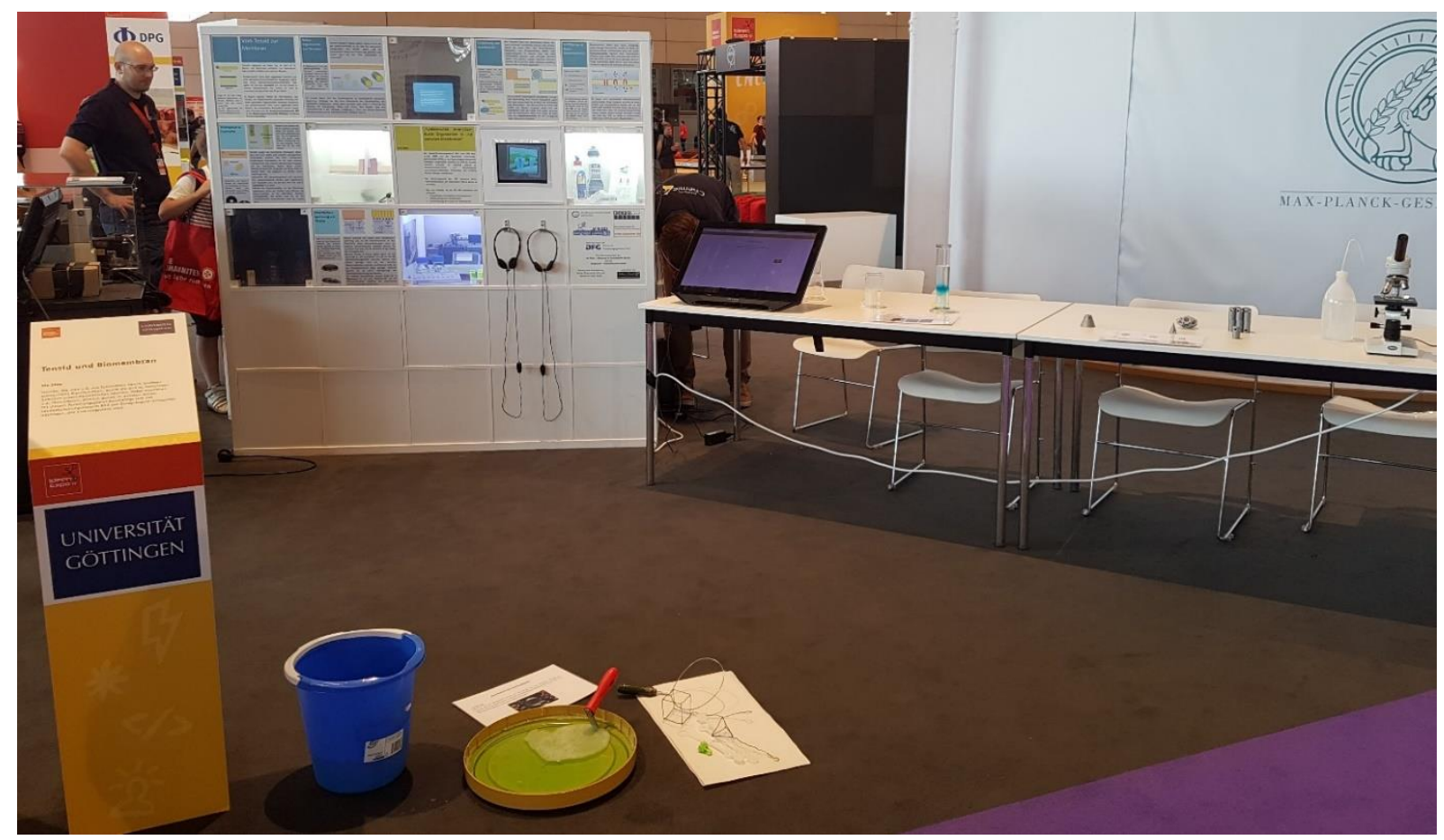

Abbildung 98: Messestand des SFB 803 bei der IDEENEXPo 2017 [375].

An neun Ausstellungstagen wurden in der Umfragenapplikation insgesamt 200 Datensätze gesammelt, von denen 178 vollständig vorlagen und somit ausgewertet werden konnten. Die Teilnahme erfolgte in den meisten Fällen im Anschluss an Gespräche mit den Standbetreuern. Anhand der Filterfrage zu Beginn wurden die Teilnehmer_innen jeweils der Gruppe der SuS (N = 85, ohne Grundschüler_innen und SuS ohne Angabe der Klassenstufe $N=74)$, der MINT-Lehrkräfte $(N=44)$, oder der interessierten Allgemeinheit $(\mathrm{N}=49)$ zugeordnet ${ }^{20}$.

${ }^{20}$ Die Evaluation des Messekonzepts während der IDEENExPO wurde zum Teil bereits veröffentlicht [179]. 


\section{Schülerinnen und Schüler}

Um im Sinne der Forschungsfrage S1 zu klären, ob es möglich ist mithilfe des Messekonzepts fachwissenschaftliche Inhalte zu vermitteln, wurden der Gruppe der SuS vier inhaltliche Fragen mit steigendem Schwierigkeitsgrad gestellt, die fachwissenschaftliche Aspekte des Ausstellungsstandes aufgriffen. Da die Hauptzielgruppe SuS der Sekundarstufe II umfasst (SuS der Klasse 10 wurden aufgrund des verkürzten Abiturs mit einbezogen), wurde die erste Frage („Der Fachbegriff für die wasserabweisende Eigenschaft von Teilchen ist...", Tabelle 20) so gewählt, dass sie von SuS der Oberstufe bereits mithilfe des unterrichtlichen Vorwissens hätte gelöst werden können, um ggf. Unterschiede zwischen Fragen mit bekannten und unbekannten Inhalten feststellen zu können.

Mit 49 von 74 Teilnehmer_innen ab Klassenstufe 5 konnten zwei Drittel der SuS die Eigenschaft richtig benennen, wobei die Wahrscheinlichkeit richtig geratener Antworten bei zwei (fachlichen) Antwortmöglichkeiten zugegebenermaßen sehr hoch ist. Da jedoch insgesamt auch sieben Mal die Antwortmöglichkeit Ich weiß es nicht angewählt wurde und diese Möglichkeit bei den nachfolgenden Fragen deutlich öfter auftrat, wird von einer verhältnismäßig geringen Quote an geratenen Antworten ausgegangen. Eine Differenzierung bezüglich der Hauptzielgruppe zeigt, dass 23 der 24 SuS die erste Frage richtig beantworteten, was auf einen Vorteil hinsichtlich des Vorwissens deuten könnte.

Tabelle 20: Verteilung der Antworten von SuS bzgl. der zielgruppenspezifischen Frage zur wasserabweisenden Eigenschaft von Teilchen.

\begin{tabular}{ll}
\hline SuS 1: Der Fachbegriff für die wasserabweisende Eigenschaft von Teilchen ist... & $\mathbf{N}=\mathbf{7 4}(\mathbf{2 4})$ \\
\hline hydrophob & $49(23)$ \\
hydrophil & $18(1)$ \\
Ich weiß es nicht & $7(0)$ \\
\hline
\end{tabular}

Die zweite und dritte Frage („Welche der beiden Abbildungen zeigt ein Tensidmolekül?“; „Was kann mit dem Tyndall-Effekt nachgewiesen werden?", Tabelle 21) beziehen sich beide auf Inhalte des EXPOneer-Regals, das in den meisten Gesprächen mit Besucher_innen für Erklärungen herangezogen wurde. Die zweite Frage wurde dabei von einer Abbildung eines Tensid- und eines Lipidmoleküls begleitet (vgl. Abbildung 13, Kapitel 5.1), die einer der Infotafeln des EXPOneers entnommen wurde. Die dritte Frage bezog sich auf das im Regal verbaute push-the-button-Experiment zur Lichtstreuung, das ebenfalls anhand von Abbildungen des EXPOneers erklärt wurde.

Beide Fragen konnten von $47 \mathrm{SuS}$ richtig beantwortet werden, was abermals rund zwei Dritteln der SuS entspricht. Während in der zweiten Frage nur zwei fachliche Antwortmöglichkeiten vorgegeben waren, gab es für die dritte Frage drei Lösungsvorschläge. Dennoch ist gerade in der dritten Frage die Zahl der falschen Antworten (7) sehr gering. Dass insgesamt $20 \mathrm{Mal}$ die Antwort Ich weiß es nicht gewählt wurde kann jedoch als Indiz dafür gewertet werden, dass die Ratetendenz gering war.

Im Gegensatz zur ersten Frage fällt die Anzahl der richtigen Antworten der älteren SuS deutlich geringer aus. Bei beiden Fragen entschied sich rund ein Viertel der Teilnehmer_innen dazu, keine der fachlichen Antwortmöglichkeiten zu wählen. Mit $63 \%$ und $75 \%$ weicht die Zahl der richtigen Antworten jedoch nicht signifikant vom Gesamtergebnis ab. Auffallend ist zudem, dass die Gesamtzahlen der richtigen Antworten für die ersten drei Fragen dicht beieinanderliegen, jedoch waren lediglich $21 \mathrm{SuS}$ in der Lage, alle drei Fragen richtig zu beantworten, wobei mit 12 SuS über die Hälfte der Hauptzielgruppe ab Klasse 10 angehören. 
Tabelle 21: Verteilung der Antworten von SuS bzgl. der zielgruppenspezifischen Frage zu Tensidmolekülen und dem Tyndall-Effekt.

\begin{tabular}{ll}
\hline SuS 2:Welche der beiden Abbildungen zeigt ein Tensidmolekül? & $\mathbf{N}=\mathbf{7 4}(\mathbf{2 4})$ \\
\hline A (Lipid) & $9(3)$ \\
B (Tensid) & $47(15)$ \\
Ich weiß es nicht & $18(6)$ \\
\hline SuS 3: Was kann man mit dem Tyndall-Effekt nachweisen? & $\mathbf{N}=\mathbf{7 4}(\mathbf{2 4})$ \\
\hline Ionen & $5(0)$ \\
Mizellen & $47(18)$ \\
Atome & $2(1)$ \\
Ich weiß es nicht & $20(5)$ \\
\hline
\end{tabular}

Die vierte Frage („Welche der Molekülgeometrien ermöglicht einen Membrantransport?“, Tabelle 22) bezog sich auf 3D-Molekülmodelle, die Vorläufermodelle der in Experiment 2.4 vorgestellten Modelle waren. In einem hands-on-Experiment konnten anhand von drei geometrischen Formen die Geometrien von Vesikel, Mizellen und Lipiddoppelschichten erarbeitet werden, wobei im Gespräch mit den Standbetreuern die Bedeutung dieser Geometrien für biologische Prozesse diskutiert wurde. Das Antwortverhalten der vierten Frage weicht jedoch interessanter Weise deutlich von den ersten drei Fragen ab. Obwohl mit 40 SuS über die Hälfte die Frage richtig beantworten konnten, wurde deutlich weniger oft die Antwortmöglichkeit „Ich weiß es nicht“ angewählt. Stattdessen hielten gut ein Drittel der SuS C für die richtige Antwort. Mit Blick auf die Hauptzielgruppe überwiegt die Wahl der Antwort C (13) sogar im Vergleich zur richtigen Antwort A (10). Eine mögliche Erklärung dafür könnte sein, dass vor allem mit den älteren SuS teilweise auch die Grenzen der Modelle diskutiert wurden. Diesbezüglich wurde hinsichtlich der Vesikelgeometrien erwähnt, dass mit den vorliegenden Modellen lediglich Hohlkugeln mit Monoschichten dargestellt werden können und dass Vesikel in der Realität ebenfalls aus Lipiddoppelschichten bestehen.

Tabelle 22: Verteilung der Antworten von SuS bzgl. der zielgruppenspezifischen Frage zu Molekülgeometrien beim Membrantransport. Die Beantwortung erfolgte anhand einer beigefügten Abbildung.

\begin{tabular}{ll}
\hline SuS 4: Welche der Molekülgeometrien ermöglicht einen Membrantransport? & N = 74 (24) \\
\hline A (Vesikel) & $40(10)$ \\
B (Mizelle) & $4(0)$ \\
C (Lipiddoppelschicht) & $25(13)$ \\
Ich weiß es nicht & $5(1)$ \\
\hline
\end{tabular}

Obwohl die Inhalte der zweiten bis vierten Frage jeweils nicht in den Kerncurricula der Chemie oder Biologie verankert sind, waren jeweils über die Hälfte und in den meisten Fällen sogar über zwei Drittel der SuS in der Lage die Fragen korrekt zu beantworten. Auch wenn anhand dieser Ergebnisse weder Aussagen bezüglich des Vorwissens der SuS, noch hinsichtlich der Tiefe des Verständnisses oder der Nachhaltigkeit des Gelernten möglich sind, scheinen zahlreiche SuS zumindest für den Moment einen Wissenszuwachs erfahren zu haben. Überraschend ist diesbezüglich, dass die Ergebnisse der Hauptzielgruppe sich nicht deutlich von denen der restlichen SuS unterscheiden, was dafür spricht, dass sich das Messekonzept durchaus auch bereits für jüngere SuS eignet.

Die Erhebung in Bezug auf die Gruppe der SuS sollte durch Leitfadeninterviews mit einzelnen Teilnehmer_innen um qualitative Daten ergänzt werden. Im Sinne einer bewussten Auswahl der Teilnehmer_innen [376] sollten diese möglichst der Hauptzielgruppe ent- 
stammen und zudem ein deutliches Interesse an den Inhalten des Exponats zeigen. Letzteres schien deswegen sinnvoll, weil das Experiment zur Oberflächenminimierung (Versuch 3.1) zahlreiche Besucher_innen dazu verleitete an den Ausstellungsstand zu kommen, in vielen Fällen jedoch eher einem kindlichen Spieltrieb nachgegangen wurde. Dabei war zu beobachten, dass das Interesse an den Hintergründen dieses Experimentes sowie den weiteren Inhalten des Exponats deutlich geringer ausfiel. Dennoch bot das initiale Interesse an den Seifenblasen einen guten Gesprächseinstieg, um die Aufmerksamkeit der Besucher_innen auf die naturwissenschaftlichen Inhalte zu lenken und sie so an den Stand zu binden.

Die meisten der Teilnehmer_innen die sich über die Hintergründe der verschiedenen Experimente informierten, nahmen im Anschluss auch an der Touchscreenumfrage teil. Abbildung 99 zeigt die Verteilung der teilnehmenden SuS nach Jahrgängen. Es wird deutlich, dass nur rund ein Drittel der Teilnehmer_innen der Hauptzielgruppe entsprachen. Da die Vormittage, an denen die meisten SuS anwesend waren, gleichzeitig auch zu den besucherstärksten Zeiten der Messe gehörten und alle Standbetreuer vermehrt eingebunden waren, war es nicht immer möglich, bereits laufende Gespräche zu unterbrechen, um potentielle Interviewteilnehmer_innen von den anderen Standbetreuern zu übernehmen. Zudem zeigten sich nach den ausführlichen Erläuterungen der Mitarbeiter_innen nur wenige SuS bereit, im Anschluss noch die Zeit für ein Interview aufzuwenden. Deshalb wurden schließlich auch interessierte SuS ab der 7. Klassenstufe für die Interviews in Betracht gezogen.

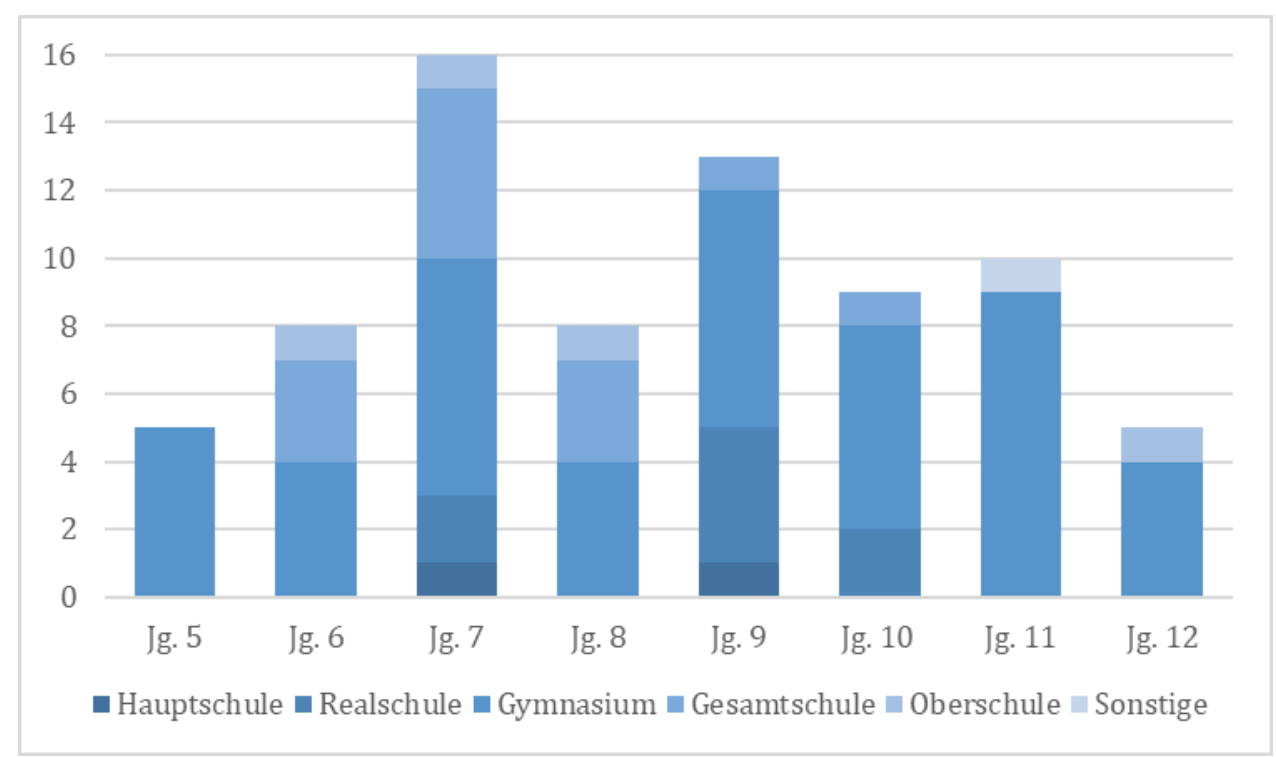

Abbildung 99: Evaluationsteilnehmer_innen (SuS) nach Jahrgang und Schulform.

Insgesamt wurden an fünf Vormittagen zehn Interviews mit SuS der Jahrgänge sieben bis zwölf geführt, die verschiedene Schulformen besuchten (Realschule, Gymnasium, IGS, Berufsbildende Schule). Die Fragen an die SuS bezogen sich auf ihren Grund für das Stehenbleiben am Ausstellungsstand, ihr Verständnis darüber, welche Inhalte das Exponat transportieren soll, ihre Vorstellungen über die Forschung an Membranen sowie ihre bisherigen schulischen Auseinandersetzungen mit Membranen und ihr Interesse daran, im Unterricht mehr über den Alltag von Wissenschaftler_innen zu erfahren.

Über die Hälfte der Teilnehmer_innen gab an, dass sie aufgrund eines allgemeinen Interesses für chemische und biologische Themen stehengeblieben waren, was sich auch in 
den naturwissenschaftlichen Berufswünschen im Bereich der Biologie, Chemie und Medizin zahlreicher der SuS widerspiegelt. Die Verortung des Exponats in diesem thematischen Bereich machten sie dabei an den Abbildungen von u. a. Tensiden und Phospholipiden, aber auch am Vorhandensein eines Mikroskops fest. Des Weiteren nannten einige SuS auch die interessant wirkenden Experimente als Grund des Stehenbleibens. Nur zwei SuS entschieden sich initial wegen der Seifenblasen zur Teilnahme, wobei eine der Schülerinnen hinzufügte, dass sie sich wegen der Alltäglichkeit von Seifenblasen fragte, warum diese auf einer Messe präsentiert werden. Ein Schüler merkte zudem an, dass auf der Messe vornehmlich Exponate zu physikalischen Themen anzufinden seien, diese jedoch nicht seinem Interessengebiet entsprächen.

Nach den Gesprächen mit den Standmitarbeiter_innen herrschte Uneinigkeit darüber, welche Inhalte mithilfe des Exponats transportiert werden sollen. Die Hälfte der Interviewten glaubte, dass es vornehmlich um Tenside und deren Eigenschaften geht. Zwar wurden in diesem Zusammenhang teilweise auch Membranen genannt, jedoch wurde auch hier eine Verbindung zu Tensiden hergestellt: „[Es geht] im Prinzip darum, mit Tensiden Zellmembranen nachzubilden und dann auch zu erforschen, wie sich diese dann verhalten, was für Einflüsse die haben können" (Jg. 11). Die anderen SuS verorteten das Exponat thematisch im Bereich der Zellmembranen, wobei insbesondere Aspekte wie die Grenzwirkung von Zellen, sowie die Selbstassemblierung und der Stofftransport genannt wurden. Diesbezüglich sei jedoch zu erwähnen, dass nur wenige Fachwörter verwendet und stattdessen vornehmlich Konzepte beschrieben wurden: „Also Membranen [...], die sich halt im Wasser bestimmt orientieren, [...] also die Moleküle an sich [...] [sind] wassermögend und wasserabweisend und orientieren sich ja im Wasser bestimmt" (Jg. 12 zur Selbstassemblierung).

Auch die Vorstellungen bezüglich dessen, woran in der Membranforschung geforscht wird, sind breit gestreut und bleiben oft wenig konkret. Die Vorschläge reichen dabei von „Nachweisen [...], dass es sowas wie Zellen und sowas gibt“ (Jg. 8) bis „Ich denke ein Membranforscher, der guckt sich die Membranen mit dem von dem Nobelpreisträger entwickelten Mikroskop an und versucht die neuen empirischen Erkenntnisse an die alten Thesen anzubinden" (Jg. 12). Viele der SuS vermuten zudem einen Anwendungsbereich in medizinischen Kontexten: „Also wahrscheinlich gucken die, wenn auch irgendwelche Krankheiten oder so da sind [...], wo die Stoffe nicht so richtig in die Zellen reinkommen [...], wie man die Medikamente oder so herstellen kann, dass die da reinkommen" (Jg 8). Neben biologischen verbinden einige der SuS die Membranforschung auch mit der Entwicklung von künstlichen Membranen, von denen sie vermuten, dass diese wiederum in anderen Techniken eingesetzt werden.

Fast alle SuS haben sich in Abhängigkeit der Klassenstufe zudem bereits im Rahmen des Unterrichts mit dem Thema der Biomembranen auseinandergesetzt. Typischerweise wurde diesbezüglich der Bau von tierischen und pflanzlichen Zellen anhand von mikroskopischen Betrachtungen verglichen und diese Erkenntnisse in der Oberstufe um Aspekte wie die Erregungsübertragung im Nervensystem erweitert. In allen Fällen wurde die Thematik jedoch lediglich im Biologieunterricht aufgegriffen. Interdisziplinären Betrachtungen sowie Einblicken in den Alltag von Wissenschaftler_innen im Unterricht gegenüber waren alle SuS generell nicht abgeneigt, wobei ein Schüler anmerkte, dass die Lehrpläne bereits sehr voll seien. Zwei weitere SuS empfanden insbesondere Einblicke in die Wissenschaft prinzipiell als sehr spannend, gaben jedoch zu bedenken, dass es für interessierte SuS bereits zahlreiche Angebote wie den Zukunftstag sowie verschiedene Messeformate gibt, die es ermöglichen sich selbstständig zu informieren. 
Das Interesse an chemischen und biologischen Themen als Begründung für das Stehenbleiben der SuS deutet an, dass das Design des Exponats bereits auf die naturwissenschaftliche Ausrichtung der Inhalte hinweist. Neben dem Mikroskop und den Experimenten werden auch die Abbildungen des EXPOneer-Regals als Indikatoren hierfür genannt. Die Antworten der SuS verdeutlichen jedoch auch, dass das Messekonzept noch nicht das zum Ausdruck bringt, was es eigentlich soll - Einblicke in die Membranforschung, insbesondere des SFB 803, geben. Gerade die Verwendung von Tensiden, die gewählt wurden, da mit ihrer Hilfe Seifenblasen als auf makroskopischer Ebene erfahrbare Membranen gedeutet werden können, scheint vom eigentlich Inhalt der Forschung, den Biomembranen, abzulenken. Entsprechend ist es nicht verwunderlich, dass den SuS zudem auch nicht klar ist, worum es in der Membranforschung geht. Trotz des prinzipiellen Interesses mehr über die Membranforschung und den Alltag von Wissenschaftler_innen zu lernen, sind die Einwände der SuS berechtigt. Umso wichtiger scheint es jedoch im Sinne der Berufsorientierung, z. B. im Rahmen von Angeboten wie Zukunftstagen und Fachpraktika, Einblicke in die Forschung zu gewähren und Konzepte für Messen oder Tage/Nächte der offenen Tür zu entwickeln, um auch weniger interessierten SuS ein realistisches Bild bieten zu können.

\section{Lehrkräfte}

Während der IDEENEXPO besuchten auch zahlreiche Lehrkräfte den Ausstellungsstand des SFB 803, von denen sich 44 bereit erklärten, an der Fragenbogenstudie teilzunehmen. Bezugnehmend auf die Fragestellungen L1 und L2 wurden drei inhaltliche Items entwickelt, von denen eines als Filterfrage designt wurde und ggf. die Beantwortung einer vierten Frage erforderlich machte. Die erste Frage („Haben Sie das Thema Biomembranen jemals in Ihrem Unterricht behandelt?", Tabelle 23) sollte in Abhängigkeit einer soziodemographischen Frage nach den durch die Lehrkraft unterrichteten MINT-Fächern Aufschluss darüber geben, ob Biomembranen bereits interdisziplinär auch in anderen Unterrichtsfächern als der Biologie thematisiert werden. Von den 44 teilnehmenden Lehrkräften gaben 25 an, dass sie das Thema Biomembranen bereits behandelt hätten. 21 dieser Lehrkräfte unterrichteten mindestens das Fach Biologie, die anderen vier Lehrkräfte jeweils zumindest Chemie. Während in Bezug auf die Allgemeinheit der Biologielehrkräfte keine Aussagen darüber getroffen werden können, ob diese Biomembranen auch außerhalb des Biologieunterrichts aufgreifen (zahlreiche unterrichten die Fächerkombination Biologie und Chemie), liegt zumindest die Vermutung nahe, dass die vier Chemielehrkräfte in ihrem Unterricht interdisziplinäre Bezüge hergestellt haben könnten.

Tabelle 23: Verteilung der Antworten von Lehrkräften bzgl. der zielgruppenspezifischen Frage zur unterrichtlichen Behandlung des Themas Biomembranen.

\begin{tabular}{ll}
\hline $\begin{array}{l}\text { Lehrkräfte 1: Haben Sie das Thema Biomembranen jemals in Ihrem Unterricht behan- } \\
\text { delt? }\end{array}$ & $\mathbf{N}=\mathbf{4 4}$ \\
\hline Ja & 25 \\
Nein & 19 \\
\hline
\end{tabular}

In Hinblick auf die Fragestellung L2 wurden die Lehrkräfte zudem gefragt, ob sie gerne mehr über die aktuelle Membranforschung erfahren würden, um diese in ihren Unterricht einzubinden (Tabelle 24). Immerhin 36 der 44 Teilnehmer_innen bejahten diese Aussage, zu denen Lehrkräfte aller Fächerkombinationen zählten, wobei an dieser Stelle Zustimmungstendenzen nicht vernachlässigt werden dürfen. Die 36 interessierten Lehrkräfte wurden im Anschluss befragt, was sie am Thema Biomembranen am meisten interessiert. Eine vierteilige Skala mit Mehrfachauswahl gab ihnen die Möglichkeit, ihr Interesse be- 
züglich Experimenten (32), Informationsmaterialien (9), ausgearbeiteten Unterrichtseinheiten (23) sowie Arbeitsblättern (25) zu bekunden. In offenen Kommentarfeldern konnten diese Wünsche konkretisiert werden, was jedoch nur vereinzelt genutzt wurde (anschauliche, leicht durchführbare Experimente, u. a. zu Vesikeln; Modelle). In einer dritten Frage wurden die Lehrkräfte zudem nach ihrem Interesse an einer entsprechenden Fortbildung zu Biomembranen gefragt (Tabelle 24), was von 30 Lehrkräften, und somit von zwei Dritteln der Teilnehmer_innen, positiv beantwortet wurde.

Tabelle 24: Verteilung der Antworten von Lehrkräften bzgl. der zielgruppenspezifischen Frage zum Interesse an Informationen zur aktuellen Membranforschung.

\begin{tabular}{lc}
\hline $\begin{array}{l}\text { Lehrkräfte 2: Würden Sie gerne mehr über die aktuelle Membranforschung erfahren, } \\
\text { um diese in Ihren Unterricht einzubinden? }\end{array}$ & $\mathbf{N}=\mathbf{4 4}$ \\
\hline Ja & 36 \\
Nein & 8 \\
\hline Lehrkräfte 3: Würden Sie an einer Lehrerfortbildung zum Thema Biomembranen teil- & $\mathbf{N}=\mathbf{4 4}$ \\
nehmen? & 30 \\
\hline Ja & 14 \\
\hline Nein & \\
\hline
\end{tabular}

In Einklang mit den Schulformen der teilnehmenden SuS, nahmen auch deutlich mehr Gymnasial- (24) und Gesamtschullehrkräfte (10) an der Umfrage teil, als Lehrkräfte anderer Schulformen. Obwohl das Thema Biomembranen wie erwartet - und im Einklang mit den Angaben der interviewten SuS - in der Regel lediglich im Fach Biologie unterrichtet wird, waren dennoch viele Lehrkräfte auch anderer Fachrichtungen interessiert, mehr über den Themenbereich zu erfahren. Erfreulich ist zudem, dass zwei Drittel auch Interesse an entsprechenden Fortbildungen zeigen. Das große Interesse an Experimenten, aber auch Arbeitsblättern und ganzen Unterrichtseinheiten könnte ein Indiz dafür sein, dass eine interdisziplinäre Einbindung prinzipiell nicht an der Motivation, sondern vielmehr an der zeitintensiven Erarbeitung eines Konzeptes und einer entsprechenden Auswahl der Materialien scheitert.

\section{Interessierte Allgemeinheit}

Neben SuS und Lehrkräften nahmen weitere 49 Personen an der Umfrage teil, die der Gruppe der interessierten Allgemeinheit zuzuordnen sind. In Bezug auf die Fragestellungen A1 bis A3 sollten die Teilnehmer_innen drei inhaltliche Fragen mit Ordinalskala beantworten und zudem sowohl ihr Alter angeben als auch auswählen, ob sie beruflich mit den Naturwissenschaften zu tun haben bzw. hatten. Im Sinne der Fragestellung A1 sollten die Teilnehmer_innen zunächst entscheiden, wie wichtig sie die naturwissenschaftliche Forschung für die Gesellschaft erachten (siehe Tabelle 25). Mit 48 Stimmen schätzte eine deutliche Mehrheit der Personen diese als sehr wichtig (40), bzw. wichtig (8) ein. Anhand einer zweiten Fragen mit fast demselben Wortlaut sollten die Teilnehmer_innen zudem die Wichtigkeit der naturwissenschaftlichen Forschung für ihre persönliche Zukunft abschätzen. Immerhin 42 Personen gaben auch in diesem Fall an, dass sie die Forschung als generell wichtig erachten, jedoch wählten lediglich noch 26 der zuvor 40 Teilnehmer_innen die Option sehr wichtig aus. 
Tabelle 25: Verteilung der Antworten der Allgemeinheit bzgl. der zielgruppenspezifischen Fragen zur Relevanz von Forschung für die Gesellschaft bzw. die persönliche Zukunft.

\begin{tabular}{ll}
\hline $\begin{array}{l}\text { Allgemeinheit 1: Die naturwissenschaftliche Forschung erachte ich für die Gesellschaft } \\
\text { als... }\end{array}$ & $\mathbf{N}=\mathbf{4 9}$ \\
\hline sehr wichtig & 40 \\
wichtig & 8 \\
wenig wichtig & 1 \\
unwichtig & 0 \\
\hline Allgemeinheit 2: Die naturwissenschaftliche Forschung erachte ich für meine persönli- & $\mathbf{N}=\mathbf{4 9}$ \\
che Zukunft als... & 26 \\
\hline sehr wichtig & 16 \\
wichtig & 6 \\
wenig wichtig & 1 \\
\hline unwichtig & 16 \\
\hline
\end{tabular}

Insgesamt stuften 16 Personen ihre Antwort der zweiten Frage im Vergleich zur ersten um mindestens eine, zum Teil auch zwei Skalenwerte herunter. Bezieht man den beruflichen Hintergrund dieser Teilnehmer_innen mit ein, fällt auf, dass 13 der 16 Personen einem Beruf ohne naturwissenschaftlichen Hintergrund nachgehen, obwohl mit 24 Personen gut die Hälfte in naturwissenschaftlichen Berufsfeldern arbeiten (vgl. Abbildung 100). Mit Blick auf das Alter gehören von diesen Personen ebenfalls $13 \mathrm{zu}$ den Gruppen der 31- bis 40- und 41- bis 50-Jährigen, jedoch nahmen letztere auch überproportional oft an der Umfrage Teil und zudem besitzen in beiden Gruppen auch vergleichsweise viele Teilnehmer_innen keinen naturwissenschaftlichen beruflichen Hintergrund.

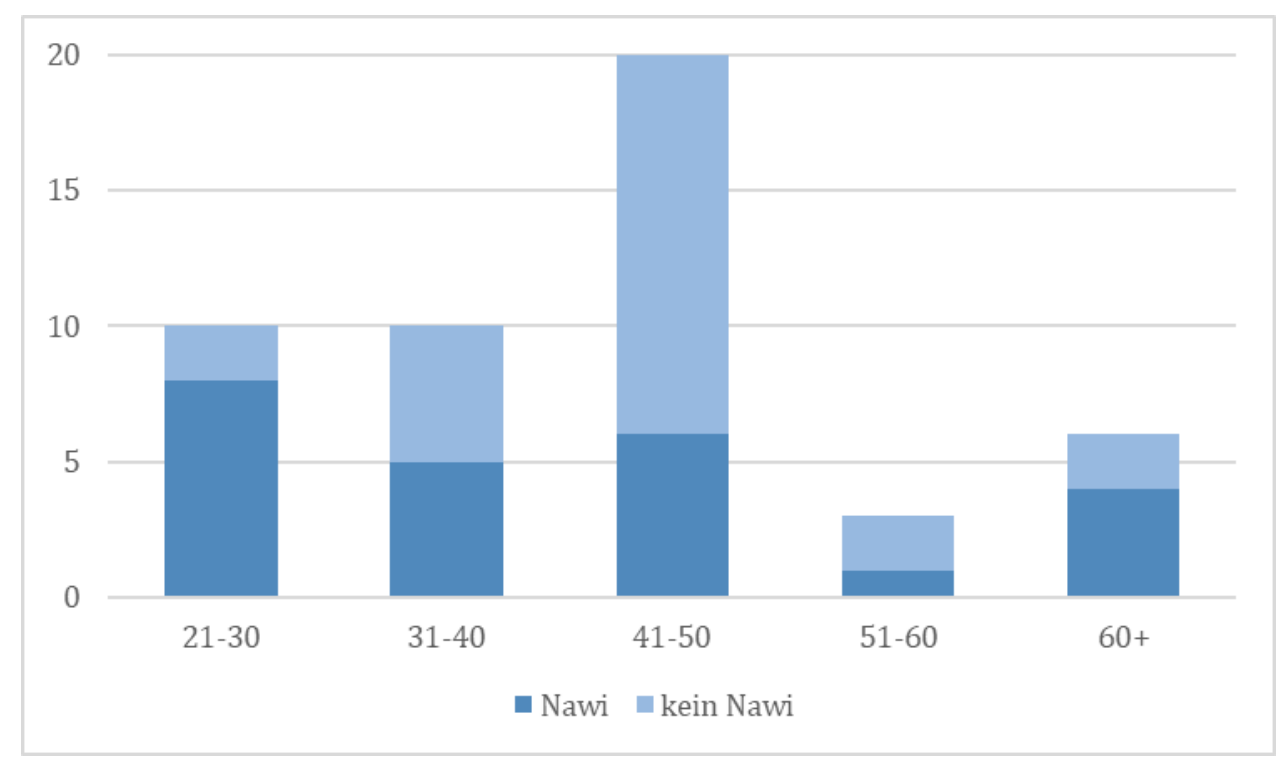

Abbildung 100: Evaluationsteilnehmer_innen (Allgemeinheit) nach Alter und beruflichem Hintergrund.

In Anlehnung an die Frage aus der eingangs erwähnten Studie der Europäischen Kommission [17], wie gut sich europäische Bürger über Entwicklungen in Wissenschaft und Technologie informiert fühlen, wurden die Teilnehmer_innen zudem gefragt, ob gesellschaftlich relevante naturwissenschaftliche Erkenntnisse in den Medien präsenter dargestellt werden sollten (Tabelle 26). Dieser Frage stimmten 47 Personen allgemein zu. Dieses Ergebnis deckt sich mit der Studie der Europäischen Kommission, in der nur knapp über $40 \%$ der Deutschen angaben, sich gut über Wissenschaft und Technologie informiert zu fühlen, wobei nur sieben der $40 \%$ sich sogar sehr gut informiert fühlen. 
Tabelle 26: Verteilung der Antworten der Allgemeinheit bzgl. der zielgruppenspezifischen Frage zur Darstellung wissenschaftlicher Erkenntnisse in den Medien.

\begin{tabular}{ll}
\hline Allgemeinheit 3: Denken Sie, dass gesellschaftlich relevante naturwissenschaftliche Er- & $\mathbf{N}=\mathbf{4 9}$ \\
kenntnisse in den Medien präsenter dargestellt werden sollten? & \\
\hline Stimme zu & 29 \\
Stimme eher zu & 18 \\
Stimme eher nicht zu & 2 \\
Stimme nicht zu & 0 \\
\hline
\end{tabular}

Gerade die unterschiedliche Gewichtung der Forschung für die Gesellschaft und die persönliche Zukunft verdeutlicht, dass vielen Menschen nicht bewusst ist, welchen Einfluss Forschungsergebnisse jeglicher Art, so abstrakt sie auch wirken mögen, auf das eigene Leben und somit die persönliche Zukunft haben können. Entsprechend scheint es umso wichtiger, wissenschaftliche Öffentlichkeitsarbeit nicht nur in Bezug auf SuS, sondern auch für die allgemeine Öffentlichkeit zu betreiben.

\subsubsection{Highlights der Physik 2018}

Unter Berücksichtigung der Erkenntnisse aus den Erhebungen der IDEENEXPO wurde das Konzept für die HDP dahingehend verändert, dass mit der Fokussierung auf den inhaltlichem Schwerpunkt Botox die Relevanz der Biomembranforschung für die Gesellschaft aufgezeigt werden sollte. Neben der Erweiterung des Konzepts um zwei EXPOneer-Regale bot vor allem das Fluoreszenzmikroskop die Möglichkeit, neben den fachlichen Inhalten auch Forschungsmethoden zu thematisieren. Zur Validierung der Forschungsergebnisse aus der ersten Erhebung wurden die o. g. Forschungsfragen erneut in den Mittelpunkt des Forschungsinteresses gerückt.

\section{Evaluationsdesign}

Für die quantitative Erhebung wurde abermals die Umfragenapplikation Limesurvey genutzt. Die Fragen für die einzelnen Zielgruppen unterlagen dabei denselben Kriterien wie zuvor, lediglich die Zahl der inhaltlichen Fragen wurde auf fünf bis sechs Fragen je Zielgruppe angehoben. Die sich am Forschungsinteresse aus Kapitel 6.4.2 orientierenden Fragen wurden dabei entsprechend der Anpassung des Messekonzept inhaltlich an das Thema Botox angepasst.

Um nicht nur in Bezug auf SuS, sondern auch im Allgemeinen qualitative Daten erheben zu können, wurde die quantitative Erhebung durch teilnehmende Beobachtungen [357] ergänzt. Zur Gewährleistung der Reliabilität der eigenen Beobachtungen wurden zusätzlich Interviews mit den zwei mitgereisten Standbetreuern geführt, die bis dato beide noch auf keiner Bildungsmesse gearbeitet hatten [377]. Um diesbezüglich ein möglichst authentisches Bild zu erhalten, wurden die Mitarbeiter_innen zuvor nicht instruiert, aktiv Beobachtungen durchzuführen, und am ersten Abend mithilfe eines Leitfadeninterviews bezüglich ihrer Eindrücke befragt. Anhand desselben Leitfadens wurde zwei Tage später ein weiteres Interview geführt, um zu überprüfen, ob sich die Beobachtungen hinsichtlich zuvor genannter Aspekte geändert oder ggf. manifestiert haben.

\section{Evaluationsergebnisse}

Der Ausstellungsstand während der HDP 2018 wurde täglich von drei Mitarbeiter_innen betreut, die allesamt Lehramtsstudierende der Chemie waren. U. a. basierend auf der Erkenntnis, dass es mithilfe des bisherigen Messekonzepts noch nicht gelungen war, Einblicke in die Forschung des SFB 803 zu geben, wurde das Konzept um zwei weitere EXPOneer-Regale sowie ein Fluoreszenzmikroskop erweitert, die den Schwerpunkt sowohl auf 
Anwendungsgebiete der Forschung als auch auf die zur Erkenntnisgewinnung verwendeten Methoden verschoben. Unter dem Titel „... alles für die Schönheit - Botox: Gift oder Heilmittel?" wurden zudem mit Abbildungen angereicherte Informationstexte für eine Stellwand konzipiert, die allen Ausstellern zur Verfügung gestellt wurden und den Besucher_innen helfen sollten, sich thematisch zu orientieren. Da die Veranstaltung von Dienstag bis Samstag stattfand, waren vor allem an den ersten vier Vormittagen vermehrt SuS der Hauptzielgruppe anwesend. Ähnlich wie zuvor verweilten die einzelnen Besucher_innen zumeist nicht länger als fünf bis zehn Minuten am Exponat.

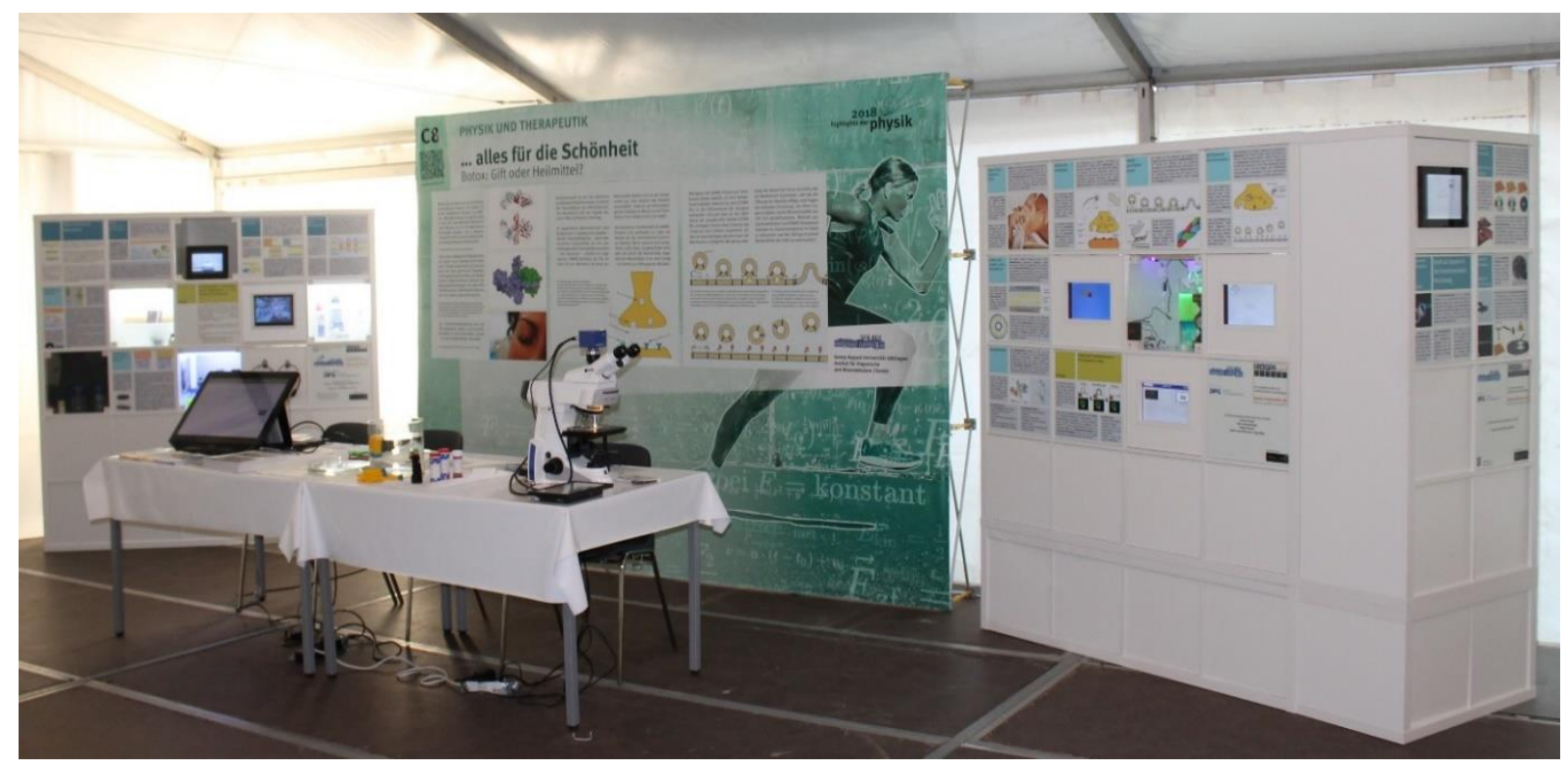

Abbildung 101: Messestand bei den Highlights der Physik 2018.

Von insgesamt 158 mithilfe der Umfragenapplikation gesammelten Datensätzen lagen 142 vollständig vor. Diese konnten jeweils den Gruppen der SuS (N = 39, ohne Grundschüler_innen und SuS ohne Angabe der Klassenstufe, N = 34), der MINT-Lehrkräfte (N = 10) sowie der interessierten Allgemeinheit $(\mathrm{N}=93)$ zugeordnet werden. Dabei fällt auf, dass im Vergleich zur vier Tage länger andauernden IdeenExpo verhältnismäßig mehr Besucher_innen an der Umfrage teilnahmen, jedoch deutlich weniger SuS und Lehrkräfte akquiriert werden konnten.

\section{Schülerinnen und Schüler}

Obwohl zunächst alle Teilnehmer_innen der Umfrage anhand einer Filterfrage den jeweiligen Fragebogentypen zugeordnet wurden, wurde jeder Gruppe zu Beginn dieselbe Frage gestellt. Dabei sollten die Teilnehmer_innen entscheiden, ob es sich bei Botox um einen Naturstoff, ein Arzneimittel, ein Gift oder ein Schönheitsmittel handelt, wobei Mehrfachnennungen möglich waren (Tabelle 27). Obwohl, soweit möglich, in nahezu allen Gesprächen alle vier Möglichkeiten explizit erwähnt wurden, geht die deutliche Mehrheit der SuS davon aus, dass es sich bei Botox sowohl um ein Gift (29) als auch um ein Schönheitsmittel (20) handelt. Die Hälfte denkt zudem, dass Botox auch als Arzneimittel verwendet wird. Jedoch glauben lediglich fünf SuS, dass es sich bei Botox ebenfalls um einen Naturstoff handelt, was damit zusammenhängen kann, dass Chemie im Allgemeinen, und mit ihr auch chemische Verbindungen wie Botox, oftmals fälschlicherweise als etwas nicht Natürliches bzw. als etwas Menschengemachtes und sogar Schlechtes wahrgenommen wird [378]. 
Tabelle 27: Verteilung der Antworten von SuS bzgl. der Frage zur Einordnung Botox. Eine Mehrfachnennung war möglich.

\begin{tabular}{ll}
\hline SuS 1: Botox ist ein... & $\mathbf{N}=\mathbf{3 4}$ (18) \\
\hline Naturstoff & $5(0)$ \\
Arzneimittel & $16(8)$ \\
Gift & $29(18)$ \\
Schönheitsmittel & $20(9)$ \\
\hline
\end{tabular}

An die erste allgemeine Frage schlossen sich für die SuS vier inhaltliche Fragen an, die sich auf Botox und dessen Wirkung auf submikroskopischer und makroskopischer Ebene sowie auf das im zweiten EXPOneer verbaute Modellexperiment zur Exocytose bezogen. Die Aussage „Botox lässt sich klassifizieren als ein...", die drei Antwortmöglichkeiten bot, konnte von etwas mehr als der Hälfte der SuS richtig vervollständigt werden (Tabelle 28). Eine Differenzierung in Bezug auf die Hauptzielgruppe (wie zuvor ab Klassenstufe 10, in diesem Fall inklusive Jahrgang 13) zeigt, dass sogar drei Viertel der SuS in der Lage waren, Botox als ein Protein zu identifizieren. Bezüglich der Aussage „Am synaptischen Spalt bewirkt Botox, dass Acetylcholin..." zeigt sich trotz einer zusätzlichen Antwortmöglichkeit ein ähnliches Bild. 17 der 34 können benennen, dass Acetylcholin aufgrund der Wirkung von Botox nicht mehr ausgeschüttet wird, bei den SuS des 10. bis 13. Jahrgangs sind es sogar zwei Drittel. Auch die Frage zum push-the-button-Experiment zur Exocytose („Mit welchem Bestandteil einer Synapse ist die im Versuch gezeigte mittlere Phase (blau) zu vergleichen?“, Tabelle 28) konnte ebenfalls von insgesamt 17 SuS und gut zwei Dritteln der Hauptzielgruppe richtig beantwortet werden. Obwohl auch hier, ähnlich wie bei der IDEENEXPO, die Anzahl der richtigen Antworten abermals dicht beieinanderliegen, beantworteten lediglich sechs SuS, ausnahmslos aus dem 12. Jahrgang, alle drei Fragen richtig.

Tabelle 28: Verteilung der Antworten von SuS bzgl. der zielgruppenspezifischen Fragen zur Klassifizierung von Botox, der Wirkung von Acetylcholin und dem Modellversuche aus dem EXPOneer Gifte für die Schönheit.

\begin{tabular}{ll}
\hline SuS 2: Botox lässt sich klassifizieren als ein... & $\mathbf{N}=\mathbf{3 4}(\mathbf{1 8})$ \\
\hline Lipid & $7(2)$ \\
Protein & $18(14)$ \\
Vesikel & $4(1)$ \\
Ich weiß es nicht & $5(1)$ \\
\hline SuS 3: Am synaptischen Spalt bewirkt Botox, dass Acetylcholin... & $\mathbf{N}=\mathbf{3 4}(\mathbf{1 8})$ \\
\hline aufgenommen wird & $1(0)$ \\
nicht mehr aufgenommen wird & $5(3)$ \\
ausgeschüttet wird & $6(2)$ \\
nicht mehr ausgeschüttet wird & $17(12)$ \\
Ich weiß es nicht & $5(1)$ \\
\hline SuS 4: Mit welchem Bestandteil einer Synapse ist die im Versuch gezeigte mittlere & $\mathbf{N}=\mathbf{3 4}(\mathbf{1 8})$ \\
Phase (blau) zu vergleichen? & \\
\hline Präsynapse (A) & $1(0)$ \\
Präsynaptische Membran (B) & $17(11)$ \\
Synaptischer Spalt (C) & $9(6)$ \\
Postsynaptische Membran (D) & $1(1)$ \\
Ich weiß es nicht & $6(0)$ \\
\hline
\end{tabular}

Hinsichtlich der letzten Frage („Welche Aussage über Botox stimmt NICHT? Botox wird eingesetzt bei...", Tabelle 29) standen den SuS vier Antwortmöglichkeiten zur Verfügung. 
Lediglich neun der 34 SuS beantworteten diese Frage korrekterweise mit Muskellähmungen, während die Mehrheit Depressionen für die richtige Antwort hielt. Auch die Antworten der Hauptzielgruppe sind diesbezüglich ähnlich verteilt. Obwohl die drei Fragen zuvor von jeweils fünf bzw. sechs SuS mit „Ich weiß es nicht“ beantwortet wurden, schienen sich die SuS gerade bei dieser Frage besonders sicher zu sein. Dafür gibt es zwei Erklärungsansätze, die jedoch lediglich auf Beobachtungen und Spekulationen beruhen. Zum einen wurde in den Gesprächen mit den SuS zunächst vor allem Wert auf die Erklärung der Exocytose und der Wirkweise von Botulinumtoxin auf die an der Exocytose beteiligten SNARE-Proteine gelegt. Dadurch könnte es vermehrt vorgekommen sein, dass mögliche Anwendungsfelder im medizinischen Bereich nicht vollständig oder gar nicht erwähnt wurden. Zum anderen handelt es sich bei Muskellähmungen, Bewegungsstörungen und vermehrter Schweißbildung um körperliche Beschwerden, während es sich bei Depressionen um eine psychische Störung handelt. Entsprechend scheint die Antwort Depressionen hinsichtlich dieser Einteilung zunächst als logisch. Tatsächlich wird Botox aber vereinzelt bei Depressionen eingesetzt, wobei sich das Toxin nicht auf molekularer Ebene auf die Depression auswirkt. Stattdessen führt die gezielte Behandlung der Zornesfalte des Patienten zu einem entspannteren Gesichtsausdruck, was nach der Facial-Feedback-Hypothese auch für positivere Emotionen sorgen soll [379].

Tabelle 29: Verteilung der Antworten von SuS bzgl. der zielgruppenspezifischen Frage zur Wirkung von Botox.

\begin{tabular}{ll}
\hline SuS 5: Welche Aussage über Botox stimmt NICHT? Botox wird eingesetzt bei... & N = 34 (18) \\
\hline Muskellähmungen & $9(6)$ \\
Depressionen & $15(7)$ \\
Bewegungsstörungen & $4(2)$ \\
vermehrter Schweißbildung & $4(2)$ \\
Ich weiß es nicht & $2(1)$ \\
\hline
\end{tabular}

Eine deutliche Mehrheit der teilnehmenden SuS besuchte die 12. Jahrgangsstufe. Wie zuvor nahmen zudem vornehmlich SuS von Gymnasien (27) an der Fragebogenstudie teil. Im Vergleich zur IDEENEXPO war jedoch der Anteil der SuS ab der 10. Klassenstufe mit über $50 \%$ der relevanten Teilnehmer_innen deutlich größer. Ähnlich wie zuvor war ein Großteil in der Lage, die meisten Fragen richtig zu beantworten, obwohl diese curricular nicht relevante Inhalte abdeckten. Obwohl das Format der Bildungsmessen weiterhin nicht geeignet ist, um diesbezüglich in Längsschnittstudien Aussagen über die inhaltliche Tiefe des Verständnisses oder die Nachhaltigkeit des Gelernten zu erheben, kann das Erzielen ähnlicher Ergebnisse wie zuvor als Indiz dafür gewertet werden, dass zahlreiche SuS den Erklärungen der Standmitarbeiter_innen in weiten Teilen folgen konnten. 


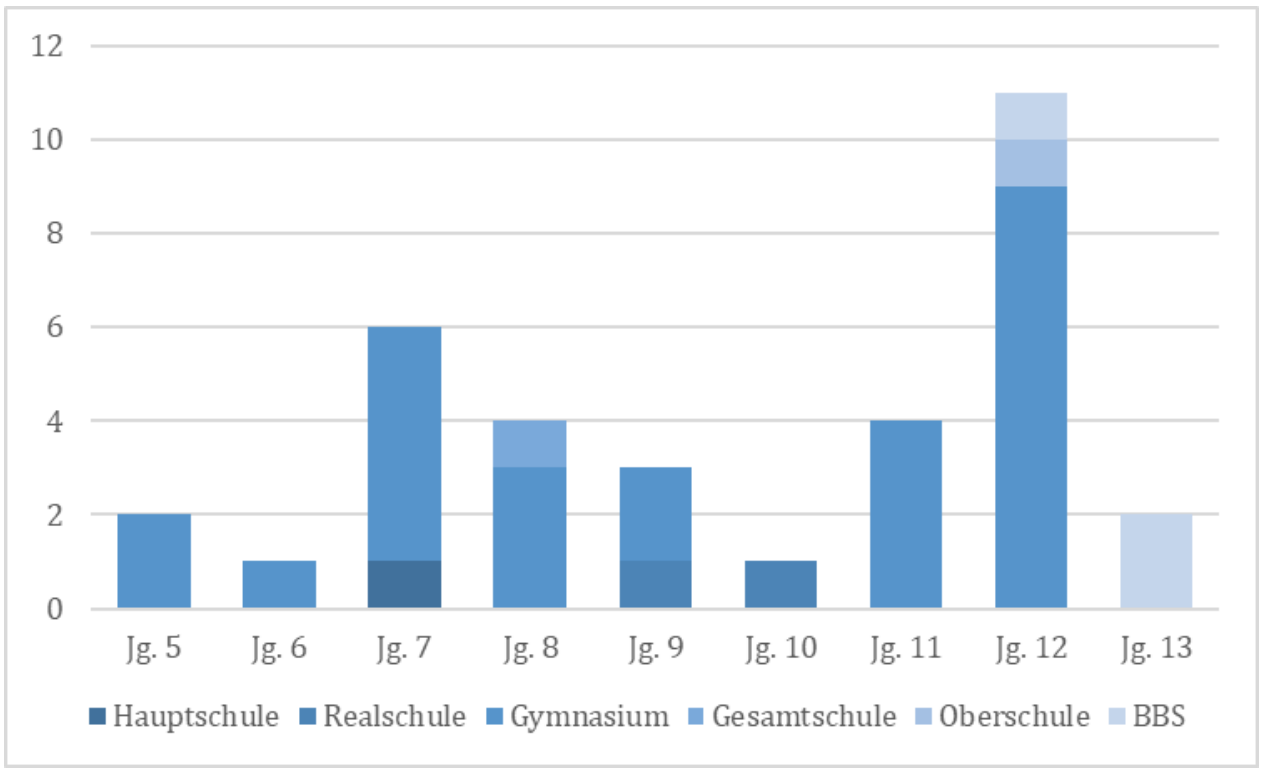

Abbildung 102: Evaluationsteilnehmer_innen (SuS) nach Jahrgang und Schulform.

\section{Lehrkräfte}

Im Vergleich zur IDEENEXPO konnten während der HDP mit zehn Lehrkräften nur verhältnismäßig wenige Personen dieser Zielgruppe gewonnen werden, was u. a. damit zusammenhängt, dass angemeldete Lehrkräfte auf der IDEENEXPO anhand eines Lehrkräfteausweises identifiziert und somit gezielt angesprochen werden konnten. Sieben der Lehrkräfte unterrichteten zudem das Fach Physik, zumeist in Kombination mit Mathematik, was sicherlich der physikalischen Ausrichtung der HDP zugeschrieben werden kann. Der Fragebogen für Lehrkräfte enthielt vier inhaltliche Fragen, von denen eine Filterfrage ggf. der Beantwortung einer weiteren Frage bedurfte. Ebenso wie den SuS wurde auch den Lehrkräften eingangs die Frage gestellt, worum es sich bei Botox handelt (Tabelle 30). Wie zuvor gaben die meisten an, dass es sich bei Botox um ein Gift handelt, die Hälfte hielt es zudem für ein Schönheitsmittel. Lediglich vier bzw. drei Lehrkräfte hielten Botox zudem für ein Arzneimittel bzw. einen Naturstoff. Während dieser Umstand nicht unbedingt zum Grundwissen einer Physiklehrkraft gezählt werden kann, ist es dennoch verwunderlich, dass zwei der drei Lehrkräfte die Biologie oder Chemie unterrichteten, Botox ebenfalls nicht als einen Naturstoff identifizierten.

Tabelle 30: Verteilung der Antworten von Lehrkräften bzgl. der Frage zur Einordnung Botox. Eine Mehrfachnennung war möglich.

\begin{tabular}{ll}
\hline Lehrkräfte 1: Botox ist ein... & N = 10 \\
\hline Naturstoff & 3 \\
Arzneimittel & 4 \\
Gift & 9 \\
Schönheitsmittel & 5 \\
\hline
\end{tabular}

Die Lehrkräfte sollten des Weiteren angeben, ob sie das Thema Botox jemals in ihrem Unterricht behandelt hätten. Lediglich eine Lehrkraft mit der Fächerkombination Biologie und Naturwissenschaften beantwortete die Frage positiv, gab jedoch in einer Anschlussfrage an, dass sie das Thema nicht im Kontext der Biomembranen unterrichtet habe. Die Hälfte der Lehrkräfte beantwortete zudem die dritte Frage („Würden Sie gerne mehr über das Thema Botox (Botulinumtoxin) erfahren, um dieses in Ihren Unterricht einzubinden?") positiv, die in den meisten Fällen u. a. eines der Fächer Biologie, Chemie oder Naturwissenschaften unterrichteten. 
Die vierte Frage zu jemals im Unterricht thematisierten Forschungsmethoden (Tabelle 31)zielte mit Blick auf die Forschungsfrage L3 darauf ab, in Erfahrung zu bringen, inwiefern Forschungsmethoden wie REM, AFM, Fluoreszenzmikroskopie und NMR bereits im Unterricht thematisiert werden, um Schlüsse darüber zu ziehen, inwiefern bereits in Ansätzen Einblicke in die Forschung gegeben werden. Obwohl alle der genannten Methoden weder in den niedersächsischen noch in den nordrhein-westfälischen Kerncurricula insbesondere der Physik [249, 380] Erwähnung finden, wurden sowohl die Rasterelektronenmikroskopie als auch die Magnetresonanzspektroskopie bereits von jeweils sechs Lehrkräften unterrichtet, die allesamt entweder Physik- oder Biologielehrkräfte waren. Die Fluoreszenzmikroskopie wurde bisher jedoch nur von drei Lehrkräften thematisiert (vornehmlich von Biologielehrkräften) und lediglich eine Physiklehrkraft gab an, bereits alle Methoden unterrichtet zu haben.

Tabelle 31: Verteilung der Antworten von Lehrkräften bzgl. der zielgruppenspezifischen Frage zur unterrichtlichen Behandlung verschiedener Forschungsmethoden.

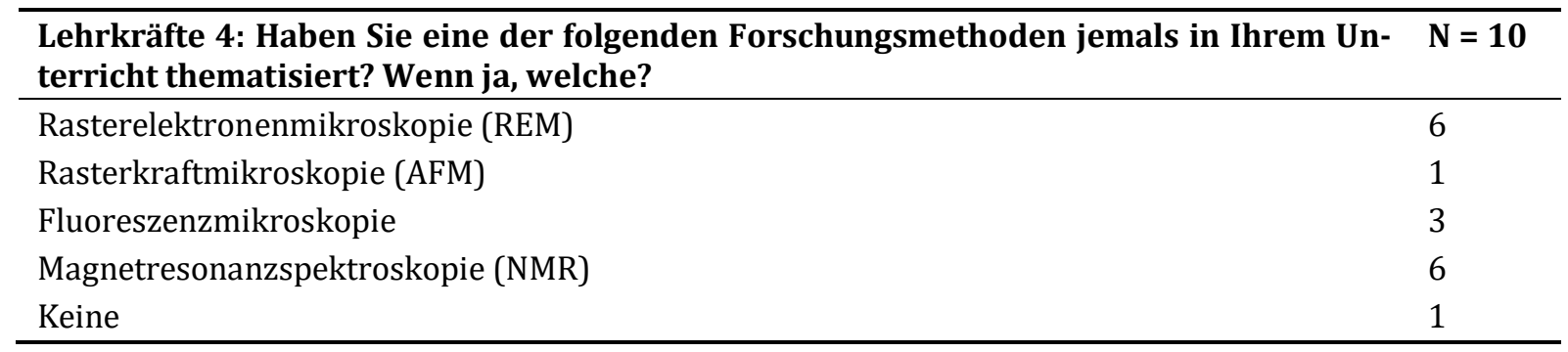

Trotz eines im Vergleich zur IDEENEXPO deutlich geringen Anteil an Biologie- und/oder Chemielehrkräften ( 3 von 10 vs. 38 von 44), für die das Thema Botox die meisten curricularen Anknüpfungspunkte bietet, zeigte die Hälfte der Lehrkräfte dennoch ein Interesse an weiterführenden Informationen. Die Zahl der Lehrkräfte, die bereits verschiedene Forschungsmethoden in ihren Unterricht einbinden, ist erfreulich, wobei der Fokus diesbezüglich deutlich auf der Rasterelektronenmikroskopie und der Magnetresonanzmikroskopie liegt. Sowohl die Rasterkraft- als auch die Fluoreszenzmikroskopie, die beide schwerpunktmäßig im Messekonzept des SFB verankert sind, finden scheinbar im Unterricht deutlich weniger Beachtung. Das Aufzeigen von Anwendungsgebieten dieser Methoden scheint gerade deshalb besonders sinnvoll.

\section{Interessierte Allgemeinheit}

Der letzten Gruppe der interessierten Allgemeinheit wurden insgesamt sechs inhaltliche Fragen gestellt, von denen die erste wie zuvor den Fragen der SuS und Lehrkräfte glich (Tabelle 32). Auch hier setzt sich der allgemeine Trend fort, dass Botox von den meisten Teilnehmer_innen als ein Gift identifiziert, und dass es nur von den wenigsten Personen auch für einen Naturstoff gehalten wird.

Tabelle 32: Verteilung der Antworten der Allgemeinheit bzgl. der Frage zur Einordnung Botox. Eine Mehrfachnennung war möglich.

\begin{tabular}{ll}
\hline Allgemeinheit 1: Botox ist ein... & N $\mathbf{9 9 3}$ \\
\hline Naturstoff & 37 \\
Arzneimittel & 55 \\
Gift & 84 \\
Schönheitsmittel & 50 \\
\hline
\end{tabular}

In einer anschließenden Frage sollten die Teilnehmer_innen angeben, ob sie eine AntiFalten-Behandlung mit Botox in Betracht ziehen würden, wenn ihre Krankenkasse die Kosten für die Behandlung übernehmen würde. Mit 88 der der 93 Teilnehmer_innen 
lehnte eine Mehrzahl der Befragten diese Aussage ab, wobei sogar 79 der Personen gar nicht zustimmten. Lediglich drei Personen stimmten dieser Aussage gänzlich zu. Zum Vergleich wurden die Teilnehmer_innen überdies gefragt, ob sie die Verwendung von Botox im Bereich der Schönheitschirurgie als gefährlich erachten (Tabelle 33). Immerhin noch 71 Teilnehmer_innen erachten diese Art der Verwendung als eher gefährlich (38) oder gefährlich (33), wobei diese Aussage in Bezug zur zweiten Frage deutlich schwächer negativ bewertet wurde. Die Personen, die dabei zuvor angaben, eine Behandlung in Betracht zu ziehen, schätzen die Verwendung von Botox auch als ungefährlich oder eher ungefährlich ein. Eine medizinische Verwendung von Botox erachten hingegen 81 Personen als allgemein sinnvoll. Eine mögliche Erklärung der unterschiedlichen Einstufung der Gefährlichkeit von Botox ist, dass aufgrund des Effekts der sozialen Erwünschtheit sowohl die Anwendung von Botox vornehmlich abgelehnt wird als auch die Anwendung in diesem Bereich als gefährlicher angesehen wird.

Tabelle 33: Verteilung der Antworten der Allgemeinheit bzgl. der zielgruppenspezifischen Fragen zur Verwendung von Botox.

\begin{tabular}{lc}
\hline $\begin{array}{l}\text { Allgemeinheit 3: Die Verwendung von Botox im Bereich der Schönheitschirurgie er- } \\
\text { achte ich als... }\end{array}$ & $\mathbf{N}=93$ \\
\hline ungefährlich & 4 \\
eher ungefährlich & 18 \\
eher gefährlich & 38 \\
gefährlich & 33 \\
\hline Allgemeinheit 4: Die Verwendung von Botox im Bereich der Medizin erachte ich als... & $\mathbf{N}=93$ \\
\hline sinnvoll & 40 \\
eher sinnvoll & 41 \\
eher nicht sinnvoll & 8 \\
nicht sinnvoll & 4 \\
\hline
\end{tabular}

Wie bereits während der IDEENEXPO wurde die interessierte Allgemeinheit erneut hinsichtlich ihrer Einschätzung der Relevanz von naturwissenschaftlicher Forschung sowohl für die Gesellschaft als auch für ihre persönliche Zukunft befragt (Tabelle 34). Ähnlich wie zuvor, beurteilten deutlich mehr Teilnehmer_innen die Forschung für die Gesellschaft als sehr wichtig (81) denn als für ihre persönliche Zukunft (57). Insgesamt schätzten dabei 29 Personen die Relevanz für die persönliche Zukunft anders ein als zuvor, wobei zwei Teilnehmer_innen ihre Angaben diesbezüglich sogar nach oben korrigierten.

Tabelle 34: Verteilung der Antworten der Allgemeinheit bzgl. der zielgruppenspezifischen Fragen zur Relevanz von Forschung für die Gesellschaft bzw. die persönliche Zukunft.

\begin{tabular}{ll}
\hline $\begin{array}{l}\text { Allgemeinheit 5: Die naturwissenschaftliche Forschung erachte ich für die Gesellschaft } \\
\text { als... }\end{array}$ & $\mathbf{N}=\mathbf{9 3}$ \\
\hline sehr wichtig & 81 \\
wichtig & 8 \\
wenig wichtig & 3 \\
unwichtig & 1 \\
\hline Allgemeinheit 6: Die naturwissenschaftliche Forschung erachte ich für meine persönli- & $\mathbf{N}=\mathbf{9 3}$ \\
che Zukunft als... & 57 \\
\hline sehr wichtig & 28 \\
wichtig & 6 \\
wenig wichtig & 2 \\
\hline unwichtig & \\
\hline
\end{tabular}


15 dieser Personen hatten dabei keinen naturwissenschaftlichen beruflichen Hintergrund (insgesamt hatten mit 50 Teilnehmer_innen knapp mehr als die Hälfte beruflich mit naturwissenschaftlichen Themen zu tun). Zudem fällt auf, dass vor allem Personen im Alter von 50+ dazu neigten, die Forschung persönlich als weniger relevant einzuschätzen. Im Vergleich dazu waren es während der IDEEnExPo eher Personen in der Altersspanne von 31 bis 50. Vergleicht man jedoch die Altersverteilung der Besucher_innen der beiden Messen, so wird deutlich, dass die entsprechenden Gruppen in beiden Fällen auch übermäßig oft vertreten waren. Dies lässt sich auch anhand von Beobachtungen während beider Messen bestätigen. Während die IDEENEXPO vor allem am Nachmittag und an den Wochenenden von Eltern und zumindest teilweise auch Großeltern mit jungen Kindern gezielt besucht wurde, waren nur selten ältere Personen ohne Kinder anzutreffen. Die HDP wurden hingegen, gefördert durch die zentrale Lage in der Innenstadt, in vielen Fällen wahrscheinlich auch spontan besucht, so dass deutlich mehr ältere Personen ohne Begleitung von Kindern beobachtet werden konnten. Gleichzeitig waren zudem auch viele Studenten zugegen, was den vergleichsweise hohen Anteil an (vornehmlich naturwissenschaftlich involvierten) 11 bis 30-jährigen Teilnehmer_innen erklären würde. Entsprechend können jedoch unter Einbeziehung der Ergebnisse von der IDEENEXPo keine Aussagen darüber getroffen werden, ob das Alter einen Einfluss auf das Relevanzempfinden bezüglich der naturwissenschaftlichen Forschung hat. Eindeutiger hingegen ist, dass gerade Personen die sich zumindest beruflich weniger mit den Naturwissenschaften auseinandersetzen eher dazu neigen, die Forschung auf persönlicher Ebene als weniger relevant einzuschätzen.

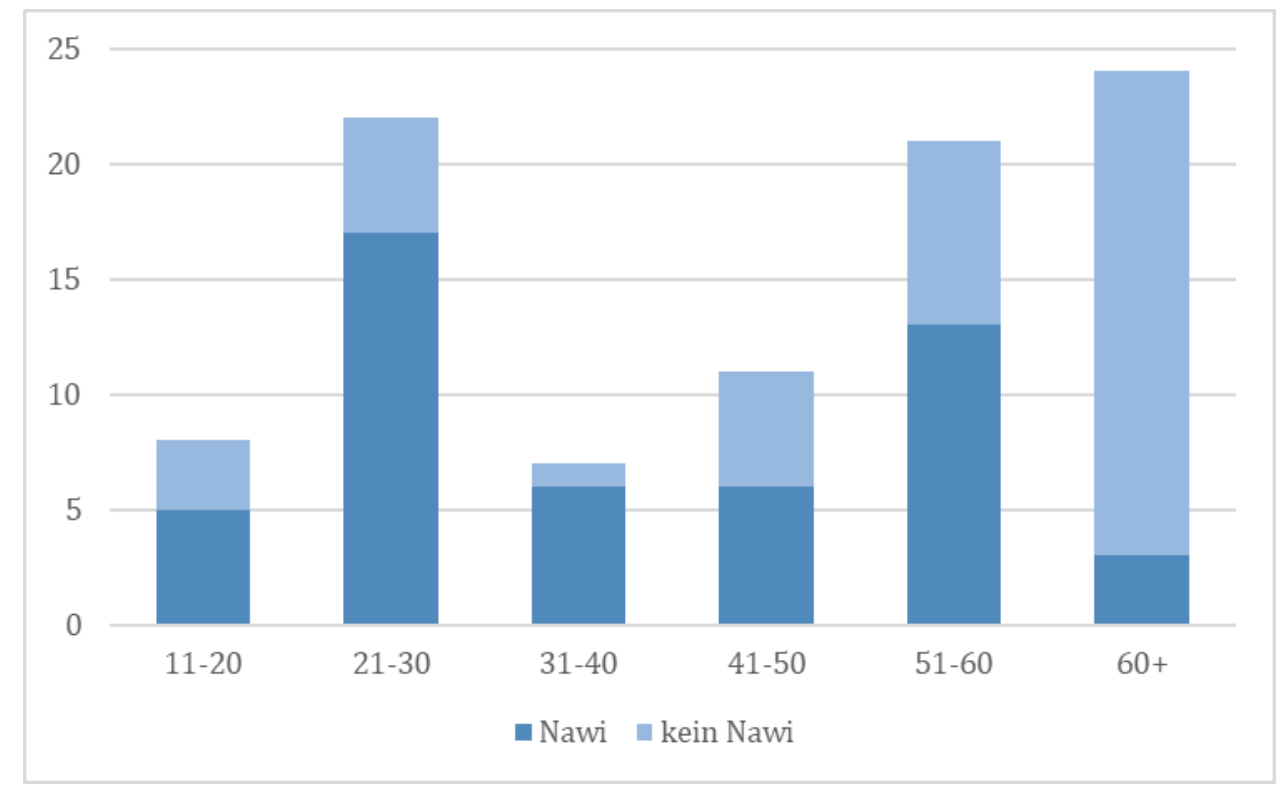

Abbildung 103: Evaluationsteilnehmer_innen (Allgemeinheit) nach Alter und beruflichem Hintergrund.

Zusätzlich zur Fragebogenerhebung wurden eigene Beobachtungen jeweils während und nach der Veranstaltung notiert, sowie die beiden Mitarbeiter_innen anhand eines Leitfadeninterviews bezüglich ihrer Eindrücke sowohl am ersten als auch am dritten Tag der Messe befragt. Die Tonaufnahmen der Interviews wurden im Nachhinein transkribiert und dahingehend untersucht, ob sich die Beobachtungen im Laufe der Zeit änderten oder festigten und ob ggf. sogar neue hinzukamen. Anhand des Leitfadens wurden die Mitarbeiter_innen gefragt, ob sie Gründe für das Stehenbleiben der Besucher_innen am Exponat ausmachen konnten und ob die sie die Personen, mit denen sie sich unterhalten haben, selber ansprechen mussten oder ob sie von diesen angesprochen wurden. Des Weiteren 
sollten die Mitarbeiter_innen erörtern, welches Vorwissen die Besucher_innen in Bezug auf das Thema Botox bereits besitzen und welche Fragen die Besucher_innen ihnen stellen. Diesbezüglich sollten sie insbesondere auch benennen, welche Fragen von Lehrkräften an sie gestellt werden. Die entsprechenden Beobachtungen werden nachfolgend stichpunktartig skizziert:

- SuS bleiben vornehmlich wegen der Experimente, des Mikroskops sowie der fluoreszierenden Flüssigkeiten im EXPOneer-Regal am Ausstellungsstand des SFB stehen.

- Dabei geht es weniger darum, ob das angebotene Thema von Interesse ist, vielmehr wird stattdessen einem kindlichen Spieltrieb nachgegangen.

- Oftmals wissen SuS, wenn sie stehenbleiben zunächst nicht, worum es an dem Ausstellungsstand geht.

- Erwachsene Besucher_innen gehen systematischer vor und lesen zunächst Überschriften und Informationstexte.

- Erwachsenen und SuS ist jedoch gemein, dass sie es meistens bei einer kurzen Betrachtung der Texte oder Experimente belassen. Entsprechend müssen sie angesprochen werden, um sie für kurze Zeit am Stand zu halten.

- SuS sind zudem oft in Kleingruppen unterwegs, von denen nur einige wenige SuS aufrichtiges Interesse zeigen, wenn sie angesprochen werden.

- Erwachsene reagieren oft ablehnend, wenn sie angesprochen werden, da sie Botox generell ablehnen und ein Verkaufsgespräch befürchten.

- Botox ist SuS zunächst kein Begriff, als Mittel zur Faltenglättung ist es den meisten SuS aber dennoch bekannt.

- Medizinische Anwendungsmöglichkeiten sind lediglich erwachsenen Besucher_innen bekannt, oftmals da sie von eigenen oder von Behandlungen von Familienmitgliedern berichten können.

- Im Allgemeinen werden von den Besucher_innen kaum Fragen gestellt. Die wenigen gestellten Fragen der Erwachsenen bezogen sich meistens auf eine mögliche Verwendung von Botox hinsichtlich verschiedener Krankheitsbilder.

- Auch Lehrkräfte stellen keine weitergehenden Fragen zum Konzept, wobei dies auch dran liegen kann, dass vornehmlich Physiklehrkräfte den Ausstellungsstand besuchten.

\subsubsection{Reflexion des Formats}

Obwohl sich das Format der Bildungsmessen im Gegensatz zu den anderen Aktivitäten nicht ausschließlich der primären Zielgruppe der SuS widmet, ist es als bedeutsames SO Konzept hervorzuheben. So bieten Bildungsmessen ganz unabhängig vom Messekonzept des SFB die Möglichkeit, das Interesses an den Naturwissenschaften und zudem auch die Scientific Literacy der Besucher_innen zu fördern. Insbesondere in Bezug auf die Zielgruppe der SuS leisten sie einen wichtigen Beitrag zur Berufsorientierung, indem Einblicke in verschiedene Berufsfelder gegeben werden. Zudem ermöglicht der Austausch mit jungen und in vielen Fällen weiblichen Wissenschaftlerinnen auf Augenhöhe einen authentischen Blick auf die Wissenschaft, der stereotypen Vorstellungen entgegenwirken kann und gerade jungen Mädchen wissenschaftliche Vorbilder bietet. Während diese Aspekte auch in die Planung des vorgestellten Messekonzepts einflossen, wurden die Messen in Bezug auf Lehrkräfte u. a. auch dazu genutzt, neue Experimente vorzustellen und mit ihnen über die Einbringung von Forschungsinhalten in den Unterricht ins Gespräch zu kommen. 
Die EXPOneer Regale, die zunächst als eigenständige interaktive Exponate gedacht waren, entwickelten sich zu wichtigen Informationsquellen, deren Abbildungen der Erklärung unterschiedlicher wissenschaftlicher Inhalte dienten. Gleichzeitig umrahmten sie im Hintergrund den jeweiligen Ausstellungsstand, sodass eine klare Abgrenzung zu anderen Exponaten möglich war. Der größte Fokus der Besucher_innen lag hingegen auf den Experimenten, die, wie im Falle geometrischer Seifenblasen, z. T. vor allem dazu dienten, situationales Interesse zu wecken, um die Besucher_innen an den Stand zu binden, aber auch explizit der Erkenntnisgewinnung dienten. Das Messekonzept entwickelte sich diesbezüglich kontinuierlich weiter, so dass weniger die Vermittlung fachlicher Inhalte und stattdessen vielmehr Anwendungsgebiete sowie Forschungsmethoden in den Vordergrund rückten. Hinsichtlich der in Kapitel 5 formulierten Leitfragen, wurde die Beantwortung der letzten Leitfrage in den Mittelpunkt des Messekonzepts gerückt.

Dabei musste jedoch festgestellt werden, dass sich das Thema „Gifte für die Schönheit“ im Rahmen von Bildungsmessen nur bedingt als Anwendungsbeispiel eignet, da insbesondere der Begriff Botox auf viele Leute abschreckend wirkte. Kamen die Besucher_innen jedoch mit den Standmitarbeiter_innen ins Gespräch zeigte sich deutlich, dass die verschiedenen Anwendungsgebiete des Toxins nur wenigen bekannt waren und sich das Beispiel dennoch eignete, um über die Forschung des SFB an SNARE-Proteinen zu informieren. Dennoch bildet das Format der Bildungsmessen einen wichtigen Baustein im SO-Konzept des SFB, da es zahlreiche Möglichkeiten bietet, die Forschung des SFB zu präsentieren und Einblicke in den Alltag und die Methoden der Wissenschaft zu geben. 


\section{Abschließende Betrachtungen und Ausblick}

Die kontinuierliche und rapide Zunahme an Erkenntnissen in naturwissenschaftlichen Forschungsfeldern macht es unabdingbar, der Gesellschaft im Rahmen einer naturwissenschaftlichen Grundbildung eine Teilhabe an wissenschaftlich motivierten Entscheidungen in Politik und Alltag zu ermöglichen. Neben einer Grundsteinlegung in der Schule bedarf es jedoch eines gewissen Maßes an Eigeninteresse jedes Einzelnen, sich mit naturwissenschaftlichen Themen auseinanderzusetzen.

Das Interesse an den Naturwissenschaften sinkt jedoch vor allem im Kontext Schule mit zunehmendem Alter kontinuierlich, auch wenn der naturwissenschaftlichen Forschung sowohl von $\mathrm{SuS}$ als auch der Allgemeinheit eine hohe gesellschaftliche Relevanz zugesprochen wird. In Bezug auf das sinkende Interesse gegenüber den naturwissenschaftlichen Schulfächern wird vor allem mangelnde Relevanzzuschreibung aufgrund fehlender Kontextualisierung moniert. Analysen von Kerncurricula sowie Schulbüchern zeigen außerdem, dass aktuelle Erkenntnisse aus der Forschung im Unterricht kaum eine Rolle spielen, oder erst nach Jahren in der Schulbuchliteratur zu finden sind. Zudem ist es auch für den interessierten Laien schwierig, sich über aktuelle wissenschaftliche Inhalte zu informieren, da diese vornehmlich für bestimmte Wissenschaftsgemeinden in oft für die Allgemeinheit kaum zugänglichen Journalen veröffentlicht werden (Kapitel 3).

Eine Möglichkeit, auch außerhalb des Unterrichts sowohl für SuS als auch die Allgemeinheit Einblicke in die Forschung zu geben, bieten verschiedene Konzepte wissenschaftlicher Öffentlichkeitsarbeit. Im Rahmen dieser Arbeit wurden die zahlreichen existierenden Projekte erstmalig hinsichtlich der intendierten Lernprozesse aus Kontext- und Subjektperspektive systematisiert. Zudem wurde mit Blick auf die Zielgruppenorientierung der verschiedenen Aktivitäten zwischen SO-Projekten, die der allgemeinen Interessenförderung, und solchen, die der Kontextualisierung wissenschaftlicher Inhalte dienen, bspw. durch Aufzeigen aktueller Forschungsinhalte, unterschieden (Kapitel 3).

Am Beispiel des Sonderforschungsbereichs 803 wurden im Anschluss unter Berücksichtigung der verschiedenen Kontext- und Subjektperspektiven verschiedene SO-Projekte entwickelt und evaluiert. Dazu wurden zunächst sechs Themenfelder identifiziert, anhand derer die Forschung des SFB an Biomembranen experimentell aufgearbeitet wurde. Ausgehend von grundlegenden Struktur-Eigenschaftsbeziehungen von Tensiden erfolgt dabei der Übergang von makroskopisch wahrnehmbaren Phänomenen aus dem Alltag zur submikroskopischen Erarbeitung des Aufbaus von Membranen, bevor schließlich Einblicke in die explizite Forschung des SFB gegeben werden (Kapitel 5).

Basierend auf dieser didaktischen Erschließung der Forschung an Membranen wurden vier SO-Aktivitäten entwickelt und - soweit möglich -erprobt und evaluiert. Dabei wurde darauf geachtet, dass die Aktivitäten verschiedene SO-Bildungskontexte berücksichtigen, so dass sie neben Unterrichtsmaterialien und einem Schülerlabor auch Adaptierte Primärliteratur und ein Bildungsmessekonzept umfassen (Kapitel 6).

Abschließend soll hier die Eignung der verschiedenen SO-Aktivitäten im Kontext der Forschung des SFB 803, aber auch hinsichtlich forschungsbasierter Öffentlichkeitsarbeit im Allgemeinen diskutiert werden. Obwohl die Membranforschung auf den ersten Blick durchaus geeignet für eine interdisziplinäre Aufarbeitung im Rahmen von SO erscheint, zeigt eine Analyse der Kerncurricula der Fächer Biologie und Chemie, dass sich die fachlichen Inhalte bezüglich Biomembranen vornehmlich auf eine modellartige Betrachtung von Lipiden und Kanälen als Bestandteile von Membranen beschränken. Dabei stehen 
Aufbau und Funktion von Membranen im Vordergrund, während in der Forschung die Interaktion einzelner Lipide und Proteine auf molekularer Ebene von Interesse ist.

Um jedoch möglichst auch im Unterricht anhand verschiedener Experimente Einblicke in die Forschung geben zu können, müssen die en Unterrichtsmaterialien deutlich über die curricular vorgeschriebenen Inhalte hinausgehen. Zum einen fehlen hierfür $u$. a. zeitliche Kapazitäten im Unterricht, zum anderen übersteigen die Forschungsinhalte in Teilen auch die fachlichen Kompetenzen der meisten Lehrkräfte. Eine alternative theoretische Auseinandersetzung der SuS mit Forschungsinhalten im Anschluss an die Vermittlung der curricularen Inhalte erscheint jedoch weder motivierend noch zielführend. So wünschenswert eine Einbettung von Forschungsinhalten in den Unterricht auch ist, scheint sich die Entwicklung von Unterrichtsmaterialien, sei es als fertige Unterrichtseinheit, oder als flexibler Materialienpool, zumindest im Fall des SFB 803 nur bedingt für eine forschungsbasierte Öffentlichkeitsarbeit zu eigenen.

Als Ergänzung zur curricularen Erarbeitung des Themas Membranen im naturwissenschaftlichen Unterricht bieten sich Einblicke in die Forschung stattdessen im Rahmen non-formal intentionaler Angebote wie Schülerlaboren an. Die freiwillige Teilnahme außerhalb des Regelunterrichts ermöglicht eine größere zeitliche und somit auch inhaltliche Flexibilität. Die Nutzung eines außerschulischen Lernorts bietet zudem die Möglichkeit, forschungsnahe Experimente anzubieten, die Chemikalien und Gerätschaften erfordern, die in Schulen in der Regel nicht vorhanden sind. Bezüglich der komplexen und sehr spezifischen Grundlagenforschung des SFB 803 ist es jedoch nötig, authentische und für die SuS relevante Kontexte zu identifizieren, anhand derer die Forschungsinteressen des SFB aufgezeigt werden können. Obwohl innerhalb des SFB nicht an der Wirkweise des Botulinumtoxins geforscht wird, eignet sich dieses aus dem Alltag als Botox bekannte Toxin dennoch, um auf die Forschung bezüglich des Mechanismus der SNARE-vermittelten Fusion von Membranen aufmerksam zu machen.

Das Konzept des Schülerlabors zu Botox wurde mittlerweile zu einem einwöchigen Science Camp für interessierte SuS ausgeweitet, innerhalb dessen unter dem Titel „Was haben Legionellen, EHEC und Botox gemeinsam?" zudem noch weitere bekannte Krankheitserreger thematisiert werden [381]. An eine experimentelle Erarbeitung verschiedener Aspekte des Aufbaus von Biomembranen und der Membranforschung, schließt sich dabei eine literaturgestützte Betrachtung verschiedener Pathogene an, die $u$. a. das Lesen von Adaptierter Primärliteratur beinhaltet.

Insbesondere mit Blick auf die stereotypen Vorstellungen, die bei SuS und in der Gesellschaft bezüglich Wissenschaftler_innen und der wissenschaftlichen Forschung vorherrschen, scheint es wichtig nicht nur für die Inhalte aktueller Forschung zu sensibilisieren, sondern vermehrt auch die Forschungsprozesse in den Vordergrund einer Öffentlichkeitsarbeit zu rücken. Entsprechend soll APL weniger als konkretes Lernmaterial verstanden werden, anhand dessen curriculare Inhalte im Kontext aktueller Forschung erarbeitet werden können. Zwar sind curriculare Anknüpfungspunkte innerhalb der APL wünschenswert und sollten bei der kriteriengeleiteten Auswahl der Originalpublikation Beachtung finden, jedoch eignet sich APL eher, um im Anschluss oder auch zu Beginn einer Sequenz die Aktualität, aber auch die Relevanz der jeweiligen Unterrichtsthematik aufzuzeigen.

In diesem Zuge ist es möglich, SuS schrittweise an das Lesen von wissenschaftlichen Artikeln heranzuführen, was insbesondere in Hinblick auf die Ergreifung eines naturwissenschaftlichen Berufs eine wichtige Kompetenz darstellt. Zudem deuten erste Evaluationen an, dass das Lesen von Adaptierter Primärliteratur das Verständnis bezüglich einzelner 
Dimensionen der Natur der Naturwissenschaften fördern kann. Im Rahmen der Öffentlichkeitsarbeit des SFB wurde deshalb bereits eine weitere APL zur „SNARE-vermittelte[n] Membranfusion an festkörpergestützten und freitragenden Lipidmembranen " in Anlehnung an KUHLMANN ET AL. ${ }^{[287]}$ erarbeitet. Zudem sind weitere Adaptionen von SFBPublikationen im Kontext der hier vorgestellten Materialien denkbar, bspw. bezüglich der Permeabilisierung von Membranen durch antimikrobielle Peptide ${ }^{[26] .}$

Die größte Reichweite der hier vorgestellten SO-Aktivitäten des SFB 803 besitzen jedoch Bildungsmessen, die deshalb auch eine zentrale Rolle im SO-Konzept des SFB spielen. Sowohl im Rahmen dieser Arbeit als auch darüber hinaus konnten sich Besucher_innen seit 2015 mittlerweile acht Mal während Veranstaltungen wie der IDEENEXPO, den HIGHLIGHTS DER PHYSIK, der GÖTTINGER NACHT DES WiSSENS oder dem NiEDERSÄCHSISCHEN FORSCHUNGSTAG bezüglich verschiedener Aspekte der Membranforschung des SFB 803 informieren. Während alle anderen hier vorgestellten Projekte sich vornehmlich an die Zielgruppe der SuS der Sekundarstufe II richten, wurden die jeweiligen Konzepte kontinuierlich sowohl an die Heterogenität der Besucher_innen als auch an die Schnelllebigkeit der Messen angepasst.

Obwohl von der Teilnahme an Bildungsmessen hinsichtlich einer wissenschaftlichen Öffentlichkeitsarbeit nicht erwartet werden kann, dass eine Mehrzahl der Besucher_innen in der kurzen Zeit am Ausstellungsstand ein tiefergreifendes Verständnis für die komplexen Forschungsinhalte des SFB erlangt, leisten Formate wie Bildungsmessen dennoch einen wichtigen Beitrag zum allgemeinen Ziel, die Gesellschaft mit einer naturwissenschaftlichen Grundbildung auszustatten. Denn anhand zahlreicher alltagsnaher Beispiele und ansprechender Experimente wird zumindest das situationale Interesse der Teilnehmer_innen gefördert, das als Grundlage für die Entwicklung eines dauerhaften individuellen Interesses gilt. Zudem trägt der Austausch mit den jungen und oftmals auch weiblichen Standbetreuerinnen dazu bei, Rollenvorbilder zu liefern, die es ermöglichen, stereotype Annahmen bezüglich Wissenschaftler_innen zu revidieren. Neben der Publikation in fachdidaktischen Journalen eignen sich die Messen zudem in besonderem Maße, um neu entwickelte Experimente und Materialien zu disseminieren, da Lehrkräfte - die ihre SuS in vielen Fällen auf die Messe begleiten - die Experimente sowohl ausprobieren als auch die Erkenntnisgewinnungsprozesse ihrer SuS beobachten können.

Aufgrund der Schwierigkeit, komplizierte fachliche Inhalte innerhalb kurzer Zeit an Standbesucher_innen zu vermitteln, deren Vorwissen stark variieren kann, rückte die Vermittlung von Forschungsmethoden zuletzt stärker in den Fokus des Bildungsmessekonzepts. Neben der anschaulichen Darstellung der Rasterkraftmikroskopie beinhaltet das Konzept vor allem verschiedene Experimente zur Fluoreszenz bzw. Fluoreszenzmikroskopie. Ausgehend von der Fluorochromierung von Pflanzenbestandteilen wird zunächst makroskopisch und anschließend mikroskopisch die Bedeutung der Fluoreszenzmikroskopie für die Forschung an Biomembranen erläutert. Für nachfolgende Bildungsmessen ist zudem eine Zusammenarbeit mit dem EXCELLENZCLUSTER der Universität Göttingen Mulitscale Bioimaging - From Molecular Machines to Networks of Excitable Cells [382] angedacht, in dessen Rahmen u. a. die Einbettung eines STED-Mikroskopmodells in das Messekonzept geplant ist.

Das in dieser Arbeit dargelegte SO-Konzept lässt sich in seiner Gesamtheit wahrscheinlich nicht ohne Weiteres auf andere forschungsorientierte Öffentlichkeitsarbeitsprojekte übertragen. Dennoch ermöglicht es eine Systematisierung geplanter Projekte und bietet Anhaltspunkte bezüglich einer möglichen Ausgestaltung der didaktischen Erschließung 
des Forschungsgebietes sowie der Entwicklung und Evaluation verschiedener SO-Konzepte. Insbesondere im Rahmen langjähriger Forschungsprojekte bietet es sich dabei an, von Beginn an eine Öffentlichkeitsarbeit zu etablieren, da zunächst die Grundlagen didaktisch erschlossen werden müssen, bevor neue Erkenntnisse und Methoden experimentell aufgearbeitet werden können. Dabei zeigte sich im Rahmen dieses Projektes immer wieder deutlich, dass die Entwicklung der Aktivitäten vom Austausch zwischen Forschern und Didaktikern lebt, aber auch die Durchführung des Engagements von Wissenschaftler_innen bedarf. Denn neben der Durchführung der Experimente verleiht vor allem die Kommunikation der Teilnehmer_innen oder Besucher_innen mit Wissenschaftler_innen der Öffentlichkeitsarbeit ihre Authentizität. 


\section{Literaturverzeichnis}

[1] J. Osborne, S. Simon, S. Collins, International Journal of Science Education 2003, 25 (9), 1049.

[2] R. Johnson, A. Watkinson, M. Mabe, The STM Report: An Overview of Scientific and Scholarly Publishing, 5th ed. 2018.

[3] O. Renn, L. Betschart, Nachr. Chem. 2020, 68 (5), 12.

[4] M. D. Olvera-Lobo, L. Lopez, Journal of Science Communication 2015, 14 (03).

[5] A. Schibany, H. Gassler, Nutzen und Effekte der Grundlagenforschung: POLICIES Research Report Nr. 98-2010, Wien 2010.

[6] European Commission, Horizon 2020 in brief: The EU Framework Programme for Research \& Innovation 2014.

[7] Bundesministerium für Bildung und Forschung (BMBF), Die Hightech-Strategie für Deutschland 2006.

[8] Bundesministerium für Bildung und Forschung (BMBF), Die Hightech-Strategie zum Klimaschutz 2007.

[9] Bundesministerium für Bildung und Forschung (BMBF), Ideen. Innovation. Wachstum: Hightech-Strategie 2020 für Deutschland 2010.

[10] Bundesministerium für Bildung und Forschung (BMBF), Die neue Hightech-Strategie: Innovationen für Deutschland 2014.

[11] Bundesministerium für Bildung und Forschung (BMBF), Forschung und Innovation für die Menschen: Die Hightech Strategie 20252018.

[12] European Commission, The Sixth Framework Programme in brief 2002.

[13] European Commission, 7th Framework Programme for Research and Technological Development 2007.

[14] European Commission, Responsible Research and Innovation: Europe's Ability to Respond to Societal Challenges 2014.

[15] European Commission, Fact sheet: Science with and for Society in Horizon 2020 2013.

[16] European Commission, Special Eurobarometer 401: Responsible Research and Innovation (RRI), Science and Technology 2013.

[17] European Commission, Special Eurobarometer 401 Summary: Responsible Research and Innovation (RRI), Science and Technology 2013.

[18] J. Baumert et al., Internationales und nationales Rahmenkonzept für die Erfassung von naturwissenschaftlicher Grundbildung in PISA, Berlin 1999. 
[19] R. C. Laugksch, Sci. Ed. 2000, 84 (1), 71.

[20] C. Anelli, American Entomologist 2011, 57 (4), 235.

[21] D. A. Roberts, Scientific Literacy / Science Literacy. In: Abell, S. K., Lederman, N. G. Handbook of Research on Science Education, Lawrence Erlbaum Associates. Mahwah, New Jersey 2007.

[22] H. E. Fischer, Zeitschrift für Didaktik der Naturwissenschaften 1998, 4 (2), 41.

[23] J. D. Miller, The Conceptualization and Measurement of Civic Scientific Literacy for the Twenty-First Century. In: Meinwald, J., Hildebrand, J. G. Science and the Educated American: A Core Component of Liberal Education. Cambridge 2010.

[24] T. Wilke, Konzeptualisierung des Themas "Nano" für den Chemieunterricht, Dissertation, Georg-August-Universität 2016.

[25] S. R. Goldman, G. L. Bisanz, Toward a Functional Analysis of Scientific Genres. In: Otero, J., León, J. A., Graesser, A. C. The Psychology of Science Text Comprehension, Lawrence Erlbaum Associates. Mahwah 2002.

[26] National Science Board, Science \& Engineering Indicators 20182018.

[27] J. B. Corbett, J. L. Durfee, Science Communication 2004, 26 (2), 129.

[28] C. Russell, Covering Controversial Science. In: Kennedy, D., Overholser, G. Science and the Media. Cambridge 2010.

[29] R. C. J. Somerville, S. J. Hassol, Physics today 2011, 64 (10), 48.

[30] K. Bultitude, The Why and How of Science Communication. In: Science Communication. Pilsen 2011.

[31] S. C. Moser, WIREs Clim Change 2010, 1 (1), 31.

[32] R. E. Dunlap, A. M. McCright, Organized Climate Change Denial. In: Dryzek, J. S., Norgaard, R. B., Schlosberg, D. The Oxford Handbook of Climate Change and Society, Oxford University Press. Oxford 2011.

[33] O. Renn, Journal of Environmental Psychology 1990, 10 (2), 151.

[34] K. E. von Mühlendahl, T. Lob-Corzilius, Pädiatrische Allergologie 2018 (1), 50.

[35] J. Osborne, Science for Citizenship. In: Osborne, J., Dillon, J. Good Practice in Science Teaching: What research has to say, Open Univ. Press. Berkshire, England 2010.

[36] Y.-Y. Lo, Online Communication Beyond the Scientific Community: Scientists' Use of New Media in Germany, Taiwan and the United States to Address the Public, Dissertation, Freie Universität Berlin 2016. 
[37] Sekretariat der Ständigen Konferenz der Kultusminister der Länder in der Bundesrepublik Deutschland, Beschlüsse der Kultusministerkonferenz: Bildungsstandards im Fach Chemie für den Mittleren Schulabschluss, Luchterhand, München 2004.

[38] S. Swarat, A. Ortony, W. Revelle, J Res Sci Teach 2012, 49 (4), 515.

[39] P. Potvin, A. Hasni, Studies in Science Education 2014, 50 (1), 85.

[40] A. Krapp, An Educational-Psychological Theory of Interest and Its Relation to SDT. In: Deci, E., Ryan, R. The Handbook of Self-Determination Research, University of Rochester Press. Rochester, NY 2002.

[41] A. Krapp, M. Prenzel, International Journal of Science Education 2011, 33 (1), 27.

[42] S. Hidi, Review of Educational Research 1990, 60 (4), 549.

[43] S. Hidi, K. A. Renninger, Educational Psychologist 2006, 41 (2), 111.

[44] A. Krapp, S. Hidi, K. A. Renninger, Interest, Learning and Development. In: Hidi, S., Krapp, A., Renninger, K. A. The Role of Interest in Learning and Development, Lawrence Erlbaum Associates. Hillsdale, NJ 1992.

[45] S. Sjøberg, C. Schreiner, The ROSE Project: An Overview and Key Findings 2010.

[46] E. W. Jenkins, N. W. Nelson, Research in Science \& Technological Education 2005, $23(1), 41$.

[47] R. Tytler, J. Osborne, Student Attitudes and Aspirations Towards Science. In: Fraser, B. J., Tobin, K., McRobbie, C. J. Second International Handbook of Science Education, Springer International Handbooks of Education, Vol. 24, Springer Science+Business Media B.V. Dordrecht 2012.

[48] A. Hasni, P. Potvin, International Journal of Environmental \& Science Education 2015, 10 (3), 337-366.

[49] G. Merzyn, Naturwissenschaften, Mathematik, Technik - immer unbeliebter?: Die Konkurrenz von Schulfächern um das Interesse der Jugend im Spiegel vielfältiger Untersuchungen, Schneider Hohengehren, Baltmannsweiler 2008.

[50] C. Murphy, J. Beggs, School Science Review 2003, 84 (308).

[51] T. Pell, T. Jarvis, International Journal of Science Education 2001, 23 (8), 847.

[52] J. Osborne, S. Collins, International Journal of Science Education 2001, 23 (5), 441.

[53] M. Jansen, U. Schroeders, P. Stanat, Motivationale Schülermerkmale in Mathematik und den Naturwissenschaften. In: Pant, H. A., Stanat, P., Schroeders, U., Roppelt, A., Siegle, T., Pöhlmann, C. IQB-Ländervergleich 2012: Mathematische und naturwissenschaftliche Kompetenzen am Ende der Sekundarstufe I, Waxmann. Münster 2013. 
[54] A. Schönborn, M. Kremer, T. Götz, Der mathematische und naturwissenschaftliche Unterricht 2012, 65 (5), 309.

[55] N. Holstermann, S. Bögeholz, Zeitschrift für Didaktik der Naturwissenschaften 2007, 13, 71.

[56] L. Hoffmann, P. Häußler, M. Lehrke, Die IPN-Interessenstudie Physik IPN, Vol. 158, IPN, Kiel 1998.

[57] Deutsche Akademie der Technikwissenschaften, Körber-Stiftung, MINT-Nachwuchsbarometer 20142014.

[58] A. Krapp, Psychologie in Erziehung und Unterricht 1998, 44, 185.

[59] Z. Daniels, Entwicklung schulischer Interessen im Jugendalter Pädagogische Psychologie und Entwicklungspsychologie, Vol. 69, Waxmann, Münster 2008.

[60] A. Krapp, Learning and Instruction 2002, 12 (4), 383.

[61] V. Christidou, International Journal of Environmental \& Science Education 2011, 6 (2), 141.

[62] A. H. Johnstone, J. Chem. Educ. 1993, 70 (9), 701.

[63] K. Salta, C. Tzougraki, Sci. Ed. 2004, 88 (4), 535.

[64] V. Christidou, International Journal of Science Education 2006, 28 (10), 1181.

[65] P. O. Dierks, T. Höffler, I. Parchmann, CHEMKON 2014, 21 (3), 111.

[66] G. Merzyn, PhyDid B - Didaktik der Physik - Beiträge zur DPG-Frühjahrstagung 2010.

[67] M. Mead, R. Métraux, Science (New York, N.Y.) 1957, 126 (3270), 384.

[68] D. W. Chambers, Sci. Ed. 1983, 67 (2), 255.

[69] K. D. Finson, School Science and Mathematics 2002, 102 (7), 335.

[70] I. Ruiz-Mallén, S. Gallois, M. Heras, Science Communication 2018, 40 (6), 749.

[71] J. Steinke et al., Science Communication 2007, 29 (1), 35.

[72] D. Elster, Plus Lucis 2007 (3).

[73] C. Schreiner, S. Sjøberg, Science Education and Young People's Identity Construction. In: Corrigan, D., Dillon, J., Gunstone, R. The Re-Emergence of Values in Science Education, Sense Publ. Rotterdam 2007.

[74] S. Sjøberg, C. Schreiner, Asia-Pacific Forum on Science Learning and Teaching 2005, 6 (2).

[75] S. Sjøberg, Acta Didactica Oslo 2002, 3 (1). 
[76] S. Sjøberg, C. Schreiner, Reaching the Minds and Hearts of Young People: What Do We Know About Their Interests, Attitudes, Values and Priorities? What About Their Interest for Space Science?, Bern 2007.

[77] J. K. Gilbert, International Journal of Science Education 2006, 28 (9), 957.

[78] M. Stuckey, A. Hofstein, R. Mamlok-Naaman, I. Eilks, Studies in Science Education 2013, 49 (1), 1.

[79] M. Stuckey et al., CHEMKON 2014, 21 (4), 175.

[80] J. Bennett, F. Lubben, International Journal of Science Education 2006, 28 (9), 999.

[81] R. Demuth, C. Gräsel, I. Parchmann, B. Ralle (Hg.), Chemie im Kontext: Von der Innovation zur nachhaltigen Verbreitung eines Unterrichtskonzepts, Waxmann, Münster, New York, München, Berlin 2008.

[82] I. Parchmann, S. Schmidt, $M N U$ 2003, 56 (4), 214.

[83] B. Krilla, Der 11. Jahrgang Chemie in Nordrhein-Westfalen: Curriculare Entwicklung im Rahmen einer chemiedidaktischen Aktionsforschungsstrategie, Dissertation, Technische Universität Dortmund 2003.

[84] A. Pilot, A. M. W. Bulte, International Journal of Science Education 2006, 28 (9), 1087.

[85] D. King, Studies in Science Education 2012, 48 (1), 51.

[86] I. Eilks, Science Education International 2000, 11 (1), 16.

[87] I. Eilks, R. Marks, M. Stuckey, Praxis der Naturwissenschaften ChiS 2016, 65 (5).

[88] R. Marks, I. Eilks, International Journal of Environmental \& Science Education 2009, $4(3), 231$.

[89] H. van Vorst et al., Zeitschrift für Didaktik der Naturwissenschaften 2015, 21 (1), 29.

[90] H. van Vorst, S. Fechner, E. Sumfleth, Zeitschrift für Didaktik der Naturwissenschaften 2018, 24 (1), 167.

[91] K. Simons, E. Ikonen, Nature 1997, 387 (6633), 569.

[92] H. Linder, H. Bayrhuber, R. Drös, W. Hauber, Linder: Biologie, 24th ed., Westermann 2019.

[93] U. Weber (Hg.), Biologie Oberstufe: Gesamtband S II, 3rd ed., Cornelsen, Berlin 2017.

[94] A. Upmeier zu Belzen, D. Krüger, Zeitschrift für Didaktik der Naturwissenschaften 2010, 16, 41. 
[95] Freie Hansestadt Hamburg, Behörde für Schule und Berufsbildung, Bildungsplan gymnasiale Oberstufe: Chemie, Hamburg 2009.

[96] J. H. Falk et al., International Journal of Science Education, Part B 2015, 6 (4), 369.

[97] J. H. Falk et al., J Res Sci Teach 2018, 55 (3), 422.

[98] J. H. Falk et al., Sci. Ed. 2016, 100 (5), 849.

[99] J. H. Falk, M. D. Needham, J Res Sci Teach 2013, 50 (4), 431.

[100] M. Bulunuz, O. S. Jarrett, International Journal of Environmental and Science Education 2010, 5 (1), 65.

[101] E. Poliakoff, T. L. Webb, Science Communication 2007, 29 (2), 242.

[102] T. W. Burns, D. J. O'Connor, S. M. Stocklmayer, Public Underst Sci 2003, 12 (2), 183.

[103] P. Bell, B. Lewenstein, A. W. Shouse, M. A. Feder, Learning Science in Informal Environments: People, Places, and Pursuits 2009.

[104] Europäische Kommission, Einen europäischen Raum des lebenslangen Lernens schaffen: Mitteilung der Kommission, Brüssel 2001.

[105] T. Rauschenbach et al., Konzeptionelle Grundlagen für einen Nationalen Bildungsbericht: Non-formale und informelle Bildung im Kindes- und Jugendalter, Berlin 2004.

[106] T. Bäumer et al., Zeitschrift für Erziehungswissenschaft 2011, 14 (2), 87.

[107] S. Maschke, L. Stecher, Non-formale und informelle Bildung. In: Lange, A., Reiter, H., Schutter, S., Steiner, C. Handbuch Kindheits- und Jugendsoziologie, Springer Reference Sozialwissenschaften, Springer VS. Wiesbaden 2018.

[108] J. Zinnecker, Zeitschrift für Pädagogik 200, 46 (5), 667.

[109] 0. J. Haupt et al., $M N U$ 2013, 66 (6), 324.

[110] M. W. Tausch, Praxis der Naturwissenschaften 2015, 64 (1), 5.

[111] A. Banerji, J. Dörschelln, D. Schwarz, Chemie in unserer Zeit 2018, 52 (1), 34.

[112] C. Bohrmann-Linde, D. Zeller, World Journal of Chemical Education 2018, 6 (1), 36.

[113] M. Klaus et al., CHEMKON 2014, 21 (2), 65.

[114] D. Quarthal, J. Novotny, M. Oetken, CHEMKON 2018, 25 (2), 74.

[115] S. Schwarzer, R. Abdelaziz, M. Elbahri, T. Wilke, CHEMKON 2016, 23 (4), 188.

[116] T. Wilke, S. Waitz, E. von Hoff, T. Waitz, CHEMKON 2018, 25 (1), 16.

[117] K. H. Lott, Journal of Science Education and Technology 2003, 12 (1), 65. 
[118] G. L. Long et al., J. Chem. Educ. 2012, 89 (10), 1249.

[119] Advanced Materials Science rano GmbH, NanoSchoolBox, http://www.amsrano.com/produkte/nanoschoolbox.html, zuletzt geprüft am 26.06.2020.

[120] Cornelsen Experimenta GmbH, Cornelsen Experimenta, https://cornelsen-experimenta.de/, zuletzt geprüft am 26.06.2020.

[121] J. Young, N. Ortiz, J. Young, International Journal of Education in Mathematics, Science and Technology 2017, 5 (1), 62.

[122] Stiftung Jugend forscht e. V., Jugend forscht: Wir fördern Talente, https://www.jugend-forscht.de/, zuletzt geprüft am 26.06.2020.

[123] S. Laursen, C. Liston, H. Thiry, J. Graf, CBE life sciences education 2007, 6 (1), 49.

[124] G. Clark et al., PLoS biology 2016, 14 (2), e1002368.

[125] N. E. Lee, K. G. Schreiber, J. Chem. Educ. 1999, 76 (7), 917.

[126] LernortLabor - Bundesverband der Schülerlabore e.V., Schülerlabor-Atlas: Die Karte für junge Forschende!, https://www.schuelerlabor-atlas.de/, zuletzt geprüft am 26.06.2020.

[127] G. Heß, YLAB: Geisteswissenschaftliches Schülerlabor, http://ylab.uni-goettingen.de/home/, zuletzt geprüft am 26.06.2020.

[128] M. Euler, T. Schüttler, D. Hausamann, Schülerlabore. In: Kircher, E., Girwidz, R., Häußler, P. Physikdidaktik: Theorie und Praxis, Springer-Lehrbuch, Springer Spektrum. Berlin 2015.

[129] M. Budke, I. Parchmann, M. Beeken, J. Chem. Educ. 2019, 96 (1), 12.

[130] J. d. Houck, N. K. Machamer, K. A. Erickson, J. Chem. Educ. 2014, 91 (10), 1606.

[131] XLAB - Göttinger Experimentierlabor für junge Leute, XLAB International Science Camp, http://www.xlab-goettingen.de/xlab_international.html, zuletzt geprüft am 26.06.2020.

[132] D. Lewalter, C. Geyer, ZfE 2009, 12 (1), 28.

[133] J. Rosendhal, P. Sakimoto, R. Pertzborn, L. Cooper, Advances in Space Research 2004, 34 (10), 2127.

[134] H. W. Kerby et al., J. Chem. Educ. 2010, 87 (10), 1024.

[135] C. Silva et al., Journal of Science Communication 2016, 15 (01).

[136] J. Silvertown, Trends in ecology \& evolution 2009, 24 (9), 467.

[137] D. K. Smith, J. Chem. Educ. 2014, 91 (10), 1594. 
[138] TED Conferences, LLC, TED Ed, https://ed.ted.com/, zuletzt geprüft am 26.06.2020.

[139] E. Jensen, N. Buckley, Public Underst Sci 2014, 23 (5), 557.

[140] K. Bultitude, D. McDonald, S. Custead, International Journal of Science Education, Part B 2011, 1 (2), 165.

[141] IdeenExpo GmbH, IdeenExpo: Die Idee, https://www.ideenexpo.de/die-idee, zuletzt geprüft am 26.06.2020.

[142] M. Hill, Science Slam und die Geschichte der Kommunikation von wissenschaftlichem Wissen an außeruniversitäre Öffentlichkeiten. In: Engelschalt, J., Maibaum, A. Auf der Suche nach den Tatsachen: Proceedings der 1. Tagung des Nachwuchsnetzwerkes "INSIST" 2014.

[143] S. Z. K. Tan, J. A. U. Perucho, Science Communication 2018, 40 (6), 819.

[144] M. Beeken, M. Budke, CHEMKON 2017, 24 (5), 381.

[145] K. K. Arcand, M. Watzke, Journal of Science Communication 2010, 9 (2), 1.

[146] B. G. Koehler, L. Y. Park, L. J. Kaplan, J. Chem. Educ. 1999, 76 (11), 1505.

[147] T. Boyette, J. Ramsey, Journal of Science Communication 2019, 18 (02).

[148] K. Woods-Townsend et al., International Journal of Science Education, Part B 2016, $6(1), 89$.

[149] The Royal Society, Science Communication: Survey of Factors Affecting Science Communication by Scientists and Engineers, London 2006.

[150] Crettaz von Roten, Fabienne, Science Communication 2011, 33 (1), 52.

[151] J. C. Besley, A. Dudo, S. Yuan, F. Lawrence, Science Communication 2018, 40 (5), 559.

[152] D. R. Johnson, E. H. Ecklund, A. E. Lincoln, Science Communication 2014, 36 (1), 81.

[153] K. P. Dabney et al., International Journal of Science Education, Part B 2012, 2 (1), 63.

[154] B. Bogue, Assessment Driven Change. In: Proceedings of the 2005 American Society of Engineering Education and Annual Conference \& Exposition 2005.

[155] J. Huwer, Nachhaltigkeit und Chemie im Schülerlabor: Forschendes Experimentieren im Kontext einer naturwissenschaftlich-technischen Umweltbildung, Dissertation, Universität des Saarlandes 2015.

[156] P. Guderian, Wirksamkeitsanalyse außerschulischer Lernorte: Der Einfluss mehrmaliger Besuche eines Schülerlabors auf die Entwicklung des Interesses an Physik, Dissertation, Humbold Universität 2006. 
[157] F. Simon, Der Einfluss von Betreuung und Betreuenden auf die Wirksamkeit von Schülerlaborbesuchen: Eine Zusammenhangsanalyse von Betreuungsqualität, Betreuermerkmalen und Schülerlaborzielen sowie Replikationsstudie zur Wirksamkeit von Schülerlaborbesuchen, Dissertation, Technische Universität Dresden 2019.

[158] C. L. Thomas, J. Chem. Educ. 2012, 89 (10), 1259.

[159] A. Brandt, J. Möller, K. Kohse-Höinghaus, Zeitschrift für Pädagogische Psychologie 2008, 22 (1), 5.

[160] I. Glowinski, Schülerlabore im Themenbereich Molekularbiologie als Interesse fördernde Lernumgebung, Dissertation, Christian-Albrecht-Universität 2007.

[161] D. G. Markowitz, Journal of Science Education and Technology 2004, 13 (3), 395.

[162] C. Pawek, Schülerlabore als interessefördernde außerschulische Lernumgebungen für Schülerinnen und Schüler aus der Mittel- und Oberstufe, Dissertation, ChristianAlbrecht-Universität 2009.

[163] R. L. Penn, L. Flynn, P. Johnson, J. Chem. Educ. 2007, 84 (6), 955.

[164] B. Priemer et al., Zeitschrift für Didaktik der Naturwissenschaften 2018, 12 (7), 411.

[165] B. Priemer, C. Pawek, Out-of-school STEM learning in Germany: Can we catch and hold students' interest?, Berlin 2014.

[166] M. Streller, The Educational Effects of Pre and Post-Work in Out-of-School Laboratories, Dissertation, Technische Universität Dresden 2015.

[167] K. Neubauer, Unterstützung naturwissenschaftlicher Grundbildung durch Schulklassenbesuche in naturwissenschaftlich-technischen Museen: Motivationale und kognitive Wirkung unterschiedlicher Besuchsformen, Dissertation, Technische Universität München 2015.

[168] M. J. Raddick et al., Astronomy Education Review 2010, 9 (1).

[169] I. Ruiz-Mallén et al., Science Communication 2016, 38 (4), 523.

[170] S. Illingworth, E. Lewis, C. Percival, Journal of Science Communication 2015, 14 (02).

[171] J. Vennix, P. den Brok, R. Taconis, International Journal of Science Education 2018, 40 (11), 1263.

[172] Deutsche Forschungsgemeinschaft e.V., Sonderforschungsbereiche, https://www.dfg.de/foerderung/programme/koordinierte_programme/sfb/, zuletzt geprüft am 26.06.2020.

[173] Deutsche Forschungsgemeinschaft e.V., Liste der laufenden Sonderforschungsbereiche, https://www.dfg.de/gefoerderte_projekte/programme_und_projekte/listen/index.jsp?id=SFB, zuletzt geprüft am 26.06.2020. 
[174] Collaborative Research Center 803, Functionality Controlled by Organization in and Between Membranes: Funding Proposal 2017-2020, Göttingen 2017.

[175] E. von Hoff et al., CHEMKON 2017, 24 (4), 165.

[176] J.-C. Petit, European Review 2004, 12 (2), 191.

[177] E. von Hoff, I. Mey, T. Waitz, Making Science Visible. In: New Perspectives in Science Education: Conference Proceedings, Vol. 6, liberiauniversitaria.it 2017.

[178] J. Dege et al., Praxis der Naturwissenschaften 2016, 65 (2), 5.

[179] E. von Hoff, T. Waitz, I. Mey, Science Outreach Activities on Biomembrane Research in Formal and Non-Formal Settings. In: New Perspectives in Science Education: Conference Proceedings, Vol. 7, liberiauniversitaria.it 2018.

[180] T. Wilke, J. Dege, T. Waitz, CHEMKON 2017, 24 (4), 209.

[181] Niedersächsisches Kultusministerium, Kerncurriculum für das Gymnasium Schuljahrgänge 5-10: Naturwissenschaften, Hannover 2015.

[182] Niedersächsisches Kultusministerium, Kerncurriculum für das Gymnasium - gymnasiale Oberstufe, die Gesamtschule - gymnasiale Oberstufe, das Berufliche Gymnasium, das Abendgymnasium, das Kolleg: Biologie, Hannover 2017.

[183] Niedersächsisches Kultusministerium, Kerncurriculum für das Gymnasium - gymnasiale Oberstufe, die Gesamtschule - gymnasiale Oberstufe, das Berufliche Gymnasium, das Abendgymnasium, das Kolleg: Chemie, Hannover 2017.

[184] C. Gräsel, I. Parchmann, Unterrichtswissenschaft 2004, 32 (3), 196.

[185] B. Alberts et al., Molekularbiologie der Zelle, 6th ed., Wiley-VCH, Weinheim 2017.

[186] H. Schmidkunz, W. Rentzsch, Chemische Freihandversuche: Band 2: Kleine Versuche mit großer Wirkung, 1st ed., Vol. 2, Aulis Verlag, Köln 2011.

[187] N. B. Vargaftik, B. N. Volkov, L. d. Voljak, Journal of Physical and Chemical Reference Data 1983, 12 (3), 817.

[188] L. D. A. Chumpitaz, L. F. Coutinho, A. J. A. Meirelles, J Amer Oil Chem Soc 1999, 76 (3), 379.

[189] J. Hecker, A. Weigend, Experimente: Den Naturwissenschaften auf der Spur Der Kinder Brockhaus, Brockhaus, Gütersloh 2010.

[190] A. Marchand, J. H. Weijs, J. H. Snoeijer, B. Andreotti, American Journal of Physics 2011, 79 (10), 999.

[191] H. Schmidkunz, W. Rentsch, Chemische Freihandversuche: Band 1, Aulis, Köln 2011. 
[192] W. Glöckner, W. Jansen, R. G. Weissenhorn, Handbuch der experimentellen Chemie Sekundarbereich II: Band 10: Funktionelle Gruppen - Fette - Farbstoffe, Aulis-Verl. Deubner, Köln 2008.

[193] J.-L. Rigaud, D. Lévy, Reconstitution of Membrane Proteins into Liposomes. In: Düzgüneș, N. Liposomes, Vol. 372, Methods in Enzymology, Vol. 372, Elsevier. Amsterdam 2003.

[194] M. K. Domanska, V. Kiessling, L. K. Tamm, Biophysical journal 2010, 99 (9), 2936.

[195] J. N. Israelachvili, Intermolecular and Surface Forces, 3rd ed., Academic Press, Burlington 2011.

[196] K. Häusler, H. Rampf, R. Reichelt, Experimente für den Chemieunterricht: Mit einer Einführung in die Labortechnik, 2nd ed., Oldenbourg, München 2005.

[197] P. A. Hassan, S. Rana, G. Verma, Langmuir the ACS journal of surfaces and colloids 2015, 31 (1), 3.

[198] M. Holzer, S. Barnert, J. Momm, R. Schubert, Journal of chromatography. A 2009, $1216(31), 5838$.

[199] C. Del Bianco, D. Torino, S. S. Mansy, J. Chem. Educ. 2014, 91 (8), 1228.

[200] E. von Hoff, S. Toy, T. Waitz, I. Mey, World Journal of Chemical Education 2019, 7 (2), 185.

[201] O. Abdulhameed, A. Al-Ahmari, W. Ameen, S. H. Mian, Advances in Mechanical Engineering 2019, 11 (2), 1.

[202] J. Gardan, International Journal of Production Research 2016, 54 (10), 3118.

[203] Deutsche Bahn AG, Innovation: 3D-Druck bei der Deutschen Bahn, https://inside.bahn.de/3d-druck/ 2017, zuletzt geprüft am 26.06.2020.

[204] Y. Huang, M. C. Leu, J. Mazumder, A. Donmez, J. Manuf. Sci. Eng 2015, 137 (1).

[205] C. S. Ong et al., pr 2018, 83 (1-2), 223.

[206] N. Noor et al., Advanced science (Weinheim, Baden-Wurttemberg, Germany) 2019, 6 (11).

[207] N. Hong, G.-H. Yang, J. Lee, G. Kim, Journal of biomedical materials research. Part B, Applied biomaterials 2018, 106 (1), 444.

[208] K. van Wieren, H. N. Tailor, V. F. Scalfani, N. Merbouh, J. Chem. Educ. 2017, 94 (7), 964.

[209] P. J. Paukstelis, J. Chem. Educ. 2018, 95 (1), 169.

[210] S. Rossi et al., J. Chem. Educ. 2015, 92 (8), 1398.

[211] S. C. Meyer, J. Chem. Educ. 2015, 92 (12), 2120. 
[212] O. A. H. Jones, M. J. S. Spencer, J. Chem. Educ. 2018, 95 (1), 88.

[213] R. de Cataldo, K. M. Griffith, K. H. Fogarty, J. Chem. Educ. 2018, 95 (9), 1601.

[214] M. J. Robertson, W. L. Jorgensen, J. Chem. Educ. 2015, 92 (12), 2113.

[215] F. A. Carroll, D. N. Blauch, J. Chem. Educ. 2017, 94 (7), 886.

[216] C. Steinem, A. Janshoff, Chemie in unserer Zeit 2008, 42 (2), 116.

[217] E. Gorter, F. Grendel, Journal of Experimental Medicine 1925, 41 (4), 439.

[218] J. F. Danielli, H. Davson, J. Cell. Comp. Physiol. 1935, 5 (4), 495.

[219] S. J. Singer, G. L. Nicolson, Science (New York, N.Y.) 1972, 175 (4023), 720.

[220] O. G. Mouritsen, L. A. Bagatolli, Life - as a Matter of Fat: Lipids in a Membrane Biophysics Perspective The Frontiers Collection, Springer, Cham 2016.

[221] C. V. Boys, Soap-Bubbles: Their Colours and the Forces Which Mould Them, Society for Protoming Christian Knowledge, London 1916.

[222] T. C. Hales, Discrete Comput Geom 2001, 25 (1), 1.

[223] M. Terasaki et al., Cell 2013, 154 (2), 285.

[224] J. Guven, G. Huber, D. M. Valencia, Physical review letters 2014, 113 (18), 188101.

[225] Y. Imura, N. Choda, K. Matsuzaki, Biophysical journal 2008, 95 (12), 5757.

[226] H. Neubacher et al., Langmuir the ACS journal of surfaces and colloids 2014, 30 (16), 4767.

[227] M. L. Rotter, Journal of Hospital Infection 2001, 48, 4-8.

[228] M. Mahlapuu, J. Håkansson, L. Ringstad, C. Björn, Frontiers in Cellular and Infection Microbiology 2016, 6.

[229] H. Plattner, J. Hentschel, Zellbiologie, 5th ed., Georg Thieme Verlag, Stuttgart 2017.

[230] D. E. Sadava, D. M. Hillis, H. C. Heller, Purves Biologie, 10th ed. 2019.

[231] R. Briones et al., Biophysical journal 2016, 111 (6), 1223.

[232] J. Friedrich, M. Oetken, Praxis der Naturwissenschaften - Chemie in der Schule 2013, $62(8), 29$.

[233] A. M. Seddon et al., Chemical Society reviews 2009, 38 (9), 2509.

[234] W. Suhr, H. J. Schlichting, Antibubbles. In: Erb, R. Didaktik der Physik: Beiträge zur DPG-Frühjahrstagung 2011.

[235] M. Ducci, CHEMKON 2016, 23 (1), 14. 
[236] N. Emmerichs, Untersuchung der Wechselwirkungen von Mangan- und Calciumionen mit Alginat von Algen und von verschiedenen mucoiden Stämmen des Bakteriums Pseudomonas aeruginosa, Dissertation, Universität Duisburg-Essen 2004.

[237] R. B. Sutton, D. Fasshauer, R. Jahn, A. T. Brunger, Nature 1998, 395 (6700), 347.

[238] N. Milsch, E. von Hoff, I. Mey, T. Waitz, Zum Interesse von Jugendlichen an Science Outreach Projekten. In: Maurer, C. Qualitätsvoller Chemie- und Physikunterricht: Normative und empirische Dimensionen, Vol. 38. Regensburg 2018.

[239] A. T. Brunger, D. J. Cipriano, J. Diao, Critical reviews in biochemistry and molecular biology 2015, 50 (3), 231.

[240] S. Takamori et al., Cell 2006, 127 (4), 831.

[241] J. Blasi et al., The EMBO journal 1993, 12 (12), 4821.

[242] J. Blasi et al., Nature 1993, 365 (6442), 160.

[243] G. Schiavo et al., Nature 1992, 359 (6398), 832.

[244] Y. A. Chen, R. H. Scheller, Nature Reviews Molecular Cell Biology 2001, 2 (2), 98.

[245] R. Jahn, D. Fasshauer, Nature 2012, 490 (7419), 201.

[246] R. Jahn, R. H. Scheller, Nature reviews. Molecular cell biology 2006, 7 (9), 631.

[247] H. J. Risselada, G. Bubnis, H. Grubmüller, Proceedings of the National Academy of Sciences of the United States of America 2014, 111 (30), 11043.

[248] K. Sommer, M. Klein, H. Steff, P. Pfeifer, Naturwissenschaften im Unterricht. Chemie 2012, 23 (132), 2.

[249] Niedersächsisches Kultusministerium, Kerncurriculum für das Gymnasium - gymnasiale Oberstufe, die Gesamtschule - gymnasiale Oberstufe, das Berufliche Gymnasium, das Abendgymnasium, das Kolleg: Physik, Hannover 2017.

[250] E. Abbe, Archivf. mikrosk. Anatomie 1873, 9 (1), 413.

[251] S. Schmitz, C. Desel, Der Experimentator Zellbiologie Experimentator, Springer Berlin Heidelberg, Berlin, Heidelberg 2018.

[252] T. A. Klar et al., Proceedings of the National Academy of Sciences of the United States of America 2000, 97 (15), 8206.

[253] F. Balzarotti et al., Science (New York, N.Y.) 2017, 355 (6325), 606.

[254] P. W. Atkins, J. de Paula, Physikalische Chemie, 4th ed., Wiley-VCH, Weinheim 2012.

[255] D. Wöhrle, M. Tausch, W.-D. Stohrer, Photochemie: Konzepte, Methoden, Experimente, Wiley-VCH, Weinheim 1998.

[256] A. Boguta, D. Wróbel, Journal of Fluorescence 2001, 11 (2), 129. 
[257] J. A. Campbell, J. Chem. Educ. 1963, 40 (11), 578.

[258] R. Christie, Colour Chemistry, Royal Society of Chemistry, Cambridge 2007.

[259] E. Gerdes, Qualitative anorganische Analyse: Ein Begleiter für Theorie und Praxis, 2nd ed., Springer, Berlin 2001.

[260] S. Höfener et al., Phys. Chem. Chem. Phys. 2013, 15 (30), 12572.

[261] A. A. Jamali, A. Tavakoli, J. Ezzati Nazhad Dolatabadi, Eur Food Res Technol 2012, $235(3), 367$.

[262] O. Oter, S. Aydogdu, Journal of Fluorescence 2011, 21 (1), 43.

[263] A. S. Roy et al., Mol. BioSyst. 2016, 12 (9), 2818.

[264] P. C. Hollman, J. M. van Trijp, M. N. Buysman, Analytical chemistry 1996, 68 (19), 3511.

[265] P. Lanfermann et al., CHEMKON, (angenommen).

[266] H. D. Hardt, A. Pierre, Z. Anorg. Allg. Chem. 1973, 402 (1), 107.

[267] P. C. Ford, A. Vogler, Acc. Chem. Res. 1993, 26 (4), 220.

[268] C. Salter, K. Range, G. Salter, J. Chem. Educ. 1999, 76 (1), 84.

[269] J. H. Gutow, J. Chem. Educ. 2005, 82 (2), 302.

[270] T. Wang, E. A. Smith, E. R. Chapman, J. C. Weisshaar, Biophysical journal 2009, 96 (10), 4122.

[271] X. Zhuang et al., Proceedings of the National Academy of Sciences of the United States of America 2000, 97 (26), 14241.

[272] R. F. Chen, J. R. Knutson, Analytical biochemistry 1988, 172 (1), 61.

[273] T. Förster, Naturwissenschaften 1946, 33 (6), 166.

[274] R. M. Clegg, Förster Resonance Energy Transfer. In: Gadella, T. Fret and Flim Techniques, Vol. 33, Laboratory Techniques in Biochemistry and Molecular Biology, Elsevier 2009.

[275] C. Berney, G. Danuser, Biophysical journal 2003, 84 (6), 3992.

[276] B. N. G. Giepmans, S. R. Adams, M. H. Ellisman, R. Y. Tsien, Science (New York, N.Y.) 2006, 312 (5771), 217.

[277] M. Şener et al., Chemphyschem a European journal of chemical physics and physical chemistry 2011, 12 (3), 518.

[278] B. Hochreiter, M. Kunze, B. Moser, J. A. Schmid, Sci Rep, 9 (1), 1. 
[279] J. Otterstrom, A. M. van Oijen, Biochemistry 2013, 52 (10), 1654.

[280] I. Mey, Praxis der Naturwissenschaften 2016, 65 (2), 10.

[281] H. Stein et al., Frontiers in physiology 2017, 8, 63.

[282] L. Qu, Y. Akbergenova, Y. Hu, T. Schikorski, The Journal of comparative neurology 2009, 514 (4), 343.

[283] K. Akashi, H. Miyata, H. Itoh, K. Kinosita, Biophysical journal 1996, 71 (6), 3242.

[284] D. S. Dimitrov, M. I. Angelova, Lipid Swelling and Liposome Formation on Solid Surfaces in External Electric Fields. In: Hoffmann, H. New Trends in Colloid Science, Vol. 73, Progress in Colloid \& Polymer Science, Vol. 73, Steinkopff. Darmstadt 1987.

[285] L. L. G. Schwenen et al., Scientific reports 2015, 5, 1.

[286] N. Teske et al., Langmuir the ACS journal of surfaces and colloids 2017, 33 (49), 14175.

[287] J. W. Kuhlmann, M. Junius, U. Diederichsen, C. Steinem, Biophysical journal 2017, 112 (11), 2348.

[288] M. Kocun, T. D. Lazzara, C. Steinem, A. Janshoff, Langmuir the ACS journal of surfaces and colloids 2011, 27 (12), 7672.

[289] L. E. Palacios, T. Wang, Journal of the American Oil Chemists' Society 2005, 82 (8), 565.

[290] R. W. Burry, Immunocytochemistry: A Practical Guide for Biomedical Research, Springer New York, New York, NY 2010.

[291] J. Schindelin et al., Nature methods 2012, 9 (7), 676.

[292] J. Engelhardt, W. Knebel, Phys. Unserer Zeit 1993, 24 (2), 70.

[293] A. Oshima, K. Sumitomo, Biochemistry and Biophysics Reports 2017, 11, 58.

[294] O. Biner, T. Schick, Y. Müller, C. von Ballmoos, FEBS Letters 2016, 590 (14), 2051.

[295] D. M. Bailer-Jones, Naturwissenschaftliche Modelle. In: Beckermann, A., Nimtz, C. Argument \& Analyse - Sektionsvorträge: Ausgewählte Sektionsvorträge des 4. Internationalen Kongresses der Gesellschaft für Analytische Philosophie, mentis. Paderborn 2002.

[296] A. Meisert, Zeitschrift für Didaktik der Naturwissenschaften 2008, 14 (243-261).

[297] Sekretariat der Ständigen Konferenz der Kultusminister der Länder in der Bundesrepublik Deutschland, Beschlüsse der Kultusministerkonferenz: Bildungsstandards im Fach Biologie für den Mittleren Schulabschluss, München 2004. 
[298] Sekretariat der Ständigen Konferenz der Kultusminister der Länder in der Bundesrepublik Deutschland, Beschlüsse der Kultusministerkonferenz: Bildungsstandards im Fach Physik für den Mittleren Schulabschluss, München 2004.

[299] A. G. Harrison, D. F. Treagust, International Journal of Science Education 2000, 22 (9), 1011.

[300] D. F. Treagust, G. Chittleborough, T. L. Mamiala, International Journal of Science Education 2002, 24 (4), 357.

[301] D. Krüger, A. Kauertz, A. Upmeier zu Belzen, Modelle und das Modellieren in den Naturwissenschaften. In: Krüger, D., Parchmann, I., Schecker, H. Theorien in der naturwissenschaftsdidaktischen Forschung, Springer Berlin Heidelberg. Berlin, Heidelberg 2018.

[302] A. Beerenwinkel, I. Parchmann, Praxis der Naturwissenschaften ChiS 2008, 57 (4), 13.

[303] d. Axelrod et al., Biophysical journal 1976, 16 (9), 1055.

[304] E. Neher, B. Sakmann, Nature 1976, 260 (5554), 799.

[305] C. Helfferich, Leitfaden- und Experteninterviews. In: Baur, N., Blasius, J. Handbuch Methoden der empirischen Sozialforschung, Springer Fachmedien Wiesbaden. Wiesbaden 2014.

[306] L. M. Groesz, M. P. Levine, S. K. Murnen, The International journal of eating disorders 2002, 31 (1), 1.

[307] M. Götz, Televizion Digital, 2019 (1).

[308] G. Holland, M. Tiggemann, Body Image 2016, 17, 100.

[309] C. E. Walker, E. G. Krumhuber, S. Dayan, A. Furnham, Current Psychology 2019.

[310] B. Frees, W. Koch, ARD/ZDF-Onlinestudie 2018: Zuwachs bei medialer Internetnutzung und Kommunikation 2018.

[311] Deutsche Gesellschaft für Ästhetisch-Plastische Chirurgie (DGÄPC), DGÄPC-Statistik 2018: Zahlen, Fakten und Trends der Ästhetisch-Plastischen Chirurgie 2018.

[312] Deutsche Gesellschaft für Ästhetisch-Plastische Chirurgie (DGÄPC), Umfrage: Motiviert der Selfie-Boom immer häufiger zu Schönheitsoperationen? 2019.

[313] 0. Rossetto, M. Pirazzini, C. Montecucco, Nature reviews. Microbiology 2014, 12 (8), 535.

[314] E. van Ermengen, Über einen neuen anaeroben Bacillus und seine Beziehungen zum Botulismus, Veit 1897.

[315] F. Chen, G. M. Kuziemko, R. C. Stevens, Infection and immunity 1998, 66 (6), 2420.

[316] C. N. Homann et al., Nervenarzt 2002, 73 (6), 519. 
[317] F. Erbguth, Akt Neurol 2015, 42 (02), 105.

[318] I. Lanzl, P. Roggenkämper, R.-L. Merté, Klin Monatsbl Augenheilkd 2014, 231 (07), 749.

[319] K. Taylor, C. Gericke, L. R. Alvarez, ALTEX 2019, 36 (1), 81.

[320] S. Fokken, Spiegel Online 2018.

[321] Bundesministerium der Justiz und für Verbraucherschutz, Ausführungsgesetz zu Artikel 26 Abs. 2 des Grundgesetzes (Gesetz über die Kontrolle von Kriegswaffen) Anlage (zu \&1 Abs. 1)Kriegswaffenliste: KrWaffKontrG 1990.

[322] F. S. Gutzwiller et al., Deutsche medizinische Wochenschrift (1946) 2008, 133 (16), 840.

[323] L. M. Wein, Y. Liu, Proceedings of the National Academy of Sciences of the United States of America 2005, 102 (28), 9984.

[324] R. L. Shapiro, JAMA 1997, 278 (5), 433.

[325] J. Holbrook, M. Rannikmae, International Journal of Science Education 2007, 29 (11), 1347.

[326] I. Eilks, Plädoyer für eine konsequentere gesellschaftliche Orientierung des Chemieund Physikunterrichts. In: Höttecke, D. Naturwissenschaftliche Bildung als Beitrag zur Gestaltung partizipativer Demokratie, Vol. 31, LIT-Verlag. Münster 2011.

[327] E. von Hoff et al., CHEMKON 2019, 26 (5), 190.

[328] U. Kessels, B. Hannover, Zeitschrift für Entwicklungspsychologie und Pädagogische Psychologie 2004, 36 (3), 130.

[329] U. Gebhard, D. Höttecke, M. Rehm, Pädagogik der Naturwissenschaften: Ein Studienbuch Lehrbuch, Springer VS, Wiesbaden 2017.

[330] W. Wentorf, T. N. Höffler, I. Parchmann, CHEMKON 2017, 24 (3), 111.

[331] N. G. Lederman, Nature of Science: Past, Present, and Future. In: Handbook of Research on Science Education.

[332] D. Urhahne, K. Kremer, J. Mayer, Unterrichtswissenschaft 2008, 36, 71.

[333] W. F. McComas, J. K. Olson, The Nature of Science in International Science Education Standards Documents. In: McComas (Hg.) 1998 - The Nature of Science.

[334] J. Osborne et al., J Res Sci Teach 2003, 40 (7), 692.

[335] M. Werner, K. Kremer, Erkenntnisweg Biologiedidaktik 2010, 9, 135.

[336] W. Wentorf, T. N. Höffler, I. Parchmann, Zeitschrift für Didaktik der Naturwissenschaften 2015, 21 (1), 207. 
[337] J. L. Holland, Making Vocational Choices: A Theory of Vocational Personalities and Work Environments, 3rd ed., Psychological Assessment Resources, Lutz, Fla. 1997.

[338] C. Tenopir, D. W. King, Communication Patterns of Engineers, IEEE Press [u.a.], Piscataway NJ 2004.

[339] L. M. Phillips, S. P. Norris, Res Sci Educ 2009, 39 (3), 313.

[340] A. Beerenwinkel, C. Gräsel, Zeitschrift für Didaktik der Naturwissenschaften 2005, 11, 21-39.

[341] G. Merzyn, Physikschulbücher, Physiklehrer und Physikunterricht: Beiträge auf der Grundlage einer Befragung westdeutscher Physiklehrer IPN, Vol. 139, IPN, Kiel 1994.

[342] D. Höttecke, Zeitschrift für Didaktik der Naturwissenschaften 2001, 7, 7.

[343] A. Yarden, G. Brill, H. Falk, Journal of Biological Education 2001, 35 (4), 190.

[344] S. P. Norris, L. M. Phillips, Sci. Ed. 2003, 87 (2), 224.

[345] A. Yarden, S. P. Norris, L. M. Phillips, Adapted Primary Literature: The Use of Authentic Scientific Texts in Secondary Schools Innovations in science education and technology, Springer, Heidelberg, New York, London 2015.

[346] J. Osborne, Res Sci Educ 2009, 39 (3), 397.

[347] K. Penney, S. P. Norris, L. M. Phillips, G. Clark, Canadian Journal of Science, Mathematics and Technology Education 2003, 3 (4), 415.

[348] G. A. Myers, English for Specific Purposes 1992, 11 (1), 3.

[349] P. Scheller-Brüninghaus, C. Schmidt, Bildung und Erziehung 2011, 64 (1), 53.

[350] F. Suppe, Philosophy of Science 1998, 65 (3), 381.

[351] H. Falk, A. Yarden, Res Sci Educ 2009, 39 (3), 349.

[352] D. J. Ford, Res Sci Educ 2009, 39 (3), 385.

[353] H. Falk, A. Yarden, Journal of Biological Education 2011, 45 (2), 77.

[354] A. Baram-Tsabari, A. Yarden, J Res Sci Teach 2005, 42 (4), 403.

[355] L. J. Patalag et al., Chembiochem 2017, 18 (21), 2171.

[356] S. P. Norris, N. Stelnicki, G. de Vries, Res Sci Educ 2012, 42 (4), 633.

[357] P. Mayring, Qualitative Sozialforschung, Beltz Verlagsgruppe, s.l. 2002.

[358] G. S. Aikenhead, Sci. Ed. 1987, 71 (4), 459.

[359] K. Marniok, C. S. Reiners, CHEMKON 2016, 23 (2), 65. 
[360] S. G. Hoskins, L. M. Stevens, R. H. Nehm, Genetics 2007, 176 (3), 1381.

[361] S. G. Hoskins, D. Lopatto, L. M. Stevens, CBE life sciences education 2011, 10 (4), 368.

[362] Arbeitsgruppe des Niedersächsischen Kultusministerium, Das Seminarfach: Hinweise und Empfehlungen für Schulen Schulverwaltungsblatt 2006.

[363] D. Braunert, 395.000 Besucher auf der IdeenExpo 2019: Schmidt: "Die Erfolgsstory wird fortgesetzt.", https://www.ideenexpo.de/sites/default/fi-

les/20190624_IdeenExpo_Bilanz_PM_final.pdf, zuletzt geprüft am 26.06.2020.

[364] Hannover Live 2019, 23, 6.

[365] Bundesministerium für Bildung und Forschung (BMBF), Mit MINT in die Zukunft!: Der MINT-Aktionsplan des BMBF 2019.

[366] A. Carl, Bericht über die "Highlights der Physik 2019 - Zeig Dich!" vom 16.09. 21.09.2019 in Bonn, Dinslagen 2019.

[367] Deutsche Physikalische Gesellschaft, Highlights der Physik, https://www.dpg-physik.de/aktivitaeten-und-programme/wissenschaftsfestivals-shows/hdp, zuletzt geprüft am 26.06.2020.

[368] L. Kampschulte, I. Parchmann, LUMAT 2015, 3 (4), 462.

[369] L. Kampschulte, S. Schwarzer, EXPOneer Austellungshandbuch: Version V1.1b, Kiel 2015.

[370] A. Schulz, Experimentierspezifische Qualitätsmerkmale im Chemieunterricht: Eine Videostudie Studien zum Physik- und Chemielernen, Vol. 113, Logos-Verl., Berlin 2011.

[371] U. Kelle, Mixed Methods. In: Baur, N., Blasius, J. Handbuch Methoden der empirischen Sozialforschung, Springer Fachmedien Wiesbaden. Wiesbaden 2014.

[372] LimeSurvey GmbH, Professionelle Online-Umfragen mit LimeSurvey, https://www.limesurvey.org/de/, zuletzt geprüft am 26.06.2020.

[373] R. Porst, Fragebogen, Springer Fachmedien Wiesbaden, Wiesbaden 2014.

[374] M. Weichbold, Pretest. In: Baur, N., Blasius, J. Handbuch Methoden der empirischen Sozialforschung, Springer Fachmedien Wiesbaden. Wiesbaden 2014.

[375] E. von Hoff, N. Milsch, T. Waitz, I. Mey, Interdisziplinäre Projekte zur Öffentlichkeitsarbeit im SFB 803. In: Maurer, C. Qualitätsvoller Chemie- und Physikunterricht: Normative und empirische Dimensionen, Vol. 38. Regensburg 2018.

[376] H. Kromrey, Empirische Sozialforschung, 10th ed., Leske + Budrich, Opladen 2002.

[377] M. Häder, Empirische Sozialforschung: Eine Einführung, 3rd ed., Springer VS, Wiesbaden 2015. 
[378] M.-D. Weitze, Nachr. Chem. 2007, 55 (2), 140.

[379] T. H. C. Kruger, M. A. Wollmer, Toxicon 2015, 107, 154.

[380] Ministerium für Schule und Weiterbildung des Landes Nordrhein-Westfalen, Kernlehrplan für die Sekundarstufe II Gymnasium/Gesamtschule in Nordrhein-Westfalen: Physik, Düsseldorf 2014.

[381] H. Aljets, L. Leibold, Mey Ingo, Waitz Thomas, What Do Legionella, EHEC and Botox Have in Common? In: New Perspectives in Science Education: Conference Proceedings, Vol. 9, liberiauniversitaria.it 2020.

[382] MBExC, Multiscale Bioimaging: Cluster of Excellence, https://mbexc.de/, zuletzt geprüft am 26.06.2020. 


\section{Abbildungsverzeichnis}

Abbildung 1: Wie gut fühlen sich europäische Bürger über Entwicklungen in Wissenschaft und Technologie informiert (aus dem Englischen übersetzt nach EUROPEAN COMMISSION [17]).

Abbildung 2: Rahmenmodell der Interessengenese nach KRAPP [58]. 8

Abbildung 3: Länderspezifische Verteilung der Antworten von Jungen und Mädchen bezügliche der Frage „Ich mag naturwissenschaftlichen Unterricht mehr als die meisten anderen Fächer" (aus dem Englischen übersetzt nach SJøBERG \& SCHREINER [76])................. 11

Abbildung 4: Modell der drei Dimension der Relevanz nach STUCKEY ET AL. [79].................... 12

Abbildung 5: Modell zur Operationalisierung von Kontexten nach VAN VORST ET AL. [89].. 13

Abbildung 6: Häufigkeit des Wortes „Forschung“ in deutschen Chemie-Kerncurricula der Sekundarstufe II.

Abbildung 7: Anzahl der in Google Scholar gelisteten Publikationen mit dem zusammenhängenden Ausdruck „Science Outreach“ seit 1990.

Abbildung 8: Verteilung der auf der Website LELA gelisteten Schülerlabore in Deutschland sowie im deutschsprachigen Raum [126]

Abbildung 9: Der INNOTRUCK des BMBF am 22.05.2019 in Pankow, Berlin, beim 12. Ausbildungstag Pankow \& Lichtenberg.

Abbildung 10: Schematische Darstellung der Interaktion von Peptiden mit Membranen (A). Weiterhin sind die Anreicherung und Insertion in die Membran (B), die Aggregation (C) und schließlich die Bildung von Kanälen (D) für den Transport von Stoffen über die Membran abgebildet [174] 33

Abbildung 11: Schematische Darstellung der SNARE-vermittelten Fusion eines Vesikels mit einer Lipidmembran. Der im Vesikel eingeschlossene Inhalt kann nach Fusion der Membranen ausgeschüttet werden [174]

Abbildung 12: Modell der Fachdidaktischen Transferforschung in Bezug auf Science Outreach adaptiert nach WILKE, DEGE \& WAITZ [180].

Abbildung 13: Skelettformel eines Phospholipids (A, Phosphatidylcholin, hier POPC) sowie eines Tensids (B, Stearinsäure) mit farblicher Unterlegung der hydrophoben Ketten (gelb) sowie der hydrophilen Kopfgruppen (blau). 39

Abbildung 14: Vor Zugabe des Spülmittels zum Wasser verbleibt das angefärbte Öl im enghalsigen Gefäß (A). Nach der Zugabe des Tensids steigt das Öl aus dem Gefäß heraus an die Wasseroberfläche (B-D).

Abbildung 15: Wassertropfen (A) sowie Tropfen einer Tensidlösung (B) auf einer 50-Cent Münze.

Abbildung 16: Eine Büroklammer liegt aufgrund der Oberflächenspannung trotz größerer Dichte auf der Oberfläche des Wassers auf.

Abbildung 17: Schematische Darstellung der anziehenden und abstoßenden Kräfte die auf Wassermoleküle an der Oberfläche und im Inneren der Flüssigkeit wirken. (A) vor, (B) nach Zugabe von Tensidmolekülen.

Abbildung 18: Ein Wassertropfen (A) sowie ein Tropfen einer Spülmittellösungen auf einem Baumwolltuch (B)... 
Abbildung 19: Ein Wollfaden bleibt auf einer Wasseroberfläche liegen (A), während er von einer Tensidlösung benetzt wird und langsam zu Boden sinkt (B).

Abbildung 20: Schematische Darstellung der Benetzung einer hydrophoben (A) sowie einer hydrophilen Oberfläche (B) durch einen Wassertropfen.

Abbildung 21: Die Benetzbarkeit einer rauen hydrophilen Oberfläche kann durch Lufteinschlüsse (A) herabgesetzt werden. Die Zugabe von Tensiden führt hingegen zu einer Erhöhung der Benetzbarkeit (B). 44

Abbildung 22: Filtrat nach Zugabe von Wasser (A) und nach Zugabe einer Tensidlösung (B), sowie schematische Darstellung des Filtrationsprozesses auf Teilchenebene (C, D)

Abbildung 23: Wasser-Öl Gemisch vor dem Schütteln (A) bzw. nach dem Schütteln (B, C), Das Reagenzglas in (C) enthält zusätzlich Spülmittel. (D, E) Schematische Darstellung der Emulsion von Öl in Wasser. 46

Abbildung 24: Änderung der Oberflächenspannung in Abhängigkeit der Tensidkonzentration. 48

Abbildung 25: Lipidgeometrien in Abhängigkeit von ihrem Packungsparameter nach ISRAELACHVILI [195].

Abbildung 26: Schematische Darstellung einer Ölschicht auf der Wasseroberfläche auf makroskopischer und submikroskopischer Ebene vor (A) und nach der Zugabe eines Tensids (B). 50

Abbildung 27: Angefärbter Wassertropfen auf der hydrophoben (A) bzw. hydrophilen Oberfläche der Stearinsäure (B). (C) Strukturformel der Stearinsäure, (D) Deutung auf Teilchenebene.

Abbildung 28: (A) Lichtstreuung in einer Tensidlösung (links). In einer Natriumchloridlösung (rechts) kann keine Lichtstreuung beobachtet werden. (B) Schematische Darstellung der Streuung des Lichts an Mizellen.

Abbildung 29: Größenausschluss-Chromatographiesäulen (Sephadex 25) unter UV-Licht von A) Pyraninlösung + Spülmittel, B) Pyraninlösung + Lecithin, C), Pyraninlösung + Ölsäure (pH = 8) und D) Pyraninlösung + Ölsäure (pH = 11) [200]

Abbildung 30: Schematische Darstellung der Auftrennung der Moleküle in den jeweiligen Säulen. (A) Lösungen A und D, (B) Lösungen B und C [200].

Abbildung 31: (A, von links nach rechts) Typisches Sulfonat-Tensid, Phosphocholin-Lipid, Ölsäuredimer bei $\mathrm{pH}=8$, deprotoniertes Ölsäuremonomer bei $\mathrm{pH}=11$. (B) Darstellung eines Tensids mit konischer Geometrie das Mizellen bildet. (C) Darstellung eines zylindrischen Lipids, das Vesikel bildet [200]. 55

Abbildung 32: 3D-gedruckte Tensid- bzw. Lipidmodelle mit zylindrischer (A) und konischer Geometrie (B).

Abbildung 33: Typische Darstellung einer Membran nach dem Flüssig-Mosaik-Modell bestehend aus Lipiden (blau-gelb), Cholesterinen (blau-rot) sowie Transmembranproteinen (braun), die durch die Membran durchreichen und Kanäle bilden können, und peripheren Proteinen (grün), die nur an einen Lipidmonolayer assoziiert sind. In orange sind Polysaccharide dargestellt, die sowohl an Lipide als auch Proteine gebunden sein können. 59 
Abbildung 35: Strukturformeln typischer Membranlipide sowie von Cholesterin. Die Glycerin- (blau) und Sphingosinbestandteile (rot) sind farbig hervorgehoben................... 59

Abbildung 36: Seifenhäute in 3D-gedruckten geometrischen Formen.

Abbildung 37: Vergleich der submikroskopischen Struktur von Seifenblasen (A) mit derer von Lipiddoppelschichten (B).

Abbildung 38: (A, B) Präparation von Zwiebelhäuten. (C, D) Lösungen entsprechend der Tabelle, links Proben mit Zwiebelhäuten, rechts Vergleichslösungen. 62

Abbildung 39: Durchlichtmikroskopische Aufnahmen einer Zwiebelhaut vor (A) bzw. nach der Behandlung mit Magainin 2 (B)..... 64

Abbildung 40: Schematische Darstellung verschiedener Membrantransportarten. (A) einfache Diffusion, (B) schnelle Diffusion durch ein Kanalprotein, (C) carriervermittelter passiver Transport, (D) carriervermittelter aktiver Transport, (E) Exocytose, (F) Endocytose.

Abbildung 41: (A) Schematische Darstellung der Anregung zur Fluoreszenz auf makroskopischer Ebene. (B, C) Submikroskopische Darstellung der Diffusion der Pyraninmoleküle durch den Dialyseschlauch. 68

Abbildung 42: Strukturformeln der Moleküle von dextrangebundenem Texas Red sowie Pyranin. Die Glucoseeinheiten können $\alpha$-1,4- oder $\alpha$-1,6-glycosidisch verbunden sein, so dass verzweigte Dextrannetzwerke entstehen.

Abbildung 43: (A, B) Die Phenolphthaleinlösung außerhalb des Dialyseschlauch entfärbt sich. (C, D) Schematische Darstellung der Diffusion von Protonen durch den Dialyseschlauch, die zur Protonierung und dadurch Entfärbung des Indikatorfarbstoffs führt. 70

Abbildung 44: (A) Versuchsaufbau für das Modellexperiment zur erleichterten carriervermittelten Diffusion. (B) Schematische Darstellung der ablaufenden Prozesse auf Teilchenebene nach FRIEDRICH \& OETKEN [232]. 71

Abbildung 45: Schematische Darstellung verschiedener carriervermittelter Membrantransportmechanismen. (A) Uniport, (B) Symport, (C) Antiport (passiv).......... 72 Abbildung 46: (A-C) Eine farbstoffgefüllte Antiblase sinkt durch die zwei oberen Lösungen mit geringerer Dichte, verbleibt kurz an der Grenzschicht zur untersten Lösung und gibt beim Platzen den eingeschlossen Farbstoff in die unterste Phase ab. (D-F) Schematische Darstellung des Modellexperiments. 73

Abbildung 47: (A-C) Eine farbstoffgefüllte Natriumalginatblase sinkt durch die zwei oberen Lösungen mit geringerer Dichte und verbleibt auch nach längerer Zeit an der Grenzschicht zur untersten Lösung derselben Dichte. (D) Strukturformel von Alginat, (E) schematische Darstellung des Alginatkomplexes [235].

Abbildung 48: Vergleich des Modellexperiments zur Exocytose (A) mit der schematische Darstellung der Ausschüttung von Neurotransmittern durch Exocytose am synaptischen Spalt (B).

Abbildung 49: Schematische Darstellung der am Fusionsprozess beteiligten Proteine SNAP-25, Synaptobrevin und Syntaxin samt der Angriffspunkte für das Tetanus- und verschiedene Botulinumtoxine. 76

Abbildung 50: Modell des coiled-coil SNARE-Komplexes [237]. Markiert sind die Angriffspunkte für verschiedene Tetanus- (TeNT) und Botulinumtoxine (BoNT). 
Abbildung 51: Schematische Darstellung der SNARE-vermittelten Membranfusion nach JAHN \& SCHELLER [246]. (A) Ausbildung von SNARE-Komplexen, (B) Hemifusion (Fusion Stalk), (C) Hemifusion (Transmembrankontakt), (D) Öffnung der Fusionspore, (E) Weitung der Fusionspore.

Abbildung 52: (A) Mikroskopische Aufnahmen des Sprossquerschnitts eines Maiglöckchens (Convallaria majalis), (B) Eigenfluoreszenz nach Anregung mit UV-Licht.

Abbildung 53: Schematische Darstellung der Anregung zur Fluoreszenz in einem vereinfachten JABŁOŃSKI-Diagramm. 80

Abbildung 54: Strukturformeln von Phenolphthalein (links, $\mathrm{pH}>8,2$ ) und Fluorescein (rechts)...

Abbildung 55: Die nach dem Schütteln blaue Lösung (A) liegt nach kurzer Zeit wieder entfärbt vor $(B, C)$.

Abbildung 56: Reaktionsschema der reversiblen Reaktion von Leukomethylenblau (links, farblos) zu Methylenblau (rechts, blau).

Abbildung 57: (A) Mit größer werdenden konjugierten $\pi$-System nimmt die absorbierte Wellenlänge zu. (B) $\beta$-Carotin besitzt ein konjugiertes $\pi$-System mit 11 Doppelbindungen, das für eine orangene Farbwahrnehmung verantwortlich ist.

Abbildung 58: (A) Gelbe Färbung der Lösung bei Tageslicht, (B) blau-grüne Fluoreszenz unter UV-Licht..

Abbildung 59: (A) Das Morinmolekül ist zunächst sterisch nicht gehindert, so dass eine Abregung über Schwingungsrelaxation möglich ist. (B) Bzgl. der Chelatierung mit Aluminiumionen werden verschiedene Bindungsstellen vorgeschlagen. Die rot markierten Bindungsstellen verhindern eine Rotation und verringern die Schwingungszustände, so dass eine Abregung mittels Fluoreszenzemission erfolgt........ 83

Abbildung 60: Trispyridinkomplexes unter UV-Licht bei Raumtemperatur (A), sowie unter Kühlung in flüssigem Stickstoff (B). (C) Vorgeschlagene Komplexstruktur des Trispiridinkomplexes [265].

Abbildung 61: (A) Energiediagramm für die möglichen Potentialverläufe der angeregten Zustände ${ }^{3}$ CC und ${ }^{3}$ XLCT des Trispyridinkupfer(I)-iodid-Komplexes. (B, C) Darstellung der Anregung in korrespondierende Jabłoński-Diagrammen [265]. 85

Abbildung 62: Beobachtete Fluoreszenz verschiedenfarbig fluoreszierender Lösungen nach Anregung mit Laserstrahlen verschiedener Wellenlängen. 86

Abbildung 63: Elektromagnetisches Spektrum des sichtbaren Lichts. Links an das Spektrum schließt sich der Bereich der ultravioletten Strahlung an (UV), rechts des Spektrums folgt der Bereich der Infrarotstrahlung (IR)..

Abbildung 64: (A) Das Emissionsspektrum ist im Vergleich zum Absorptionsspektrum deutlich rotverschoben. (B) Aufgrund des STOKES-Shift reichen die Energien grüner bzw. roter Wellenlängenbereiche nicht aus, um eine grün fluoreszierende Substanz zur Fluoreszenz anzuregen.

Abbildung 65: (A) Verschiedenfarbige Knicklichter im ungeknickten Zustand unter UVLicht. (B) Schematische Darstellung der Funktion von Knicklichtern. 88

Abbildung 66: Allgemeiner Reaktionsmechanismus für die Reaktion eines Oxalsäureesters mit Wasserstoffperoxid in einem Knicklicht. 88 
Abbildung 67: Fluoreszenz in vormals farbigen Textmarkerlösungen nach mehrmaliger Verdünnung.

Abbildung 68: Chininhaltige Lösung unter UV-Licht vor (A) und nach Zugabe von Natriumchlorid (B). 90

Abbildung 69: Pyraninlösung unter UV-Licht bei sukzessiver Zugabe von Pyraninkristallen.

Abbildung 70: (A) JABŁOŃSKI-Diagramm für die strahlungslose Energieübertagung auf einen Stoßpartner mit unterschiedlichem Energieprofil. (B) Veranschaulichung einer möglichen Erklärung für das Phänomen des Self-quenching-Effekts.

Abbildung 71: Beobachtete Fluoreszenz bei Anregung einer grün fluoreszierenden (links), einer gelb fluoreszierenden (Mitte) und einer Mischung aus den ersten beiden Lösungen (rechts) mit einem violetten und einem grünen Laserstrahl. 92

Abbildung 72: Das Emissionsspektrum des ersten Farbstoffs $\left(E_{1}\right)$ überlappt mit dem Absorptionsspektrum des zweiten Farbstoffs $\left(\mathrm{A}_{2}\right)$. Dies ermöglicht eine Energieübertragung vom ersten auf den zweiten Farbstoff, so dass es bei der Farbstoffmischung bei Anregung mit beiden Lasern zu einer gelben Fluoreszenz kommt.

Abbildung 73: Darstellung des FRET-Effektes im JABŁOŃSKI-Diagramm. (A) der grün fluoreszierende Farbstoff kann nur mithilfe des violetten Lasers zur Fluoreszenz angeregt werden. (B) Der gelb fluoreszierende Farbstoff kann von beiden Lasern zu Fluoreszenz angeregt werden. (C) Bei Anregung der Farbmischung mit einem violetten Laser kommt es zur Energieübertragung auf das zweite Fluorophor, so dass eine Emission gelben Lichts folgt. 94

Abbildung 74: Emissionsspektren der Farbstoffmischungen nach Anregung mit einem violetten Laser nach HOCHREITER ET AL. [278]. $E_{1}$ und $E_{2}$ sind theoretische Emissionsspektren für die einzelnen Farbstoffe. $\mathrm{E}_{3}$ verdeutlicht den FRET-Effekt. Das Integral von $\mathrm{E}_{3}$ entspricht dem von $\mathrm{E}_{1}$, der Peak bei $\mathrm{E}_{1}$ ist jedoch abgeschwächt, dafür bei $\mathrm{E}_{2}$ deutlich höher. 94

Abbildung 75: (A) freistehende Membran, (B) festkörperunterstützte Membran, (C) schwarze Membran, (D) porenüberspannende Membran (nach STEIN ET AL. [281]). ............ 96

Abbildung 76: Eigelb wird zunächst mit Ethanol homogenisiert (A). Nach Zugabe von nHeptan wird abfiltriert (B) und das Lösemittel eingedampft (C). Die entstandene Emulsion (D) nach dreimaligen Waschen mit Aceton (E)... 97

Abbildung 77: (A) Durchlichtmikroskopische Aufnahmen von Vesikeln. (B) Fluoreszenzmikroskopische Aufnahmen Texas Red-markierter Vesikel..

Abbildung 78: Mikroskopische Aufnahmen eines farbstoffgefüllten Vesikels vor (A) und nach Zugabe von Salzsäurelösung (B). (C) und (D) zeigen korrespondierende schematische Darstellungen des Versuchs auf Teilchenebene.

Abbildung 79: Schematische Darstellung der Vesikelpräparation durch Quellen nach STEIN ET AL. [281]. (A) Lipiddoppelschichten nach Verblasen des Lösungsmittels, (B) Zugabe einer Pufferlösung, (C) Aufquellen der Lipidschichten zu Vesikeln. . .100

Abbildung 80: Schematische Darstellung der Vesikelpräparation in einer Elektroformationskammer nach STEIN ET AL. [281]). .100 
Abbildung 81: Vergleich fluoreszenzmarkierter Pflanzenbestandteile bei Tageslicht (oben) bzw. unter UV-Licht (unten). In (D) und (E) ist das Leitgewebe anhand der Fluoreszenz deutlich zu erkennen. In (F) fluoreszieren hydrophile Trichome. .101

Abbildung 82: Konfokalmikroskopische Aufnahmen von Fibroblasten. In (A) wurden die Aufnahmen des rot markierten Aktin-Zytoskeletts (B), der grün markierten Membran (C) und des blau markierten Zellkerns (D) überlagert. .103

Abbildung 83: (A) Konfokal-Fluoreszenzmikroskop (CLSM; FluoView1200; Olympus, Tokyo, Japan), Hoch-leistungsobjektiv (UPLFLN 100xO2PH, NA = 1,3; Olympus, Tokyo, Japan). (B) schematische Darstellung des Aufbaus eines Fluoreszenzmikroskops..........104

Abbildung 84: (A) Mit Pyranin versetze Seifenblase. (B) Beim Aufeinanderlegen zweier Seifenblasen, wird der fluoreszierende Farbstoff der oberen Seifenblase auf die untere Seifenblase übertragen (C). .105

Abbildung 85: Zeitabhängige fluoreszenzspektroskopische Messung der Intensität in Mischungen kationischer und anionischer Vesikellösungen. Dabei entspricht der schwarze Graph der ersten, der rote der zweiten Messung. .106

Abbildung 86: (A) Gequenchte Vesikelpopulationen mit kationischen und fluoreszenzmarkierten Lipiden fusionieren mit Vesikelpopulationen anionischer Vesikel, wodurch es zum Dequenching kommt. Entsprechend ist spektroskopisch ein Anstieg der Intensität zu beobachten (B).

Abbildung 87: (A) In ionischen Lösungen bilden sich elektrochemische Doppelschichten an den Phasengrenzen der Vesikelpopulationen, so dass die Wahrscheinlichkeit für Fusionen sinkt. Die Intensität während der spektroskopischen Messung nimmt wegen Bleaching-Effekten kontinuierlich ab. .108

Abbildung 88: Ausschnitte aus dem in Kooperation mit BEILSTEIN TV produzierten Film zur Forschung des SFB 803.

Abbildung 89: (A) Bändermodell des Botulinumtoxins (BoNT/A1). (B) Schematische Darstellung des Wirkmechanismus von Botulinumtoxin (in Anlehnung an RossETTO, PiRAZZini \& MONTECUCCO [313]).

Abbildung 90: Dimensionen des naturwissenschaftlichen Verständnisses, in Anlehnung an URHAHNE, KREMER \& MAYER [332], sowie WERNER \& KREMER [335].

Abbildung 91: Übersicht über die verschiedenen Arten naturwissenschaftlicher Literatur und ihrer Zielgruppen [327]

Abbildung 92: Beispiele zur Adaptierung von Abbildungen (oben) sowie Textausschnitten (unten) aus dem Originalartikel. Abbildung (A) ${ }^{[231]}$ zeigt sowohl die Struktur des Kanals VDAC-1 sowie den verwendeten Simulationsaufbau. In der APL werden Struktur und Aufbau schematisiert dargestellt (B). Der Textausschnitt (C) [231] zum Patch-ClampVerfahren enthält zahlreiche, für das Grundverständnis irrelevante, technische Angaben. In (D) wird das Verfahren in einer didaktisch reduzierten Form zur Messung eines Ionenstroms in Abhängigkeit eines angelegten Potentials dargestellt [327]. 135

Abbildung 93: Besucher_innenzahlen der IDEENEXPo[141] (links) und der HighLIGHTS DER PHYSIK ${ }^{[366]}$ (rechts).

Abbildung 94: Schematische Darstellung der Inhalte der einzelnen Fächer des EXPOneers „Vom Tensid zur Biomembran“ (links), sowie dessen Einsatz auf Bildungsmessen (rechts). 
Abbildung 95: Schematische Darstellung der Inhalte der einzelnen Fächer des EXPOneers „Gifte für die Schönheit“ (links) sowie dessen Einsatz auf Bildungsmessen (rechts).....151 Abbildung 96: Schematische Darstellung der Inhalte der einzelnen Fächer des EXPOneers „Rasterkraftmikroskopie“ (links), sowie dessen Einsatz auf Bildungsmessen (rechts). 151

Abbildung 97: SuS unterschiedlicher Altersgruppen führen unter Anleitung Experimente zur Fluorochromierung von Blüten (A), zur Exocytose (B), sowie zur Oberflächenminimierung durch $(C)$ und betrachten Pflanzenzellen unter dem Mikroskop (D) . .153

Abbildung 98: Messestand des SFB 803 bei der IdeenExpo 2017 [375].................................156

Abbildung 99: Evaluationsteilnehmer_innen (SuS) nach Jahrgang und Schulform..........159 Abbildung 100: Evaluationsteilnehmer_innen (Allgemeinheit) nach Alter und beruflichem Hintergrund. 163

Abbildung 101: Messestand bei den Highlights der Physik 2018. .165

Abbildung 102: Evaluationsteilnehmer_innen (SuS) nach Jahrgang und Schulform.......168 Abbildung 103: Evaluationsteilnehmer_innen (Allgemeinheit) nach Alter und beruflichem Hintergrund. 


\section{Tabellenverzeichnis}

Tabelle 1: Lernprozesse aus Subjekt- und Kontextperspektive nach MASCHKE \& STECHER [107].

Tabelle 2: Einteilung von SO-Aktivitäten in sechs SO-Bildungskontexte hinsichtlich (vorherrschenden) Lernprozessen aus Subjekt und Kontextperspektive

Tabelle 3: Vergleich verschiedener Evaluationsformen hinsichtlich der unterschiedlichen SO-Bildungskontexte 29

Tabelle 4: SO-Projekte des SFB 803 differenziert hinsichtlich der sechs SOBildungskontexte. 35

Tabelle 5: Nach Kompetenzen geordnete Lernziele aus dem niedersächsischen Kerncurriculum Biologie [181, 182], die bezüglich der SO-Aktivitäten des SFB 803 von Relevanz sind. EP = Einführungsphase, $\mathrm{QP}=$ Qualifikationsphase. 36

Tabelle 6: Nach Kompetenzen geordnete Lernziele aus dem niedersächsischen Kerncurriculum Chemie [181, 183], die bezüglich der SO-Aktivitäten des SFB 803 von Relevanz sind. EP = Einführungsphase, $\mathrm{QP}=$ Qualifikationsphase. 37

Tabelle 7: Inhalte der anhand der Leitfragen abgeleiteten Themenfelder. 38

Tabelle 8: Ansätze für die Versuchsreihe mit und ohne Zugabe von Zwiebelhaut. 62

Tabelle 9: Schlussfolgerungen bezüglich der Wirkung verschiedener Substanzen auf Lipidmembranen.

Tabelle 10: Mischungsverhältnisse zur Herstellung zweier unterschiedlicher Vesikelpopulationen .106

Tabelle 11: SO-Projekte des SFB 803 differenziert hinsichtlich der sechs SOBildungskontexte. Die hervorgehobenen Projekte werden nachfolgend vorgestellt. .....110

Tabelle 12: Verschiedene Niveaus der naturwissenschaftlichen Modellkompetenz unterteilt in fünf Teilkompetenzen UPMEIER ZU BELZEN \& KRÜGER [94]

Tabelle 13: Inhalte und korrespondierende Experimente der verschiedenen Sequenzen der Unterrichtseinheit Vom Tensid zur Biomembran.

Tabelle 14: Inhalte und korrespondierende Experimente der verschiedenen Sequenzen des Materialienpools mit dem Titel Vom Tensid zur Biomembran.

Tabelle 15: Vier-Säulen-Modell des gesellschaftskritisch-problemorientierten Chemieunterrichts nach EILKS [326]. 124

Tabelle 16: Inhalte und korrespondierende Experimente der verschiedenen Sequenzen des Schülerlabors Gifte für die Schönheit.

Tabelle 17: Ausgewählte Schritte des Adaptierungsprozesses von Originaltexten zu APL .

Tabelle 18: Differenzierung verschiedener Lehr-/Lernmodelle zur Einführung von APL hinsichtlich ihrer ihre Vorteile und Grenzen (aus dem Englischen übersetzt nach FALK \& YARDEN [353]).

Tabelle 19: Verknüpfung des modularen Fragenkatalogs mit dem niedersächsischen Kerncurriculum der gymnasialen Oberstufe im Fach Chemie [183]. Die kursiv gedruckten Lernziele entsprechen dem erhöhten Anforderungsniveau. $\mathrm{S}-\mathrm{T}=$ Stoff-Teilchen, $\mathrm{CR}=$ 
Chemische Reaktion, S-E = Struktur-Eigenschaft, E = Energie, EP = Einführungsphase, $\mathrm{QP}$ $=$ Qualifikationsphase.

Tabelle 20: Verteilung der Antworten von SuS bzgl. der zielgruppenspezifischen Frage zur wasserabweisenden Eigenschaft von Teilchen.

Tabelle 21: Verteilung der Antworten von SuS bzgl. der zielgruppenspezifischen Frage zu Tensidmolekülen und dem Tyndall-Effekt. 158

Tabelle 22: Verteilung der Antworten von SuS bzgl. der zielgruppenspezifischen Frage zu Molekülgeometrien beim Membrantransport. Die Beantwortung erfolgte anhand einer beigefügten Abbildung.

Tabelle 23: Verteilung der Antworten von Lehrkräften bzgl. der zielgruppenspezifischen Frage zur unterrichtlichen Behandlung des Themas Biomembranen. 161

Tabelle 24: Verteilung der Antworten von Lehrkräften bzgl. der zielgruppenspezifischen Frage zum Interesse an Informationen zur aktuellen Membranforschung. 162

Tabelle 25: Verteilung der Antworten der Allgemeinheit bzgl. der zielgruppenspezifischen Fragen zur Relevanz von Forschung für die Gesellschaft bzw. die persönliche Zukunft. .163

Tabelle 26: Verteilung der Antworten der Allgemeinheit bzgl. der zielgruppenspezifischen Frage zur Darstellung wissenschaftlicher Erkenntnisse in den Medien. . .164

Tabelle 27: Verteilung der Antworten von SuS bzgl. der Frage zur Einordnung Botox. Eine Mehrfachnennung war möglich.

Tabelle 28: Verteilung der Antworten von SuS bzgl. der zielgruppenspezifischen Fragen zur Klassifizierung von Botox, der Wirkung von Acetylcholin und dem Modellversuche aus dem EXPOneer Gifte für die Schönheit. 166

Tabelle 29: Verteilung der Antworten von SuS bzgl. der zielgruppenspezifischen Frage zur Wirkung von Botox.

Tabelle 30: Verteilung der Antworten von Lehrkräften bzgl. der Frage zur Einordnung Botox. Eine Mehrfachnennung war möglich. 168

Tabelle 31: Verteilung der Antworten von Lehrkräften bzgl. der zielgruppenspezifischen Frage zur unterrichtlichen Behandlung verschiedener Forschungsmethoden.

Tabelle 32: Verteilung der Antworten der Allgemeinheit bzgl. der Frage zur Einordnung Botox. Eine Mehrfachnennung war möglich. 169

Tabelle 33: Verteilung der Antworten der Allgemeinheit bzgl. der zielgruppenspezifischen Fragen zur Verwendung von Botox.

Tabelle 34: Verteilung der Antworten der Allgemeinheit bzgl. der zielgruppenspezifischen Fragen zur Relevanz von Forschung für die Gesellschaft bzw. die persönliche Zukunft. 


\section{Eidesstattliche Erklärung}

Hiermit bestätige ich, dass die Arbeit mit dem Titel

ENTWICKLUNG UND EVALUATION VON KONZEPTEN UND FORMATEN ZUM WISSENSTRANSFER VON DER FORSCHUNG IN DIE SCHULE UND ÖFFENTLICHKEIT - AM BEISPIEL DES SFB 803

von mir selbstständig angefertigt wurde und dass die Stellen der Arbeit, die in wesentlichen Teilen anderen Werken entnommen sind, mit genauer Angabe der Quelle gekennzeichnet sind. Weiterhin bestätige ich, dass benutzte Hilfsmittel an entsprechender Stelle von mir angegeben wurden. Die Dissertation ist weder in Teilen noch in ihrer Gesamtheit einer anderen wissenschaftlichen Hochschule zur Begutachtung in einem Promotionsverfahren vorgelegt worden.

Göttingen, den 24.08.2020

Elena von Hoff 